\title{
Comparative gene expression to study the developmental basis of organ diversification.
}

\author{
Dissertation \\ for the award of the degree \\ Doctor rerum naturalium (Dr.rer.nat.)
}

\begin{abstract}
Division of Mathematics and Natural Sciences of the Georg-August-Universität Göttingen within the doctoral program Genes and Development of the Georg-August University School of Science (GAUSS)
\end{abstract}

Submitted by

Elisa Buchberger

from Vils, Tirol, Austria

Göttingen, July 2019 


\section{Thesis Advisory Committee}

\section{Dr. Nico Posnien (Supervisor)}

Dep. of Developmental Biology, Johann-Friedrich-Blumenbach-Institute of Zoology and Anthropology, Georg-August-University Göttingen

Prof. Daniel J. Jackson

Dep. of Geobiology, Geoscience Centre, Georg-August-University Göttingen

\section{Prof. Steven A. Johnsen}

Dep. of Gastroenterology and Hepatology; Mayo Clinic - Rochester, Minnesota

\section{Members of the Examination Board}

First Reviewer: Dr. Nico Posnien

Dep. of Developmental Biology, Johann-Friedrich-Blumenbach-Institute of Zoology and Anthropology, Georg-August-University Göttingen

Second Reviewer: Prof. Daniel J. Jackson

Dep. of Geobiology, Geoscience Centre, Georg-August-University Göttingen

\section{Extended Examination Board}

Prof. Steven A. Johnsen

Dep. of Gastroenterology and Hepatology; Mayo Clinic - Rochester, Minnesota

\section{Prof. Christoph Bleidorn}

Dep. of Animal Evolution and Biodiversity, Johann-Friedrich-Blumenbach-Institute of Zoology and Anthropology, Georg-August-University Göttingen

\section{Prof. Argyris Papantonis}

Institute for Pathology, University Medical Center Göttingen

Dr. Gerd Vorbrüggen

RG Molecular Cell Dynamics, Max Planck Institute for Biophysical Chemistry

Date of oral examination: September $3^{\text {rd }}, 2019$ 


\section{Declaration}

I herewith declare, that I prepared the Dissertation 'Comparative gene expression to study the developmental basis of organ diversification' on my own and with no other sources and aids than quoted.

Elisa Buchberger 
Prudens interrogatio quasi dimidium sapientiae.

(Francis Bacon) 


\section{Acknowledgements}

First and foremost, I would like to thank Dr. Nico Posnien for giving me the opportunity to work on this exciting project, but especially for his optimism and trust in me and my work. I would like to thank you for always taking my opinion serious, and supporting me in every way possible, be it by scientific discussions, encouraging me to attend courses and conferences or giving me the freedom to decide in which direction the project is going. I consider myself extremely lucky to be a member of the Posnien Lab.

I thank the members of my Thesis Committee, Prof. Daniel Jackson and Prof. Stephen Johnsen. Your interest in my work and helpful discussions made the TAC meetings something I was always looking forward to. I would also like to thank Prof. Christoph Bleidorn, Prof. Argyris Papantonis and Dr. Gerd Vorbrüggen for agreeing to serve in my Extended Examination Board. I would like to thank Prof. Ernst Wimmer for hosting me in his department for more than four years. Him, Prof. Gregor Bucher, Prof. Sigrid Hoyer-Fender, Dr. Gerd Vorbrüggen and Dr. Ufuk Günesdogan I would like to thank for the relaxed atmosphere in the department and their scientific input and advice.

A special thanks goes to Max Farnworth, for countless coffee and fruit breaks, for many deep discussions and silly jokes (and GIFs) and for always being there when needed. The PhDjourney, especially the last months, would have been so much harder and less fun without you as a friend.

I am extremely grateful to Dr. Micael Reis. Without your constant scientific and non-scientific advice this work wouldn't have turned out the way it has. Every PhD student can only wish for having such a dedicated and skilled post-doc and friend by his or her side. I learned a lot from you!

I thank Amel Chtioui, Ting-Hsuan Lu and Gordon Wiegleb for being such joyful lab mates. I would like to thank my students, especially Anıl Bilen, Sanem Ayaz, Cristina Matas de las Heras and Armin Nikšić. The times when we worked together on the project were the times when it made the biggest progress.

I would like to thank all the people in the Department of Developmental Biology, including PhD colleagues, post-docs and technicians who made working there such a fun experience. I am 
especially grateful to Felix Quade, Beate Preitz and Marita Büscher for always helping when help was needed. I highly appreciate the constant support from Merle Eggers, Birgit Rossi and Bettina Hucke. Thanks to Hassan Mutasim Mohammed Ahmed for our chats not only during the weekends, Bibi Atika for always asking how I am and so many others in the lab whom I cannot all mention here. I would also like to thank Montserrat Torres-Oliva for all the help when I joined the lab during my Masters. And thanks to Max, Peter Kitzmann, Salim Ansari and Nico for our sports sessions.

I thank our collaborators Prof. Alistair McGregor, Prof. Fernando Casares, Dr. Montserrat Torres-Oliva and Dr. Isabel Almudi, for sharing their data and their valuable scientific input. I thank Dr. Barbora Konopová for our fruitful collaboration on the Schistocerca project. Also, this work would not have been possible without the bioinformatics community and all the researchers, that publish their codes and programs open access.

I am very grateful to the team of the GGNB office. Their ongoing support with all organizational things makes everything much easier. I also would like to acknowledge the opportunity of being a member of the GGNB Times Newsletter editorial board for three years.

I would like to thank Maria for being the best flat mate ever. Thanks for the countless chats at the kitchen table or on the balcony but mostly for becoming my family here in Göttingen. And thank you for proofreading the thesis! I thank the rest of 'Der Harte Kern' - Lisa and Tina, and Jule for being by my side since my beginnings here in Germany. I'm very thankful for all the nice memories we share. A big thanks goes to Britta for the many hours of Volleyball that got me out of the lab and library. And to Christian - Thank you for all the wonderful distractions and for making the last months - despite writing the thesis - so special.

Agnes, Theresa, Laura and Alexandra, I thank you so much for your long-standing friendship, for making Austria a home to me and for your genuine interest in what life will bring next for me.

Meiner Familie gebührt der größte Dank. David - Ich bin unglaublich froh einen Bruder an meiner Seite zu haben und ich bin extrem stolz auf dich! Mama und Papa - eure Unterstützung und die Möglichkeit zu studieren hat diese Arbeit erst möglich gemacht. Danke für euer Vertrauen in meine Entscheidungen, dass ihr jede einzelne davon unterstützt und das Wissen, dass ich immer heim kommen kann. Diese Arbeit ist euch gewidmet. 


\section{Table of Contents}

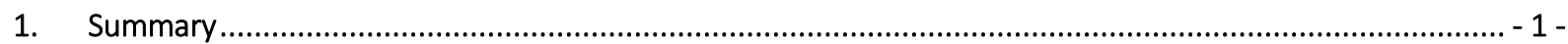

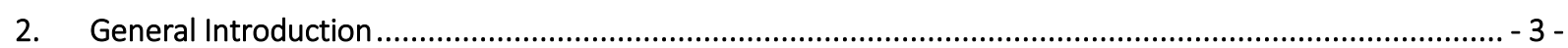

2.1. Development, function and evolution of body structures are governed by tightly regulated gene

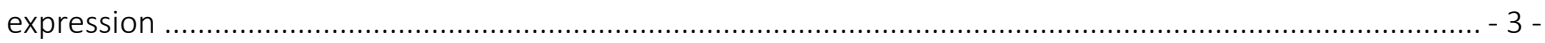

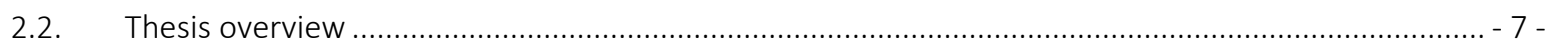

2.3. Comparative gene expression studies in development ................................................................ 8 -

2.3.1. Schistocerca gregaria as a model to study the role of pleuropodia in insect embryogenesis. - 10 -

2.4. Comparative gene expression studies in phenotypic evolution ...................................................... - 13 -

2.4.1. Drosophila melanogaster as a model species to study head size and shape evolution............ - 15 -

2.4.2. Mechanisms underlying context dependent gene expression divergence ................................. 19 -

3. Chapter I - Transcriptomics supports that pleuropodia of insect embryos function in degradation of

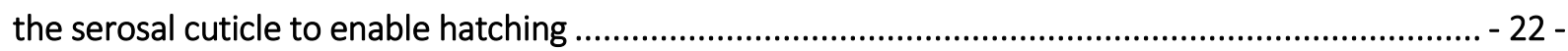

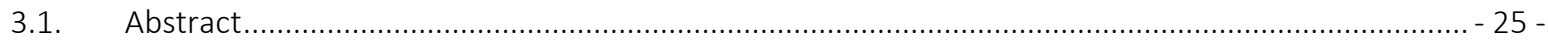

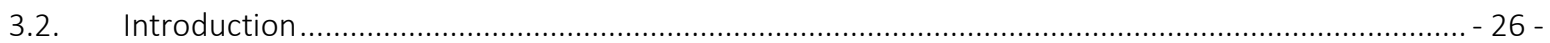

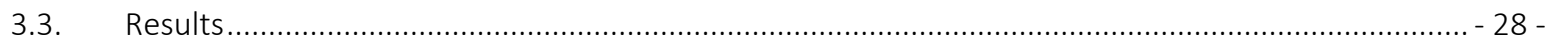

3.3.1. Development of pleuropodia in the course of Schistocerca embryogenesis ............................. 28 -

3.3.2. Generation of a comparative RNA-seq dataset from developing pleuropodia and legs of

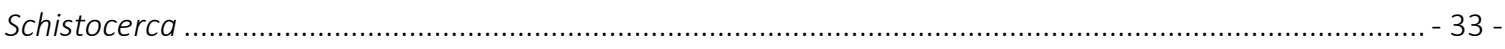

3.3.3. Identification of genes upregulated in the intensively secreting pleuropodia ....................... - 35 -

3.3.4. The pleuropodia upregulate genes for cuticular chitin degrading enzymes ......................... - 39 -

3.3.5. Pleuropodia upregulate transcripts for some proteases that could digest a cuticle ............... - 42 -

3.3.6. Pleuropodia are enriched in transcripts for immunity-related proteins................................. - 44 -

3.3.7. The pleuropodia do not upregulate the pathway for ecdysone biosynthesis ......................... - 46 -

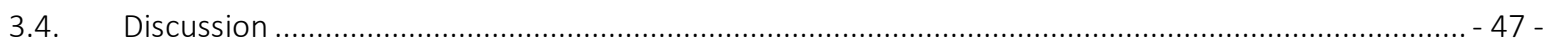

3.4.1. Pleuropodia of Schistocerca express genes for the "hatching enzyme" ............................... 47 -

3.4.2. Pleuropodia in some other insects could secrete the "hatching enzyme" and their function may

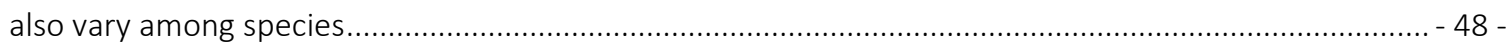

3.4.3. The pleuropodia of Schistocerca are enriched in transcripts for enzymes functioning in immunity - 49 -

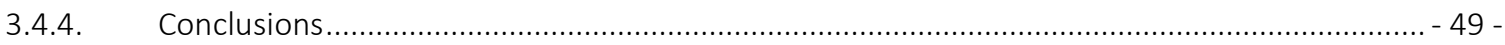

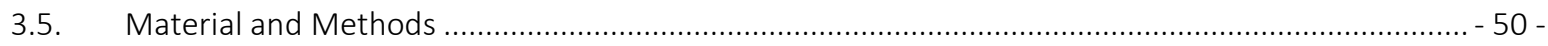

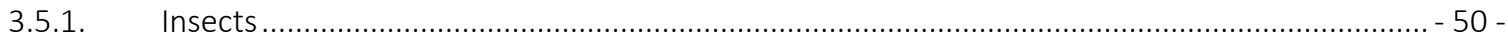

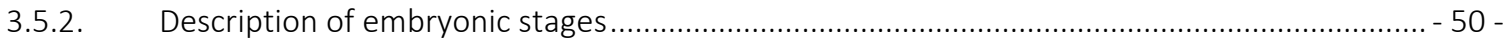

3.5.3. Immunohistochemistry on paraffin sections ............................................................... 50

3.5.4. Transmission (TEM) and scanning (SEM) electron microscopy ............................................ 51 - 
3.5.5. Preparation of the reference transcriptome …............................................................. 51 -

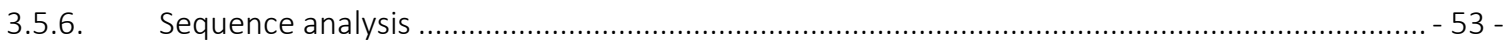

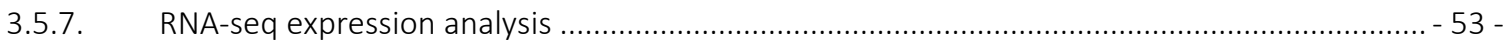

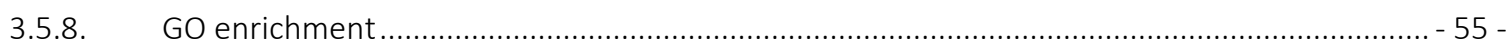

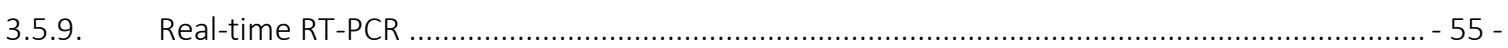

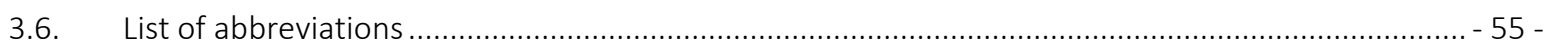

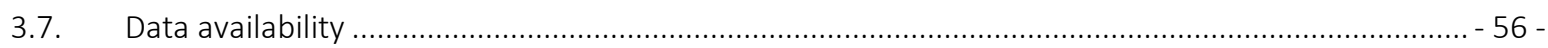

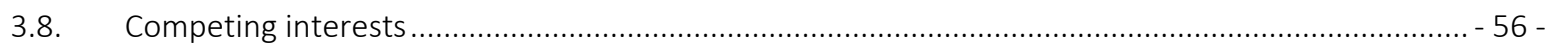

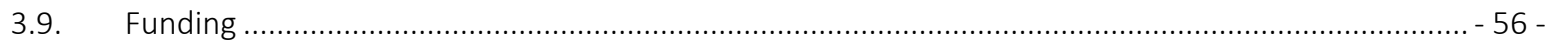

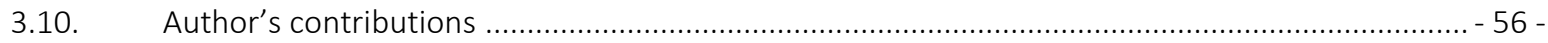

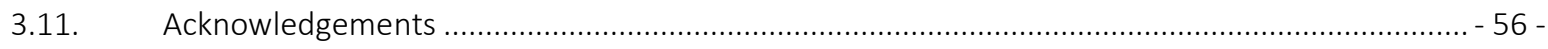

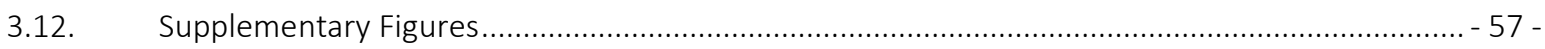

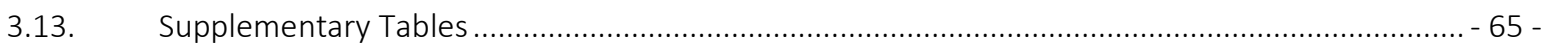

4. Chapter II - Variation in a pleiotropic regulatory module drives evolution of head shape and eye size in Drosophila ................................................................................................................ 110 -

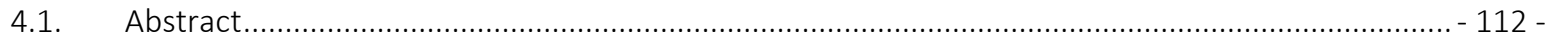

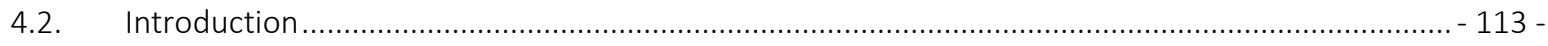

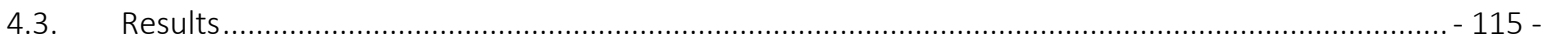

4.3.1. Drosophila melanogaster and D. mauritiana exhibit differences in dorsal head shape .........- 115 -

4.3.2. Difference in the transcriptomics landscape recapitulate observed morphological differences between D. melanogaster and D. mauritiana ........................................................................... 118

4.3.3. Central transcription factors regulate differentially expressed genes ................................... 119 -

4.3.4. Pannier regulates genes that are differentially expressed between $D$. melanogaster and $D$.

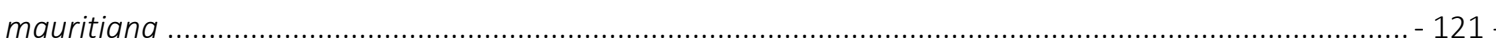

4.3.5. Pnr activates and represses target genes in the eye-antennal disc .................................... 124 -

4.3.6. Pannier and its co-repressor U-shaped participate in the same regulatory network during eyeand head development in Drosophila

$-126-$

4.3.7. Overexpression of pannier phenocopies aspects of the differences observed between $D$.

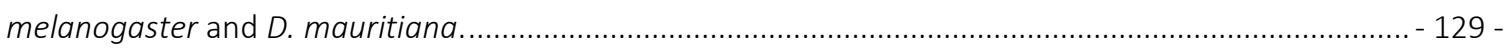

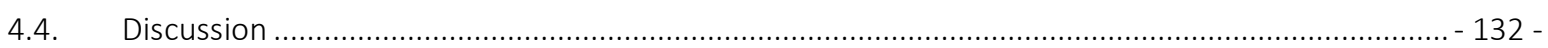

4.4.1. A developmental model for natural variation in head shape and eye size............................ - 132 -

4.4.2. Pnr and Ush represent a functionally linked pleiotropic module in the GRN underlying head and

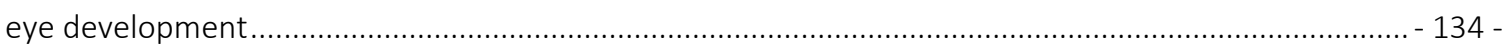

4.4.3. GRN rewiring facilitates natural variation in pleiotropic developmental factors ................... - 136 -

4.4.4. Evolution of GRNs and implications for convergent evolution of head shape and eye size... - 137 -

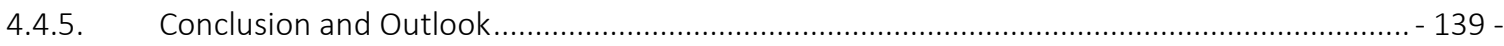

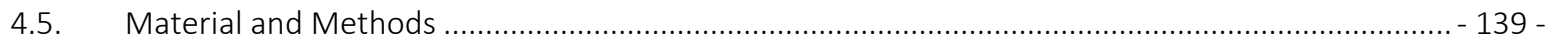

4.5.1. Generation of the transcriptomic dataset ............................................................. $139-$ 
4.5.2. Generation of the ATAC-seq dataset .................................................................... $141-$

4.5.3. Bioinformatics processes of the ATAC-seq dataset..................................................... 142 -

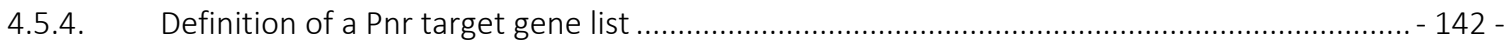

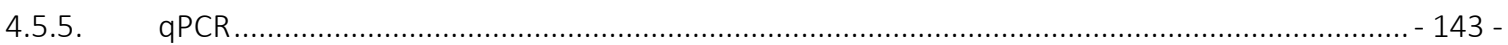

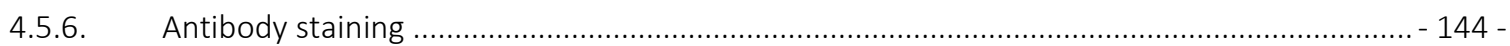

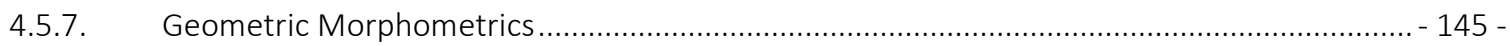

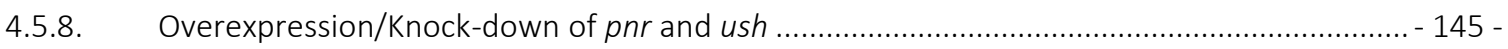

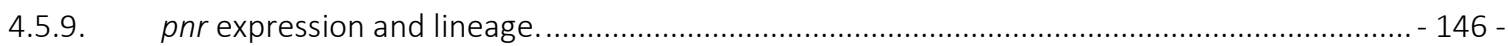

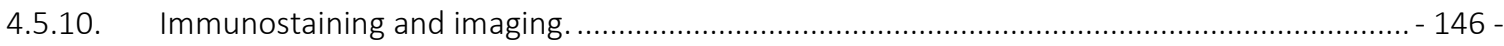

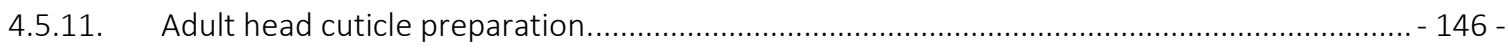

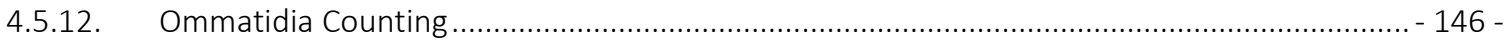

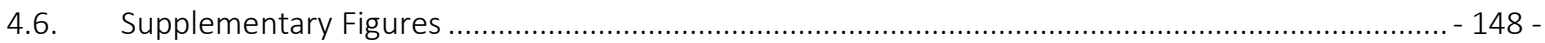

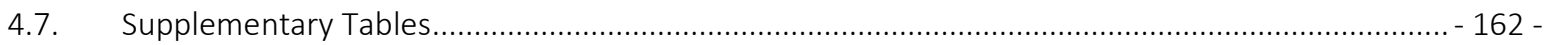

5. Chapter III - Regulatory Divergence in the Drosophila melanogaster subgroup ....................... - 169 -

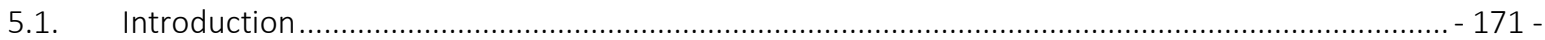

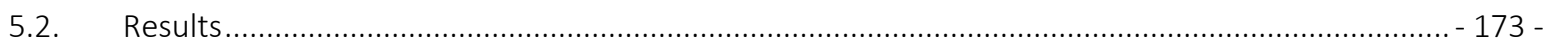

5.2.1. Regulatory Divergence in the D. melanogaster subgroup ............................................ $173-$

5.2.2. A comparative ATAC-seq dataset of three closely related Drosophila species ...................... - 176 -

5.2.3. Genes with species specific regulatory regions are more often regulated in cis ................... - 178 -

5.2.4. Regulatory regions of genes, diverging in cis, show a higher sequence divergence............... 180 -

5.2.5. Regulatory divergence in transcription factors .............................................................. $182-$

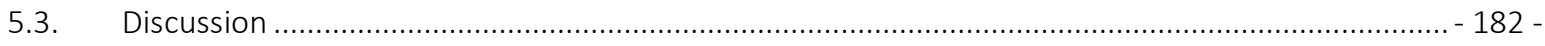

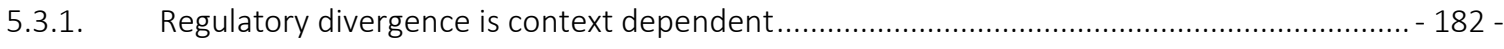

5.3.2. cis- regulatory divergence is due to changes in chromatin accessibility and sequence divergence$185-$

5.3.3. Compensation and conservation of gene expression ................................................... $187-$

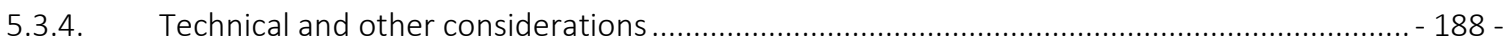

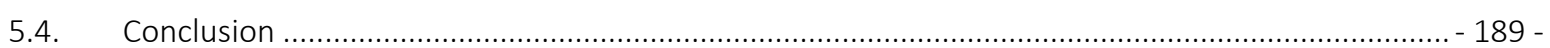

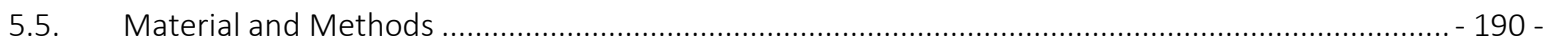

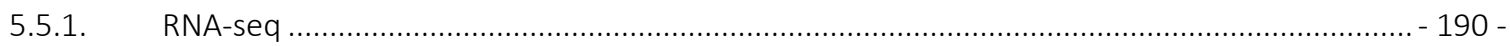

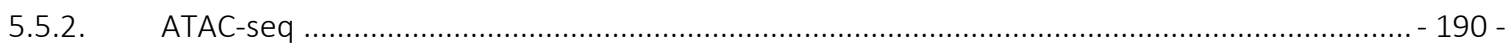

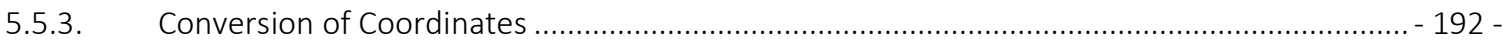

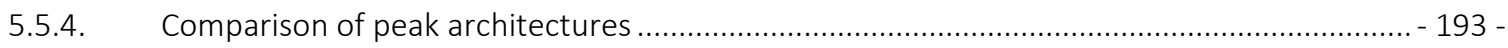

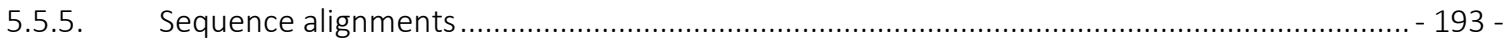

5.5.6. Overlap with DrolD database .......................................................................................... $194-$

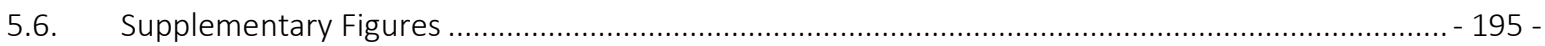

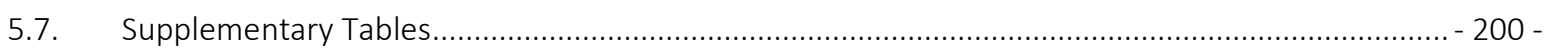




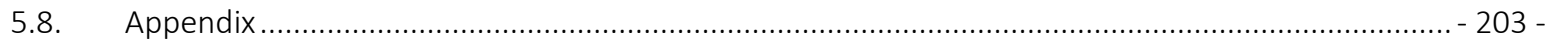

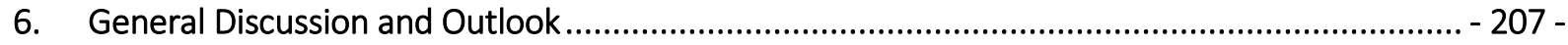

6.1. Integration of different datasets in comparative biological studies......................................... 207 -

6.2. Comparative gene expression studies and gene regulatory networks in development ................ $210-$

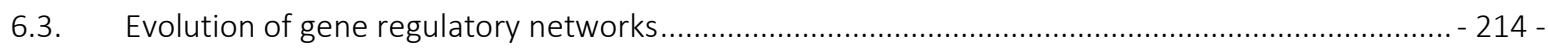

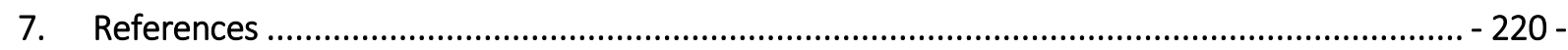




\section{List of Abbreviations}

\begin{tabular}{|c|c|}
\hline (qRT) PCR & (quantitative real time) polymerase chain reaction \\
\hline $3 \mathrm{D}$ & 3-dimentional \\
\hline AEL & after egg laying \\
\hline agouti-related peptide2 & agrp2 \\
\hline Ance & Angiotensin-converting enzyme \\
\hline ASE & allele specific expression \\
\hline ATAC-seq & Assay for Transposase-Accessible Chromatin \\
\hline ato & atonal \\
\hline BMP4 & bone morphogenetic protein 4 \\
\hline bp & base pair \\
\hline CaM & calmodulin \\
\hline CDS & coding sequence \\
\hline ChIP-seq & Chromatin Immuno Precipitation - sequencing \\
\hline $\mathrm{CHT}$ & chitinase \\
\hline CpG & C-phosphate-G \\
\hline $\mathrm{Ct}$ & Cut \\
\hline DEG & differentially expressed gene \\
\hline DF & dorsal frons \\
\hline DHS & DNase hyperactive sites \\
\hline$d l$ & dorsal \\
\hline$d l l$ & distal-less \\
\hline DNA & deoxyribonucleic acid \\
\hline$d p p / D p p$ & decapentaplegic/Decapentaplegic \\
\hline EC1/EC2 & first and second embryonic cuticle \\
\hline EcR & Ecdysone receptor \\
\hline EGFR & Epidermal growth factor receptor \\
\hline Evo-Devo & Evolutionary Developmental Biology \\
\hline ey/Ey & eyeless/Eyeless \\
\hline eyg & eyegone \\
\hline F1 hybrid & filial 1 hybrid \\
\hline FAIRE-seq & Formaldehyde-Assisted Isolation of Regulatory Elements \\
\hline GAL & galactose \\
\hline GO & gene ontology \\
\hline GRN & gene regulatory network \\
\hline GWAS & genome-wide association study \\
\hline Hth & Homothorax \\
\hline i.e. & id est \\
\hline in. prep. & in preparation \\
\hline JNK & c-Jun $\mathrm{N}$-terminal kinases \\
\hline Jra & Jun-related antigen \\
\hline LEG & hind legs \\
\hline IncRNA & long non-coding RNA \\
\hline Mc1r & Melanocyte-stimulating hormone receptor \\
\hline
\end{tabular}




\begin{tabular}{|c|c|}
\hline Mef2 & Myocyte enhancer factor 2 \\
\hline MF & morphogenetic furrow (OR moulting fluid in Chapter I) \\
\hline $\operatorname{miR}-92 a$ & micro RNA $92 a$ \\
\hline miRNA & micro RNA \\
\hline mRNA & messenger RNA \\
\hline NAG & $\beta$-N-acetyl-hexosaminidase \\
\hline Nej & Nejire \\
\hline NGS & next generation sequencing \\
\hline OC & orbital cuticle \\
\hline$O C$ & ocelliless \\
\hline Pax6 & Paired box protein 6 \\
\hline Pc & Polycob \\
\hline PC & principal component \\
\hline PCA & principal component analysis \\
\hline Pitx1 & Paired Like Homeodomain 1 \\
\hline PLP & pleuropodium \\
\hline pMad & phosphorylated Mothers against dpp \\
\hline pnr/Pnr & pannier/Pannier \\
\hline QTL & quantitative trait locus \\
\hline RNA & ribonucleic acid \\
\hline RNAi & RNA interference \\
\hline RPKM & Reads Per Kilobase Million \\
\hline SC & serosal cuticle \\
\hline sd & scalloped \\
\hline SEM & scanning electron microscopy \\
\hline Sfmbt & $\begin{array}{l}\text { Sex comb on midleg-related gene containing four mbt } \\
\text { domains }\end{array}$ \\
\hline SNP & single nucleotide polymorphism \\
\hline So & Sine Oculis \\
\hline$s v b / S v b$ & shavenbaby/Shavenbaby \\
\hline TEM & transmission electron microscopy \\
\hline TF & transcription factor \\
\hline Tin & Tinman \\
\hline $\operatorname{trn}$ & tartan \\
\hline tsh & teashirt \\
\hline TSS & transcription start site \\
\hline Ttk & Tramtrack \\
\hline UAS & upstream activation sequence \\
\hline$w g / W g$ & wingless/Wingless \\
\hline
\end{tabular}




\section{Summary}

The striking diversity in adult morphologies is the result of millions of years of adaptation of species to different environments and habitats. Fixed changes in populations or species are the consequence of mutations in the genome and thus in the developmental programs of body plans, their structures and organs. Years of studies in the field of 'Evo-Devo' have revealed that there exists only a limited number of genes, governing basic developmental processes, and that these so-called 'toolkit genes' are highly conserved even between distantly related species. It is nowadays accepted, that morphological diversification is often driven by changes in gene expression and subsequently the interplay of gene products. Since the expression of genes is tightly controlled in a spatiotemporal manner on several molecular levels, also the wiring of such gene regulatory networks is highly context dependent. Therefore, single cells, tissues and organs are characterized by a unique set of expressed transcripts and proteins which are specifically intertwined and govern their developmental programs. The advent of high throughput sequencing techniques provides nowadays the opportunity to analyze the transcriptome of developing structures in a highly specific manner and opens the possibility to understand how these toolkit genes are differentially used and rewired in different developmental and evolutionary contexts. In Chapter I of this thesis, I studied gene expression in a developmental context, using the emerging model species Schistocerca gregaria to understand the development and function of pleuropodia - small glandular structures forming on the first abdominal segment of many insect embryos. In Chapter II, I used a comparative transcriptomic dataset of developing eye-antennal discs in two closely related species of the Drosophila melanogaster subgroup to study the molecular basis of evolution of complex traits. The size and shape of the compound eyes and head structures vary extensively between $D$. melanogaster and D. mauritiana and show a typical trade-off between eye-size and head width. I could show that differential expression of pannier (pnr) underlies natural variation of eye size, ommatidia number and head width between these two species. In Chapter III, I combined an allele specific expression dataset of F1 hybrids between D. melanogaster vs. D. mauritiana and D. simulans vs. D. mauritiana with a newly generated comparative ATAC-seq dataset, to study gene expression divergence and sought to recapitulate the observed patterns in terms of nucleotide turnover and accessibility of regulatory regions. In summary, this works shows that 
the combination of methods and various datasets allows to gain major insights into development, function, and evolution of morphological traits. 


\section{General Introduction}

\subsection{Development, function and evolution of body structures are} governed by tightly regulated gene expression

The information how we and all other organisms develop, function and interact with our environment is encoded in our DNA which lies tightly packed as chromosomes in the nuclei of each of our cells (Figure 1A). During a process called transcription the genetic information encoded in genes is transcribed into messenger RNA (mRNA). The mRNA provides the template for the translational machinery, which translates the mRNA into amino acid sequences and eventually functional proteins (Figure 1C).

A typical eukaryotic gene locus is composed of several elements. The protein information is encoded in one or more exons, which together form the coding region (CDS), and are separated by introns. Transcription is initiated by the assembly of a basal transcription machinery at the promoter region, mostly located $5^{\prime}$ upstream, close to the transcription start site (TSS) of the respective gene. This protein complex recruits the RNA polymerase that synthesizes the pre-mRNA. Where, when and how strong a gene is transcribed is though in the first place controlled by regulatory intronic or intergenic DNA regions, so called enhancers or cis-regulatory regions ((Davidson, 2001; Wray, 2003), Figure 1C). Therefore the respective genomic regions must be depleted of nucleosomes, which otherwise confer tight DNA packing. Hence, regulatory sequences must be accessible for transcription factors (TFs) that physically interact with the DNA by recognizing sequence-specific TF-binding motifs. This in turn leads to recruitment of additional TFs and co-factors. Enhancer sequences, are often of modular nature, meaning that several, locally separated regulatory regions modulate the expression of a single gene (e.g. (Adachi et al., 2003; Davidson, 2001; Stanojevic et al., 1991)). The advances in high throughput sequencing methods nowadays allow to reliably define the location of open chromatin regions in the genome. Approaches like ChIP-seq (Johnson et al., 2007; Robertson et al., 2007), FAIRE-seq (Giresi et al., 2007) or ATAC-seq (Buenrostro et al., 2013) are frequently used to define putative regulatory regions and allow to link them to gene expression, if combined with other methods like RNA-seq (Wang et al., 2009). However, how exactly enhancers carry out their regulatory function is not yet completely understood and different models of enhancer function have been proposed (Buffry et al., 2016). Chromosome 
conformation capture methods combined with high throughput sequencing such as $\mathrm{Hi}-\mathrm{C}$ (van Berkum et al., 2010) allow resolving the 3-dimensional chromatin states and are used to study how distantly located regulatory sequences exert their regulatory function (Furlong and Levine, 2018).

Each cell type of an organism is characterized by a certain combination of expressed genes and the defined interplay of their gene products. Since different cell types have to carry out distinct functions for a long period of time (depending on the life span of an organism), this function is ensured by tissue or even cell-specific gene expression (Lübbe and Schaffner, 1985). Traditional methods to quantify the expression levels of single genes include quantitative realtime PCR (qRT PCR,(Bustin, 2000)) and Northern Blotting (Alwine et al., 1977). The spatial distribution of transcripts can be studied by in-situ hybridization (Pardue and Gall, 1969). Nevertheless, only the advent of next generation sequencing (NGS) like RNA-seq facilitated the efficient genome wide assessment of gene expression by quantifying the complete mRNA content that is expressed at a certain time point in a cell or tissue (Wang et al., 2009). Disturbance of gene expression, and thus function, eventually leads to disease or death of the respective organism (e.g. (Dermitzakis, 2008; Emilsson et al., 2008)). For instance, in humans, the formation and progression of cancer is tightly linked to aberrant gene expression and regulation (e.g. (Liang and Pardee, 2003)). Therefore, the expression of genes has to be under tight spatial and temporal regulation, which is ensured on several molecular and cellular levels (Figure 1C). The accessibility of regulatory regions for instance is highly dependent on the tissue and developmental stage (e.g. Bozek et al., 2019). Furthermore, biochemical modifications of DNA (methylation) and histone proteins (methylation, acetylation, phosphorylation and many others) influence gene expression (Kouzarides, 2007; Lawrence et al., 2016) (Figure 1C). In Drosophila dosage compensation relies for example on the acetylation of lysine 16 residues on the $\mathrm{H} 4$ histones of the $\mathrm{X}$-chromosome, allowing the increase of transcription in males by decondensation of the chromosomes (e.g. (Akhtar and Becker, 2000; Turner et al., 1992)). Additionally, methylation of Cytosines has been linked to repression of transcription (reviewed in Bird and Wolffe, 1999). In vertebrates for example, promoter or enhancer regions, often containing so-called CpG-islands are usually depleted of methylated CpGs and hyperacetylated histones, marking actively transcribed genes. 
The spatially and temporally restricted availability of TFs and co-factors that bind to accessible regulatory regions further represents a level of context specific gene regulation. One example of transcriptional co-regulation, which will be introduced in Chapter II in more detail can be found in the developing Drosophila wing disc. Pannier (Pnr), a GATA transcription factor which usually activates expression of its target genes, interacts in a spatially defined manner with U-shaped (Ush) (Fromental-Ramain et al., 2010, 2008). The resulting heterodimer loses the activating role of Pnr but acquires a repressing function (Haenlin et al., 1997). Also, posttranscriptional processes can modulate gene expression in a context dependent manner. For instance, the context dependent expression of small regulatory RNA molecules such as microRNAs (miRNAs) modifies the stability of mRNA or the efficiency with which an mRNA molecule is translated (reviewed in Bartel, 2018; Kittelmann and McGregor, 2019). Also, for long-non-coding RNAs (IncRNAs) it has been established that they are transcribed in a highly spatially and temporally controlled manner and are suggested to influence for example the expression of genes in their close genomic vicinity (Kopp and Mendell, 2018; Ponting et al., 2009; Sarropoulos et al., 2019). These are only few of the many examples that show that tissue and stage specific gene expression is orchestrated on different levels of the gene regulation machinery.
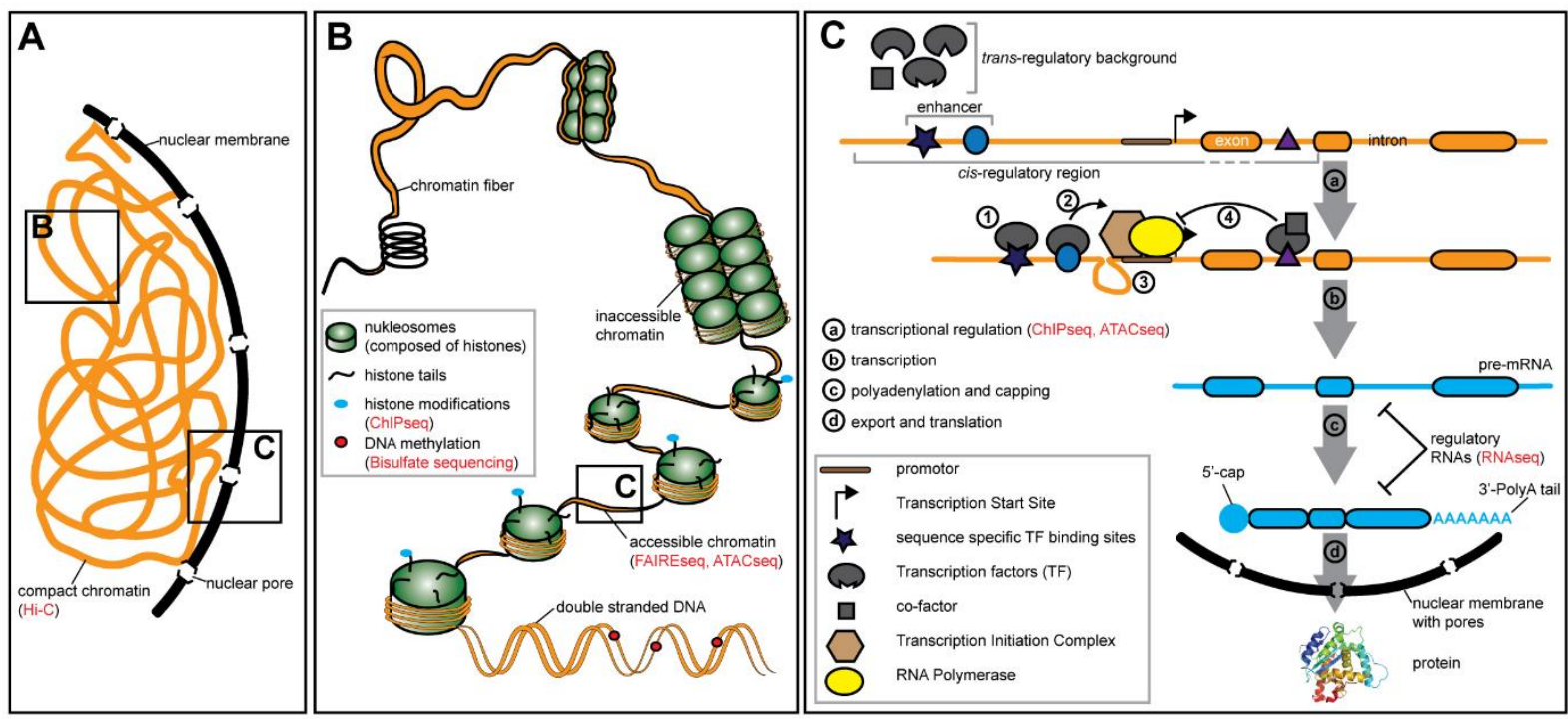

Figure 1. Gene expression is tightly controlled. A. The DNA lies heavily packed as so-called chromatin in the nuclei of eukaryotic cells. B. Formation of chromatin is carried out by wrapping DNA around histones, which are composed of nucleosomes. Regions of loose packing, characterized by nucleosome depletion, are in general more accessible for transcription factors (TFs) and loci in these regions are mostly actively transcribed. In contrast, tightly packed DNA is inaccessible to regulatory proteins and subsequent transcription. Biochemical modification of histones or cytosines provide another level of gene regulation. C. A eukaryotic gene locus is composed of one or more exons, which make up the CDS of the gene. Regulatory regions are located in introns, separating the exons, or in intergenic regions. Transcription is initiated at the promoter region, $5^{\prime}$ upstream of 
the transcription start site (TSS), and TFs bound to enhancer regions further regulate gene expression. The figure is taken from (Buchberger et al., 2019).

While gene expression has to be tightly controlled to ensure proper organ development and function, many evolutionary studies revealed that divergence in gene expression is a key driver for phenotypic evolution (Alvarez et al., 2015; Carroll, 2005; King and Wilson, 1975; Todd et al., 2016). One of the most classical examples, where differences in morphologies were associated with differential gene expression is the work of Abzhanov and colleagues, who linked higher expression of bone morphogenetic protein 4 (BMP4) to wide beak morphology in ground finches (Abzhanov, 2004), whereas development of long beaks of cactus finches is mainly driven by higher levels of calmodulin (CaM) (Abzhanov et al., 2006). In East African cichlid fish it has recently been revealed, that changes in the expression of the agrp2 gene, defines the pigmentation pattern of different radiations (Kratochwil et al., 2018). Similarly, adaptive changes in abdominal pigmentation of African Drosophila populations are caused by expression variation of the ebony gene (Pool and Aquadro, 2007; Rebeiz et al., 2009). Changes in gene expression levels could be due to changes in a gene's own regulatory regions (cis-regulatory divergence) or due to divergence of upstream regulators, such as transcription factors or regulatory RNAs (trans-regulatory divergence) (Cowles et al., 2002; Wittkopp et al., 2004). For many simple traits, including pigmentation, trichome formation or loss of specific skeletal structures, it has been shown that the causative underlying mutations are often located in the non-coding, regulatory regions of the locus (e.g. Chan et al., 2010; McGregor et al., 2007; Prud'homme et al., 2006; Rebeiz et al., 2009), which would eventually affect the expression of the respective gene. If this also applies to quantitative, complex traits like size and shape of organs and structures remains to be established.

In summary, gene expression is a central biological process that transfers the information stored in the genome of an organism to its development, function and evolution. 


\subsection{Thesis overview}

During my PhD work I applied comparative gene expression studies to gain new insights into:

I. developmental processes and organ function,

II. the evolution of complex morphological traits and

III. molecular mechanisms underlying gene expression divergence.

Chapter I 'Transcriptomics supports that pleuropodia of insect embryos function in degradation of the serosal cuticle to enable hatching' resulted from a collaboration with Dr. Barbora Konopová and Dr. Alastair Crisp. Applying comparative RNA-seq, we provide strong evidence that pleuropodia in the locust Schistocerca gregaria indeed participate directly in the digestion of the serosal cuticle during embryogenesis and reveal that they also might take over a role in insect immunity.

In Chapter II 'Variation in a pleiotropic regulatory module drives evolution of head shape and eye size in Drosophila' I studied differences in gene expression dynamics between $D$. melanogaster and D. mauritiana and show that differential expression of the conserved transcription factor Pnr underlies variation in head shape and ommatidia number between the two species. Additionally, I found that the co-factor of Pnr, Ush is expressed and functional in the developing eye-antennal discs of Drosophila and therefore represents a new player in the eye and head GRN.

For Chapter III 'Regulatory divergence in the Drosophila melanogaster subgroup' I combined previous knowledge about regulatory divergence in three species of the $D$. melanogaster subgroup with a newly generated ATAC-seq dataset to study if patterns of cisand trans-regulatory divergence can be recapitulated on the basis of open and accessible chromatin regions.

In the following paragraphs I will provide an overview of the current knowledge to introduce each of the three chapters. 


\subsection{Comparative gene expression studies in development}

The goal of molecular studies in developmental biology is to understand how gene products work together to provide instructive signals that control developmental processes (Wolpert and Tickle, 2011). The context dependency of gene expression ensures that specific cell types and tissues are characterized by the expression of a unique set of transcripts which are then translated into transcription factors and structural proteins, making up the building blocks of the respective cell, tissue and organ. Assessing and comparing mRNA composition and gene expression levels across developmental time points provides therefore the chance to better understand the molecular underpinnings of developmental processes and eventually organ functions.

Much of our detailed knowledge about the genes coordinating developmental processes in insects is deduced from studies in the model species $D$. melanogaster. In this species, for instance, the establishment of the body axis, was first studied and understood in great detail: The translation of maternally deposited mRNA leads to the activation of a hierarchical gene activation cascade and subsequently to anterior-posterior segmentation of the complete developing embryo (e.g. Johnston and Nüsslein-Volhard, 1992). Since the advent of RNA-seq, major effort has been made to characterize not only the location and role of single genes, but to establish a complete catalog of transcripts and their expression dynamics in developing and adult tissues (e.g. Brown et al., 2014; Graveley et al., 2011). One major drawback of focusing developmental studies on established model systems is that derived structures or organs that are not present in the vinegar fly are less well studied and understood. Easy accessibility and constant reduction of costs for next generation sequencing techniques nowadays allow to explore the development and function of single organs in nearly every species, including plants and animals (Wang et al., 2009) and has greatly expanded the use of emerging model organisms in developmental biology (Ellegren, 2014).

Since genomic or transcriptomic resources are usually sparse in emerging model systems, the first step often includes the de-novo assembly of reference genomes or transcriptomes against which the short reads can subsequently be mapped (reviewed in Cheng et al., 2018). Depending on the species, de-novo transcriptome assembly can be achieved with the help of a reference genome, or if not available by using the short reads directly for assembly (Cheng et al., 2018). Blasting the de-novo assembled transcriptome against databases like 
UniProt/Swiss-Prot, allows to assign putative functions to transcripts and by this allows to retrieve such information also for transcriptome datasets of non-model species ("UniProt," 2019).

Once references are established, genome wide gene expression can be compared across different conditions of interest, which can include the comparison of different stages during development of a certain organ, or the comparison of different tissues. Such an analysis usually results in long lists of differentially expressed genes. Depending on the exact research question, a major challenge is to extract meaningful information from such large datasets. A first helpful step is often the integration of prior molecular, cellular or functional knowledge. This information can be retrieved from the Gene Ontology (GO) database, which links a particular gene to its function by annotating it to one or more defined GO-terms. Using a statistical framework, it allows to understand in which molecular functions, biological processes and cellular components differentially expressed genes are enriched in (Ashburner et al., 2000; The Gene Ontology Consortium, 2019). If, for instance, different developmental stages are studied, the expression dynamics can be analyzed in more detail by clustering genes that share a similar expression profile. It has been proposed, that such co-expressed genes are often coregulated by the same upstream transcription factors and are involved in related biological functions (Yu et al., 2003). Following that assumption, clustering algorithms that group genes with similar expression levels over a certain period of time, combined with GO-enrichment analysis and an upstream search for transcription factor binding motifs, provides a meaningful tool for the reconstruction of developmental gene regulatory networks (GRNs). Note that the direct search for transcription factor binding motifs works well for established model systems, where databases of TF binding profiles exist. These include e.g. humans and mouse as representatives of vertebrates, D. melanogaster for insects or Arabidopsis thaliana representing plants (Khan et al., 2018). Nevertheless, for non-model systems a so-called denovo motif search can be useful to find overrepresented motifs in regulatory regions of coexpressed genes, followed by a subsequent comparison to known motifs (e.g. Bailey et al., 2009), since transcription factor binding domains are often conserved along large phylogenetic distances.

Studying the development and function of organs in classical model organisms like Drosophila has brought major insights in many aspects of biology. However, for some questions 
in developmental or evolutionary biology, the selection of a handful of established model species does not necessarily represent the best systems. Developmental processes that are highly derived in Drosophila are for example insect head development (Davis and Patel, 2002; Grossniklaus et al., 1994) or the embryonic development of insects. Extraembryonic membranes that usually protect insect eggs from desiccation have been secondarily reduced in higher flies (Schizophora) (Glaser-Schmitt and Parsch, 2018; Jacobs et al., 2013; Schmidt-Ott, 2000) and certain structures which play a role during hatching of the embryo, like pleuropodia (see next section) are missing in the model species Drosophila. Studying traits which are not present in classical model species requires therefore to establish morphological and genomic resources in a variety of species. We applied a comparative RNA-seq approach to pleuropodia and leg buds of the desert locust Schistocerca gregaria (S. gregaria) and combined this with a thorough description of their ultrastructure throughout development to understand their function during insect embryogenesis. We further provide a transcriptomic resource to understand appendage differentiation by comparing two serially homologous structures.

\subsubsection{Schistocerca gregaria as a model to study the role of pleuropodia in insect embryogenesis.}

Insects are the most species-rich animal group on this planet and their success is the result of several evolutionary specializations which allowed them to conquer all environments such as air, water and land (Losos, 2014). These include for instance the emergence of wings in pterygotes (winged insects), the development of three life stages in holometabolous species or eusociality in several insect lineages (Losos, 2014). The colonization of land also required protection against desiccation, especially during embryonic development (Jacobs et al., 2013). Most insect embryos possess two membranes, the amnion and the serosa (Figure 2B), which do not directly contribute to the formation of the insect body, but often cover the entire embryo and take part in crucial developmental processes including - amongst many others cuticle production, immune responses, or hatching (e.g.(Jacobs et al., 2015, 2014, 2013; Panfilio, 2008)). A non-cellular, three-layered serosal cuticle, which is secreted by the serosa itself, lies between this non-embryonic membrane and the eggshell ((Goltsev et al., 2009; Jacobs et al., 2015) Figure 2B). The serosal cuticle has to be digested prior to hatching of the embryo. In grasshoppers and glowworms for instance, the two inner layers of the serosal cuticle merge and decay shortly before hatching, whereas the eggshell and the remaining layer of the 
serosal cuticle layer is mechanically torn by the mandibles (H. Slifer, 1937; Kobayashi et al., 2003). The pair of pleuropodia develops in a plethora of insects at the first abdominal segment of the embryo (Figure 2A; (Wheeler, 1890)) and degenerates at the end of embryogenesis. Orthopterans have proven to be a valuable model to study these small organs, since they are due to their large embryos - easily accessible. Consequently, it was already shown 80 years ago in grasshoppers, that pleuropodia are involved in the digestion of the serosal cuticle $(\mathrm{H}$. Slifer, 1937; Slifer, 1938). However, the clear mechanism how these organs are involved in this process has remained elusive so far. It was suggested, that they facilitate digestion indirectly via secretion of the ecdysone hormone (Novak and Zambre, 1974), or directly by secreting the so-called 'hatching enzyme' (H. Slifer, 1937; Louvet, 1975). Up to now, a thorough description of pleuropodia development, their function and transcriptomic landscape is still missing. Furthermore, since pleuropodia are serially homologous to leg buds, these two structures provide an excellent model to study when and how initially similar structures differentiate during the development of an organism. The proper development of body structures is highly dependent on tissue and stage specific gene expression and the correct interplay of the translated proteins. The methods described in the latter section nowadays allow to generate relatively easily transcriptomes from different organs of non-model species, taking the spatiotemporal gene expression into account. With this they provide the basis for comparative gene expression approaches, which permit to recapitulate and understand the developmental programs of differentiating, serially homologous organs. 
A

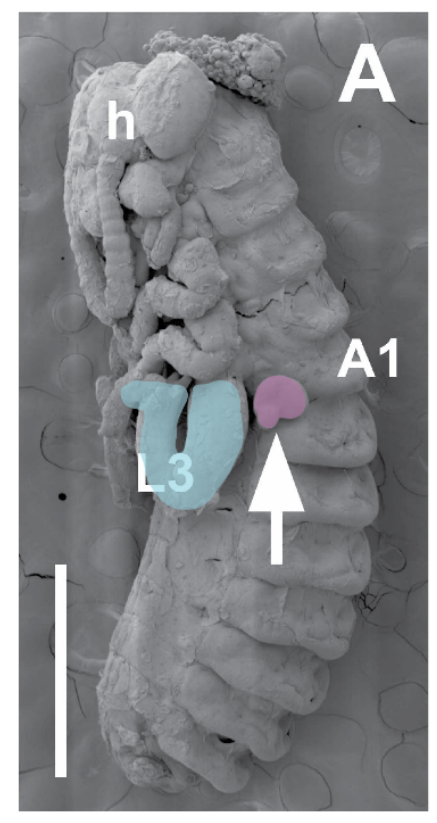

B
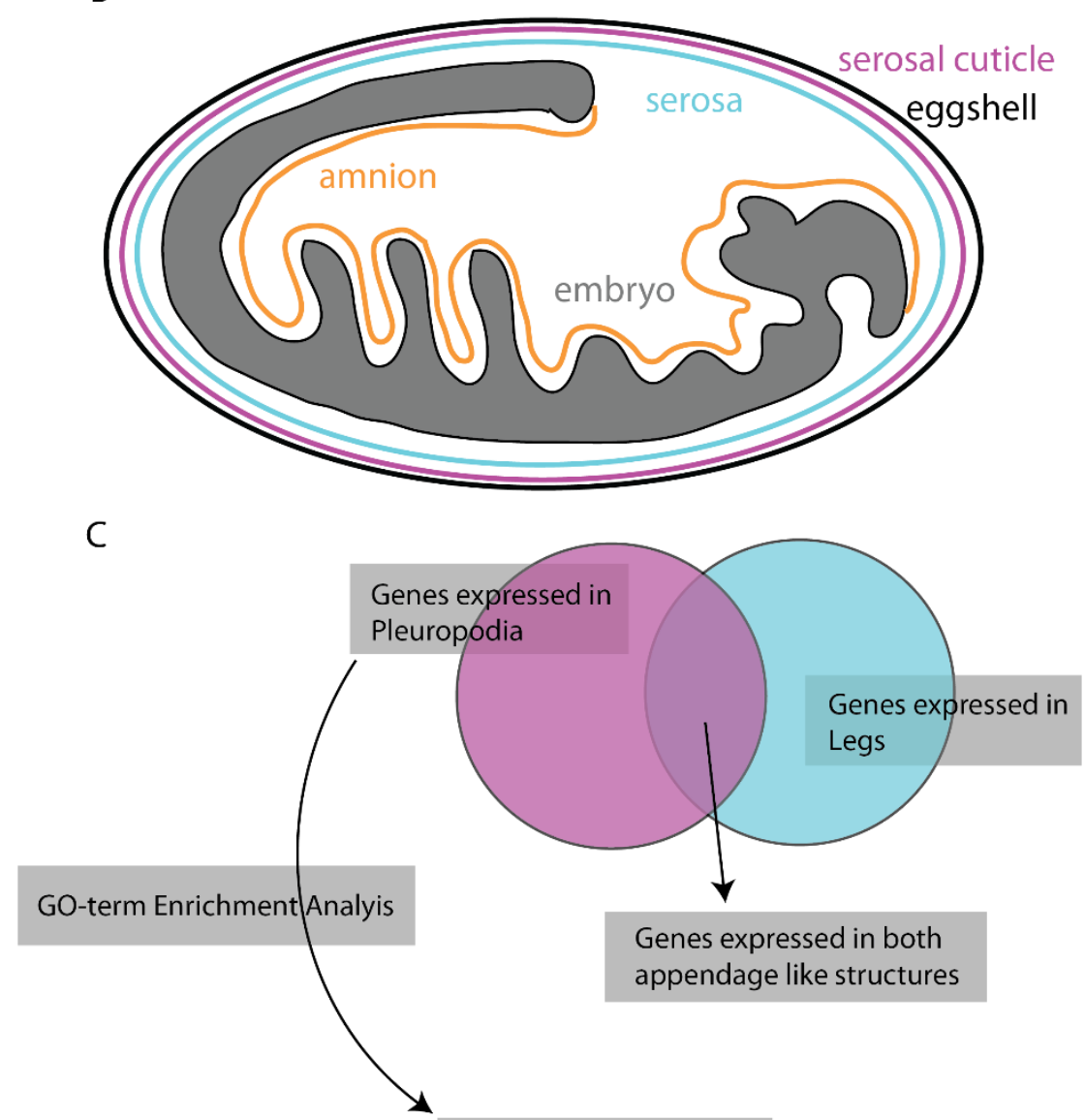

Function of pleuropodia during Development

Figure 2. Pleuropodia and their role during insect embryogenesis. A. A pair of pleuropodia develops at the first abdominal segment in insect embryos (here marked with the white arrow and pink labelled in an embryo of $S$. gregaria). Pleuropodia and the third leg pair (in blue) were dissected to generate a comparative transcriptomic dataset (adapted from (Konopová et al., 2019)). B. Schematic representation of an insect embryo (germband stage). The embryo (in grey) is covered by the amnion (in orange). The serosa (in blue) envelopes the complete embryo and secretes the serosal cuticle (in pink) which lies between the serosa and the egg shell (in black) (after (Panfilio, 2008)) C. Experimental set-up of the comparative gene expression study to analyze function and putative new roles of pleuropodia during insect embryogenesis.

We therefore generated a comparative embryonic RNA-seq dataset of Schistocerca gregaria (S. gregaria) pleuropodia and legs (Figure $2 \mathrm{~A}$ and C), to investigate on a transcriptional level how the pleuropodia facilitate hatching of the embryo. The possibility to dissect pleuropodia and legs provided the opportunity to generate tissue specific datasets at 10 timepoints, also accounting for the temporal context dependency of gene expression. Combined with an in-depth morphological characterization, our results provide interesting insights into the development of pleuropodia, their function during hatching and putative roles in the embryo's immunity and are described in Chapter I of this thesis. 


\subsection{Comparative gene expression studies in phenotypic evolution}

Besides far-reaching novelties, the adaptation to different environments is also facilitated by the ability to change the size and shape of organs and other body parts. The most classic example for natural variation in size and shape are the various beak forms of Galápagos finches, where changes in beak morphology were fundamental for the adaptation to different environments and food sources (Abzhanov, 2004; Abzhanov et al., 2006; Schluter, 2000). Morphological differences that are fixed across populations or species are the result of heritable changes in the genome (Figure 3). Even though this fact is widely accepted, pinpointing the exact molecular changes has been shown to be rather difficult and only few studies succeeded in resolving the causative genomic changes that underlie variation in adult phenotypes. This is mainly due to two reasons. First, variation in many traits, but especially complex traits like size and shape, are influenced by several genomic loci, i.e. they are polygenic (Boyle et al., 2017). Second, causative changes are not always found in the coding region of a gene (CDS), potentially changing the function of the resulting protein, but it is nowadays believed, that many changes occur in so-called cis-regulatory regions, affecting the expression of the respective gene (Wray, 2007).

While selection mostly acts on adult structures, developmental processes define the size and shape of the respective organ. Therefore, fixed changes in adult structures are the result of variation in developmental processes (Figure 3). By comparing the development of organisms one can thus reveal mechanisms underlying morphological divergence. The task of finding the genetic causes for phenotypic variation is usually addressed in the field of evolutionary developmental biology ('Evo-Devo'), the combination of evolutionary studies and developmental biology. 'Evo-Devo' aims to assess conserved aspects as well as differences in developmental programs between species that eventually result in variation in adult morphology (e.g.(Hall, 2003; Raff, 2000), Figure 3).

Environment

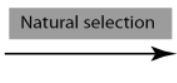

Natural Variation in Morphology

Figure 3. Genetic changes in the genome, which can occur in coding regions but also regulatory regions (light blue box) underlie changes in development by rewiring developmental gene regulatory networks (dark blue box) and subsequent variation in adult morphology (yellow box). If a certain phenotype provides an advantage in fitness in a specific environment (green box), these specific phenotypes will eventually be more common than others ('natural selection', grey box). 
Numerous studies in this field resulted in exciting findings, such as the observation that a set of highly conserved transcription factors and signaling pathways governs the development of organisms over large phylogenetic distances from invertebrates to vertebrates. This was impressively shown in the case of HOX genes, a cluster of homeobox transcription factors, which define the anterior-posterior axes of all metazoans (Duboule and Dollé, 1989; Graham et al., 1989; McGinnis and Krumlauf, 1992; Scott and Weiner, 1984). Another well-described example is the Pax6 gene, which is conserved in all organisms with light sensitive cells. Loss of function of this gene results in a no-eye phenotype in mouse embryos as well as in the vinegar fly Drosophila, where the gene was typically called eyeless (ey) (Hill et al., 1991; Quiring et al., 1994). The coding sequences of the two homologous proteins are strikingly similar, illustrated by the observation that the mouse protein can rescue mutants in the fly (Halder et al., 1995). Therefore, despite the diversity present in nature, the development of organisms is controlled by a limited set of highly conserved regulators, the so called 'developmental toolkit' (Carroll et al., 2001). Consequently, one major question in evolutionary biology is to understand how phenotypic diversity can arise in the light of generally highly conserved developmental regulators. In some cases, the causative mutations underlying phenotypic variation have been identified in protein coding sequences. Hoekstra and colleagues linked a fixed mutation in the gene, encoding for the receptor Mc1r, to differences in color patterns between subspecies of the beach mouse, Peromyscus polionotus (Hoekstra et al., 2006). Additionally, variation in HOX proteins has been shown to drive body plan diversification (Grenier and Carroll, 2000). However, many genetic variants identified for instance by quantitative genetics approaches mapped to non-coding regions (Dixon et al., 2007; Gilad et al., 2008; Jia and Xu, 2007). Already King and Wilson concluded in 1975 that much of the variation that can be observed between species, must be rather based on the way how genes are expressed than on changes in protein sequences themselves (King and Wilson, 1975). Therefore, variation in gene expression underlies phenotypic evolution. Here, we address the question how gene expression diverges in closely related species, and we use Drosophila head and eye development as a model to understand how body structures change their size and shape during evolution. 


\subsubsection{Drosophila melanogaster as a model species to study head size and shape evolution}

Many studies assess the consequences of gene expression divergence by studying classical, discrete traits, like trichome patterns (e.g. McGregor et al., 2007), coloring patterns (e.g.(Gautier et al., 2018; Kratochwil et al., 2018; Prud'homme et al., 2006)) or the loss or gain of skeletal structures (Chan et al., 2010; Xie et al., 2019). However, in recent years, researchers also started to focus on the genomic basis underlying complex trait evolution, such as changes in size and shape of adult structures. The vinegar fly $D$. melanogaster but also its closely related sister species, $D$. simulans and $D$. mauritiana regularly serve as model species to study evolution of organ size. Hagen et al. showed for example that differences in the expression of tartan (trn) underlies the evolution of male genitalia size between D. simulans and D. mauritiana (Hagen et al., 2018). Especially variation in head and eye structures of Drosophila has been of particular interest in recent years (Arif et al., 2013a; Gaspar et al., 2019; Hilbrant et al., 2014; Keesey et al., 2019; Norry et al., 2000; Posnien et al., 2012). In comparison to its sister species, D. melanogaster has very small eyes with a broad interstitial face cuticle. In contrast, D. mauritiana has bigger eyes with a reduced face cuticle (Figure 4A and B, (Posnien et al., 2012)). Interestingly, it has recently been shown in a large-scale screen covering more than 60 Drosophila species that the trade-off between eye and head size is a common feature of Drosophila and most likely represents a functionally relevant subdivision of the visual and olfactory system (Keesey et al., 2019).

A

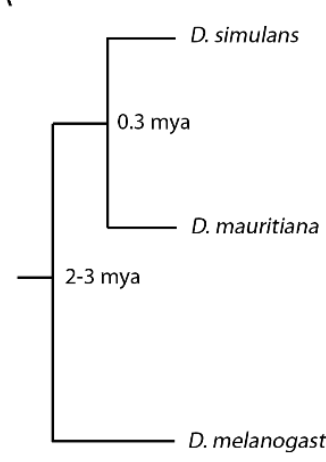

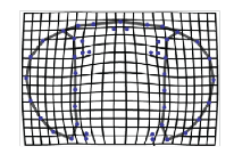

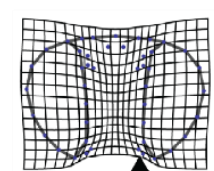

.

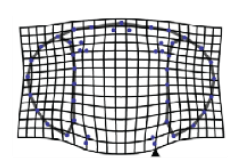

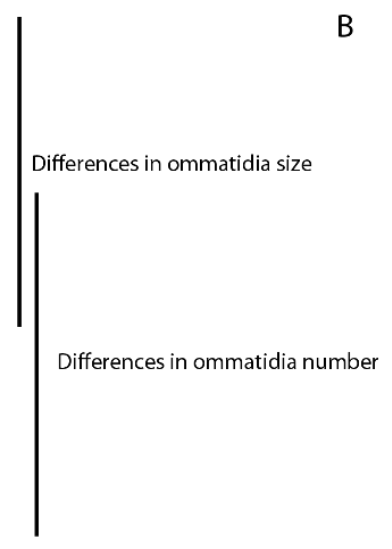

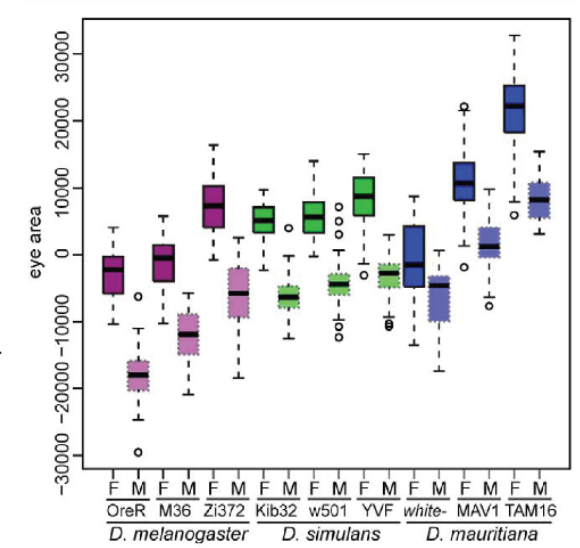

Figure 4. Natural Variation in eye size and head shape between closely related Drosophila species. A. Species in the D. melanogaster subgroup show extensive variation in eye size and head shape. They display the typical trade-off between the head capsule and the compound eye, where a larger eye area goes hand in hand with a narrower interstitial face cuticle and vice-versa. D. melanogaster has very small eyes, and a broad face, whereas D. mauritiana has very large eyes, which is especially pronounced in the dorsal part of the compound eyes. 
Differences in eye size can either arise due to variation in ommatidia number, which is the case between $D$. melanogaster and D. mauritiana, or due to changes in ommatidia size, as observed for D. mauritiana vs. D. simulans. B. Eye area differences in different strains of $D$. melanogaster, $D$. simulans and $D$. mauritiana. OregonR (D. melanogaster) and TAM16 (D. mauritiana) show the most extreme phenotypes on both ends of the spectrum. Figure adapted from (Posnien et al., 2012).

The natural variation in Drosophila eye size and head shape provides an excellent model to study evolution of complex traits, since the GRNs that govern the development of these structures were already extensively studied. The Drosophila head develops from so-called eyeantennal imaginal discs which reside in the larva, attached to the mouth hooks and the two brain lobes. These paired epithelial cell sheets eventually give rise to several distinct adult head structures, including the head capsule, eyes, antennae and mouthparts (Haynie and Bryant, 1986). They have been used to study basic questions in developmental biology, including pattern formation, organ growth or the establishment of compartment boundaries (reviewed in Kumar, 2018). The developing eye-antennal imaginal disc grows homogeneously during the first two larval stages. Only at the end of the second instar the so-called morphogenetic furrow (MF) starts sweeping across the tissue, commencing at the posterior end of the disc. Cells in front of the MF stop dividing after a final mitotic wave. Cells posterior to the MF undergo a second and final round of mitosis, generating the cells, that make up each ommatidium, including for instance photoreceptors and cone cells (Wolpert and Tickle, 2011). Therefore, at the end of larval development the number of ommatidia in the adult compound eye is already defined.

All imaginal discs are formed by two layers, the disc proper and the peripodial epithelium. Both layers are connected via the cuboidal marginal cells ((Lim and Choi, 2004), reviewed in (Gibson and Schubiger, 2001; Kumar, 2018)). The squamous peripodial epithelium is defined by its large cell nuclei that can easily be distinguished from the columnar epithelial cells in the disc proper ((Auerbach, 1936), Figure 5A and B). The disc proper gives rise to most of the adult head structures, whereas the peripodial epithelium is thought to contribute to parts of the body wall cuticle (Figure 5C, (Fristrom et al., 1993; Milner et al., 1984)). It is nowadays accepted that the peripodial epithelium is essential for proper eye development, playing a role for instance in coordinating signaling pathways involved in dorsal-ventral patterning or MF progression, as well as disc growth via microtubule-based extension signaling through the lumen between the two layers (Gibson and Schubiger, 2000). This second epithelium is also important during pupal stages, where the two eye discs evert and finally fuse 
to form the adult head structures. Mechanistic analyses suggested that the reduction of the peripodial epithelium area pushes the eye over the antennal area and by this facilitates morphogenesis of the head (Milner et al., 1984).
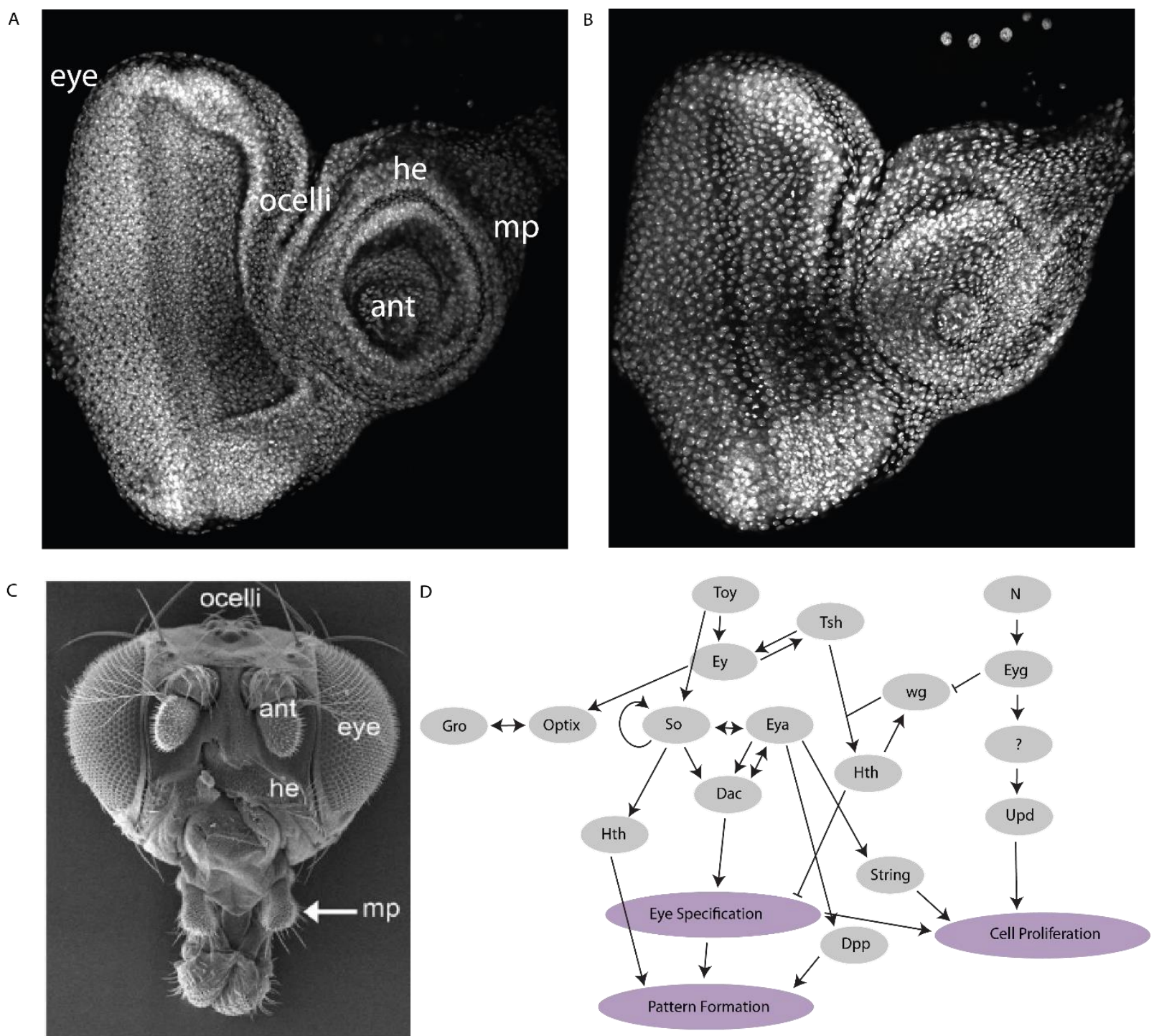

Figure 5. Eye and head development in Drosophila. A. In the third instar eye-antennal disc it can already be determined which part will give rise to which adult structure (eye, ocelli, head (he), antenna and maxillary palp (mp). B. Same eye-antennal disc as in A., focusing on the peripodial epithelium, characterized by large nuclei, stained with DAPI. C. Adult Drosophila head, the structures are labelled as in A. D. A simplified scheme of the GRN network governing eye development in Drosophila (Figure adapted from (Kumar, 2009)).

The GRN governing eye and head development is among the best studied in Drosophila. It is composed of a set of genes, the so-called retinal determination genes. On top of this cascade stands the famous Pax6 homolog ey as a master regulator for eye development (Callaerts et al., 1997). Besides the retinal determination genes, important signaling pathways, including Wnt-, Dpp- and Notch signaling are part of the GRN and are involved in eye/head specification and cell proliferation (reviewed in (Kumar, 2009), Figure 5D). 
The retinal determination genes get restricted to the posterior part of the developing eye-antennal disc during the second larval instar and by this stage ey is not expressed in the antennal part anymore. Instead, expression of the transcription factor Cut (Ct) can be detected in the anterior part of the disc. One important hallmark of this interplay of transcription factors is that they are not activated in a hierarchical cascade but interact in different GRNs which are themselves interconnected (Kumar, 2009; Treisman, 2013). These GRNs do not only include activation between transcription factors and their target genes but also involve feedback loops and repression of locally restricted GRNs: Wang and Sun showed that the expression of ey in the antennal part is repressed by $\mathrm{Ct}$ and Homothorax (Hth), whereas Sine oculis (So) is activated by Ey in the eye disc and represses Hth and So (Wang and Sun, 2012). Also, the growth of the final adult structures and therefore the size relationship among them are controlled via the repressing function of specific transcription factors. For instance, Wingless (Wg) signaling is important for defining the head cuticle fate by repressing retinal development and in turn promotes dorsal head specification (Magri et al., 2018; Treisman and Rubin, 1995). Therefore, in order to ensure the development of several functional organs and structures from one single epithelium, the underlying, intertwined GRNs must be tightly controlled and regulated.

Given the observed variation in eye size and head width within the D. melanogaster subgroup, we sought to study the evolution of this trade-off in D. melanogaster and $D$. mauritiana and focused on recapitulating where GRNs in closely related species evolve. Following the assumption that variation in gene expression is a major driver of phenotypic evolution, we generated a comparative transcriptomic dataset covering three distinct stages during eye and head development (72h after egg laying (AEL), 96h AEL and 120h AEL) in both species. Differential expression analysis together with a transcription factor binding site analysis showed that the GATA factor Pannier (Pnr) regulates many genes that are differentially expressed between D. melanogaster and D. mauritiana. We found that the transcript of pnr itself is differentially expressed in the two species during eye development. Additionally, our genome wide approach allowed us to characterize U-shaped (Ush), a co-factor of Pnr, as a previously unknown player in the GRN of the developing eye-antennal disc and could show that they genetically interact. Overall, we show in Chapter II that higher expression of pnr in D. mauritiana underlies part of the observed natural variation in eye size and head shape between D. mauritiana and D. melanogaster. 


\subsubsection{Mechanisms underlying context dependent gene expression divergence}

While gene expression represents a great intermediate phenotype to study development and the molecular basis of phenotypic variation, it is also of major interest to gain comprehensive insights into the mechanisms underlying gene expression divergence itself. Divergent gene expression can arise due to two different mechanisms; either due to differences in the regulatory region of the differentially expressed gene itself (cis-regulatory divergence, Figure 6) or due to changes in an upstream regulator (trans-regulatory divergence, Figure 6) (Cowles et al., 2002; Wittkopp, 2005; Wittkopp et al., 2004). cis-regulatory divergence is the result of variation in a gene's regulatory region, caused by nucleotide changes in promoter or enhancer sequences that lead for instance to divergence in transcription factor binding (Wittkopp, 2013). trans-regulatory divergence is caused by changes in the upstream gene regulatory cascade, for instance in an upstream transcription factors, which would onsequently affect the transcriptional response following its binding to regulatory regions. Differences in the functionality of such an upstream factor can either be due to changes in the coding region, affecting for instance DNA-binding affinity, or due to differences in its expression, influencing the amount of available transcription factor in a given cell or tissue (Wittkopp, 2005). Even though trans-regulatory changes are mostly referred to as changes in transcription factors, it is noteworthy to mention, that upstream changes can occur on all levels of the upstream gene regulatory cascade, including for instance regulatory miRNAs (Figure 1).

Allele specific expression analysis (ASE) has been used to gain mechanistic insights into gene expression divergence. This approach makes use of an F1 hybrid generation by comparing gene expression in homozygous parent species with expression of their alleles in the same trans-regulatory background of the heterozygous hybrid ((Cowles et al., 2002; Wittkopp et al., 2008 , 2004) Figure 6). Is a specific allele still differentially expressed in the hybrid background, then the causative mutation underlying differential expression of the respective genes in the parentals is thought to be located in the gene's own cis-regulatory region (Figure 6). If the two alleles do not show differential expression in the hybrids anymore, then the differential expression in the parental species is due to changes in upstream trans-regulatory factors, which are neutralized in the common hybrid background. The approach also reveals genes, whose expression is kept conserved in all conditions, i.e. neither the genes in the parental species, nor the alleles in the hybrid are differentially expressed. ASE also gives insights into compensatory 
mechanisms, for instance when the expression of a gene is conserved in the parental species but the two allelic variants in the hybrid do show significant differential expression ((McManus et al., 2010) see Figure 6). Even though ASE is a valuable tool to gain genome wide insights into the mechanisms that underly gene expression divergence, the causative locus underlying differential gene expression cannot be directly inferred (reviewed in (Buchberger et al., 2019)). Additionally, one can only reveal changes over short evolutionary distances, since they rely on the ability of two parental species to produce viable hybrids.

conserved

D. melanogaster

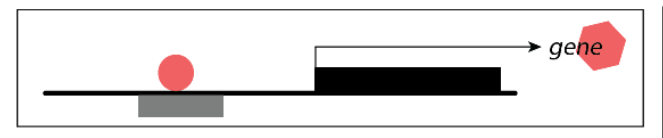

D. mauritiana
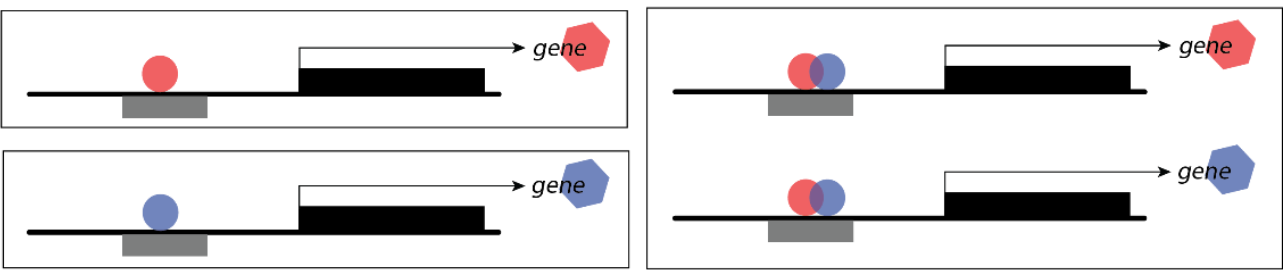

D. melanogaster

D. mauritiana

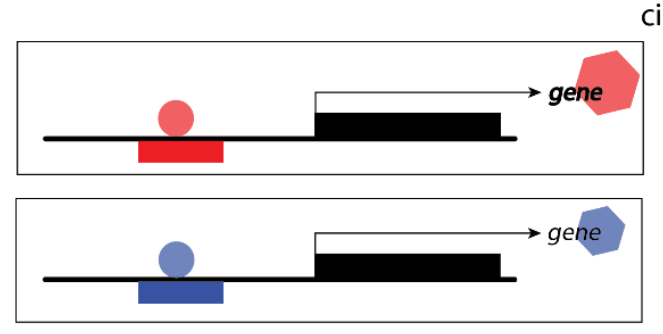

cis

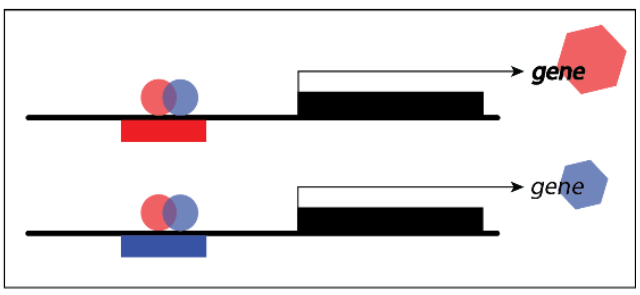

trans

D. melanogaster
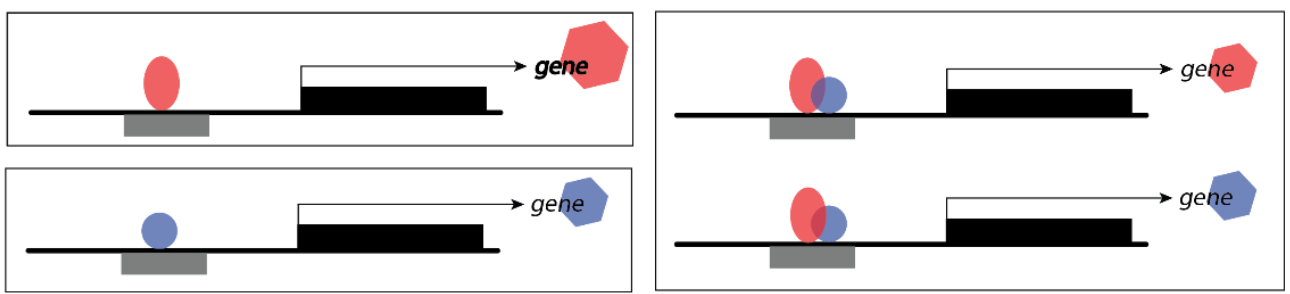

D. mauritiana

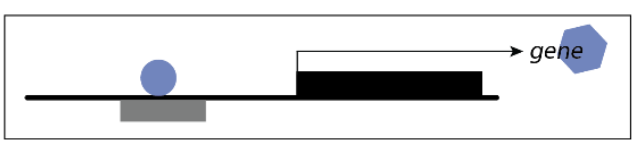

compensatory

D. melanogaster

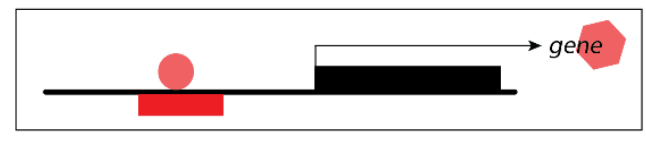

D. mauritiana
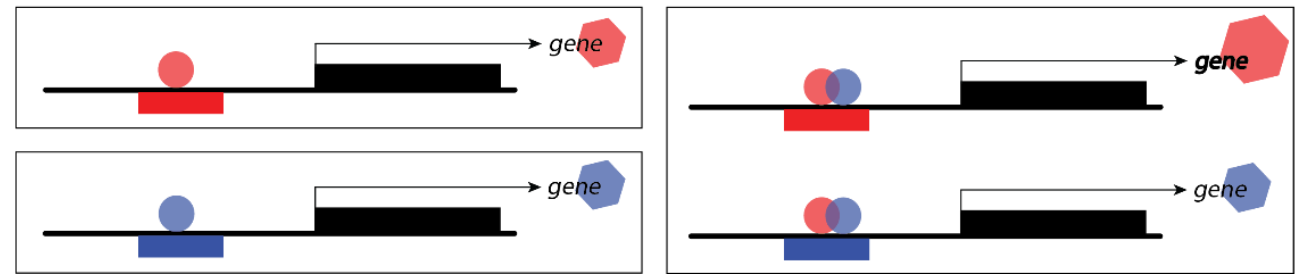

Figure 6. Allele specific expression analysis to study gene expression divergence. Parental species are shown on the left side: Red $-D$. melanogaster and blue $-D$. mauritiana. The colored bars represent the cis-regulatory elements of the respective alleles. In the F1 hybrid the trans background (TFs and co-factors) contains factors from both parents, therefore only differences in the cis-regulatory regions of the two alleles will influence differences in allelic expression. A gene is called 'conserved' if neither the genes in the parental species, nor the two alleles in the hybrids are differentially expressed. A gene is differentially expressed due to cis-regulatory changes, if it is higher expressed in one of the two parental species, and if the allele coming from the same parent is equally higher expressed in the hybrid. A gene is differentially expressed due to trans-regulatory changes, if it is differentially expressed in the parental species, but the alleles do not show differential expression in the hybrid 
offspring. 'Compensatory' describes the situation, if the gene is not differentially expressed between the parentals but the alleles in the hybrids show differential expression. Figure adapted from (McManus et al., 2010).

Even though it is clear from the literature that both cis- and trans-regulatory changes contribute to the evolution of phenotypic traits (Hoekstra and Coyne, 2007; Stern and Orgogozo, 2008), genome-wide ASE studies found cis-regulatory changes to be more prevalent. This fact is usually explained by the argument that trans-regulatory changes would potentially act in a highly pleiotropic manner (Wittkopp et al., 2008). The rational is that mutations in transcription factors or enzymes which are involved in many biological processes, would impact not only one evolving structure but many (He and Zhang, 2006). In contrary, mutations in a cisregulatory region of a gene could have a more tissue-specific function due to the modular nature of the regulatory landscape (reviewed in (Stern and Orgogozo, 2008). Up to now, the question if gene expression divergence results mainly from cis- or trans-regulatory changes has mostly been tackled by studying adult tissue (e.g. (Coolon et al., 2015; Graze et al., 2009; Wittkopp et al., 2004)). However, since gene expression changes during developmental processes shape adult morphology, it is important to study the mechanisms underlying gene expression divergence also during these early stages.

Even though they are closely related, we found many genes to be differentially expressed between species of the D. melanogaster subgroup (Buchberger et al. in prep. (Chapter II), Almudi et al. in prep.) and we used here this model system to study the evolution of gene expression divergence during head and eye development. We combined previous knowledge about regulatory divergence in three species of the $D$. melanogaster subgroup ( $D$. melanogaster, D. simulans and D. mauritiana) with a newly generated ATAC-seq dataset to study, if patterns of cis- and trans-regulatory divergence can be recapitulated on the basis of open and accessible chromatin regions. Preliminary data surprisingly showed, that gene expression divergence during eye and head development is mainly cause by trans-regulatory divergence. Additionally, we describe in Chapter III that the combination of ASE with ATAC-seq datasets indeed allowed to partly recapitulate regulatory divergence by analysing species, stage and tissue specific open chromatin architecture. We revealed, that nucleotide changes in regulatory regions but also their differential accessibility explains parts of the observed cisregulatory divergence. Additionally, not only the coding regions but also the cis-regulatroy regions of conserved or trans-regulated upstream factors are highly constraint on a sequence level. 
3. Chapter I - Transcriptomics supports that pleuropodia of insect embryos function in degradation of the serosal cuticle to enable hatching

The manuscript 'Transcriptomics supports that pleuropodia of insect embryos function in degradation of the serosal cuticle to enable hatching' is the result of a collaboration with Dr. B. Konopová and Dr. A. Crisp.

The work was conceived and coordinated by Dr. B. Konopová. The manuscript was written by Dr. B. Konopová. I was involved in revising the manuscript.

My contribution for this manuscript includes the following bioinformatic analyses:

- Final cleaning of the transcriptome (filtering, incl. testing for completeness) and blast against Uniprot databases

- Quality assessment of the RNA-seq dataset (quality inspection of raw data, preparation of reads for mapping, principal component analysis (PCA))

- Mapping of RNA-seq reads to the transcriptome

- Differential Expression Analysis

- GO enrichment analysis

- $\quad$ Editing of the draft

I prepared the following figures for the manuscript:

- Figure 5 A: Legs and pleuropodia become genetically more different as development progresses

- Figure 6 A and B: Dot plot visualization of GO terms enriched in DEGs in the highly secreting pleuropodia

The following figures and tables were summarized and prepared by B. Konopová with the data resulting from my bioinformatics analyses:

- Figure 5 B: Legs and pleuropodia become genetically more different as development progresses

- Figure 7 (only RNA-seq): Expression profiles of NAGs and CHTs upregulated in the pleuropodia of Schistocerca across development 
- Table 1: Top ten percent of the most abundant transcripts upregulated in the highly secreting pleuropodia of Schistocerca

- Table 2: RNA-seq differential gene expression of cuticular chitin degrading enzymes in the highly secreting pleuropodia of Schistocerca

- Table 3: MF proteases that were upregulated in the highly secreting pleuropodia of Schistocerca

- Table 4: RNA-seq differential gene expression of Schistocerca lysozymes in the highly secreting pleuropodia.

- Table 5: RNA-seq differential gene expression of Schistocerca ecdysone biosynthesis enzymes in the highly secreting pleuropodia.

- Table S1: Embryonic transcriptome of Schistocerca: numbers of sequenced reads and assembled transcripts.

- Table S2: RNA-seq expression analysis: numbers of sequenced and mapped reads.

- Table S3: Number of differentially expressed genes at selected levels of stringency.

- Table S4: Differential expression of genes, whose expression dynamics in the early stages is known.

- Table S5 (only RNA-seq): Comparison between differential expression of selected genes obtained by RNA-seq and real-time RT-PCR.

- Table S6 - S9: GO enrichment analyses

- Table S10 - S15: transcript annotations

- Table S16: RNA-seq differential gene expression of Schistocerca ecdysone biosynthesis enzymes in the pleuropodia at diverse stages.

- Table S17: Schistocerca genes with GO terms "hormone biosynthetic process" upregulated in the highly secreting pleuropodia.

$\underline{\text { Status of the manuscript: }}$

Published on bioRxiv (doi: http://dx.doi.org/10.1101/584029)

In preparation for submission to Scientific Reports 
Title

Transcriptomics supports that pleuropodia of insect embryos function in degradation of the serosal cuticle to enable hatching.

Authors

Barbora Konopová ${ }^{1,2},{ }^{*}$, Elisa Buchberger ${ }^{2}$, Alastair Crisp ${ }^{3}$

${ }^{1}$ Department of Zoology, University of Cambridge, United Kingdom

${ }^{2}$ Department of Developmental Biology, University of Göttingen, Germany

${ }^{3}$ MRC Laboratory of Molecular Biology, Cambridge, United Kingdom

* author for correspondence: barbora.konopova@biologie.uni-goettingen.de

Keywords

insect, Orthoptera, RNA-seq, pleuropodia, embryonic organ, gland, moulting fluid, chitinase, immunity, ecdysone 


\subsection{Abstract}

\section{Background}

Pleuropodia are limb-derived vesicular organs that transiently appear on the first abdominal segment of embryos from the majority of insect "orders". They are missing in the model Drosophila and little is known about them. Experiments carried out on orthopteran insects eighty years ago indicated that the pleuropodia secrete a "hatching enzyme" that at the end of embryogenesis digests the serosal cuticle to enable the larva to hatch. This hypothesis contradicts the view that insect cuticle is digested by enzymes produced by the tissue that deposited it.

\section{Results}

We studied the development of the pleuropodia in embryos of the locust Schistocerca gregaria (Orthoptera) using transmission electron microscopy. RNA-seq was applied to generate a comprehensive embryonic reference transcriptome that was used to study genome wide gene expression of 10 stages of pleuropodia development. We show that the mature and secretion releasing pleuropodia are primarily enriched in transcripts associated with transport functions. They express genes encoding enzymes capable of digesting cuticular protein and chitin. These include the potent cuticulo-lytic Chitinase 5, whose transcript rises just before hatching. The pleuropodia are also enriched in transcripts for immunity-related enzymes, including the Toll signaling pathway, melanization cascade and lysozymes.

\section{Conclusions}

These data provide transcriptomic evidence that the pleuropodia of orthopterans produce the "hatching enzyme", whose important component is the Chitinase 5. They also indicate that the organs facilitate epithelial immunity and may function in embryonic immune defence. Based on their gene expression the pleuropodia appear to be an essential part of insect physiology. 


\subsection{Introduction}

An integral part of insect embryogenesis is the transient appearance of enigmatic glandular organs on the first abdominal segment (A1) that are called the pleuropodia (Rathke, 1844; Wheeler, 1890) (Figure 7A-C). These are paired structures that form external vesicles in some species while in others they sink down into the body wall (reviewed in e.g. (Hussey, 1926; Roonwal Mithan Lal and Imms Augustus Daniel, 1936; Wheeler, 1890)). The pleuropodia are peculiarly modified limbs (Bennett et al., 1999; Lewis et al., 2000; Machida, 1981) (Figure 7D,E): their buds emerge in a line with the buds for the walking legs, but unlike the legs, the pleuropodia remain short, the majority of their cells massively enlarge and develop into a transporting-like and secretory epithelium (Bullière, 1970; Louvet, 1975, 1973; Stay, 1977). The pleuropodia degenerate before hatching and are absent in larvae. They have been found in at least some species of nearly all insect "orders" (Figure 7F), but are absent in others, like Diptera, Hymenoptera and advanced Lepidoptera such as silkworms (e.g. (Ando, 1962; Ando and Haga, 1974; Bedford, 1978; Fraulob et al., 2015; Graber, 1889; Hagan, 1931; Heming, 1993; Hussey, 1926; Kamiya and Ando, 1985; Kobayashi et al., 2003; Kobayashi and Ando, 1990; Lambiase et al., 2003; Larink, 1983; Louvet, 1983; Machida, 1981; Machida et al., 2004; Mashimo et al., 2014; Miller, 1940; Miyakawa, 1979; Norling, 1982; Roonwal Mithan Lal and Imms Augustus Daniel, 1936; Rost et al., 2004; S. MILLAM STANLEY and W. GRUNDMANN, 1970; Tanaka et al., 1985; Tsutsumi, 2008; Uchifune and Machida, 2005)). Perhaps because the pleuropodia are missing in the genetic model Drosophila, they have been neglected in recent decades. Their function has remained unclear and the genes expressed during their active stages are unknown.

Eighty years ago Eleanor Slifer ( $H$. Slifer, 1937; Slifer, 1938) demonstrated that the pleuropodia of grasshoppers (Orthoptera) are necessary for the digestion of the serosal cuticle (SC) before hatching, to enable the larva to get out of the egg. The SC is a chitin and proteincontaining sheet structurally similar to the larval or adult cuticles and is produced by the extraembryonic serosa in early embryogenesis (Goltsev et al., 2009; Jacobs et al., 2015). Shortly before hatching the inner layer of the SC (procuticle) disappears. Slifer (H. Slifer, 1937) showed that when the pleuropodia are removed from fully developed embryos, the SC remains thick and the larva stays arrested in the egg. She proposed that the pleuropodia secrete the "hatching enzyme", a substance likely similar to the cuticle degrading moulting fluid (MF) that is released by the larval epidermis under the old cuticle when the insect is preparing to moult 
(Reynolds and Samuels, 1996). The exact molecular composition of this "hatching enzyme" is unknown.

The endocrinologists Novak and Zambre (Novak and Zambre, 1974) argued that this would be an unusual way to digest a cuticle. During larval moulting (Nijhout, 1998) the larval epidermal cells deposit a cuticle and subsequently it is the same epidermal cells, not a special gland that secretes the cuticle degrading MF. Therefore, they proposed that the SC degrading enzymes would most probably be secreted by the serosa itself. They proposed that the pleuropodia instead secrete the moulting hormone "ecdysone", which then stimulates the serosa to secrete the "hatching enzyme". They also suggested that the pleuropodia reach the peak of their activity in very young embryos during katatrepsis when the serosa is still present (Panfilio, 2008).
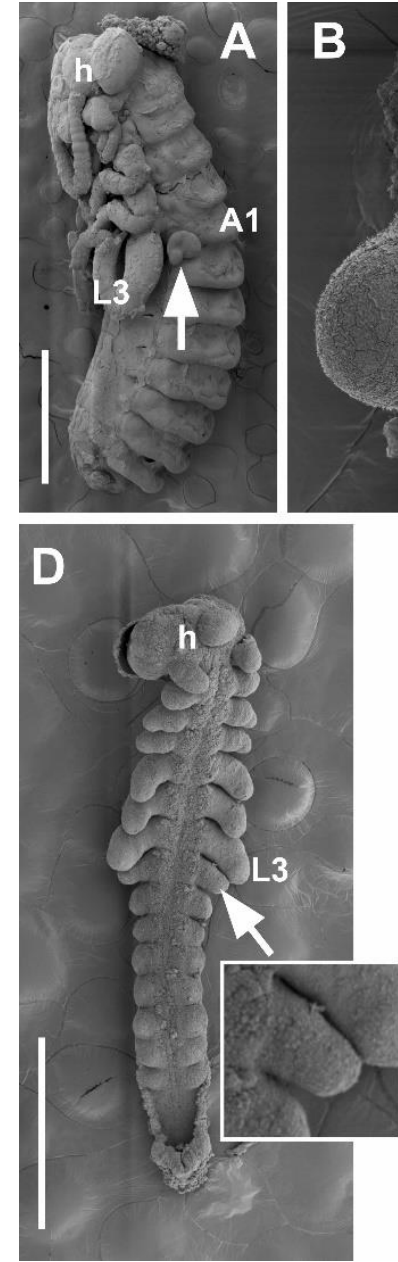
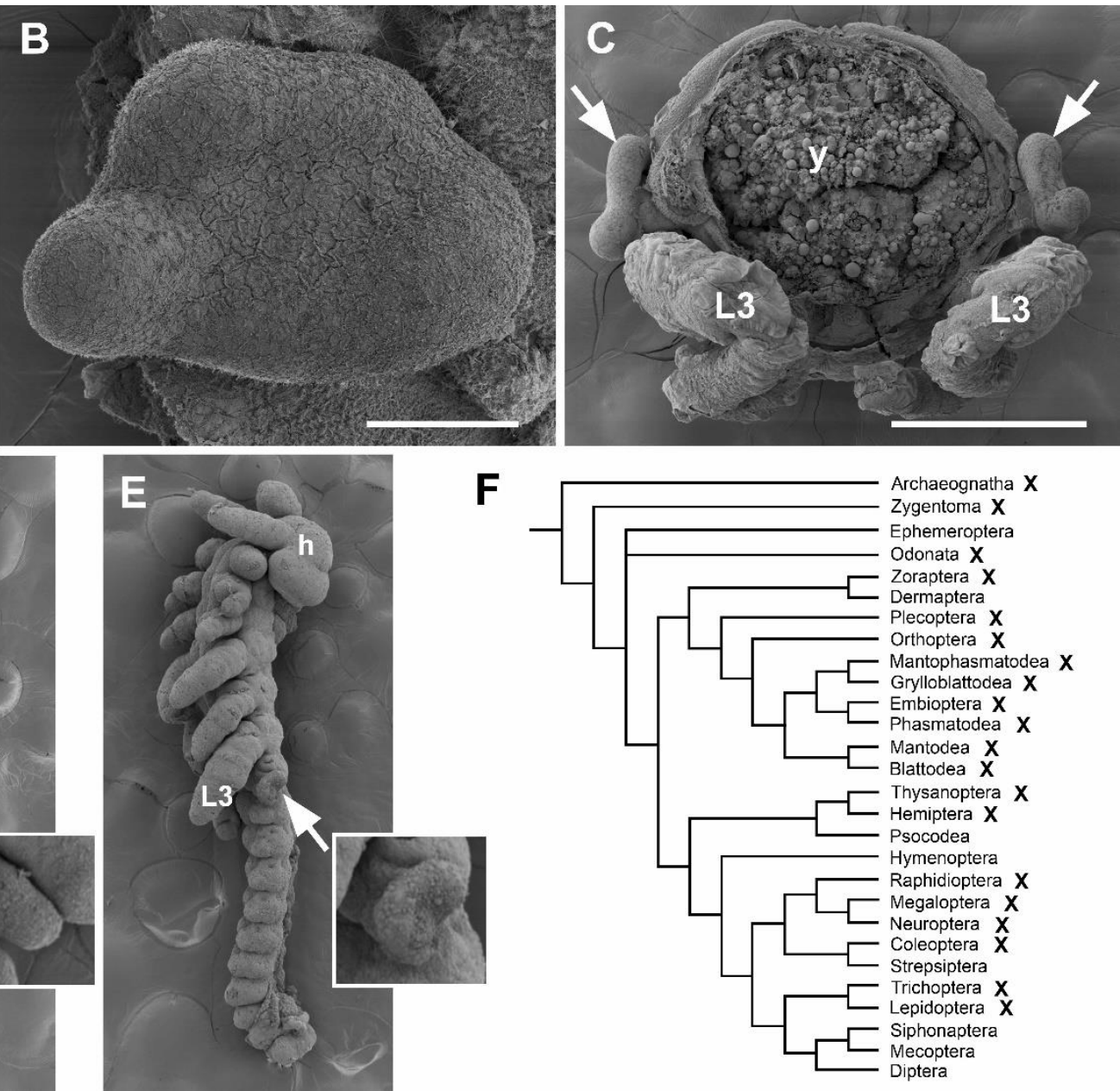

Figure 7. Pleuropodia are limb-derived organs on the first abdominal segment of insect embryos. A-C. External morphology of fully developed pleuropodia of Schistocerca gregaria. A. Embryo before dorsal closure (yolk was removed). B. Enlarged left pleuropodium. C. Cross section through A1. D. and E.: Pleuropodia originate by a modification of a limb bud. D. Early embryo: all appendages are similarly looking buds. E. Older embryo: future legs elongate and the buds on A1 start to take shape of pleuropodia. F. Insect phylogenetic tree showing the 
presence of pleuropodia among "orders". The cross marks "orders" where at least some species develop pleuropodia. Phylogeny from (Kjer et al., 2016), other references in the text. A-E are SEM micrographs. Pleuropodium is marked with an arrow. A1, the first abdominal segment; h, head; L3, hind third. leg; y, yolk. Scale bars: in $\mathbf{A}_{\text {. }} 1 \mathrm{~mm}$; in B., $100 \mu \mathrm{m}$; in C.; $500 \mu \mathrm{m}$; in $\mathbf{D}$., for $\mathbf{D}_{\text {. and }}$., $500 \mu \mathrm{m}$.

In some insects, including locusts, ultrastructural studies (Bullière, 1970; Louvet, 1975, 1973; Rost et al., 2004; Viscuso and Sottile, 2008) have indeed shown that the pleuropodia secrete granules similar to the "ecdysial droplets" carrying the MF (Locke and Krishnan, 1973). Some of the Slifer's experiments (H. Slifer, 1937) were successfully repeated by others (Jones, 1956) and a substance capable of digesting pieces of SC was even isolated from the pleuropodia (Shutts, 1952). But a proper validation by the state-of-the-art genetic methods that the pleuropodia express genes for enzymes capable to digest the SC is missing.

Here, we identified the mRNAs expressed in the pleuropodia of the locust Schistocerca gregaria (Orthoptera). We chose Schistocerca as an ideal model, because it has large embryos (eggs over $7 \mathrm{~mm}$ ) and external pleuropodia that can easily be dissected out, and because the previous experiments testing the function of pleuropodia were carried out in orthopterans. We studied the development of the pleuropodia including using transmission electron microscopy (TEM), and by high-throughput RNA sequencing (RNA-seq) generated transcriptomes from 10 morphologically defined stages. We performed differential gene expression analysis between the pleuropodia and similarly aged hind legs. For mapping of reads we assembled a transcriptome from whole embryos. The goal of this paper was to investigate whether the observed gene expression profile of the pleuropodia is consistent with the idea that these are organs for the secretion of the "hatching enzyme". We show that during their high secretory activity the pleuropodia express genes for cuticle degrading chitinase and proteases that were previously identified in the moulting fluid. This supports the "hatching enzyme hypothesis" $(\mathrm{H}$. Slifer, 1937; Slifer, 1938).

\subsection{Results}

\subsubsection{Development of pleuropodia in the course of Schistocerca embryogenesis}

Before we could start exploring the genes expressed in the pleuropodia of Schistocerca we needed to understand how these organs develop in the locust, i.e. when they are fully differentiated and show activity. Cytological study of developing pleuropodia in grasshopper embryos was previously carried out by Slifer (Slifer, 1938), but the light microscopy that she used does not provide sufficient resolution to distinguish the fine ultrastructure of the cells. 
Ultrastructure of pleuropodia by TEM has been described for several insects (Bullière, 1970; Louvet, 1983, 1975, 1973; Rost et al., 2004; Stay, 1977; Viscuso and Sottile, 2008), but a chronological study is missing for Schistocerca or any other orthopteran.

Under our conditions Schistocerca embryogenesis lasts 14.5 days (100\% developmental time, DT) (Figure 8A, S1). We followed the development of the pleuropodia from the age of 4 days (27.6\% DT), when all appendages are similar looking short buds, until just before hatching, day 14 (Figures 8B, S2-3). Simultaneously, we followed the development of the hind leg, which we used for comparison (because pleuropodia are peculiarly modified legs).

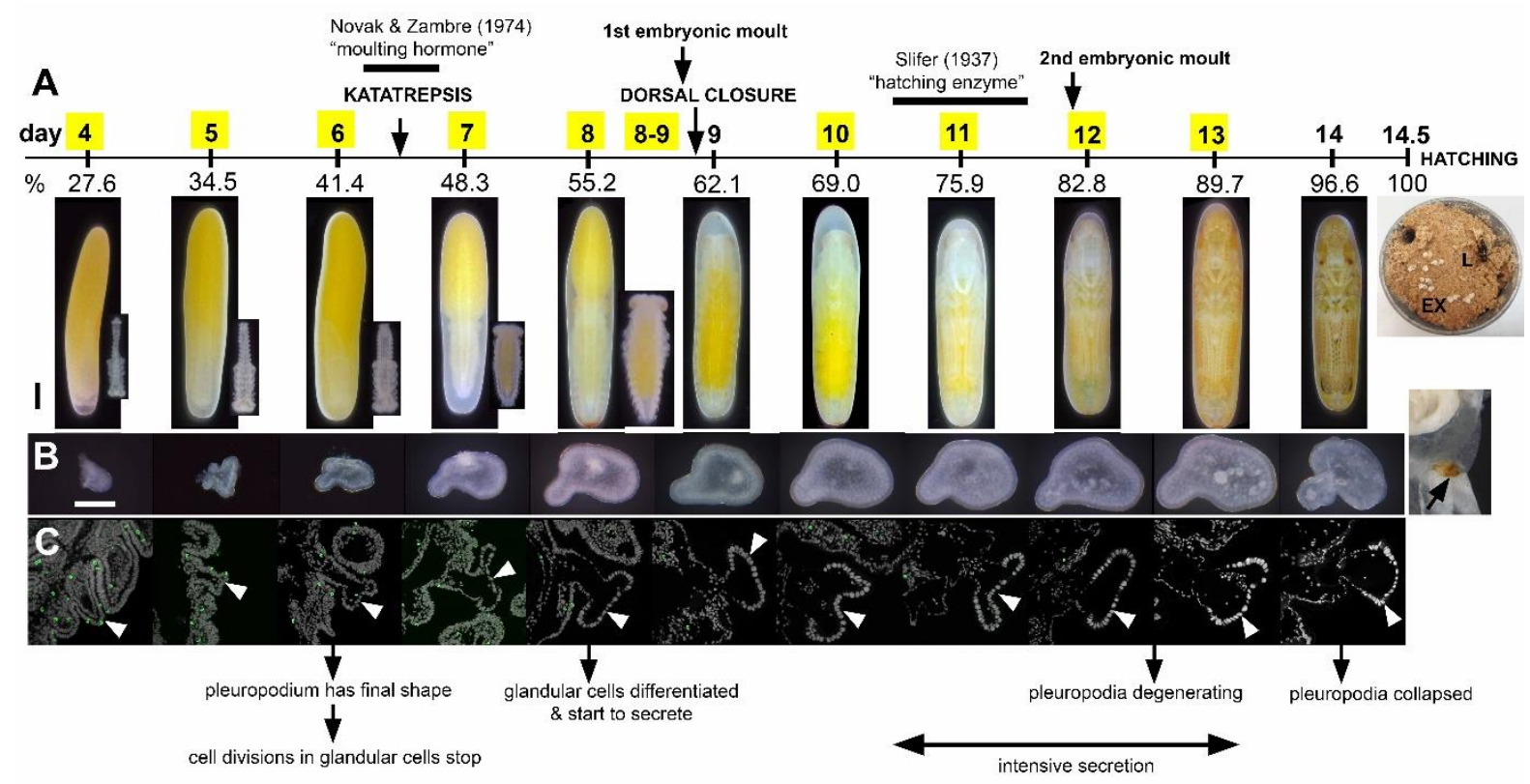

Figure 8. Summary of the development of pleuropodia in Schistocerca embryos. A. Scheme of Schistocerca embryogenesis marking the key developmental events in the embryos and timing of the two experiments on pleuropodia. Numbers above the scale are days from egg-laying, numbers below the scale are percent of embryonic developmental time. Yellow boxes indicate the stages that were sampled for RNA-seq. Eggs with the developing embryos at each stage are shown below the scale, insets for the 4-8 day stages show the embryo dissected out from the egg. B. External features of the developing pleuropodia; after hatching part of the stretched exuvia is shown; the degenerated pleuropodium is marked with an arrow. C. Paraffin sections through the pleuropodium and surrounding tissue. Pleuropodia are marked with arrowheads. PH3 (green) detects cell divisions in the immature glandular cells (tip of appendage bud) on day 4 and 5, not in later stages. The pleuropodial stalk cells, haemocytes entering the pleuropodia and cells in other tissues were labeled. Nuclei (grey) enlarge from day 6 . The text below the pictures refers to the main events in the glandular cells. EX, exuvia; L, larva. Scale bars: in A. (eggs), $1 \mathrm{~mm}$; in B., $0.2 \mathrm{~mm}$. Background was cleaned in photos in A (see Materials and Methods).

We traced cell divisions in the pleuropodia by using Phosphohistone- 3 as a marker (Figure $8 \mathrm{C}$ ). The glandular cells were labeled only in the days 4 and 5 . From day 6 onwards no cell divisions were detected and the nuclei started to enlarge as the cells became polyploid 
(Grellet, 1971). The pleuropodial stalk cells, haemocytes entering the pleuropodia and cells in the other embryonic tissues kept dividing.

Although the pleuropodia get their final external mushroom-like shape just before the embryos undergo katatrepsis (day 6; 41.4\% DT) (Figure 8A,B), we found by TEM (Figure 9) that the glandular cells fully differentiate only later, shortly before dorsal closure (day 8; 55.2\% DT) (compare the undifferentiated cells in Figure 9F-I, with differentiated cells in Figure 9J-P). At that time these cells form a single-layered transporting-like epithelium (Berridge and Oschman, 1972) and secretion granules inside and outside the cells become visible (Figure 9A-E, J). The granules outside of the cells first appear at the base and in between the long apical microvilli (brush-border) (Figure 9E,J). The whole pleuropodium is covered with a thin embryonic cuticle ("the first embryonic cuticle", EC1); the tips of the microvilli produce fibrous material that is a part of this cuticle (Figure 9E) (compare with similar fibers above the leg epidermis Figure S4). 

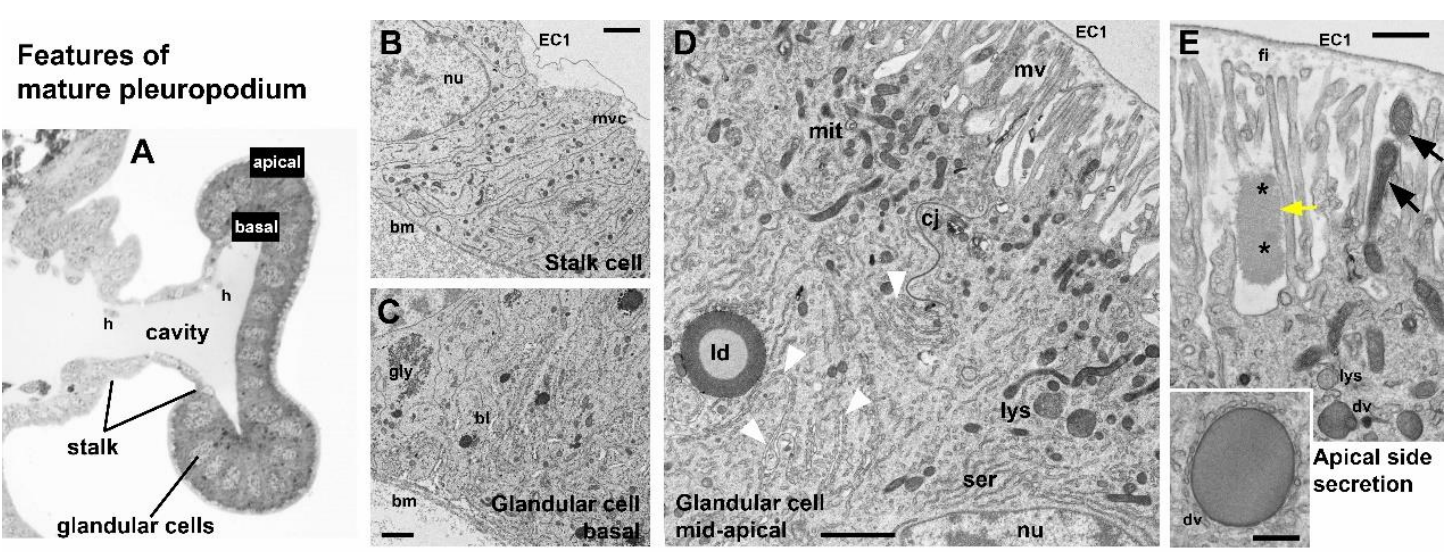

Development of secretory activity in glandular cells
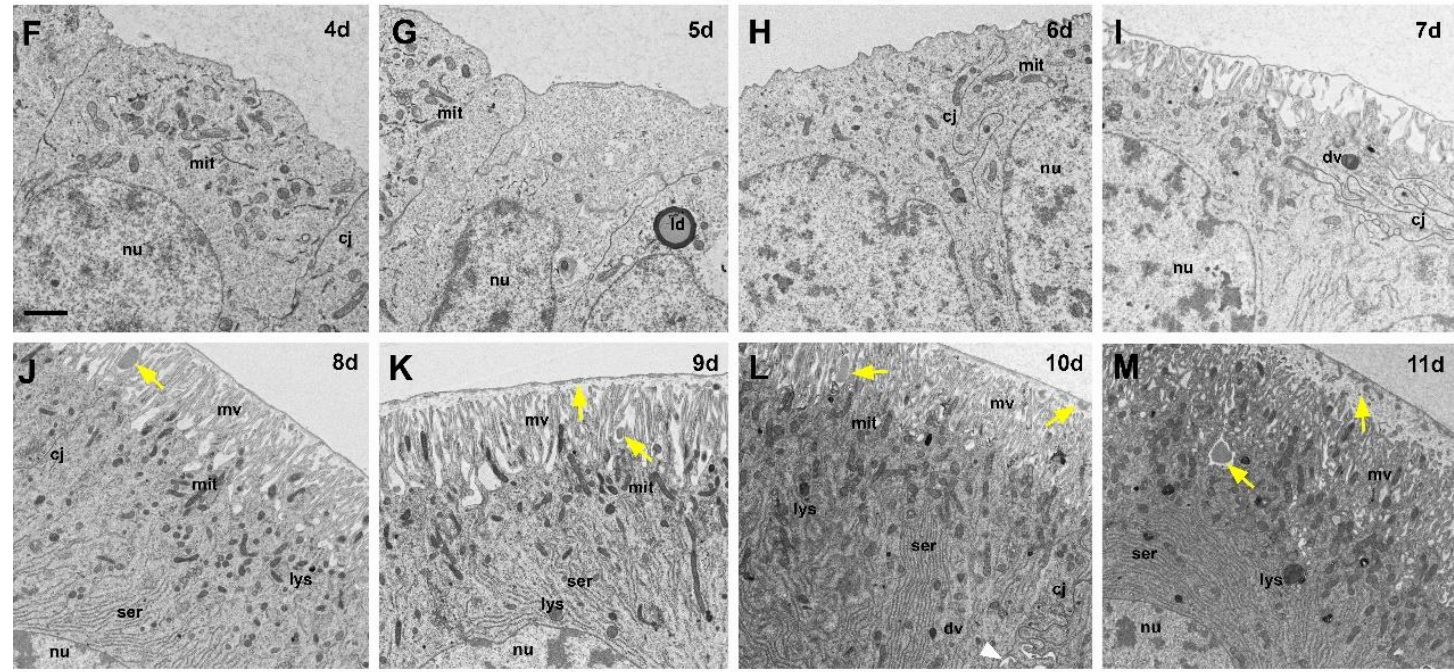

K

9d
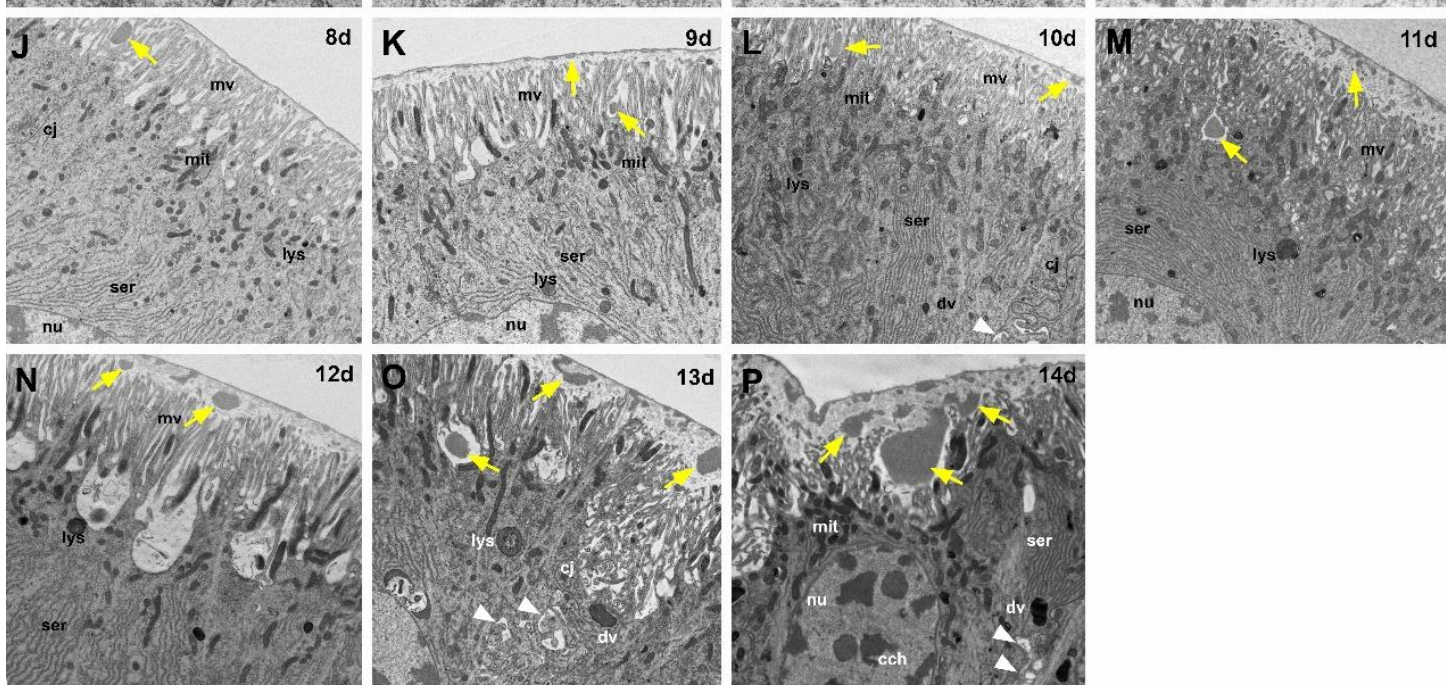

Figure 9. Ultrastructure of the Schistocerca pleuropodia. A.-E. Main features of the cells in the fully formed pleuropodia. Pleuropodia just before dorsal closure are shown. A. Cross section through the pleuropodium. B. Stalk cell. The short microvilli at the apical side are associated with the deposition of fibres in the embryonic cuticle ("the first embryonic cuticle", EC1). C.-E. Glandular cells. In D. the white arrowheads mark the spaces between neighboring cells. In $\mathbf{E}$. the black arrows mark mitochondria inside the microvilli and the asterisks mark spots of different electron-density in the secreted granule. Note that the secretion granule is located at the base of the microvilli (brush-border); the tips of the microvilli produce fibrous material that is a part of the embryonic cuticle EC1. F.-P. Ontogenesis of the glandular cells. Note the development of the microvilli (brush border) and the onset of secretion (appearance of secretion granules within and above the microvilli). On day 8 (J.) the glandular cells are differentiated, on day 12 (N.) patches of the apical side elevate, on day 13 (O.) the organelles are disorganized, on day 14 (P.) cytoplasm is electron dense (cells shrink), chromatin condensed, but large secretion granules are still present at the base of microvilli and above them. $\mathbf{A}$. is a toluidine blue stained semithin section, B.-P. TEM micrographs. Secretion granules are marked with yellow arrows. bm, basement membrane; bl, basal labyrinth (infolding of the basal plasma membrane); cj, cell junction; dv, dense vesicle; EC1, the first embryonic cuticle; gly, glycogene; Id, lipid droplet; mit, mitochondria; mv, microvilli; nu, nucleus; ser, smooth endoplasmic reticulum. Scale bars: in B., C., D., E. and F. for F.-P., $2 \mu \mathrm{m}$; inset in E., $500 \mathrm{~nm}$. 
As development progresses the secretion granules (inside and outside the cells) become more abundant and are present also above the microvilli (Figure 9K-P). On day 12 the apical side of the glandular cells changes: clusters of microvilli (usually at the borders between cells) elevate (Figure 9N). Later the cells show signs of degeneration, the chromatin condenses and the cell content becomes disorganized (Figure 90,P). Large secretion granules are still abundant and probably released even on the last day before hatching, when the pleuropodia have shrunk and collapsed (Figures 8B, 9P).

When the embryo moults (apolyses a cuticle and secretes a new one), first at about 8.5 days and again just before 12 days (Figures 8A, S4), ecdysial droplets are present below the apolysed cuticle. These droplets are very similar at both moults (compare Figures S4F and I). They are very similar, but not identical to the granules released by the pleuropodia (Figure $10 \mathrm{~A}, \mathrm{~B})$. The glandular cells of the pleuropodia do not moult and keep the first embryonic cuticle (EC1) their whole life-time.
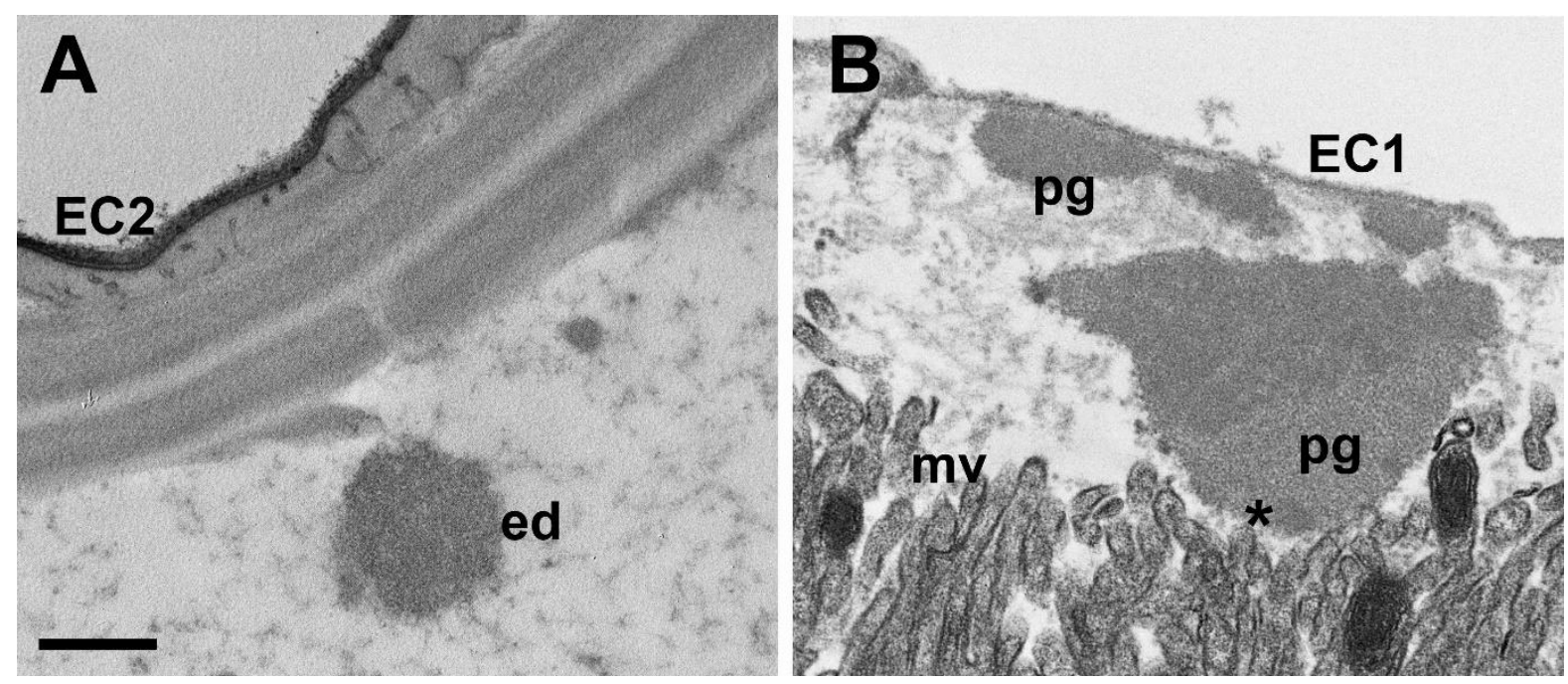

Figure 10. Granules secreted from the pleuropodia resemble ecdysial droplets. A. Ecdysial droplet secreted during the second embryonic moult by hind leg epidermis. B. Granules secreted from pleuropodia at the same developmental stage. The pleuropodial granules are typically larger, less compact and with non-homogeneous electrondensity. The "spot" of a different electron-density in the pleuropodial granules is marked with an asterisk. EC1, EC2, the first and second embryonic cuticles; ed, ecdysial droplets; mv, microvilli; pg, granules secreted from the pleuropodia. Scale bar: for A. and B., $500 \mathrm{~nm}$.

At hatching, the larva enclosed in the (now apolysed) second embryonic cuticle (EC2) leaves the eggshell and digs through the substrate up to the surface (Bernays, 1971; Konopová and Zrzavý, 2005). Here the EC2 is shed and the degenerated pleuropodia are removed with it ((Roonwal ML and Imms AD, 1936); Figure 8A). 
Therefore our observations show that the timing of the high secretory activity corresponds to the stages when Slifer ( $\mathrm{H}$. Slifer, 1937) demonstrated the presence of the "hatching enzyme" (Figure 8A). Next we looked at what genes are expressed in the pleuropodia at this time.

\subsubsection{Generation of a comparative RNA-seq dataset from developing pleuropodia and legs of Schistocerca}

To find out what genes are upregulated in the pleuropodia of Schistocerca, we applied a comparative genome wide expression analysis using RNA-seq. We generated a comprehensive embryonic transcriptome (see details in Materials and Methods) that served as reference for the analysis. This transcriptome consists of 20834 transcripts (Table S1). Its completeness was assessed using the open-source software BUSCO (Simão et al., 2015; Waterhouse et al., 2017). 95.6\%, 96.3\% and 94.6\% of the Metazoa, Arthropoda and Insecta orthologs, respectively, were found, a level comparable to published "complete" transcriptomes.

To gain insights into the gene expression dynamics of pleuropodia development, we dissected pleuropodia from 10 embryonic stages and isolated their mRNAs. In parallel, we dissected hind legs for the same 10 stages to generate a comparative transcriptomic dataset. In total we sequenced pairs of samples (pleuropodia and legs) from 10 developmental stages and performed a differential expression analysis between legs and pleuropodia for each stage (Figure 8A, Table S2). A principal component analysis (PCA) confirmed that legs and pleuropodia are not only morphologically very similar at early stages, but share a common transcriptomic landscape as well (Figure 11A). The number of differentially expressed genes (DEGs) rises as development progresses (Figure 11B, Table S3). 


\section{A PCA plot, rlog transformed raw data}

$150-$

$100-$

LEG-12d

LEG-13d
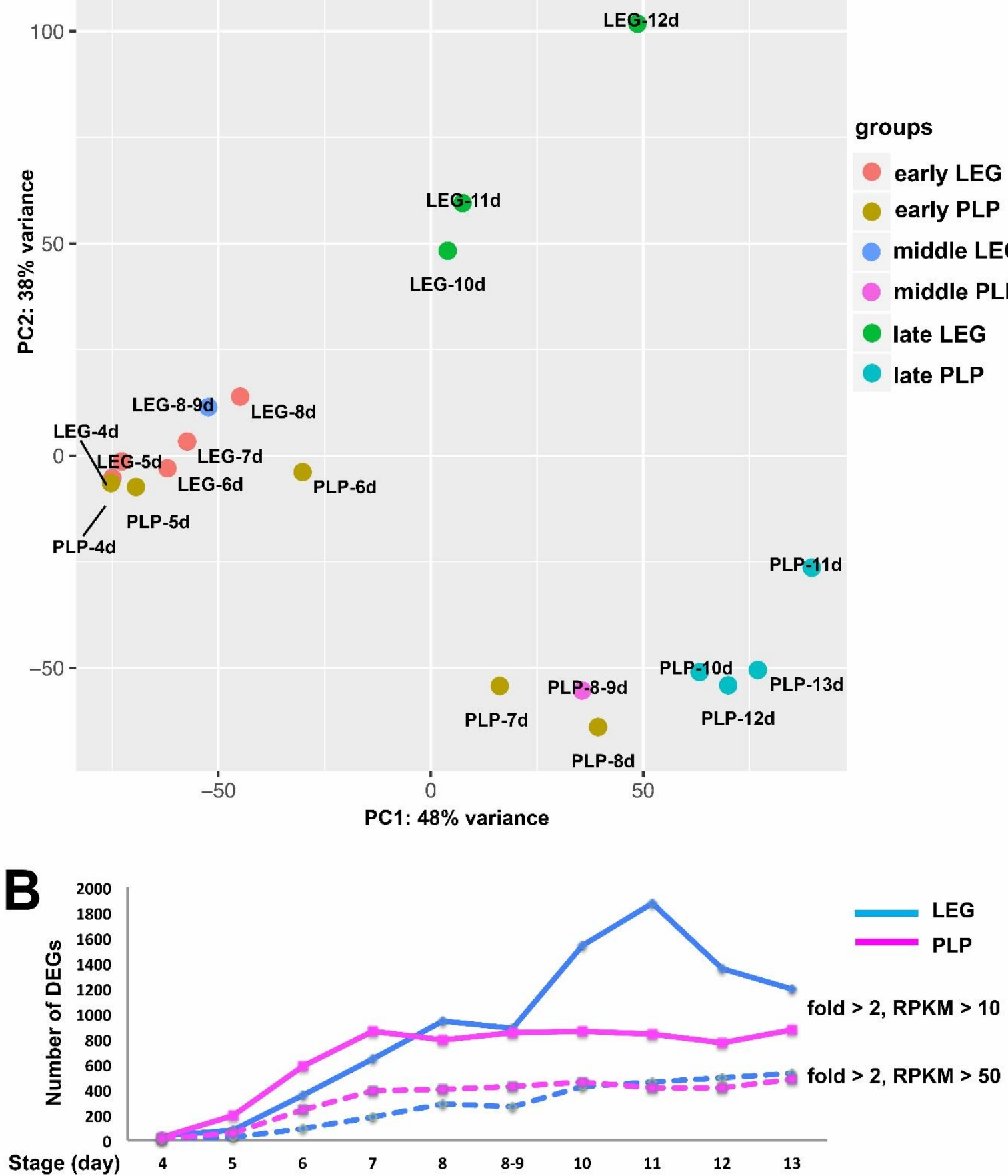

Figure 11 Legs and pleuropodia become genetically more different as development progresses. A. PCA on genes expressed in legs and pleuropodia at 10 embryonic stages (rlog transformed read counts). The expression profile diversifies with development, consistent with the observation that the two tissues develop into two different structures (starting from day 6). Samples from young embryos are genetically more similar and cluster together, while samples from advanced stages are genetically more distant and also separated on the plot. B. Number of 
DEGs at two levels of stringency (RPKM $\geq 10$ and fold change $\geq 2$ was considered as a threshold for a gene to be differentially expressed). LEG, DEGs downregulated in pleuropodia and upregulated in legs, PLP, DEGs upregulated in pleuropodia and downregulated in legs.

For several genes whose expression dynamics in the pleuropodia were already known, such as Ubx, abd-A, dll and dac (Angelini et al., 2005; Bennett et al., 1999; Hughes and Kaufman, 2002; Prpic et al., 2001; Tear et al., 1990; Zhang et al., 2005), we confirmed that they were upor downregulated in our RNA-seq data as predicted (Table S4). To further validate the RNA-seq dataset, we carried out real-time RT-PCR on 46 selected genes in several stages (in total in 176 cases) and got results consistent with the sequencing data (Table S5). Therefore, we are confident that we can identify important factors that are relevant for pleuropodia function and development.

\subsubsection{Identification of genes upregulated in the intensively secreting pleuropodia}

Since we wanted to focus specifically on the pleuropodia with high secretory activity we pooled the data from the samples 10,11 and 12 days together, separately for pleuropodia and legs, and treated them as triplicates. These three samples cover the stages from the embryos after the dorsal closure, when the pleuropodia intensively release secretion granules, but are not in advanced state of degeneration (day 13) (Figures 10A, 9L-N). We performed differential expression analysis and gene ontology (GO) enrichment analysis with genes upregulated in legs and pleuropodia. We identified 781 transcripts upregulated in the pleuropodia (compared to the legs) and 1535 downregulated (Table S3). Table 1 shows the top 10\% of the most highly abundant transcripts (measured in RPKM units, "reads per kilobase of transcript per million reads mapped") that we found upregulated in the pleuropodia.

Table 1. Top ten percent of the most abundant transcripts upregulated in the highly secreting pleuropodia of Schistocerca.

\begin{tabular}{|c|c|c|c|c|c|c|c|}
\hline \multirow[b]{2}{*}{ Transcript ID } & \multirow[b]{2}{*}{ Protein } & \multirow[b]{2}{*}{ Characteristics } & \multirow[b]{2}{*}{ Immunity ${ }^{a}$} & \multirow{2}{*}{$\begin{array}{l}\text { Cuticle } \\
\text { digestion }^{\mathrm{b}}\end{array}$} & \multicolumn{2}{|c|}{ RPKM } & \multirow{2}{*}{$\begin{array}{l}\text { Fold } \\
\text { change }\end{array}$} \\
\hline & & & & & legs & pleuropodia & \\
\hline SgreTa0017702 & $\mathrm{x}$ & & & & 23.07 & 15186.05 & 658.36 \\
\hline SgreTa0007897 & C-type lysozyme & anti-bacterial protein & $\mathrm{x}$ & & 42.93 & 14452.15 & 336.64 \\
\hline SgreTa0002988 & $\begin{array}{l}\text { Uncharacterized, contains DUF } 4773 \\
\text { domain }\end{array}$ & & & & 15.16 & 9112.05 & 601.19 \\
\hline SgreTa0005052 & $\mathrm{x}$ & & & & 13.37 & 7950.98 & 594.48 \\
\hline SgreTa0001636 & Serine protease & proteolysis & $\mathrm{x}$ & $\mathrm{x}$ & 49.38 & 7578.31 & 153.48 \\
\hline SgreTa0008851 & Chitin binding Peritrophin-A & perotrophic matrix protein & & & 9.12 & 6836.42 & 749.88 \\
\hline SgreTa0017707 & I-type lysozyme & anti-bacterial protein & $\mathrm{x}$ & & 12.20 & 6712.31 & 550.26 \\
\hline SgreTa0007042 & $\mathrm{x}$ & & & & 7.04 & 6650.18 & 944.25 \\
\hline SgreTa0004599 & Alpha-tocopherol transfer protein & $\begin{array}{l}\text { intermembrane lipid } \\
\text { transfer }\end{array}$ & & & 8.99 & 5848.12 & 650.71 \\
\hline SgreTa0009217 & $\mathrm{x}$ & & & & 5.03 & 5384.56 & 1070.14 \\
\hline SgreTa0003175 & Collagen & & & & 32.25 & 5220.96 & 161.87 \\
\hline SgreTa0007886 & Alpha-N-acetylgalactosaminidase & carbohydrate catabolism & & & 3.85 & 4372.63 & 1134.69 \\
\hline SgreTa0002109 & $\mathrm{x}$ & & & & 2.20 & 3016.31 & 1372.07 \\
\hline SgreTa0017715 & Serine protease, Snake-like & proteolysis, Toll signaling & $\mathrm{x}$ & $\mathrm{x}$ & 70.55 & 2947.46 & 41.78 \\
\hline SgreTa0017664 & Chitinase 5 & cuticular chitin degradation & & $x$ & 79.32 & 2620.11 & 33.03 \\
\hline SgreTa0002467 & Neutral endopeptidase 24.11 & proteolysis & & $\mathrm{x}$ & 62.26 & 2282.01 & 36.66 \\
\hline
\end{tabular}


degradation of the serosal cuticle to enable hatching

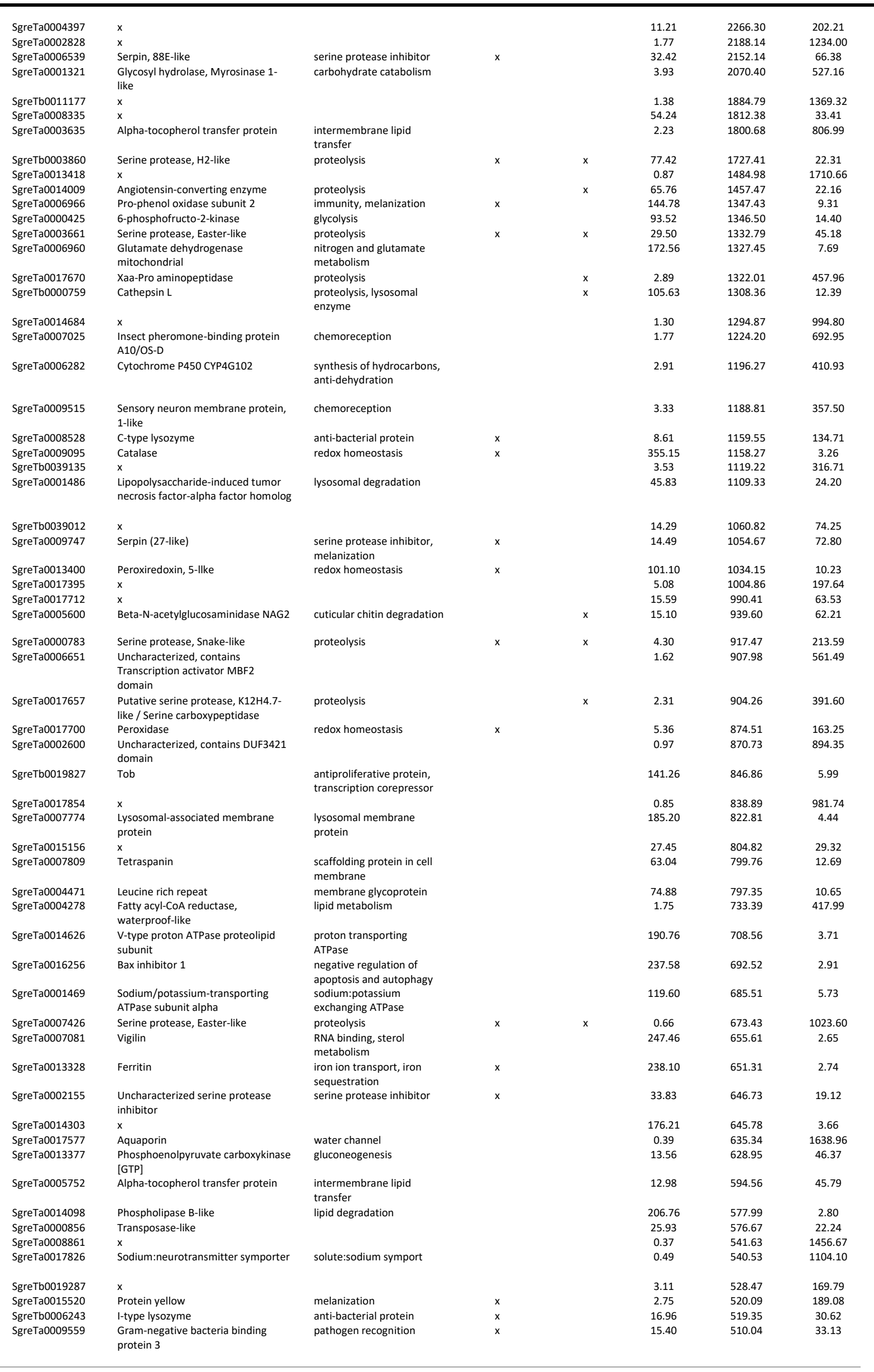


a proteins related to immune response

${ }^{b}$ proteins that participate in larval moulting; some of them are known, other anticipated to digest cuticular chitin and protein (e.g., present in the MF)

For the sake of clarity we summarized redundant GO terms in representative GO-groups (Figure 12; the full set of enriched GO terms are presented in Tables S6,S7; GOs enriched at each developmental stage separately are in Tables S8,S9) (see Materials and Methods). Our results show that the genes downregulated in the pleuropodia (upregulated in the legs) are enriched in GO terms associated with development and function of muscle tissue, cell division and DNA synthesis. This is in agreement with our and previous observations that the pleuropodia lack muscles, while at these stages the legs are differentiating, developing muscles and their cells are still dividing (Figure $8 \mathrm{C}$ ). The pleuropodia downregulate genes for the development of mesoderm, which is consistent with the morphological observation that they are formed by ectodermal cells (Figure 9A). 


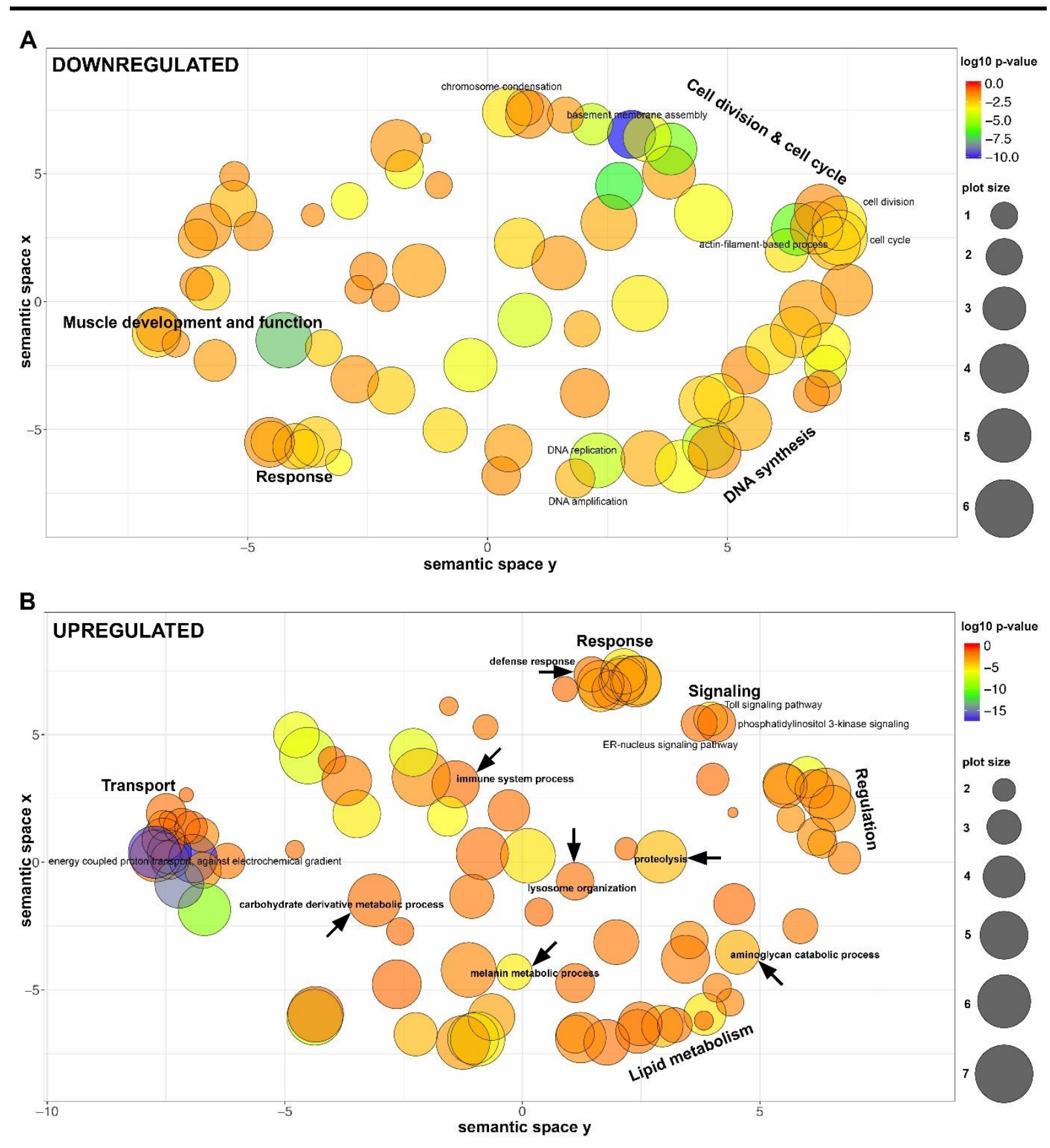

Figure 12. Dot plot visualization of GO terms enriched in differentially expressed genes in highly secreting pleuropodia. Representative groups of GO terms enriched in genes that are A. downregulated in pleuropodia (in comparison to legs) and B. upregulated in pleuropodia. Major clusters are labeled. Relevant GOs are marked with an arrow. Bubble color indicates the p-value of the GO term, the size indicates the frequency of the GO term in the underlying Gene Ontology Annotation (GOA) database (bubbles of more general terms are larger).

The upregulated genes are primarily enriched in GO terms (Figure 12, Table S7) associated with transport thus genetically confirming the morphological observations that the pleuropodia are transporting organs. These include genes for transporters present in typical insect transporting epithelia (Chintapalli et al., 2013), such as the energy providing V-ATPase and $\mathrm{Na}^{+}$, $\mathrm{K}^{+}$ATPase (Table S10). We found enriched GO terms linked with lysosome organization, consistent with the observation that the pleuropodia contain numerous lysosomes (Figure 9, 
(Louvet, 1975)). We also found a large cluster of GO terms associated with lipid metabolism, consistent with the abundant smooth endoplasmic reticulum in the cells- Therefore, the pool of genes expressed in the pleuropodia is in agreement with the morphology of the organs. Among the novel findings are upregulation of genes associated with immunity, as well as with carbohydrate derivative metabolism, aminoglycan catabolic process and proteolysis: these might contain genes for degradation of the SC. Next we looked at selected genes in a detail.

\subsubsection{The pleuropodia upregulate genes for cuticular chitin degrading enzymes}

Insect cuticle is digested by a cocktail of chitin and protein degrading enzymes (Reynolds and Samuels, 1996; Zhang et al., 2014). Cuticular chitin is hydrolyzed by a two-enzyme system composed of a $\beta-N$-acetyl-hexosaminidase (NAG) and a chitinase (CHT) (Zhu et al., 2007). Both types of enzymes, a NAG and a chitinase, have to be simultaneously present for efficient hydrolysis of chitin (Fukamizo and Kramer, 1985). Previous studies have shown that only particular NAGs and CHTs are capable of efficiently digesting the type of chitin present in the insect cuticle (see below).

Insect NAGs were classified into 4 major classes, of which chitinolytic activity was demonstrated for group I and II (Table 2) (Hogenkamp et al., 2008; Rong et al., 2013). Our transcriptome contains 4 NAG transcripts, each representing one group (Table 2, Figures 13AD, S5A, S6A). All were upregulated in the pleuropodia. Among them the Sg-nag2 for the chitinolitic NAG group II had the highest expression (among 46 most highly "expressed" genes, Table 1) and fold change between legs and pleuropodia. The abundance of transcripts for the chitinolitic NAGs starts to rise from day 6 (Figure 13A, B) when the glandular cells in the pleuropodia begin to differentiate morphologically (Figs 7, 9). The expression profile of Sgnag2, that we have chosen for validation, was similar by RNA-seq and real-time RT-PCR (compare Figure $13 \mathrm{~B}$ and $\mathrm{B}^{\prime}$ ).

Table 2. RNA-seq differential gene expression of cuticular chitin degrading enzymes in highly secreting pleuropodia of Schistocerca.

\begin{tabular}{|c|c|c|c|c|c|c|}
\hline Family & Group & Protein & $\begin{array}{l}\text { Schistocerca } \\
\text { gene }\end{array}$ & UP/DOWNa & $\begin{array}{c}\text { Fold } \\
\text { change }\end{array}$ & Expression $^{b}$ \\
\hline \multirow[t]{3}{*}{ ß-N-acetylhexosaminidase } & 1 & NAG1 & Sg-nag1 & UP & 7.85 & $124(15.88 \%)$ \\
\hline & ॥ & NAG2 & Sg-nag2 & UP & 62.21 & $46(5.89 \%)$ \\
\hline & III & Fused lobes & $S g-f d l$ & UP & 14.18 & $592(75.8 \%)$ \\
\hline
\end{tabular}




\begin{tabular}{|c|c|c|c|c|c|c|}
\hline & IV & Hex & Sg-hex & UP & 47.37 & $306(39.18 \%)$ \\
\hline \multirow[t]{17}{*}{ chitinase-like } & I-Major "moulting" chitinases & Chitinase 5 & sg-cht5-1 & UP & 33.03 & $15(1.92 \%)$ \\
\hline & & & Sg-cht5-2 & UP & 234.78 & $400(51.21 \%)$ \\
\hline & II-"Moulting" chitinases & Chitinase 10 & Sg-cht10-1 & $n a^{c}$ & & \\
\hline & & & Sg-cht10-2 & $n s^{d}$ & & \\
\hline & III-Cuticle assembly chitinases & Chitinase 7 & Sg-cht7-1 & ns & & \\
\hline & & & Sg-cht7-1 & ns & & \\
\hline & & & sg-cht7-1 & ns & & \\
\hline & IV-Gut, fat body and other & Chitinase 8 & Sg-cht8-1 & na & & \\
\hline & chitinases & & & & & \\
\hline & & & Sg-cht8-1 & na & & \\
\hline & & & Sg-cht8-1 & na & & \\
\hline & & Chitinase 6 & Sg-cht6-1 & ns & & \\
\hline & & & Sg-cht6-2 & $\mathrm{ns}$ & & \\
\hline & & Chitinase 2 & Sg-cht2 & UP & 2.81 & $188(24.07 \%)$ \\
\hline & V-Imaginal disc growth factors & IDGF & Sg-idgf-1 & UP & 20.97 & $391(50.06 \%)$ \\
\hline & & & $S g-i d g f-2$ & ns & & \\
\hline & & & Sg-idgf-3 & $\mathrm{ns}$ & & \\
\hline \multicolumn{7}{|c|}{ a upregulated (UP)/ downregulated (DOWN) } \\
\hline \multicolumn{7}{|c|}{$\begin{array}{l}\text { b the DEGs were ranked according to their RPKM (in descending order), the number describes the position of th } \\
\text { DEG in the ranked table; top } 25 \% \text { highlighted in black, others in descending level of grey }\end{array}$} \\
\hline
\end{tabular}

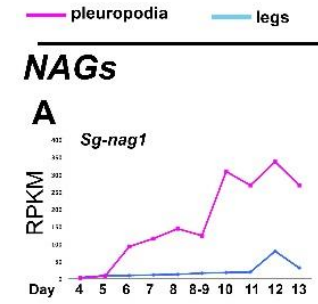

RNA-seq

real-time RT-PCR
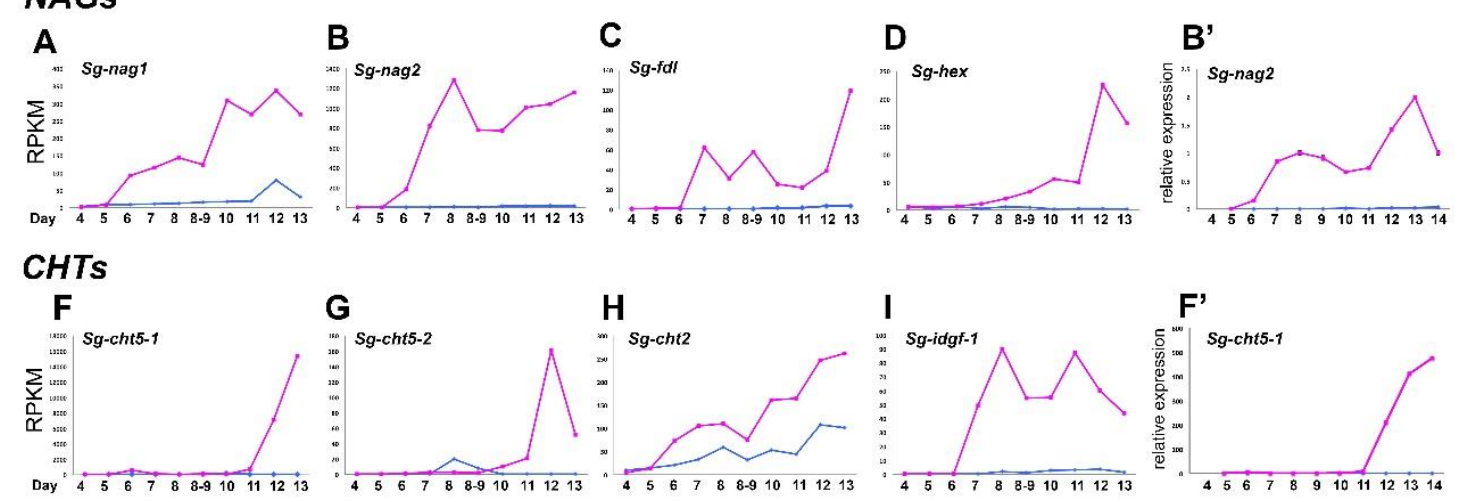

Figure 13. Expression profiles of NAGs and CHTs upregulated in the pleuropodia of Schistocerca across development. Top row: NAGs, bottom row: CHTs. A-D. and F-I. RNA-seq, Expression in single-sample sequencing is shown. B'. and $\mathbf{F}^{\prime}$. real-time RT-PCR. B'. is the same gene as in $\mathbf{B}$. and $\mathbf{F}^{\prime}$. is the same gene as in F. Analysis of 34 technical replicates is shown. Expression in day 8 was set as 1. 
To see if the pleuropodia are the major source of the $S g$-nag2 transcript in the embryo, we looked at its expression in various parts of the body (head, thorax, abdomen with pleuropodia, abdomen from which pleuropodia were removed) using real-time RT-PCR (Figure $14 A, B)$. We performed this analysis in embryos on day 6 , when the pleuropodia are still immature, day 8, just at the onset of the secretory activity, day 10 and day 12 during active secretion. During all of the stages the abdomen with pleuropodia had the highest expression (A+ in Figure 14B), although the expression was lower in the youngest sample (day 6) compared to the samples from older embryos (day 8, 10 and 12). This shows that the pleuropodia are the major source of mRNAs for this cuticle-degrading NAG.
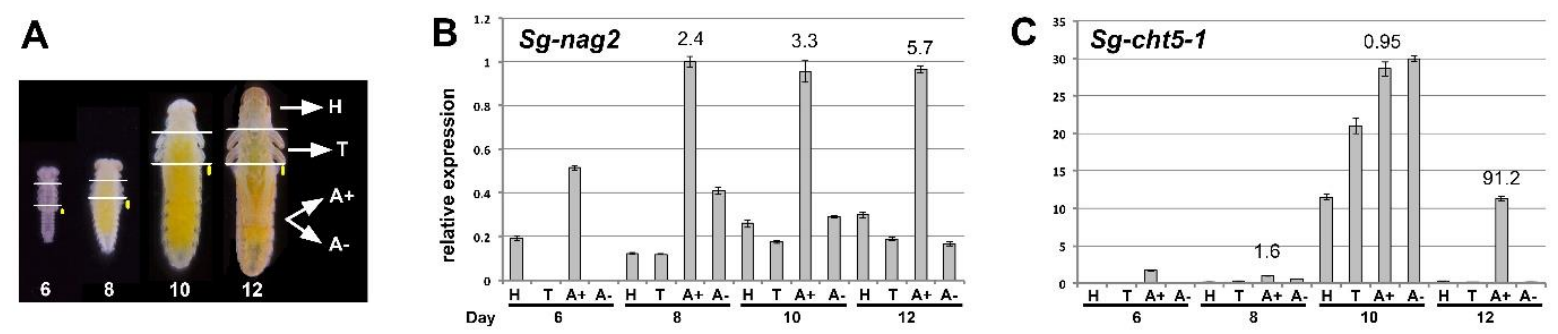

Figure 14. Real-time RT-PCR expression analysis of Sg-nag2 and Sg-cht5-1 on cDNA from parts of Schistocerca embryos. A. cDNA was prepared from mRNAs isolated from parts of embryos at the age of 6, 8, 10 and 12 days: $H$, head; $T$, thorax; $A+$, abdomen with pleuropodia; $A-$, abdomen without pleuropodia. For each age the same number of body parts was used (5-10) and RNA was resuspended in the same volume of water. The size of the pleuropodium is indicated by the yellow dot. B. and C. expression of Sg-nag2 and Sg-cht5-1, respectively. Analysis of 3-4 technical replicates is shown. Expression in A+8 (abdomen with pleuropodia at stage when the organs first become differentiated) was set as 1 . Numbers above A+ expression is fold change from A- of the same age.

The insect CHTs have been classified into several groups (Noh et al., 2018; Zhu et al., 2016), of which the major role in the digestion of cuticular chitin is played by Chitinase 5 and (perhaps with a secondary importance) by Chitinase 10 (Qu et al., 2014; Zhu et al., 2008) (Table 2; the classification of CHTs into 5 major groups that we use here is based on (Zhu et al., 2008)). Some chitinases, for example, are expressed in the gut, trachea and fat body, where they are likely involved in digestion of dietary chitin, turnover of peritrophic matrix and immunity, other chitinases organize assembly of new cuticle (Merzendorfer, 2013; Noh et al., 2018; Pesch et al., 2016).

Our transcriptome contains 16 full or partial transcripts of CHTs representing all of the major CHT groups (Table 2, Figure S5B, S6B). The pleuropodia specifically upregulate both of the genes for Chitinase 5, homologs of cht5-1 and cht5-2 from the locust Locusta migratoria (Li et al., 2015). One of the transcripts, Sg-cht5-1, was among the top 15 most highly expressed genes (Table 1). The predicted amino acid sequence contains a conserved catalytic domain and 
a signal peptide, and thus is likely to be active and secreted, respectively (Figure S5B). The other upregulated CHTs were homologs of Cht2 and Idgf. By contrast, the Schistocerca homolog of cht-10 that also has a role in cuticular chitin hydrolysis and required for larval moulting (Pesch et al., 2016; Zhu et al., 2008) had low expression in both legs and pleuropodia.

We next focused on the transcript of the major chitinase, Sg-cht5-1. Unlike the NAGs, both RNA-seq and real-time RT-PCR have shown that the expression of this CHT is low in the early secreting stages, rises only later around day 12 and reaches highest levels when the pleuropodia are already degenerating (day 13 and 14) (Figure 13 F,G,F'). Also real-time RT-PCR on cut embryos has shown that the pleuropodia are a major source of the Sg-cht5-1 mRNA on day 12 but not before (the high expression in the whole embryo on day 10 could be linked to the second embryonic moult and was also observed with Sg-cht7, although not with Sg-cht10, Figure S8). These data show that the pluropodia before hatching express a cuticle-degrading chitinase.

\subsubsection{Pleuropodia upregulate transcripts for some proteases that could digest a cuticle}

Our GO enrichment analysis has shown that the secreting pleuropodia are enriched in transcripts for genes associated with proteolysis (Figure 12, Table S11). Transcripts for proteases and their inhibitors are abundant among the top 10 per cent of the most highly "expressed" upregulated DEGs (Table 1). To see if the upregulated transcripts encode enzymes that are associated with digestion of insect cuticle, we compared our data with the enzymes identified in the complete proteomic analysis of the MF from the lepidopteran Bombyx mori (Liu et al., 2018; Zhang et al., 2014). Out of 69 genes that we searched, we found homologs or very similar genes in Schistocerca transcriptome for half of them (35). This made in total 75 transcripts, of which 27 were upregulated (7 among the top 10 per cent most highly expressed) and 15 downregulated (Table 3, S12). The prominent MF protease Carboxypeptidase A (Sui et al., 2009; Zhang et al., 2014) and the Trypsin-like serine protease known to function in locust moulting (Wei et al., 2007) were not upregulated in the pleuropodia. These data indicate that the pleuropodia upregulate transcripts for proteolytic enzymes associated with the degradation of the cuticle and would be able to contribute to digest the SC.

Table 3. MF proteases that were upregulated in the highly secreting pluropodia of Schistocerca.

\begin{tabular}{lllll}
\hline & Schistocerca & & \\
MF protein $^{\mathrm{a}}$ & Blast query & & \\
& transcript ID & homolog/similar & RPKM PLP & Fold change UP \\
\hline
\end{tabular}




\subsection{1}

Putative peptidase

Aminopeptidase $\mathrm{N}-12$

Neutral endopeptidase

Ecdysteroid-inducible

angiotensin-converting

enzyme

Carboxypeptidase E-like

Angiotensin-converting

enzyme-like

Aminopeptidase $\mathrm{N}$-like

Digestive cysteine protease

1, cathepsin L

Serine carboxypeptidase

Serine protease HP21

precurso

Trypsin-like serine protease

- fibroin heavy chain

Serine protease, Easter-like
SgreTa0000627

I3VR83 SgreTb0018983

Q9BLH1

SgreTa0002467

Q9BLH1

SgreTa0017692

similar

133.30

219.35

1457.47

similar

Q9NDS8

SgreTa0014009

Q9NDS8

SgreTa0017728

H9IST0 SgreTa0000925

H9IZ41 SgreTa0003298

H9JEW9 SgreTa0017219

H9JHZ1

SgreTa0000627

H9J242

SgreTa0017657

H9JJA9

SgreTa0017649

H9JPA8

SgreTa0001636

homolog

7578.31

153.48

Q2VG86

SgreTa0003188

homolog

485.97

837.45

\begin{tabular}{|c|c|c|c|c|c|}
\hline & Q2VG86 & SgreTa0003661 & homolog & 1332.79 & 45.18 \\
\hline & Q2VG86 & SgreTa0006780 & homolog & 103.37 & 14.76 \\
\hline & Q2VG86 & SgreTa0007424 & homolog & 29.62 & 79.13 \\
\hline & Q2VG86 & SgreTa0007425 & homolog & 123.69 & 72.31 \\
\hline & Q2VG86 & SgreTb0037249 & homolog & 21.76 & 249.74 \\
\hline & Q2VG86 & SgreTb0039879 & homolog & 305.63 & 544.04 \\
\hline & H9JLZ4 & SgreTa0010219 & similar & 46.12 & 20.75 \\
\hline & H9JLZ4 & SgreTb0039024 & similar & 11.71 & 22.11 \\
\hline Serine protease 1 & H9JXY6 & SgreTb0003860 & homolog & 1727.41 & 22.31 \\
\hline
\end{tabular}


a proteomic sequencing of MF of the lepidopteran Bombyx mori (Zhang et al., 2014; Liu et al., 2018)

${ }^{\mathrm{b}}$ Uniprot ID for blast on Schistocerca transcriptome

${ }^{\mathrm{c}}$ transcripts in bold were among the top $10 \%$ most highly "expressed" upregulated DEGs (Table 1)

${ }^{d}$ considered as homologous, if reciprocal blast retrieved the query sequence

\subsubsection{Pleuropodia are enriched in transcripts for immunity-related proteins}

An observation that was not anticipated was the upregulation of genes for proteins involved in immunity (Buchon et al., 2014; Lemaitre and Hoffmann, 2007) (Figures 12, 15, Table S13). This is especially interesting, because immunity related proteins have been found in the MF (Zhang et al., 2014). It is in agreement with that the cells in the pleuropodia are a type of barrier epithelium (Bergman et al., 2017; Buchon et al., 2014; Lemaitre and Hoffmann, 2007), which enables the contact between the organism and its environment. Barrier epithelia (e.g., the gut, Malpighian tubules or tracheae) constitutively express genes for immune defense.

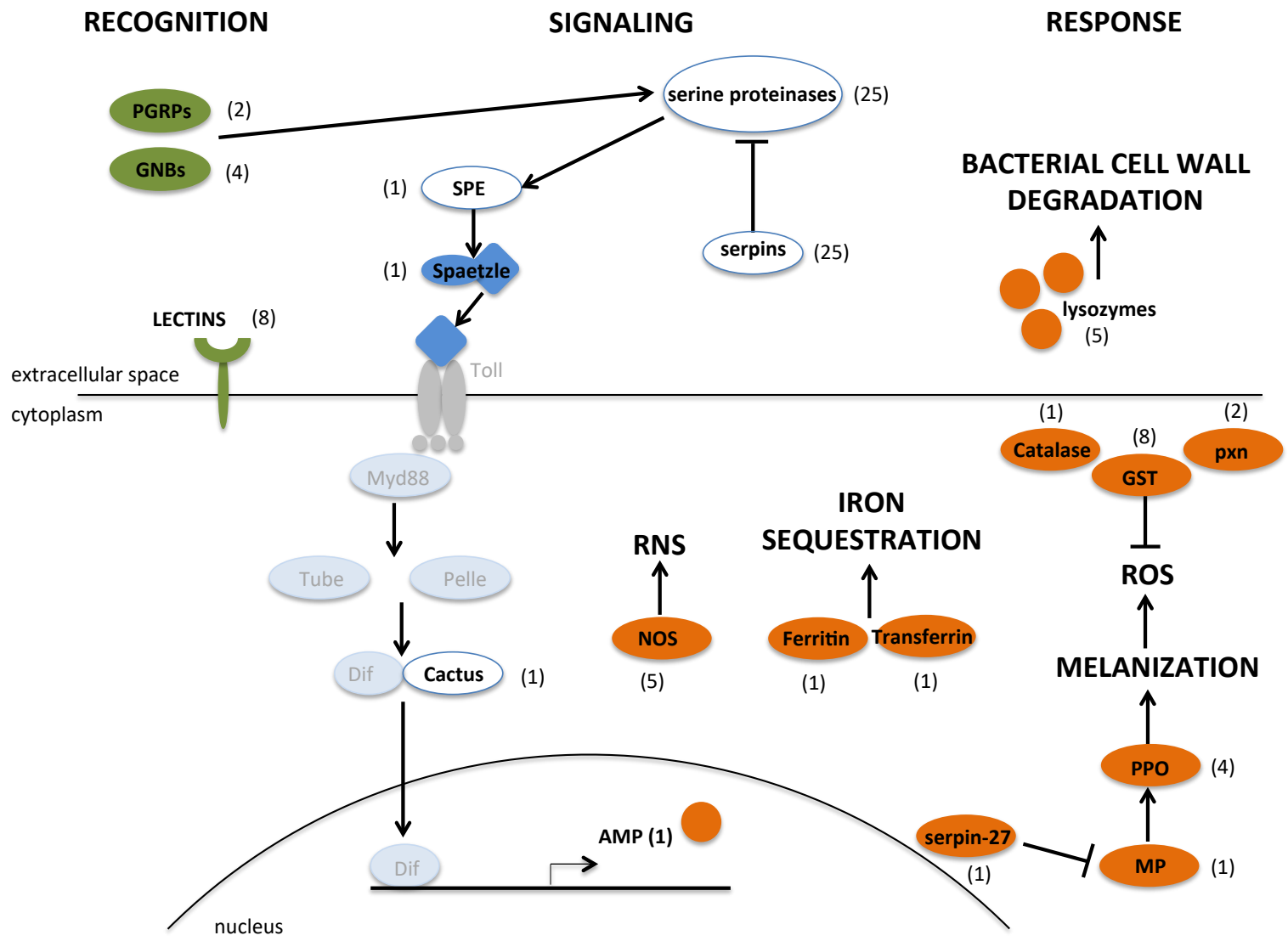

Figure 15. Schematic representation of the key immunity-related genes expressed in the highly secreting pleuropodia of Schistocerca. Proteins whose transcripts were found in the pleuropodia are in black, number in the brackets is the number of upregulated transcripts. Proteins whose transcripts were not upregulated are in 
grey. Out of the total 25 serine proteases and 25 serpins, 14 and 15 are known to function in Toll signaling, respectively. AMP, antimicrobial peptide; GNBP, gram-negative bacteria-binding protein; GST, glutathione Stransferase; MP, melanization protease; NOS, nitric oxide synthase; PGRP, peptidoglycan recognition preotein; PPO, pro-phenoloxidase; pxn, peroxiredoxin; RNS, reactive nitrogen species; ROS, reactive oxygen species; SPE, Spaetzle-processing enzyme.

In total we found upregulated 99 transcripts (13 per cent of the upregulated genes) for immunity-related proteins. These include proteins at all three levels, the pathogen recognition, signaling and response (Figure 15, Table S13). From the four signaling pathways, Toll was upregulated, but not IMD or JAK/STAT, and from the JNK signaling we found c-Jun. Genes for a range of immune responses were upregulated, including production of reactive nitrogen species (RNS), melanization, genes for lysozymes and one antimicrobial peptide (AMP) similar to Diptericin.

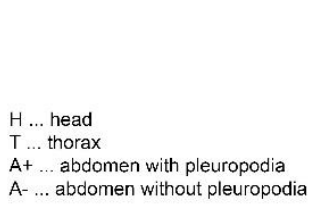

A
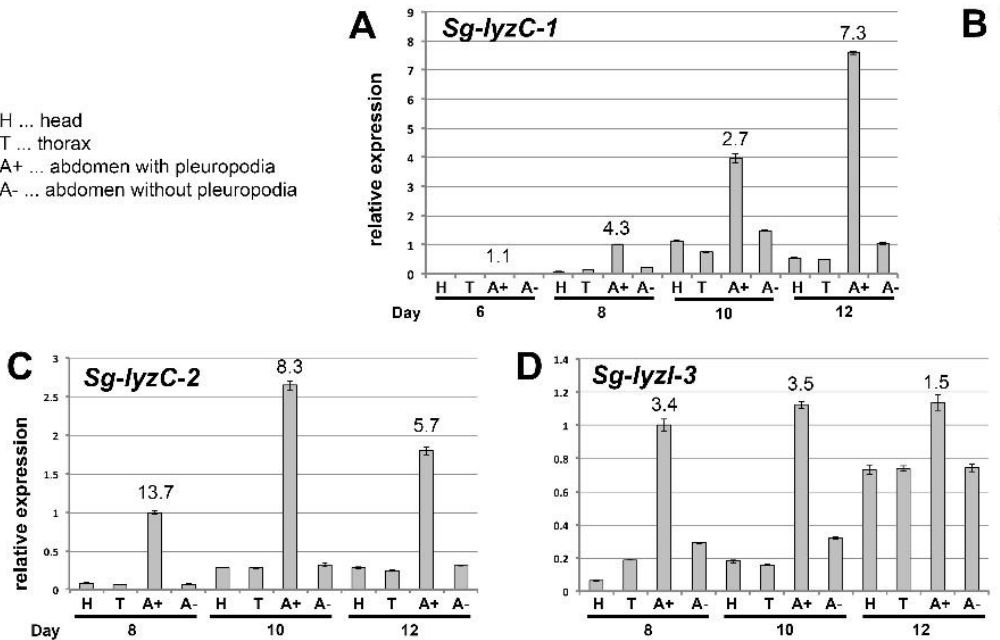
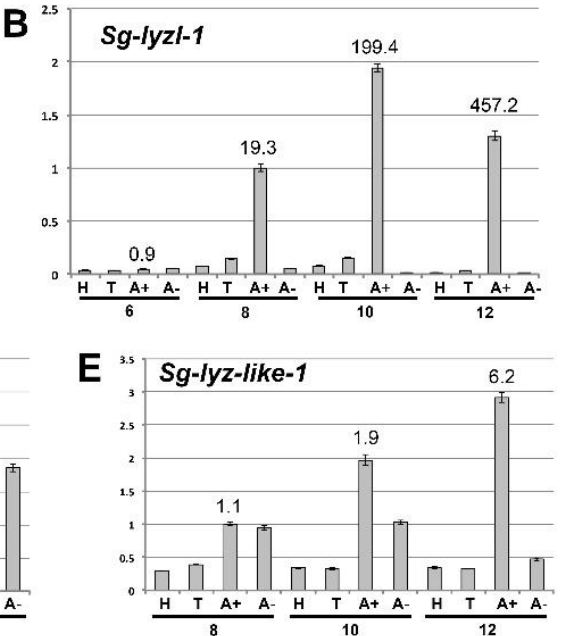

Figure 16. Real-time RT-PCR expression analysis of genes for lysozymes on CDNA from parts of Schistocerca embryos. cDNA was prepared from mRNAs isolated from parts of embryos at the age of 6, 8, 10 and 12 days. For each age the same number of body parts was used (5-10) and RNA was resuspended in the same volume of water. Analysis of 3-4 technical replicates is shown. Expression in A+8 (abdomen with pleuropodia at stage when the organs first become differentiated) was set as 1 . Numbers above A+ expression is fold change from A- of the same age.

The transcripts for lysozymes were among the most highly expressed (Table 1) and we chose to focus on them. Lysozymes are secreted proteins that kill bacteria by breaking down their cell wall. Our Schistocerca transcriptome contains 9 genes for lysozymes, 7 of which were upregulated (Table 4, Table S14). The second most highly expressed DEG was a transcript for a C-type lysozyme $(S g L y z C-1)$ that was previously shown to have anti-bacterial properties in Schistocerca (Mohamed et al., 2016) (Table 1). We examined expression of 5 selected genes on cut embryos by real-time RT-PCR (Figure 15). Our data showed that the pleuropodia are the major source of mRNAs for these genes. 
Table 4. RNA-seq differential gene expression of Schistocerca lysozymes in the highly secreting pleuropodia.

\begin{tabular}{|c|c|c|c|c|}
\hline Lysozyme type & Gene & UP/DOWN ${ }^{a}$ & Fold change & Expression $^{b}$ \\
\hline \multirow[t]{2}{*}{ C-type lysozyme } & $S g L y z C-1$ & UP & 336.64 & $2(0.26 \%)$ \\
\hline & $S g L y z C-2$ & UP & 134.71 & $37(4.74 \%)$ \\
\hline \multirow[t]{5}{*}{ I-type lysozyme } & SgLyzl-1 & UP & 550.26 & $7(0.90 \%)$ \\
\hline & $S g L y z I-2$ & $n s^{c}$ & & \\
\hline & SgLyzI-3 & UP & 30.62 & $76(9.73 \%)$ \\
\hline & SgLyzI-4 & DOWN & -34.41 & $1251(81.50 \%$ \\
\hline & SgLyzI-5 & ns & & \\
\hline \multirow[t]{2}{*}{ Lysozyme-like } & SgLyz-like-1 & UP & 192.68 & 150 (19.21\%) \\
\hline & SgLyz-like-2 & ns & & \\
\hline
\end{tabular}

\footnotetext{
a upregulated (UP)/ downregulated (DOWN)

${ }^{b}$ the DEGs were ranked according to their RPKM (in descending order), the number describes the position of the DEG in the ranked table; shading as in Table 2
}

${ }^{c}$ not significant

\subsubsection{The pleuropodia do not upregulate the pathway for ecdysone biosynthesis}

Previous work has suggested that pleuropodia may be embryonic organs producing the moulting hormone ecdysone (Novak and Zambre, 1974). During post-embryonic stages, ecdysone is synthesized in the prothoracic glands and several other tissues by a common set of enzymes (Niwa and Niwa, 2014; Ou et al., 2016), some which have been characterized in the locusts (Lenaerts et al., 2016; Marchal et al., 2012, 2011; Sugahara et al., 2017). As shown in Drosophila, these genes are expressed in diverse cell types in embryos, and when the larval prothoracic glands are formed their expression co-localizes there (Chávez et al., 2000; Niwa et al., 2004; Petryk et al., 2003; Warren et al., 2004, 2002).

Out of the nine genes critical for ecdysone biosynthesis, only one (dib) was upregulated in the highly secreting pleuropodia (Table 5, S15). One gene (spo) was downregulated. The pleuropodia are not enriched in the whole pathway at any time of development, including around katatrepsis, in which experiments supporting the synthesis of moulting hormone were carried out (Table S9, S16). Under the GO term "hormone biosynthetic process" enriched in the 
intensively secreting pleuropodia (Table S7, S17) we found a gene Npc2a that encodes a transporter of sterols including precursors of ecdysone. It is also required for ecdysone biosynthesis, but indirectly and in the cells it functions as a general regulator of sterol homeostasis (Huang et al., 2007). We conclude that our transcriptomic data provide little evidence that the pleuropodia are involved in ecdysone biosynthesis.

\subsection{Discussion}

\subsubsection{Pleuropodia of Schistocerca express genes for the "hatching enzyme"}

The first demonstration of the physiological role of the pleuropodia comes from the experiments carried out on a grasshopper Melanoplus (closely related to Schistocerca), by Eleanor Slifer (H. Slifer, 1937). When she took embryos before hatching (Figure 8) and separated anterior and posterior halves by ligation the SC was digested only in the part of the egg with the pleuropodia. Surgical removal of the pleuropodia prevented SC digestion in the whole egg. Slifer's hypothesis that the pleuropodia secrete the "hatching enzyme" was criticized by Novak and Zambre (Novak and Zambre, 1974): if the deposition and digestion of the $\mathrm{SC}$ is similar to the cuticle turnover during larval moulting, then the "hatching enzyme" is produced by the serosa. They believed that the pleuropodia reach the peak of their activity in embryos during katatrepsis (45\% development) and participate on SC digestion indirectly by secreting ecdysone to stimulate the serosa.

Our ultrastructural observations on staged pleuropodia of Schistocerca have shown that the glandular cells only begin to differentiate just at the time of katatrepsis (45\% DT) and do not secrete at that time. This would explain why no digestive effect on the SC was detected by Novak and Zambre (Novak and Zambre, 1974) using a homogenate from Schistocerca pleuropodia isolated at this stage. The release of granular secretion starts just before the dorsal closure (55\% DT) and intensifies before hatching. This is in agreement with previous observations on some stages of the pleuropodia in other orthopterans (Louvet, 1975; Viscuso and Sottile, 2008).

Our RNA-seq analysis revealed that the secreting pleuropodia highly express genes encoding enzymes that are capable of digesting a typical chitin-protein insect cuticle. These include genes for proteolytic enzymes similar to those present in the moulting fluid and cuticular chitin-degrading NAGs and Chitinase 5. The pleuropodia also express genes for Chitinase 2 and Idgf, which have low effect on cuticular chitin digestion, but were shown to 
organize proteins and chitin fibres during cuticle deposition (Pesch et al., 2016). These CHTs may organize the fibres in the cuticle secreted by the pleuropodia (Figure 9).

In combination with RT-PCR we showed that, while the expression of the Sg-nag1 and Sgnag2 started to rise in parallel with the differentiation of the glandular cells, the Sg-cht5-1 and Sg-cht5-2 transcripts raised shortly before hatching. Chitinase 5 is a critical chitin-degrading chitinase in insects: it is highly abundant in the moulting fluid and its silencing in diverse insects including locusts leads to failure in larval moulting (Li et al., 2015; Pesch et al., 2016; Xi et al., 2015; Zhang et al., 2014; Zhu et al., 2008). Our data indicate that the sudden rise in the expression of cht5 in the pleuropodia at the end of embryogenesis and presumably secretion of this $\mathrm{CHT}$ into the extraembryonic space is the key component of the "hatching enzyme" effect (H. Slifer, 1937; Slifer, 1938) in locusts and grasshoppers.

\subsubsection{Pleuropodia in some other insects could secrete the "hatching enzyme" and their} function may also vary among species

There is evidence to suggest that the process occurs similarly in some insect. As in orthopterans, the pleuropodia of the rhagophthalmid beetle Rhagophthalmus ohbai release secretion soon after katatrepsis and SC rapidly degrades just shortly before hatching (Kobayashi et al., 2003). In the large water true bugs from the family Belostomatidae, the male carries a batch of eggs on his back. It is believed that the detachment of the eggs just before hatching is also caused by the secretion from the pleuropodia (Tanizawa et al., 2007).

The molecular mechanism of SC degradation may also vary between insects and as previously hypothesized (Novak and Zambre, 1974) the serosa may also contribute to the SC degradation. The serosa of the beetle Tribolium, expresses cht10 and cht7 (Jacobs et al., 2015), of which the former $\mathrm{CHT}$ is important for cuticular chitin digestion. Silencing of cht10, but not cht5 prevented larvae from hatching (Zhu et al., 2008). Transcripts for cht10 were not upregulated in the pleuropodia of Schistocerca. This suggests that the SC is degraded by enzymes produced by both, the serosa and the pleuropodia and that the indispensable roles in cuticle digestion are played by different enzymes in different insects.

In some insects the pleuropodia may not be involved in hatching but have another function. In the viviparous cockroach Diploptera punctata (Stay, 1977), the secretion from the pleuropodia is very low and the large pleuropodia of the melolonthid beetle Rhizotrogus majalis have not been observed to produce any secretion granules at all (Louvet, 1983). In dragonflies, 
one of the more basal lineages of insects, the secretion likely has a different function than in orthopterans, because their SC is not digested before hatching (Andō, 1962). The special epithelium in the pleuropodia shares features with transporting epithelia (Louvet, 1973; Stay, 1977) that function in water transport and ion balance (Berridge and Oschman, 1972). Our data do not exclude this function, but it is yet to be tested.

3.4.3. The pleuropodia of Schistocerca are enriched in transcripts for enzymes functioning in immunity

We found that many of the genes expressed in the pleuropodia encode proteins involved in immunity (Lemaitre and Hoffmann, 2007). This indicates that the pleuropodia are also organs of epithelial immunity, similar to other barrier epithelia in postembryonic stages (such as the gut) (Bergman et al., 2017), which are in a constant contact with microorganisms. The pleuropodia differ from such tissues in that they are not directly exposed to the environment, but enclosed in the eggshell, seemingly limiting their contact with microorganisms. Proteins associated with immune defense are also found in the MF (Zhang et al., 2014), where they prevent invasion of pathogens through a "naked" epidermis after the separation of the old cuticle from the epidermis in the process of apolysis. As found in the beetle Tribolium, during the early embryonic stages the frontier epithelium providing the egg with an immune defense (Jacobs et al., 2014) is the extraembryonic serosa. The serosa starts to degenerate after katatrepsis and disappears at dorsal closure (Panfilio, 2008). The pleuropodia of Schistocerca differentiate just before dorsal closure, suggesting that they take over this defense function in late embryogenesis. It will be interesting to clarify in the upcoming research whether apart from their role in hatching the pleuropodia are important organs for fighting against potential pathogens that have gained access to the space between the embryo and the eggshell.

\subsubsection{Conclusions}

The pleuropodia of Schistocerca have morphological markers of high secretory activity in the second half of embryogenesis after the definitive dorsal closure is finished. Transcriptomic profiling indicate that the conclusions that Eleanor Slifer drew from her experiments over eighty years ago that the pleuropodia secrete cuticle degrading enzymes, were correct. The pleuropodia likely have other functions, such as in immunity. The pleuropodia are specialized embryonic organs and an important though neglected part of insect physiology. 


\subsection{Material and Methods}

\subsubsection{Insects}

Schistocerca gregaria (gregarious phase) were obtained from a long-term, partly inbred colony at the Department of Zoology, University of Cambridge. Eggs were collected into aluminium pots filled with damp sand. The pots were picked up after 2 (most samples) or 4 hours and incubated at $30^{\circ} \mathrm{C}$.

\subsubsection{Description of embryonic stages}

Embryos and appendages were dissected in phosphate buffer saline (PBS). Whole eggs were bleached in 50 per cent household bleach to dissolve the chorion. All were photographed in water or PBS using the Leica M125 stereomicroscope equipped with DFC495 camera and associated software. Photos were processed using Adobe Photoshop CC 2017.1.1. Photos of eggs and embryos that illustrate the stage (Figure 8A and S1) had the background cleaned using the software (removal of the tools that hold the photographed objects in place).

\subsubsection{Immunohistochemistry on paraffin sections}

Embryos were dissected in PBS and pieces including posterior thorax and anterior abdomen (older embryos) or mid thorax plus whole abdomen (young embryos) were fixed in PEMFA (4\% formaldehyde in PEM buffer: $100 \mathrm{mM}$ PIPES, $2.0 \mathrm{mM} \mathrm{EGTA}, 1.0 \mathrm{mM} \mathrm{MgSO} 4$ ) at room temperature (RT) for 15-30 minutes, then washed in PBT (PBS with $0.1 \%$ Triton-X 100) and stored in ethanol at $-20^{\circ} \mathrm{C}$.

Samples were cleared in 3×10 minutes in Histosol (National Diagnostics) at RT, infiltrated with paraffin at $60^{\circ} \mathrm{C}$ for $2-3$ days, embedded in moulds and hardened at RT. Sections $6-8 \mu \mathrm{m}$ thick were prepared on a Leica RM2125RTF microtome. The slides with sections were washed with Histosol, ethanol, then step wise re-hydrated to PBT. Incubations were carried out in a humidified chamber. Slides were blocked with 10\% sheep serum (Sigma-Aldrich) in PBT for 30 minutes at RT, incubated with Phospho-Histone H3 antibody (Invitrogen) diluted with PBT 1:130 at $4^{\circ} \mathrm{C}$ overnight, washed and incubated with Alexa Fluor 568 anti-rabbit secondary antibody (Invitrogen) diluted 1:300 at RT for 2 hours, washed and incubated with DAPI (Invitrogen) diluted 1:1000. Sections were imaged with a Leica TCS SP5 confocal microscope and photos processed using Fiji (https://fiji.sc). 
3.5.4. Transmission (TEM) and scanning (SEM) electron microscopy

For TEM embryos were removed from the chorion in PBS and pieces of posterior thorax to anterior abdomen were fixed in 2.5-3.0\% glutaraldehyde in $0.1 \mathrm{M}$ phosphate buffer $\mathrm{pH} 7.2$ for a few hours at room temperature and then at $4^{\circ} \mathrm{C}$ for several days. Each pleuropodium and leg were then separated and embedded into $2 \%$ agar. Small cubes of agar with the tissue were incubated in osmium ferrocyanide solution (3 \% potassium ferricyanide in cacodylate buffer with $4 \mathrm{mM}$ calcium chloride) for $1-2$ days at $4^{\circ} \mathrm{C}$, then in thiocarbohydrazide solution $(0.1 \mathrm{mg}$ thiocarbohydrazide from Sigma-Aldrich, and $10 \mathrm{ml}$ deionized water dissolved at $60^{\circ} \mathrm{C}$ ) and protected from light for 20-30 minutes at RT, then in 2\% aqueous osmium tetroxide $30-45$ minutes at $\mathrm{RT}$ and in $1 \%$ uranyl acetate (maleate buffered to $\mathrm{pH} 5.5$ ) at $4^{\circ} \mathrm{C}$ overnight. Washing between each step was done with deionized water. Samples were dehydrated in ethanol, washed with dry acetone, dry acetonitrile, infiltrated with Quetol 651 resin (Agar Scientific) for 4-6 days and hardened in moulds at $60^{\circ} \mathrm{C}$ for $2-3$ days. Semithin sections were stained with toluidine blue. Ultrathin sections were examined in the Tecnai G280 microscope.

For SEM whole embryos were dissected out of the chorion in PBS, fixed in $3 \%$ glutaraldehyde in phosphate buffer similarly as above. They were post-fixed with osmium tetroxide, dehydrated through the ethanol series, critical point dried, gold coated, and observed in a FEI/Philips XL30 FEGSEM microscope. Photos from TEM and SEM were processed using Adobe Photoshop CC 2017.1.1.

\subsubsection{Preparation of the reference transcriptome}

Whole embryo transcriptome: Eggs from each 1-day egg collection incubated for the desired time were briefly treated with $50 \%$ bleach, washed in distilled water and frozen in liquid nitrogen. Total RNA was isolated with TRIzol reagent (Invitrogen), treated with TURBO DNase (Invitrogen) and purified on a column supplied with the RNAeasy Kit (Quiagen). The purified RNA from each day (14 samples) was pooled into 4 samples: day 1-4, 5-7, 8-10 and 11-14. 10 $\mu \mathrm{g}$ of RNA from each of the 4 samples was sent to BGI (Hong Kong). The total RNA was enriched in mRNA by using the oligo(dT) magnetic beads and cDNA library was prepared. 100 bp pairedend (PE) reads were sequenced on Illumina HiSeq 2000; numbers of the reads obtained are in Table S2. Non-clean reads were filtered using filter_fq software (removes reads with adaptors, reads with unknown nucleotides larger than $5 \%$ and low quality reads). Transcripts from all samples were assembled separately using the Trinity software (release 20130225) (Grabherr et 
al., 2011) with parameters: --seqType fq --min_contig_length 100; --min_glue 4 -group_pairs_distance 250; --path_reinforcement_distance 95 --min_kmer_cov 4. Transcriptes from the 4 assemblies were then merged together to form a single set of non-redundant transcripts using TGICL software (v2.1) (Pertea et al., 2003) with parameters: -I 40 -c 10 -v 20.

Legs and pleuropodia transcriptome (age about 8.5-8.75 days): The appendages were dissected in cold RNase-free PBS (treated with diethyl pyrocarbonate) and total was RNA isolated and cleaned as described above. $10 \mu \mathrm{g}$ of RNA from each leg sample and pleuropodium sample were transported to the Eastern Sequence and Informatics Hub (EASIH), Cambridge (UK). cDNA libraries were prepared including mRNA enrichment. 75 bp PE reads were sequenced on Illumina GAIIX; numbers of the reads obtained are in Table S2. The reads were trimmed to the longest contiguous read segment for which the quality score at each base is greater than a Phred quality score of $Q=13$ (or 0.05 probability of error) using the program DynamicTrim (v. 1.7) from the package SolexQA ((Cox et al., 2010) http://solexaqa.sourceforge.net/). The trimmed reads were then filtered to remove sequence adapter using the program cutadapt (v. 0.9; http://code.google.com/p/cutadapt/). Sequences shorter than 40 base pairs were discarded. Trimmed reads were used to de novo assemble the transcriptome using Velvet (v. 1.1.07; (Zerbino and Birney, 2008); http://www.ebi.ac.uk/ zerbino/velvet/) (commands: -shortPaired -fastq; -short2 -fastq; read_trkg yes) and Oases (v. 0.2.01; (Schulz et al., 2012); http://www.ebi.ac.uk/ zerbino/oases/) (commands: -ins_length 350). Velvet is primarily used for de-novo genome assembly; here, the contigs that were output by Velvet were used by the complementary software package Oases to build likely transcripts from the RNA-seq dataset. K-mer sizes of 21, 25 and 31 were attempted for the two separate samples as well as the combined samples and optimal K-mer sizes of 21 were found for both samples.

Transcripts for the reference transcriptome were selected from the embryonic and legs and pleuropodia transcriptome. The transcripts were first merged with evigene ((Gilbert, 2013) version 2013.03.11) using default parameters. Because this selection of transcripts eliminated some genes (gene represented by zero transcripts, although the transcripts were present in the original transcriptomes), we repeated the step with less strict parameters (cd-hit-est - version 4.6, with -c 0.80 -n 5). This second selection contained several genes represented by more transcripts, thus we aligned selection 1 and 2 to each other to identify, which genes in selection 
1 were missing. Selection 1 was then completed with the help of selection 2 by adding the missing transcripts. The quality and completeness of the resulting transcriptome was assessed and edited in the following steps. First, we removed several redundant transcripts manually: these were found by blasting diverse insect sequences (queries) against the Schistocerca transcriptome using the local ViroBLAST interface (Deng et al., 2007). Some transcripts were edited manually, such as when we found that two transcripts were combined into one, resulting in an alignment against two protein sequences (Schistocerca transcript blasted against NCBI database) we split the respective transcripts. Second, we blasted the whole transcriptome against itself and removed redundant sequences, if the alignment was spanning at least 300bp with a sequence identity of at least 98\% (Blast+ suite, version 2.6.0) (Camacho et al., 2009). The longer transcript was kept in all cases. Transcripts shorter than 200 bp were discarded. All these steps were carried out in R (R Development Core Team, 2008) and sequences were handled using the Biostrings package (Pagès et al., 2017).

\subsubsection{Sequence analysis}

Basic transcript analysis was done by CLC Sequence Viewer7 (QIAGEN). Signal peptide and transmembrane regions were predicted by Phobius (Käll et al., 2007); http://phobius.binf.ku.dk/index.html). To annotate the newly assembled transcriptome, the freely available annotation pipeline Trinotate (version 3.1.1) was used (Haas et al., 2013). The longest candidate ORF of each sequence was identified with the help of the inbuilt TransDecoder (Haas et al., 2013); https://github.com/TransDecoder/TransDecoder/wiki) software.

A blast was run against Uniprot sequences specific for Schistocerca gregaria, Locusta migratoria, Apis melifera, Tribolium castaneum, Bombyx mori and Drosophila melanogaster (blastx with default parameter and -max_target_seqs 1) and against nr database using Blast2GO (Götz et al., 2008).

\subsubsection{RNA-seq expression analysis}

Pleuropodia and hind legs from embryos at the same age (day 4, 5, 6, 7, 8, 10, 11, 12 and 13) were dissected in cold RNase-free PBS and total RNA was isolated as described for samples for the reference transcriptome, but cleaned with RNA Clean \& Concentrator (Zymo Research). $1 \mu \mathrm{g}$ of RNA from each sample was sent to BGI (Hong Kong). The mRNA enrichment 
and cDNAs preparation as described above. 50 bp single-end (SE) reads were sequenced on Illumina HiSeq 2000. Over 45 million reads were sequenced from each sample (Table S2).

A pair of samples from mixed embryos 8-9 days that was used for the preparation of the reference transcriptome (described above) was also included in the expression analysis, but prior to mapping, the 75bp PE reads were trimmed to $50 \mathrm{bp}$, using Trimmomatic in the pairedend mode (version 0.36) using the CROP function (CROP:50) (Bolger et al., 2014). A single pleuropodium or leg sample was sequenced from each stage.

The quality of the sequenced reads was assessed with the help of the FastQC software. All samples consistently showed a Per base sequence quality of $>30$. Reads were mapped to the Reference transcriptome with Bowtie2 (version 2.2.5) using default parameter and the local alignment mode (Langmead et al., 2009). The trimmed pairs of reads were concatenated for each stage and treated as single reads. A PCA plot was generated to assess if differences in sequencing type and processing (SE samples and PE samples day 8-9) had an effect, which was not the case. This plot was prepared by using the plotPCA() function in the DESeq2 R package (Love et al., 2014); the count matrix was transformed with the rlog() function. The R package HTSFilter (Rau et al., 2013) was used with default parameters to filter constantly low expressed genes and 12988 transcripts were left.

The differential expression analysis was performed with the NOISeq R package (2.22.1; (Tarazona et al., 2011). Reads were first normalized using the RPKM method (Mortazavi et al., 2008). We used NOISeq-sim to find the differentially expressed genes between legs and pleuropodium for each stage with the following parameters: $k=N U L L$, norm $=" n ", p n r=0.2$, nss $=5, v=0.02, \mathrm{l}=1$, replicates $="$ no", following the recommendations by the authors for simulation of "technical replicates" prior to differential expression analysis without replicates. Additionally differentially expressed genes between active pleuropodia and legs at the same stage were assessed (treating samples from day 10, 11 and 12 as replicates) using the NOISeqreal algorithm with the following parameters: $k=0.5$, norm=" $n "$, factor="type", nss=0, $\mid c=1$, replicates = "technical". To define significantly, differentially expressed genes, the probability ("prob") threshold was set at 0.7 for single stage comparisons and 0.8 for the triplicated comparison, RPKM $\geq 10$ and fold change $\geq 2$ for both single stage and triplicated comparisons (based on the expression of the genes whose expression dynamics in the pleuropodia were already known, Table S4). 


\subsubsection{GO enrichment}

The transcriptome was blasted against the whole UniProt/Swiss-Prot database to assess the corresponding GO terms. Only blast hits with an e-value $<=1 \mathrm{e}-5$ were considered for the subsequent GO annotation. GO enrichment of differentially expressed genes was performed using the R package GOSeq (version 1:30.0, (Noh et al., 2018) implemented in the Trinotate pipeline (see above). Enriched GO-terms were summarized and visualized with REVIGO (Supek et al., 2011). Dot plots were prepared from DEGs selected at thresholds: RPKM $>50$, fold change $>3$.

\subsubsection{Real-time RT-PCR}

Tissues were dissected, total RNA was isolated and DNase treated the same way as for sequencing and cleaned with RNA Clean \& Concentrator (Zymo Research). cDNA was synthesized with oligo-dT primer (Invitrogen) $0.5 \mu \mathrm{g}$ (legs, pleuropodia) or $1 \mu \mathrm{g}$ (pieces of embryos) of the RNA using ThermoScript RT-PCR System (Invitrogen) at $55^{\circ} \mathrm{C}$. The cDNA was diluted to concentration $40 \mathrm{ng} / \mu \mathrm{l}$ and $5 \mu \mathrm{l}$ was used in a reaction containing $10 \mu \mathrm{l}$ of SYBR Green PCR Master Mix (Applied Biosystems) and $5 \mu$ l of a 1:1 mix of forward and reverse primers (each $20 \mathrm{nM}$ in this mix). Reactions were run in the LightCycler480 (Roche) and analyzed using the associated software (release 1.5.0 SP1) according to the comparative Ct method and normalized to the eEF1 $\alpha$ gene. Primers (Table S18) were designed with Primer3PLUS program (Untergasser et al., 2007). To check for the presence of a single PCR product, the melting curve was examined after each run and for each pair of primers at least 2 finished runs were visualized on a $2 \%$ agarose gel.

The program was: denaturation: $95^{\circ} \mathrm{C}$ for 10 minutes $(1 \mathrm{cycle})$, amplification: $95^{\circ} \mathrm{C}$ for 10 seconds, $60^{\circ} \mathrm{C}$ for 15 seconds, $72^{\circ} \mathrm{C}$ for 12 seconds ( 40 cycles) melting: $95^{\circ} \mathrm{C}$ for 5 seconds, $60^{\circ} \mathrm{C}$ for 1 minute, $95^{\circ} \mathrm{C}$.

\subsection{List of abbreviations}

CHT: chitinase, DEG: differentially expressed gene; EC1, EC2: the first and the second embryonic cuticle, respectively; GO: gene ontology; LEG: hind leg(s); MF: moulting fluid; NAG: B-N-acetyl-hexosaminidase; PCA: principal component analysis; PLP: pleuropodium (pleuropodia); RPKM: reads per kilobase of transcript per million reads mapped; SC: serosal cuticle 


\subsection{Data availability}

The sequencing data generated and analyzed during the study are available in the NCBI repository, BioProject ID PRJNA524786 (the reference transcriptome has the accession number GHHP00000000, the version described in this paper is the first version, GHHP01000000).

\subsection{Competing interests}

The authors declare that they have no competing interests.

\subsection{Funding}

This work was supported by Human Frontier Science Program (Long-Term postdoctoral fellowship LT000733/2009-L), Biotechnology and Biological Sciences Research Council (grant number grant BB/ K009133/1), Isaac Newton Trust (University of Cambridge) and BalfourBrowne Fund (University of Cambridge).

\subsection{Author's contributions}

BK initiated the study, designed research, carried out all experimental work, supervised the bioinformatics analysis, interpreted the data and wrote the paper; EB performed majority of the bioinformatics analysis and edited the draft; $A C$ carried out the initial steps in the selections of transcripts for the reference transcriptome and did a preliminary expression analysis. All authors read and approved the manuscript.

\subsection{Acknowledgements}

Majority of the work was carried out in the lab of Michael Akam (University of Cambridge) and the data analysis was finished in the lab of Gregor Bucher (University of Göttingen); BK thanks to both for hosting and financial support. Electron microscopy was done at the Cambridge Advanced Imaging Centre (University of Cambridge). Immunolabeling was done in the lab and with help of Andrew Gillis. Stereomicroscopic pictures were taken in the lab of Paul Brakefield. We also thank for help and advice to Ken Siggens, Jenny Barna, Jeremy Skepper and lab, Steven Van Belleghem, Barry Denholm, Jan Sobotnik, and Gareth Griffiths, for scripts to Erik Clark and Simon Martin. We thank to Michael Akam, Siegfried Roth, Stuart Reynolds, Nico Posnien and Maurijn van der Zee for comments on the manuscript. 


\subsection{Supplementary Figures}

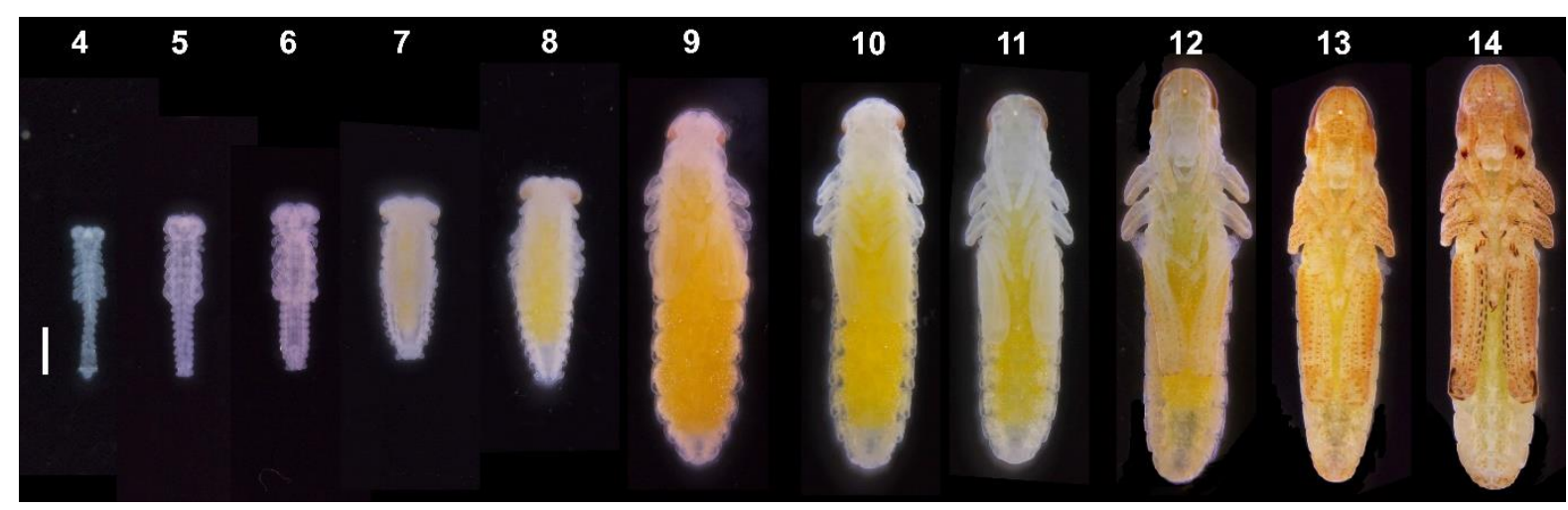

Supplementary Figure 1. Schistocerca embryonic stages used in this study. Images of live embryos dissected out of the eggs; imaged under a stereomicroscope. Eggs and embryos of Schistocerca typically slightly vary in size. Numbers indicate age in days. Scale bar: $1 \mathrm{~mm}$. Background in photos was cleaned (see Materials and Methods).

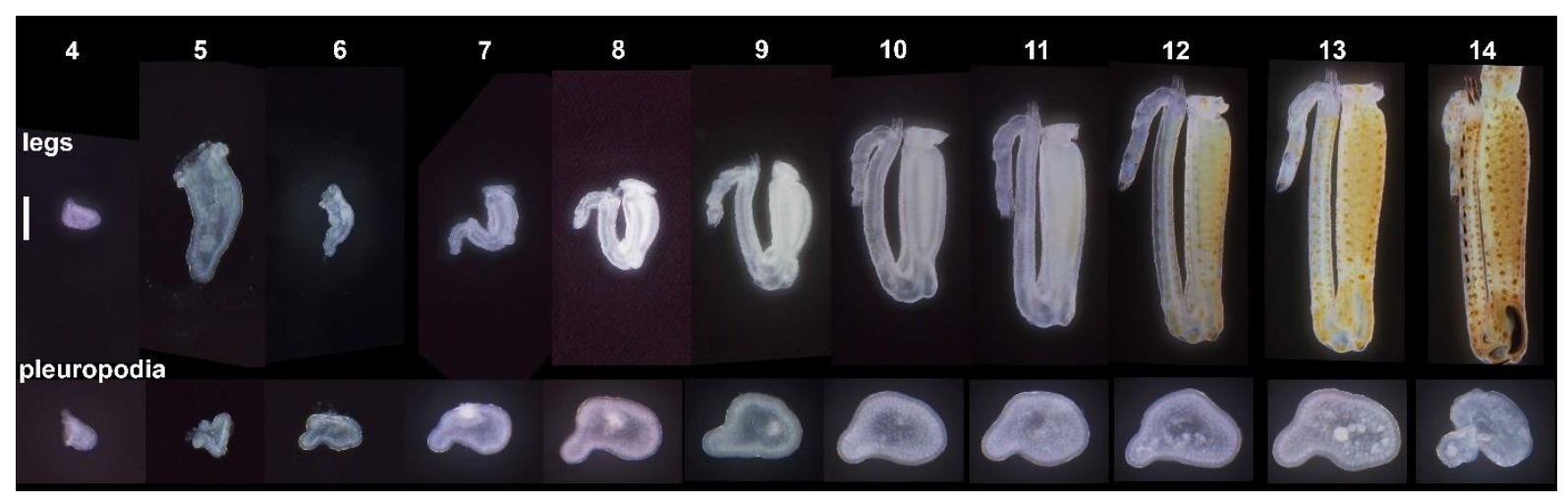

Supplementary Figure 2. External features of developing hind legs and pleuropodia. Compare the sizes of the appendages; imaged under a stereomicroscope. Numbers indicate age in days. Scale bar: $0.2 \mathrm{~mm}$ for all pleuropodia and for legs at days 4 and 5; $0.5 \mathrm{~mm}$ for legs at days 6-14. 


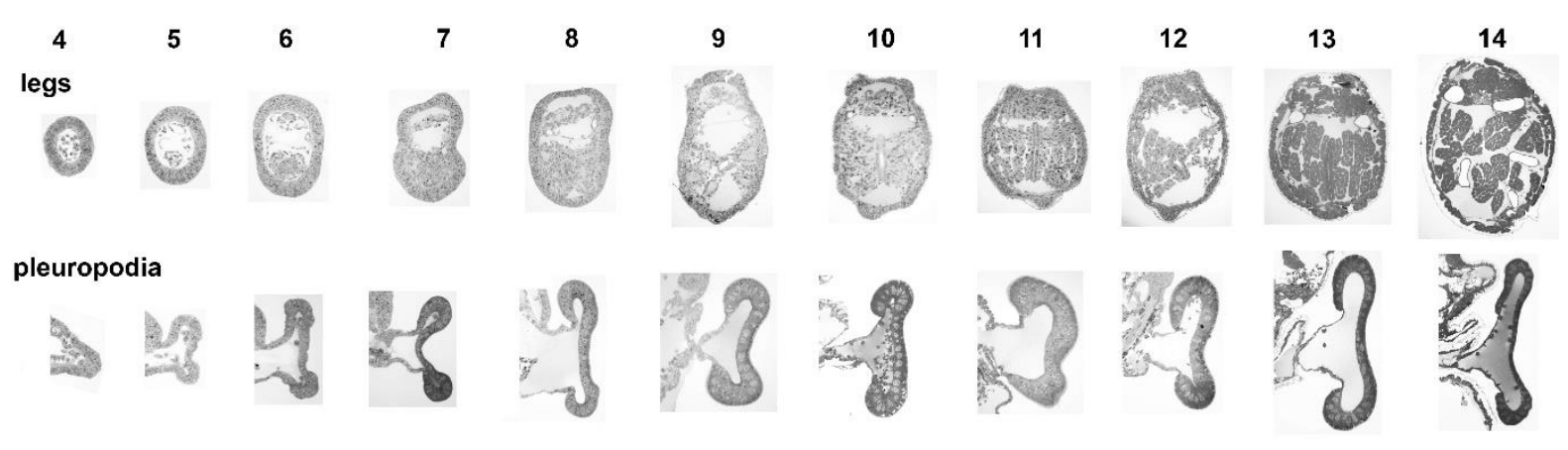

Supplementary Figure 3. Figure S3. Cross-sections through developing hind legs and pleuropodia. Toluidine blue stained semi-thin sections of appendages embedded in epoxy resin. Numbers indicate age in days.
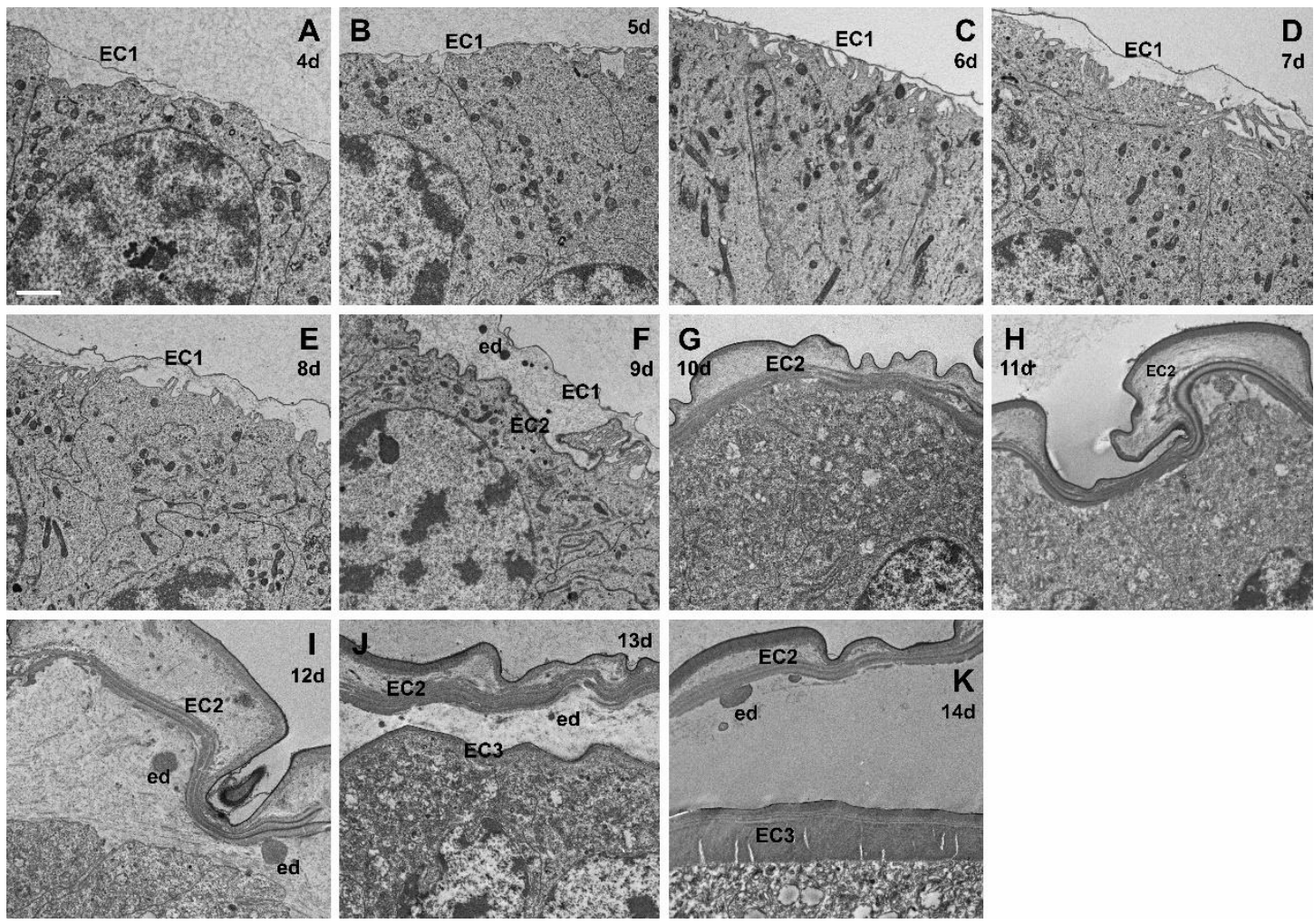

Supplementary Figure 4. Ultrastructure of epidermal cells in developing hind legs. TEM micrographs. Compare with pleuropodia in Figure 3. Note the three different cuticles and appearance of ecdysial droplets (ed) during embryonic moulting. EC1, EC2, EC3, the first, the second and the third embryonic cuticle, respectively (EC3 becomes the cuticle of the first instar larva). Scale bar: $2 \mu \mathrm{m}$. 
Chapter I - Transcriptomics supports that pleuropodia of insect embryos function in degradation of the serosal cuticle to enable hatching

(A)

Sg-nag1

MSVISTTVLVFALYGIFSCFATQAEEERPVWTWECRESRCEKVAAGEGEAQSLGACRLSCDPWATLWPRPRGGLQRTPGRLLALNPYSVSVEAAGRDLQP GVRQLLQEAGRIFHRKVERKARTGAKLRSAGERRSLFVTLTVSDGQTRSFHTDTSEAYSLSISEVTAGRVNAAVTADTFFGARHALETLYQLIVYDDINKQLLL LSEINLSDSPAFPHRAIALDTARSYFSVASIKRTIDAMAANKLNTFHWHITDSHSFPFVSETFPKLSQYGAYSPEKVYTPDEIKSVVEYARVRGVRIIPEFDAPAH VGEGWOWVGDNATVCFKADPWSOYCVEPPCGQLNPTSEKMYQVLAGIYKDMLNVFDSDVFHMGGDEVNMNCWNTSEVITDWMDANGIPRTEEGL HELWDRFQSRAYSLLAEANGKKELPVILWTSTLTDVAHVDKYLDNKRYIIQIWTRGTDLVIPELIRKGFRVIFSNYDALYFDCGFGAWIGSGNNWCSPYIGW QKVYDNNVWDLLSAFGIDVGEGSEARKLVLGSEAALWSEQADEFALDGRLWPRAAALAERLWTDPVEGWMSAEHRFLIQRQRLVDEGIAADTIEPEWCL QNQGHCYA*

Sg-nag2

MAPAPPAPHLLALTLLLTLLPSPPVVWANSPRWOWTCDSGLCVRSEAPPEPRLDAELEETVVQRSVHRLRPPWPSHELCRLTCGPYGALWPRPTGHTLIA DALVPFNPATARFDLSAVAGEQGRELVDAASRRWVRDLQHALAASGGHGGGGEVAGAAAGAGTDVLVTVLTRDSPQALSWETDETYTLDVASSGHEVR VTVSAQTVWGALHGLTSLRQLVGCCSEDGAALMVAEARIVDGPVYAHRGLLLDTARNFLPVETMMATMDAMAASKLNVLHWHATDSQSFPLLLPRVP QLARWGAFSARETYSSQQVSALLGYAHARGIRLLLELDAPAHSGQGWQWGEAEGLGALALCVGQQPWRRLCIQPPCGQLNPANPRLVGVLADVYRDVV DLWPPGQPLHMGGDEVSYSCWNSSAEVLEYMSKRRWDRSQDGFLRLWAEFQQAALEALDAARGSSDVPAILWSSHLTRPGNIERFLNSSRYVIETWVE GGDPLPQQLLALGYRLVVATKDAWYLDHGFWGSTRYHDWKAVYSNRLPGSMAQGVLGGEVASWGELVDDQSLDARLWPRAAALAERLWSNPGASAR EAEPRLHAHRARLVAAGVRPEALAPRYCVLNEGACQ*

\section{$S g-f d l$}

MSRQRLLWRLLGAALALTVAGLAAPPLFRLLVSPHSAANSVAGRRVYSSDPGPWTWSCESGRCVRALWQGGTQVSLDTCQWTCAGWEAPLWPRPTGA LRLANSTAALPEDLDVRLRLSGPQHEDTRGLLAAATERLARHLQLVRPAWAGRVACDAARGATVARLTVFVKLDADGSRPTGQLTLDTDESYRLQVRRESO DLQAEIDARSFFGARHALETLSQLAWWDPVSGCVHILDSAIVKDAPKFRHRGLMVDTARNFIPLEALQRTVDAMASNKLNTLHWHLTDSTSFPYLSRALPT MARYGAYSPEQVYSMEDVSRLAEFARERGVRLVVELDVPAHAAAGWPTEQVSCSEQRGSAANAPLVQQQQHRQNEDNGLQYRQEERRERRAQHGGE QQPAWWELCGQPPCGQLPPADEAAFGTLRTLYQELRQASGASDVAHLGGDEVSAECWGGVRGERLWSLWGGFMRRAHRELVAASQGNPPTAVLVW SSELTAPHNLRRYFDPSTHVVQVWGGSKWNETLPVLLAGFRAVVSHVDAWYLDCGWGDFRSGGPGPCGPVATWQTVYSHRPWAAFPPGARSRLLGGE ACLWSEKVDDQTLDVRLWPRAAALAERLWSDPPAGVHPDLPPPGSPQRDEPTLRRAYQRLSHHRERLVARGVRAEAMWPRYCHLNPGACF*

Sg-hex

MGKKVEVVLCACVCVGLLLTVTAAEPLPRYITEPGPTVKATQGAVWPKPQNEQRFGGSVLIVPGNFTFQVEGPECDILSEAVSRYEAILKEEAAIKGPRNASE ASTQLSALLVRLDGECGDRPVFGMDESYELRINSPDLPGAMLLTSASVWGILRGLETFSQVATRVKTADALILDNLAIADIPRFSHRGLLLDTSRHFIPVSYIKK TLDAMAYNKMNVFHWHIVDDQSFPYOSAAFPLLSEKGSYDPERFVYSPADVAEVIEYARVRGIRVVPEFDTPGHTRSWGEAYPDLLTPCYNATGSPDGTY GPIDPTKNFTYEFLQTLFEEIVNVFPDEYFHLGGDEVGFECWESNQDILDFMSEHNITESKDLESYYIQKIVDIASNLNSKSIVWQEVFDNEVRLSADTVVHIW TGDRNEELDSVTAAGHYTLLSOCYYLDRFRYFGGDWHKFYNCEPLDFSADNVYQYDLVIGGEAAMWSEFVDESNVESRVWPRASAVAERLWSPMNVTD IDEAATRIEEHYCRLRRRGINAQPPNGPGYCV*

\section{(B)}

Sg-cht5-1

MRTSAAWFLAVAGLCVVFCPPLVSGNVGDRGRVVCYFSNWAIYRPGIGRYGIDDVPASMCTHLVYSFIGVSNVTWGVLVIDPENDVENHGFANFTALKSK YPGLKTQLAIGGWAEGGRKYSAMAAVPARRRSLIASVVEYMKRYGFDGFDLDWEYPGAADRGGSFSDKNHFKCFVQELREAFDAEGQGWEITMAVPLA KFRLQEGYHVPELCELVDAIHVMSYDLRGNWAGFADTHSPLYKRPHDQWAYEKLNVHDGLKLWQDMGCPAHKLVVGVPFYGRSFTLSAGNKDYKLGTY INKEAGGGKPGNYTQAKGFLAYYEICLEIQEVGGWTEKWDEAGKVPYAYKGTQWVGFENPKSVQIKMDFIKAKGYGGAMTWAIDMDDFRGVCGPKDA LISVMYNNMKDYIVPDIQYSTTKRPDWDRPPPCDGKKPGAAPASTTTRRPTAAPTQSTTRRPAPTTTAAPSSSSSTTTTRRTTTASRPSTQPPPPPAAPDDN ELPPAAIDCSDGDFVPHHDCSKYYRCVYGKPVEFSCYEGTVWNPQLRVCDRPNDVHRTDCSMAKLHS*

Sg-cht5-2

MRAATQVGLLLAVALALAAASDEDTTPLDSSTGSPTNSVDEESSSSENAAVLSGGQRRGRVTCYFESWAVYRKRLRYGIEDIPGDMCTHIIYSFVGLNNVT WELOVLDEKLDVQDGGFENFTALROEFPGVRLQVALGGWAEGGHNYSAMVGDPARRASLVRSAVAFLHRYG FDGFDV WWEYPGNAPRGGVPEDKDD FLCFMQELRVAFDAEGLGWELTMAVPLTEDKLRDGFHVPQLCSIVDAVHVMAYDLRGEWDHFADVHSPLYRRPHDTGAYAKINTHDGLLLWEQLGGSS WGCHSTATPTNCVPTSPTTLPVLASFRAPEMTSAE*

(based on alignment with homologous sequences this transcript might be misassembled and the amino acid sequenced prematurely terminated by introduction of a stop codon)

Sg-cht10-1

MWRPVALSLWLLLATSRGLHVPPADEPSFVRDAVEAPPGQSLALRRSATASRPRLPAFGTRQLPLRQAVESPPMAARLRSSERLPLRDAVEHVPYEALPGA PTASEAFSLWRGFGDWLPENLPSTRQFNHSFAWWHDAIIAKLSLGGPRTKPPSLQAPSTHTSGIRQFKVVCFVEGWAGYRRDPMRFTTADIDPFACTHIIY AFAVMDPHDLHIKPQDEQYDIIQGGYRSIVGLKRQNPQLKVMISVGGWPEERRKFAEMTASASTRREFIRSVLHFIDEYGFDGIDLDWEYPGAADMGGSA REKEHFSLLVEELAEAFAPRGSVLSASVSPSRFRVEDGYDVPRLARRLDFLNLMAFDLLTEQDAAADHHAPLTQRKHDYGLAVFYNVDYAVRYWLRKGARR DQLVVGIPFHGHSFTLQDEAKNSPGAPVKGLGKEGPYTQEKGFLAYFEILQLLEEGHWMKATDDVGSPYMVKGNOWIGYEDQRSIATKVMYIKKNLLGG AMVWALDLDDFEGAYGQKWPLLSVVKKGLLETTPQSDQQQASQEPTHVTPPIAGVPVSVDSSQYNCSGRGYVRDSASCQIYHRCEWGMKHTYICPEGL 
Chapter I - Transcriptomics supports that pleuropodia of insect embryos function in degradation of the serosal cuticle to enable hatching

HYDSRTQLCDWPQIANCPMDNSSQRIEQENQSEVACNEEGLMEDPKDCNRYYMCHKGVAQHYSCMLGQYFNVQKGICEYGSCMPKAPQDNIPSSQTR NLVGEDHYKVVCYYASWAWYRKEGGKFVPEHIDPTLCTHIVYAYASLDPNTLTMIKYFDERADKENNFYERLTELPKKSGHHQQHASDVTVMIGLGGWTD SAGDKYSRLVSEGSARRRFVSKAVEFLHRHQFGGLHLDWDYPRCWQSNCGRGPTSDKPNFTKLVQELRQAFKKQNPPLALAISISGYHEVIDEAYDLAELG RNTDFMSVMTYDYHGSWEKSTGHVSPLYHRNGDIFPMYNTNDTMEYLVNKGAPRDKLLVGIPFYGQSYTLENPSNHDIGAPATGPGLAGEFTMQPGML. AYYEICDRVRNNFWKIGRDRFGATGPFAYAGNQWVSFEDTKSVKEKAKYIKNMGYGGAMTFTLDLDDFENRCCRGAFPLLRSINRVFGRIPDSAEPSGDD CTRPPPPVTPPPPTYTTGVDSGDHRPTTPISTTHQHPTSPKPSTTEYPWW

Sg-cht10-2

PSTTTSTTTSTTTTTTTTTTSTTTTPRPTTRPTTMSTTEYPWWTPSTTSTTRKPPTTRPTTTSTTEEYPWWTPPSTTKKPTTSSTTEQPWWTPSTTSTTSTAAP TTTMTTTEKPWWSTTPQKPLPPDSGPCEAGVYYPDPTNCNAYYRCVLGELRKEFCAGGLHWNPDKKVCDWPSESKCDTKEPSETTVGSTTSSTTENPWW TPSKPSETQATTTTTEVPWWSTTRPPRPPTTEGNSEWVTTSRPTTTQQPSEEVSECMNGQYYPVAGSCKSFYICVNGRLIKQTCAPGLVWNQDQTMCD WGFNVKCADDSEREAVHKAQPDDPCNQGALNPYPGDCTRYLYCQWGRYHEADCAAGLHWNEMEKICDWPENAKCTDMESGSEAPAASSQKPVTEM STSWTTAAPTTKPPWTWATTTTVKPVTTTSTRAPPAQGPPISGYFKVVCYFTNWAWYRRGLGKYVPEDIDANLCTHIVYGFAVLDYENLIIKAHDSWADFD NKFYERVVAYKKKKGLKVSLAIGGWNDSAGDKYSRLVNSPSARRRFIKHVLEFLEKYGFDGLDLDWEYPVCWQVDCAKGPASDKSSFAALVKELRQAFEPKE LLLSSAVSPSKTVIDAGYDVKTLAENLDWIAVMTYDFHGQWDKKTGHVAPLYFHPDDDFYFFNANFSINYWISEGAPRRKIVMGMPLYGQSFQLEKASTN GLNARSTGPGQAGEFTRAAGFLAYYEICDRIKNKGWTVVQDPERRMGPYAFKGNQWVSFDDVAMIQQKSEYIRKMGLGGGMIWALDLDDFRNRCGG GTHPLLNTIRTVLAAPPGGDGATEMPPSWSTPGGGQPTMSTEEWMSSTSISSTEITDSGHHSTQDSGGEVTSVSPAITTTNRPAHPGTSSSPPPPPSQGE KVVCYFTNWAWYRQGVGKYLPNEIDPDLCTHIVYGFAVLNGDRLTIKPHDTWADYDNKFYEKVVTEYKKKGIKVLVAIGGWNDSAGDKYSRLVNSPGARRR FIEDVIDFIEQNNFDGLDLDWEYPKCWQVDCKKGPDSDKEAFAAFVRELRAAFNPKGLLLTAAVSPSKAVVDAGYDVPTLSQNLDWIAVMTYDFHGQWD KITGHVAPMYTHPEDVDVTFNANFSIHYWIQKGASPKKIVMGMPMYGQSFSLADNSDHGLNAPTYGGGEAGESTRARGFLSYYEICTNIQKKGWRVVKD PEGRMGPYAYLRDQWVSFDDTSMIRYKSNFIRRMGLGGGMIWALDLDDFRNVCSCEKYPLLKTINRVLRGYPGPGPNCDIEATEKPGSEETDNRIHPTIPP TSSTNNWNVISGGGGIVPKDPTCGNRLFAPHDKDCNKYYLCQYGDFMEQSCPQGLYWNKDHCDWPSNTDCSKEDSSVINPAPIASTQEPEMSSTTENIH MSESTVTTSIRPSEPGTSTVMTPSGDYMVVCYFTNWAWYRQGLGKYLPSDIDTSLCTHIAYGFAVLDGNSLTIKPHDSWADLDNEFYTKVSGLKKKGIKVLL AIGGWNDSLGDKYSRLANNPSARRKFVEHVVKFIEKYGEGLDLDWEYPKCWQVDCNAGPDSDKQGFADLVKELSMAFKPRGLLLSSAVSPSKVVIDSGY DVPVLSQYFDYISVMTYDFHGHWDKQTGHVAPLYYYPGDTYDYFNANFTMHYWIEKGADRKKLIMGMPMYGQSFSLADAKNHGLNAKSYGPGEAGEF TRAGGFMAYYEICYNVKSKGWTTVRDPEGRIGPYAYRGNOWVSYDDVSDIRRKTQFIKELGLGGGMIWALDLDDFRNRCGCGTYPLLRTINSELRGLTAN THDCT*

Sg-cht7-1

MIAPRCVWRAALWCVVIILLADLVYSASSTGRRRLRRPGGSSSSSTTSSSSSTSTKVRTRDQETSASVNRFRVRNRLTPPGANRKSGSGSAVAAASDKSGG KVVCYYTNWSOYRTAHGKFLPEDITPDLCTHIIYAFGWLKKGKLTSFEGNDETKDGKVGLYERVMALKKANPKLKVLLALGGWSFGTQKFKAMSETRYTRO TFIYSAIPYLRKHDFDGLDMDWEYPKGTDDKKNFVLLLKELREAFEAEAQEVKQSRLLLSAAVPVGPDNVRGGYDVPAVASYLDFINLMAYDFHGKWERET GHNAPLYAPSSDSEWRKQLSVDHAATMWVKLGAPKEKLVIGMPTYGRTFTLSNPSNFKVNAPASGGGKAGDFTKEGGFLAYYEVCDMLKKGATYIWDD EMKVPYAVMGDQWVGFDDERSIRHKMKWLKEGGYGGAMVWTVDMDDFTGTVCGGGVKYPLIGAIREELRGVSRGPNAKDVDWSKVARTVSLEATT KPAPIKIDVSEVLNRVRKPTKQAPADLSNEVIDLNSRPAQVFCYMTSWSGKRPGAGKFSPEDVDPSLCTHVVFAFATLKDHKLAPANDKDDGLYERVIALRE KNPQLKVLLAIGGWAFGSTPFKELTSNVFRMN QFVYDAIELLRDFKFDGLDVDWEYPRGADDRAAYVSLLKELRIMAFEGEAKTAEQPRLLLSAAVPASFEAI AAGYDVPEISKYLDFINVMTYDFHGQWERQVGHNSPLYPLESATSYOKKLTVDFSAREWVKQGAPKEKLLIGMPTYGRSFTLVDTSKFDIGAPASGGGAAG RYTAEAGFMAYYEVCDFLHHDNTTLVWDNEQQVPFAYRGDQWVGFDDERSLKTKMGWLKELGFGGIMVWSVDMDDFRGQCGAGKYPLLTSMRQEL RDYRVQLEYDGPYESRGPLGAYTTKDPTSVSCEEEDGHISYHPDKADCTMYYMCEGERKHHMPCPSNLVFNPNENVCDWPENVEGCMHHTQAPPAAR $\mathrm{RR}^{*}$

Sg-cht7-2

MTWPPPPLLLSLLVLLATSASARFVSTHDVTPCAVEALAPSDKALLCYYEGRLSVYQLDPCLCTHIVFKDAAVVSDNFGLKIVSDVSGASLLRARSPSLRTVLGL RLSGAVARAALASPSRRLALARDAARRLYAHHLDGIELSVDDDEAASAAAADAAPAATARQGLVALLKALRTALDSHGREKRDYLVSEQVFDDFTTQEYEPT WSDGSSRKSRRRATTTTTTSTTESPEETAARYLELERDAQNAQLLLSLPTKPETIAKRYDVKNITRYVDYVVLRTQAMTDDSERGLVYHPSRLMGLDDMLN ADAVVDLVTSLGASPAQLVITLPGQATAFELRREDRTEPRSPASGAPRTISQPELCRALSRGNWTLERDEDQTAPYAYSGRRWIAFDDALSASIKGKYAVVR GLAGTAVDAADALDWQGTCGAPASQLRALHSALAQLRRSSRGALLHGLE

Sg-cht7-3

DKGMPKNKIIVGIPTYGHSFRLINAENHGWSAPASGYGKIGSKGFVSYPEVCQFLHSTGSKYIFDKNFEVPYAYQGLEWISYDDECSVMYKAKYIASSSYGGA MVFSLNVDDHQGVCAGTTFLLTTQIRNILGVSWQ*

Sg-cht2

MQQLAPLAFVLAFLAAAFAASPLGHNKAVVCYVSSWAVYRPGNGVFTVSDINPNICSHLVYAFAGLNATDNTIITLDKYNDLEEDYGKGNYKKITGLKNQYP HLKVSIAIGGWNEGSANYSHMASTPTTRQQFIRSVVNFLRKYNFDGLDLDWEYPTQRGGVPSDRENFVALVRELRQEFDKNGWLLTAALGASTAVIEKAY DVPMLGKYLDYMHIMCYDYHGTWDKMTGANAPLYGSSPSDTLSVDNSIRYYLKLGAPAKKLLMGVPLYGRTFMSDANANMGGLGAPAEEKSFQGPYTK EDGYMGYNEICLELKTNSSMWTIMWDDKSSTPYAVSTNKVIVYDNAKSLTEKVNLAMKLELGGIMVWPLDTDDFRGECSEGIYPLMHTINKAIVQSSQQK SDSSGMKVPDSTAAASCGCASLIFLSFLYLFQL*

Sg-cht6-1

VCYYTNWSVYRPGTAKFTPQNINPYLCTHLIYAFGGLSRENGLRPFDKYQDIEQGGYAKFTGLKTYNKDLKTMLAIGGWNEGSTRFSPLVADAERRKEFVKN VLRFLRQNHFDGLDLDWEYPAFRDGGKSRDRDNYALLVKELREEFDRESEKTGRPRLLLTMAVPAGIEYIDKGFDIASMNKHLDFMNILSYDYHSAFEPAVN HHSPLYSMEEDDEYNFDAQLTIDHTVNHYMKSGADRNKLVLGIPTYGRSYTLFNPLATELGSPADGPGEQGDSTREKGYLAYYEICENLQSDDWKVVQPN 
Chapter I - Transcriptomics supports that pleuropodia of insect embryos function in degradation of the serosal cuticle to enable hatching

PSAMGPYAYKGNQWVSYDDMDIIKKKAQYVNDNGLGGIMFWAIDN DFRGKCHGRPYPLIEAGKEAMLKGVKRSNNEIETTPVQNNRQSSRKRNRNR SKGNARGRTRTTASTSTVVTTTTTTTTTTAAPLITPSYTTPEPPTTPDPGSDFKCKDEGFFPHPRDCKKYFWCLDSGPSNLGIVAHQFTCPSGLFFNKAADSC DYARNVVCNKKSKSQGGSSSTLPPIKAATSSTTRFSTSPSTKLTTKLTTTTTTEPPPVLDDDDDDD

\section{Sg-cht6-2}

MNIRVKQPVIIGNCYRGQPNRLWEVFILKWFLVAVACLIAAGAVTVYLAHYFMKTRYTSTNVTGVTGQHSDLNTYKGQLQDMGDGYSLFKQEDMTQICK TDELTGSQQMRKQSTKLVCYYTFP GPGGLVPDKIDPFLCTHINIAAVGINNSKLEPLCEERKEVIKSLVGLKTRNKNLKVILSVIGMPGGFGDMVSKSSSRRM $\underline{F I K D L}$

Sg-cht8-1

MSHFWLRLAVILGVSLSICGAEDKKVVVYHGSWSAYRNGNGRFEIEYIQPELCTHLIYTFVGITSAGEVRILDEWLDLPSGKNAYNRFNALKSSNTKTLVAIGG WNEGSATYSAVMNDASLRAKFVQNVVNFVKTYGFDGFDLDWEYPANRGGSPGDLTAFVELIKELRTEFDKYGYLLTAAVGVGRYLIGTAYDVPQISKYLDF INLMTYDLHGSWDGKTGQNAPLYASSAD KTEAERQLNVDSSVRYWIQNGADPSKLVLGMGTYGRTFTLSSAANTGVGAPATAPGTNGPYTMESGMMG YNEICEKINAGGWTVVWDEEQKVPYAVNGNQWIGYDNEESIRLKSQYVLDMGLAGGMIWSLETDDFKGLCGSKTYPLLSTINEVLRGITSTNSGSSSSSSSS SSSSSSSSSSSSSSSSSSSNTAASASSSSGVCSSAGYVRDPSDCGVFYLCTASGSGYTASKFTCPGDLVFDESSSACNYKSLVAC*

Sg-cht8-2

MSPFLSGLLLLLGVLNICGADEKKVVCYHGSWSAYRNGNGRFEIEYIRPELCTHMIYSFVGITSAGEVRILDEWLDLASGKNAYNRFNKLKSSNTKTLVAIGG WNEGSATYSAVMNNAALRQKFVQNVVNFVKTYGFDGFDLDWEYPANRGGSPGDLRAYVELLKELRAEFDKHGFILSAAVGVGRYLIGSAYDVPQLSKYLD $\underline{F I N L}$

Sg-cht8-3

ESGMMGYNEICEKIKAGGWKVTWDDEQKVPYAVSGNQWVGYDNEESIKLKSOYVLDMGLGGGMIWSLETDDFKGVCGAGTFPLLSAINQVLRGAAAT SSAGSSTSGSSSGSSGSSSSSSASSGGSSSGTSSGSSTTSSGASADSSGSSASSGSSSSGSSATSVSSGSNSSGVCNSAGYARDPSDCGV

Sg-idgf-1

MAELPLLLLLLAAAATCWTSAAALGATRVVCYLDGGALRRPEPHRMLVSEIEPSLTYCTHLIYGYATIDTDSYKAVPRHEGEGTNYTSVVALKRRFPALNVLLS IGGGSADSGQREKYLHLLESDEHRRTFVKSAKDLLKKYYHFDGIDIAWEFP MNKEKKERSTLGSFWHGFKKVIGLAHSHKDEKADEHRREFSSLIQELKTSLKT ENALLTLSVIPYINHTLYYDCSALSPHVDHLHLLAYDYHTPQRTPNTADYPAPLYVAGKRDPDLTADGNVRWFLERGFPSRKIILGIPTFARTWKLDDDSRVS GVPPIEADGAGDTDNIANTAGIMAFQTVCMLLPNAGNAGYKTTLSRVTDPTDRLGSYGFRLPSGEVTGLWVGYEDPDVAQYKAAYAKIKSLGGIAFSDLSL DYHGICTGDKYPIVRAGTLKLRYK*

Sg-idgf-2

MQSFARLLLLSACCWSAALAATTKVVCYFNTSALKRPESSRMLLSQIEPSFSYCTHLVVGYATINTETYKAVPPSEDEHTTYTNIVALKRRFPSLKILLSIGGGAA DTDTREKYFELLESDEHRTTFVSSAKSLLKQHGFDGIDIAWEFPKNKAKKDRGTFGSIWHGIKKAVGAAHSHTDEKADEHKSOFSALIRELRTSLRNENALLTL SVIPYINQSLYYDPTALNQQIDELHVLAFDYRNPERDSQGGRLPCAALPSRAEGLRPLGRREHPLVPRELIPS*

\section{Sg-idgf-3}

TDSYKAVPRYQDDTTKYTSLVALKERFPSLKVLLSIGGGGADADQRKKYLELLESDEHRRTFVDSVKELLQQNRFDGIDIAWEFPFSKEKKDRGNVWHGVKKVLGY AHSHRDENPDEHRRQFSALIRELKSSLKTQNALLTLSVIPYINHSLYYDCASLSPEIDQLHLLAYDYHSPTRTPKKADYPAPLYRAGERSADLTVDGNVRWFLEKGFPS RKIILGIPTFARTWKLTKDSRITGVPPIDADGPGVAGSIANISGLLAYQTVCTLLPNDANAAYRTTLRRVTDPTDRLGSYGFRLPTREVSGLWVGYENQHSAEYKAAY ARKKSLGGIAFSDLSLDDYNGVCTGEKFPIVRAGTLKLLSTSV*

Supplementary Figure 5 Amino acid sequences and conserved domains of Schistocerca chitin degrading enzymes. A. NAGs, B. CHTs. Signal peptide and transmembrane region identified by Phobius (http://phobius.binf.ku.dk/index.html) and conserved domains identified by SMART (http://smart.emblheidelberg.de/) are underlined and coloured. In A. and B. signal peptide: magenta, transmembrane region: dark blue. In A. Glycohydro 20b2 domain (N-terminal domain of the eukaryotic beta-hexosaminidases): light green, Glyco hydro 20 domain (glycoside hydrolase family 20 catalytic domain): grey. In B. Glyco 18 domain (catalytic domain): light blue, Chitin-binding domain type 2 (ChBD2): green; catalytically critical consensus sequence in the Glyco 18 domain, FDG(L/F)DLDWE(Y/F)P, is highlighted in yellow and amino acid changes from the consensus are coloured in orange. 


\section{FIGURE S6}

A

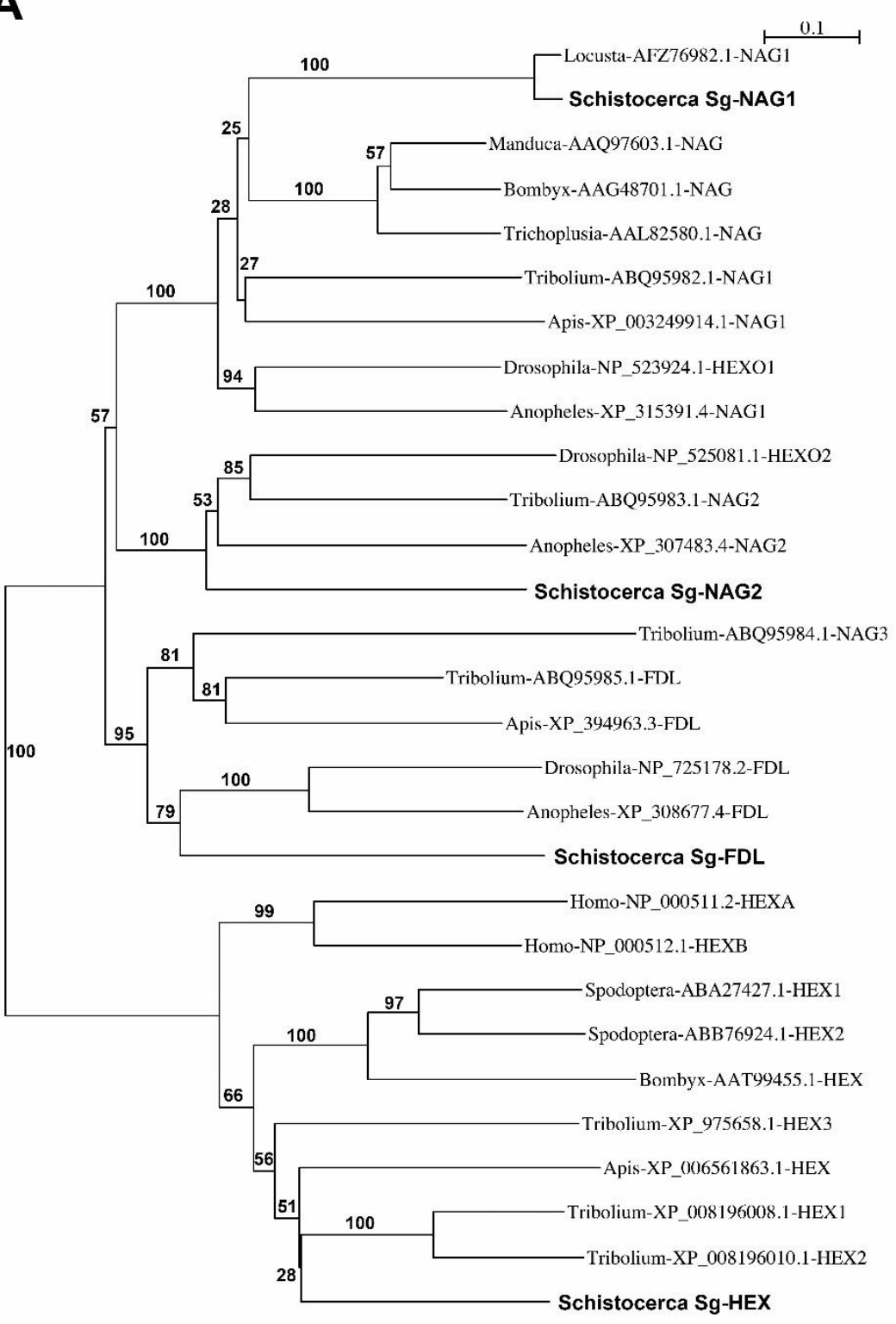

\section{group}

I

II

III

IV 
B

\section{group}
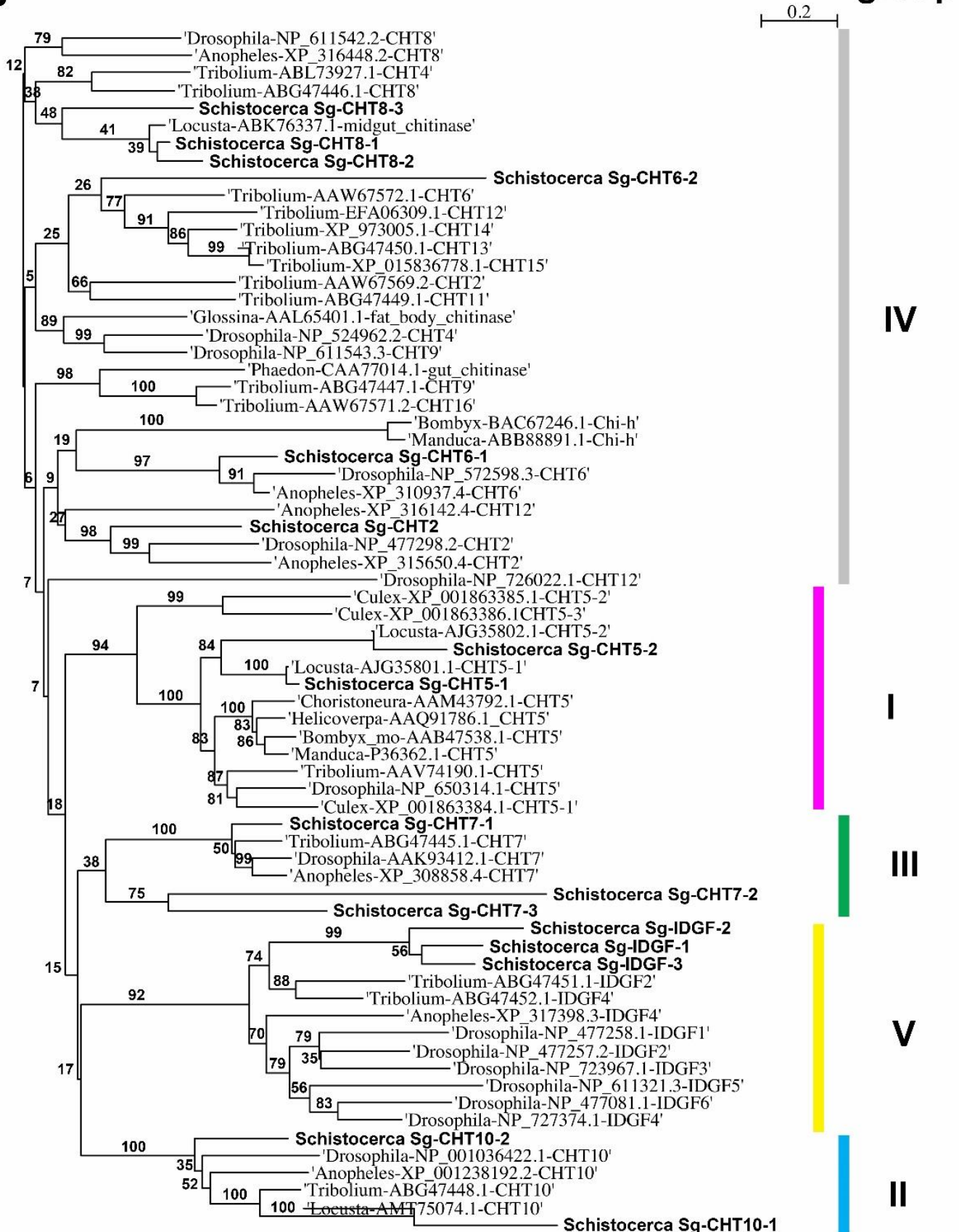

III

Supplementary Figure 6. Phylogenetic trees of chitin degrading enzymes in Schistocerca and other insects. A. NAGs, B. CHTs. Schistocerca sequences are in bold. Amino acid sequences were extracted from NCBI GenBank. The numbers above the branches are bootstrap support. The marker shows a branch length. Both trees are unrooted. The tree in A. was prepared using the SeaView software (version 4.6.1; (Gouy et al., 2010); http://doua.prabi.fr/software/seaview): alignment with default parameters, tree using the Neighbor Joining method, Poisson distribution, 5000 bootstrap replicates. The tree in B. was prepared using the CLC Sequence Viewer (version 7.8.1; https://www.qiagenbioinformatics.com/products/clc-sequence-viewer/): alignment with default parameters except gap open cost 3.0 and gap extension cost 3.0, tree using Neighbor Joining method, Kimura model, 1000 bootstrap replicates. 


\section{FIGURE S7}
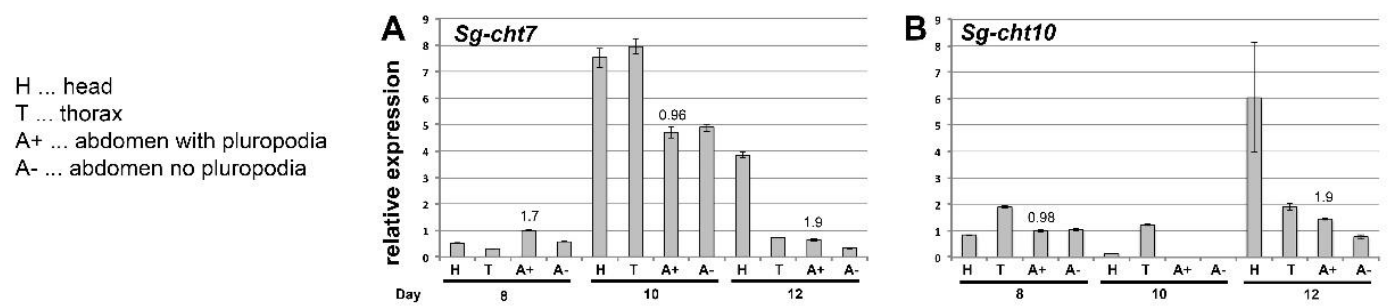

Supplementary Figure 7. Real-time RT-PCR expression analysis of Sg-cht7 and Sg-cht10-1 on cDNA from parts of Schistocerca embryos. cDNA was prepared from mRNAs isolated from parts of embryos at the age of 8, 10 and 12 days: $H$, head; $T$, thorax; $A+$, abdomen with pleuropodia; $A-$, abdomen without pleuropodia. Analysis of 3-4 technical replicates is shown. Expression in A+8 (abdomen with pleuropodia when they first become differentiated) was set as 1 . Numbers above A+ expression is fold change from A- of the same age. 


\subsection{Supplementary Tables}

Supplementary Table 1. Embryonic transcriptome of Schistocerca: numbers of sequenced reads and assembled transcripts.

\begin{tabular}{|c|c|c|c|}
\hline Samples $^{a}$ & Reads total & Unique transcripts & $\begin{array}{l}\text { Transcripts in reference } \\
\text { transcriptome }^{b}\end{array}$ \\
\hline 1-4d embryos & $96,907,644$ & \multirow{4}{*}{70,529} & \multirow{6}{*}{20,834} \\
\hline 5-7d embryos & $92,825,202$ & & \\
\hline 8-10d embryos & $99,198,014$ & & \\
\hline 11-14d embryos & $96,759,706$ & & \\
\hline 8-9d legs & $38,919,230$ & \multirow{2}{*}{40,143} & \\
\hline 8-9d pleuropodia & $22,302,378$ & & \\
\hline
\end{tabular}

a in "embryo" samples the mRNA was isolated from whole eggs collected at each day, then in indicated age groups pooled together for sequencing

${ }^{\mathrm{b}}$ see Materials and Methods how transcripts for the reference transcriptome were selected

Supplementary Table 2. RNA-seq expression analysis: numbers of sequenced and mapped reads.

\begin{tabular}{|l|r|l|}
\hline Sample & Reads total & Reads mapped \\
\hline 4d LEG & $50,592,896$ & $38.404 .015(75.91 \%)$ \\
\hline 4d PLP & $47,004,156$ & $35.905 .385(76.39 \%)$ \\
\hline 5d LEG & $49,391,167$ & $35.559 .693(75.11 \%)$ \\
\hline 5d PLP & $49,002,608$ & $36.095 .324(73.66 \%)$ \\
\hline 6d LEG & $50,647,001$ & $37.684 .851(74.41 \%)$ \\
\hline 6d PLP & $49,111,150$ & $37.490 .747(76.34 \%)$ \\
\hline 7d LEG & $47,410,277$ & $35.958 .856(75.85 \%)$ \\
\hline 7d PLP & $47,275,171$ & $35.971 .381(76.09 \%)$ \\
\hline 8d LEG & $49,998,624$ & $38.119 .439(76.24 \%)$ \\
\hline 8d PLP & $48,420,404$ & $37.706 .738(77.87 \%)$ \\
\hline 8-9d LEG & $38,919,230$ & $29.467 .879(75,72 \%)$ \\
\hline 8-9d PLP & $22,302,378$ & $16.152 .357(72.42 \%)$ \\
\hline 10d LEG & $49,170,085$ & $37.814 .977(76.91 \%)$ \\
\hline 10d PLP & $46,901,233$ & $35,403,192(75.48 \%)$ \\
\hline 11d LEG & $49,472,441$ & $37.815 .815(76.44 \%)$ \\
\hline 11d PLP & $48,516,135$ & $36.818 .833(75.89 \%)$ \\
\hline 12d LEG & $47,068,033$ & $34.117 .674(72.49 \%)$ \\
\hline 12d PLP & $46,801,370$ & $34.936 .272(74.65 \%)$ \\
\hline 13d LEG & $46,658,116$ & $33.454 .889(71.70 \%)$ \\
\hline 13d PLP & $49,776,232$ & $37.167 .588(74.67 \%)$ \\
\hline & &
\end{tabular}


Supplementary Table 3. Number of differentially expressed genes at selected levels of stringency.

\begin{tabular}{|c|c|c|c|c|c|c|c|c|c|c|c|}
\hline \multirow{2}{*}{\multicolumn{2}{|c|}{$\begin{array}{ll} & \text { Day } \\
\text { RPKM } & \text { Fold change }\end{array}$}} & \multicolumn{2}{|c|}{4} & \multicolumn{2}{|c|}{5} & \multicolumn{2}{|c|}{6} & \multicolumn{2}{|c|}{7} & \multicolumn{2}{|c|}{8} \\
\hline & & DOWNa $^{a}$ & UP & DOWN & UP & DOWN & UP & DOWN & UP & DOWN & UP \\
\hline$>10$ & $>2$ & 29 & 19 & 77 & 195 & 360 & 589 & 649 & 857 & 944 & 791 \\
\hline$>50$ & $>2$ & 5 & 6 & 18 & 63 & 97 & 241 & 181 & 394 & 289 & 403 \\
\hline$>100$ & $>2$ & 2 & 3 & 7 & 26 & 31 & 130 & 70 & 238 & 111 & 265 \\
\hline \multicolumn{2}{|r|}{$8-9$} & \multicolumn{2}{|c|}{10} & \multicolumn{2}{|c|}{11} & \multicolumn{2}{|c|}{12} & \multicolumn{2}{|c|}{13} & \multicolumn{2}{|c|}{$10+11+12$} \\
\hline DOWN & UP & DOWN & UP & DOWN & UP & DOWN & UP & DOWN & UP & DOWN & UP \\
\hline 890 & 850 & 1538 & 857 & 1874 & 842 & 1358 & 772 & 1196 & 871 & 781 & 1535 \\
\hline 259 & 430 & 427 & 454 & 457 & 411 & 492 & 408 & 523 & 478 & 451 & 484 \\
\hline 108 & 256 & 215 & 301 & 216 & 292 & 287 & 286 & 350 & 312 & 327 & 277 \\
\hline
\end{tabular}

a DOWN: downregulated, UP: upregulated 

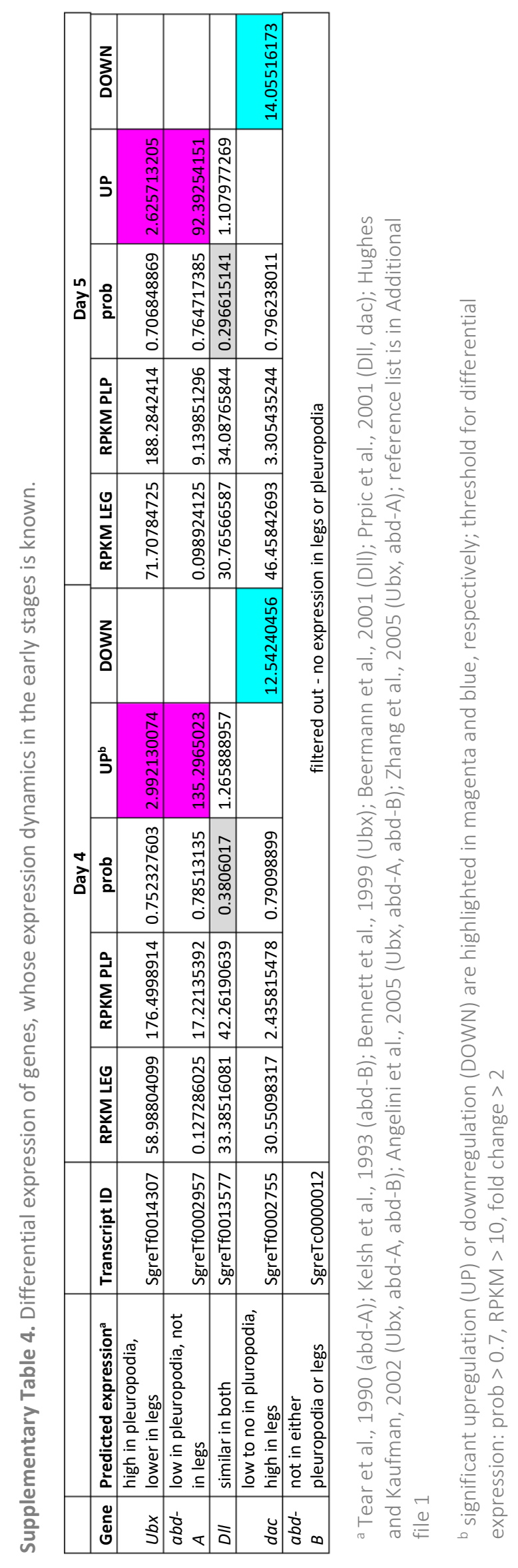
Supplementary Table 5. Comparison between differential expression of selected genes obtained by RNA-seq and real-time RT-PCR.

\begin{tabular}{|c|c|c|c|c|c|c|c|c|}
\hline \multirow[b]{2}{*}{ Day } & \multirow[b]{2}{*}{ Transcript ID } & \multicolumn{2}{|c|}{ Real-time RT-PCR ${ }^{a}$} & \multicolumn{2}{|c|}{ RNA-seq ${ }^{b}$} & \multicolumn{3}{|c|}{ Details RNA-seq } \\
\hline & & UP & DOWN & UP & DOWN & RPKM leg & RPKM plp & prob \\
\hline 4 & SgreTa0007432 & & 1.058 & 1.064 & & 11.177 & 11.888 & 0.261 \\
\hline 4 & SgreTa0001469 & 1.303 & & 1.214 & & 96.102 & 116.656 & 0.359 \\
\hline 4 & SgreTa0005616 & not & cted & 6.282 & & 0.002 & 0.012 & 0.261 \\
\hline 4 & SgreTa0013453 & 1.734 & & 1.543 & & 75.079 & 115.851 & 0.521 \\
\hline 4 & SgreTa0008219 & not & cted & & 3.584 & 0.232 & 0.065 & 0.261 \\
\hline 4 & SgreTa0001661 & 1.058 & & & 1.035 & 38.974 & 37.662 & 0.265 \\
\hline 4 & SgreTa0014626 & 1.055 & & & 1.022 & 145.383 & 142.194 & 0.263 \\
\hline 5 & SgreTa0007432 & 1.099 & & 1.011 & & 11.303 & 11.430 & 0.266 \\
\hline 5 & SgreTa0001469 & 2.060 & & 1.510 & & 84.745 & 127.987 & 0.515 \\
\hline 5 & SgreTa0015941 & 210.358 & & 168.642 & & 0.276 & 46.467 & 0.797 \\
\hline 5 & SgreTa0007802 & $\begin{array}{c}3.726 \\
\text { UP }\end{array}$ & & 5.914 & & 0.450 & 2.659 & 0.543 \\
\hline 5 & SgreTa0005616 & indefinitely & & 2.473 & & 0.056 & 0.138 & 0.266 \\
\hline 5 & SgreTa0017664 & not & cted & $N A^{c}$ & NA & NA & NA & NA \\
\hline 5 & SgreTa0009118 & & 1.823 & & 1.972 & 119.291 & 60.507 & 0.579 \\
\hline 5 & $\begin{array}{c}\text { SgreTa0000088 } \\
\text { SgreTd000275 }\end{array}$ & 1.074 & & & 1.171 & 55.737 & 47.592 & 0.333 \\
\hline 5 & 5 & & 10.247 & & 14.055 & 46.458 & 3.305 & 0.796 \\
\hline 5 & SgreTa0001341 & & 8.662 & & 10.941 & 22.491 & 2.056 & 0.790 \\
\hline 5 & SgreTf0013577 & $\begin{array}{l}1.015 \\
\text { UP }\end{array}$ & & 1.108 & & 30.766 & 34.088 & 0.297 \\
\hline 5 & SgreTa0005600 & indefinitely & & 5.204 & & 0.526 & 2.739 & 0.543 \\
\hline 5 & SgreTa0013453 & 2.469 & & 2.368 & & 81.519 & 193.025 & 0.689 \\
\hline 5 & SgreTa0008219 & & 1.123 & 2.095 & & 0.314 & 0.657 & 0.266 \\
\hline 5 & SgreTa0008219 & & 1.120 & 2.095 & & 0.314 & 0.657 & 0.266 \\
\hline 5 & SgreTf0014307 & 2.661 & & 2.626 & & 71.708 & 188.284 & 0.707 \\
\hline 5 & SgreTa0001661 & 1.237 & & 1.238 & & 38.483 & 47.658 & 0.360 \\
\hline 5 & SgreTa0014626 & 1.427 & & 1.408 & & 142.712 & 200.902 & 0.454 \\
\hline 5 & SgreTa0007477 & 4.762 & & 5.104 & & 52.030 & 265.551 & 0.789 \\
\hline 6 & SgreTa0007432 & & 1.181 & & 1.258 & 10.152 & 8.069 & 0.369 \\
\hline 6 & SgreTa0001469 & 2.475 & & 2.182 & & 85.763 & 187.142 & 0.680 \\
\hline 6 & SgreTa0002409 & 30.406 & & 33.056 & & 8.717 & 288.152 & 0.794 \\
\hline 6 & SgreTa0015941 & 12.189 & & 22.566 & & 0.040 & 0.907 & 0.289 \\
\hline 6 & SgreTa0007802 & $\begin{array}{l}3.463 \\
\text { UP }\end{array}$ & & 3.248 & & 0.809 & 2.627 & 0.448 \\
\hline 6 & SgreTa0005616 & $\begin{array}{c}\text { indefinitely } \\
\text { UP }\end{array}$ & & $\begin{array}{l}901.151 \\
2717.49\end{array}$ & & 0.198 & 178.706 & 0.971 \\
\hline 6 & SgreTa0017664 & indefinitely & & 5 & & 0.211 & 572.225 & 0.999 \\
\hline 6 & SgreTa0009118 & & 3.750 & & 4.200 & 117.188 & 27.902 & 0.776 \\
\hline 6 & $\begin{array}{c}\text { SgreTa0000088 } \\
\text { SgreTd000275 }\end{array}$ & & 1.065 & & $\begin{array}{c}1.136 \\
275.79\end{array}$ & 46.963 & 41.325 & 0.329 \\
\hline 6 & 5 & & 320.639 & & 5 & 74.322 & 0.269 & 0.794 \\
\hline
\end{tabular}




\begin{tabular}{|c|c|c|c|c|c|c|c|c|}
\hline 6 & $\begin{array}{c}\text { SgreTb000624 } \\
3\end{array}$ & $\begin{array}{c}\text { UP } \\
\text { indefinitely }\end{array}$ & & 2.287 & & 0.442 & 1.011 & 0.289 \\
\hline 6 & SgreTa0017707 & & 8.595 & & 2.935 & 4.258 & 1.451 & 0.528 \\
\hline 6 & SgreTa0017736 & 8.122 & & 7.221 & & 0.156 & 1.129 & 0.289 \\
\hline 6 & SgreTa0008528 & & $\begin{array}{c}\text { DOWN } \\
\text { indefinitely }\end{array}$ & & 1.681 & 3.778 & 2.247 & 0.396 \\
\hline 6 & SgreTa0005600 & $\begin{array}{c}\text { UP } \\
\text { indefinitely }\end{array}$ & & 97.272 & & 1.880 & 182.830 & 0.794 \\
\hline 6 & SgreTa0013453 & 2.602 & & 2.513 & & 76.870 & 193.188 & 0.707 \\
\hline 6 & SgreTa0013453 & 2.602 & & 2.513 & & 76.870 & 193.188 & 0.707 \\
\hline 6 & SgreTa0008219 & & 2.208 & & 1.516 & 0.752 & 0.496 & 0.289 \\
\hline 6 & SgreTa0008219 & & 1.327 & & 1.516 & 0.752 & 0.496 & 0.289 \\
\hline 6 & SgreTf0014307 & & 1.193 & & 1.082 & 112.101 & 103.605 & 0.308 \\
\hline 6 & SgreTa0001661 & 1.488 & & 1.259 & & 40.953 & 51.580 & 0.398 \\
\hline 6 & SgreTa0014626 & 2.116 & & 1.868 & & 162.842 & 304.203 & 0.585 \\
\hline 7 & SgreTa0007432 & & 1.035 & & 1.217 & 10.039 & 8.247 & 0.347 \\
\hline 7 & SgreTa0001469 & 5.404 & & 2.822 & & 82.172 & 231.897 & 0.718 \\
\hline 7 & SgreTa0007802 & $\begin{array}{c}563.365 \\
\text { UP }\end{array}$ & & 300.262 & & 1.148 & 344.621 & 0.795 \\
\hline 7 & SgreTa0005616 & $\begin{array}{c}\text { indefinitely } \\
\text { UP }\end{array}$ & & 619.842 & & 0.242 & 149.935 & 0.927 \\
\hline 7 & SgreTa0017664 & indefinitely & & 251.604 & & 0.330 & 83.089 & 0.794 \\
\hline 7 & SgreTa0009118 & & 4.507 & & 6.370 & 98.857 & 15.520 & 0.791 \\
\hline 7 & SgreTa0000088 & 1.266 & & & 1.005 & 45.617 & 45.398 & 0.303 \\
\hline 7 & $\begin{array}{c}\text { SgreTa0014975 } \\
\text { SgreTb001997 }\end{array}$ & 4.777 & & 3.028 & & 17.526 & 53.072 & 0.752 \\
\hline 7 & $\begin{array}{c}3 \\
\text { SgreTb000624 }\end{array}$ & 87.226 & & 63.539 & & 18.121 & 1151.375 & 0.795 \\
\hline 7 & 3 & 1268.530 & & 524.381 & & 0.555 & 290.855 & 0.927 \\
\hline 7 & SgreTa0017707 & 52.614 & & 25.430 & & 18.890 & 480.378 & 0.795 \\
\hline 7 & SgreTa0017736 & 54.154 & & 16.270 & & 0.719 & 11.703 & 0.769 \\
\hline 7 & SgreTa0007897 & 391.606 & & 160.119 & & 0.530 & 84.880 & 0.794 \\
\hline 7 & SgreTa0008528 & 229.010 & & 147.348 & & 3.858 & 568.467 & 0.795 \\
\hline 7 & SgreTa0005600 & 966.179 & & 279.788 & & 2.927 & 819.045 & 0.795 \\
\hline 7 & SgreTa0013453 & 9.930 & & 8.482 & & 61.614 & 522.632 & 0.794 \\
\hline 7 & SgreTa0008219 & 145.183 & & 143.957 & & 0.797 & 114.758 & 0.794 \\
\hline 7 & SgreTa0006308 & 8.248 & & 9.049 & & 0.111 & 1.008 & 0.303 \\
\hline 7 & SgreTa0001661 & 4.515 & & 3.163 & & 38.206 & 120.862 & 0.754 \\
\hline 7 & SgreTa0014626 & 8.443 & & 6.388 & & 160.490 & 1025.240 & 0.791 \\
\hline 8 & SgreTa0007432 & & 1.189 & & 1.296 & 9.430 & 7.276 & 0.395 \\
\hline 8 & SgreTa0001469 & 4.905 & & 3.109 & & 89.858 & 279.396 & 0.749 \\
\hline 8 & SgreTa0007802 & 2052.856 & & 652.039 & & 1.063 & 693.338 & 0.788 \\
\hline 8 & SgreTa0005616 & 552.396 & & 234.275 & & 0.489 & 114.514 & 0.788 \\
\hline 8 & SgreTa0017664 & 26.449 & & 7.225 & & 7.118 & 51.426 & 0.785 \\
\hline 8 & SgreTa0009118 & & 8.144 & & 9.556 & 82.176 & 8.599 & 0.787 \\
\hline 8 & SgreTa0000088 & & 1.443 & & 1.496 & 34.508 & 23.071 & 0.526 \\
\hline 8 & SgreTa0014975 & 3.818 & & 2.915 & & 18.201 & 53.056 & 0.710 \\
\hline
\end{tabular}




\begin{tabular}{|c|c|c|c|c|c|c|c|}
\hline 8 & $\begin{array}{c}\text { SgreTb001997 } \\
3 \\
\text { SgreTb000624 }\end{array}$ & 29.591 & 20.892 & & 55.364 & 1156.651 & 0.788 \\
\hline 8 & 3 & 537.340 & 297.963 & & 2.284 & 680.550 & 0.788 \\
\hline 8 & SgreTa0017707 & 344.572 & 219.619 & & 16.926 & 3717.263 & 0.788 \\
\hline 8 & SgreTa0017736 & 20.254 & 11.347 & & 1.017 & 11.544 & 0.763 \\
\hline 8 & SgreTa0007897 & 116.944 & 69.794 & & 8.998 & 628.012 & 0.788 \\
\hline 8 & SgreTa0008528 & 420.588 & 251.695 & & 3.422 & 861.195 & 0.788 \\
\hline 8 & SgreTa0005600 & 318.652 & 179.306 & & 7.170 & 1285.584 & 0.788 \\
\hline 8 & SgreTa0013453 & 5.827 & 5.301 & & 73.218 & 388.135 & 0.780 \\
\hline 8 & $\begin{array}{c}\text { SgreTa0008219 } \\
\text { SgreTd000888 }\end{array}$ & 133.217 & 91.006 & & 1.483 & 134.976 & 0.788 \\
\hline 8 & $\begin{array}{c}6 \\
\text { SgreTd001487 }\end{array}$ & 1.776 & & 2.530 & 1.933 & 0.764 & 0.467 \\
\hline 8 & 5 & 1.260 & 1.143 & & 10.234 & 11.697 & 0.345 \\
\hline 8 & SgreTa0006386 & 6.385 & & 23.445 & 18.820 & 0.803 & 0.779 \\
\hline 8 & SgreTa0006977 & 5.063 & & 13.209 & 9.183 & 0.695 & 0.750 \\
\hline 8 & SgreTa0006308 & 2.995 & & 1.383 & 5.007 & 3.620 & 0.390 \\
\hline 8 & SgreTa0002186 & 820.939 & 463.758 & & 2.510 & 1164.179 & 0.788 \\
\hline 8 & $\begin{array}{c}\text { SgreTa0001661 } \\
\text { SgreTb001604 }\end{array}$ & 5.725 & 4.409 & & 42.609 & 187.852 & 0.771 \\
\hline 8 & $\begin{array}{c}7 \\
\text { SgreTb001604 }\end{array}$ & 5.226 & 4.234 & & 30.761 & 130.242 & 0.771 \\
\hline 8 & 7 & 5.048 & 4.234 & & 30.761 & 130.242 & 0.771 \\
\hline 8 & SgreTa0014626 & 7.975 & 4.671 & & 199.900 & 933.699 & 0.773 \\
\hline 8 & SgreTa0008504 & 8.862 & 6.990 & & 76.345 & 533.636 & 0.786 \\
\hline 10 & SgreTa0007432 & 1.999 & & 1.396 & 9.752 & 6.988 & 0.420 \\
\hline 10 & SgreTa0001469 & 5.781 & 5.850 & & 109.290 & 639.310 & 0.773 \\
\hline 10 & SgreTa0007802 & 132.766 & 250.286 & & 2.403 & 601.355 & 0.781 \\
\hline 10 & $\begin{array}{l}\text { SgreTa0005616 } \\
\text { SgreTa0011044 }\end{array}$ & $\begin{array}{l}64.339 \\
\text { not detected }\end{array}$ & 58.145 & & $\begin{array}{l}0.902 \\
\text { filtered } \\
\text { out }\end{array}$ & 52.426 & 0.780 \\
\hline 10 & SgreTa0006252 & 1.805 & 3.043 & & 52.999 & 161.261 & 0.741 \\
\hline 10 & SgreTa0017664 & 9.079 & & 3.391 & 166.597 & 49.135 & 0.742 \\
\hline 10 & SgreTa0005054 & $\begin{array}{l}2.158 \\
\text { DOWN }\end{array}$ & & 1.237 & 34.081 & 27.554 & 0.396 \\
\hline 10 & SgreTa0002027 & indefinitely & & 87.018 & 3.994 & 0.046 & 0.642 \\
\hline 10 & SgreTa0009118 & 45.352 & & 33.703 & 69.800 & 2.071 & 0.781 \\
\hline 10 & SgreTa0000088 & 11.293 & & 9.208 & 43.460 & 4.720 & 0.779 \\
\hline 10 & SgreTa0014975 & 3.435 & 4.368 & & 11.542 & 50.413 & 0.762 \\
\hline 10 & SgreTa0001826 & 20.587 & 24.972 & & 11.469 & 286.400 & 0.781 \\
\hline 10 & SgreTa0000488 & 12.479 & 34.903 & & 9.829 & 343.044 & 0.781 \\
\hline 10 & SgreTa0009559 & 29.148 & 56.862 & & 5.898 & 335.386 & 0.781 \\
\hline 10 & $\begin{array}{c}\text { SgreTa0003305 } \\
\text { SgreTd000394 }\end{array}$ & 3.697 & 5.089 & & 42.278 & 215.172 & 0.773 \\
\hline 10 & $\begin{array}{c}9 \\
\text { SgreTb000624 }\end{array}$ & 14.818 & 20.871 & & 2.640 & 55.104 & 0.780 \\
\hline 10 & 3 & 87.476 & 113.407 & & 5.083 & 576.448 & 0.781 \\
\hline 10 & SgreTa0017707 & 1001.576 & 756.230 & & 9.643 & 7292.232 & 0.919 \\
\hline
\end{tabular}




\begin{tabular}{|c|c|c|c|c|c|c|c|c|}
\hline 10 & SgreTa0017736 & 22.014 & & 47.457 & & 1.186 & 56.284 & 0.780 \\
\hline 10 & SgreTa0007897 & 103.446 & & 136.655 & & 48.453 & 6621.339 & 0.781 \\
\hline 10 & SgreTa0008528 & $\begin{array}{c}180.946 \\
\text { UP }\end{array}$ & & 202.895 & & 8.199 & 1663.469 & 0.781 \\
\hline 10 & SgreTa0001449 & indefinitely & & 582.222 & & 0.369 & 214.943 & 0.919 \\
\hline 10 & SgreTa0005600 & $\begin{array}{l}56.826 \\
\text { UP }\end{array}$ & & $\begin{array}{c}62.483 \\
2844.05\end{array}$ & & 12.381 & 773.605 & 0.781 \\
\hline 10 & SgreTc0000004 & indefinitely & & 0 & & 0.154 & 439.013 & 0.998 \\
\hline 10 & SgreTc0000003 & 3.309 & & 4.751 & & 2.622 & 12.459 & 0.740 \\
\hline 10 & SgreTc0000003 & 3.687 & & 4.751 & & 2.622 & 12.459 & 0.740 \\
\hline 10 & SgreTa0013453 & 2.349 & & 3.157 & & 82.567 & 260.688 & 0.741 \\
\hline 10 & SgreTa0008219 & 36.004 & & 37.305 & & 4.463 & 166.492 & 0.781 \\
\hline 10 & SgreTa0008497 & 63.642 & & 55.465 & & 2.680 & 148.627 & 0.781 \\
\hline 10 & SgreTa0002186 & 225.140 & & 195.257 & & 2.298 & 448.716 & 0.781 \\
\hline 10 & $\begin{array}{c}\text { SgreTa0001661 } \\
\text { SgreTb001604 }\end{array}$ & 3.569 & & 4.482 & & 34.555 & 154.888 & 0.764 \\
\hline 10 & 7 & 6.146 & & 6.000 & & 21.481 & 128.879 & 0.777 \\
\hline 10 & SgreTa0014626 & 3.451 & & 5.045 & & 167.728 & 846.249 & 0.773 \\
\hline 11 & SgreTa0007432 & & 1.320 & & 1.732 & 10.454 & 6.036 & 0.514 \\
\hline 11 & SgreTa0001469 & 5.808 & & 5.190 & & 117.377 & 609.215 & 0.769 \\
\hline 11 & SgreTa0007802 & 100.910 & & 143.963 & & 2.751 & 396.066 & 0.776 \\
\hline 11 & SgreTa0005616 & 18.886 & & 58.723 & & 0.980 & 57.520 & 0.775 \\
\hline 11 & SgreTa0017664 & 1256.088 & & 12.672 & & 55.939 & 708.860 & 0.776 \\
\hline 11 & $\begin{array}{c}\text { SgreTa0014975 } \\
\text { SgreTb000624 }\end{array}$ & 4.698 & & 4.449 & & 14.202 & 63.189 & 0.758 \\
\hline 11 & 3 & 57.231 & & 108.492 & & 5.052 & 548.138 & 0.776 \\
\hline 11 & SgreTa0017707 & 1216.859 & & 358.692 & & 18.704 & 6708.830 & 0.776 \\
\hline 11 & SgreTa0017736 & 133.453 & & 210.082 & & 1.312 & $\begin{array}{c}275.635 \\
17085.54\end{array}$ & 0.776 \\
\hline 11 & SgreTa0007897 & 428.417 & & 352.913 & & 48.413 & 2 & 0.776 \\
\hline 11 & SgreTa0008528 & 78.880 & & 73.748 & & 12.770 & 941.764 & 0.776 \\
\hline 11 & SgreTa0005600 & 79.572 & & 68.596 & & 14.671 & 1006.405 & 0.776 \\
\hline 11 & SgreTa0013453 & 3.110 & & 2.931 & & 85.577 & 250.815 & 0.699 \\
\hline 11 & SgreTa0008219 & 19.265 & & 24.288 & & 4.807 & 116.746 & 0.776 \\
\hline 11 & SgreTa0001661 & 4.935 & & 4.377 & & 34.422 & 150.654 & 0.759 \\
\hline 11 & SgreTa0014626 & 4.514 & & 4.126 & & 161.557 & 666.631 & 0.759 \\
\hline 12 & SgreTa0007432 & 1.306 & & 1.009 & & 5.591 & 5.640 & 0.368 \\
\hline 12 & SgreTa0001469 & 10.590 & & 6.115 & & 132.137 & 808.004 & 0.754 \\
\hline 12 & SgreTa0007802 & 30.338 & & 19.349 & & 12.445 & 240.795 & 0.756 \\
\hline 12 & SgreTa0005616 & 13.108 & & 3.231 & & 16.053 & 51.872 & 0.720 \\
\hline 12 & $\begin{array}{c}\text { SgreTa0017664 } \\
\text { SgreTb000624 }\end{array}$ & 2689.973 & & 460.128 & & 15.436 & 7102.347 & 0.756 \\
\hline 12 & 3 & 11.666 & & 10.639 & & 40.743 & 433.469 & 0.756 \\
\hline 12 & SgreTa0017707 & 4391.195 & & 743.840 & & 8.249 & 6135.875 & 0.916 \\
\hline 12 & SgreTa0017736 & 964.373 & & 291.207 & & 1.516 & $\begin{array}{c}441.598 \\
19649.55\end{array}$ & 0.756 \\
\hline 12 & SgreTa0007897 & 2008.506 & & 615.488 & & 31.925 & 9 & 0.916 \\
\hline
\end{tabular}




\begin{tabular}{|c|c|c|c|c|c|c|}
\hline 12 & SgreTa0008528 & 506.989 & 179.902 & 4.855 & 873.417 & 0.756 \\
\hline 12 & SgreTa0005600 & 56.899 & 56.893 & 18.258 & 1038.782 & 0.756 \\
\hline 12 & SgreTa0013453 & 2.626 & 1.967 & 126.721 & 249.303 & 0.575 \\
\hline 12 & SgreTa0008219 & 15.694 & 8.224 & 16.314 & 134.174 & 0.756 \\
\hline 12 & SgreTa0001661 & 6.011 & 3.578 & 39.526 & 141.416 & 0.725 \\
\hline 12 & SgreTa0014626 & 3.395 & 2.522 & 242.996 & 612.785 & 0.681 \\
\hline 13 & SgreTa0007432 & 1.771 & 1.474 & 3.521 & 5.189 & 0.431 \\
\hline 13 & SgreTa0001469 & 10.436 & 5.266 & 136.452 & 718.578 & 0.732 \\
\hline 13 & SgreTa0007802 & 18.212 & 15.472 & 6.595 & 102.045 & 0.737 \\
\hline 13 & SgreTa0005616 & 7.423 & 2.137 & 14.119 & 30.177 & 0.641 \\
\hline 13 & $\begin{array}{c}\text { SgreTa0017664 } \\
\text { SgreTb000624 }\end{array}$ & 1748.639 & 576.461 & 26.670 & 4 & 0.911 \\
\hline 13 & 3 & 46.612 & 47.622 & 6.077 & 289.402 & 0.737 \\
\hline 13 & SgreTa0017707 & 8527.308 & 469.508 & 7.262 & 3409.772 & 0.911 \\
\hline 13 & SgreTa0017736 & 1344.749 & 658.489 & 0.672 & $\begin{array}{c}442.276 \\
17116.00\end{array}$ & 0.911 \\
\hline 13 & SgreTa0007897 & $\begin{array}{c}1243.649 \\
\text { UP }\end{array}$ & 456.919 & 37.460 & 2 & 0.911 \\
\hline 13 & SgreTa0008528 & indefinitely & 433.678 & 2.621 & 1136.614 & 0.738 \\
\hline 13 & SgreTa0005600 & 83.004 & 72.572 & 15.901 & 1153.927 & 0.738 \\
\hline 13 & SgreTa0013453 & 2.524 & 1.873 & 112.755 & 211.156 & 0.565 \\
\hline 13 & SgreTa0008219 & 29.562 & 10.248 & 13.991 & 143.386 & 0.737 \\
\hline 13 & SgreTa0001661 & 14.842 & 5.235 & 33.357 & 174.626 & 0.731 \\
\hline 13 & SgreTa0014626 & 5.341 & 3.954 & 164.431 & 650.184 & 0.711 \\
\hline
\end{tabular}

a "UP indefinitely": not detected in the legs after 35 cycles, "DOWN indefinitely": not detected in the pleuropodia; compare with the low RPKM in LEG and PLP samples, respectively

${ }^{\mathrm{b}}$ significant upregulation (UP) or downregulation (DOWN) (fold change between expression in pleuropodia and legs) are highlighted in magenta and blue, respectively (thresholds: prob > 0.7, RPKM > 10, fold change $>2$; prob below threshold highlighted in grey)

c not applicable - expression too low 
Supplementary Table 6. GOs enriched in the downregulated DEGs from the highly secreting pleuropodia (joined sample 10, 11 and 12 days) - First 100 terms are shown.

\begin{tabular}{|c|c|c|c|c|c|c|}
\hline category & $\begin{array}{c}\text { over } \\
\text { represented } \\
\text { pvalue }\end{array}$ & $\begin{array}{c}\text { num } \\
\text { DEInCat }\end{array}$ & $\begin{array}{c}\text { num } \\
\text { InCat }\end{array}$ & term & ontology ${ }^{a}$ & $\begin{array}{l}\text { over } \\
\text { represented } \\
\text { FDR }\end{array}$ \\
\hline GO:0048856 & $2.00 \mathrm{E}-20$ & 320 & 2000 & anatomical structure development & $\mathrm{BP}$ & $3.36 \mathrm{E}-16$ \\
\hline GO:0051301 & $1.75 \mathrm{E}-19$ & 88 & 292 & cell division & $\mathrm{BP}$ & $1.47 \mathrm{E}-15$ \\
\hline GO:0007010 & $1.56 \mathrm{E}-17$ & 112 & 446 & cytoskeleton organization & $\mathrm{BP}$ & $6.54 \mathrm{E}-14$ \\
\hline GO:0022402 & $2.10 \mathrm{E}-16$ & 126 & 567 & cell cycle process & $\mathrm{BP}$ & $5.89 \mathrm{E}-13$ \\
\hline GO:0031032 & $1.25 \mathrm{E}-15$ & 37 & 81 & actomyosin structure organization & $\mathrm{BP}$ & $3.00 \mathrm{E}-12$ \\
\hline GO:0007049 & $5.33 \mathrm{E}-15$ & 81 & 301 & $\begin{array}{c}\text { cell cycle } \\
\text { single-organism developmental }\end{array}$ & $\mathrm{BP}$ & $1.12 \mathrm{E}-11$ \\
\hline GO:0044767 & $1.21 \mathrm{E}-14$ & 375 & 2646 & process & $\mathrm{BP}$ & $2.26 \mathrm{E}-11$ \\
\hline GO:0051276 & $1.35 \mathrm{E}-14$ & 61 & 195 & chromosome organization & $\mathrm{BP}$ & $2.27 \mathrm{E}-11$ \\
\hline GO:0048513 & $3.75 E-14$ & 151 & 838 & animal organ development & BP & $5.72 \mathrm{E}-11$ \\
\hline GO:0032502 & $1.16 \mathrm{E}-13$ & 389 & 2822 & developmental process & $\mathrm{BP}$ & $1.62 \mathrm{E}-10$ \\
\hline GO:1903047 & $4.45 \mathrm{E}-13$ & 90 & 395 & mitotic cell cycle process & $\mathrm{BP}$ & $5.49 \mathrm{E}-10$ \\
\hline GO:0009888 & $4.57 \mathrm{E}-13$ & 80 & 348 & tissue development & $\mathrm{BP}$ & $5.49 \mathrm{E}-10$ \\
\hline GO:0071840 & $7.41 \mathrm{E}-13$ & 380 & 2732 & $\begin{array}{c}\text { cellular component } \\
\text { organization or biogenesis }\end{array}$ & $\mathrm{BP}$ & 8.31E-10 \\
\hline GO:0016043 & $1.26 \mathrm{E}-12$ & 374 & 2689 & cellular component organization & $\mathrm{BP}$ & $1.25 \mathrm{E}-09$ \\
\hline GO:0045214 & $1.60 \mathrm{E}-12$ & 25 & 51 & sarcomere organization & $\mathrm{BP}$ & 1.49E-09 \\
\hline GO:0022414 & $2.36 \mathrm{E}-12$ & 169 & 999 & reproductive process & $\mathrm{BP}$ & $2.08 \mathrm{E}-09$ \\
\hline GO:0097435 & $1.50 \mathrm{E}-11$ & 58 & 217 & supramolecular fiber organization & $\mathrm{BP}$ & 1.27E-08 \\
\hline GO:0071103 & $3.16 \mathrm{E}-11$ & 28 & 64 & DNA conformation change & $\mathrm{BP}$ & $2.53 \mathrm{E}-08$ \\
\hline GO:0007017 & $1.35 \mathrm{E}-10$ & 79 & 377 & $\begin{array}{l}\text { microtubule-based process } \\
\text { microtubule cytoskeleton }\end{array}$ & BP & $1.03 \mathrm{E}-07$ \\
\hline GO:0000226 & $1.51 \mathrm{E}-10$ & 54 & 202 & organization & $\mathrm{BP}$ & 1.10E-07 \\
\hline GO:0006996 & $1.99 \mathrm{E}-10$ & 196 & 1224 & organelle organization & $\mathrm{BP}$ & 1.39E-07 \\
\hline GO:0006323 & $3.62 \mathrm{E}-10$ & 21 & 40 & DNA packaging & $\mathrm{BP}$ & $2.44 \mathrm{E}-07$ \\
\hline GO:0006260 & $3.91 \mathrm{E}-10$ & 37 & 114 & DNA replication & $\mathrm{BP}$ & 2.53E-07 \\
\hline GO:0030261 & $1.55 \mathrm{E}-09$ & 20 & 39 & chromosome condensation & $\mathrm{BP}$ & 7.92E-07 \\
\hline GO:0000278 & 1.70E-09 & 30 & 83 & mitotic cell cycle & $\mathrm{BP}$ & 8.42E-07 \\
\hline GO:0035295 & 3.63E-09 & 47 & 187 & tube development & $\mathrm{BP}$ & $1.69 \mathrm{E}-06$ \\
\hline GO:0007444 & 5.94E-09 & 29 & 89 & $\begin{array}{l}\text { imaginal disc development } \\
\text { anatomical structure }\end{array}$ & BP & $2.56 \mathrm{E}-06$ \\
\hline GO:0009653 & $1.39 \mathrm{E}-08$ & 157 & 1030 & morphogenesis & $\mathrm{BP}$ & $5.72 \mathrm{E}-06$ \\
\hline GO:0007517 & 2.61E-08 & 25 & 70 & muscle organ development & $\mathrm{BP}$ & $1.04 \mathrm{E}-05$ \\
\hline GO:0042127 & $3.83 \mathrm{E}-08$ & 88 & 474 & regulation of cell proliferation & $\mathrm{BP}$ & $1.43 \mathrm{E}-05$ \\
\hline GO:0010564 & $5.03 E-08$ & 69 & 338 & regulation of cell cycle process & $\mathrm{BP}$ & $1.76 \mathrm{E}-05$ \\
\hline GO:0030036 & $6.24 \mathrm{E}-08$ & 45 & 175 & actin cytoskeleton organization & BP & 2.14E-05 \\
\hline GO:1903046 & 7.39E-08 & 33 & 113 & meiotic cell cycle process & $\mathrm{BP}$ & 2.49E-05 \\
\hline GO:0032501 & $1.53 \mathrm{E}-07$ & 240 & 1835 & multicellular organismal process & $\mathrm{BP}$ & 5.04E-05 \\
\hline GO:0051726 & $1.63 \mathrm{E}-07$ & 95 & 533 & $\begin{array}{l}\text { regulation of cell cycle } \\
\text { regulation of developmental }\end{array}$ & $\mathrm{BP}$ & $5.28 \mathrm{E}-05$ \\
\hline GO:0050793 & $1.67 \mathrm{E}-07$ & 153 & 989 & process & BP & $5.28 \mathrm{E}-05$ \\
\hline
\end{tabular}




\begin{tabular}{|c|c|c|c|c|c|c|}
\hline GO:0009886 & 2.02E-07 & 44 & 182 & $\begin{array}{l}\text { post-embryonic animal } \\
\text { morphogenesis }\end{array}$ & BP & $6.28 \mathrm{E}-05$ \\
\hline GO:0030029 & 3.27E-07 & 46 & 193 & actin filament-based process & $\mathrm{BP}$ & $9.37 E-05$ \\
\hline GO:0006270 & $3.29 \mathrm{E}-07$ & 14 & 28 & $\begin{array}{l}\text { DNA replication initiation } \\
\text { single organism reproductive }\end{array}$ & $\mathrm{BP}$ & 9.37E-05 \\
\hline GO:0044702 & $3.52 \mathrm{E}-07$ & 123 & 766 & $\begin{array}{l}\text { process } \\
\text { regulation of mitotic cell }\end{array}$ & $\mathrm{BP}$ & $9.87 \mathrm{E}-05$ \\
\hline GO:1901990 & 4.50E-07 & 41 & 165 & cycle phase transition & BP & 0.000122089 \\
\hline GO:0030703 & 4.60E-07 & 9 & 12 & eggshell formation & BP & 0.00012278 \\
\hline GO:0044699 & $4.86 \mathrm{E}-07$ & 678 & 6032 & single-organism process & $\mathrm{BP}$ & 0.000127791 \\
\hline GO:0060429 & $5.28 \mathrm{E}-07$ & 39 & 161 & epithelium development & $\mathrm{BP}$ & 0.000136482 \\
\hline GO:0006275 & $5.68 \mathrm{E}-07$ & 17 & 41 & regulation of DNA replication & BP & 0.000138504 \\
\hline GO:0007498 & $6.68 \mathrm{E}-07$ & 18 & 46 & mesoderm development & $\mathrm{BP}$ & 0.00015832 \\
\hline GO:0051783 & $8.24 \mathrm{E}-07$ & 42 & 174 & regulation of nuclear division & $\mathrm{BP}$ & 0.000192552 \\
\hline GO:0007346 & 1.00E-06 & 65 & 338 & $\begin{array}{l}\text { regulation of mitotic cell cycle } \\
\text { regulation of mitotic nuclear }\end{array}$ & $\mathrm{BP}$ & 0.000231045 \\
\hline GO:0007088 & $1.20 \mathrm{E}-06$ & 40 & 163 & $\begin{array}{c}\text { division } \\
\text { single-multicellular organism }\end{array}$ & BP & 0.000262458 \\
\hline GO:0044707 & $1.25 \mathrm{E}-06$ & 209 & 1578 & $\begin{array}{c}\text { process } \\
\text { regulation of cell cycle phase }\end{array}$ & $\mathrm{BP}$ & 0.000270362 \\
\hline GO:1901987 & $1.28 \mathrm{E}-06$ & 42 & 176 & transition & BP & 0.000272555 \\
\hline GO:0007076 & $1.36 \mathrm{E}-06$ & 12 & 22 & mitotic chromosome condensation & $\mathrm{BP}$ & 0.00028512 \\
\hline GO:2000026 & $1.61 \mathrm{E}-06$ & 115 & 715 & $\begin{array}{l}\text { regulation of multicellular } \\
\text { organismal development } \\
\text { positive regulation of cell cycle }\end{array}$ & BP & 0.000330645 \\
\hline GO:0090068 & $1.82 \mathrm{E}-06$ & 34 & 128 & process & $\mathrm{BP}$ & 0.000368697 \\
\hline GO:0051239 & 2.61E-06 & 153 & 1042 & $\begin{array}{l}\text { regulation of multicellular } \\
\text { organismal process }\end{array}$ & BP & 0.000521508 \\
\hline GO:0061077 & $2.80 \mathrm{E}-06$ & 13 & 33 & $\begin{array}{l}\text { chaperone-mediated protein folding } \\
\text { chorion-containing eggshell }\end{array}$ & $\mathrm{BP}$ & 0.000547652 \\
\hline GO:0007304 & $2.84 \mathrm{E}-06$ & 8 & 11 & formation & BP & 0.000549565 \\
\hline GO:0035220 & $3.44 \mathrm{E}-06$ & 19 & 59 & wing disc development & $\mathrm{BP}$ & 0.000646364 \\
\hline GO:0048869 & $3.46 \mathrm{E}-06$ & 201 & 1476 & cellular developmental process & $\mathrm{BP}$ & 0.000646364 \\
\hline GO:0032989 & $3.86 \mathrm{E}-06$ & 74 & 451 & cellular component morphogenesis & BP & 0.000704622 \\
\hline GO:0043062 & 4.36E-06 & 25 & 90 & $\begin{array}{l}\text { extracellular structure organization } \\
\text { regulation of DNA-dependent DNA }\end{array}$ & $\mathrm{BP}$ & 0.000779886 \\
\hline GO:0090329 & 4.91E-06 & 11 & 23 & $\begin{array}{c}\text { replication } \\
\text { pteridine-containing compound }\end{array}$ & $\mathrm{BP}$ & 0.000868797 \\
\hline GO:0042559 & 5.27E-06 & 8 & 14 & $\begin{array}{l}\text { biosynthetic process } \\
\text { columnar/cuboidal epithelial }\end{array}$ & $\mathrm{BP}$ & 0.000923292 \\
\hline GO:0002066 & $6.43 \mathrm{E}-06$ & 22 & 76 & $\begin{array}{l}\text { cell development } \\
\text { cellular process involved in } \\
\text { reproduction }\end{array}$ & $\mathrm{BP}$ & 0.001114131 \\
\hline GO:0022412 & 6.67E-06 & 72 & 405 & $\begin{array}{l}\text { in multicellular organism } \\
\text { neurofilament cytoskeleton }\end{array}$ & BP & 0.001145056 \\
\hline GO:0060052 & $9.62 \mathrm{E}-06$ & 7 & 10 & organization & BP & 0.001586311 \\
\hline GO:0007552 & $1.05 \mathrm{E}-05$ & 12 & 40 & $\begin{array}{l}\text { metamorphosis } \\
\text { regulation of chromosome }\end{array}$ & $\mathrm{BP}$ & 0.001685364 \\
\hline GO:0051983 & 1.10E-05 & 19 & 58 & $\begin{array}{c}\text { segregation } \\
\text { imaginal disc-derived appendage }\end{array}$ & BP & 0.00175249 \\
\hline GO:0035114 & $1.13 \mathrm{E}-05$ & 24 & 88 & morphogenesis & $\mathrm{BP}$ & 0.001777181 \\
\hline GO:0007015 & 1.19E-05 & 30 & 122 & actin filament organization & BP & 0.001832454 \\
\hline
\end{tabular}




\begin{tabular}{|c|c|c|c|c|c|c|}
\hline GO:0000904 & $1.26 \mathrm{E}-05$ & 23 & 84 & $\begin{array}{l}\text { cell morphogenesis involved in } \\
\text { differentiation }\end{array}$ & $\mathrm{BP}$ & 0.001926008 \\
\hline GO:0045297 & $1.34 \mathrm{E}-05$ & 8 & 25 & post-mating behavior & $\mathrm{BP}$ & 0.002030503 \\
\hline GO:0061061 & $1.59 \mathrm{E}-05$ & 33 & 162 & muscle structure development & $\mathrm{BP}$ & 0.002373115 \\
\hline GO:0048646 & $1.61 \mathrm{E}-05$ & 74 & 433 & $\begin{array}{l}\text { anatomical structure formation } \\
\text { involved in morphogenesis }\end{array}$ & BP & 0.002378056 \\
\hline GO:0002064 & $1.77 \mathrm{E}-05$ & 27 & 108 & $\begin{array}{l}\text { epithelial cell development } \\
\text { pteridine-containing }\end{array}$ & $\mathrm{BP}$ & 0.002539527 \\
\hline GO:0042558 & $1.77 \mathrm{E}-05$ & 10 & 23 & compound metabolic process & $\mathrm{BP}$ & 0.002539527 \\
\hline GO:0006281 & $1.90 \mathrm{E}-05$ & 60 & 335 & DNA repair & $\mathrm{BP}$ & 0.002689969 \\
\hline GO:0030071 & $2.12 \mathrm{E}-05$ & 15 & 41 & $\begin{array}{l}\text { regulation of mitotic metaphase/ } \\
\text { anaphase transition }\end{array}$ & $\mathrm{BP}$ & 0.002893062 \\
\hline GO:1902099 & $2.12 \mathrm{E}-05$ & 15 & 41 & $\begin{array}{l}\text { regulation of metaphase/ } \\
\text { anaphase transition of cell cycle }\end{array}$ & BP & 0.002893062 \\
\hline GO:0030198 & $2.57 \mathrm{E}-05$ & 22 & 82 & extracellular matrix organization & $\mathrm{BP}$ & 0.003437198 \\
\hline GO:0044763 & $2.58 \mathrm{E}-05$ & 548 & 4818 & single-organism cellular process & $\mathrm{BP}$ & 0.003437198 \\
\hline GO:0030707 & $2.60 \mathrm{E}-05$ & 19 & 63 & ovarian follicle cell development & $\mathrm{BP}$ & 0.003439415 \\
\hline GO:0007527 & $2.74 \mathrm{E}-05$ & 7 & 10 & adult somatic muscle development & $\mathrm{BP}$ & 0.003439415 \\
\hline GO:0045168 & $2.74 \mathrm{E}-05$ & 19 & 65 & $\begin{array}{l}\text { cell-cell signaling involved } \\
\text { in cell fate commitment }\end{array}$ & BP & 0.003439415 \\
\hline GO:0046331 & $2.74 \mathrm{E}-05$ & 19 & 65 & $\begin{array}{l}\text { lateral inhibition } \\
\text { negative regulation of mitotic }\end{array}$ & $\mathrm{BP}$ & 0.003439415 \\
\hline GO:0045841 & $2.74 \mathrm{E}-05$ & 10 & 20 & $\begin{array}{c}\text { metaphase/anaphase transition } \\
\text { negative regulation of } \\
\text { metaphase/anaphase transition of }\end{array}$ & $\mathrm{BP}$ & 0.003439415 \\
\hline GO:1902100 & $2.74 \mathrm{E}-05$ & 10 & 20 & $\begin{array}{c}\text { cell cycle } \\
\text { negative regulation of }\end{array}$ & $\mathrm{BP}$ & 0.003439415 \\
\hline GO:1905819 & $2.74 \mathrm{E}-05$ & 10 & 20 & $\begin{array}{l}\text { chromosome separation } \\
\text { negative regulation of mitotic }\end{array}$ & $\mathrm{BP}$ & 0.003439415 \\
\hline GO:2000816 & $2.74 \mathrm{E}-05$ & 10 & 20 & $\begin{array}{l}\text { sister chromatid separation } \\
\text { multicellular organismal }\end{array}$ & $\mathrm{BP}$ & 0.003439415 \\
\hline GO:0048609 & $2.90 \mathrm{E}-05$ & 68 & 409 & $\begin{array}{l}\text { reproductive process } \\
\text { regulation of sister chromatid }\end{array}$ & $\mathrm{BP}$ & 0.003612766 \\
\hline GO:0033045 & $3.02 \mathrm{E}-05$ & 17 & 52 & segregation & $\mathrm{BP}$ & 0.003706573 \\
\hline GO:0010965 & $3.11 \mathrm{E}-05$ & 15 & 42 & $\begin{array}{l}\text { regulation of mitotic sister } \\
\text { chromatid separation } \\
\text { regulation of chromosome }\end{array}$ & BP & 0.0037589 \\
\hline GO:1905818 & $3.11 \mathrm{E}-05$ & 15 & 42 & separation & $\mathrm{BP}$ & 0.0037589 \\
\hline GO:0007519 & $3.20 \mathrm{E}-05$ & 11 & 24 & skeletal muscle tissue development & $\mathrm{BP}$ & 0.003837808 \\
\hline GO:0032467 & $3.41 \mathrm{E}-05$ & 8 & 13 & positive regulation of cytokinesis & $\mathrm{BP}$ & 0.003984256 \\
\hline GO:0042335 & $3.67 E-05$ & 20 & 78 & $\begin{array}{c}\text { cuticle development } \\
\text { negative regulation of RNA }\end{array}$ & $\mathrm{BP}$ & 0.004254892 \\
\hline GO:0051253 & $3.69 \mathrm{E}-05$ & 77 & 460 & $\begin{array}{l}\text { metabolic process } \\
\text { post-embryonic appendage }\end{array}$ & $\mathrm{BP}$ & 0.004254892 \\
\hline GO:0035120 & $3.97 E-05$ & 22 & 82 & $\begin{array}{c}\text { morphogenesis } \\
\text { negative regulation of }\end{array}$ & $\mathrm{BP}$ & 0.004537709 \\
\hline GO:0033046 & 4.09E-05 & 11 & 25 & $\begin{array}{l}\text { sister chromatid segregation } \\
\text { negative regulation of }\end{array}$ & $\mathrm{BP}$ & 0.004610621 \\
\hline GO:0051985 & $4.09 \mathrm{E}-05$ & 11 & 25 & chromosome segregation & $\mathrm{BP}$ & 0.004610621 \\
\hline
\end{tabular}

a BP, biological process; CC, cellular component; MF, molecular function 
Supplementary Table 7. GOs enriched in the upregulated DEGs from the highly secreting pleuropodia (joined sample 10, 11 and 12 days).

\begin{tabular}{|c|c|c|c|c|c|c|}
\hline category & $\begin{array}{l}\text { over } \\
\text { represented } \\
\text { pvalue }\end{array}$ & $\begin{array}{c}\text { num } \\
\text { DEInCat }\end{array}$ & $\begin{array}{l}\text { num } \\
\text { InCat }\end{array}$ & term & ontology & $\begin{array}{l}\text { over } \\
\text { represented } \\
\text { FDR }\end{array}$ \\
\hline GO:0006811 & $1.47 \mathrm{E}-19$ & 77 & 534 & ion transport & BP & $1.24 \mathrm{E}-15$ \\
\hline GO:0034220 & $2.23 E-16$ & 43 & 221 & ion transmembrane transport & $\mathrm{BP}$ & $4.69 \mathrm{E}-13$ \\
\hline GO:0090662 & $7.94 \mathrm{E}-16$ & 16 & 24 & $\begin{array}{l}\text { ATP hydrolysis coupled } \\
\text { transmembrane transport }\end{array}$ & BP & $1.48 \mathrm{E}-12$ \\
\hline GO:0015672 & 4.65E-15 & 33 & 149 & $\begin{array}{c}\text { monovalent inorganic cation } \\
\text { transport }\end{array}$ & $\mathrm{BP}$ & $6.76 \mathrm{E}-12$ \\
\hline GO:0055085 & $4.82 \mathrm{E}-15$ & 56 & 381 & transmembrane transport & $\mathrm{BP}$ & $6.76 \mathrm{E}-12$ \\
\hline GO:0015988 & $6.36 \mathrm{E}-15$ & 14 & 19 & $\begin{array}{c}\text { energy coupled proton } \\
\text { transmembrane transport, } \\
\text { against electrochemical gradient }\end{array}$ & $\mathrm{BP}$ & 7.64E-12 \\
\hline GO:0015991 & $6.36 \mathrm{E}-15$ & 14 & 19 & $\begin{array}{l}\text { ATP hydrolysis coupled } \\
\text { proton transport }\end{array}$ & $\mathrm{BP}$ & $7.64 \mathrm{E}-12$ \\
\hline GO:0099131 & $4.55 \mathrm{E}-14$ & 14 & 21 & $\begin{array}{l}\text { ATP hydrolysis coupled } \\
\text { ion transmembrane transport }\end{array}$ & BP & 4.50E-11 \\
\hline GO:0099132 & $4.55 \mathrm{E}-14$ & 14 & 21 & $\begin{array}{l}\text { ATP hydrolysis coupled } \\
\text { cation transmembrane transport }\end{array}$ & $\mathrm{BP}$ & $4.50 \mathrm{E}-11$ \\
\hline GO:0006820 & $1.20 \mathrm{E}-13$ & 43 & 253 & anion transport & $\mathrm{BP}$ & $1.06 \mathrm{E}-10$ \\
\hline GO:0006818 & $8.74 \mathrm{E}-13$ & 17 & 41 & hydrogen transport & $\mathrm{BP}$ & 7.00E-10 \\
\hline GO:0015711 & $1.50 \mathrm{E}-12$ & 37 & 207 & organic anion transport & BP & $1.14 \mathrm{E}-09$ \\
\hline GO:0015992 & $7.45 \mathrm{E}-12$ & 16 & 40 & proton transport & $\mathrm{BP}$ & $5.45 \mathrm{E}-09$ \\
\hline GO:0044765 & $8.20 E-11$ & 92 & 961 & single-organism transport & $\mathrm{BP}$ & $4.90 E-08$ \\
\hline GO:0007311 & $1.21 \mathrm{E}-10$ & 12 & 24 & $\begin{array}{c}\text { maternal specification of } \\
\text { dorsal/ventral } \\
\text { axis, oocyte, germ-line encoded }\end{array}$ & $\mathrm{BP}$ & $6.77 \mathrm{E}-08$ \\
\hline GO:0006812 & $1.34 \mathrm{E}-10$ & 41 & 302 & cation transport & $\mathrm{BP}$ & $7.26 \mathrm{E}-08$ \\
\hline GO:1902600 & $2.07 \mathrm{E}-10$ & 14 & 35 & $\begin{array}{c}\text { hydrogen ion transmembrane } \\
\text { transport }\end{array}$ & $\mathrm{BP}$ & $1.06 \mathrm{E}-07$ \\
\hline GO:1902578 & $6.71 \mathrm{E}-10$ & 95 & 1043 & single-organism localization & BP & $3.05 \mathrm{E}-07$ \\
\hline GO:0098655 & $1.92 \mathrm{E}-09$ & 24 & 133 & cation transmembrane transport & $\mathrm{BP}$ & 7.67E-07 \\
\hline GO:0008063 & 7.06E-09 & 15 & 50 & Toll signaling pathway & $\mathrm{BP}$ & $2.58 \mathrm{E}-06$ \\
\hline GO:1901615 & $1.75 \mathrm{E}-08$ & 36 & 273 & $\begin{array}{l}\text { organic hydroxy compound } \\
\text { metabolic process }\end{array}$ & $\mathrm{BP}$ & 5.65E-06 \\
\hline GO:0007310 & $1.82 \mathrm{E}-08$ & 13 & 39 & $\begin{array}{l}\text { oocyte dorsal/ventral axis } \\
\text { specification }\end{array}$ & $\mathrm{BP}$ & 5.76E-06 \\
\hline GO:0098660 & $2.46 \mathrm{E}-08$ & 24 & 145 & $\begin{array}{c}\text { inorganic ion transmembrane } \\
\text { transport }\end{array}$ & $\mathrm{BP}$ & 7.34E-06 \\
\hline GO:0098662 & 3.09E-08 & 21 & 117 & $\begin{array}{c}\text { inorganic cation transmembrane } \\
\text { transport }\end{array}$ & $\mathrm{BP}$ & 8.80E-06 \\
\hline GO:0009950 & 4.22E-08 & 15 & 55 & dorsal/ventral axis specification & $\mathrm{BP}$ & $1.14 \mathrm{E}-05$ \\
\hline GO:0015849 & $5.06 \mathrm{E}-08$ & 23 & 132 & organic acid transport & $\mathrm{BP}$ & $1.31 \mathrm{E}-05$ \\
\hline GO:0046942 & $5.06 \mathrm{E}-08$ & 23 & 132 & carboxylic acid transport & $\mathrm{BP}$ & $1.31 \mathrm{E}-05$ \\
\hline GO:0006865 & $6.50 \mathrm{E}-08$ & 17 & 76 & amino acid transport & BP & $1.58 \mathrm{E}-05$ \\
\hline GO:0003333 & $1.02 \mathrm{E}-07$ & 12 & 37 & $\begin{array}{c}\text { amino acid transmembrane } \\
\text { transport }\end{array}$ & $\mathrm{BP}$ & $2.42 \mathrm{E}-05$ \\
\hline GO:0006629 & $1.29 \mathrm{E}-07$ & 64 & 687 & lipid metabolic process & BP & 3.02E-05 \\
\hline
\end{tabular}




\begin{tabular}{|c|c|c|c|c|c|c|}
\hline GO:0007309 & 1.69E-07 & 14 & 52 & oocyte axis specification & $\mathrm{BP}$ & $3.80 \mathrm{E}-05$ \\
\hline GO:0006814 & 3.19E-07 & 15 & 73 & sodium ion transport & $\mathrm{BP}$ & $6.96 \mathrm{E}-05$ \\
\hline GO:0007370 & 4.49E-07 & 8 & 17 & ventral furrow formation & $\mathrm{BP}$ & 9.44E-05 \\
\hline GO:0006809 & 5.39E-07 & 5 & 7 & nitric oxide biosynthetic process & $\mathrm{BP}$ & 0.000109252 \\
\hline GO:0046209 & $5.39 \mathrm{E}-07$ & 5 & 7 & nitric oxide metabolic process & $\mathrm{BP}$ & 0.000109252 \\
\hline GO:0044281 & $1.22 \mathrm{E}-06$ & 85 & 1048 & small molecule metabolic process & $\mathrm{BP}$ & 0.000226884 \\
\hline GO:0044710 & $1.23 \mathrm{E}-06$ & 144 & 2090 & single-organism metabolic process & $\mathrm{BP}$ & 0.000226884 \\
\hline GO:1903825 & $1.91 \mathrm{E}-06$ & 12 & 47 & $\begin{array}{c}\text { organic acid transmembrane } \\
\text { transport }\end{array}$ & $\mathrm{BP}$ & 0.00032152 \\
\hline GO:1905039 & $1.91 \mathrm{E}-06$ & 12 & 47 & $\begin{array}{c}\text { carboxylic acid transmembrane } \\
\text { transport }\end{array}$ & $\mathrm{BP}$ & 0.00032152 \\
\hline GO:0098656 & $2.65 \mathrm{E}-06$ & 15 & 75 & anion transmembrane transport & $\mathrm{BP}$ & 0.000423703 \\
\hline GO:0006810 & 4.16E-06 & 136 & 1930 & transport & $\mathrm{BP}$ & 0.000641496 \\
\hline GO:0044699 & 4.20E-06 & 346 & 6032 & single-organism process & $\mathrm{BP}$ & 0.000642071 \\
\hline GO:0051234 & 4.73E-06 & 139 & 1983 & establishment of localization & $\mathrm{BP}$ & 0.000714453 \\
\hline GO:0006885 & $4.76 \mathrm{E}-06$ & 10 & 35 & regulation of $\mathrm{pH}$ & $\mathrm{BP}$ & 0.000714453 \\
\hline GO:0044703 & 5.37E-06 & 13 & 67 & $\begin{array}{l}\text { multi-organism reproductive } \\
\text { process }\end{array}$ & $\mathrm{BP}$ & 0.00079928 \\
\hline GO:2001057 & $6.39 E-06$ & 5 & 9 & $\begin{array}{l}\text { reactive nitrogen species } \\
\text { metabolic process }\end{array}$ & $\mathrm{BP}$ & 0.000925751 \\
\hline GO:0006026 & 8.56E-06 & 11 & 47 & aminoglycan catabolic process & $\mathrm{BP}$ & 0.001199491 \\
\hline GO:0055067 & 8.96E-06 & 10 & 37 & $\begin{array}{c}\text { monovalent inorganic cation } \\
\text { homeostasis }\end{array}$ & $\mathrm{BP}$ & 0.001245784 \\
\hline GO:1903409 & 1.93E-05 & 5 & 11 & $\begin{array}{l}\text { reactive oxygen species } \\
\text { biosynthetic process }\end{array}$ & $\mathrm{BP}$ & 0.002598165 \\
\hline GO:0051453 & 2.03E-05 & 9 & 33 & regulation of intracellular $\mathrm{pH}$ & $\mathrm{BP}$ & 0.002715202 \\
\hline GO:0046348 & $2.30 \mathrm{E}-05$ & 7 & 19 & amino sugar catabolic process & $\mathrm{BP}$ & 0.003041764 \\
\hline GO:0030641 & 2.74E-05 & 9 & 34 & regulation of cellular $\mathrm{pH}$ & $\mathrm{BP}$ & 0.003577987 \\
\hline GO:0045851 & $2.88 \mathrm{E}-05$ & 6 & 15 & $\mathrm{pH}$ reduction & $\mathrm{BP}$ & 0.003727792 \\
\hline GO:1901136 & $3.44 \mathrm{E}-05$ & 15 & 93 & $\begin{array}{c}\text { carbohydrate derivative catabolic } \\
\text { process }\end{array}$ & $\mathrm{BP}$ & 0.004385845 \\
\hline GO:0050801 & 3.60E-05 & 26 & 237 & ion homeostasis & $\mathrm{BP}$ & 0.00454847 \\
\hline GO:0030004 & 3.68E-05 & 9 & 35 & $\begin{array}{c}\text { cellular monovalent inorganic } \\
\text { cation homeostasis }\end{array}$ & $\mathrm{BP}$ & 0.004619921 \\
\hline GO:0042940 & $3.92 \mathrm{E}-05$ & 4 & 6 & D-amino acid transport & $\mathrm{BP}$ & 0.004883535 \\
\hline GO:0006869 & 4.07E-05 & 18 & 130 & lipid transport & $\mathrm{BP}$ & 0.004979036 \\
\hline GO:0019835 & 4.09E-05 & 5 & 13 & cytolysis & $\mathrm{BP}$ & 0.004979036 \\
\hline GO:0005975 & $4.52 \mathrm{E}-05$ & 35 & 364 & carbohydrate metabolic process & $\mathrm{BP}$ & 0.005466278 \\
\hline GO:0006582 & $5.02 \mathrm{E}-05$ & 10 & 47 & melanin metabolic process & $\mathrm{BP}$ & 0.005988923 \\
\hline GO:0007035 & $5.38 \mathrm{E}-05$ & 5 & 10 & vacuolar acidification & $\mathrm{BP}$ & 0.006373312 \\
\hline GO:0009617 & 5.70E-05 & 17 & 123 & response to bacterium & $\mathrm{BP}$ & 0.006708191 \\
\hline GO:0048878 & $5.84 \mathrm{E}-05$ & 33 & 341 & chemical homeostasis & $\mathrm{BP}$ & 0.006773732 \\
\hline GO:0043207 & $5.84 \mathrm{E}-05$ & 33 & 369 & $\begin{array}{l}\text { response to external biotic } \\
\text { stimulus } \\
\text { glucosamine-containing }\end{array}$ & BP & 0.006773732 \\
\hline GO:1901072 & 7.99E-05 & 6 & 16 & $\begin{array}{l}\text { compound } \\
\text { catabolic process }\end{array}$ & $\mathrm{BP}$ & 0.008951938 \\
\hline GO:0072593 & 8.36E-05 & 9 & 43 & $\begin{array}{l}\text { reactive oxygen species metabolic } \\
\text { process }\end{array}$ & $\mathrm{BP}$ & 0.009311797 \\
\hline
\end{tabular}




\begin{tabular}{|c|c|c|c|c|c|c|}
\hline GO:0009607 & $9.01 \mathrm{E}-05$ & 33 & 377 & response to biotic stimulus & $\mathrm{BP}$ & 0.009925828 \\
\hline GO:0035006 & $9.47 E-05$ & 8 & 32 & melanization defense response & $\mathrm{BP}$ & 0.010268044 \\
\hline GO:0055088 & 0.0001012 & 12 & 70 & lipid homeostasis & BP & 0.010843485 \\
\hline GO:0051179 & 0.0001095 & 148 & 2272 & localization & $\mathrm{BP}$ & 0.011649984 \\
\hline GO:0044706 & 0.0001168 & 10 & 55 & $\begin{array}{l}\text { multi-multicellular organism } \\
\text { process }\end{array}$ & BP & 0.012352242 \\
\hline GO:0050830 & 0.0001252 & 9 & 43 & $\begin{array}{l}\text { defense response to Gram- } \\
\text { positive bacterium }\end{array}$ & BP & 0.013158789 \\
\hline GO:0009798 & 0.0001292 & 16 & 109 & axis specification & $\mathrm{BP}$ & 0.013446589 \\
\hline GO:0030001 & 0.0001295 & 23 & 222 & metal ion transport & $\mathrm{BP}$ & 0.013446589 \\
\hline GO:0009620 & 0.0001398 & 9 & 42 & response to fungus & BP & 0.014424836 \\
\hline GO:0009605 & 0.0001444 & 51 & 666 & response to external stimulus & $\mathrm{BP}$ & 0.014716665 \\
\hline GO:0006003 & 0.0001636 & 3 & 3 & $\begin{array}{l}\text { fructose } 2,6 \text {-bisphosphate } \\
\text { metabolic process }\end{array}$ & BP & 0.016276968 \\
\hline GO:0006665 & 0.0001704 & 11 & 61 & sphingolipid metabolic process & $\mathrm{BP}$ & 0.016755635 \\
\hline GO:0051704 & 0.0001748 & 44 & 549 & multi-organism process & $\mathrm{BP}$ & 0.016992012 \\
\hline GO:0018958 & 0.0001763 & 13 & 88 & $\begin{array}{l}\text { phenol-containing compound } \\
\text { metabolic process }\end{array}$ & $\mathrm{BP}$ & 0.017039357 \\
\hline GO:0051707 & 0.0001907 & 28 & 311 & response to other organism & $\mathrm{BP}$ & 0.018223573 \\
\hline GO:0065008 & 0.0001928 & 103 & 1526 & regulation of biological quality & $\mathrm{BP}$ & 0.018318887 \\
\hline GO:0006066 & 0.0002057 & 20 & 175 & alcohol metabolic process & $\mathrm{BP}$ & 0.019211887 \\
\hline GO:0051452 & 0.0002413 & 5 & 14 & intracellular $\mathrm{pH}$ reduction & BP & 0.02229664 \\
\hline GO:0006563 & 0.0002432 & 4 & 7 & L-serine metabolic process & BP & 0.022346163 \\
\hline GO:0010817 & 0.0003176 & 22 & 213 & regulation of hormone levels & $\mathrm{BP}$ & 0.028404042 \\
\hline GO:0071825 & 0.0003465 & 6 & 22 & $\begin{array}{l}\text { protein-lipid complex subunit } \\
\text { organization }\end{array}$ & $\mathrm{BP}$ & 0.030502766 \\
\hline GO:0071827 & 0.0003465 & 6 & 22 & $\begin{array}{c}\text { plasma lipoprotein particle } \\
\text { organization }\end{array}$ & BP & 0.030502766 \\
\hline GO:0006032 & 0.0004406 & 5 & 14 & chitin catabolic process & BP & 0.03838474 \\
\hline GO:0034368 & 0.0004595 & 5 & 14 & protein-lipid complex remodeling & $\mathrm{BP}$ & 0.039247694 \\
\hline GO:0034369 & 0.0004595 & 5 & 14 & $\begin{array}{l}\text { plasma lipoprotein particle } \\
\text { remodeling }\end{array}$ & $\mathrm{BP}$ & 0.039247694 \\
\hline GO:0034375 & 0.0004595 & 5 & 14 & $\begin{array}{l}\text { high-density lipoprotein particle } \\
\text { remodeling }\end{array}$ & BP & 0.039247694 \\
\hline GO:0042742 & 0.0004629 & 14 & 109 & defense response to bacterium & $\mathrm{BP}$ & 0.039308893 \\
\hline GO:0034374 & 0.0005063 & 4 & 9 & $\begin{array}{l}\text { low-density lipoprotein particle } \\
\text { remodeling }\end{array}$ & BP & 0.042777281 \\
\hline GO:0045087 & 0.0005142 & 18 & 162 & innate immune response & BP & 0.043231252 \\
\hline GO:0019752 & 0.0005449 & 43 & 513 & carboxylic acid metabolic process & $\mathrm{BP}$ & 0.04558214 \\
\hline GO:0006564 & 0.0005656 & 3 & 4 & L-serine biosynthetic process & BP & 0.047084223 \\
\hline GO:0032367 & 0.0005741 & 5 & 18 & intracellular cholesterol transport & $\mathrm{BP}$ & 0.047557237 \\
\hline GO:0032787 & 0.0005854 & 26 & 263 & $\begin{array}{c}\text { monocarboxylic acid metabolic } \\
\text { process }\end{array}$ & $\mathrm{BP}$ & 0.048015835 \\
\hline
\end{tabular}

a BP, biological process; CC, cellular component; MF, molecular function 
Supplementary Table 8. GOs enriched in the downregulated DEGs from each developmental stage $(F D R<e-5)$; Only the first $10 \mathrm{GO}$ terms of each time-point are shown.

\begin{tabular}{|c|c|c|c|c|c|c|c|}
\hline day & category & $\begin{array}{c}\text { over } \\
\text { represented } \\
\text { pvalue }\end{array}$ & $\begin{array}{l}\text { num } \\
\text { DEInCat }\end{array}$ & $\begin{array}{c}\text { num } \\
\text { InCat }\end{array}$ & term & ontology $\mathrm{y}^{\mathrm{a}}$ & $\begin{array}{l}\text { over } \\
\text { represented } \\
\text { FDR }\end{array}$ \\
\hline \multirow[t]{2}{*}{7} & GO:0051301 & $1.50 \mathrm{E}-11$ & 23 & 292 & cell division & $\mathrm{BP}$ & $2.52 \mathrm{E}-07$ \\
\hline & GO:0007049 & $1.34 \mathrm{E}-09$ & 21 & 301 & cell cycle & $\mathrm{BP}$ & $1.13 E-05$ \\
\hline \multirow[t]{10}{*}{8} & GO:0051301 & $2.76 \mathrm{E}-11$ & 28 & 292 & cell division & BP & $4.65 \mathrm{E}-07$ \\
\hline & GO:0005488 & $2.99 \mathrm{E}-10$ & 185 & 7055 & binding & MF & $1.76 \mathrm{E}-06$ \\
\hline & GO:0007049 & $3.14 \mathrm{E}-10$ & 27 & 301 & $\begin{array}{c}\text { cell cycle } \\
\text { chaperonin-containing T- }\end{array}$ & BP & $1.76 \mathrm{E}-06$ \\
\hline & GO:0005832 & $2.00 \mathrm{E}-09$ & 6 & 8 & complex & CC & $6.33 \mathrm{E}-06$ \\
\hline & GO:0101031 & 2.00E-09 & 6 & 8 & chaperone complex & CC & $6.33 \mathrm{E}-06$ \\
\hline & GO:0016043 & $2.55 \mathrm{E}-09$ & 93 & 2689 & $\begin{array}{l}\text { cellular component organization } \\
\text { cellular component organization }\end{array}$ & $\mathrm{BP}$ & $6.33 E-06$ \\
\hline & GO:0071840 & $2.63 \mathrm{E}-09$ & 94 & 2732 & or biogenesis & BP & $6.33 \mathrm{E}-06$ \\
\hline & GO:0005634 & $1.30 \mathrm{E}-08$ & 95 & 2897 & nucleus & CC & 2.74E-05 \\
\hline & GO:0097159 & $3.75 \mathrm{E}-08$ & 117 & 4057 & $\begin{array}{l}\text { organic cyclic compound binding } \\
\text { pteridine-containing compound }\end{array}$ & MF & 7.00E-05 \\
\hline & GO:0042559 & $5.81 \mathrm{E}-08$ & 6 & 14 & biosynthetic process & BP & $9.76 E-05$ \\
\hline \multirow[t]{10}{*}{$8-9$} & GO:0051301 & $9.46 \mathrm{E}-16$ & 32 & 292 & cell division & BP & $1.59 \mathrm{E}-11$ \\
\hline & GO:0005634 & $1.43 \mathrm{E}-13$ & 100 & 2897 & nucleus & CC & $1.20 \mathrm{E}-09$ \\
\hline & GO:0005488 & $1.18 \mathrm{E}-11$ & 172 & 7055 & binding & MF & $6.62 E-08$ \\
\hline & GO:0007049 & $2.34 \mathrm{E}-11$ & 27 & 301 & cell cycle & BP & $9.82 \mathrm{E}-08$ \\
\hline & GO:0044427 & $1.03 \mathrm{E}-09$ & 30 & 473 & chromosomal part & CC & $3.46 \mathrm{E}-06$ \\
\hline & GO:0071103 & $2.76 \mathrm{E}-09$ & 12 & 64 & $\begin{array}{l}\text { DNA conformation change } \\
\text { anatomical structure }\end{array}$ & BP & 7.74E-06 \\
\hline & GO:0048856 & $5.90 \mathrm{E}-09$ & 68 & 2000 & development & BP & $1.32 \mathrm{E}-05$ \\
\hline & GO:0051276 & $6.93 \mathrm{E}-09$ & 19 & 195 & $\begin{array}{l}\text { chromosome organization } \\
\text { cellular component organization }\end{array}$ & BP & $1.32 \mathrm{E}-05$ \\
\hline & GO:0071840 & $7.43 E-09$ & 85 & 2732 & or biogenesis & BP & $1.32 \mathrm{E}-05$ \\
\hline & GO:0016043 & 7.83E-09 & 84 & 2689 & cellular component organization & BP & $1.32 \mathrm{E}-05$ \\
\hline \multirow[t]{10}{*}{10} & GO:0005198 & $2.07 E-15$ & 45 & 502 & structural molecule activity & MF & 3.47E-11 \\
\hline & GO:0042302 & $1.47 \mathrm{E}-14$ & 20 & 113 & structural constituent of cuticle & MF & $1.08 \mathrm{E}-10$ \\
\hline & GO:0071103 & $2.56 \mathrm{E}-14$ & 19 & 64 & DNA conformation change & BP & $1.08 \mathrm{E}-10$ \\
\hline & GO:0006323 & $2.58 \mathrm{E}-14$ & 16 & 40 & DNA packaging & BP & $1.08 \mathrm{E}-10$ \\
\hline & GO:0044427 & $1.57 \mathrm{E}-13$ & 46 & 473 & chromosomal part & CC & $5.26 \mathrm{E}-10$ \\
\hline & GO:0051276 & $1.95 \mathrm{E}-13$ & 30 & 195 & chromosome organization & BP & $5.47 \mathrm{E}-10$ \\
\hline & GO:0030261 & $3.22 \mathrm{E}-13$ & 15 & 39 & chromosome condensation & BP & $7.73 \mathrm{E}-10$ \\
\hline & GO:0051301 & $5.77 \mathrm{E}-13$ & 36 & 292 & $\begin{array}{l}\text { cell division } \\
\text { cellular component organization }\end{array}$ & BP & $1.21 \mathrm{E}-09$ \\
\hline & GO:0071840 & $1.07 \mathrm{E}-12$ & 135 & 2732 & or biogenesis & BP & $2.01 \mathrm{E}-09$ \\
\hline & GO:0016043 & $1.58 \mathrm{E}-12$ & 133 & 2689 & $\begin{array}{l}\text { cellular component organization } \\
\text { cellular component organization }\end{array}$ & BP & $2.66 \mathrm{E}-09$ \\
\hline \multirow[t]{3}{*}{11} & GO:0071840 & $5.54 \mathrm{E}-16$ & 153 & 2732 & or biogenesis & BP & $9.32 \mathrm{E}-12$ \\
\hline & GO:0016043 & $1.73 \mathrm{E}-15$ & 150 & 2689 & cellular component organization & BP & $1.46 \mathrm{E}-11$ \\
\hline & GO:0044427 & $3.31 \mathrm{E}-15$ & 51 & 473 & chromosomal part & CC & $1.85 \mathrm{E}-11$ \\
\hline
\end{tabular}




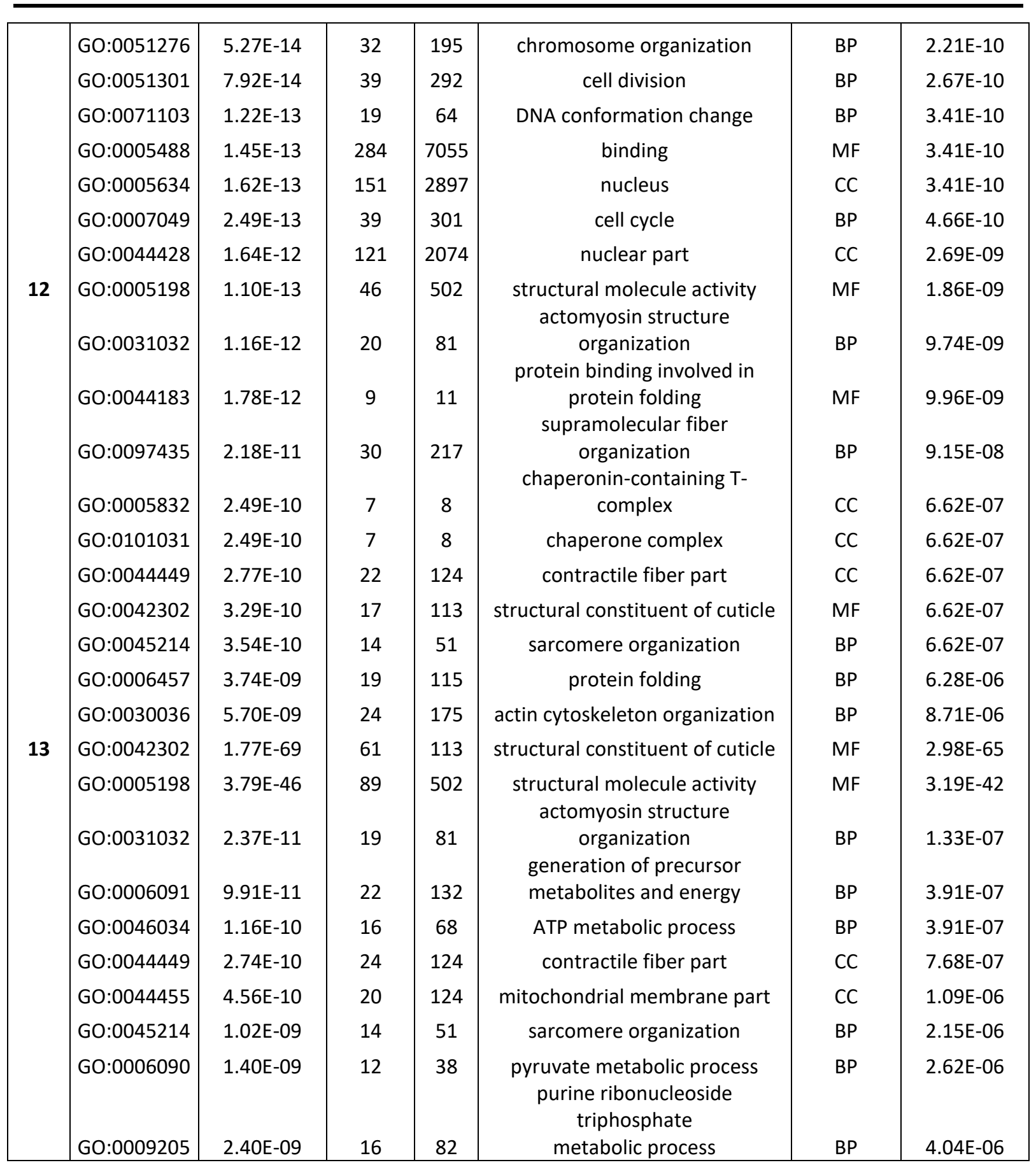

a BP, biological process; CC, cellular component; MF, molecular function 
Supplementary Table 9. GOs enriched in the upregulated DEGs from each developmental stage $(F D R<e-5)$; Only the first $10 \mathrm{GO}$ terms of each time-point are shown.

\begin{tabular}{|c|c|c|c|c|c|c|c|}
\hline day & category & $\begin{array}{l}\text { over } \\
\text { represented } \\
\text { pvalue }\end{array}$ & $\begin{array}{c}\text { num } \\
\text { DEInCat }\end{array}$ & $\begin{array}{l}\text { num } \\
\text { InCat }\end{array}$ & term & ontology ${ }^{a}$ & $\begin{array}{l}\text { over } \\
\text { represented } \\
\text { FDR }\end{array}$ \\
\hline \multicolumn{8}{|l|}{4} \\
\hline \multirow[t]{2}{*}{5} & GO:0044420 & $4.846 \mathrm{E}-09$ & 8 & 66 & extracellular matrix component & $\mathrm{CC}$ & 8.14836E-05 \\
\hline & GO:0005604 & $9.752 \mathrm{E}-09$ & 7 & 46 & basement membrane & $\mathrm{CC}$ & 8.19932E-05 \\
\hline \multirow[t]{4}{*}{6} & GO:0006030 & $1.032 \mathrm{E}-13$ & 11 & 26 & chitin metabolic process & BP & 1.73589E-09 \\
\hline & GO:1901071 & $1.561 \mathrm{E}-11$ & 11 & 37 & compound metabolic process & BP & 1.31253E-07 \\
\hline & GO:0006040 & $2.608 \mathrm{E}-11$ & 12 & 50 & amino sugar metabolic process & $\mathrm{BP}$ & $1.46188 \mathrm{E}-07$ \\
\hline & GO:0044421 & $2.251 \mathrm{E}-10$ & 51 & 1255 & $\begin{array}{l}\text { extracellular region part } \\
\text { ATP hydrolysis coupled }\end{array}$ & $\mathrm{CC}$ & 9.46145E-07 \\
\hline \multirow[t]{10}{*}{7} & GO:0090662 & 7.616E-20 & 16 & 24 & $\begin{array}{l}\text { transmembrane transport } \\
\text { energy coupled proton } \\
\text { transmembrane transport, }\end{array}$ & BP & 1.28067E-15 \\
\hline & GO:0015988 & $2.554 \mathrm{E}-18$ & 14 & 19 & $\begin{array}{l}\text { against electrochemical gradient } \\
\text { ATP hydrolysis coupled proton }\end{array}$ & BP & $1.43142 \mathrm{E}-14$ \\
\hline & GO:0015991 & $2.554 \mathrm{E}-18$ & 14 & 19 & $\begin{array}{c}\text { transport } \\
\text { ATP hvdrolvsis coupled ion }\end{array}$ & BP & $1.43142 \mathrm{E}-14$ \\
\hline & GO:0099131 & $1.415 \mathrm{E}-17$ & 14 & 21 & $\begin{array}{l}\text { transmembrane transport } \\
\text { ATP hydrolysis coupled cation }\end{array}$ & BP & 4.75697E-14 \\
\hline & GO:0099132 & $1.415 \mathrm{E}-17$ & 14 & 21 & transmembrane transport & BP & 4.75697E-14 \\
\hline & GO:0044425 & $1.846 \mathrm{E}-17$ & 153 & 3119 & membrane part & $\mathrm{CC}$ & 5.17435E-14 \\
\hline & GO:0016021 & $3.583 \mathrm{E}-16$ & 124 & 2321 & membrane & CC & 8.60757E-13 \\
\hline & GO:0006818 & 7.457E-16 & 16 & 41 & NA & NA & $1.56745 \mathrm{E}-12$ \\
\hline & GO:0031224 & $1.117 \mathrm{E}-15$ & 125 & 2397 & $\begin{array}{l}\text { Intrinsic component of } \\
\text { membrane }\end{array}$ & $\mathrm{CC}$ & 2.08631E-12 \\
\hline & GO:0015992 & 1.137E-14 & 15 & 40 & NA & NA & 1.91225E-11 \\
\hline \multirow[t]{10}{*}{8} & GO:0090662 & $4.98 \mathrm{E}-20$ & $1.60 \mathrm{E}+01$ & $2.40 \mathrm{E}+01$ & $\begin{array}{l}\text { transmembrane transport } \\
\text { energy coupled proton } \\
\text { transmembrane transport, }\end{array}$ & BP & 8.37E-16 \\
\hline & GO:0015988 & $1.49 \mathrm{E}-18$ & $1.40 \mathrm{E}+01$ & $1.90 \mathrm{E}+01$ & $\begin{array}{l}\text { against electrochemical gradient } \\
\text { ATP hydrolysis coupled proton }\end{array}$ & BP & 8.37E-15 \\
\hline & GO:0015991 & $1.49 \mathrm{E}-18$ & $1.40 \mathrm{E}+01$ & $1.90 \mathrm{E}+01$ & $\begin{array}{c}\text { transport } \\
\text { ATP hydrolysis coupled ion }\end{array}$ & BP & 8.37E-15 \\
\hline & GO:0099131 & $9.69 \mathrm{E}-18$ & $1.40 \mathrm{E}+01$ & $2.10 \mathrm{E}+01$ & $\begin{array}{l}\text { transmembrane transport } \\
\text { ATP hydrolysis coupled cation }\end{array}$ & $\mathrm{BP}$ & $3.26 \mathrm{E}-14$ \\
\hline & GO:0099132 & $9.69 \mathrm{E}-18$ & $1.40 \mathrm{E}+01$ & $2.10 \mathrm{E}+01$ & transmembrane transport & $\mathrm{BP}$ & $3.26 \mathrm{E}-14$ \\
\hline & GO:0044425 & $5.63 \mathrm{E}-17$ & $1.50 \mathrm{E}+02$ & $3.12 E+03$ & membrane part & $\mathrm{CC}$ & $1.58 \mathrm{E}-13$ \\
\hline & GO:0006818 & $7.48 \mathrm{E}-16$ & $1.60 \mathrm{E}+01$ & $4.10 \mathrm{E}+01$ & NA & NA & $1.80 \mathrm{E}-12$ \\
\hline & GO:0044710 & $1.06 \mathrm{E}-15$ & $1.13 \mathrm{E}+02$ & $2.09 E+03$ & NA & NA & $2.23 \mathrm{E}-12$ \\
\hline & GO:0015992 & $1.15 \mathrm{E}-14$ & $1.50 \mathrm{E}+01$ & 4.00E+01 & $\begin{array}{c}\text { NA } \\
\text { integral component of }\end{array}$ & NA & $2.14 \mathrm{E}-11$ \\
\hline & GO:0016021 & $2.10 \mathrm{E}-14$ & $1.18 \mathrm{E}+02$ & $2.32 E+03$ & membrane & $\mathrm{CC}$ & $3.54 \mathrm{E}-11$ \\
\hline $8-9$ & GO:0090662 & $1.72 \mathrm{E}-19$ & $1.60 \mathrm{E}+01$ & $2.40 \mathrm{E}+01$ & transmembrane transport & $\mathrm{BP}$ & $2.88 \mathrm{E}-15$ \\
\hline
\end{tabular}




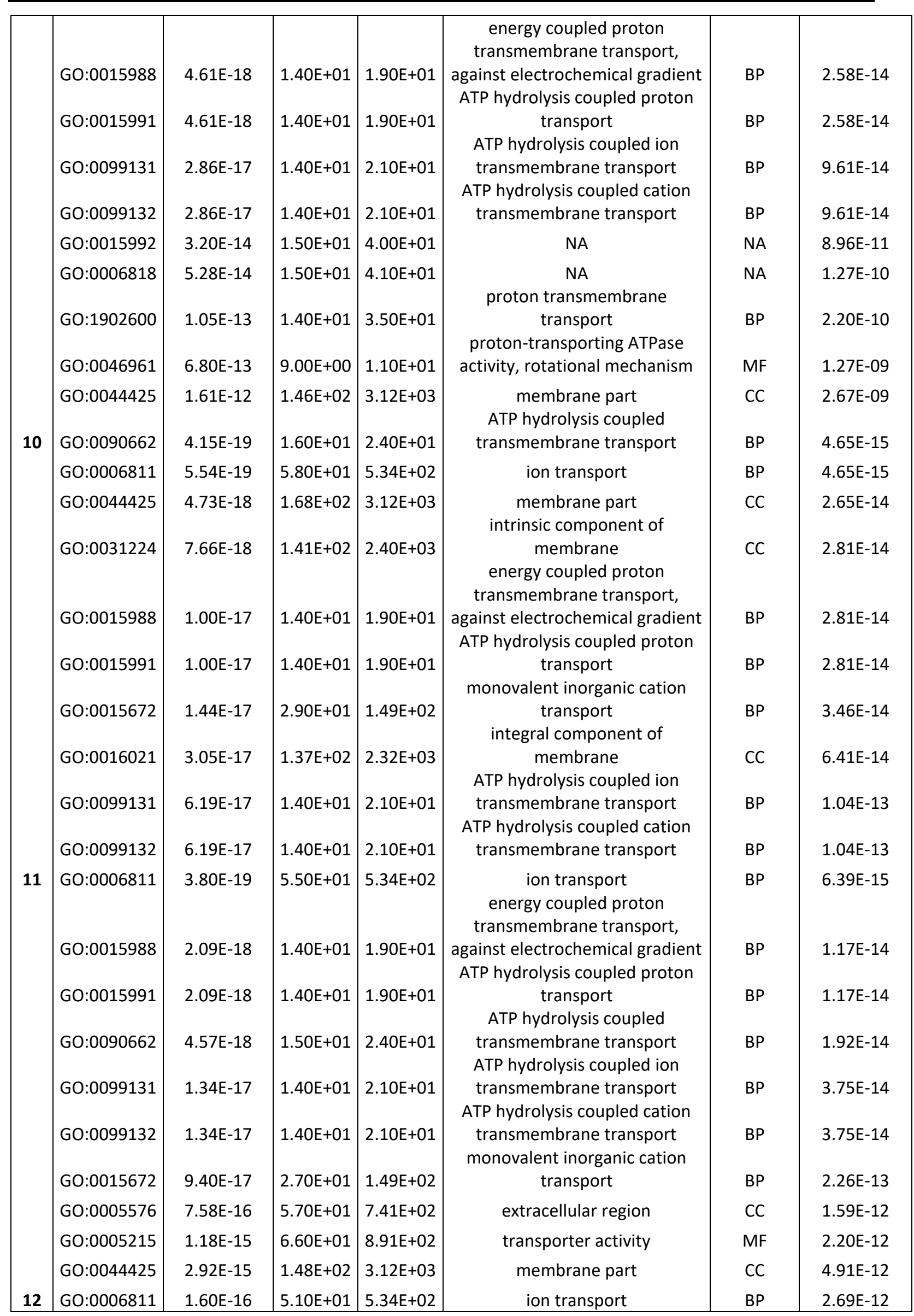




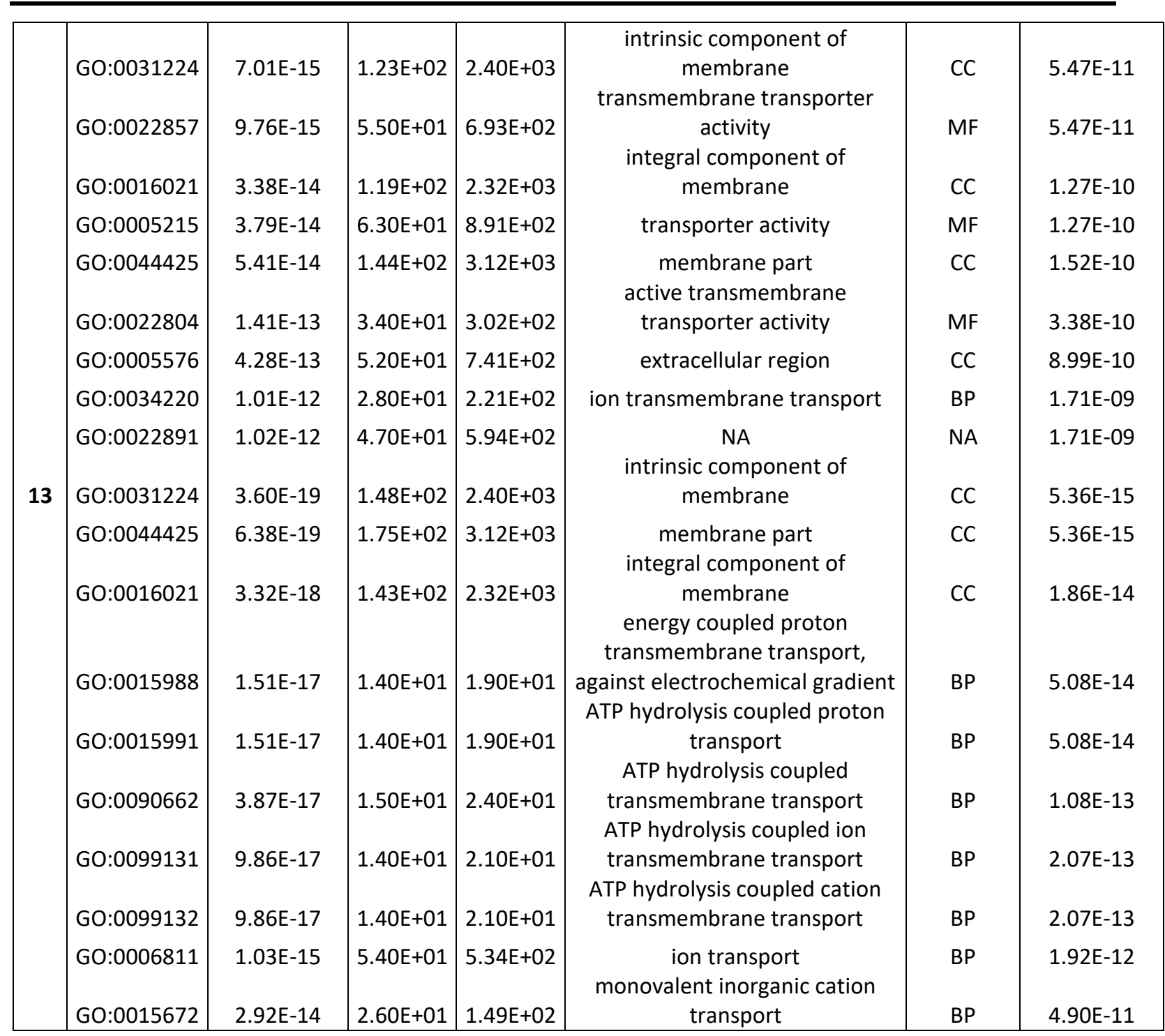




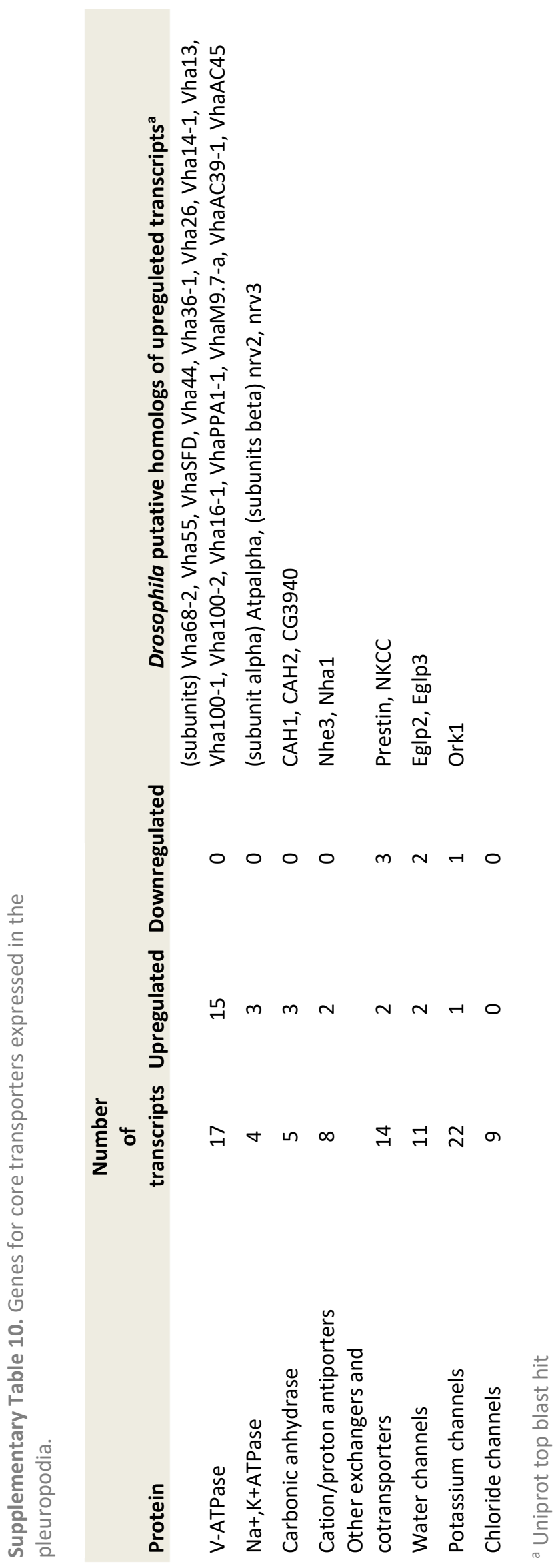


Supplementary Table 11. Schistocerca gene for proteins with GO "proteolysis" that were upregulated in the highly secreting pleuropodia.

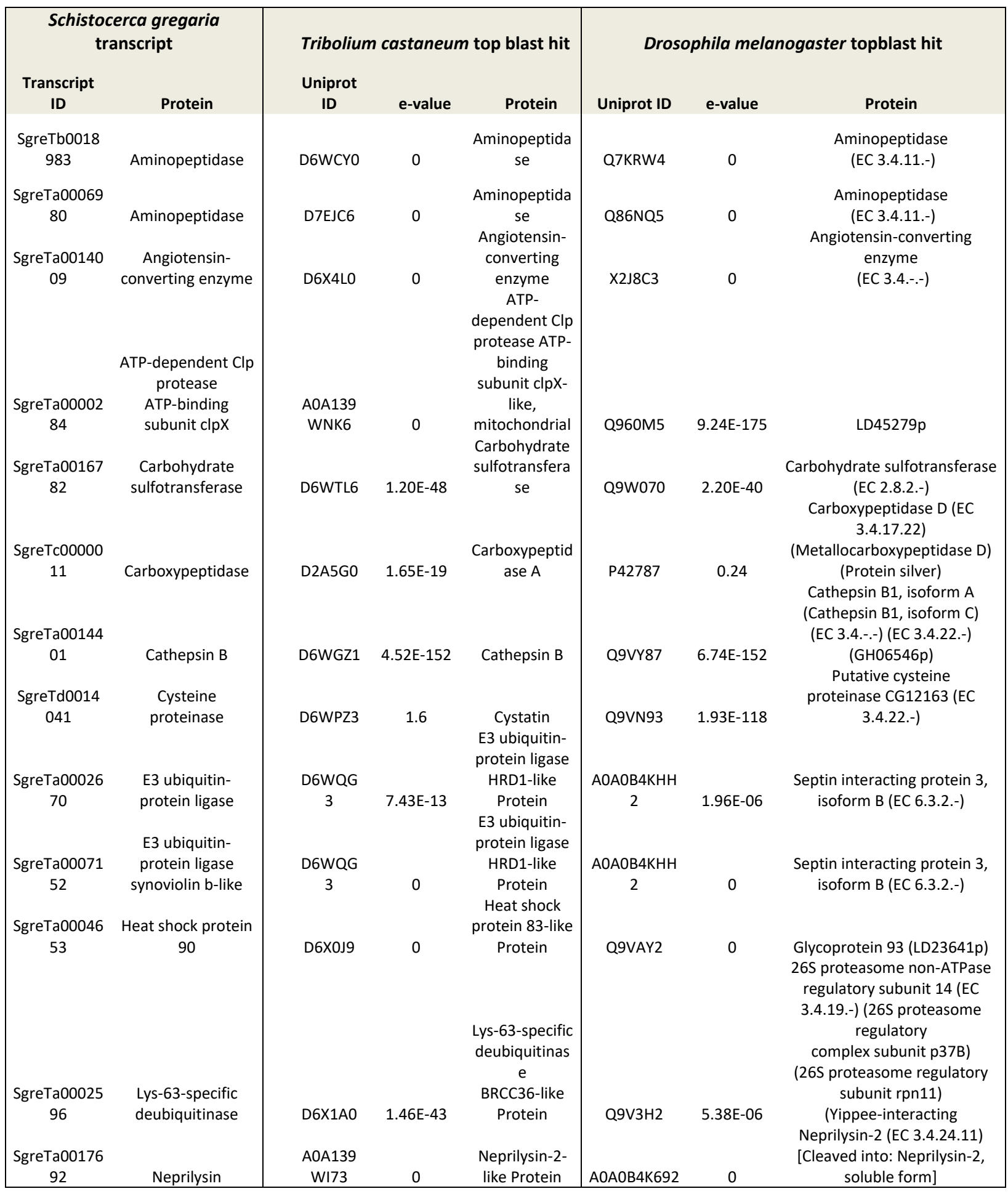




\begin{tabular}{|c|c|c|c|c|c|c|c|}
\hline & & & & & & & Neprilysin-2 (EC 3.4.24.11) \\
\hline SgreTa001769 & & & & Neprilysin-2- & & & [Cleaved into: Neprilysin-2, soluble \\
\hline 2 & Neprilysin & A0A139WI73 & 0 & like Protein & АОАОВ4К692 & 0 & form] \\
\hline SgreTa001774 & & & $1.37 \mathrm{E}$ & Neprilysin-2- & & $1.38 \mathrm{E}$ & $\begin{array}{c}\text { Neprilysin-2 (EC 3.4.24.11) } \\
\text { [Cleaved into: Neprilysin-2, soluble }\end{array}$ \\
\hline 6 & Neprilysin & A0A139WHPO & -54 & like Protein & АОАОВ4K692 & -52 & form] \\
\hline & & & & & & & Neprilysin-2 (EC 3.4.24.11) \\
\hline SgreTa000246 & & & & Neprilysin-2- & & & [Cleaved into: Neprilysin-2, soluble \\
\hline 7 & Neprilysin & A0A139WHP0 & 0 & like Protein & АОАОВ4К692 & 0 & form] \\
\hline & & & & & & & Neprilysin-2 (EC 3.4.24.11) \\
\hline SgreTb003904 & & & $6.32 \mathrm{E}$ & Neprilysin-2- & & $1.08 \mathrm{E}$ & [Cleaved into: Neprilysin-2, soluble \\
\hline 5 & Neprilysin & A0A139WI73 & -14 & like Protein & АОАОВ4К692 & -12 & form] \\
\hline SgreTb003912 & & & $3.79 \mathrm{E}$ & Neprilysin-2- & & $3.90 \mathrm{E}$ & $\begin{array}{c}\text { Neprilysin-2 (EC 3.4.24.11) } \\
\text { [Cleaved into: Neprilysin-2, soluble }\end{array}$ \\
\hline 3 & Neprilysin & A0A139WHP0 & -23 & like Protein & АОАОВ4К692 & -26 & form] \\
\hline SgreTa001658 & Protein & A0A139WMR & $2.72 \mathrm{E}$ & Protein roadkill- & & $1.02 \mathrm{E}$ & \\
\hline 2 & $\begin{array}{l}\text { roadkill-like } \\
\text { Putative } \\
\text { serine }\end{array}$ & 0 & -12 & $\begin{array}{c}\text { like Protein } \\
\text { Putative serine } \\
\text { protease }\end{array}$ & C7LAF6 & -15 & RE09961p \\
\hline SgreTa001765 & protease, & & $2.66 \mathrm{E}$ & K12H4.7-like & & $5.88 \mathrm{E}$ & \\
\hline 7 & K12H4.7-like & D6WGL2 & -101 & Protein & Q9VS02 & -90 & CG9953 \\
\hline SgreTa000493 & Rhomboid- & & $1.61 \mathrm{E}$ & Rhomboid-like & & $1.39 \mathrm{E}$ & \\
\hline 9 & like protein & D6WUJ2 & -105 & protein & Q9VYW6 & -86 & Rhomboid-like protein (EC 3.4.21.-) \\
\hline SgreTb002374 & $\begin{array}{l}\text { RING finger } \\
\text { domain }\end{array}$ & & $3.52 \mathrm{E}$ & $\begin{array}{l}\text { RING-box } \\
\text { protein }\end{array}$ & & $2.50 \mathrm{E}$ & $\begin{array}{c}\text { MIP07211p (RE61847p) } \\
\text { (Regulator of cullins 2, isoform A) }\end{array}$ \\
\hline 5 & protein & $\mathrm{D} 2 \mathrm{~A} 2 \mathrm{~S} 1$ & -13 & 1A-like Protein & Q7JWH5 & -32 & (Regulator of cullins 2, isoform B) \\
\hline SgreTa000590 & Selenoprotei & & $2.27 \mathrm{E}$ & Uncharacterize & & & \\
\hline 5 & nS & D6WGX2 & -06 & $\mathrm{~d}$ protein & Q9W0D3 & 6.6 & GH15728p \\
\hline SgreTa001764 & Serine & & $1.55 \mathrm{E}$ & Serine protease & & $5.03 E$ & \\
\hline 9 & $\begin{array}{l}\text { protease } \\
\text { Serine }\end{array}$ & A0A139W9L3 & -74 & P43 & Q9VAQ3 & -52 & GH18608p \\
\hline SgreTa001021 & protease, & & $1.90 \mathrm{E}$ & Serine protease & & $4.44 \mathrm{E}$ & Serine_protease_easter_(EC_3.4.21. \\
\hline 9 & $\begin{array}{l}\text { Easter-like } \\
\text { Serine }\end{array}$ & D6WUF6 & -20 & P136 & P13582 & -18 & $-)$ \\
\hline SgreTb003724 & protease, & & 9.05E & Serine protease & AOAOB4KGQ & $4.24 \mathrm{E}$ & Easter, isoform B (EC 3.4.-.-) (EC \\
\hline 9 & $\begin{array}{l}\text { Easter-like } \\
\text { Serine }\end{array}$ & D6WUF7 & -54 & H137 & 4 & -52 & 3.4.21.-) \\
\hline SgreTb003902 & protease, & & $2.22 \mathrm{E}$ & Serine protease & & $1.35 \mathrm{E}$ & \\
\hline 4 & $\begin{array}{l}\text { Easter-like } \\
\text { Serine }\end{array}$ & D6WGT8 & -26 & H33 & P13582 & -21 & Serine protease easter (EC 3.4.21.-) \\
\hline SgreTb003987 & protease, & & $2.25 \mathrm{E}$ & Serine protease & & $7.93 E$ & \\
\hline 9 & $\begin{array}{l}\text { Easter-like } \\
\text { Serine }\end{array}$ & D6WP87 & -16 & P90 & P13582 & -14 & Serine protease easter (EC 3.4.21.-) \\
\hline SgreTa000366 & protease, & & $5.95 \mathrm{E}$ & Serine protease & & $2.76 \mathrm{E}$ & \\
\hline 1 & $\begin{array}{l}\text { Easter-like } \\
\text { Serine }\end{array}$ & D6WUF6 & -90 & P136 & P13582 & -82 & Serine protease easter (EC 3.4.21.-) \\
\hline SgreTa000742 & protease, & & 2.87E & Serine protease & & $3.76 \mathrm{E}$ & \\
\hline 4 & $\begin{array}{l}\text { Easter-like } \\
\text { Serine }\end{array}$ & D6WUF6 & -75 & P136 & P13582 & -76 & Serine protease easter (EC 3.4.21.-) \\
\hline SgreTa000742 & protease, & & $1.68 \mathrm{E}$ & Serine protease & & $2.34 \mathrm{E}$ & \\
\hline 5 & $\begin{array}{l}\text { Easter-like } \\
\text { Serine }\end{array}$ & D6WUF6 & -73 & P136 & P13582 & -75 & Serine protease easter (EC 3.4.21.-) \\
\hline SgreTa000742 & protease, & & $3.37 \mathrm{E}$ & Serine protease & A0A126GUP & $5.60 \mathrm{E}$ & Melanization protease 1 (EC 3.4.21.- \\
\hline 6 & $\begin{array}{l}\text { Easter-like } \\
\text { Serine }\end{array}$ & D6WUF6 & -60 & P136 & 6 & -61 & ) \\
\hline SgreTa000106 & protease, gd- & & $3.73 \mathrm{E}$ & Serine protease & & $2.70 \mathrm{E}$ & \\
\hline 5 & $\begin{array}{l}\text { like } \\
\text { Serine }\end{array}$ & D6WYU8 & -56 & P69 & A4V9W2 & -37 & CG9649_protein \\
\hline SgreTb000386 & protease, $\mathrm{H} 2$ - & & $1.02 \mathrm{E}$ & Serine protease & & $1.14 \mathrm{E}$ & \\
\hline 0 & like & D6WBT0 & -81 & $\mathrm{H} 2$ & Q86PE8 & -69 & SD23103p \\
\hline
\end{tabular}




\begin{tabular}{|c|c|c|c|c|c|c|c|c|c|c|c|c|c|c|}
\hline$\dot{d} \frac{\mathscr{2}}{\frac{\pi}{2}}$ & 0 & \multicolumn{6}{|c|}{ 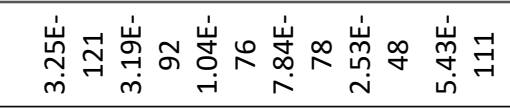 } & \multicolumn{3}{|c|}{$\circ \circ \underset{m}{\stackrel{\dot{山}}{\infty} \underset{m}{\infty} \delta}$} & \multicolumn{4}{|c|}{ 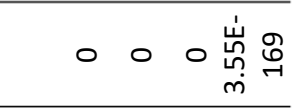 } \\
\hline 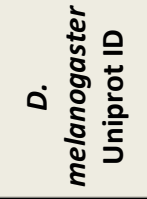 & 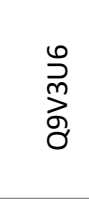 & \begin{tabular}{l}
$\stackrel{n}{n}$ \\
\multirow{5}{*}{} \\
0 \\
0
\end{tabular} & $\begin{array}{l}\stackrel{\infty}{\overrightarrow{0}} \\
\text { ơ }\end{array}$ & $\begin{array}{l}\infty \\
\stackrel{\infty}{7} \\
\stackrel{0}{0}\end{array}$ & $\begin{array}{l}\infty \\
\stackrel{\infty}{7} \\
\stackrel{8}{\sigma}\end{array}$ & \begin{tabular}{l}
$\stackrel{n}{5}$ \\
\multirow{2}{*}{} \\
$\delta$
\end{tabular} & $\stackrel{\infty}{\sigma}_{0}^{\infty}$ & $\frac{8}{8}$ & 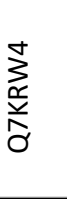 & 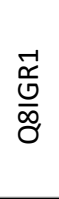 & 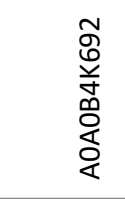 & 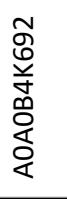 & 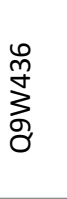 & 总 \\
\hline$\dot{d} \frac{\frac{\pi}{\pi}}{2}$ & 0 & \multicolumn{6}{|c|}{ 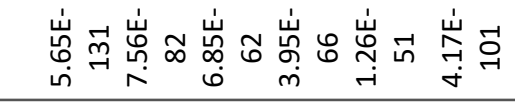 } & \multicolumn{3}{|c|}{ 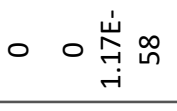 } & 0 & $\stackrel{\infty}{m}$ & \multicolumn{2}{|c|}{ 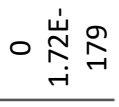 } \\
\hline 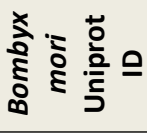 & 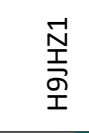 & 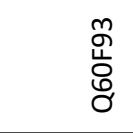 & 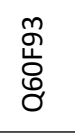 & 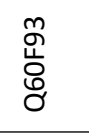 & 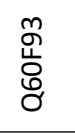 & 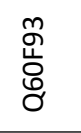 & 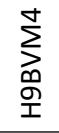 & 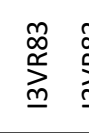 & 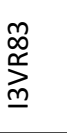 & 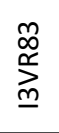 & $\begin{array}{l}\stackrel{9}{\stackrel{\leftrightarrow}{5}} \\
\text { ำ }\end{array}$ & 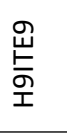 & 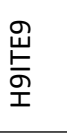 & 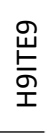 \\
\hline 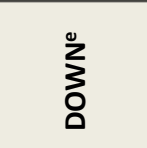 & $\begin{array}{c}\infty \\
\stackrel{m}{0} \\
\stackrel{0}{0}\end{array}$ & $\begin{array}{l}m \\
\tilde{\omega} \\
\dot{\varphi} \\
b\end{array}$ & $\stackrel{\stackrel{p}{?}}{r}$ & & & $\underset{\sim}{\stackrel{I}{i}}$ & & 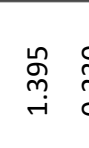 & 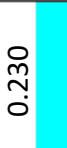 & $\begin{array}{l}\text { ô } \\
-1 \\
0 \\
0 \\
-1\end{array}$ & §̃ & : & 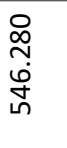 & $\begin{array}{l}\overrightarrow{-} \\
\stackrel{0}{0} \\
\stackrel{-}{N}\end{array}$ \\
\hline$\stackrel{\check{L}}{5}$ & $\underset{m}{\mathcal{I}}$ & 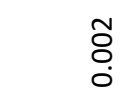 & $\stackrel{\stackrel{n}{m}}{\stackrel{0}{0}}$ & & & 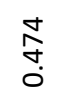 & & \begin{tabular}{ll}
\multirow{H}{A}{} \\
$\vdots$ \\
$\vdots$
\end{tabular} & 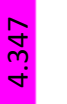 & $\begin{array}{l}0 \\
0 \\
0 \\
0\end{array}$ & 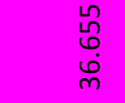 & 雍 & ס̊ & $\begin{array}{l}\mathscr{l}_{0}^{\circ} \\
0\end{array}$ \\
\hline 苗 & 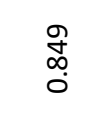 & 离 & 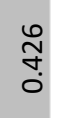 & & & $\stackrel{0}{\stackrel{9}{7}}$ & & $\underset{\substack{n \\
0}}{0}$ & 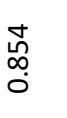 & $\begin{array}{c}\infty \\
\substack{+\infty \\
0}\end{array}$ & $\begin{array}{l}\hat{o} \\
\text { ồ } \\
0\end{array}$ & 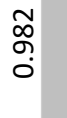 & $\underset{\substack{f \\
\vdots}}{\stackrel{n}{0}}$ & $\begin{array}{l}\infty \\
\infty \\
0 \\
0\end{array}$ \\
\hline $\begin{array}{l}\frac{0}{0} \\
\sum_{\frac{1}{2}} \\
\frac{0}{x}\end{array}$ & $\begin{array}{l}\stackrel{\infty}{N} \\
\stackrel{-}{m} \\
\rightarrow-1\end{array}$ & $\begin{array}{l}\stackrel{n}{n} \\
0 \\
0\end{array}$ & $\underset{\substack{-1 \\
-1 \\
0}}{-1}$ & & & नें & & 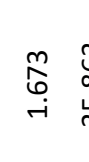 & 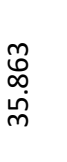 & $\underset{7}{ت}$ & $\begin{array}{l}\hat{o} \\
\underset{\text { D}}{\infty} \\
\underset{\sim}{\sim}\end{array}$ & 令 & $\begin{array}{l}0 \\
\stackrel{0}{0} \\
0 \\
0\end{array}$ & 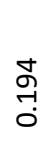 \\
\hline$\sum_{\substack{a \\
\alpha}}$ & 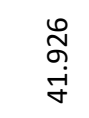 & 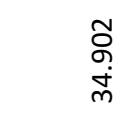 & $\begin{array}{l}m \\
\stackrel{m}{q} \\
m \\
i \\
i\end{array}$ & 总 & & 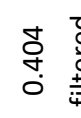 & & 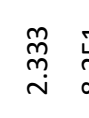 & $\underset{\infty}{\stackrel{1}{\sim}}$ & $\begin{array}{l}\vec{H} \\
\stackrel{-1}{7}\end{array}$ & 总 & 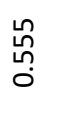 & 品 & $\underset{+}{\stackrel{न}{+}}$ \\
\hline 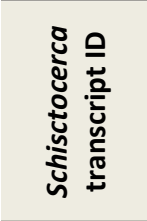 & 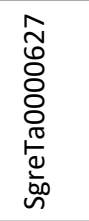 & 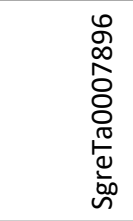 & 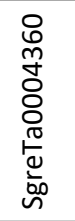 & 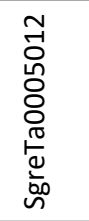 & 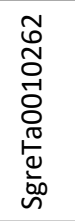 & 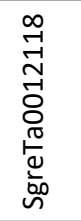 & 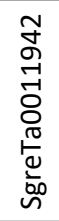 & 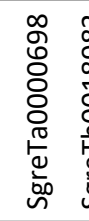 & 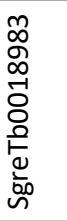 & 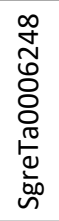 & 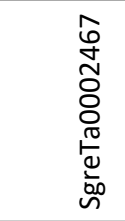 & 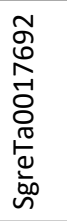 & 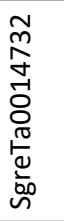 & 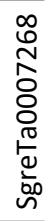 \\
\hline 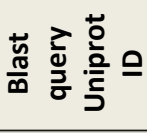 & 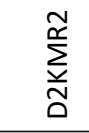 & $\begin{array}{l}n \\
0 \\
\vdots \\
0 \\
0\end{array}$ & & & & & 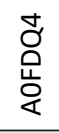 & $\begin{array}{c}\substack{\infty \\
\infty \\
\stackrel{\infty}{\infty} \\
\text { m }} \\
\end{array}$ & & & 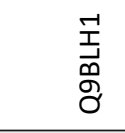 & & & \\
\hline 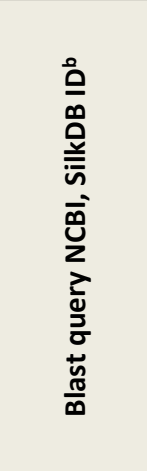 & 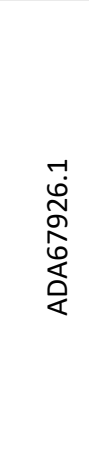 & 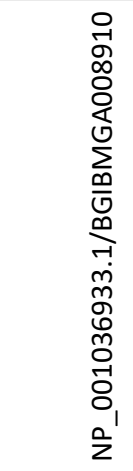 & & & & & $\begin{array}{l}\overrightarrow{1} \\
\infty \\
o \\
-1 \\
0 \\
0 \\
0 \\
8 \\
8 \\
\frac{1}{2}\end{array}$ & 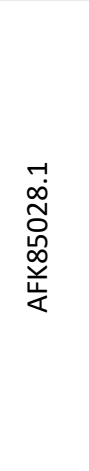 & & & 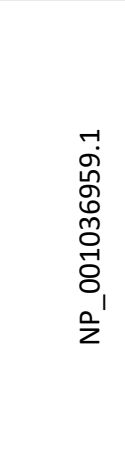 & & & \\
\hline 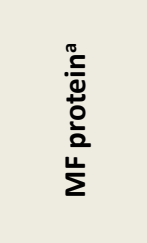 & 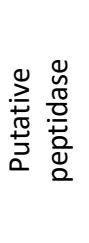 & 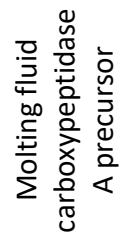 & & & & & 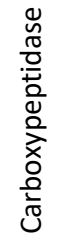 & 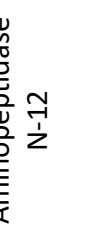 & & & 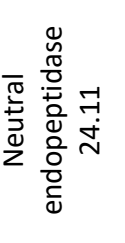 & & & \\
\hline
\end{tabular}




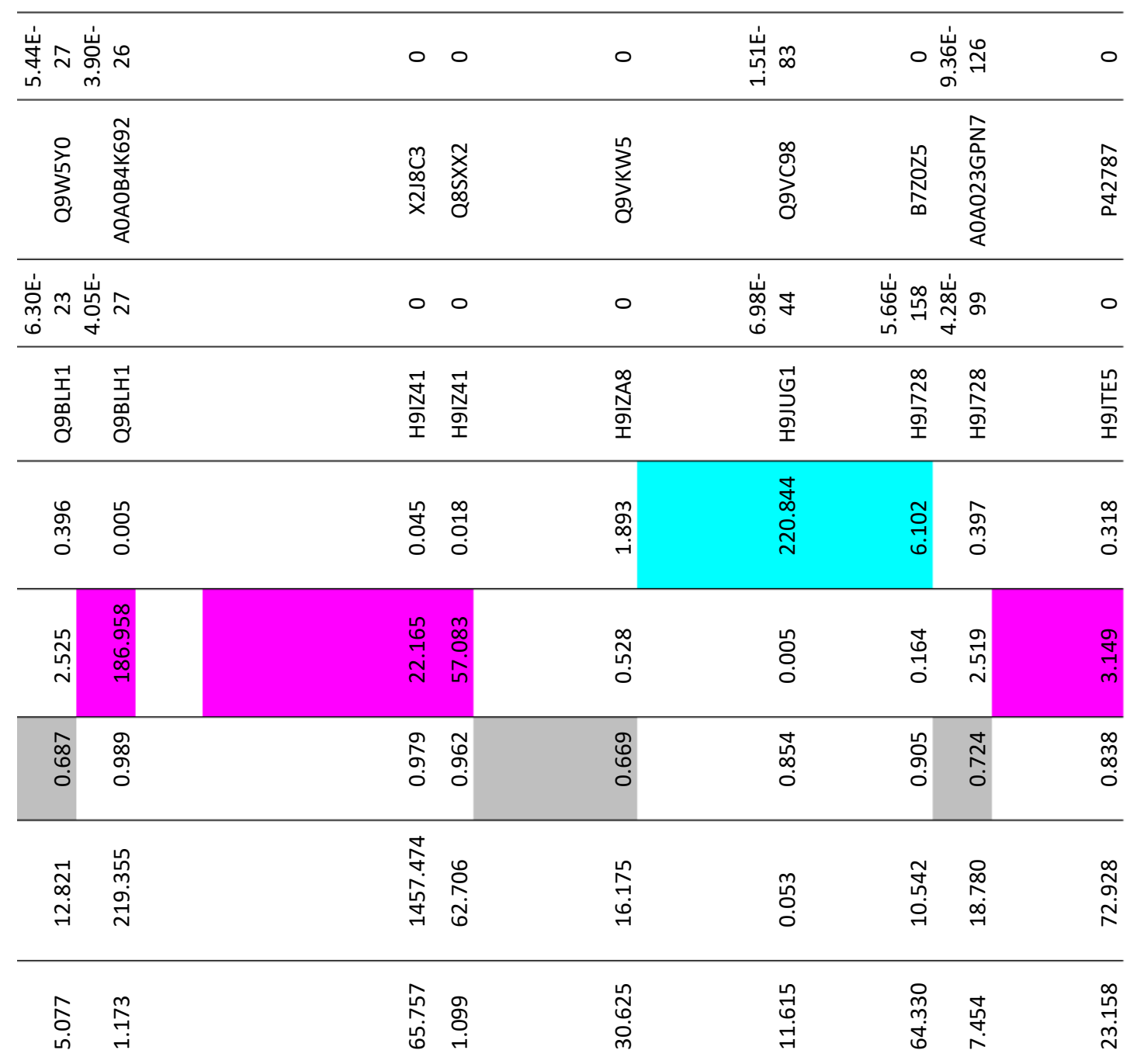

\begin{tabular}{|c|c|c|c|c|c|c|c|}
\hline 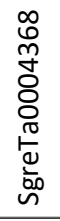 & 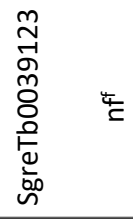 & 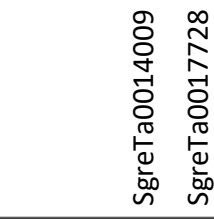 & 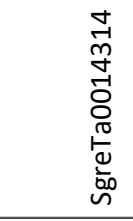 & 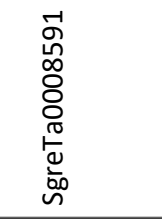 & 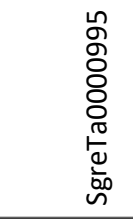 & 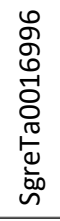 & 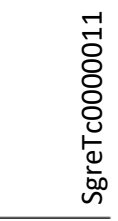 \\
\hline & $\begin{array}{l}\text { o } \\
\infty \\
0 \\
0\end{array}$ & 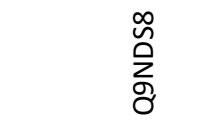 & $\begin{array}{l}\frac{\infty}{N} \\
\frac{N}{\text { के }}\end{array}$ & $\begin{array}{l}\overrightarrow{\mathrm{O}} \\
\text { 옾 }\end{array}$ & $\begin{array}{l}\stackrel{\infty}{N} \\
\stackrel{\widehat{\sigma}}{\mathbf{T}}\end{array}$ & & $\begin{array}{l}\text { 岕 } \\
\text { 옴 }\end{array}$ \\
\hline & 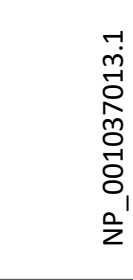 & 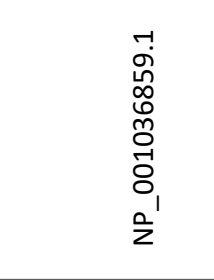 & 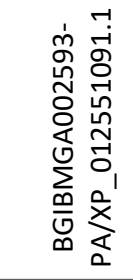 & 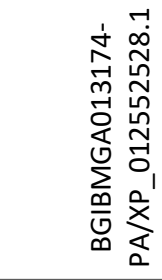 & 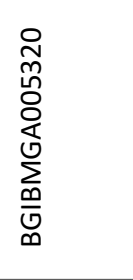 & & 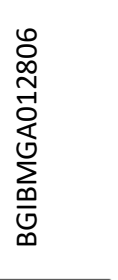 \\
\hline & 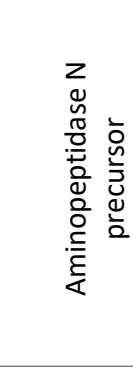 & 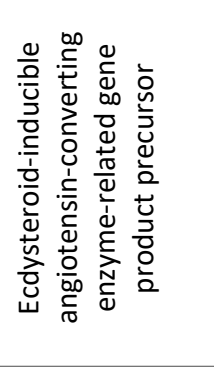 & 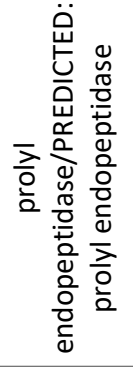 & 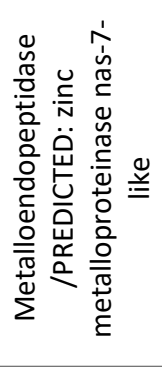 & 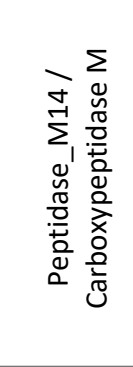 & & 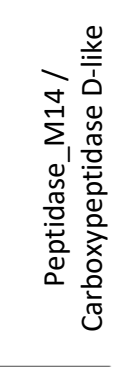 \\
\hline
\end{tabular}




\begin{tabular}{|c|c|c|c|c|c|c|c|c|c|}
\hline $\begin{array}{l}\widehat{\infty} \\
\dot{1} \\
\vec{\omega} \\
\infty \\
\infty \\
\infty\end{array}$ & & $\begin{array}{l}\hat{\infty} \\
\dot{1} \\
\stackrel{\omega}{0} \\
\infty \\
\infty \\
\end{array}$ & 0 & 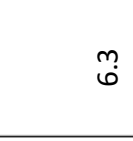 & $\begin{array}{l}\stackrel{N}{N} \\
\dot{山} \\
0 \\
\infty \\
\end{array}$ & 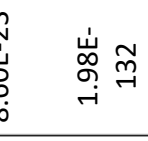 & & & 0 \\
\hline$\stackrel{\mathscr{m}}{\stackrel{\mathscr{D}}{\tilde{O}}}$ & & 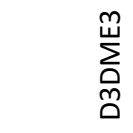 & 景 & 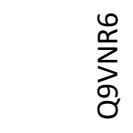 & $\begin{array}{l}\text { 岁 } \\
\text { ò } \\
\Sigma\end{array}$ & 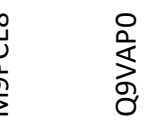 & & & $\begin{array}{l}0 \\
\text { m. } \\
\text { वे }\end{array}$ \\
\hline 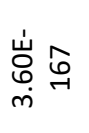 & & 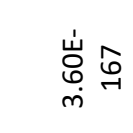 & 0 & $\stackrel{d}{\stackrel{d}{0}}$ & $\begin{array}{l}\stackrel{\sim}{\sim} \\
\stackrel{\sim}{\sim} \\
\stackrel{\sim}{+}\end{array}$ & $\begin{array}{l}\hat{\sim} \\
\stackrel{\sim}{\tilde{O}} \\
\dot{\sim}\end{array}$ & & & 0 \\
\hline $\begin{array}{l}\frac{0}{5} \\
\frac{5}{9} \\
\frac{5}{1}\end{array}$ & & $\frac{\circ}{\frac{0}{5}}$ & 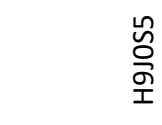 & $\frac{\stackrel{\infty}{\times}}{\frac{\infty}{x}}$ & 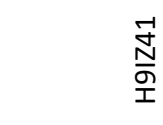 & 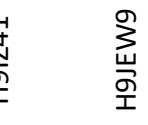 & & & $\begin{array}{l}\bar{N} \\
\text { I } \\
\text { İ }\end{array}$ \\
\hline $\overrightarrow{0}$ & & ö & $\begin{array}{l}\tilde{0} \\
\stackrel{0}{0} \\
0\end{array}$ & & $\stackrel{\text { f }}{\stackrel{1}{0}}$ & õ & & & $\stackrel{\infty}{m}$ \\
\hline $\begin{array}{l}0 \\
\text { مू } \\
\text { Oे } \\
\text { - }\end{array}$ & & $\begin{array}{l}\text { 요 } \\
\text { مे } \\
0 \\
\text {-े }\end{array}$ & $\begin{array}{l}\stackrel{8}{\circ} \\
\stackrel{\leftrightarrow}{-}\end{array}$ & & $\begin{array}{l}\text { gे } \\
\text { : } \\
\text { iे }\end{array}$ & 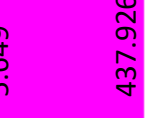 & & & $\stackrel{\sim}{\stackrel{\sim}{m}}$ \\
\hline 亩 & & 今̊ & $\begin{array}{l}\text { O⿱丶万} \\
0 \\
0\end{array}$ & & 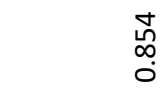 & $\begin{array}{l}\text { मे } \\
\text { g. }\end{array}$ & & & 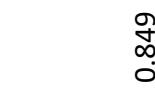 \\
\hline $\begin{array}{l}\hat{o} \\
\infty \\
\text { Oे } \\
\vec{\sigma}\end{array}$ & & 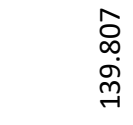 & $\begin{array}{l}\text { p } \\
\infty \\
0 \\
\infty \\
\infty\end{array}$ & & $\begin{array}{l}\hat{\tilde{O}} \\
\ddot{\sim} \\
\dot{\sim}\end{array}$ & 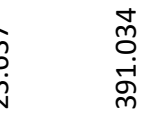 & & & $\begin{array}{l}\stackrel{\infty}{N} \\
\stackrel{-}{m}\end{array}$ \\
\hline 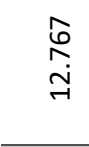 & & $\begin{array}{l}\hat{\sigma} \\
\stackrel{i}{7}\end{array}$ & 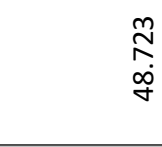 & 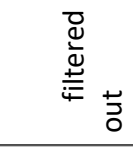 & $\stackrel{+}{\stackrel{+}{+}}$ & $\begin{array}{l}\mathscr{D} \\
\infty \\
0 \\
0\end{array}$ & & & 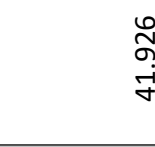 \\
\hline \multirow[t]{4}{*}{ 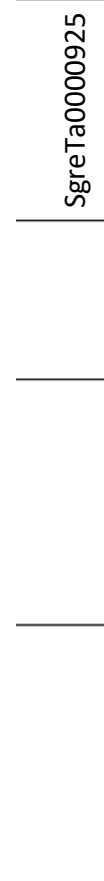 } & $\stackrel{4}{J}$ & 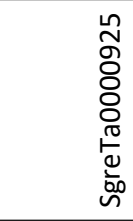 & $\begin{array}{l}\text { I } \\
\infty \\
0 \\
0 \\
0 \\
\sigma \\
\vdots \\
0 \\
0\end{array}$ & 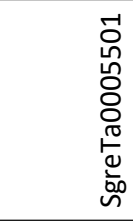 & 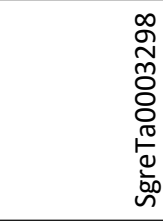 & 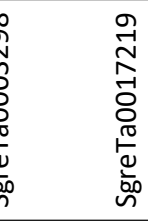 & $U^{L}$ & 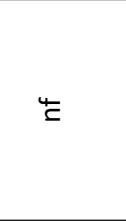 & 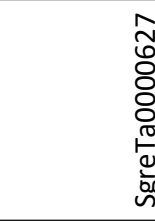 \\
\hline & 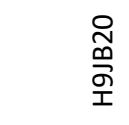 & $\frac{ㅇ}{\frac{0}{5}}$ & 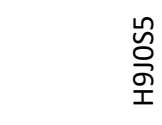 & 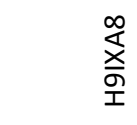 & 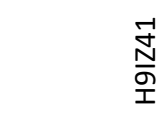 & $\begin{array}{l}\sum_{\stackrel{9}{u}} \\
\text { 定 }\end{array}$ & 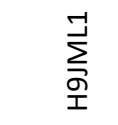 & $\begin{array}{l}\frac{-1}{\overline{7}} \\
\frac{\sigma}{1}\end{array}$ & $\begin{array}{l}\bar{N} \\
\text { 옴 }\end{array}$ \\
\hline & 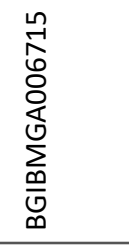 & 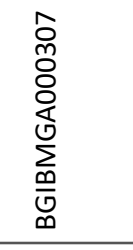 & 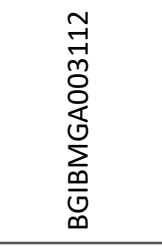 & 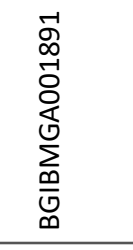 & 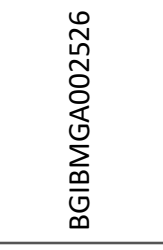 & $\begin{array}{l}0 \\
\stackrel{0}{0} \\
0 \\
0 \\
0 \\
0 \\
\sum_{0}^{0} \\
\\
\infty\end{array}$ & $\begin{array}{l}0 \\
0 \\
0 \\
0 \\
0 \\
01 \\
0 \\
\sum_{0}^{0} \\
0 \\
0\end{array}$ & 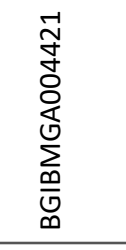 & 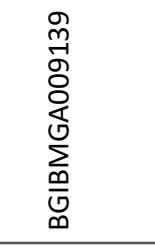 \\
\hline & 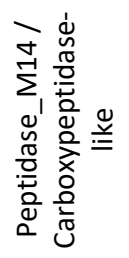 & 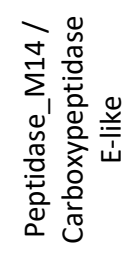 & 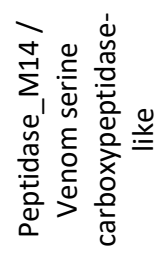 & 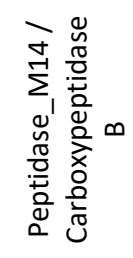 & 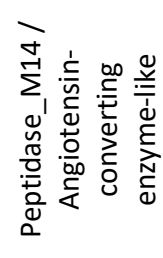 & 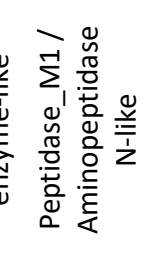 & 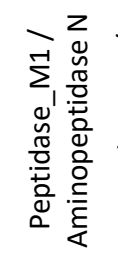 & 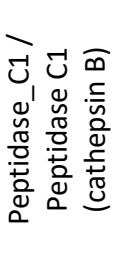 & 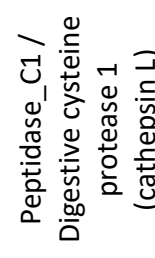 \\
\hline
\end{tabular}




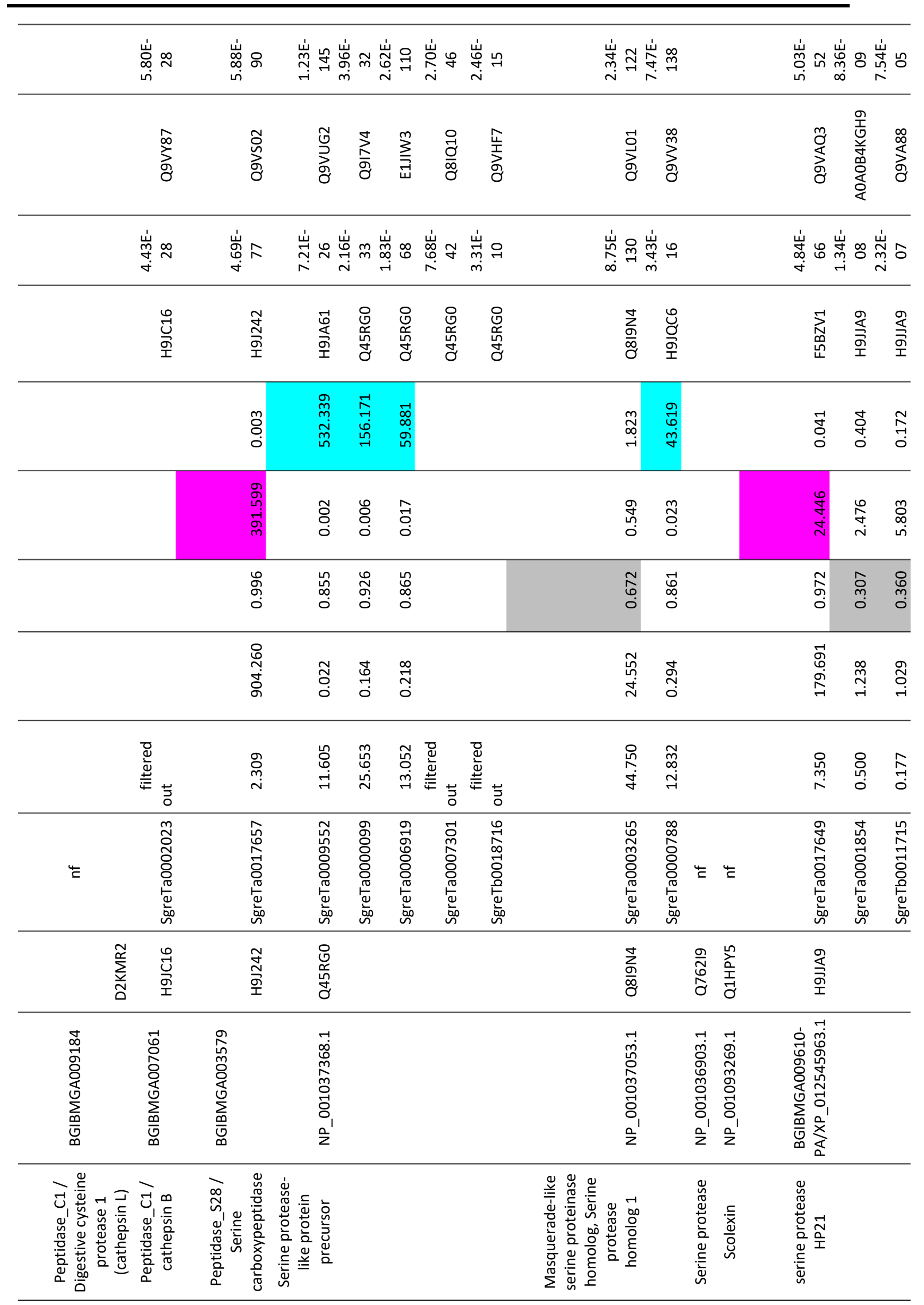




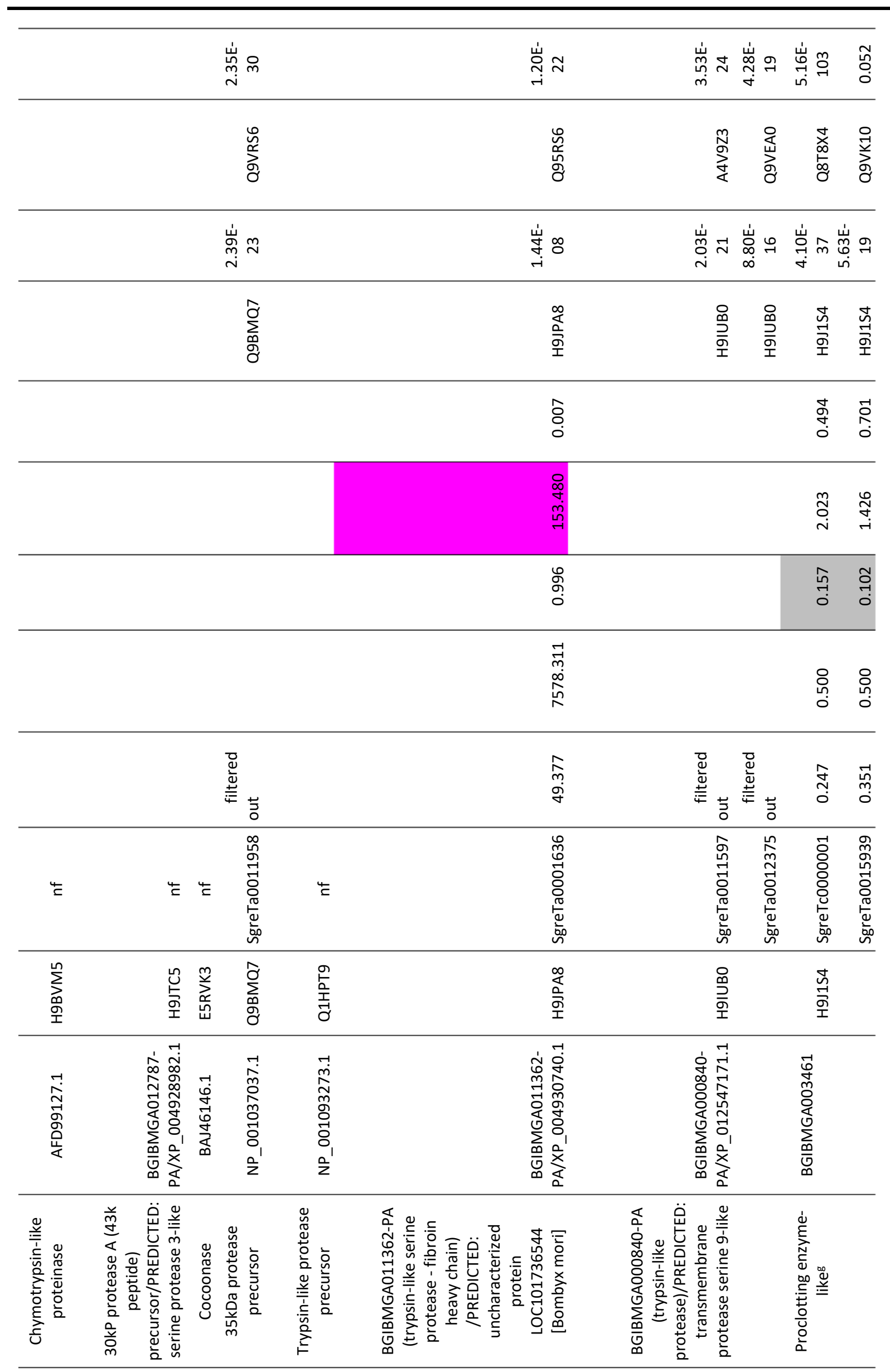




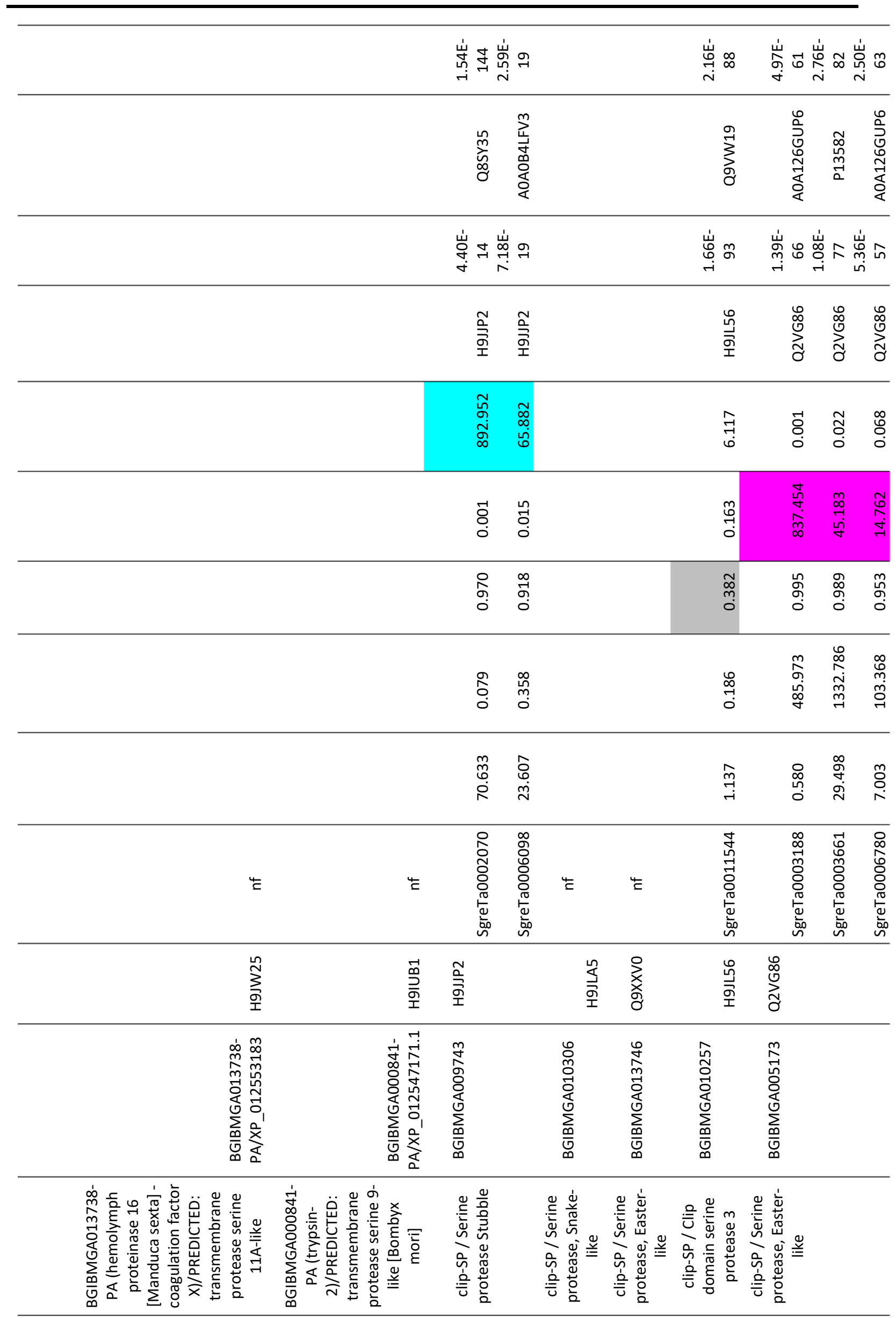




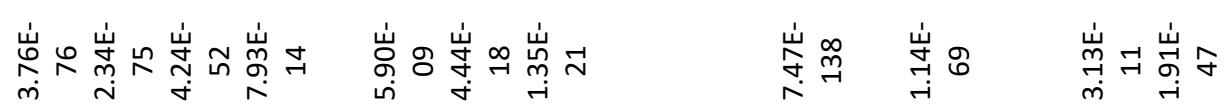

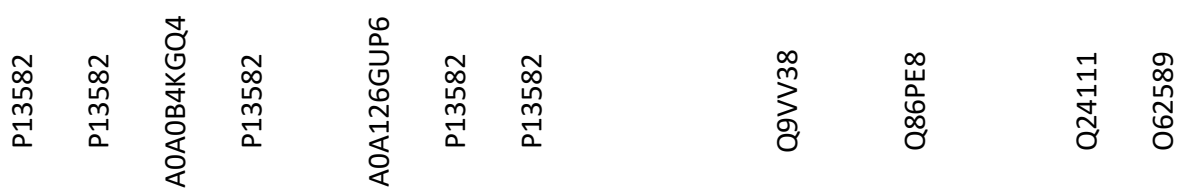

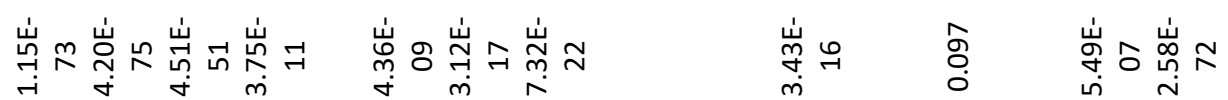

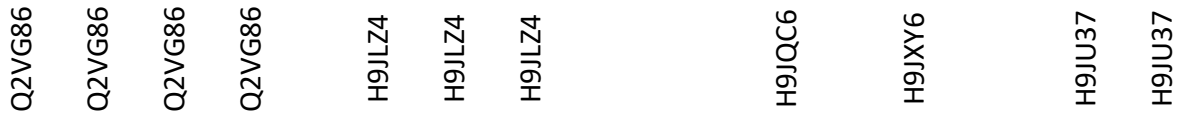

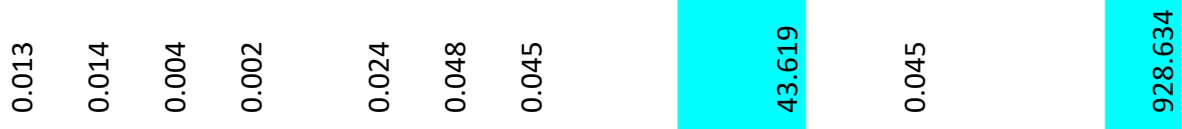

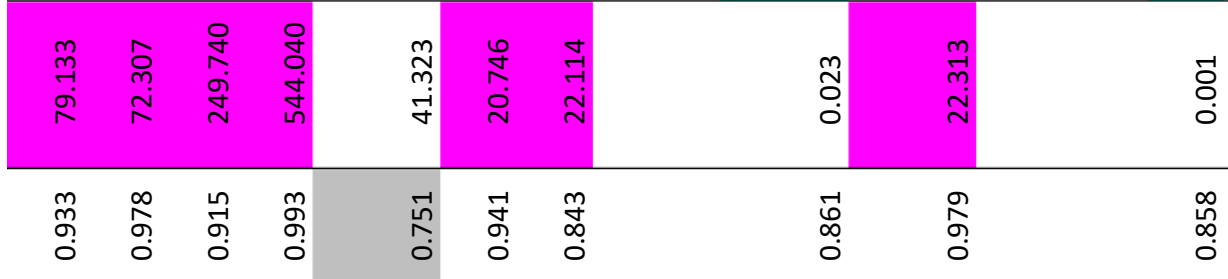

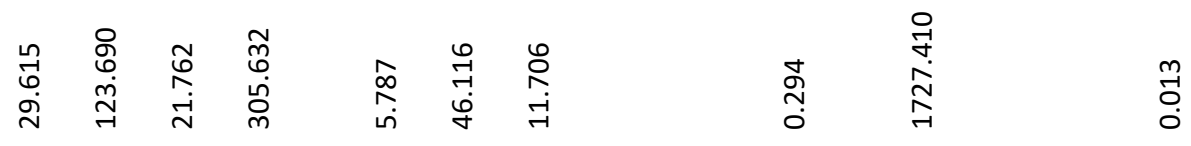

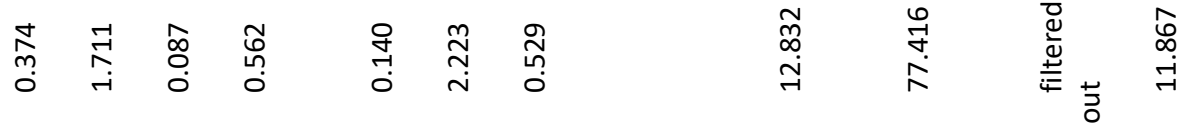

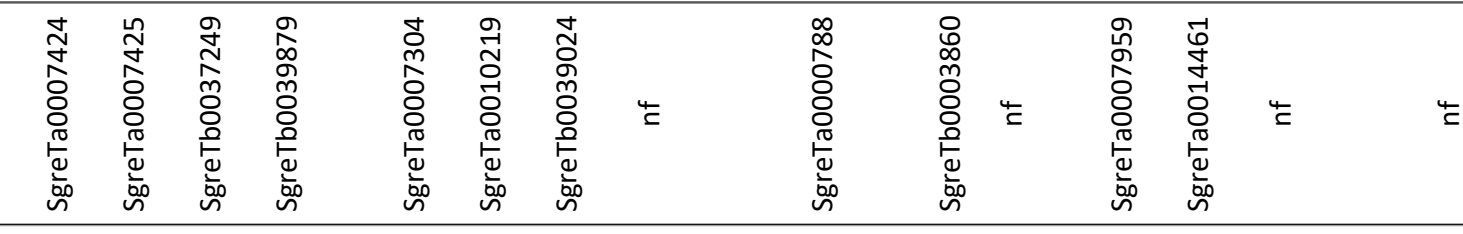

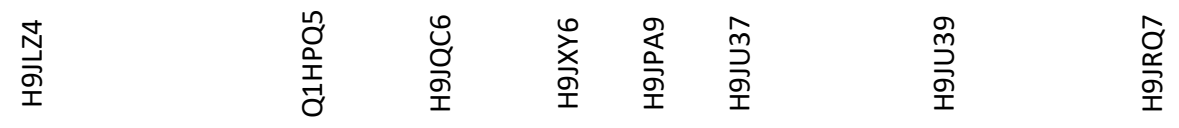

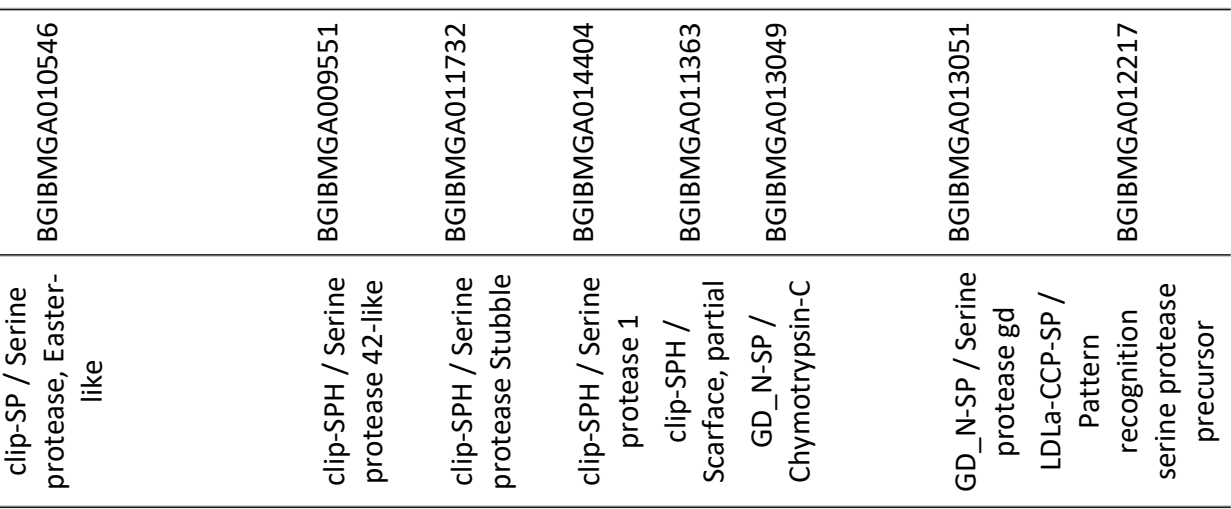




\begin{tabular}{|c|c|c|c|c|c|c|c|c|c|c|c|c|}
\hline 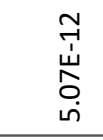 & 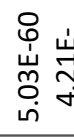 & $\vec{n}$ & & & 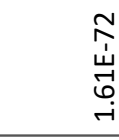 & 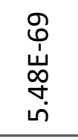 & 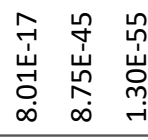 & 黛 & & & \multicolumn{2}{|l|}{ 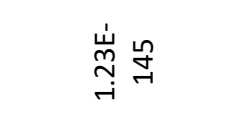 } \\
\hline 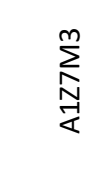 & 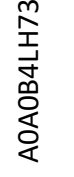 & 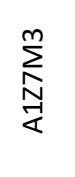 & & & $\begin{array}{l}\mathbb{N} \\
\underset{0}{\infty} \\
\underset{\leftarrow}{\infty}\end{array}$ & $\begin{array}{l}\mathbb{N} \\
\underset{\alpha}{\alpha}\end{array}$ & 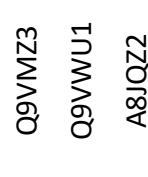 & 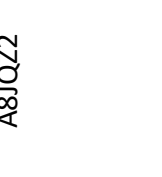 & & & กิ & \\
\hline $\begin{array}{l}\stackrel{0}{1} \\
\stackrel{1}{\dot{H}} \\
0 \\
\dot{m} \\
\dot{m}\end{array}$ & 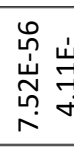 & & & & 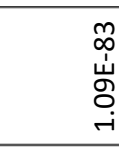 & $\begin{array}{l}\stackrel{\circ}{\$} \\
\stackrel{\leftrightarrow}{N} \\
\dot{b}\end{array}$ & 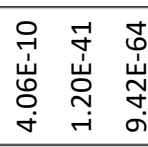 & 范 & & & 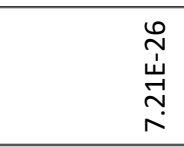 & \\
\hline 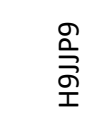 & $\begin{array}{l}\text { প } \\
\text { 옺 }\end{array}$ & $\begin{array}{l}\stackrel{9}{9} \\
\frac{9}{1}\end{array}$ & & & $\sum_{\frac{3}{1}}^{N}$ & $\sum_{\substack{\mathbf{9} \\
\text { के }}}^{N}$ & 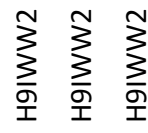 & 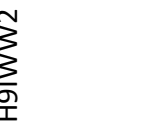 & & & 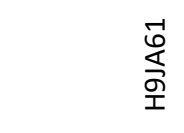 & \\
\hline $\begin{array}{l}\tilde{m} \\
\vec{m}\end{array}$ & 章 & 豙 & & & $\underset{\sim}{\stackrel{\infty}{N}}$ & $\stackrel{\mathscr{O}}{0}$ & 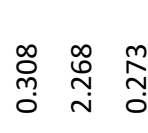 & 管 & & & 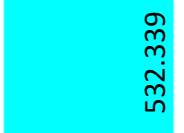 & \\
\hline $\begin{array}{l}\tilde{o} \\
0 \\
0\end{array}$ & $\stackrel{n}{n}$ & $\begin{array}{l}\stackrel{\infty}{0} \\
0 \\
0\end{array}$ & & & ڤ̊. & مी & 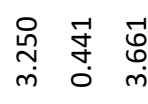 & $\begin{array}{l}-1 \\
6 \\
0 \\
n\end{array}$ & & & రి & \\
\hline ồ & 年 & $\begin{array}{l}\stackrel{n}{n} \\
\stackrel{0}{0}\end{array}$ & & & 웜 & น̊ & 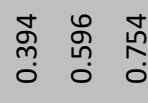 & 雍 & & & 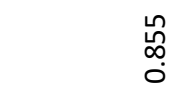 & \\
\hline $\begin{array}{l}\stackrel{-1}{\stackrel{2}{2}} \\
\stackrel{0}{0}\end{array}$ & $\stackrel{\text { ㄱ. }}{\stackrel{\sim}{i}}$ & $\begin{array}{l}\vec{H} \\
\text { مُ } \\
0\end{array}$ & & & ণ্ড & $\begin{array}{l}\stackrel{n}{f} \\
\stackrel{+}{\tilde{\sigma}} \\
\sigma\end{array}$ & 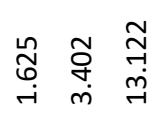 & 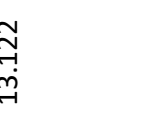 & & & $\begin{array}{l}\tilde{\Xi} \\
\text { Oे } \\
0\end{array}$ & \\
\hline $\begin{array}{l}\vec{g} \\
\stackrel{+}{+} \\
\stackrel{+}{ }\end{array}$ & 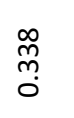 & $\begin{array}{l}\stackrel{n}{g} \\
\stackrel{i}{-} \\
\stackrel{m}{m}\end{array}$ & & & 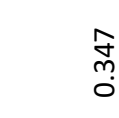 & 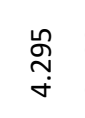 & 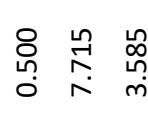 & 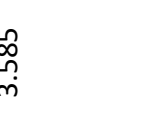 & & & $\begin{array}{l}\stackrel{n}{0} \\
\stackrel{6}{-} \\
\stackrel{-}{\rightarrow}\end{array}$ & \\
\hline 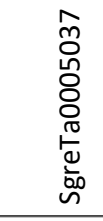 & 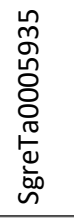 & 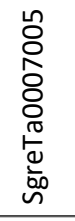 & $\stackrel{4}{\Sigma}$ & $\stackrel{4}{\Sigma}$ & 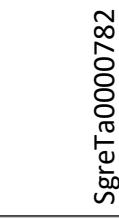 & 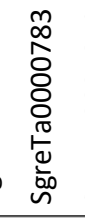 & 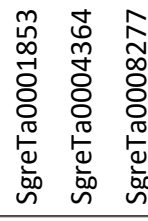 & 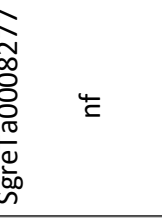 & te & $\stackrel{t}{t}$ & 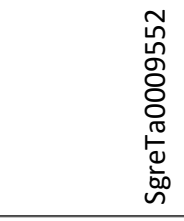 & $\stackrel{\omega}{J}$ \\
\hline $\begin{array}{l}\text { 음 } \\
\text { ㅇㅗㅗ }\end{array}$ & & & $\begin{array}{l}0 \\
\stackrel{0}{0} \\
\frac{\omega}{1}\end{array}$ & 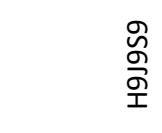 & $\sum_{\frac{3}{1}}^{N}$ & & & 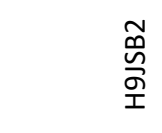 & $\begin{array}{l}\overrightarrow{9} \\
\stackrel{\overrightarrow{0}}{9} \\
\text { के }\end{array}$ & $\sum_{\substack{\infty \\
\text { के }}}^{\infty}$ & 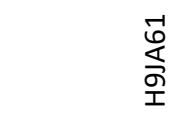 & 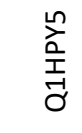 \\
\hline 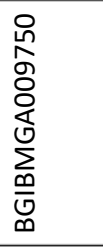 & & & 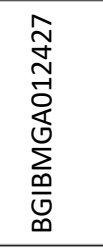 & 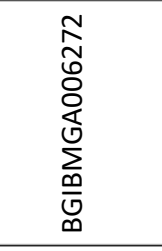 & 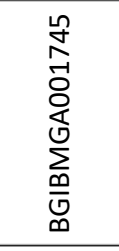 & & & 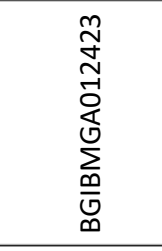 & 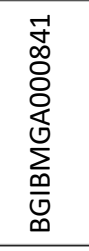 & 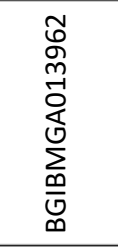 & $\begin{array}{l}0 \\
o \\
o \\
o \\
o \\
\frac{1}{0} \\
\sum_{0}^{0} \\
0 \\
\infty\end{array}$ & 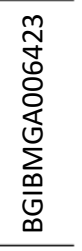 \\
\hline 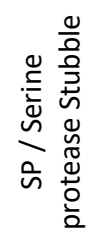 & & & 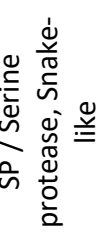 & 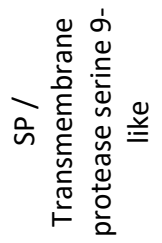 & 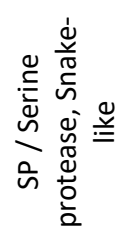 & & & 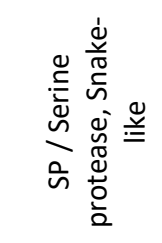 & 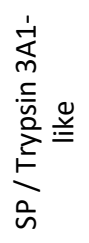 & 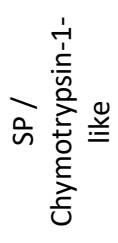 & 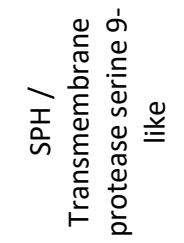 & $\begin{array}{l}\infty \\
\cdot \frac{c}{x} \\
\frac{0}{0} \\
u \\
u \\
\frac{1}{0} \\
\vdots\end{array}$ \\
\hline
\end{tabular}




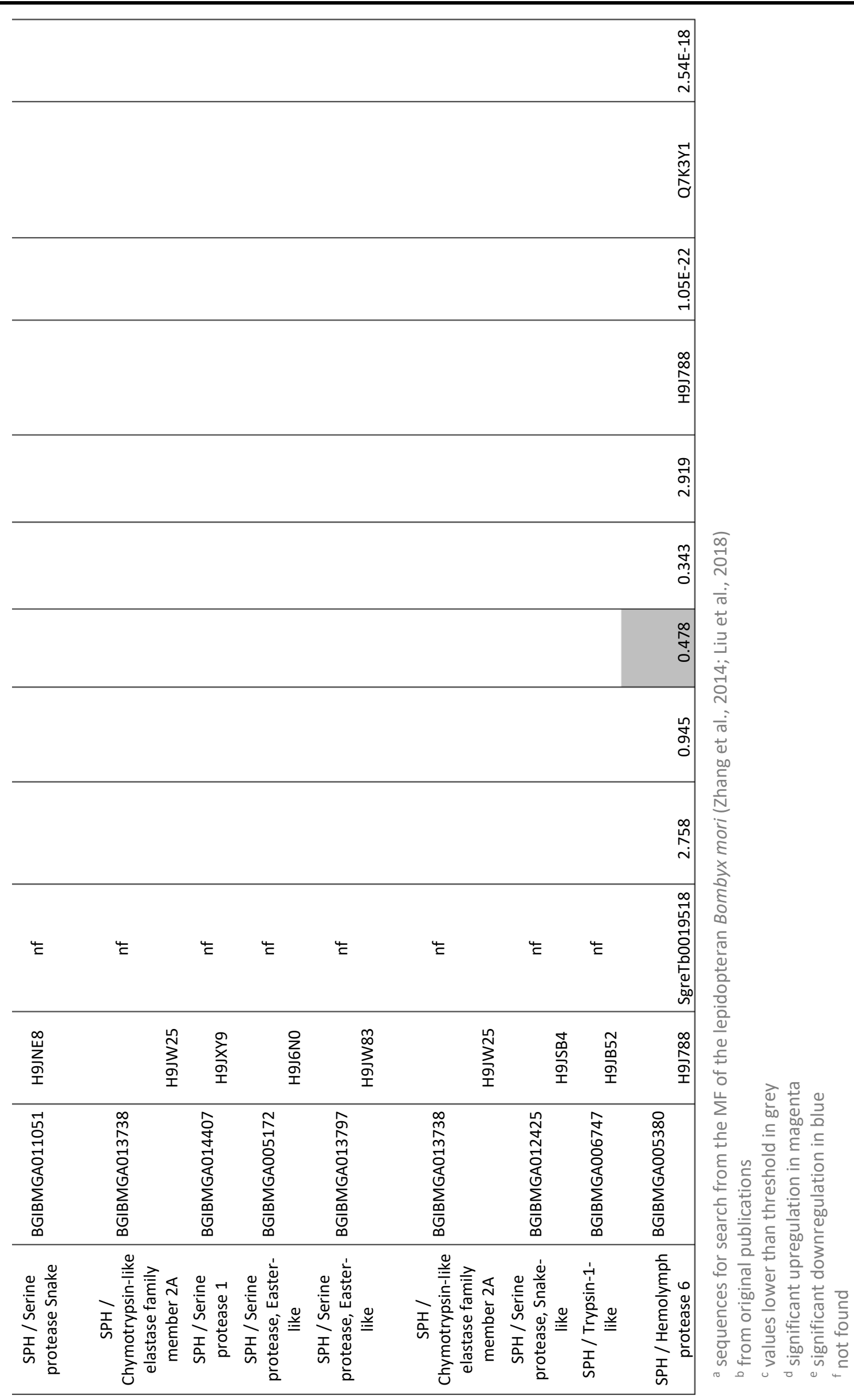




\begin{tabular}{|c|c|c|c|c|c|c|c|c|c|c|c|c|c|c|c|c|c|c|}
\hline$\frac{\frac{9}{\pi}}{3}$ & 0 & 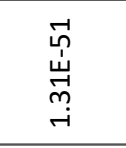 & 岕 & 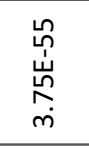 & 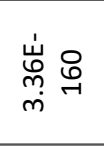 & : & 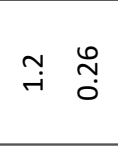 & $\stackrel{\substack{0 \\
0}}{0}$ & $\stackrel{\sim n}{m}$ & $\stackrel{\stackrel{n}{m}}{m}$ & ن & 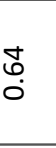 & $\begin{array}{l}\infty \\
\dot{m}\end{array}$ & $\stackrel{m}{i}$ & $\stackrel{\substack{m \\
0}}{0}$ & $\stackrel{n}{r}$ & 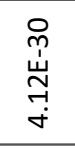 & $\begin{array}{l}\mathcal{F} \\
\stackrel{\mathcal{H}}{N} \\
\substack{\infty \\
\infty}\end{array}$ \\
\hline 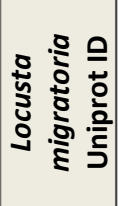 & $\sum_{\substack{\infty \\
\infty}}^{\infty}$ & $\sum_{\substack{\infty \\
\text { D }}}^{-1}$ & $\sum_{\frac{\infty}{\infty}}^{\infty}$ & 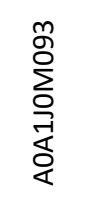 & 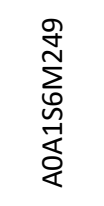 & 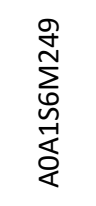 & 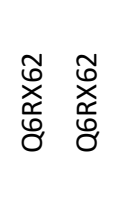 & 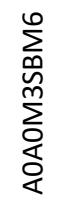 & 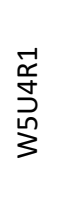 & 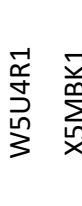 & 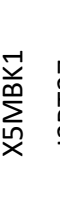 & 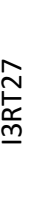 & 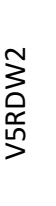 & 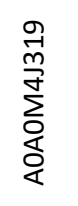 & $\sum_{\substack{n \\
\vdots}}^{\infty}$ & 怘 & $\begin{array}{l}\stackrel{n}{0} \\
\stackrel{0}{0} \\
\stackrel{\infty}{<}\end{array}$ & 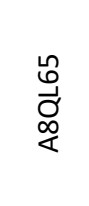 \\
\hline$\frac{\stackrel{9}{\frac{\pi}{N 0}}}{\sum_{d}^{d}}$ & 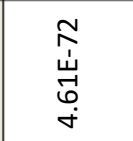 & 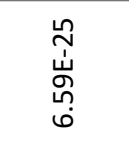 & 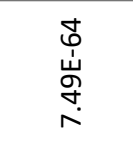 & $\stackrel{m}{\rightarrow}$ & 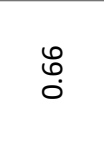 & 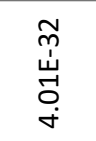 & 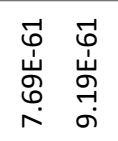 & 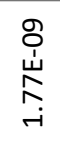 & $\begin{array}{l}\infty \\
0 \\
\stackrel{4}{0} \\
\stackrel{-}{-1}\end{array}$ & 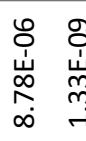 & 惫 & $\begin{array}{l}8 \\
0 \\
0\end{array}$ & 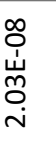 & 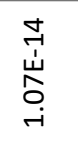 & 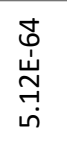 & 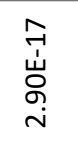 & $\begin{array}{l}\vec{y} \\
\dot{U} \\
\dot{0} \\
\dot{n}\end{array}$ & 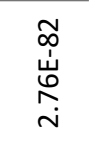 \\
\hline 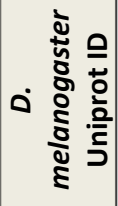 & $\begin{array}{l}\infty \\
\infty \\
\substack{\infty \\
<}\end{array}$ & $\begin{array}{l}\infty \\
\infty \\
\substack{\infty \\
<}\end{array}$ & 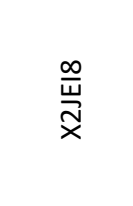 & 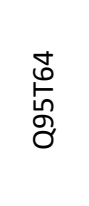 & $\begin{array}{l}\text { Na } \\
\sum_{2} \\
\text { on }\end{array}$ & $\begin{array}{l}\infty \\
\stackrel{\infty}{\beth} \\
\uplus\end{array}$ & 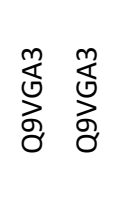 & $\begin{array}{l}\stackrel{n}{\hat{O}} \\
\text { Oे }\end{array}$ & $\begin{array}{l}\text { भ̂े } \\
\text { Oे } \\
\text { ठे }\end{array}$ & $\begin{array}{ll} & \bar{y} \\
\text { के } & \vdots \\
\text { oे } & \bar{\partial}\end{array}$ & $\sum_{0}^{-1}$ & $\sum_{\substack{N \\
\Sigma}}$ & 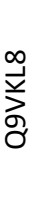 & $\begin{array}{l}\hat{m} \\
\stackrel{5}{\sigma}\end{array}$ & 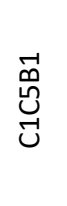 & $\sum_{\infty}^{\infty}$ & 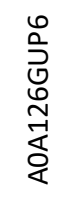 & 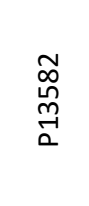 \\
\hline $\begin{array}{l}\frac{2}{2} \\
\frac{0}{0}\end{array}$ & $\begin{array}{l}\stackrel{\infty}{\sim} \\
\stackrel{\sim}{m} \\
m\end{array}$ & $\begin{array}{l}\infty \\
\stackrel{\infty}{o} \\
m\end{array}$ & $\begin{array}{l}\stackrel{+}{D} \\
\stackrel{-}{\forall}\end{array}$ & প్ & $\begin{array}{l}\stackrel{\infty}{0} \\
\stackrel{\sim}{0} \\
0\end{array}$ & $\begin{array}{l}\stackrel{0}{0} \\
\infty \\
\infty\end{array}$ & 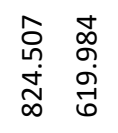 & 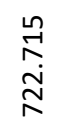 & $\begin{array}{l}\infty \\
\infty \\
\infty \\
\dot{o} \\
\dot{a}\end{array}$ & 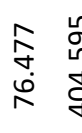 & 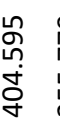 & 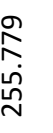 & $\begin{array}{l}\infty \\
\stackrel{0}{0} \\
\stackrel{\sigma}{\sigma}\end{array}$ & $\underset{i}{\stackrel{i}{i}}$ & $\begin{array}{l}\stackrel{\circ}{\infty} \\
\stackrel{m}{m}\end{array}$ & $\begin{array}{l}\tilde{N} \\
\stackrel{m}{\infty} \\
\infty \\
\rightarrow\end{array}$ & 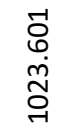 & 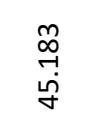 \\
\hline 움 & 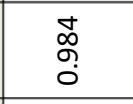 & \begin{tabular}{l}
\multirow{d}{\Delta}{} \\
0 \\
0 \\
0
\end{tabular} & $\begin{array}{l}0 \\
0 \\
0 \\
0 \\
\end{array}$ & $\begin{array}{l}\text { 员 } \\
\text { on } \\
0 \\
0\end{array}$ & $\begin{array}{l}\text { Oి } \\
\infty\end{array}$ & $\begin{array}{l}\infty \\
\infty \\
\infty \\
\infty \\
0 \\
\end{array}$ & \begin{tabular}{ll}
0 & $\infty$ \\
\multicolumn{1}{c}{} & 0 \\
0 & 0 \\
0 & 0 \\
\end{tabular} & $\begin{array}{l}-1 \\
\text { å } \\
0\end{array}$ & $\begin{array}{l}\text { \& } \\
\text { ọ } \\
0\end{array}$ & \begin{tabular}{ll} 
& 5 \\
\multirow{2}{*}{} & 5 \\
0 & 0 \\
0 & 0 \\
\end{tabular} & $\begin{array}{l} \\
\\
0 \\
0\end{array}$ & ğ & $\begin{array}{l}8 \\
0 \\
0 \\
0 \\
0\end{array}$ & $\begin{array}{l}\tilde{N} \\
\infty \\
0 \\
0 \\
\end{array}$ & 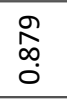 & $\begin{array}{l}n \\
0 \\
0 \\
0 \\
0\end{array}$ & $\begin{array}{l}\circ \\
\text { Я̆ } \\
0 \\
0\end{array}$ & $\begin{array}{l}\text { Oे } \\
\text { ô. } \\
0\end{array}$ \\
\hline$\sum_{\frac{1}{\alpha}} \frac{a}{a}$ & $\begin{array}{l}\text { 年 } \\
\text { : } \\
\text { in }\end{array}$ & $\underset{\substack{\stackrel{q}{N} \\
m}}{m}$ & $\begin{array}{l}\stackrel{0}{9} \\
m \\
\stackrel{0}{0} \\
-1\end{array}$ & $\begin{array}{l}\tilde{N} \\
\stackrel{N}{\dot{N}}\end{array}$ & $\begin{array}{l}\overrightarrow{0} \\
0 \\
0 \\
\rightarrow\end{array}$ & 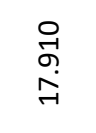 & 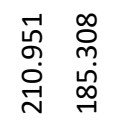 & 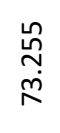 & \begin{tabular}{l}
\multirow{H}{0}{} \\
$\dot{0}$ \\
$\dot{H}$
\end{tabular} & 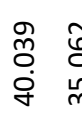 & 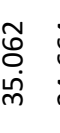 & ل & $\begin{array}{l}\vec{r} \\
\vec{n} \\
\stackrel{i}{m}\end{array}$ & 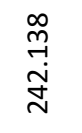 & $\begin{array}{l}\infty \\
\stackrel{+}{+} \\
\stackrel{్}{+}\end{array}$ & 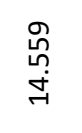 & & $\begin{array}{l}\stackrel{\infty}{\infty} \\
\stackrel{\sim}{i} \\
\stackrel{m}{\rightarrow}\end{array}$ \\
\hline$\sum_{\frac{i}{\alpha}}$ & 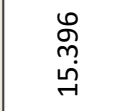 & مે & 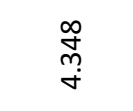 & ウે & $\begin{array}{l}\overrightarrow{-} \\
\stackrel{\infty}{-}\end{array}$ & $\begin{array}{l}\infty \\
\ddot{o} \\
i\end{array}$ & $\begin{array}{ll}\stackrel{0}{N} & \text { शे } \\
0 & 0\end{array}$ & 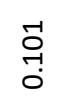 & ڤ్రి & $\begin{array}{cc}\stackrel{D}{N} & a \\
& a \\
o & c\end{array}$ & $\begin{array}{l}\hat{\infty} \\
0 \\
0 \\
0\end{array}$ & $\underset{m}{m}$ & $\begin{array}{l}\frac{0}{7} \\
\stackrel{-}{m}\end{array}$ & 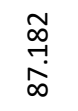 & $\begin{array}{l}\infty \\
0 \\
0 \\
0 \\
0\end{array}$ & 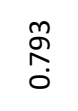 & $\begin{array}{l}\stackrel{\infty}{0} \\
0 \\
0\end{array}$ & $\begin{array}{l}\infty \\
\stackrel{\infty}{+} \\
\text { ণे }\end{array}$ \\
\hline 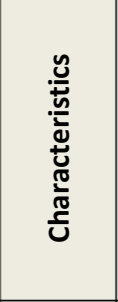 & 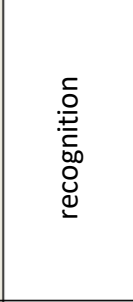 & 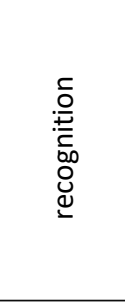 & 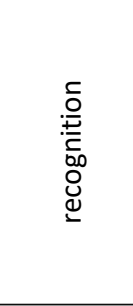 & 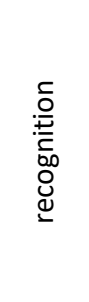 & 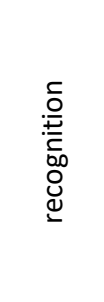 & 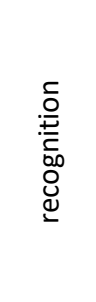 & 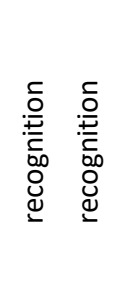 & 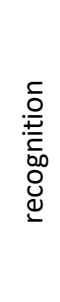 & 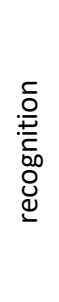 & 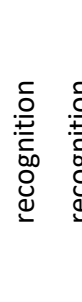 & 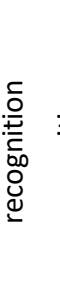 & 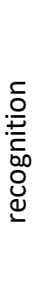 & סू. & 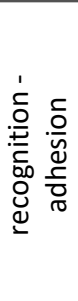 & 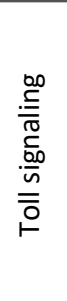 & 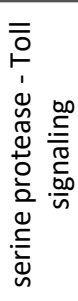 & 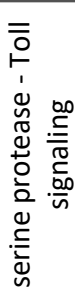 & 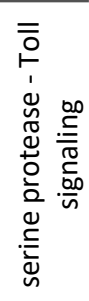 \\
\hline $\begin{array}{l}\frac{5}{\mathbb{N}} \\
\frac{0}{2} \\
\frac{0}{2}\end{array}$ & 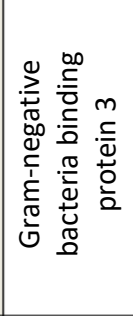 & 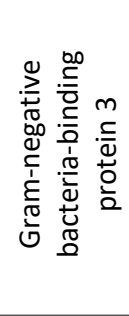 & 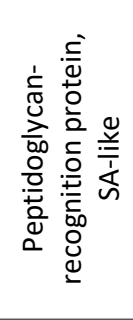 & 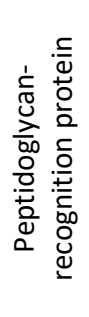 & 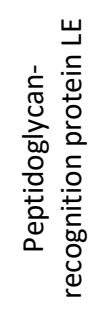 & 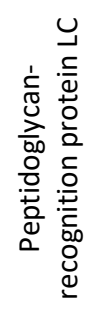 & 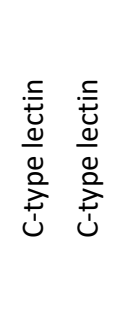 & 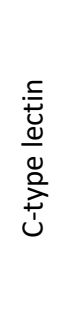 & 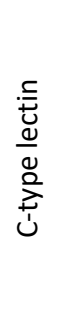 & 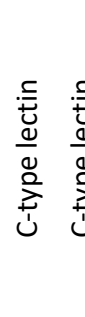 & 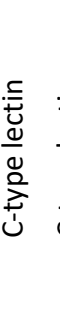 & 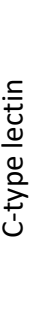 & 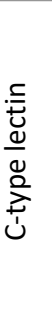 & 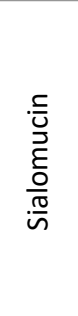 & 荧 & $\begin{array}{l}\frac{0}{N} \\
\frac{0}{0} \\
\frac{0}{n} \\
\frac{n}{n}\end{array}$ & 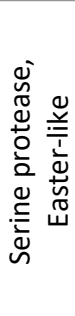 & 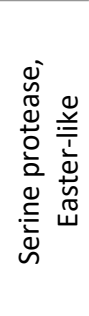 \\
\hline 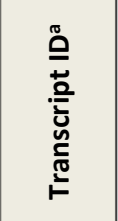 & 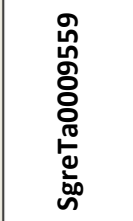 & 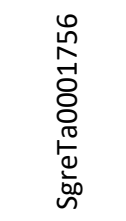 & 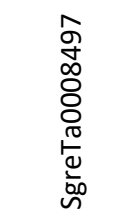 & 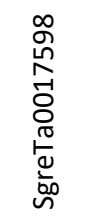 & 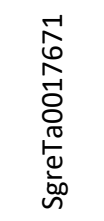 & 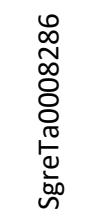 & 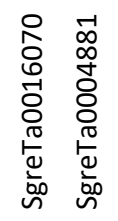 & 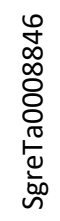 & 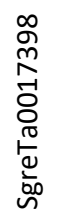 & 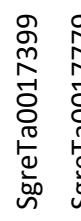 & 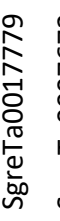 & ¿ & $\stackrel{m}{m}$ & 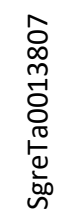 & 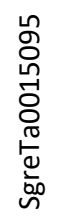 & 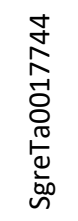 & 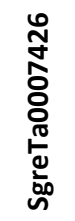 & 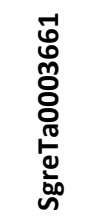 \\
\hline
\end{tabular}




\begin{tabular}{|c|c|c|c|c|c|c|c|c|c|c|c|c|c|}
\hline $\begin{array}{l}\stackrel{n}{\dot{\alpha}} \\
\stackrel{\sim}{\tilde{b}} \\
\dot{m}\end{array}$ & $\begin{array}{l}\hat{n} \\
\stackrel{\leftrightarrow}{\sim} \\
\stackrel{\sim}{\sim}\end{array}$ & 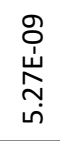 & $\begin{array}{l}\stackrel{0}{N} \\
\dot{山} \\
\infty \\
\infty \\
m \\
m\end{array}$ & 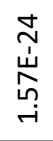 & 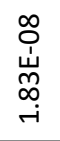 & 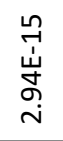 & $\begin{array}{l}\stackrel{9}{0} \\
\stackrel{\leftrightarrow}{N} \\
\stackrel{\sigma}{N}\end{array}$ & $\begin{array}{l}\vec{T} \\
\stackrel{\vec{U}}{\hat{L}} \\
\infty\end{array}$ & 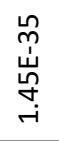 & 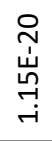 & 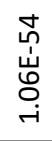 & 0 & 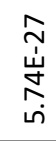 \\
\hline $\begin{array}{l}\stackrel{0}{0} \\
\stackrel{0}{\infty}\end{array}$ & $\begin{array}{l}\mathscr{0} \\
\stackrel{0}{0} \\
\stackrel{\infty}{<}\end{array}$ & $\sum_{\substack{n \\
x}}^{n}$ & $\begin{array}{l}\stackrel{n}{0} \\
\stackrel{0}{0} \\
\stackrel{\infty}{<}\end{array}$ & 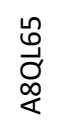 & 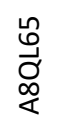 & \begin{tabular}{l}
$\stackrel{0}{0}$ \\
$\stackrel{0}{0}$ \\
\multirow{\infty}{\infty}{}
\end{tabular} & $\begin{array}{l}\tilde{0} \\
\stackrel{0}{0} \\
\infty\end{array}$ & 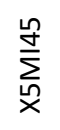 & $\begin{array}{l}\stackrel{0}{0} \\
\stackrel{0}{0} \\
\stackrel{\infty}{<}\end{array}$ & $\begin{array}{l}\tilde{0} \\
0 \\
0 \\
\infty\end{array}$ & 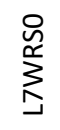 & 疋 & $\begin{array}{l}0 \\
0 \\
\mathbb{O} \\
\infty \\
\alpha\end{array}$ \\
\hline
\end{tabular}

\begin{tabular}{|c|c|c|c|c|c|c|c|c|c|c|c|c|c|c|}
\hline 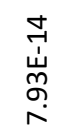 & $\begin{array}{l}\stackrel{n}{\sim} \\
\stackrel{\dot{\Psi}}{N} \\
\stackrel{N}{N}\end{array}$ & $\begin{array}{l}\stackrel{\infty}{\vec{山}} \\
\stackrel{y}{\forall} \\
\stackrel{+}{+}\end{array}$ & $\begin{array}{l}0 \\
\stackrel{1}{u} \\
\stackrel{0}{m} \\
\dot{m}\end{array}$ & N & 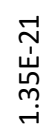 & $\begin{array}{l}\vec{n} \\
\stackrel{\leftrightarrow}{\sim} \\
\vec{\sigma}\end{array}$ & $\stackrel{\sim}{\sim}$ & 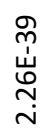 & 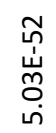 & $\begin{array}{l}\hat{n} \\
\stackrel{\leftrightarrow}{پ} \\
\stackrel{\sim}{N}\end{array}$ & 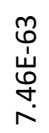 & 0 & $\begin{array}{l}\vec{b} \\
\dot{W} \\
\hat{\sigma} \\
\dot{\sigma}\end{array}$ & $\begin{array}{l}\tilde{\vartheta} \\
\stackrel{\leftrightarrow}{0} \\
\stackrel{0}{N}\end{array}$ \\
\hline
\end{tabular}

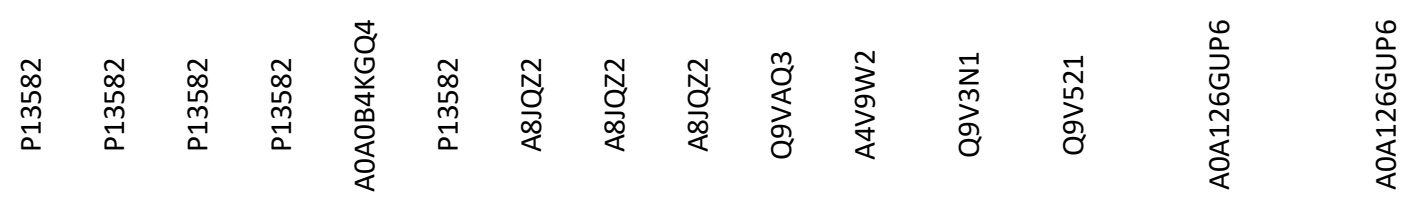

\begin{tabular}{|c|c|c|c|c|c|c|c|c|c|c|c|c|c|c|}
\hline 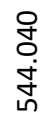 & 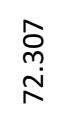 & $\begin{array}{l}\mathscr{0} \\
\stackrel{i}{i} \\
\dot{d}\end{array}$ & $\begin{array}{l}\stackrel{m}{\eta} \\
\stackrel{\sim}{r} \\
\stackrel{r}{r}\end{array}$ & 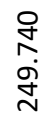 & $\begin{array}{l}\underset{J}{J} \\
\underset{I}{\sim}\end{array}$ & $\stackrel{\infty}{\stackrel{\infty}{i}}$ & $\begin{array}{l}\text { O } \\
\text { ஸn } \\
\stackrel{n}{N} \\
\stackrel{n}{n}\end{array}$ & $\begin{array}{l}\hat{A} \\
\stackrel{1}{0} \\
0 \\
o \\
g\end{array}$ & $\begin{array}{l}\mathscr{J} \\
\dot{J}\end{array}$ & 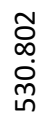 & $\begin{array}{l}\text { ¿ } \\
\infty \\
\stackrel{N}{N}\end{array}$ & $\begin{array}{c}\hat{0} \\
\tilde{\sigma} \\
\end{array}$ & 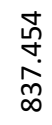 & $\begin{array}{l}\tilde{D} \\
\stackrel{+}{+}\end{array}$ \\
\hline $\begin{array}{l}\text { m } \\
\text { } \\
0\end{array}$ & $\begin{array}{l}\infty \\
\hat{\sigma} \\
0\end{array}$ & $\begin{array}{l}\vec{\sigma} \\
\text { హ. }\end{array}$ & $\begin{array}{l}m \\
\tilde{n} \\
0 \\
0\end{array}$ & $\begin{array}{l}\text { ñ } \\
\sigma \\
0 \\
0\end{array}$ & $\begin{array}{l}m \\
⿱ m \\
\infty \\
0\end{array}$ & 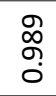 & $\begin{array}{l}\text { นn } \\
\text { Оे }\end{array}$ & $\begin{array}{l}\text { m. } \\
\text { Oे } \\
0\end{array}$ & \begin{tabular}{l}
\multirow{2}{\alpha}{} \\
0 \\
0
\end{tabular} & $\begin{array}{l}\text { o } \\
\text { o. }\end{array}$ & $\begin{array}{l}\text { సू } \\
\text { Оू }\end{array}$ & $\begin{array}{l}\text { 㞫 } \\
\text { On }\end{array}$ & $\begin{array}{l}\text { nू } \\
\text { on }\end{array}$ & $\begin{array}{l}\text { ñ } \\
\text { مू } \\
0\end{array}$ \\
\hline $\begin{array}{l}\tilde{\tilde{n}} \\
\dot{\omega} \\
\dot{m} \\
\tilde{m}\end{array}$ & 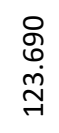 & $\begin{array}{l}0 \\
\underset{7}{-1} \\
\dot{\sigma}\end{array}$ & $\begin{array}{l}n \\
0 \\
0 \\
\dot{N}\end{array}$ & \begin{tabular}{l}
\multirow{2}{N}{} \\
$\stackrel{-}{N}$
\end{tabular} & $\begin{array}{l}\stackrel{\varrho}{2} \\
\stackrel{-}{-}\end{array}$ & 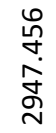 & $\begin{array}{l}\stackrel{n}{f} \\
\stackrel{-}{-} \\
\sigma\end{array}$ & $\begin{array}{l}0 \\
\hat{6} \\
\stackrel{i}{n} \\
m\end{array}$ & 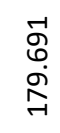 & $\begin{array}{l}\infty \\
\varnothing \\
i \\
m\end{array}$ & 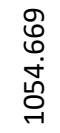 & 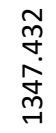 & $\begin{array}{l}n \\
\hat{\sigma} \\
\dot{\infty} \\
\infty \\
\sigma\end{array}$ & $\begin{array}{l}\infty \\
0 \\
\tilde{N} \\
\tilde{n} \\
\tilde{\gamma}\end{array}$ \\
\hline
\end{tabular}

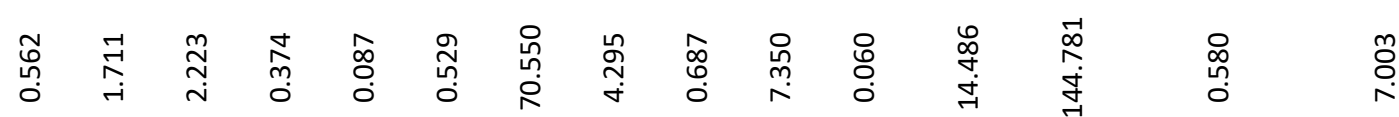

\begin{tabular}{|c|c|c|c|c|c|c|c|c|c|c|c|c|}
\hline 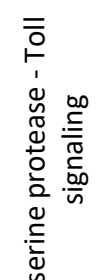 & 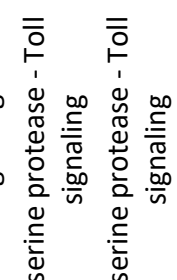 & 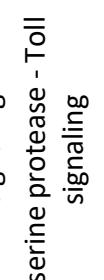 & 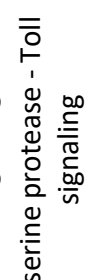 & 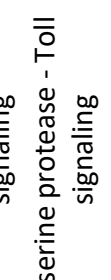 & 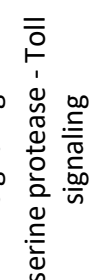 & 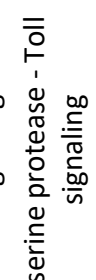 & 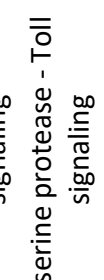 & 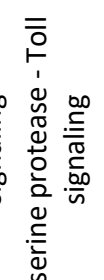 & 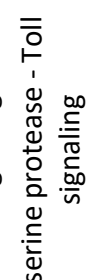 & 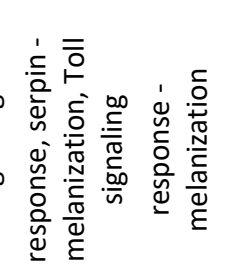 & 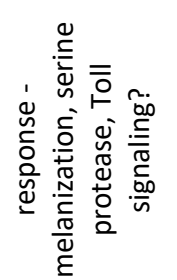 & 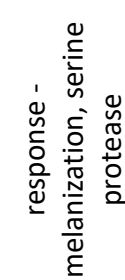 \\
\hline
\end{tabular}

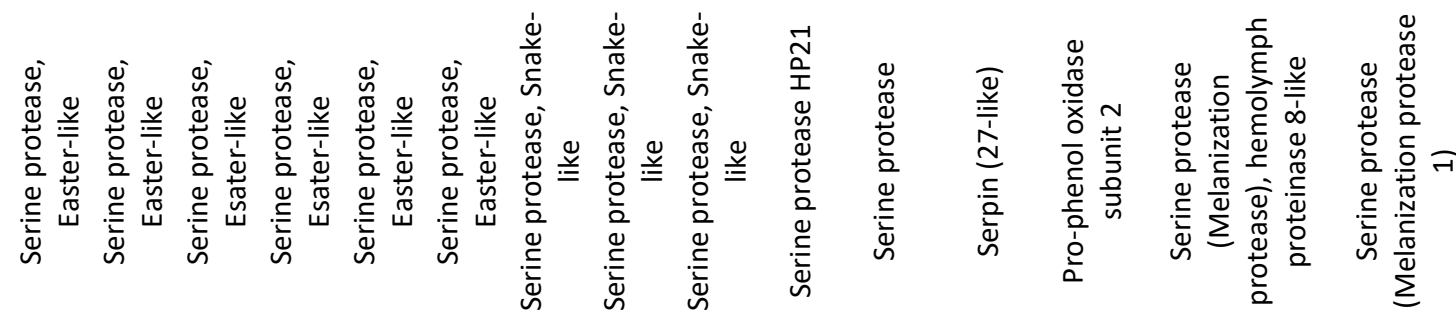

\begin{tabular}{|c|c|c|c|c|c|c|c|c|c|c|c|c|c|c|}
\hline 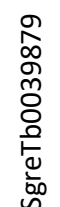 & 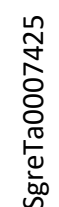 & 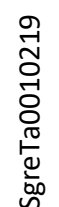 & 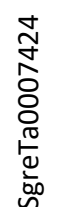 & 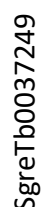 & 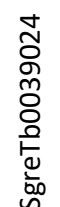 & 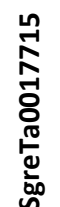 & 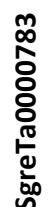 & 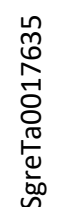 & 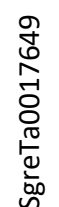 & 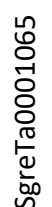 & 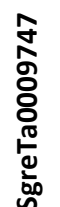 & 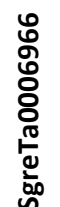 & 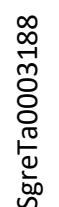 & 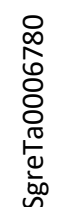 \\
\hline
\end{tabular}




\begin{tabular}{|c|c|c|c|c|c|c|c|c|c|c|c|c|c|c|}
\hline 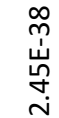 & 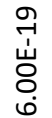 & $\stackrel{n}{\rightarrow}$ & 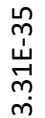 & 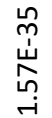 & 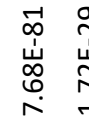 & 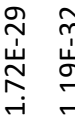 & 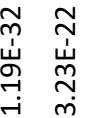 & 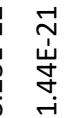 & $\begin{array}{l}\tilde{N} \\
\stackrel{\sharp}{\sharp} \\
\\
\dot{n}\end{array}$ & 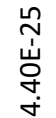 & $\stackrel{\text { ?ี }}{0}$ & $\begin{array}{l}\stackrel{L}{\infty} \\
0 \\
0\end{array}$ & $\begin{array}{l}0 \\
0 \\
0\end{array}$ & 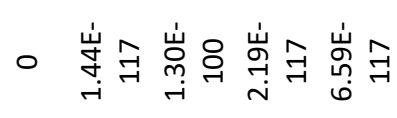 \\
\hline
\end{tabular}

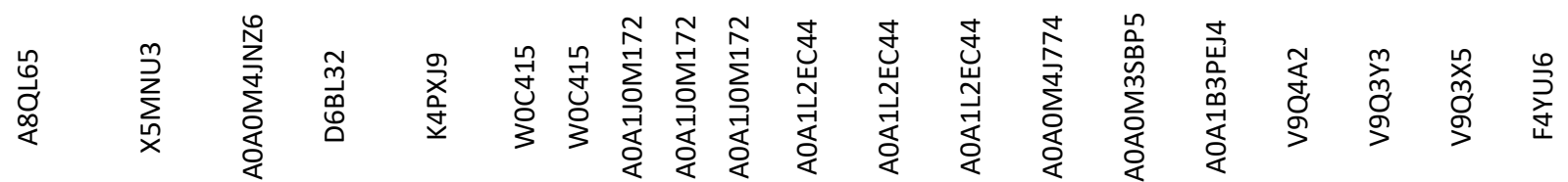

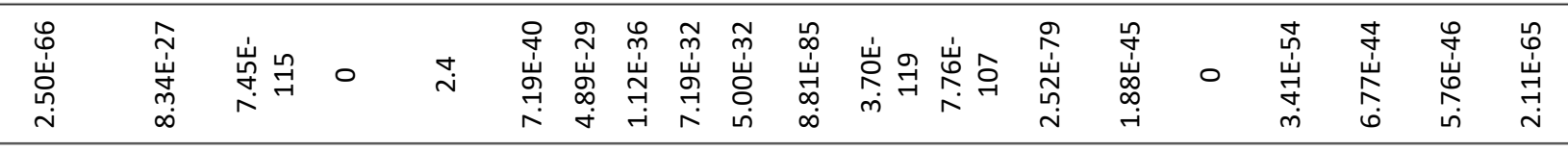

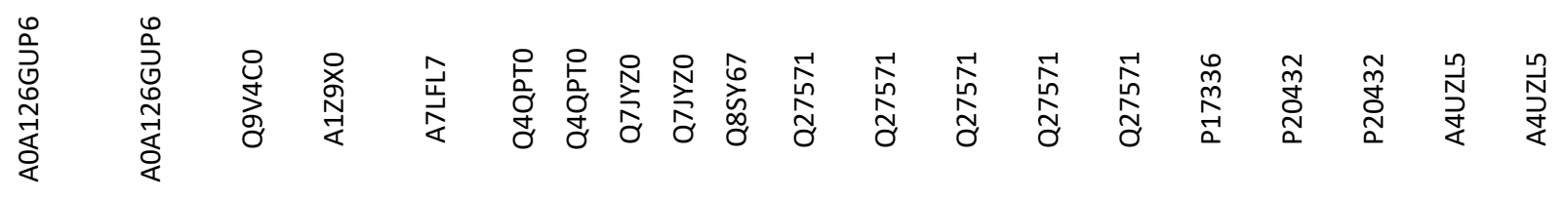

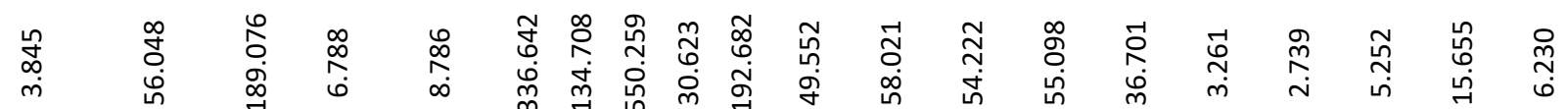

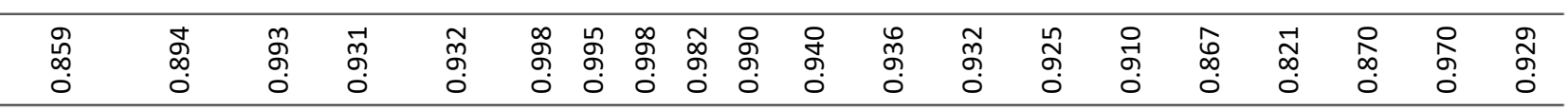

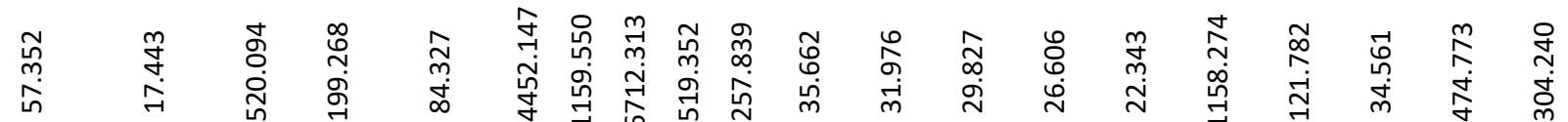

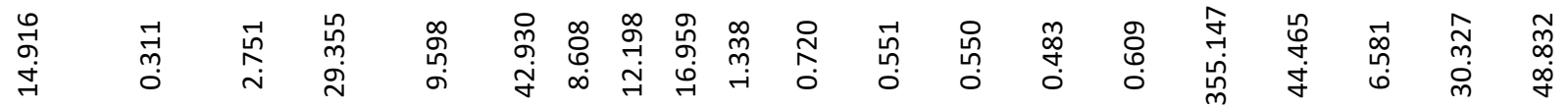

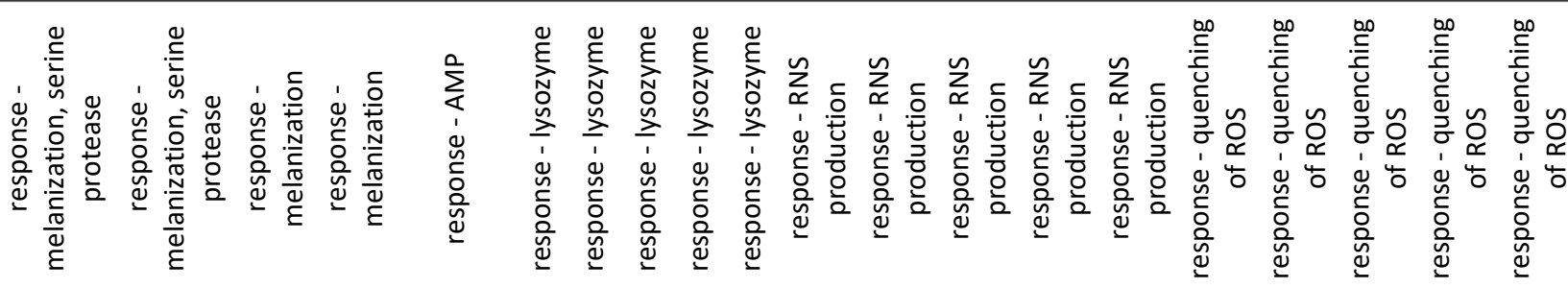

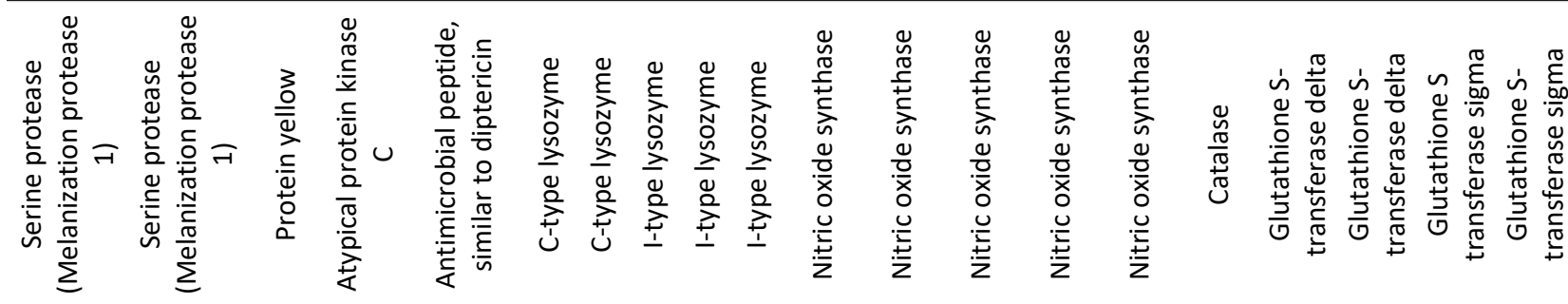

\begin{tabular}{|c|c|c|c|c|c|c|c|c|c|c|c|c|c|c|c|c|c|c|c|}
\hline 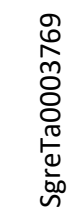 & 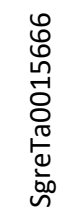 & 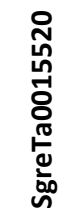 & 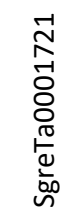 & 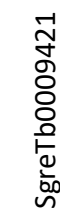 & 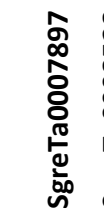 & 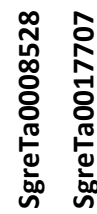 & 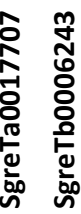 & 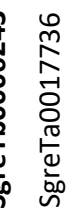 & 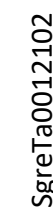 & & & 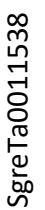 & & & & & & & 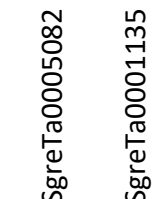 \\
\hline
\end{tabular}




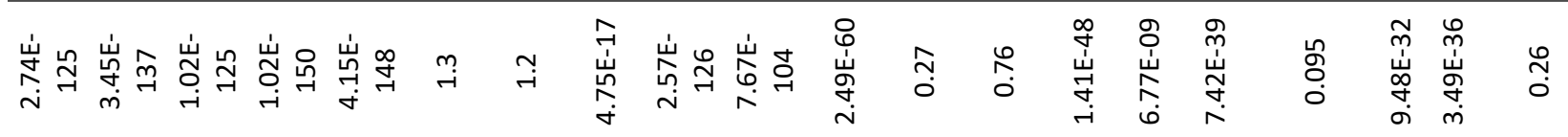

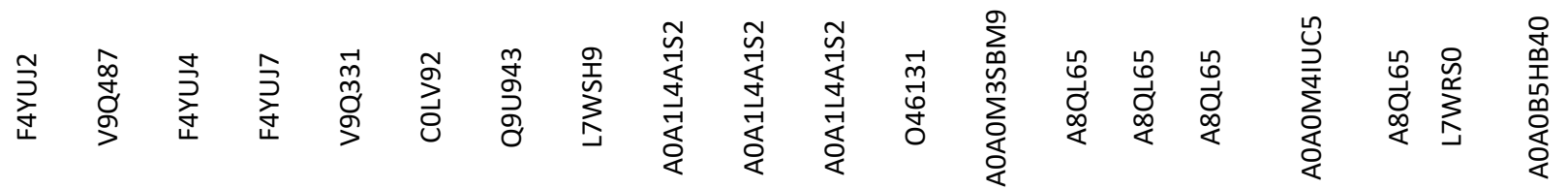

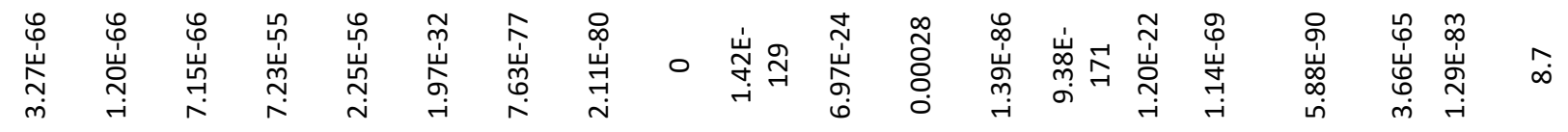

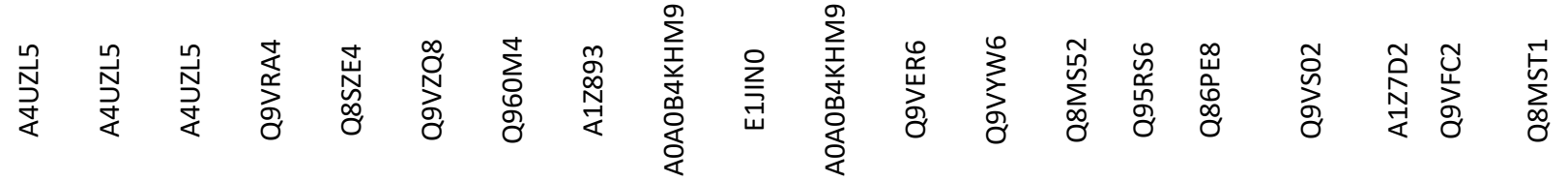

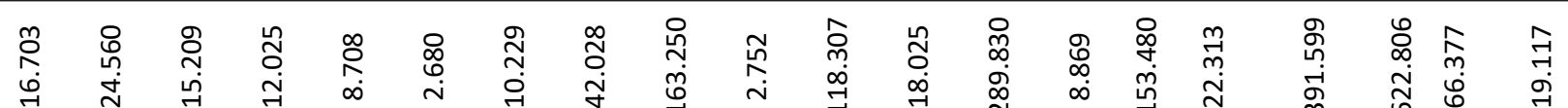

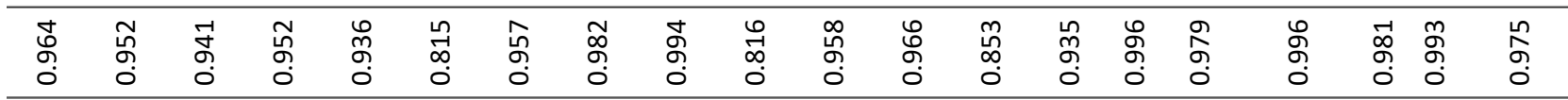

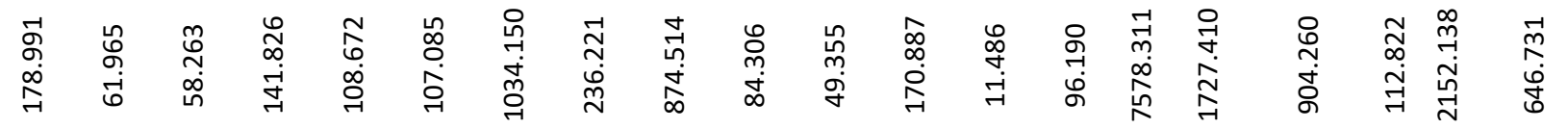

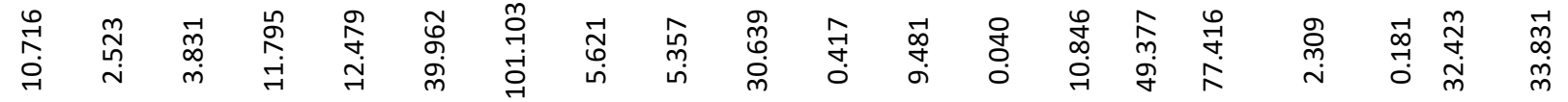

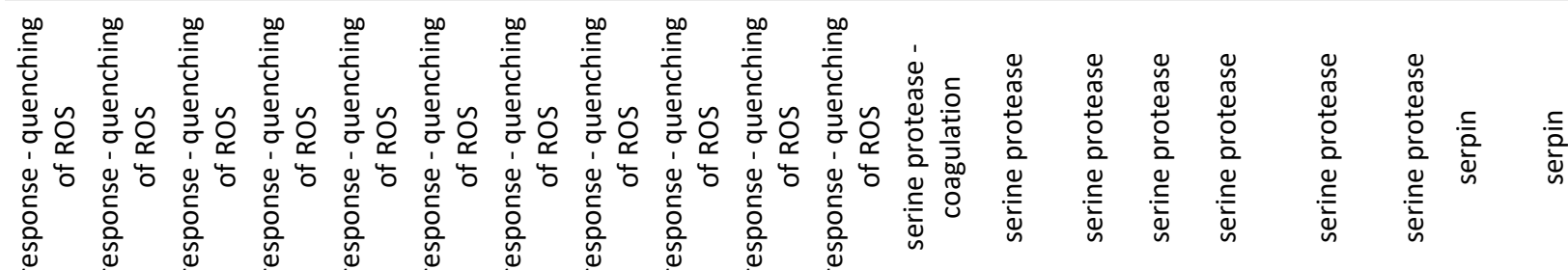

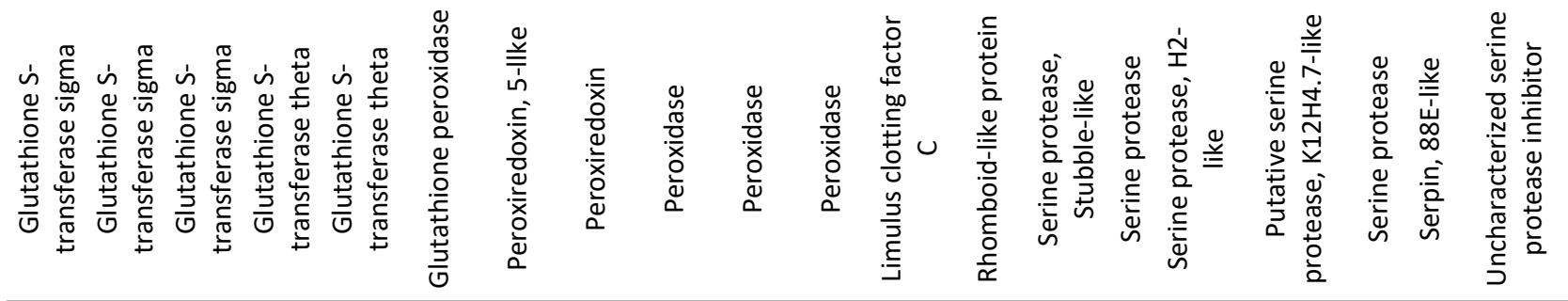

\begin{tabular}{|c|c|c|c|c|c|c|c|c|c|c|c|c|c|c|c|c|c|c|}
\hline 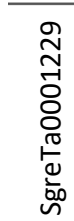 & 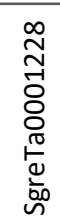 & 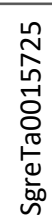 & 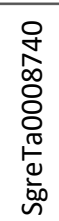 & 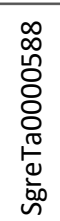 & 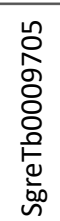 & 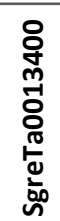 & 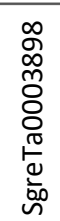 & 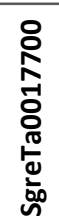 & 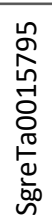 & 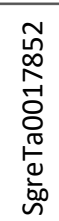 & 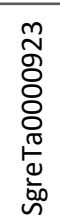 & 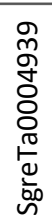 & 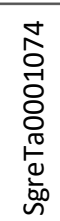 & 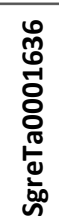 & 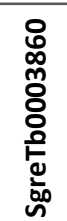 & 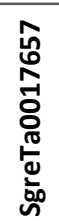 & 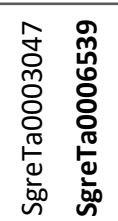 & 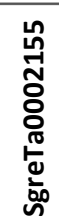 \\
\hline
\end{tabular}




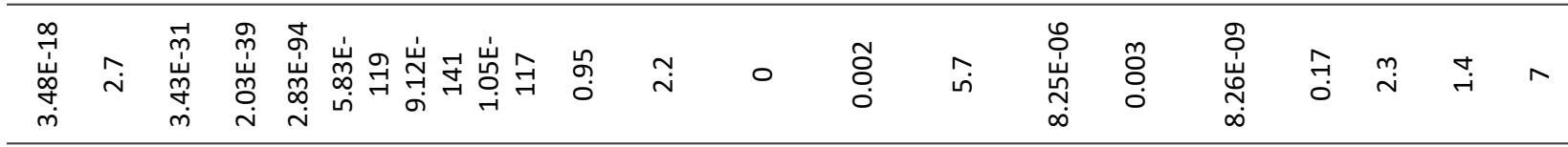

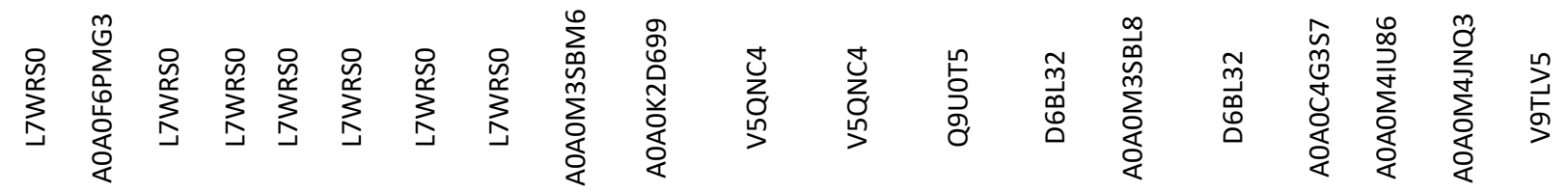

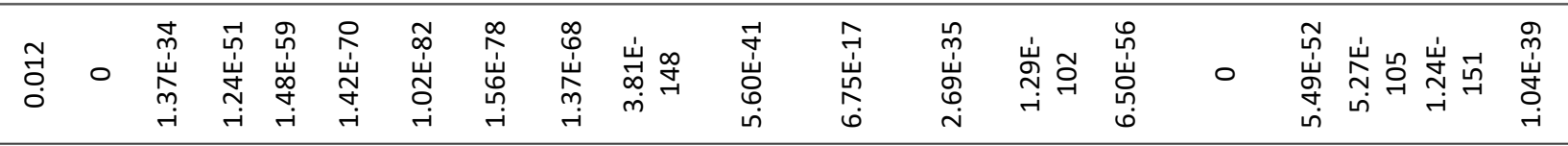

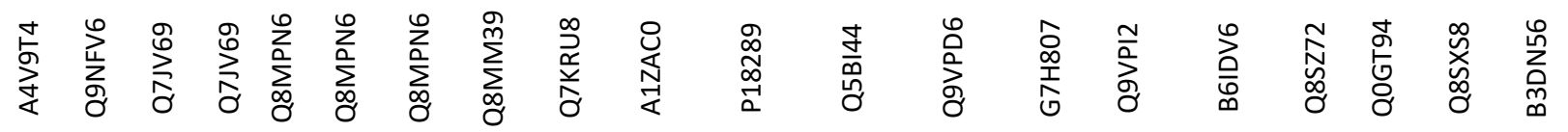

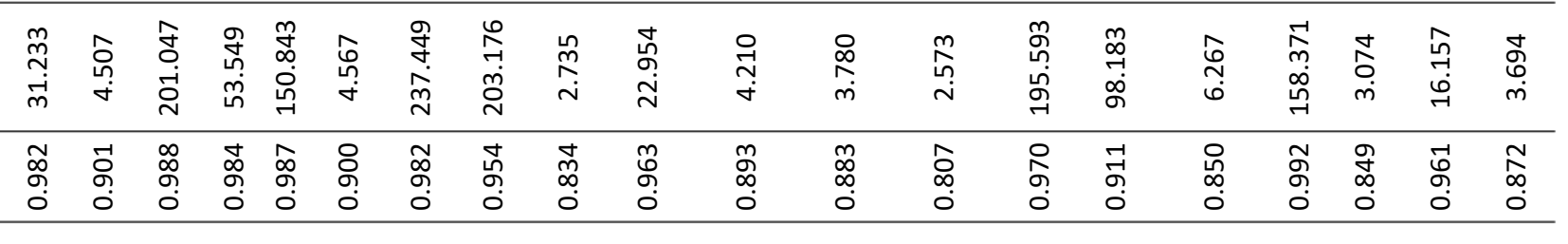

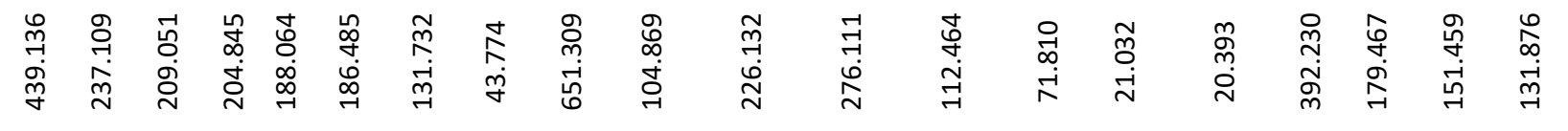

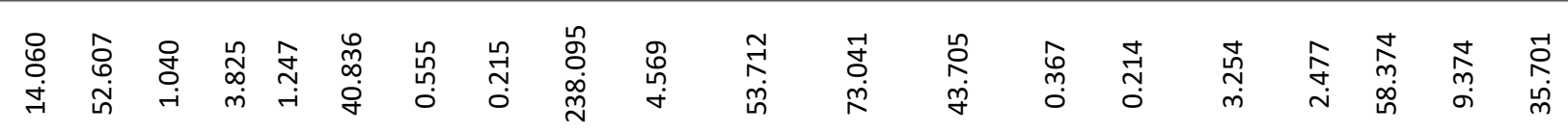

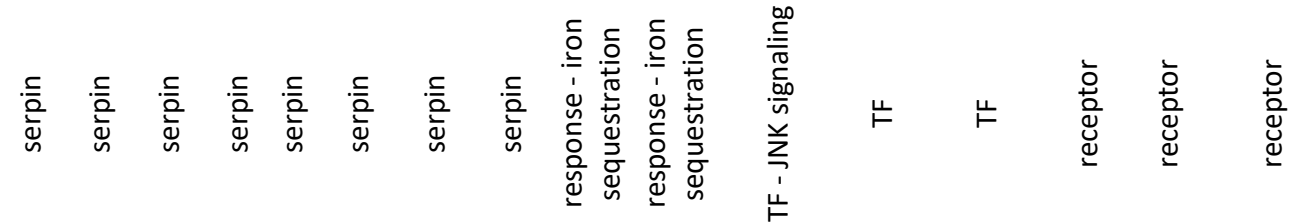

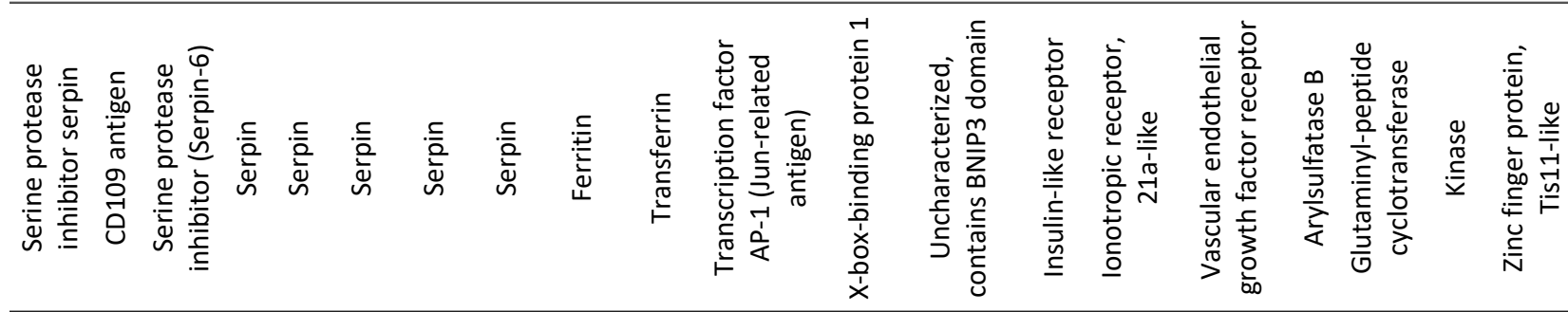

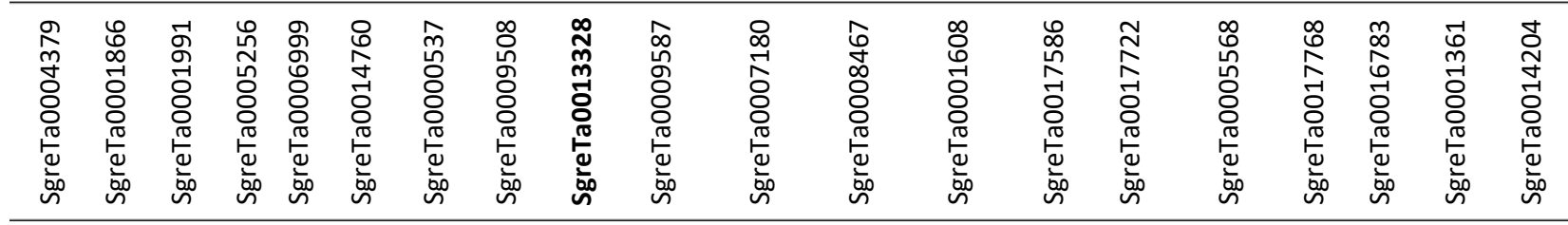




\begin{tabular}{|c|c|c|c|c|}
\hline 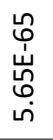 & 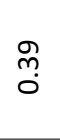 & $\stackrel{m}{m}$ & $\begin{array}{l}\text { f } \\
0 \\
0 \\
0 \\
0\end{array}$ & $\stackrel{+}{+}$ \\
\hline $\begin{array}{l}\hat{0} \\
\frac{\hat{d}}{0}\end{array}$ & 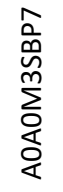 & $\begin{array}{l}\frac{-1}{\infty} \\
\stackrel{W}{\infty} \\
3\end{array}$ & $\begin{array}{l}\vec{r} \\
\stackrel{y}{5} \\
\stackrel{3}{\zeta}\end{array}$ & $\begin{array}{l}\vec{m} \\
\vec{b} \\
\dot{\sigma}\end{array}$ \\
\hline 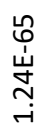 & 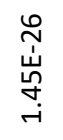 & 0 & 0 & $\stackrel{\nabla}{\sigma}$ \\
\hline $\begin{array}{l}\mathscr{0} \\
\stackrel{0}{\infty} \\
\stackrel{0}{0} \\
0\end{array}$ & $\begin{array}{l}\text { 岦 } \\
\text { ठे }\end{array}$ & 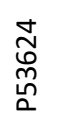 & 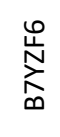 & 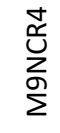 \\
\hline 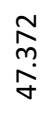 & 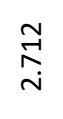 & 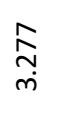 & $\stackrel{\stackrel{n}{n}}{\stackrel{\leftrightarrow}{r}}$ & $\begin{array}{l}\text { O̊ } \\
\text { Ln } \\
\text { in }\end{array}$ \\
\hline $\begin{array}{l}\Delta \\
\vdots \\
0 \\
0\end{array}$ & $\begin{array}{l}0 \\
0 \\
0 \\
0 \\
0\end{array}$ & $\begin{array}{l}\text { m } \\
\infty \\
\infty \\
0\end{array}$ & $\begin{array}{l}\text { o̊ } \\
\text { on } \\
\end{array}$ & $\begin{array}{l}\text { f } \\
\infty \\
0 \\
0\end{array}$ \\
\hline 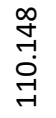 & $\underset{\stackrel{n}{N}}{\stackrel{N}{i}}$ & 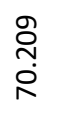 & \begin{tabular}{l}
$\stackrel{\infty}{\infty}$ \\
$\stackrel{\sim}{*}$ \\
\multirow{F}{*}{}
\end{tabular} & 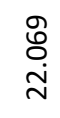 \\
\hline$\stackrel{\stackrel{\sim}{\sim}}{\underset{N}{N}}$ & $\begin{array}{l}\stackrel{\stackrel{n}{N}}{\leftrightarrow} \\
\stackrel{\omega}{N}\end{array}$ & $\begin{array}{l}\underset{\sim}{\sim} \\
\stackrel{\sim}{\sim}\end{array}$ & $\begin{array}{l}\stackrel{\overbrace{}}{~} \\
\stackrel{9}{6}\end{array}$ & $\begin{array}{l}\stackrel{\circ}{\circ} \\
\stackrel{\leftrightarrow}{n}\end{array}$ \\
\hline
\end{tabular}

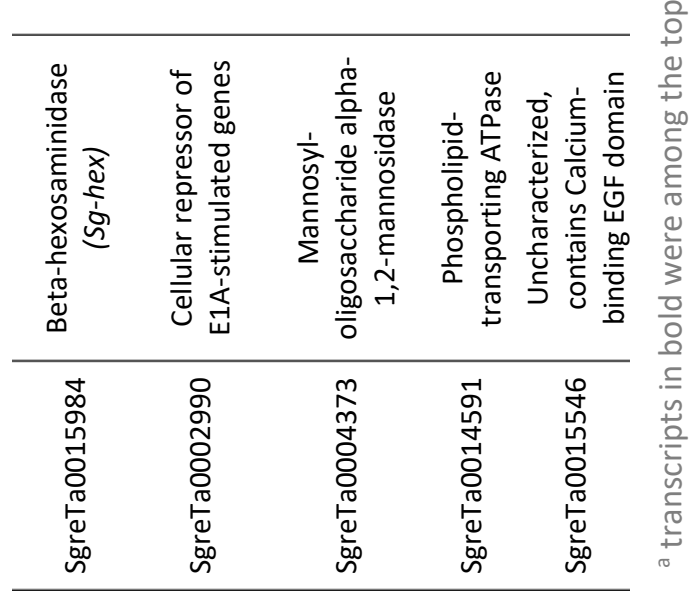




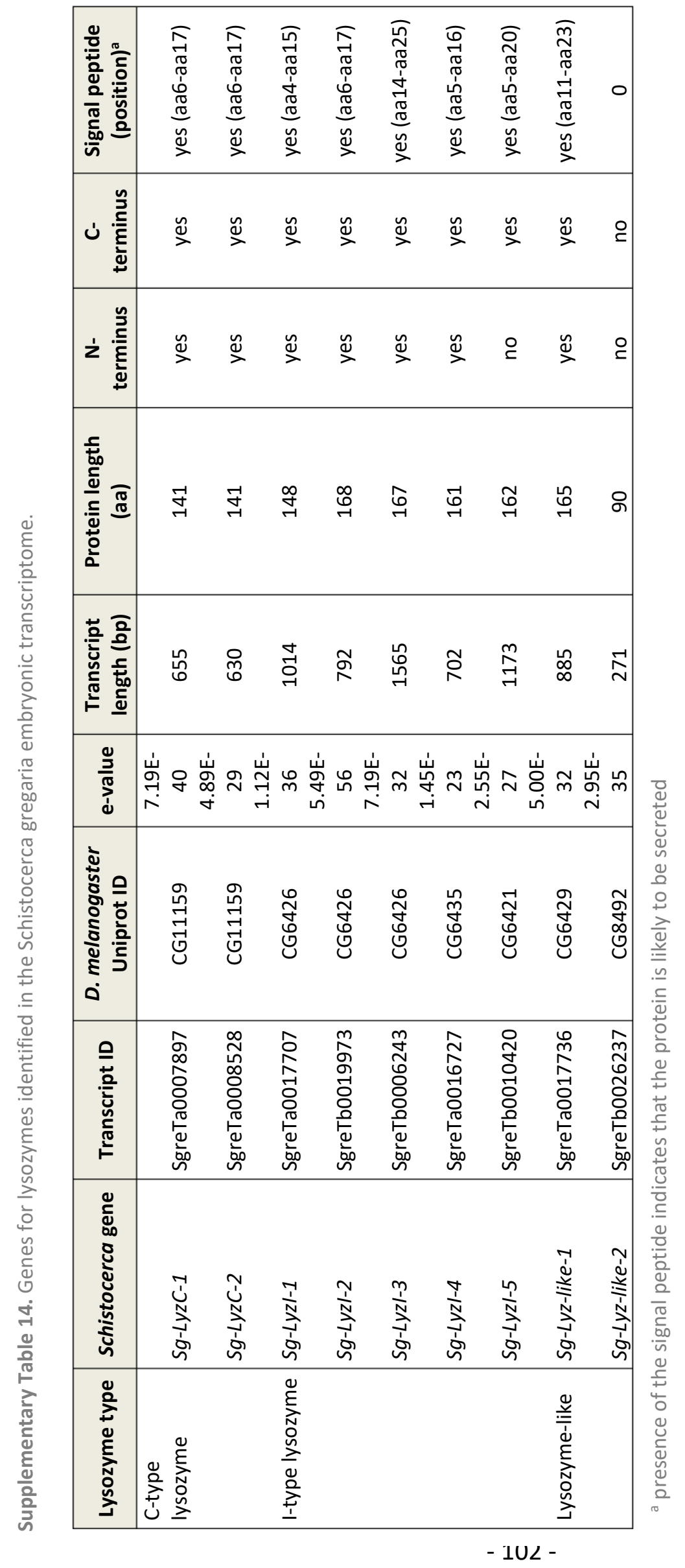


Supplementary Table 15. Genes for ecdysone biosynthesis enzymes identified in the Schistocerca embryonic transcriptome.

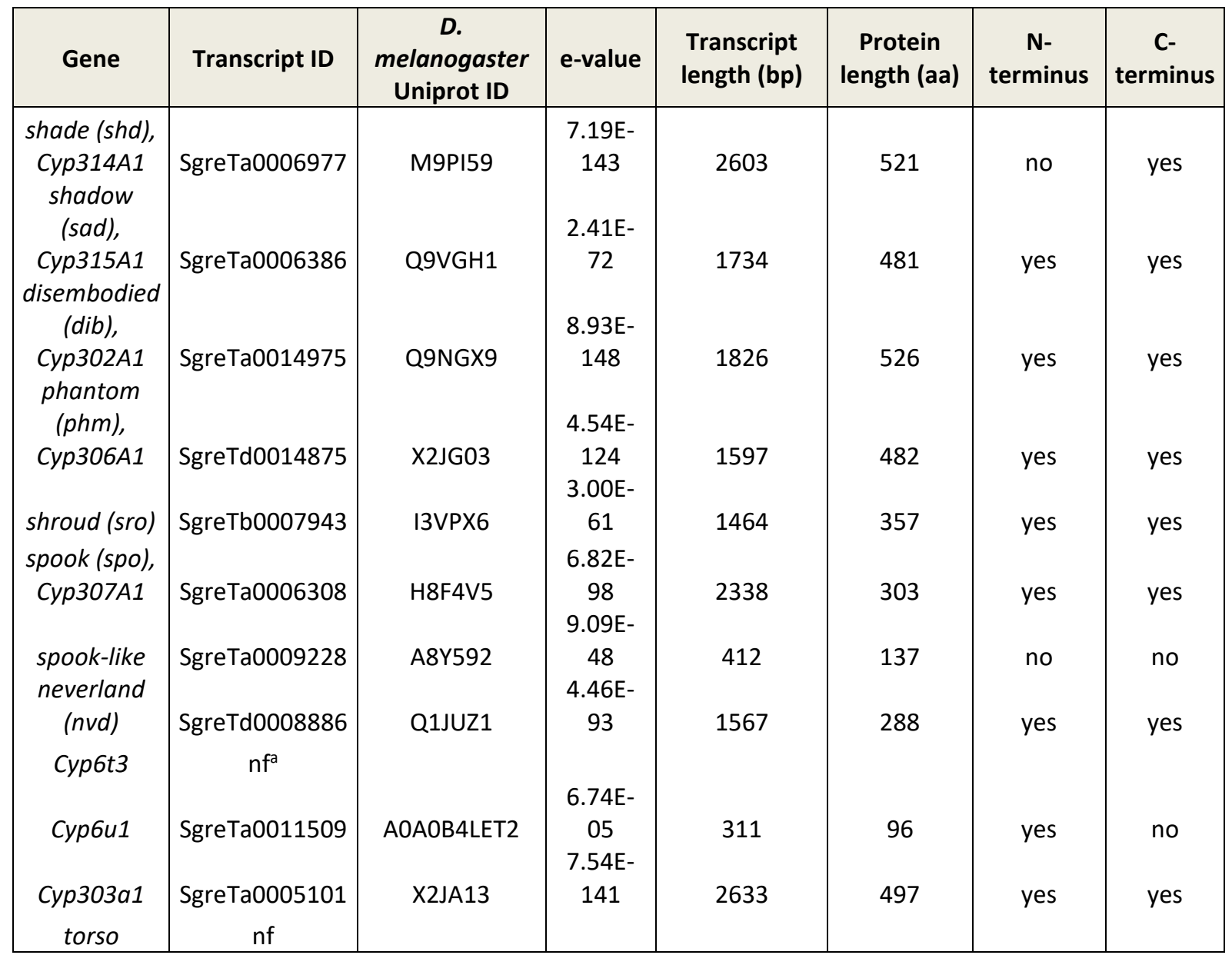

a not found 


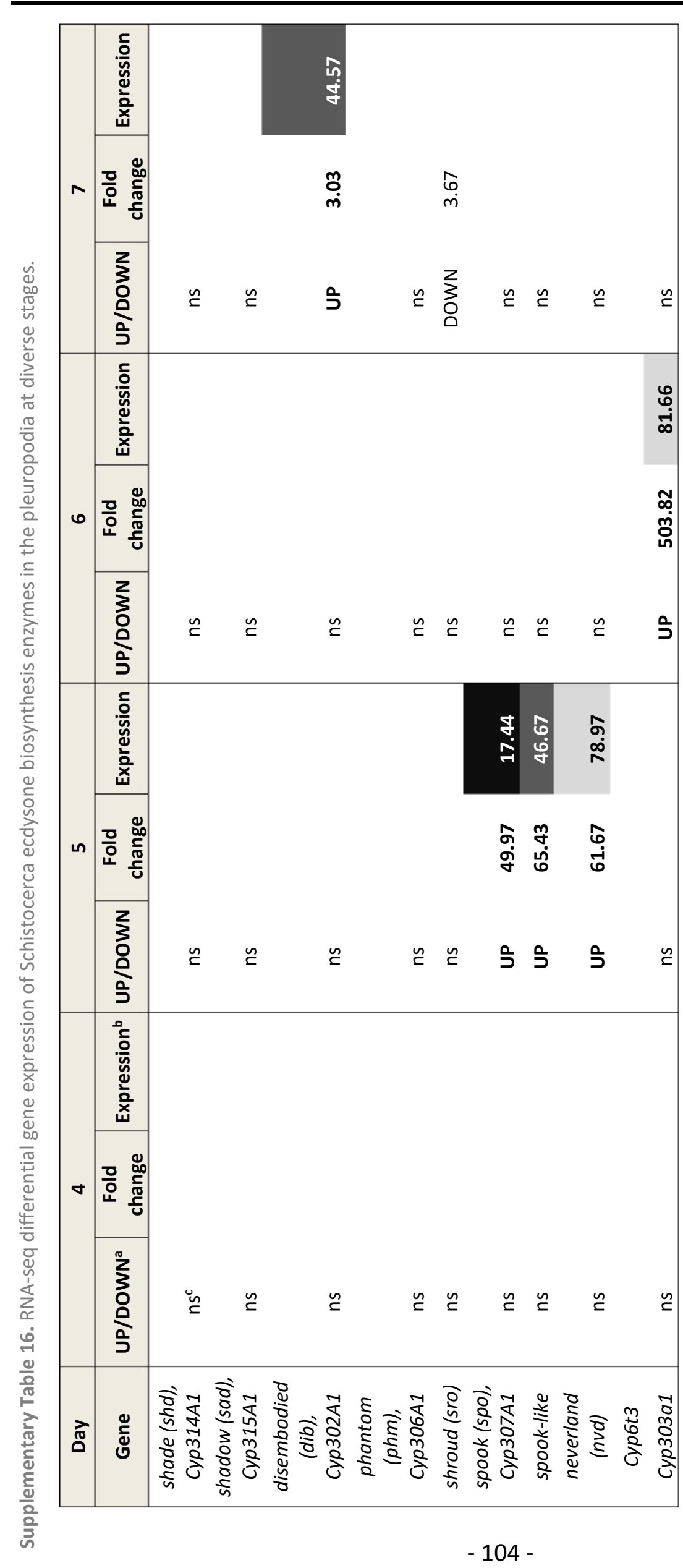




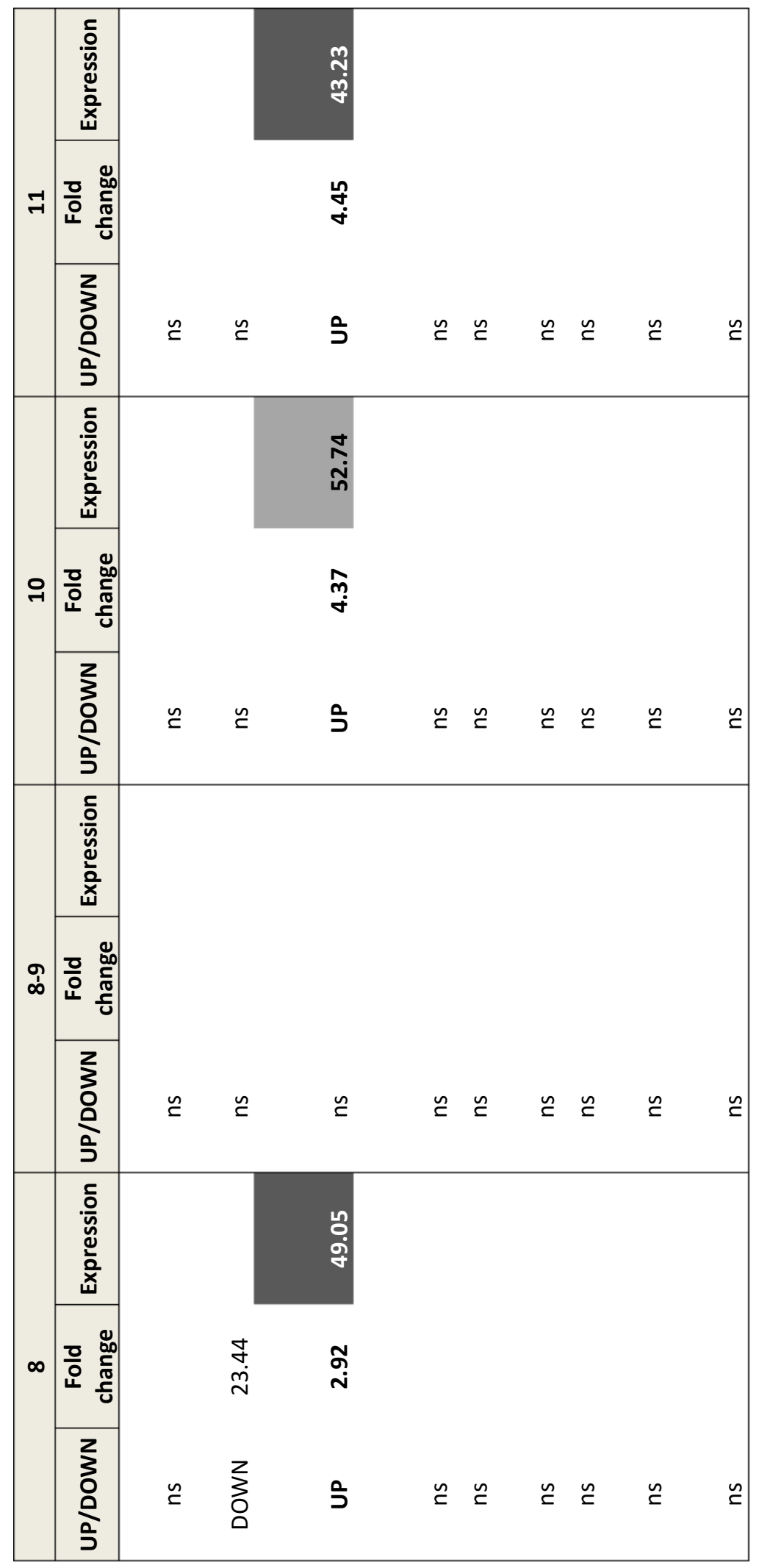




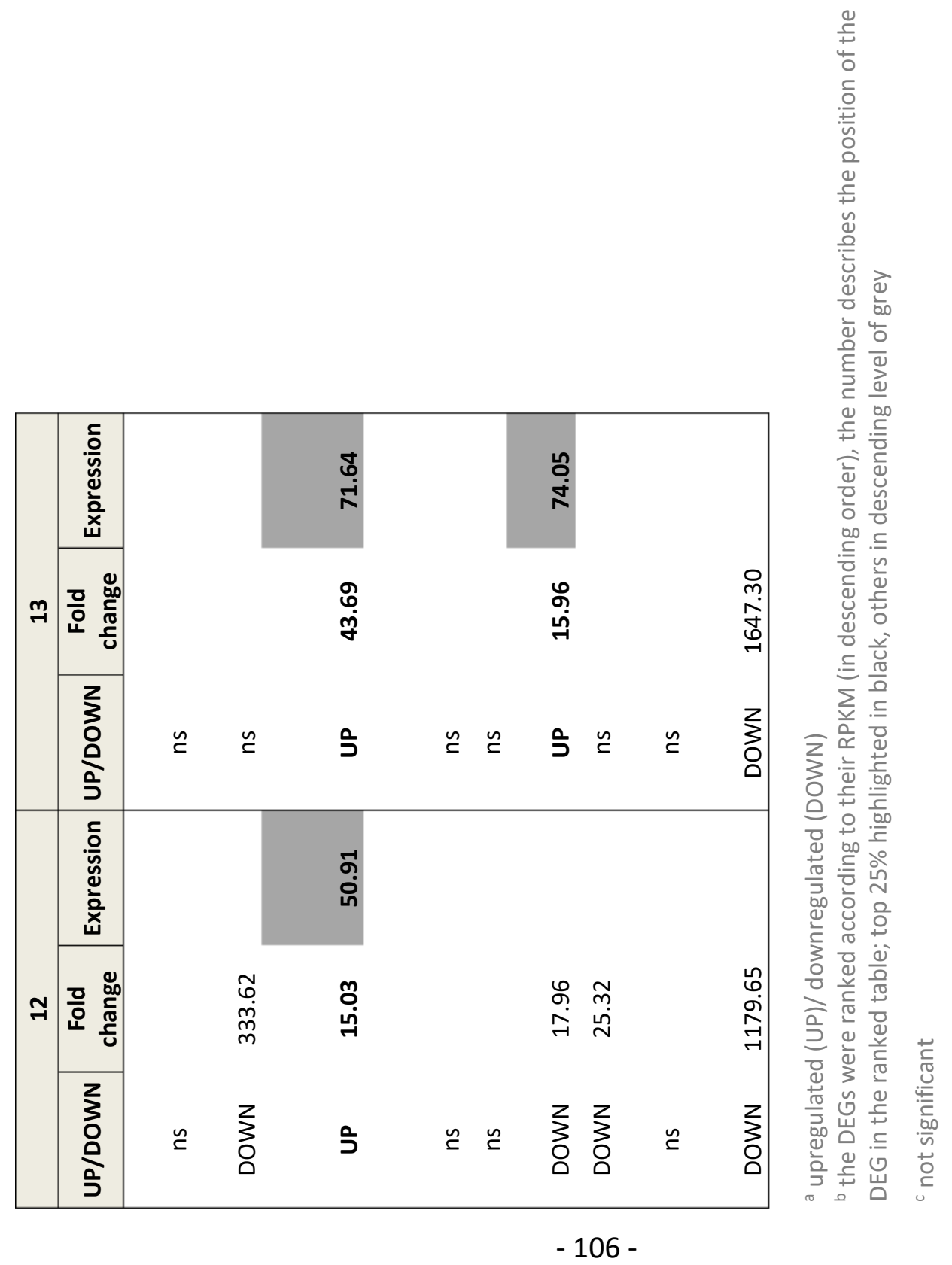


Supplementary Table 17. Schistocerca genes with GO terms "hormone biosynthetic process" upregulated in the highly secreting pleuropodia.

\begin{tabular}{|c|c|c|c|c|c|}
\hline \multicolumn{2}{|c|}{ S. gregaria transcript } & \multirow[b]{2}{*}{ Note } & \multirow[b]{2}{*}{$\begin{array}{l}\text { Functions in } \\
\text { ecdysone } \\
\text { biosynthesis }\end{array}$} & \multicolumn{2}{|c|}{$\begin{array}{c}\text { D. melanogaster top } \\
\text { hit }\end{array}$} \\
\hline Transcript ID & Protein & & & $\begin{array}{c}D . \\
\text { melanogaster } \\
\text { Uniprot ID }\end{array}$ & e-value \\
\hline SgreTa0013987 & $\begin{array}{l}\text { Juvenile hormone } \\
\text { acid O- } \\
\text { methyltransferase }\end{array}$ & $\begin{array}{l}\text { methyl transferase, in the } \\
\text { corpora allata functions in } \\
\text { juvenile hormone } \\
\text { biosynthesis }\end{array}$ & & Q9VJK8 & $\begin{array}{c}1.38 \mathrm{E}- \\
32\end{array}$ \\
\hline SgreTa0014975 & $\begin{array}{l}\text { Cytochrome P450 } \\
\text { 302A1 (dib) }\end{array}$ & $\begin{array}{l}\text { ecdysone biosynthesis in } \\
\text { prothoracic glands and } \\
\text { other ecdysone producing } \\
\text { tissues }\end{array}$ & $x$ & Q9NGX9 & $\begin{array}{c}8.93 \mathrm{E}- \\
148\end{array}$ \\
\hline SgreTa0016782 & $\begin{array}{l}\text { Carbohydrate } \\
\text { sulfotransferase }\end{array}$ & $\begin{array}{c}\text { carbohydrate } \\
\text { biosynthetic process }\end{array}$ & & Q.9W070 & $\begin{array}{c}2.20 \mathrm{E}- \\
40\end{array}$ \\
\hline SgreTa0017764 & $\begin{array}{l}\text { Uncharacterized } \\
\text { Short-chain } \\
\text { dehydrogenase- } \\
\text { reductase }\end{array}$ & & & Q9VDC0 & $\begin{array}{c}1.23 \mathrm{E}- \\
46\end{array}$ \\
\hline SgreTb0017908 & $\begin{array}{l}\text { Niemann Pick } \\
\text { type C2 protein } \\
\text { homolog (Npc2) }\end{array}$ & $\begin{array}{l}\text { regulates sterol } \\
\text { homeostasis and by this } \\
\text { also ecdysteroid } \\
\text { biosynthesis }\end{array}$ & $x$ & Q9VQ62 & $\begin{array}{c}7.98 \mathrm{E}- \\
35\end{array}$ \\
\hline SgreTa0002115 & $\begin{array}{c}\text { Dopamine } \mathrm{N} \text { - } \\
\text { acetyltransferase }\end{array}$ & melatonin biosynthesis & & Q94521 & $\begin{array}{c}1.62 \mathrm{E}- \\
21\end{array}$ \\
\hline SgreTa0002227 & $\begin{array}{c}\text { Cytochrome P450 } \\
\text { 305A1 }\end{array}$ & $\begin{array}{l}\text { may be involved in the } \\
\text { metabolism of insect } \\
\text { hormones by sequence } \\
\text { similarity (Uniprot) }\end{array}$ & & Q9VW43 & $\begin{array}{c}4.39 \mathrm{E}- \\
107\end{array}$ \\
\hline SgreTa0007915 & $\begin{array}{l}\text { Juvenile hormone } \\
\text { acid O- } \\
\text { methyltransferase }\end{array}$ & $\begin{array}{c}\text { methyl transferase, in the } \\
\text { corpora allata functions in } \\
\text { juvenile hormone } \\
\text { biosynthesis }\end{array}$ & & Q9VJK8 & $\begin{array}{c}1.14 \mathrm{E}- \\
31\end{array}$ \\
\hline
\end{tabular}


Supplementary Table 18. Sequnces of Primers

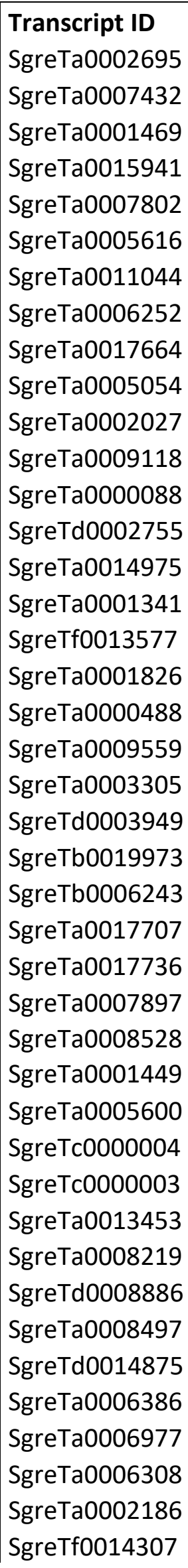

Forward primer (5'-3')

ATGCCTGGGTGTTGGATAAG AAGGTTCTTGCAGGATGGTG TCATCACTGGCATCTTCTCG AACACCGCTACAGGAAATGG ATGAGGGCTCTTTGACAACC GAAGGATTCGCTTACGAAGG TGTGAAGGGCCTAGGAAAAG TCCAACACAAAGAGGTGGTG GGACAGAAGACGACACACAGG TCGGCACACAGAAGTTCAAG ACCCGACATCCTCAAACTTC AGGTATCGCCAAGCACAAAG TGTGTCCATTGGATGTCACC GGTCCGGTATTTGGGAAAAC TGGATTCCATGTACCAGCAG GGATTCGATCTCAACGCAAG ACGATGCACCAGAACTACCC ATGCGTCCATACTTGTGGTG ACCTGTTCTGATGGCGAATC CCCTGAGATTTGGCTTGAAC AATGGCTCCAAGACAAGTGG TGAGAAGGCAGACGAACATC TCCAGTGATGACACACACACAG CCATGACTTCGCTTTGATCC TTACGTGCGATGTTCGTCAG ACTCCTCAACGATGCTTTCG TCAGGAACTGGGTATGCTTG AATTGCCAGGAGTGGATAGG GGAAAGATTGCTCTGGATGG AACTTCCTGCCAGTGGAGAC AAGGCCCAGTGTCTGTTTTC AGTGCTTTGCCTTGTTGGAC AAGGCTGCATTGTGGATACC CAAGTCGAGCAATTCTACGC GGAGCGGTGTTCAAAAAGAC GGAAACAGTGAGGCGAAAAC AGCCCGGACAACACTTCTAC GACCTCAGCAGCGATCATTC CTTGCAGATGCAGTCAATGG GTGCATCAAATGCTCACTCG ACTTTTGTGGACCCCTCATC CAAGATGCCGACTGTGAGTG
Reverse primer (5'-3') GGAGCATCTATGATGGTCACG AGCTCCACAAATCTGCCTTC TTTTCACCTCCACGGAGAAC TGCACCTTGAGGTTTGACAG ACAGCGCAGACTACGAAATG TATCGGGCTCTGGTACTTGC TCAGTTGCCTTCATCCAGTG TGCTGCAGTAAGCAACCAAC ACACGCAGGACAATGAGGAC TCCATCGAAGTCGTGCTTTC TTTGGCTGACTCCCAGAAAC GAGTTCTTATCTTGGGGTGCAG CACATGCTGCTGGATCATTC AACTGAGGTCTCGCACCTTG TGTCCTTTCAGCCACCTTTC AGGACAGCGTGTTGTTGTTG TTATTCCCTTCCCGTACAGC ATGAACAGCAGCTGGAAAGC GCCCCGTCTTCTTTTCTTG CTTCATTTCCTCGTGCCATC TCACTTGGAGATGCTGAAGG AGGGTCAGCAGTGCATTTTC CGAAATGAGGCGAGAGAAAC TAAGGCTGGTTGAGCACTTC AATGGCTGCATAGTCGAAGC GTTGCAATCCTTGCGATACC TGATCTGGAACAAGCCGTAG ATTGTAGGCCAGAGCCAAAC ATTCCAAGCTGACCACGAAG AGTGCAGCACATTCAGCTTG TTTCTCGGGGATGTACTTGG GTTCACGGAAACGATTGCAC TGGACGTGAACGATTGTAGC TCTCGGGGTTCCATAAGAAG GAAACAGCCGTGTTCCTTTC AGTTGTTCTGGGCATTAGCC CCATCATGAGCAGGAACCAG CACACGCAGGTACATATGAAGG TGGCAGTATCTTCCAGAATGG TGGACGCTAGCACTCTCTAATG AGTGGACCAGCCTTTCATAGAC GGCGGTAACAGAAACAAAGC 


\begin{tabular}{|l|l|l|}
\hline SgreTa0001661 & AGGATTGGTCCAGTTTCGTG & TCCATCTCGTCACATCTTCG \\
SgreTb0016047 & ACGTAATTGACAGCCACTCG & ATCGAGTCTTTGGTGGCATC \\
SgreTa0014626 & ATTTACGGCTTGGTCGTAGC & GATGCCGATAGCAAATCCTG \\
SgreTa0008504 & GAGAAATCATCCGGTTGGAG & AAGATGCTGCCCATGATACC \\
SgreTa0007477 & GAGCAGCATTTCCACAAGC & TCATGCGCTTCTCCTTCTG \\
\hline
\end{tabular}


4. Chapter II - Variation in a pleiotropic regulatory module drives evolution of head shape and eye size in Drosophila

The manuscript 'Variation in a pleiotropic regulatory module drives evolution of head shape and eye size in Drosophila.' is the main project of my PhD thesis.

My contributions for this manuscript includes the following parts:

- Conceptualization of project and experiments (together with Dr. Nico Ponsien)

- Bioinformatics analyses (RNA-seq and ATAC-seq)

- Planning and performing experiments (Experimental lab work was supported by Bilen A., Matas de las Heras C., Ayaz S., Niksic A.)

- Data interpretation (together with Dr. Nico Posnien)

- Writing of the first manuscript draft and editing (together with Dr. Nico Posnien)

- Visualization (together with Dr. Nico Posnien)

Contribution of other authors includes:

- Dr. Torres-Oliva, M. and Dr. Almudi, I. generated the transcriptomic dataset

- Prof. Casares, F. provided the analysis and figures for the lineage tracing experiment and the staining of pnr-expressing cells during pupal stages.

Status of the manuscript:

In preparation for submission 
Title

Variation in a pleiotropic regulatory module drives evolution of head shape and eye size in

\section{Drosophila.}

\section{Authors}

Buchberger E. ${ }^{1}$, Bilen A. ${ }^{1}$, Matas de las Heras $\mathrm{C} .^{1}$, Ayaz S. ${ }^{1}$, Niksic A. ${ }^{1}$, Almudi I. ${ }^{3}$, Torres-Oliva M. ${ }^{1,2}$, Casares F. ${ }^{3}$, Posnien N. ${ }^{1, *}$

1 Universität Göttingen, Department of Developmental Biology, Justus-von-Liebig-Weg 11, 37077 Göttingen, Germany

2 Institute of Clinical Molecular Biology, Christian-Albrechts-University of Kiel, University Hospital Schleswig-Holstein, Kiel, Germany

${ }^{3}$ CABD (CSIC/UPO/JA), DMC2 Unit, Pablo de Olavide University campus, Ctra. Utrera km1, 41013 Seville, Spain

* author for correspondence: nposnie@gwdg.de 


\subsection{Abstract}

Insect compound eyes are highly complex organs, which are composed of individual subunits, so called ommatidia. We have recently shown that closely related Drosophila species show remarkable differences in eye size and head shape. The eye size differences between $D$. melanogaster and D. mauritiana are the result of differences in the number of ommatidia. We use this model to identify the molecular changes underlying the observed morphological variation in adult structures and try to understand how gene regulatory networks (GRNs) in closely related species evolve.

A comparative developmental transcriptomic dataset combined with a transcription factor binding site analysis showed that the GATA factor Pannier (Pnr) regulates many genes that are differentially expressed between $D$. melanogaster and D. mauritiana and that the transcript of $p n r$ itself is differentially expressed in the two species during eye development. Additionally, we could show that $u$-shaped (ush), coding for a co-factor of Pnr, is transcribed and translated in the developing eye-antennal disc. We used the binary GAL4-UAS system and subsequent antibody staining to reveal that the two factors regulate each other. To test, if the regulatory module composed of Pnr and Ush may represent a flexible node in the eye and head developmental GRN, we overexpressed pnr and ush, respectively in the eye-antennal disc in $D$. melanogaster. We indeed were able to phenocopy aspects of the differences observed between $D$. melanogaster and D. mauritiana, showing that higher levels of Pnr lead to a bigger eye area, due to a higher number of ommatidia and a narrower, interstitial face cuticle. In summary, our data suggests that differences in the expression of pnr and ush might explain part of the variation observed between the head shapes of $D$. melanogaster and D. mauritiana. 


\subsection{Introduction}

The capacity of organisms to generate new forms is a key prerequisite for the adaptation to an ever-changing environment. One of the major goals in biological research is to understand the intrinsic and extrinsic forces shaping this morphological variability. Since the genome of an organism contains instructive information about its morphology, generally a first crucial step is the establishment of the genotype-phenotype map for a given morphological trait. The genetic architecture of relatively simple traits has been successfully determined at a high resolution. For instance, natural variation in body pigmentation in the vinegar fly Drosophila melanogaster or the beach mouse Peromyscus polionotus has been mapped to individual nucleotides affecting the expression of the underlying gene (Jeong et al., 2006) or protein function (Hoekstra et al., 2006), respectively. Also, the genetic basis of the gain or loss of structures like trichomes in Drosophila (Arif et al., 2013b; McGregor et al., 2007), pelvic spines in stickleback fish populations (Chan et al., 2010; Xie et al., 2019) or the repeated loss of eyes in cave fish (reviewed in (Krishnan and Rohner, 2016)) has been successfully revealed. However, the genetic changes underlying the evolution of complex traits, such as the size and shape of organs remain largely elusive to date. This is in part due to the polygenic nature of complex quantitative traits. This means that the final observable variation is influenced by many genetic changes with small effect sizes, which are spread throughout various genomic locations, significantly hampering their detection (Boyle et al., 2017; Mackay, 2001). Additionally, quantitative traits are highly context dependent, i.e. time and tissue specific and often influenced by environmental factors like temperature or food availability (e.g. (Casasa and Moczek, 2018; Siomava et al., 2016)). Despite these difficulties, the genetic basis of variation in complex traits has started to be elucidated in recent years. For instance, mandible and craniofacial shape differences between mouse strains are influenced by loci located on most of the chromosomes (Boell et al., 2011; Boell and Tautz, 2011; Burgio et al., 2009; Maga et al., 2015; Pallares et al., 2014). Several studies in Drosophila revealed, that loci on several chromosomes underly differences in eye size and head shape (Arif et al., 2013a; Gaspar et al., 2019; Norry and Gomez, 2017). These examples confirm that the genetic architecture of such traits is rather complex and individual causative molecular changes are difficult to determine.

While the genetic architecture of morphological trait variation is being revealed, a mechanistic understanding of the impact of the genomic changes is still missing to date. For 
instance, the size and shape of an organ is often exposed to selection pressures at the adult stage when it is functional. However, its appearance is defined during embryonic and postembryonic development. Therefore, it is conceivable that genetic variation underlying complex trait diversity has a direct impact on its development. The development of an organism and its organs relies on the correct activation and repression of developmental genes which is orchestrated by a complex interplay between gene products in developmental gene regulatory networks (GRNs). These GRNs must be tightly controlled because changes at any node of this network will eventually influence the interaction with its downstream target genes. A balance between a constraint network architecture and flexibility is thus important for allowing changes in size and shape of a certain organ to occur throughout evolution, but at the same time keeping the resulting adult organ fully functional. For the gain or loss of simple morphological traits, a few studies so far have established a clear link between causative genetic variation and GRN architecture. For instance, genetic variation that changes the expression of the zinc finger transcription factor Shavenbaby (Svb) is associated with the presence of trichomes in Drosophila larvae (McGregor et al., 2007), while natural variation in adult trichome patterns is explained by genetic variants affecting the expression of the micro RNA miR-92a (Arif et al., 2013b). A thorough analysis of the GRNs governing larval and adult trichome development, revealed fundamental differences in the interplay of key developmental regulators (Kittelmann et al., 2018). This data strongly suggests that the GRN architecture poses constraints on the nodes within the network that change during evolution. Due to the polygenic nature of complex morphological traits, the link between genetic variation and GRN architecture is more complicated to establish.

A typical approach to address this gap could be to first identify genetic variants associated with morphological diversity and place the candidates into the GRN context in a second step. As an alternative, we propose here to first identify putative flexible nodes within the GRN governing the development of a variable morphological trait. We suggest that the data obtained from this first step can subsequently be used in follow-up studies to reveal the causative genetic variation associated with trait variation. To identify 'flexible nodes' in an otherwise constraint GRN, we studied genome wide patterns of developmental gene expression variation. We assume that flexible nodes can be identified by their effects on downstream target genes. As model we compared eye and head development in the two closely related Drosophila species D. melanogaster and D. mauritiana, which vary extensively 
in adult eye size and head shape (Posnien et al., 2012). It has recently been shown that $D$. mauritiana develops larger compound eyes due to a higher number of individual ommatidia especially in the dorsal eye. The bigger eyes of $D$. mauritiana are accompanied by a narrower interstitial head cuticle (Posnien et al., 2012), recapitulating the common origin of eye and head cuticle tissue from the same eye-antennal imaginal disc during larval development (Haynie and Bryant, 1986). Since the GRN governing eye-antennal disc development is extensively studied and well understood in D. melanogaster (Kumar, 2009; Potier et al., 2014; Treisman, 2013), this process represents an excellent model to link morphological diversification to developmental and genetic variation.

We applied RNA-seq at different developmental stages of eye-antennal discs in $D$. melanogaster and D. mauritiana. A systematic co-expression and transcription factor enrichment analysis revealed that many differentially expressed genes were regulated by the GATA transcription factor Pannier (Pnr). Our results suggest that Pnr plays a dual role in the underlying GRN since it activates and represses its target genes. The repressive role is most likely mediated by its co-factor U-shaped (Ush) which is, in contrast to previous reports, coexpressed with Pnr during eye-antennal disc development. We applied functional genetics approaches to establish that Ush and Pnr interact genetically during eye-antennal disc development and are thus involved in the same regulatory module. Finally, we show quantitative expression differences of pnr and ush between D. melanogaster and D. mauritiana and that the overexpression of pnr in D. melanogaster phenocopies aspects of the $D$. mauritiana like head shape and eye size. Our data confirms a role of Pnr in morphological differences observed between D. melanogaster and D. mauritiana and therefore suggest that Pnr might be one flexible node in the conserved eye-antennal GRN.

\subsection{Results}

\subsubsection{Drosophila melanogaster and D. mauritiana exhibit differences in dorsal head shape}

Eye size and head shape vary extensively between Drosophila melanogaster and D. mauritiana with the latter having bigger eyes due to more ommatidia at the expense of interstitial face cuticle (Arif et al., 2013a; Hilbrant et al., 2014; Posnien et al., 2012). Since eye size differences are most pronounced in the dorsal part (Posnien et al., 2012), we proposed that the shape of the dorsal interstitial cuticle may vary as well. To test this hypothesis, we 
comprehensively quantified differences in the dorsal head morphology among the two sister species.

We placed 57 landmarks on pictures of dorsal heads (Figure 17A) covering the main dorsal cuticle regions (Figure 17A, (Haynie and Bryant, 1986)) and we applied a geometric morphometrics analysis. A discriminate function analysis clearly distinguished the head shapes of D. melanogaster and D. mauritiana (Figure 17B). In accordance with previous data (Posnien et al., 2012), we found main differences in dorsal eye size with the eye area protruding more towards the back of the head in D. mauritiana (Figure 17B). The posterior expansion of the eye area in $D$. mauritiana was accompanied by a narrower dorsal head region, which affected both the orbital cuticle (OC) and the dorsal frons (DF) region (compare to Figure 17A). The ocellar complex was slightly shifted ventrally. In D. melanogaster, the eye area was clearly smaller, whereas both dorsal head regions (OC and DF) were larger and the ocellar complex was shifted dorsally (Figure 17B).

In summary, we found that $D$. melanogaster and $D$. mauritiana do not only differ in the size of the dorsal eye area, but also exhibit variation in the relative contribution of different head regions to the dorsal head capsule. 

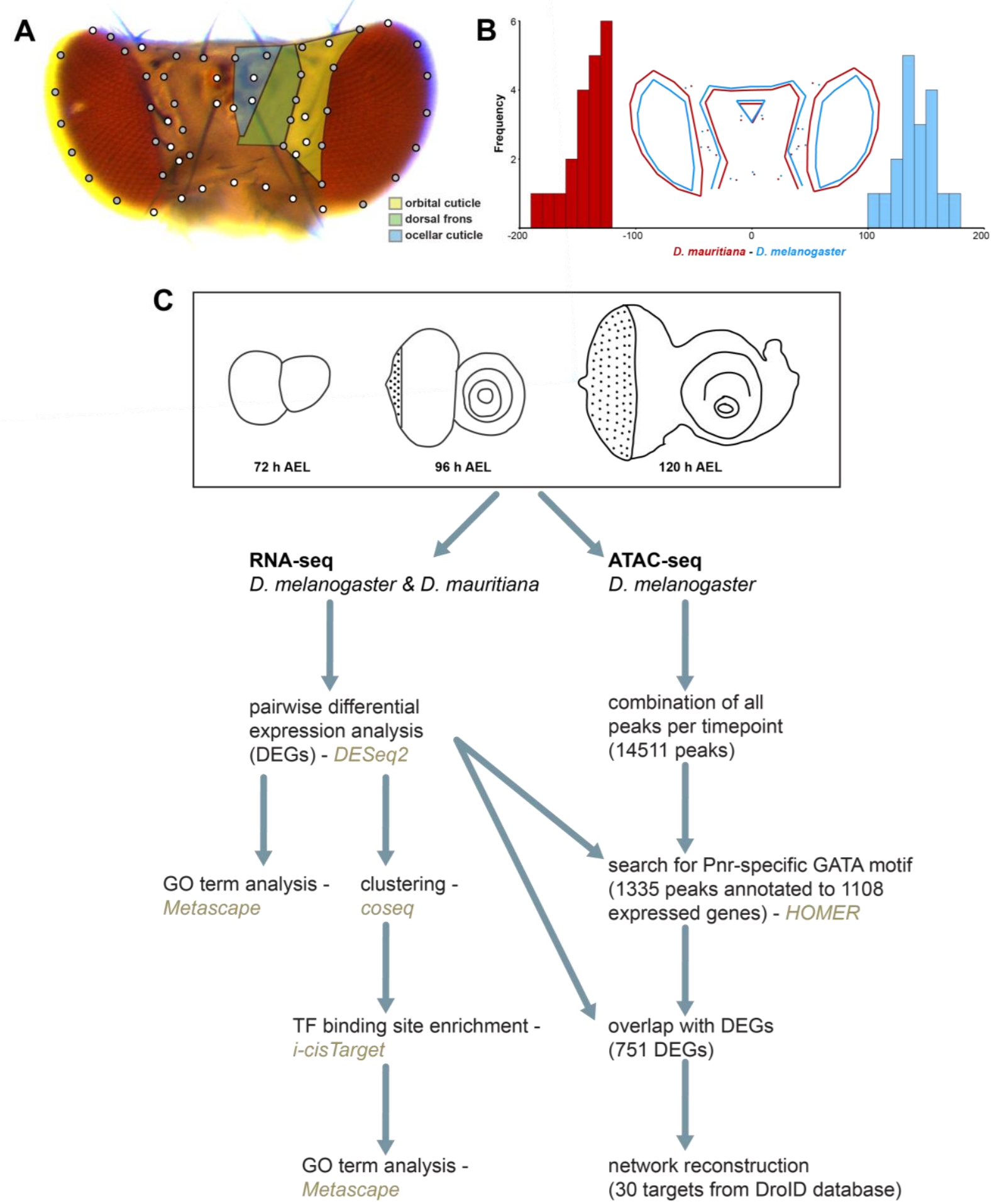

Figure 17. A. Dorsal view of a Drosophila head and schematic representation of the dorsal head structures in Drosophila. The dorsal Drosophila head cuticle consists of three morphologically distinguishable regions, namely the orbital cuticle next to the compound eye (yellow), the dorsal frons (green) and the ocellar cuticle (blue). The dots show the 57 landmarks that were used to analyze head shape, where the white landmarks represent fixed landmarks and the grey ones represent sliding landmarks. B. Mean head shape of D. melanogaster (blue) and $D$. mauritiana (red) after discriminate function analysis, which clearly distinguished the two groups based on their dorsal head shapes. C. Experimental setup of the bioinformatics analysis. Arrows point to each step in the pipeline; Left side: Transcriptomic datasets were generated for developing eye-antennal discs in both species at three developmental stages, namely 72h AEL (after egg laying; late L2), 96h AEL (mid L3) and 120h AEL (late L3). The scheme shows the workflow from data generation to, differential expression analysis to clustering of the 
differentially expressed genes. Right side: An ATAC-seq dataset was generated for developing eye-antennal discs in D. melanogaster at the same three stages. We defined a list of potential Pnr target genes, using motif search in open chromatin sequences and combined this approach with data from the DrolD database, to reconstruct the close network around the GATA-factor.

\subsubsection{Difference in the transcriptomics landscape recapitulate observed morphological}

\section{differences between D. melanogaster and D. mauritiana}

To reveal the molecular basis of the size and shape differences in dorsal head structures, we obtained comparative transcriptomes for three stages of eye-antennal discs development. The stages represented the onset of differentiation ( $72 \mathrm{~h} \mathrm{AEL}$ ), the progression ( $96 \mathrm{~h} \mathrm{AEL}$ ) and termination of differentiation (120 h AEL), respectively (Figure 17C) (Torres-Oliva et al., 2018).

A global analysis of the expression data showed that $72 \%$ of variation in the dataset was due to differences between $72 \mathrm{~h}$ and $96 \mathrm{~h}$ AEL (Supplementary Figure 8). This observation was confirmed by a pairwise differential expression analysis to determine the number of genes that were differentially expressed between D. melanogaster and D. mauritiana for each developmental stage. At $72 \mathrm{~h} \mathrm{AEL}$ we found the highest number of differentially expressed genes (DEGs), namely 6,683. This number decreased in later stages with 3,260 and 2,380 DEGs at $96 \mathrm{~h} \mathrm{AEL}$ and $120 \mathrm{~h} \mathrm{AEL}$, respectively (Supplementary Figure 9A). We did not find a biased expression difference between species since we observed a more or less equal number of DEGs with higher expression in D. melanogaster and D. mauritiana, respectively (Supplementary Figure 9A). To test whether the DEGs may be enriched for genes with specific cellular or molecular functions, we performed a gene ontology (GO) enrichment analysis. Indeed, we saw that stage specific DEGs are enriched in GO categories that can recapitulate the cellular events that happen at each respective stage. At 72h AEL we found DEGs upregulated in D. mauritiana and enriched in establishment and maintenance of cell polarity, a process which is highly important for overall disc growth and the mirror arrangement of the future ommatidia (e.g. (Jenny, 2010)). Also, DEGs were enriched in signal transduction pathways, for instance protein kinase A signalling (e.g. (Chanut and Heberlein, n.d.; Domínguez, 1999; Pan and Rubin, 1995; Strutt et al., 1995)), Inositol phosphate metabolism (e.g. (Seeds et al., 2015; Tsui and York, 2010)), and TORC signalling (e.g. (Wang and Huang, 2009)), all of which have shown to be involved in Drosophila eye development. In D. melanogaster, genes were predominantly enriched in cell cycle processes, consistent with the proliferation going on during this early stage (Casares and Almudi, 2016; Kenyon et al., 2003) (Supplementary Figure 9B). At 96h AEL differentiation events with more specific functions related to neural and photoreceptor 
development were captured using GO enrichment analysis, like R7 cell development and neural-related GO terms, reflecting the onset of the morphogenetic furrow at this time point, leaving behind differentiated ommatidia (Bonini and Choi, 1995; Heberlein and Moses, 1995; Treisman and Heberlein, 1998) (Supplementary Figure 9C). At 120h AEL we found, among many genes involved in metabolic pathways, differences and genes important for cuticle development. Overall, we were able to recapitulate the differences we observe in the adult flies already in the developing larval tissue (Supplementary Figure 9D).

Overall, we found a substantial number of DEGs between D. mauritiana and D. melanogaster during eye-antennal disc development, suggesting that we were able to recapitulate the differences we observe in the adult flies already in the developing larval tissue. The observation that these DEGs are involved in crucial developmental processes and molecular pathways suggests that various developmental mechanisms may contribute to morphological diversification between species. Also, this vast range of processes clearly reflects the development of various head regions and sensory organs from one single tissue.

\subsubsection{Central transcription factors regulate differentially expressed genes}

Since genes involved in central developmental processes are differentially expressed between $D$. melanogaster and D. mauritiana, we hypothesized that also key transcriptional regulators may be involved in their differential regulation. To get a global overview of differential gene expression dynamics across both species and time points, we clustered all genes that were differentially expressed in at least one stage according to their expression dynamics. This analysis resulted in 15 unique clusters based on 8,350 genes. Each cluster thus contained genes that share expression profiles across species and developmental stages. A gene ontology $(\mathrm{GO})$ enrichment analysis supported the specificity of the clustering approach (Supplementary Figure 10).

Assuming that co-expressed genes could be regulated by the same transcription factors, we identified putative shared transcription factor binding sites enriched in the regulatory regions of genes present in each expression cluster (see Materials and Methods for details). The unique expression dynamics of each cluster was recapitulated by a specific set of transcription factors involved in the regulation of genes in each cluster (Supplementary Figure 10). Among the enriched motifs, we found binding sites for transcription factors which have previously been described to be involved in eye-antennal disc development. For instance, in 
cluster 6 and 7 we found motifs for Lola that regulates ocelli (Mishra et al., 2016), photoreceptor and cone cell development (Zheng and Carthew, 2008). In cluster 10 we found motifs for Blimp-1, a transcriptional repressor associated with Ecdysone signalling (Neto et al., 2017), that has been shown to control the progression of the morphogenetic furrow and thus differentiation in the eye-antennal disc (Brennan et al., 1998). Intriguingly, genes in the same cluster were enriched for Ecdysone receptor (EcR) motifs, further supporting the cooperation of Blimp-1 and Ecdysone signalling (Agawa et al., 2007; Akagi and Ueda, 2011). Transcription factors that have been shown to be involved in photoreceptor development were enriched in cluster 11. For instance, Nejire (Nej) is involved in determination of photoreceptor cell fate (Kumar et al., 2004) and Jun-related antigen (Jra), a member of the c-Jun N-terminal kinase (JNK) pathway, is involved in establishment cell polarity and R3/R4 photoreceptor development (Ciapponi, 2001; Mlodzik, 2002; Weber et al., 2000). In cluster 15 we found an enrichment for the binding sites of Tramtrack (Ttk), a transcriptional repressor that negatively influences the Epidermal growth factor receptor (EGFR) signalling pathway in the eye-antennal disc (Kumar and Moses, 2001). Additionally, Ttk is involved in cone cell (Shi and Noll, 2009) photoreceptor development (Xiong and Montell, 1993). A strong enrichment of GATA motifs was observed in clusters 2, 3, 5 and 8. Motifs of the GATA transcription factor Pnr, that is playing a role in the establishment of the early dorsal ventral axis of the eye and later dorsal head development (Maurel-Zaffran and Treisman, 2000; Singh and Choi, 2003) were enriched in all four clusters with the strongest enrichment in cluster 8.

Intriguingly, 12 of the 20 identified transcription factors (60\%) were also differentially expressed between D. melanogaster and D. mauritiana (labelled transcription factors in Supplementary Figure 10), suggesting that variation in expression of these central regulators had a major impact on the transcriptomics landscape of developing eye-antennal discs among species.

In summary, we could show that interspecific variation in expression of central transcription factors very likely drive the differential expression of a high number of target genes which control important developmental processes during eye-antennal disc development. 
4.3.4. Pannier regulates genes that are differentially expressed between D. melanogaster and

D. mauritiana

Pnr is an interesting candidate transcription factor that may be involved in the development of differences in dorsal head morphology as well as eye size observed between D. melanogaster and D. mauritiana for the following reasons: 1 . Our global clustering and motif enrichment analyses suggest that Pnr regulates many DEGs between both species. 2. pnr itself is differentially expressed between D. melanogaster and D. mauritiana. 3. Pnr is known to be expressed in the dorsal portion of the eye-antennal disc (Maurel-Zaffran and Treisman, 2000; and see below) and it determines the dorsal-ventral axis of the retinal field in the early L2 discs (Maurel-Zaffran and Treisman, 2000; Singh et al., 2005; Singh and Choi, 2003). Additionally, later during eye-antennal disc development, Pnr influences the ratio of retinal and head cuticle fate in the dorsal disc by repressing retinal determination genes (Oros et al., 2010). Therefore, we sought to validate and refine our global differential expression data focusing on Pnr.

First, we asked at what stage of eye-antennal disc development pnr was differentially expressed between species. Based on our transcriptomic dataset we found significantly higher expression in D. mauritiana at 120h AEL (Figure 18A). This trend was further confirmed by realtime qPCR (Supplementary Figure 11). 
A

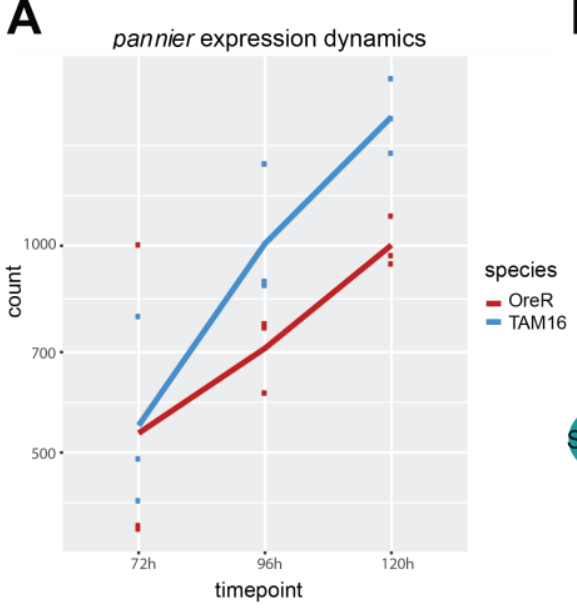

B

\section{CtBP L Doc2 Ssdp caup} ara Zfrps Chi - wg tou Bx Hand 三 direct interaction (Pnr-gene)

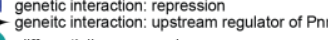
differentially expressed not differentially expressed

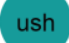

MESR4 ras

Atx2 chn glec

Sin3A RhoGEF64C pum Mef2

shn osa lap kibra

TMEM216

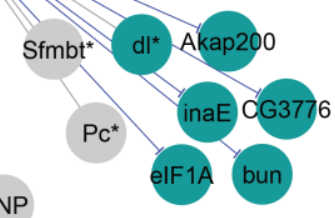

Dek stg XNP

Iswi NFAT TIk

C
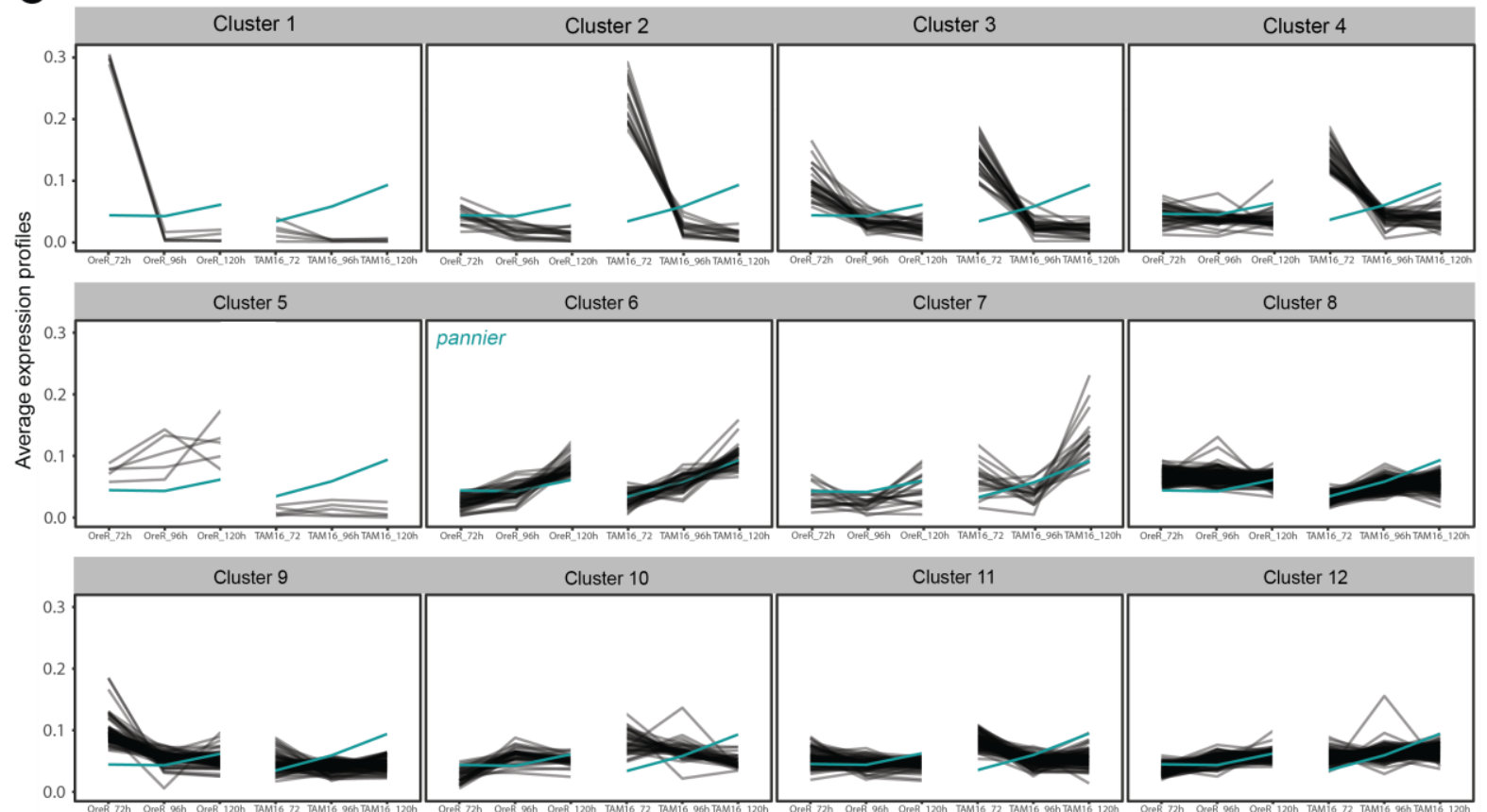

Figure 18. A. Expression dynamics of the pnr transcript at the three developmental stages in D. melanogaster (red) and D. mauritiana (blue). B. Network reconstruction of known interactions upstream and downstream targets of Pnr (DrolD (Yu et al., 2008)) that overlap with our Pnr target gene list. Cyan nodes represent target genes that are differentially expressed between $D$. melanogaster and $D$. mauritiana in at least one of the three studied developmental stages. Grey nodes represent predicted targets of Pnr based on our target gene list but are not differentially expressed. Black edges describe potential upstream regulators of Pnr based on DrolD. Red arrows point towards Pnr target genes that are annotated as being 'activated' by Pnr in DrolD, whereas blue edges point to genes where the interaction between Pnr and the gene is annotated as 'repressing'. Grey edges describe interactions that are annotated as direct TF-gene interactions in DrolD. C. Hierarchical clustering of read counts of predicted Pnr target genes (based on our target gene list) which were found to be differentially expressed in at least one developmental stage. The cyan line in each cluster represents pnr expression, which itself is a member of Cluster 6. Left side of each cluster: Expression dynamics of genes in D. melanogaster (OreR), Right side of each cluster: Expression dynamics of genes in D. mauritiana (TAM16).

Next, we wanted to define a list of putative direct Pnr target genes. This was crucial since the database used to infer motif enrichment was based on ChIP-Chip and ChIP-seq 
experiments that were not conducted in Drosophila eye-antennal discs (Herrmann et al., 2012; Imrichová et al., 2015). To obtain tissue and stage specific putative target genes, we assessed accessible chromatin regions by generating an ATAC-seq dataset for D. melanogaster eyeantennal discs covering the same three time points used for the transcriptomic dataset (Figure 17C). We found 14,511 unique peaks across all three timepoints. In the open chromatin regions, we revealed 1,335 Pnr-specific GATA motifs associated with 1108 genes expressed in our RNA-seq dataset (see Materials and Methods for details), suggesting that they were active during eye and head development. A cross validation of the putative Pnr target genes using the i-cisTarget tool confirmed an enrichment for Pnr, Nej, pMad and Mef2 binding sites (Supplementary Figure 12A). The identification of putative pMad target genes among Pnr targets may recapitulate the previous observation that both proteins interact physically during larval development (Kim et al., 2017). The putative Pnr target genes were highly enriched in GO terms like signal transduction, development, growth and cell cycle progression as well as in very specific terms such as compound eye development (Supplementary Figure 12B), recapitulating known functions of Pnr during eye-antennal disc development.

We further assessed the reliability of our target gene identification by searching for known target genes of Pnr. Among the putative target genes, we found Angiotensin-converting enzyme (Ance) (Supplementary Table 19), which is regulated by Pannier and pMad during Drosophila larval development (Kim et al., 2017). pnr itself is autoregulated in the wing imaginal disc (Fromental-Ramain et al., 2010, 2008). Accordingly, we found pnr as target gene as well (Figure 18C, Supplementary Table 19). We did not find $w g$ as putative target gene, which is consistent with the study of Pereira and collegues, who suggested that Pnr does not activate wg expression in the peripodial membrane (Pereira et al., 2006). Conserved GATA motifs, that were though shown to be not responsive to Pnr bining (Pereira et al., 2006) lie indeed between significantly called peaks of a highly accessible intergenic region between the $w g$ and $w g 6$ loci (Supplementary Figure 13). Overall, we were able to obtain a high confidence Pnr target gene list.

Our initial cluster analysis suggested that Pnr may regulate many genes that are differentially expressed between D. melanogaster and D. mauritiana. We could confirm this observation because $67.8 \%$ (751 of the 1,108) of the expressed target genes showed expression differences between D. melanogaster and D. mauritiana in at least one stage. 
In summary, we could show that pnr expression was significantly higher in D. mauritiana at $120 \mathrm{~h} \mathrm{AEL}$ and we identified a list of high confidence Pnr target genes which are mainly involved in signalling and developmental processes, cell cycle progression and growth. Most of the Pnr target genes were differentially expressed between D. melanogaster and D. mauritiana.

\subsubsection{Pnr activates and represses target genes in the eye-antennal disc}

To gain more detailed insights into the expression dynamics throughout eye-antennal disc development, we next clustered the differentially expressed Pnr target genes according to their expression profiles (Figure 18C). Among the 12 obtained clusters, we found pnr itself in cluster 6 . While the other genes in cluster 6 as well as genes in clusters 7, 8 and 12 showed a similar expression dynamics as pnr, we also found clusters in which the expression of the target genes showed the exact opposite trend. For instance, the Pnr target genes in cluster 3 were highly expressed at $72 \mathrm{~h} \mathrm{AEL}$ in D. mauritiana, while pnr itself showed a relatively low expression (Figure 18C). The expression of the same target genes decreased at $120 \mathrm{~h} \mathrm{AEL} \mathrm{with} \mathrm{pnr}$ expression increasing at the same time. This contrasting expression profile suggests that those target genes may be repressed by Pnr action. In contrast, genes in clusters that show the same dynamics as pnr may be positively regulated by Pnr. 

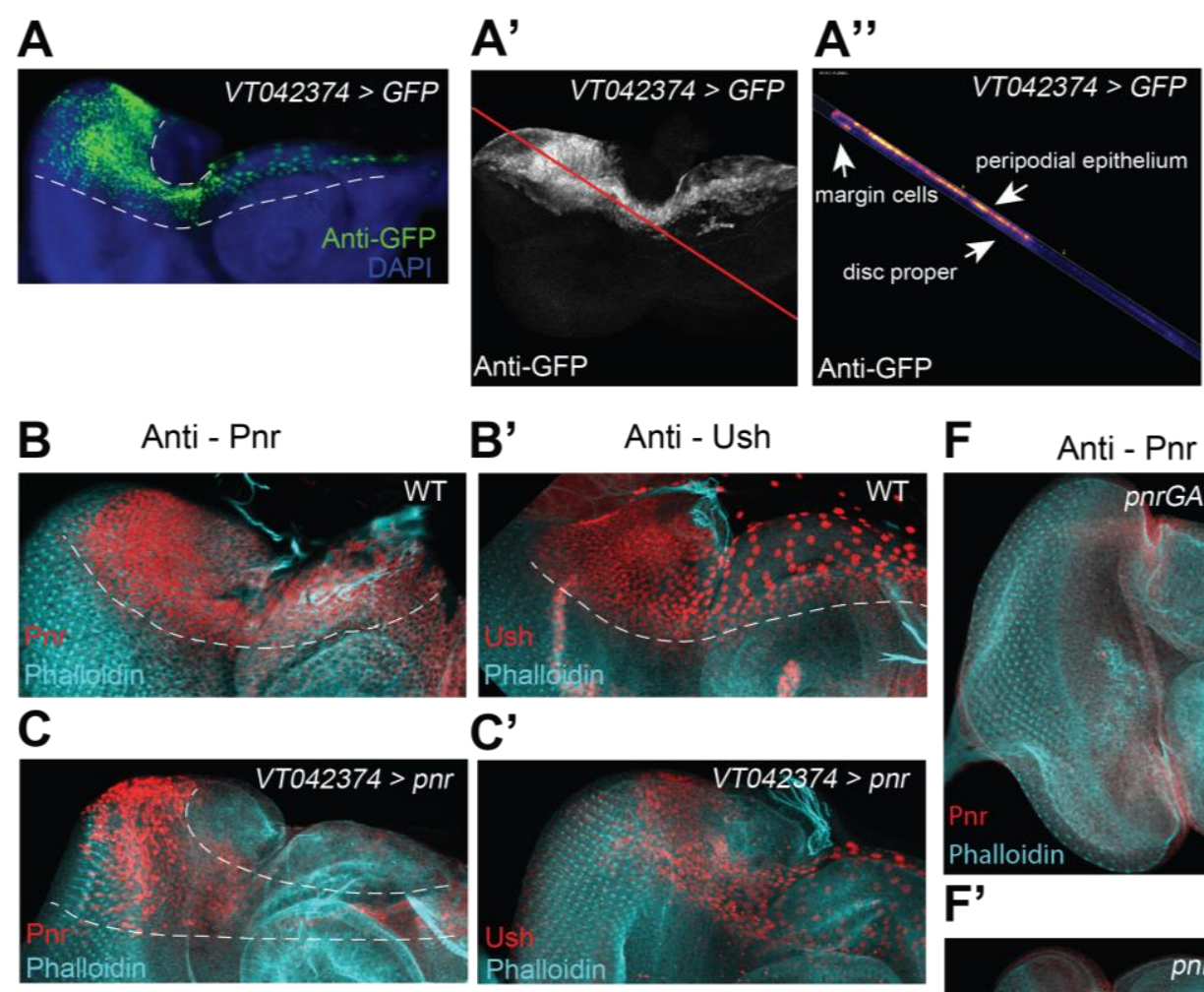

$C^{\prime}$
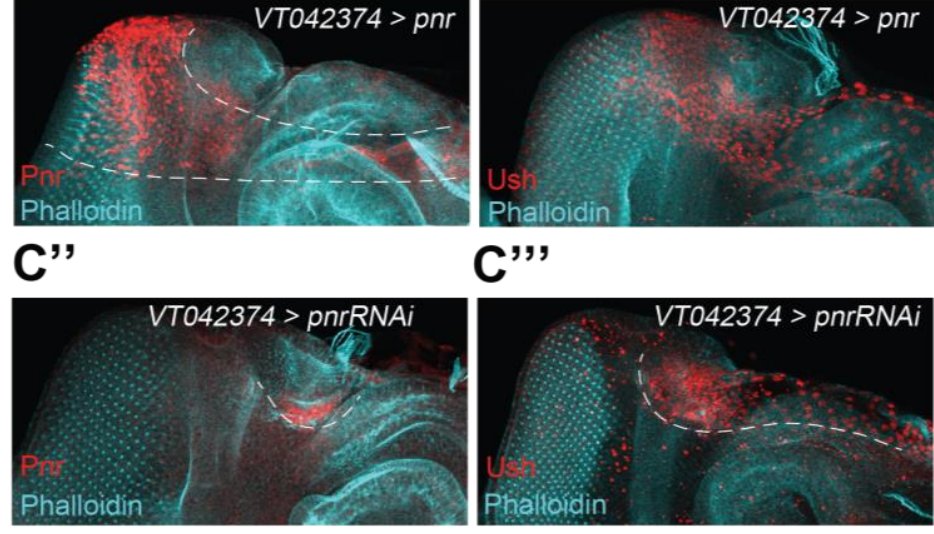

C"'

D
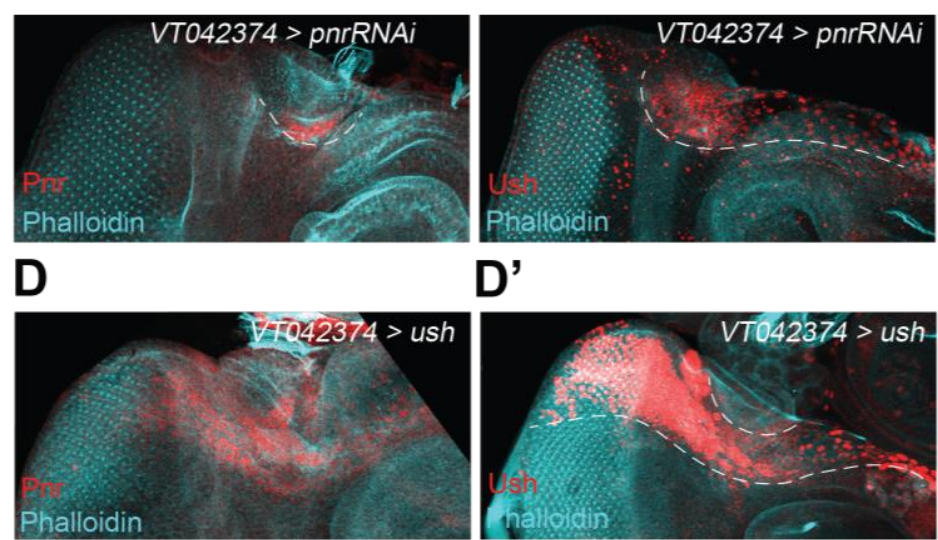

D'

F Anti - Pnr
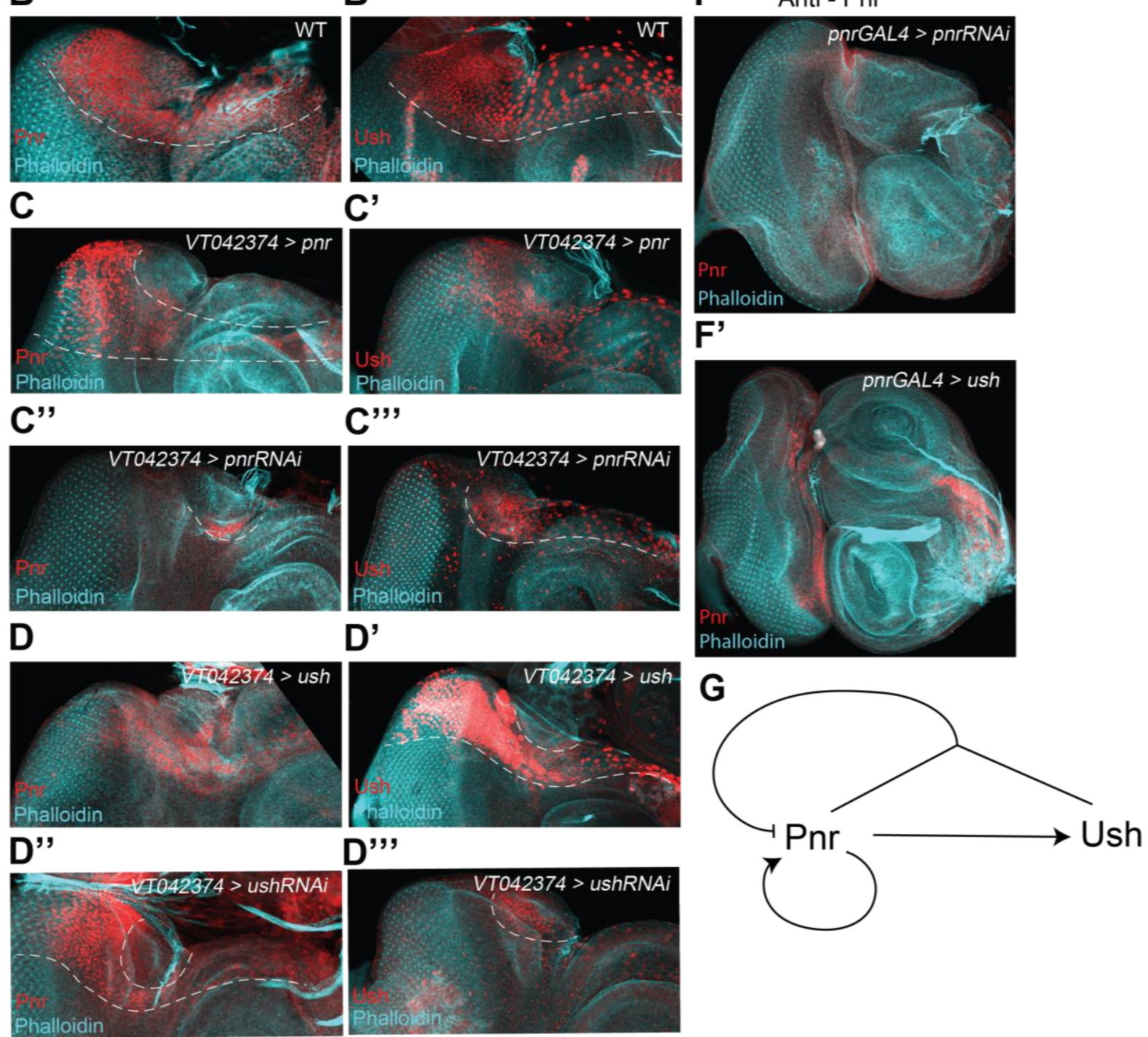

Figure 19 A. VT042374 drives expression in the dorsal part of the developing eye-antennal disc being partially reminiscent of the endogenous pnr expression. A'. Vertical section of VTO42374>GFP. VT042374 drives predominantly in the cells of the peripodial epithelium and in a few cells of the margin cells, which connect the peripodial epithelium with the disc proper. B. Pnr is localized in the dorsal part of the developing eye-antennal disc of $D$. melanogaster (detected with $\alpha$-Pnr antibody). White, dotted lines mark the area where antibody staining could be detected. Phalloidin (in cyan) was used to show the structures of the eye-antennal discs. B'. Ush was detected in the same dorsal region of the eye-antennal disc (detected with $\alpha$-Ush antibody). C - C'"': Overexpression and knock-down of pnr. C. Pnr localization after overexpression of pnr using the VT042374 driver line. C'. Ush localization after overexpression of pnr using the VT042374 driver line. C'. Pnr localization after knock-down of pnr using the VT042374 driver line and the pnrRNAi2 effector line. C'"'. Ush localization after knock-down of pnr using the VT042374 driver line and the pnrRNAi2 effector line. D-D"': Overexpression and 
knock-down of ush. D. Pnr localization after overexpression of ush using the VT042374 driver line. D'. Ush localization after overexpression of ush using the VT042374 driver line. D". Pnr localization after knock-down of ush using the VT042374 driver line. D'”. Ush localization after knock-down of ush using the VT042374 driver line. $\mathbf{F}, \mathbf{F}^{\prime}$. Overexpression of ush using pnrGAL4 $\left(\mathbf{F}^{\prime}\right)$ recapitulates knock-down of $p n r(\mathbf{F})$, resulting in duplication of the antennal part of the disc. Pnr is only detectable in a few remaining cells. G. Proposed model of how Pnr and its co-factor Ush interact in the developing eye-antennal disc.

To get a clearer picture of whether Pnr may indeed be involved in activation and repression of target genes, we integrated known interactions from the DrolD interaction database (Yu et al., 2008). We selected all known target genes of Pnr from this database and overlapped them with our list of putative Pnr target genes. We found three target genes in our list for which the direct interaction of Pnr and the target genes (i.e. Pnr-regulatory sequence interaction) was already shown ( $d l, P c$ and $S f m b t)$. Additionally, we found 25 of our high confidence target genes in the list of known genetic interactions (Figure 18B). The fact that we found GATA motifs in the putative regulatory regions of these genes, suggests that they might be direct Pnr target genes. Since the DrolD database contains the information, whether interactions are "suppressible" or "enhanceable", we tested if target genes of both categories were present in our dataset. Indeed, 14 of the 29 target genes showed "enhanceable" and 8 showed "suppressible" interactions with Pnr, respectively. 6 target genes showed both types of interactions. Intriguingly, 21 of the 30 putative Pnr target genes (68\%) found in the DrolD database were differentially expressed (Figure 18B).

The clustering analysis of differentially expressed target genes suggests that Pnr activates and represses its targets in the eye-antennal disc. An in-depth analysis of previously known interactions strongly supported that Pnr target genes are under positive as well as negative transcriptional control.

\subsubsection{Pannier and its co-repressor U-shaped participate in the same regulatory network during eye- and head development in Drosophila}

Our observation and previous reports of a dual regulatory role of Pnr during eyeantennal disc development may be mediated by the presence of a co-factor that modulates its regulatory role. In the developing wing imaginal disc, it has been shown that Pnr acquires a repressing mode of regulation upon heterodimerization with its co-factor U-shaped (Ush) (Fossett et al., 2001; Haenlin et al., 1997; Sorrentino et al., 2007). It has previously been stated that Ush is not expressed in the eye-antennal disc (Fossett et al., 2001; Maurel-Zaffran and Treisman, 2000). However, in our RNA-seq data we found the transcript of ush being expressed 
during eye-antennal disc development (Supplementary Figure 14A). Therefore, we hypothesized that Ush may act as a co-factor in this tissue.

A role of Ush as co-factor of Pnr requires both proteins to be present in the same cells of the eye-antennal disc. Since pnr expression in the eye-antennal disc has only been studied based on Gal4 driver lines, we first characterized the localization of Pnr using a newly generated antibody. We found that the protein is located, as previously reported, in the large nuclei of the dorsal peripodial epithelium (Figure 19B). Additionally, Pnr was detected in a few cell rows in the disc proper, most probably in a subset of the cuboidal margin cells (Supplementary Figure 15B-B" $)$. In later stages, the Pnr staining was less intense in the future ocellar complex region (Supplementary Figure 15AA"). Lineage tracing experiments showed that descendants of pnrpositive cells extend further ventrally in the peripodial epithelium (Supplementary Figure 15C$\left.C^{\prime \prime \prime}\right)$. Additionally, we observed descendants of pnr-positive cells in the dorsal disc margin as well as in the disc proper (Supplementary Figure 15D-D'”'). Using a newly generated antibody against Ush, we confirmed the presence of the Ush protein during eye-antennal disc development in D. melanogaster (Figure 19B'). As shown for Pnr, the Ush protein is localized in the nuclei of the peripodial epithelium in the dorsal part of the eye-antennal disc, spanning the antennal, the ocellar and parts of the future head cuticle regions (Figure 19B'). We also observed Ush expression in potential adjacent cuboidal margin cells (Supplementary Figure 16). Therefore, Ush and Pnr expression largely overlaps in the dorsal region of the eye-antennal disc (see also Supplementary Figure 14B), suggesting that they could indeed interact in the developing head. Please note that Ush is not only expressed during eye-antennal disc development, but also necessary for proper head development. Knockdown of ush in the dorsal developing eye-antennal disc consistently led to the loss of posterior vertical bristles (pVT (Chyb and Gompel, 2013)), and irregularities at the border of orbital cuticle and dorsal frons (Supplementary Figure 14C), while the upregulation of ush affected the overall head shape and loss or gain of the pVT and adjacent bristles (Supplementary Figure 14C'). The effect on bristle patterns is consistent with the reported role of Ush in bristle formation on the thorax (Cubadda et al., 1997; Haenlin et al., 1997).

The co-expression of Pnr and Ush suggested that both genes may interact genetically. To test this, we assessed the effect of gain- and loss of function of both genes on each other using the binary GAL4-UAS system in combination with Immunohistology. Since we aimed at 
modulating the expression of both genes within the endogenous domains, we used GAL4 driver lines, which drive expression in different dorsal regions of the developing eye-antennal disc. VT042374 activity was reminiscent of the pnr expression domain in the peripodial epithelium and in marginal cells except for a small region in the presumptive ocelli domain (Figure 19A). Regulatory elements of this line overlap with two open chromatin ATAC-seq peaks in an intronic region of the pnr locus (Supplementary Figure 17), suggesting that indeed partial endogenous pnr expression is reported. Additionally, we used the oc-GAL4 driver line that drove expression in the ocellar complex region that was not covered by the VT042374 line (Supplementary Figure 18 A-A").

Knock-down of pnr in the eye-antennal disc using VT042374 led to depletion of both, Pnr protein and Ush protein (Figure 19C'-C $C^{\prime \prime \prime}$ ). This finding showed on the one hand that the pnr knock-down worked efficiently and suggests on the other hand that Pnr is necessary for the expression of ush. Note that this result could also be observed using the oc-GAL4 driver line, where even though the discs show great deformation after pnr knockdown, the Ush protein was clearly detected only in a smaller region (Supplementary Figure 18D'). Pnr was upregulated upon overexpression using the VT042374 driver (Figure 19C) and the oc driver (Supplementary Figure 18C). While the effect on Ush was not obvious after pnr overexpression using the VT042374 driver (Figure 19C'), slight upregulation was observed when the oc driver was used (Supplementary Figure 18C').

The knockdown of ush using the VTO42374 driver line resulted in a complete loss of Ush protein in the expected region (Figure 19D'”'), confirming that the knock-down worked efficiently. Conversely, we observed upregulation of Pnr expression in the region where RNAi against ush was driven (Figure 19D"), suggesting that the presence of Ush results in pnr repression. Overexpression of ush using the VT042374 driver line resulted in a reduction of Pnr expression (Figure 19D). To confirm this observation, we made use of a previously reported double antenna phenotype upon loss of Pnr function (Oros et al., 2010), that we also found after pnr RNAi (Figure 19F). Intriguingly, overexpression of ush using a stronger pnr driver line (pnr-GAL4, (Fossett et al., 2001; Heitzler et al., 1996)) resulted in the same double antenna phenotype (Figure 19F'), supporting the observation that Ush is involved in repression of pnr expression. 
In summary, we could show that Ush and Pnr are spatially co-expressed during eyeantennal disc development. Our gain- and loss of function experiments showed that Ush is necessary for proper head development. Furthermore, we found evidence for genetic interactions between Ush and Pnr during eye-antennal disc development (Figure 19G), implying that both participate in the same regulatory network.

\subsubsection{Overexpression of pannier phenocopies aspects of the differences observed between D. melanogaster and D. mauritiana.}

The findings obtained so far strongly suggest that Pnr and Ush may contribute to the morphological differences observed between D. melanogaster and D. mauritiana adult heads: 1) pnr and ush showed higher expression in D. mauritiana during eye-antennal disc development. 2) Both genes are expressed in the dorsal region of the disc and they crossregulate each other. 3) Many target genes of Pnr are differentially expressed between both species. To test if changes in pnr expression indeed have the potential to explain naturally occurring differences in eye size and head shape we quantitatively analysed the shape of fly heads originating from gain- and loss of function experiments.

We crossed the VT042374 driver line to a UAS-pnr overexpression line to mimic higher pnr expression in D. melanogaster as observed in D. mauritiana. Additionally, we crossed the VT042374 line to two UAS-pnrRNAi lines. Overexpression of pnr led to a duplication of one of the posterior vertical bristles at the eye rim, while pnr RNAi resulted in a loss of bristles (Figure 20A). This observation is consistent with the reported role of Pnr in governing bristle pattern formation (Heitzler et al., 1996) and confirms the specificity of the performed gain- and loss of function experiments. 

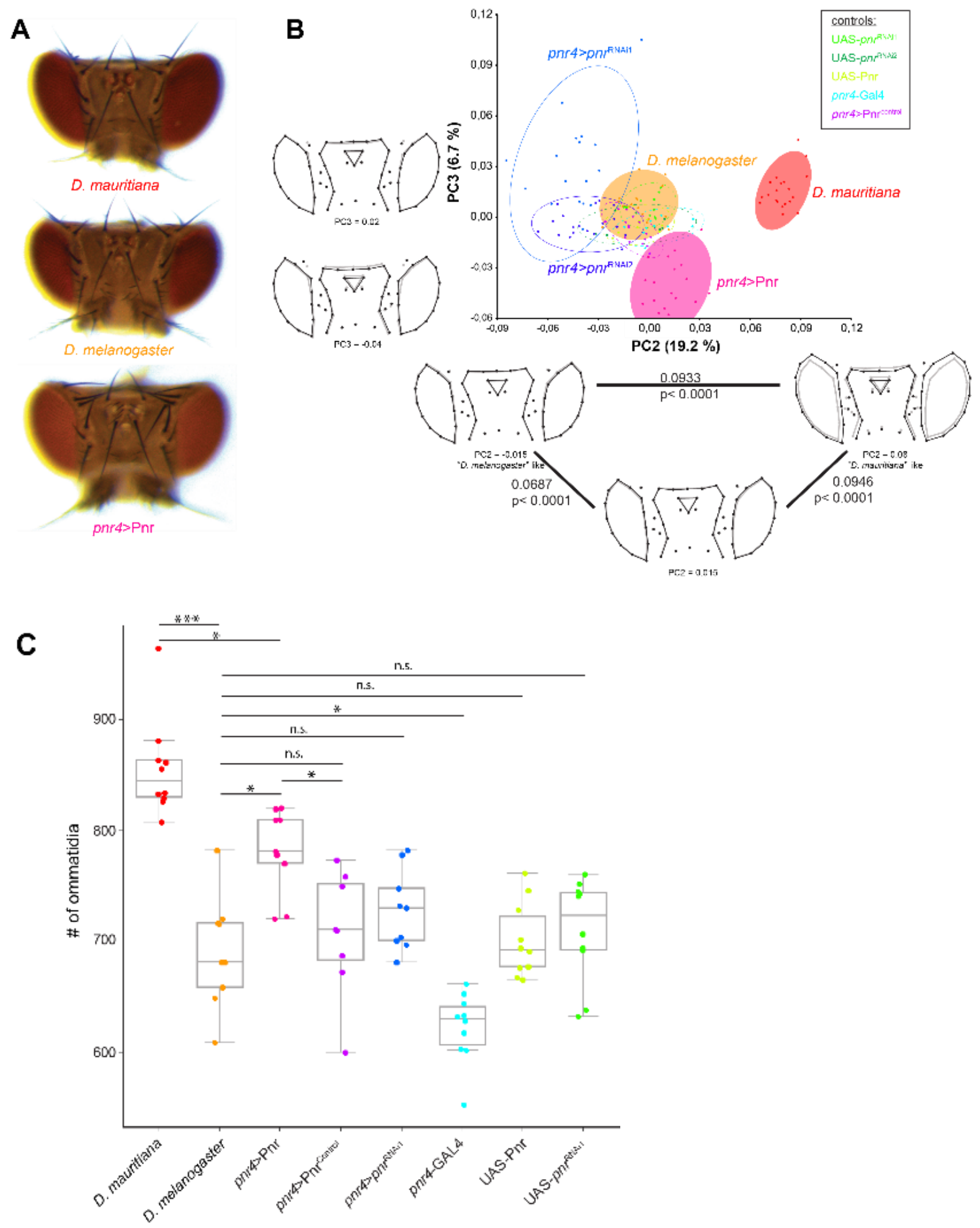

Figure 20. A. Dorsal view of heads of D. mauritiana (top), D. melanogaster (middle) and VT042374 ('pnr4') >pnr (pnr overexpression) flies. B. Principle component analysis of dorsal head shapes. Shown are PC2 against PC3. Red and orange clouds represent the 'WT-like' head shapes (D. melanogaster in orange and D. mauritiana in red). Overexpression of $p n r$ is represented in pink. The blue empty circles represent knock-down of pnr, with a weak effector line in dark blue and a strong effector RNAi line in light blue. The dotted lined circles represent head shapes of the parental UAS- and GAL4 fly lines, respectively, that were used to set up overexpression and knock-down of pnr. Extracting Procrustes distances between the groups showed that head shapes of $D$. melanogaster, D. mauritiana and flies upon pnr overexpression are all significantly different from each other. $\mathbf{C}$. 
Boxplot of ommatidia numbers in each of the lines (same color-code as in B.). Statistical comparisons represent pair-wise comparisons after Tukey HSD test: *** $p<0.0001$; ${ }^{*} p<0.05$.

Apart from extra setae at the rim of the eye, overexpression of pnr in the dorsal head region did not result in major morphological perturbations (Figure 20A). To quantitatively compare head shapes, we applied geometric morphometrics based on 57 landmarks placed on the dorsal head pictures. A principal component analysis showed that $40.9 \%$ of the observed variation in head shape could be assigned to technical artefacts related to the positioning of the heads (PC1, Supplementary Figure 19). Therefore, we excluded the first principal component (PC1) and analysed PC2 and PC3 in more detail. PC2 explained $19.2 \%$ of the observed variation in the head shape dataset and PC3 explained 6.7\% (Figure 20B). Variation along PC2 mainly captured differences in the proportion of eye vs. cuticle tissue in the dorsal head, as well as the location of the ocellar region. PC3 explained mostly differences in the dorsal-posterior head cuticle and the location of the ocellar region (Figure 20B). The overexpression of pnr in the dorsal head region resulted in a shift from a "D. melanogaster"like shape towards a more "D. mauritiana"-like shape along PC2. The shape analysis revealed an enlargement of the eyes in the dorsal head region that was accompanied by a slight reduction of the head cuticle between the two eyes (Figure 20B). Ommatidia counting in entire eyes confirmed that the increase in eye area upon pnr overexpression observed in our shape analysis was indeed due an increase in number of ommatidia (Figure 20C). Note that pnr RNAi influenced overall head shape (Figure 20B), but no impact on the number of ommatidia was observed (Figure 20C).

We also observed that the occipital region of the posterior head was more convex upon overexpression (Supplementary Figure 20A), whereas downregulation consistently led to an enlargement of these regions (Supplementary Figure 20B). To test, whether the occipital region also showed differences between $D$. melangaster and D. mauritiana, we performed a shape analysis with additional landmarks. Intriguingly, the occipital region was clearly convex in $D$. mauritiana and more concave in D. melanogaster (Supplementary Figure 20C-E). Detection of pnr expression in pupae stages (Supplementary Figure 20F-F") as well as the analysis of pnrexpressing clones in adult heads (Supplementary Figure 20G) confirmed that pnr is indeed expressed in the future occipital region. 
In summary, the upregulation of pnr expression in the developing eye-antennal disc led to larger eyes due to a higher number of ommatidia and a smaller dorsal head cuticle. Therefore, we were able to phenocopy aspects of the "D. mauritiana"-like head shape and eye size.

\subsection{Discussion}

While the genetic architecture of variation in complex morphological traits is being revealed these days (Arif et al., 2013a; Boell and Tautz, 2011; Gaspar et al., 2019; Norry and Gomez, 2017; Pallares et al., 2014; Ramaekers et al., 2018), a mechanistic understanding of how genetic variation affects trait evolution remains largely elusive to date. Here we addressed this gap by combining thorough quantitative phenotyping with comparative transcriptomics, GRN reconstruction and functional genetics to study natural interspecific variation in head shape and eye size between the two closely related Drosophila species D. melanogaster and D. mauritiana.

\subsubsection{A developmental model for natural variation in head shape and eye size}

Comparative morphology studies revealed that natural intra- and interspecific variation in head shape and eye size is pervasive among species of the D. melanogaster subgroup (Gaspar et al., 2019; Hilbrant et al., 2014; Norry et al., 2000; Posnien et al., 2012; Ramaekers et al., 2018). Previous shape analyses suggested that the eyes of D. mauritiana are predominantly larger in the dorsal region when compared to D. melanogaster (Posnien et al., 2012). Therefore, we restricted our geometric morphometrics analysis to the dorsal head region and found significant natural variation in dorsal head shape. We could confirm that increased eye size in D. mauritiana is due to a higher number of ommatidia and goes hand in hand with a reduction of the dorsal interstitial cuticle and a convex bending of the occipital head region. This tradeoff between eye size and head cuticle seems to be a common feature of Drosophila (Keesey et al., 2019; Norry et al., 2000). Previous attempts to disentangle the genetic architecture of eye and head cuticle size variation did not yet converge on a clear idea, whether the evolution of both structures is linked or not.

Morphological differences in adult traits are a result of variation in developmental processes (Carroll, 2005; Raff, 2000). Since GRNs that regulate such processes are extensively wired, the impact of variation in one node can be elucidated by extensive variation in gene expression (Thompson et al., 2015). Therefore, we applied comparative RNA-seq to reveal 'flexible nodes' in the GRN underlying head and eye development. In accordance with the 
previous observation of highly dynamic gene expression throughout eye-antennal disc development (Torres-Oliva et al., 2018), our comparative transcriptomics approach revealed stage-specific interspecific expression divergence. Intriguingly, many of the differentially expressed genes were enriched for binding sites of the GATA transcription factor Pnr that has previously been shown to be involved in dorsal head development (Maurel-Zaffran and Treisman, 2000; Oros et al., 2010). Our finding that pnr expression was higher in D. mauritiana suggests that natural variation in pnr expression may cause extensive remodelling of the transcriptional landscape downstream of this transcription factor.

We could establish a functional link between enhanced pnr expression and morphological differences, because overexpression in the dorsal eye-antennal disc of $D$. melanogaster phenocopied major aspects of D. mauritiana head shape and eye size. In particular, we observed an enlargement of the dorsal eye area due to increased ommatidia number as well as a reduction of the dorsal interstitial cuticle. Additionally, overexpression of pnr resulted in typical convex bending of the occipital region. In contrast, knockdown of pnr resulted in the opposite phenotype, characterized by reduction of the eye area, increase of the interstitial cuticle size and massive enlargement of the occipital region. The fact that the strength of the phenotype depended on the RNAi line used, strongly suggests that indeed quantitative differences in pnr expression seem to be relevant for phenotypic variation.

Our phenocopy experiment suggests that Pnr is involved in specifying the ratio between retinal tissue and head cuticle. Indeed, at least two major roles of Pnr during D. melanogaster eye-antennal disc development have been established. From the late second instar stage on, Pnr regulates the ratio of retinal cells vs. head cuticle cells by suppression of the eye fate in the dorsal region of the eye-antennal disc. This suppression is either accomplished by directly repressing members of retinal determination network as for instance teashirt (tsh) or indirectly via activation of wingless (wg) (Oros et al., 2010). Our results combining transcriptomics, ATACseq and transcription factor binding motif enrichment did not identify tsh as a direct target gene of Pnr, suggesting that the observed repression of tsh by Pnr (Oros et al., 2010) may be indirect. Interestingly, with eyeless (ey) and eyegone (eyg) we found two other members of the retinal determination network among the putative Pnr targets. Whether potential direct interactions are negative and may be linked to the repression of retinal fate in the dorsal disc remains to be determined. This later role in defining the dorsal cuticle vs. retinal fate is well in 
line with the observed trade-off between eye size and interstitial cuticle. Additionally, our tracing experiment revealed that pnr-expressing cells contribute to the dorsal occipital head region. Therefore, a direct effect on the morphological differences in this region is likely.

During early eye-antennal disc development, Pnr plays a pivotal role in defining the dorsal/ventral boundary and is therefore responsible for overall tissue growth (Maurel-Zaffran and Treisman, 2000; Singh et al., 2005; Singh and Choi, 2003). Our result that ey is among the putative direct Pnr target genes offers now an exciting and yet unpredicted early role of Pnr in ey activation in the peripodial epithelium and in margin cells. It has recently been shown that Ey activity in the peripodial epithelium and the margin cells is necessary for decapentaplegic $(d p p)$ induction and subsequent initiation of the morphogenetic furrow (Baker et al., 2018). Loss of Ey function also interferes with the placement of the dorsal/ventral boundary (Baker et al., 2018) providing a functional link to this well-established early role of Pnr. Throughout the third larval instar Pnr is predominantly expressed in the peripodial epithelium and our lineage tracing experiment showed that during earlier stages pnr must be expressed in cells that contribute to the dorsal posterior margin where the morphogenetic furrow is initiated. Therefore, Pnr is expressed in the right cells at the right time to act upstream of ey during dorsal/ventral boundary establishment and the initiation of the morphogenetic furrow, suggesting that differences in early pnr expression could have a direct effect on retinal development.

In summary, we provide a comprehensive developmental model suggesting that variation in expression of a pleiotropic central transcription factor is responsible for the concerted diversification of a complex morphological trait.

4.4.2. Pnr and Ush represent a functionally linked pleiotropic module in the GRN underlying head and eye development

Our developmental data showed that natural variation in pnr expression influences different developmental processes. Our combinatorial RNA-seq and ATAC-seq data revealed that more than 1,000 putative Pnr target genes expressed during eye-antennal disc development, further substantiating its central role during head development. Some of the target genes showed expression profiles in agreement with an activating role of Pnr, while some targets showed signatures of a negative relationship. This observation suggests that the dual regulatory role of Pnr observed in the wing disc (Fromental-Ramain et al., 2010, 2008) may be 
true for the eye-antennal disc as well. The repressive role of Pnr in the wing imaginal disc is realized upon heterodimerization with its co-factor Ush (Cubadda et al., 1997; García-García et al., 1999; Haenlin et al., 1997). However, it was thought that ush was not expressed in the developing eye-antennal disc (Fossett et al., 2001) or non-functional (Maurel-Zaffran and Treisman, 2000). Following this assumption, ush overexpression was in fact mainly used to mimic pnr knock-down (Fossett et al., 2001). Based on our RNA-seq data we show for the first time that ush is transcribed in the eye-antennal disc. Additionally, we confirm that ush transcripts are translated and that the protein is co-localized with Pnr in the squamous cells of the dorsal peripodial epithelium and in the cuboidal cells of the disc margin. Furthermore, ush expression is necessary for proper head development, since knock down in the dorsal part of the eye-antennal disc resulted in irregularities in adult dorsal head cuticle and head bristle pattern. The latter effect has been previously described for ush hypomorphs (Cubadda et al., 1997). Intriguingly, overexpression of Ush in the dorsal eye-antennal disc resulted in a double antenna phenotype reminiscent of that observed upon loss of Pnr function (Oros et al., 2010).

The co-expression as well as similar functions of pnr and ush strongly suggest that they interact during eye-antennal disc development. This hypothesis is further supported by a clear genetic interaction between both factors. We showed that Pnr is involved in ush activation. Since we did not find ush as a potential target gene of Pnr, the activation of ush may be indirect. Furthermore, we identified an autoregulatory loop of Pnr that seems to be negatively modulated by the presence of Ush. Since we found pnr in our list of putative Pnr target genes, we propose here that the expression level of pnr is kept in balance via activation by Pnr alone and repression by the Pnr-Ush heterodimer.

This model suggests that the various roles of Pnr during eye-antennal disc development could be facilitated by the stoichiometry between Pnr and its co-factor Ush. For instance, the early function of Pnr in dorsal/ventral boundary establishment and morphogenetic furrow initiation is most likely independent of Ush (i.e. mainly activating role of Pnr). This is supported by our observation that reduction of pnr expression via RNAi did not influence the final ommatidia number in the adult eyes. In the absence of Ush the reduced pnr expression can be compensated by an increased auto-activation to restore normal retinal development. Additionally, the effect of ush RNAi was mostly restricted to the dorsal head cuticle, suggesting that it might not play a major role during retina development. However, the later function in 
head cuticle development and sensory bristle formation most likely depends on the ratio of Pnr and Ush. This is supported by a similar expression profile of pnr and ush during third instar development. Additionally, it has been shown that sensory bristles in the thorax arise at regions with high pnr and low ush expression (Cubadda et al., 1997; Heitzler et al., 1996). Our overexpression of Pnr using the VT042374 driver line consistently resulted in duplication of the posterior vertical bristles, underpinning the role of Pnr in sensory bristle formation (Heitzler et al., 1996; Ramain et al., 1993). Interestingly, this is reminiscent of the phenotype described for a dominant $p n r^{D}$ allele (Heitzler et al., 1996), which is characterized by a loss of the ability to dimerize with Ush. Since Ush antagonizes bristle formation (Haenlin et al., 1997), the duplication of the posterior vertex bristles is most likely the result of overexpression in the posterior part of the dorsal peripodial epithelium where endogenous ush is not expressed anymore. In the anterior region, the endogenous ush expression is sufficient to block the development of additional sensory bristles. In contrast, overexpression of ush in most of the dorsal peripodial epithelium did not only result in the loss of the posterior vertical bristles, but also in the loss of the anterior vertical bristles, suggesting that extra Ush above a certain threshold completely antagonizes sensory bristle formation. Hence, the correct stoichiometry between Pnr and its co-factor Ush is crucial for proper dorsal head and sensory bristle formation. This notion is further supported by our observation that also ush to be slightly upregulated in D. mauritiana, recapitulating the expression dynamics of pnr.

In summary, we identified variation in expression of a highly pleiotropic regulatory module composed of Pnr and Ush that causes the differential expression of a plethora of potential target genes. Therefore, we conclude that this regulatory module might be a flexible node in the GRN underlying head and eye development in Drosophila.

\subsubsection{GRN rewiring facilitates natural variation in pleiotropic developmental factors}

Eye-antennal disc development is highly complex and the underlying GRN is extensively rewired both throughout time (Torres-Oliva et al., 2018) and in different parts of the disc (Potier et al., 2014). For instance, genes of the retinal determination network are required for the initial proliferation and growth of the entire eye-antennal disc and later they play a pivotal role in retinal specification (Baker et al., 2018; Bessa, 2002; Lopes and Casares, 2010; Neto et al., 2017). It has been suggested that the retinal determination genes are part of different GRNs during these events and extensive rewiring of the GRNs allows them to fulfil temporally 
restricted tasks (Palliyil et al., 2018). Similarly, the integration of gene products in spatially restricted GRNs may also explain why some genes are broadly expressed in the eye-antennal disc although they regulate different processes in different parts of the disc, which give rise to the various head structures (Palliyil et al., 2018; Potier et al., 2014). It seems therefore that rewiring of GRNs facilitates the use of the same developmental gene products in different contexts.

The various described roles for Pnr (summarized in (Oros et al., 2010)), its continuous expression in the eye-antennal disc and the observation that variation in pnr expression affects overall head shape and eye size simultaneously, strongly suggest that Pnr is involved in several GRNs during eye and head development. The interaction with co-factors, such as Ush provides a mechanism facilitating such network rewiring by modulating the role of Pnr from an activating to a repressing transcription factor. We conclude that the dynamic nature of GRNs may explain how interspecific variation in expression of a highly pleiotropic and central transcription factor such as Pnr can result in extensive remodelling of the transcriptomic landscape in an otherwise tightly controlled GRN.

Intriguingly, Pnr is not the only central and pleiotropic factor implicated in natural variation in head shape and eye size. It has recently been shown that a single point mutation in the cisregulatory sequence of ey, one of the most upstream factors of the retinal determination network (Callaerts et al., 1997), leads to heterochronic changes in its regulation and subsequent variation in eye size among $D$. melanogaster laboratory strains. This polymorphism segregates in natural D. melanogaster populations and it shows signatures of longitudinal cline in Europe, suggesting that it may provide some selective advantage in certain environmental conditions (Ramaekers et al., 2018). In summary, we hypothesize that the modularity of regulatory interactions during development may allow selection to act on highly pleiotropic developmental factors to drive diversification of complex morphological traits.

\subsubsection{Evolution of GRNs and implications for convergent evolution of head shape and eye} size

A trade-off between the size of the compound eye and other head structures is common in Drosophila (Keesey et al., 2019; Norry et al., 2000). Depending on the environment, enlargement or reduction of the eye is most probably selected, since smaller eyes and less ommatidia lead indeed to poorer temporal acuity (Currea et al., 2018; Ramaekers et al., 2018) 
and has ecological implications (Currea et al., 2018). This assumption is also supported by the fact that an enlargement of the compound eye is associated with increased optic lobe size (Keesey et al., 2019). However, functional sensory systems consume tremendous amounts of energy (Niven and Laughlin, 2008; Tan et al., 2005) suggesting that their size must be tightly controlled. It has been proposed that the common origin of the adult visual (i.e. compound eyes) and olfactory (i.e. antennae) system from the same imaginal disc provides an opportunity to balance the energy investment either in olfactory or in visual structures (Keesey et al., 2019). Although D. mauritiana was not included in this large-scale survey, it is likely that the resource allocation hypothesis applies to this species as well. However, it remains to be studied how temporal acuity and the size of visual neuropils coevolved with head shape variation between D. melanogaster and D. mauritiana.

In the light of a pervasive trade-off between eye and head cuticle in Drosophila it is tempting to ask whether this morphological trait evolves through the same or different nodes of the underlying GRN among different populations or species. Between D. melanogaster and D. simulans different QTL regions were identified for eye size and the width of the interstitial cuticle. This observation was supported by quantitative developmental data showing that the anlagen for the head cuticle start to diverge in size prior to the retinal tissue (Arif et al., 2013a). Therefore, the trade-off seems to be regulated by independent factors in these two species. However, recent quantitative genetics analyses identified some loci that affect eye size and head cuticle in opposite directions in intraspecific comparisons in D. melanogaster and $D$. simulans (Gaspar et al., 2019; Norry and Gomez, 2017). Additionally, our finding that variation in pnr expression influences both traits simultaneously further suggests that they may be genetically linked in D. melanogaster and D. mauritiana. Therefore, a convergent evolution of the trade-off in Drosophila is likely. A detailed analysis of the morphological basis of eye size differences showed that bigger eyes can be the result of differences in ommatidia number (e.g. between $D$. melanogaster and D. mauritiana) or ommatidia size (e.g. between $D$. simulans and D. mauritiana) (Posnien et al., 2012). Since these two features are regulated at different timepoints and developmental processes (reviewed in (Amore and Casares, 2010; Domínguez and Casares, 2005)) it is conceivable that the molecular and developmental basis of eye size differences varies in different groups. In summary, our current knowledge based on quantitative genetics, developmental as well as morphological data suggests that different 
nodes within the GRN underlying head and eye development may evolve to give rise to variation in head morphology in Drosophila.

\subsubsection{Conclusion and Outlook}

We provide here a methodological framework to reveal flexible nodes within GRNs and to subsequently validate these findings. Our comparative transcriptomics approach can be used as entry point to study the evolution of complex morphological traits or it can be applied to link already identified genetic variation to nodes within developmental GRNs and to developmental processes. It is important to note, however, that this approach unfolds its full potential if complemented with quantitative genetics data that allows identifying exact genetic variants associated with trait variation. The fact that we were not able to phenocopy the $D$. mauritiana head shape and eye size entirely, suggests that multiple genomic loci are responsible for the observed morphological divergence between D. melanogaster and D. mauritiana. Furthermore, it remains to be established, whether the pnr and/or ush loci contain genetic variants associated with eye size and head shape differences. Quantitative genetics approaches are not applicable since interspecific crosses between $D$. melanogaster and D. mauritiana result in infertile F1 females. However, reciprocal hemizygosity tests (Stern, 2014) for Pnr, Ush and putative regulators of these two factors represent a powerful tool to further dissect the causative genetic variants in the future. Overall, much more genetic as well as developmental data from different groups is necessary to draw a full picture of this exciting morphological phenomenon. Eventually, it remains to be established, whether similar functional requirements and ecological forces are involved in shaping the Drosophila head morphology.

\subsection{Material and Methods}

\subsubsection{Generation of the transcriptomic dataset}

Flies from the following strains were raised at $25^{\circ} \mathrm{C}$ at a $12: 12$ dark:light cycle for at least two generations and their eggs were collected on agar plates for one hour: D. melanogaster (OreR), D. mauritiana (TAM16). 30 L1 larva were collected in vials and developing eye-antennal discs were dissected at 72h AEL (120-130 discs; m and f), 96h AEL (80-90 discs; f) or 120h AEL (40-50 discs; f) and stored in RNALater (Quiage, Venlo, Netherlands). For each species and stage 3 biological replicates were generated. Total RNA was isolated using the Trizol (Invitrogen, Thermo Fisher Scientific, Waltham, Massachusetts, USA) method according to the manufacturer's recommendations and the 
samples were DNAsel (Sigma, St. Louis, Missouri, USA) treated in order to remove DNA contamination. RNA quality was determined using the Agilent 2100 Bioanalyzer (Agilent Technologies, Santa Clara, CA, USA) microfluidic electrophoresis. Only samples with comparable RNA integrity numbers were selected for sequencing. Library preparation for RNAseq was performed using the TruSeq RNA Sample Preparation Kit (Illumina, catalog ID RS-1222002) starting from 500 ng of total RNA. Accurate quantification of cDNA libraries was performed using the QuantiFluordsDNA System (Promega, Madison, Wisconsin, USA). The size range of final CDNA libraries was determined, applying the DNA 1000 chip on the Bioanalyzer 2100 from Agilent (280 bp). cDNA libraries were amplified and sequenced (50 bp single-end reads) using cBot and HiSeq 2000 (Illumina). Sequence images were transformed to bcl files using the software BaseCaller (Illumina). The bcl files were demultiplexed to fastq files with CASAVA (version 1.8.2)

\subsubsection{Mapping}

The reads were mapped against strain-specific transcriptomes of $D$. melanogaster and $D$. mauritiana, including CDS and UTRs (Torres-Oliva et al., 2016) using Bowtie2 v. 2.3.4.1 with the following parameters: -very-sensitive-local -N1 (Langmead et al., 2009). Samtools version 1.9 was used to further process the reads, and count the reads mapped to each transcript (idxstats) (Li et al., 2009).

\subsubsection{DEA and data visualization}

A principal component analysis was done using the regularized log (rlog) transformation from the DESeq2 package (DESeq2_1.22.2; R version 3.5.2) (Love et al., 2014).

We then used DeSeq2 (Love et al., 2014) to perform a pairwise differential expression analysis between the two species at each time point (D. melanogaster $72 \mathrm{~h}$ vs. D. mauritiana 72h, D. melanogaster $96 \mathrm{~h}$ vs. D. mauritiana 96h, D. melanogaster $120 \mathrm{~h}$ vs. D. mauritiana 120h). We used the online tool Metascape (Zhou et al., 2019) to perform GO enrichment analysis for each time point. All genes that were significantly differentially expressed (log2FC $>0 \mid \log 2 \mathrm{FC}$ $<0$ and padj < e0.05) in at least one stage (8350 unique genes) were combined and clustered using the coseq package (version 1.6.1; (Rau et al., 2013)) with the following parameters: $\mathrm{K}=2: 25$, transformation="arcsin", norm="TMM", model="Normal". We searched for potential upstream factors in $5 \mathrm{~kb}$ upstream of the TSS, 5'UTR regions and $1^{\text {st }}$ introns using the i-cisTarget tool (Imrichová et al., 2015; Pereira et al., 2006) keeping the default parameters: 
Minimum fraction of overlap: 0.4., NES: 3.0, ROC threshold for AUC calculation: 0.01 . Metascape was used to analyse differential enrichment of GO terms for each pairwise comparison.

\subsubsection{Generation of the ATAC-seq dataset}

For the generation of ATAC-seq datasets we followed (Buenrostro et al., 2013). Developing eye-antennal discs of D. melanogaster were dissected in ice-cold PBS at 72h, $96 \mathrm{~h}$ and $120 \mathrm{~h} \mathrm{AEL}$. PBS was removed and exchanged for $50 \mu$ l lysis buffer (10 mM Tris- $\mathrm{HCl}(\mathrm{pH}=7.4) ; 10 \mathrm{mM} \mathrm{NaCl}$; $3 \mathrm{mM} \mathrm{MgCl} ; 0.1 \%$ IGEPAL). The mixture was pipetted several times up and down to lyse the cells and then split into micro centrifuge tubes. Centrifugation for $10 \mathrm{~min}$ at $500 \mathrm{~g}$ and $4{ }^{\circ} \mathrm{C}$. The cell number was assessed in one of the samples and between 50,000 and 80,000 nuclei were used in subsequent steps. The supernatant was removed and the pellet(s) dissolved in $47.5 \mu \mathrm{l}$ 1X tagmentation buffer (20 mM Tris- $\mathrm{CH} 3 \mathrm{COOH}(\mathrm{pH}=7.6) ; 10 \mathrm{mM} \mathrm{MgCl} 2 ; 20 \%$ (vol/vol) dimethylformamide) with $2.5 \mu \mathrm{l}$ Tn5 Transposase and then incubated for $30 \mathrm{~min}$ at $37^{\circ} \mathrm{C}$. For purification we used the QIAGEN MinElute Kit and eluted in $10 \mu$ Elution Buffer (10 mM Tris, $\mathrm{pH}=8)$. For the PCR amplification was done as follows:

- $10 \mu$ tagmented chromatin

- $10 \mu \mathrm{H} 2 \mathrm{O}$

- $2.5 \mu \mathrm{l}$ Nextera PCR primer $1 *$

- $2.5 \mu \mathrm{l}$ Nextera PCR primer $2^{* *}$

- 25 l NEBNext High-Fidelity 2X PCR Master Mix (Cat \#M0541)

We used the following program:
(1) $72^{\circ} \mathrm{C} 5 \mathrm{~min}$
(2) $98^{\circ} \mathrm{C} \quad 30 \mathrm{sec}$
(3) $98^{\circ} \mathrm{C} 10 \mathrm{sec}$
(4) $63{ }^{\circ} \mathrm{C} \quad 30 \mathrm{sec}$
(5) $72{ }^{\circ} \mathrm{C} 1 \mathrm{~min}$
(6) repeat 3-5 13 times
(7) hold at $4{ }^{\circ} \mathrm{C}$

followed by another 2x purification step with the QIAGEN MinElute Kit: elution in $2 \times 10 \mu \mathrm{l}$ Elution Buffer (10 mM Tris, $\mathrm{pH}=8$ ). 
* AATGATACGGCGACCACCGAGATCTACACTCGTCGGCAGCGTCAGATGTG

** Ad2.2_CGTACTAG

CAAGCAGAAGACGGCATACGAGATCTAGTACGGTCTCGTGGGCTCGGAGATGT

Ad2.3_AGGCAGAA CAAGCAGAAGACGGCATACGAGATTTCTGCCTGTCTCGTGGGCTCGGAGATGT

Ad2.4_TCCTGAGC

Ad2.5_GGACTCCT

Ad2.6_TAGGCATG

CAAGCAGAAGACGGCATACGAGATGCTCAGGAGTCTCGTGGGCTCGGAGATGT

CAAGCAGAAGACGGCATACGAGATAGGAGTCCGTCTCGTGGGCTCGGAGATGT

CAAGCAGAAGACGGCATACGAGATCATGCCTAGTCTCGTGGGCTCGGAGATGT

Ad2.7_CTCTCTAC

CAAGCAGAAGACGGCATACGAGATGTAGAGAGGTCTCGTGGGCTCGGAGATGT

\subsubsection{Bioinformatics processes of the ATAC-seq dataset}

We performed quality checks of the sequenced reads using FASTQC (https://www.bioinformatics.babraham.ac.uk/projects/fastqc/). The reads were trimmed, using Trimmomatic (version 0.36) (Bolger et al., 2014) appyling a sliding window trimming with the parameters slidingwindow 4:15 and minlen 30. Trimmed reads were mapped to the D. melanogaster genome (version 6.13) after discarding the mitochondrial genome, using Bowtie2 (version 2.3.4.3) (Langmead et al., 2009), with the commands: - - no-unal and X2000. Samtools version 1.9 (Li et al., 2009) were subsequently used to convert the sam to bam files, and to sort and index bam files. We removed duplicates using PICARD (version 2.1.1, https://github.com/broadinstitute/picard) with default parameters and converted the resulted bam files to bed files. Reads were then centered as described in (Buenrostro et al., 2013). We used MACS2 (version 2.1.2) (Zhang et al., 2008, p. 2) with the following commands -g dm - nomodel --shift -100 --extsize 200 - q 0.01 -bdg to call significant peaks. We used the Integrated Genome Browser (IGB, (Freese et al., 2016)) to visualize the read depth and peaks. Peaks were annotated to the closest gene using the annotatePeaks . pl program from the HOMER software package (v4.8.3) using $\mathrm{dm} 6$ as genomic input.

\subsubsection{Definition of a Pnr target gene list}

As a basis for the high confidence list of putative Pnr target genes a Chip-chip dataset was used (downloaded on 1st of July, 2015 from http://furlonglab.embl.de/data/download, (Junion et al. 2012), which comprises ChIP-chip experiments in the Drosophila embryo with several transcription factors, including Pnr at two time points (4-6h AEL and 6-8h AEL). All Pnr-binding regions from both time points were selected with a Tile-Map score of $<5.5$. and where the distance of the center of the peak to the TSS was $-1000 \mathrm{bp}$ and $+1000 \mathrm{bp}$. This resulted in a gene list of 4009 putative Pnr targets (Figure 10). The peak regions of these genes were used to search for the Pnr GATA motif, resulting in a list of 1675 putative target genes. We restricted the list of potential Pnr target genes to those genes which are expressed $(>=10$ reads on average for each stage in D. melanogaster) in our transcriptomic dataset and 
performed hierarchical clustering using coseq (Rau and Maugis-Rabusseau, 2017) according to their expression dynamics with the following parameters: $K=2: 25$, transformation="arcsin", norm="TMM", model="kmeans". We downloaded a list of all known direct (TF-gene) or genetic interactions of Pnr from the DrolD database (Yu et al., 2008) and found an overlap of 30 genes, of which 21 are differentially expressed between the D. melanogaster and D. mauritiana. We used Cytoscape (Shannon et al., 2003) to visualize the interaction between these target genes and potential upstream regulators found in the database.

\subsection{5. $q P C R$}

Discs from D. melanogaster and D. mauritiana larva were dissected in ice-cold PBS at $96 \mathrm{~h}$ AEL and 120h AEL and collected in TRIZOL. The samples were then homogenized using a Tissuelyser and total mRNA was extracted using the Phenol/Chloroform extraction method. We then used TurboDNAse to remove potential gDNA contamination. Concentration was measured using Nanodrop and the Maxima First Strand cDNA Synthesis Kit for RT-qPCR kit was used for cDNA preparation. To test the efficiency of primers, we prepared four 1:4 dilutions of a pool of all RNA samples per species $(1: 2,1: 6,1: 18,1: 54$; for calculations see Supplementary Figure 11). Real-time qPCR was then performed using the HOT FIREpol EvaGreen qPCR Mix Plus (ROX) (Solis BioDyne, Tartu, Estland) and a CFX96 Real-time PCR System (Bio-Rad Laboratories, Hercules, CA, USA). Log2 fold changes in expression were calculated using the $\triangle \triangle C T$ method (Livak and Schmittgen, 2001) with actin79b as a reference gene.

Following primer pairs were used:

pnrB: f: CGCAGACGAATCAAACG, r: TCACGTTCTGATAGTCGC

actin 79b: f: CGCAAGGATCTGTATGCCAAC, r: TCTTGATGGTGGACGGGG

The following temperatures were used:

1) $95^{\circ} \mathrm{C}-15: 00$

2) $95^{\circ} \mathrm{C}-00: 30$

3) $56^{\circ} \mathrm{C}-0: 30$

4) $72^{\circ} \mathrm{C}-0: 30$

5) Repeat step 2-4 for 39x

6) $65^{\circ} \mathrm{C}-0: 05$ 
7) $95^{\circ} \mathrm{C}$

\subsubsection{Antibody staining}

Developing eye-antennal discs were dissected in ice-cold PBS and fixed for 30 min in $4 \%$ paraformaldehyde (PFA). The discs were then washed 3 times in 0.03\% PBT (Phosphate buffered saline 1\%, Triton X-100) before blocked in 5\% goat serum for 30min. Incubation with the Primary Antibody was done for 90 min, before 3 additional washing steps with PBT and one round of blocking for $30 \mathrm{~min}$. The tissue was then incubated overnight with the Secondary Antibody on a rocking plate on $4^{\circ} \mathrm{C}$. If needed, Phalloidin-488 (1:100) was added. After 3 washing steps with PBT, the discs were incubated with DAPI (1:1000) for $10 \mathrm{~min}$, followed by one washing step with PBT and one washing step with PBS. Subsequently, the discs were mounted in mounting medium (80\% glycerol $+4 \%$ n-propyl-galate) and kept at least one night on $4^{\circ}$ degree before imaging.

Antibodies used: We generated polyclonal, primary antibodies against Pnr (Junion et al., 2012)

(Pnr_B

(TPLWRRDGTGHYLCNACGLYHKMNGMNRPLIKPSKRLVSATATRRMGLCCTNCGTRTTTLWRRNNDG EPVCNACGLYYKLHGVNRPLAMRKDGIQTRKRKPKKTGSGSAVGAGTGSGTGSTLEAIKECKEEHDLKPSL SLERHSLSKLHTDMKSGTSSSSTLMGHHSAQQ) and Pnr_B (206-336) (GVNRPLAMRKDGIQTRKRKPKKTGSGSAVGAGTGSGTGSTLEAIKECKEEHDLKPSLSLERHSLSKLHTDM KSGTSSSSTLMGHHSAQQQQQQQQQQQQQQQQQQQQSAHQQCFPLYGQTTTQQQHQQHGH)) and Ush (Fossett et al., 2001) based on previous knowledge, against the peptide sequences: Ush-(231-250) (CSHRIKDTDEAGSDKSGAGG) and Ush-(1174-1191) (VGGHGQQKNKENLQEAAI). Before usage, both antibodies were preabsorbed overnight on Drosophila embryos on $4^{\circ} \mathrm{C}$.

Please note that we confirmed the specificity of the Ush antibody by recapitulating known Ush expression domains in the wing imaginal disc (Supplementary Material Figure 1A) and during embryonic development (Supplementary Material Figure 1B) (Muratoglu et al., 2006; Tomoyasu et al., 2000)). For test stainings in embryos we collected embryos for several hours on apple agar plates, removed the chorion with $50 \%$ Klorix and rinsed them $3 x$ with $0.03 \%$ PBT (Phosphate buffered saline 1\%, Triton X-100). We fixed the embryos with heptane and $2 \%$ formaldehyde for $20 \mathrm{~min}$ and washed with $\mathrm{MeOH}$, followed by washing steps with PBT. The embryos were then blocked in 3\% BSA for one hour, followed by incubation with the primary $A B$ overnight. After two washing steps with PBT, we added HRP-coupled secondary AB for 90 
min. After three washing steps with PBT we performed a DAB (3'-3diaminobenzidine) staining. The embryos were then washed again 2 times in PBT and mounted in glycerol.

Concentrations Primary AB's: Anti-Pnr (rabbit): 1:200, Anti-Ush (rabbit): 1:2000, Anti-GFP (chicken): 1:1000; Concentrations Secondary AB's: Anti-Chicken-488; Anti-Rabbit-Cy3: 1:500; Anti-rabbit-HRP-coupled (1:1000).

Pictures of eye-antennal discs upon antibody staining were taken using a Zeiss LSM 710 confocal microscope. Antibody stainings were visualized and processed with Fiji software (Schindelin et al., 2012). Vertical section of the confocal pictures were generated using the Volume Viewer plugin (https://imagej.nih.gov/ij/plugins/volume-viewer.html) with the following parameters: Display Mode: Slice and Boarders, Interpolation: Nearest Neighbour, Transfer Function: Fire LUT.

\subsubsection{Geometric Morphometrics}

We imaged WT species, each parental line and the offspring of the respective crosses from the dorsal view of the head using a Leica M205 FA stereo microscope. We placed 64 landmarks on pictures of these dorsal heads using the tpsDig2 software. We then defined 23 fixed landmarks and 41 sliding landmarks using tpsUtil. tpsRelw32 was used to calculate the consensus (i.e. Procrustes superimposition), partial warps and relative warps (https://life.bio.sunysb.edu/morph/). Using MorphoJ (Klingenberg, 2011) for visualization, we performed Procrustes Fit and generated a covariance matrix. To analyse differences in dorsal head shapes in WT D. melanogaster and D. mauritiana, we performed discriminate function analysis using MorphoJ. We further performed a principal component analysis (PCA) to analyse difference in head shapes upon knock-down or up-regulation of pnr.

\subsubsection{Overexpression/Knock-down of phr and ush}

To overexpress or knock-down pnr and ush, the following fly lines were used:

D. melanogaster (Oregon R) and D. mauritiana (TAM 16) (both kindly provided by Prof. Alistair McGregor, Oxford Brookes University), pnr GAL4/TM6B (kindly provided by Prof. Marc Haenlin), pnr GAL4>UAS2YFP/TM6B (kindly provided by Prof. Marc Haenlin, CBI Toulouse), ush GAL4 26662 (y[1] w[*]; P\{w[+mW.hs]=GawB\}ush[MD751]), Bloomington; UAS ush 14\|A/CyO (kindly provided by Prof. Marc Haenlin, CBI Toulouse); ush-RNAi 3622 (y[1] v[1]; P\{y[+t7.7] v[+t1.8]=TRiP.HM05193\}attP2/TM3, Sb[1]), Bloomington;UAS pnr (w; UAS-pnr/CyO; 
TM2/TM6B) (kindly provided by Prof. Fernando Casares); pnr-RNAi (VT101522/KK, \#108962 VDRC Stock Center and VT6224/GD, \#1511 VDRC Stock Center); oc2-Gal4/CyO (kindly provided by Prof. Fernando Casares).

All crosses were performed at $25^{\circ} \mathrm{C}$ and at a constant $12 \mathrm{~h} / 12 \mathrm{~h}$ light/dark cycle. Since Pnr and Ush are crucial during embryonic development, we chose combinations of GAL4/UAS lines, that resulted in a phenotype but were not lethal during embryonic, larval of pupal stages. We used a set of GAL4 lines, that overlap a range of weak to strong driving capacity and in the case of pnrRNAi we used two RNAi lines with different effector strengths.

\subsection{9. pnr expression and lineage.}

The pnr-GAL4 line pnrMD237 (Calleja et al., 1996), recombined with UAS-GFP, was used to follow pnr expression in imaginal and pupal eye-antennal discs. Adult pnr expression domain in adult heads was monitored in y; pnr-GAL4/UAS-y+ flies (Calleja et al., 1996) as the cuticle region with y-rescued pigmentation. To follow the pnr-GAL4 lineage, pnr-GA4, UAS-GFP flies were crossed to UAS-flipase; act5c>stop< nuc-lacZ flies (Struhl and Basler, 1993). In the discs of the progeny, pnr expression was visualized with anti-GFP and its lineage with anti $\beta$-galactosidase.

\subsubsection{Immunostaining and imaging.}

Third instar or pupal discs were processed as in (Magri et al., 2018). Primary antibodies were chicken anti-GFP (1/500; ab13970, Abcam), rabbit anti- $\beta$-galactosidase (1/1000; Cappel) and mouse anti-Eya (1/500; 10H6, Developmental Studies Hybridoma Bank, lowa University). Secondary antibodies at 1/400 were from Molecular Probes. Imaging was carried out on a Leica SPE confocal setup (ALMI, CABD).

\subsubsection{Adult head cuticle preparation.}

Dissected adult heads in PBS were mounted in Hoyer's mountant: Lactic Acid (50:50) as in (Magri et al., 2018).

\subsubsection{Ommatidia Counting}

To estimate ommatidia number of single fly eyes, we took pictures of one eye per fly (50 stacks/eye) using a Leica M205 FA stereo microscope and an external light source, which resulted in reflection of light by each ommatidium. We used FIJI to perform the following analyses. We performed Z-projection using maximum intensity and then transformed each picture, so that the single reflection of each ommatidium is represented by a black dot. We 
then used the ICTN cell counter tool (with the following parameters: Width: 7, Minimum Distance: 17 , Threshold: 1.5$)$ to estimate the number of black dots, i.e. the number ommatidia. Statistical analysis of ommatidia number was done using a one-way ANOVA and pair-wise comparisons were calculated using Tukey HSD test. 
Chapter II - Variation in a pleiotropic regulatory module drives evolution of head shape and eye size in Drosophila

\subsection{Supplementary Figures}

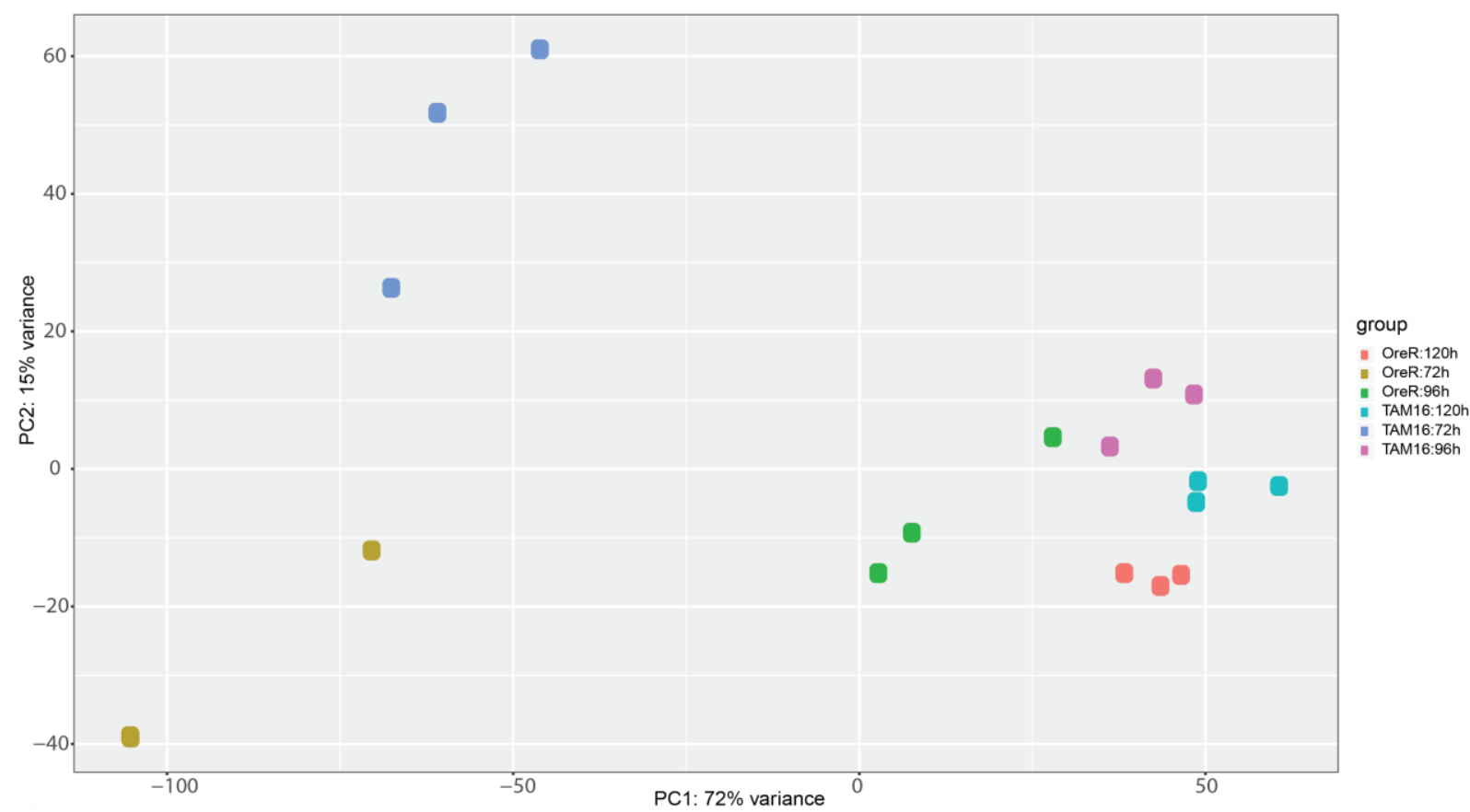

Supplementary Figure 8. Principal component analysis of all RNA-seq samples based on rlog transformed read counts. PC1 separates the samples according to time-points, whereas PC2 separates the data mainly by species. D. melanogaster (OreR), D. mauritiana (TAM16). 
A

\begin{tabular}{|c|c|c|c|}
\hline & upregulated in D. melanogaster & upregulated in D. mauritiana & total \\
\hline 72h AEL & 3244 & 3439 & 6683 \\
\hline 96h AEL & 1622 & 1638 & 3260 \\
\hline 120h AEL & 1130 & 1250 & 2380 \\
\hline
\end{tabular}

B

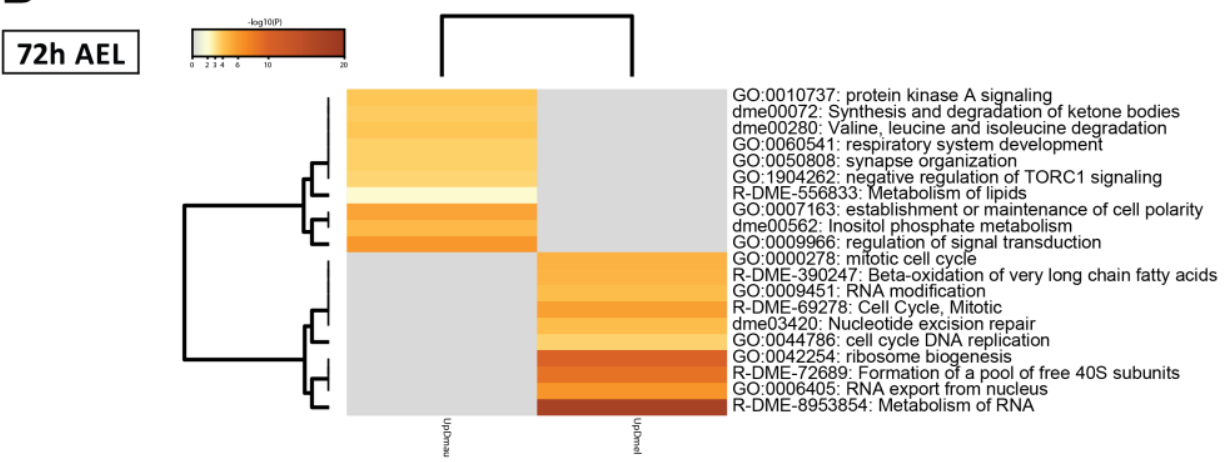

C

96h AEL

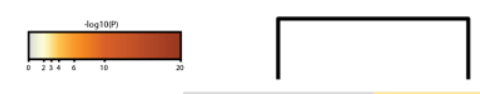

D
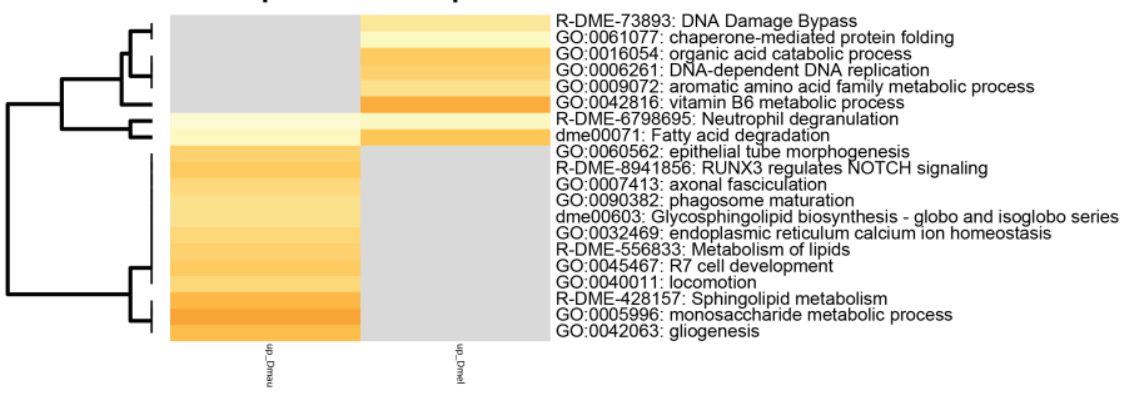

120h AEL
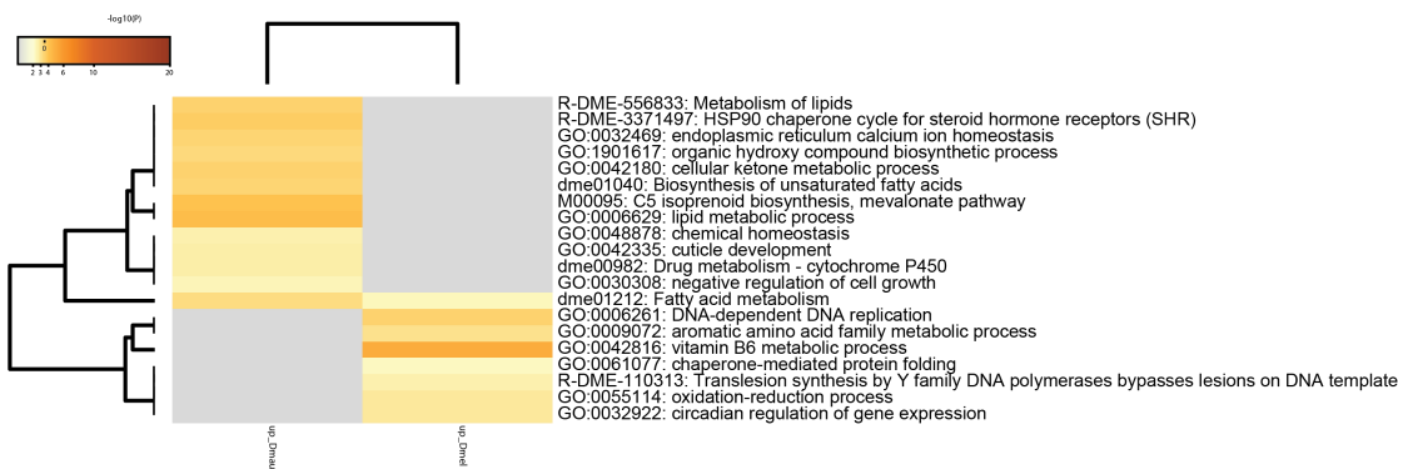

Supplementary Figure 9. A. Number of genes that are significantly differentially expressed (padj $<=0.05$ ) between the two species for each time-point. D. melanogaster is depicted in red and D. mauritiana in blue. Total number of differentially expressed gene that are upregulated are shown in the third column, as a sum of genes, upregulated in each species. B. Differential GO-term analysis of genes that are upregulated in each species per time-point. 

eye size in Drosophila

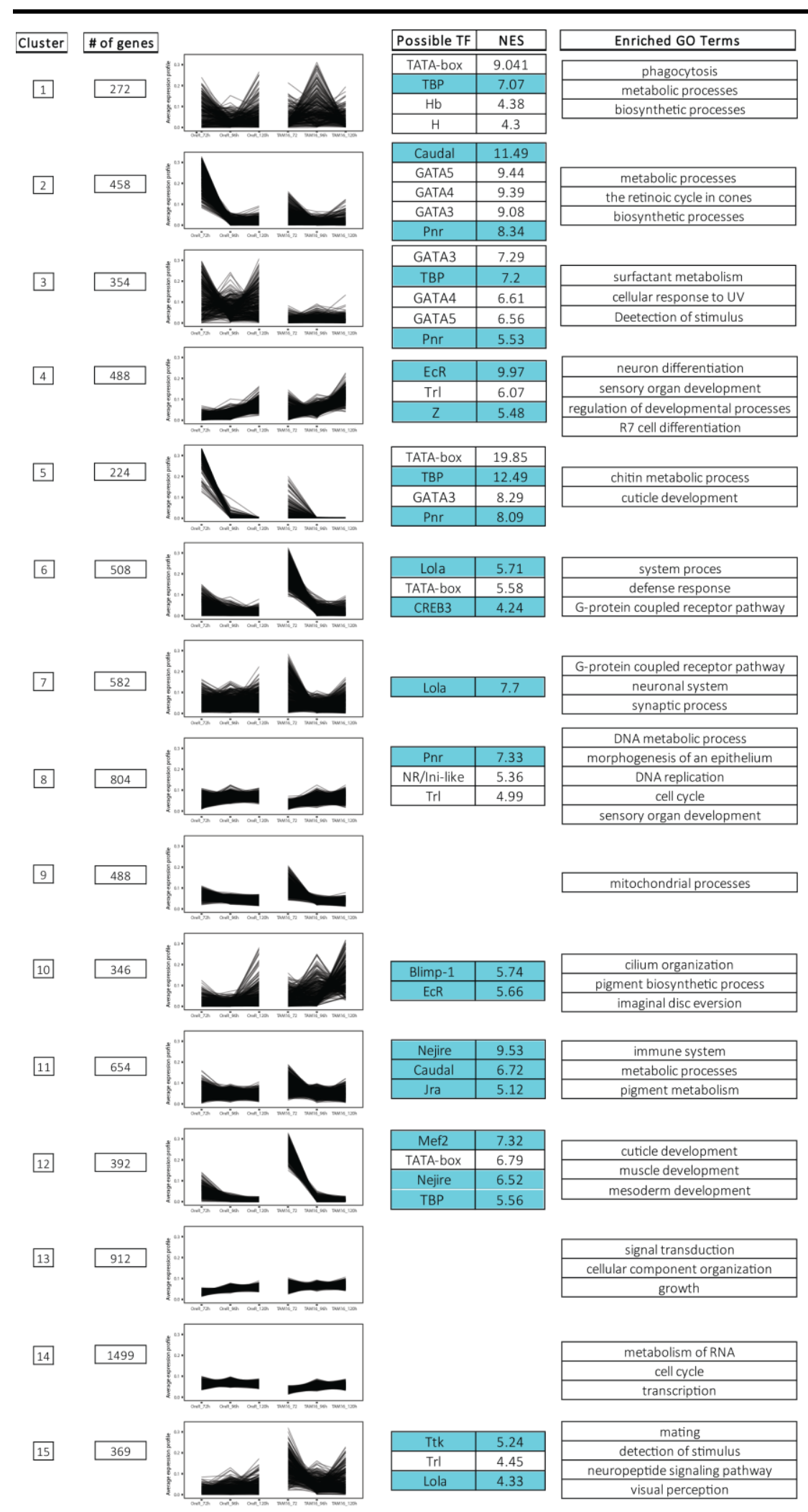

Supplementary Figure 10. Clustering of all genes that are significantly differentially expressed (padj $<=0.05$ ) between the two species in at least one time-point resulted in 15 distinct co-expression profiles. Shown is the number of genes in each cluster and the expression profile plot. The tables show transcription factors whose transcription factor binding motifs were enriched $5 \mathrm{~kb}$ upstream of the TSS, $5^{\prime}$ UTR regions and $1^{\text {st }}$ introns of the clustered genes. The NES-factors are shown in the second column. Transcription factors labelled in cyan are themselves significantly differentially expressed in at least one stage. GO-terms enriched in each cluster are given in the last column. 

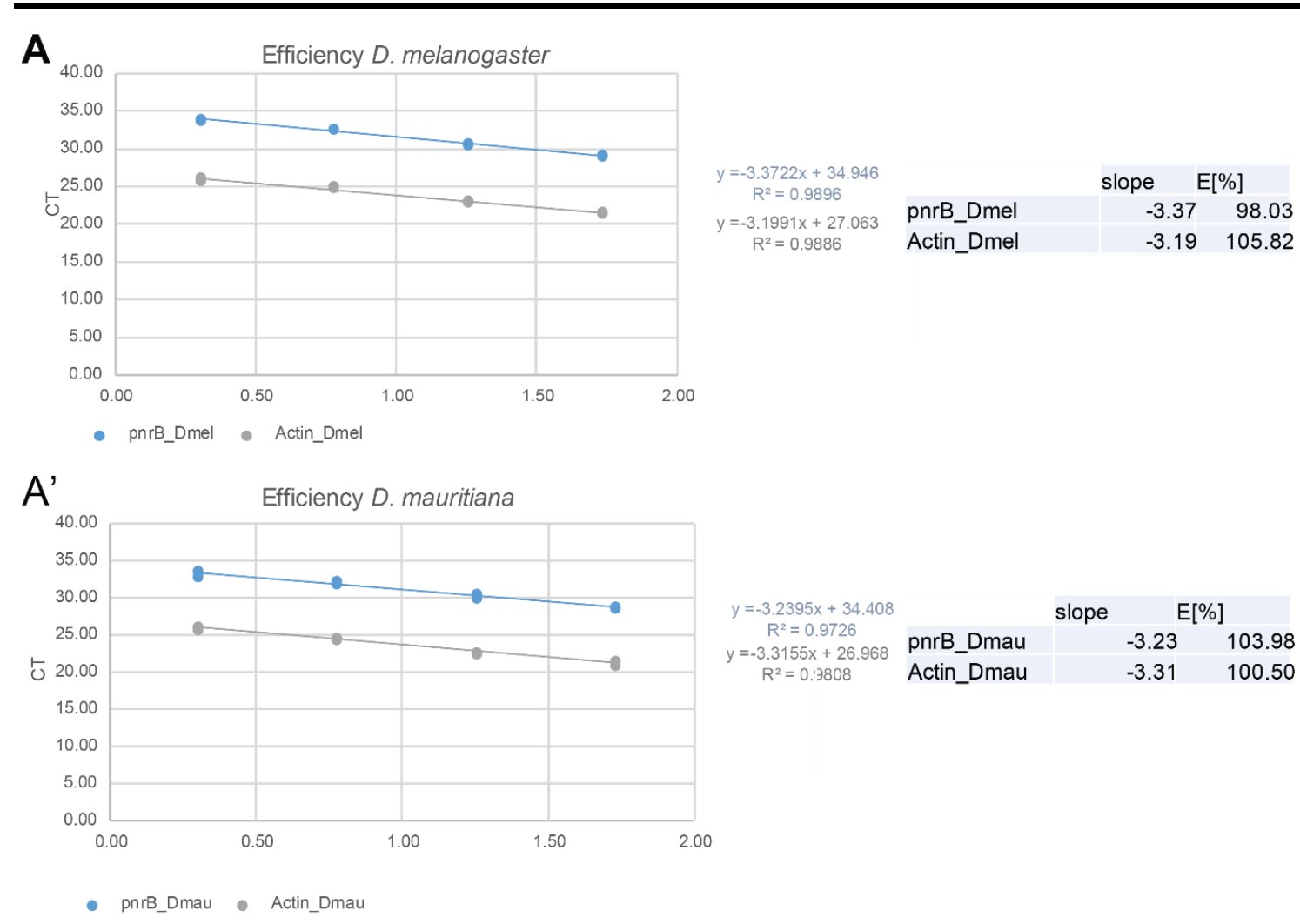

B

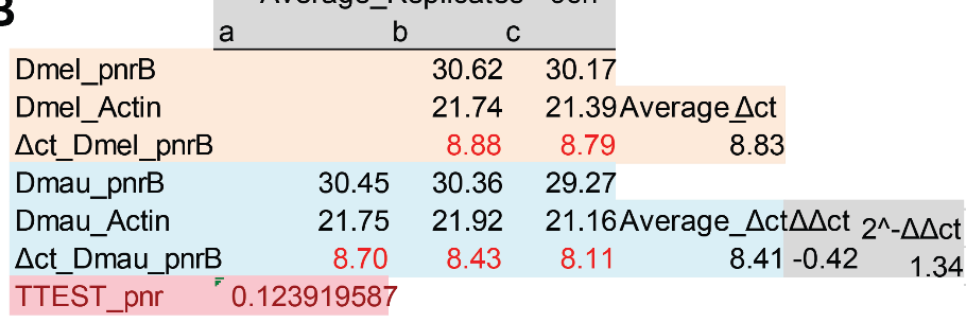

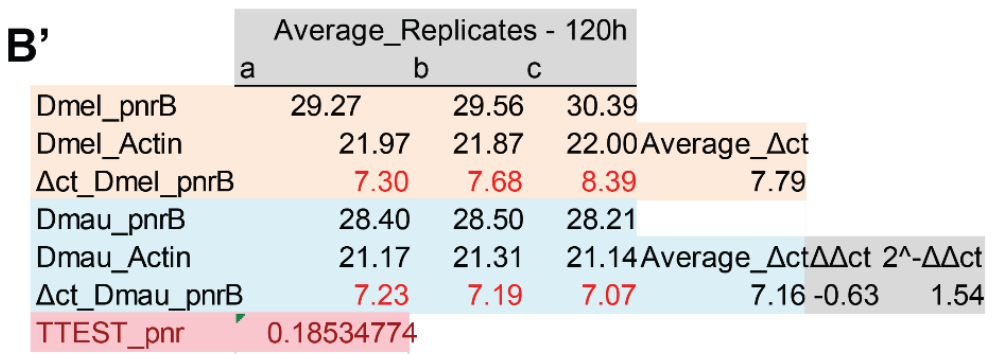

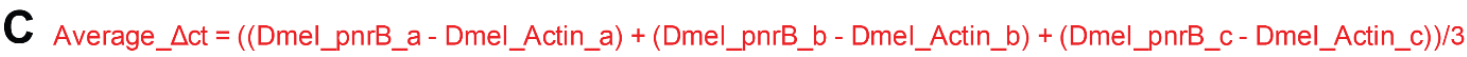
$\Delta \Delta c t=\Delta$ ct_Dmel $\_$pnrB $-\Delta$ ct_Dmau $\_$pnrB

Supplementary Figure 11. A. q-RT PCR for pnrB in D. melanogaster and D. mauritiana. Efficiency of the used primers in D. melanogaster for pnrB. we prepared four 1:4 dilutions of a pool of all RNA samples per species (1:2, $1: 6,1: 18,1: 54)$. The primer pair for pnrB yielded an efficiency of $98 \%$ in $D$. melanogaster and the primer pair for actin yielded an efficiency of $106 \%$. B. Same efficiency calculations for the same genes as in A for D. mauritiana. In this species the primer pair for pnrB showed an efficiency of $104 \%$ and $100.5 \%$ for actin. C. Comparison of pnr expression levels in D. melanogaster and D. mauritiana at $96 \mathrm{~h}$ AEL. actin was in all cases used as reference gene. Log2fold changes were calculated using the $\triangle \Delta C T$ method. At $96 \mathrm{~h} A E L$, expression of pnr was $1.3 x$ higher than in D. melanogaster at the same time point. D. Comparison of pnr expression levels in $D$. melanogaster and $D$. mauritiana at $120 \mathrm{~h}$ AEL. Expression of pnr was 1.5x higher than in D. melanogaster at the same time point. Even though the difference in expression is not significant we observe the same trend as in the transcriptomics dataset. 


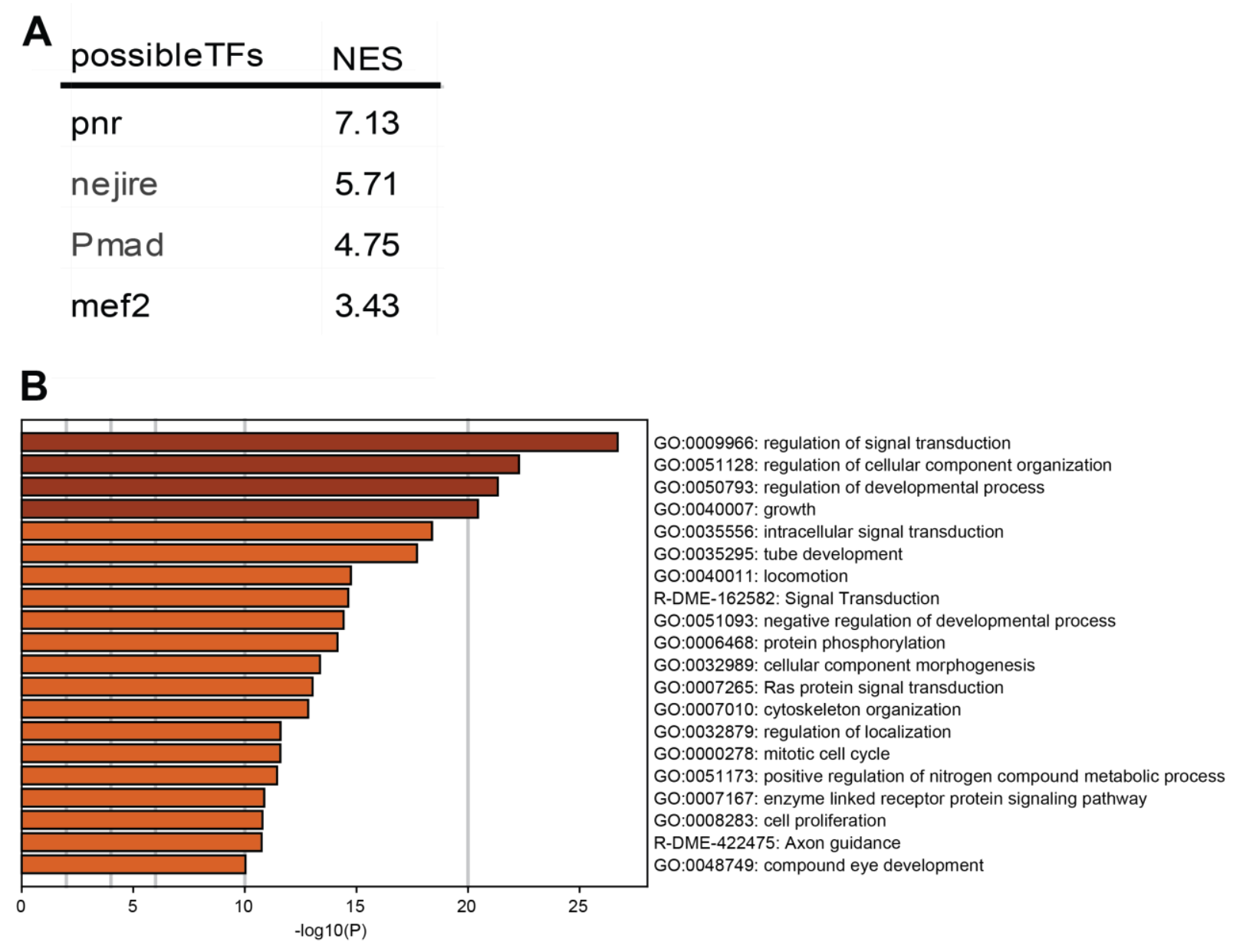

Supplementary Figure 12. A. Cross validation of TFBS enrichment in 5kb upstream of the TSS, 5'UTR regions and 1st introns of all predicted Pnr target genes, NES-values are given in the second column. B. GO-term enrichment analysis of all predicted Pnr target genes are enriched in processes like signal transduction, growth, cell cycle but also in more specific terms like compound eye development. 

eye size in Drosophila

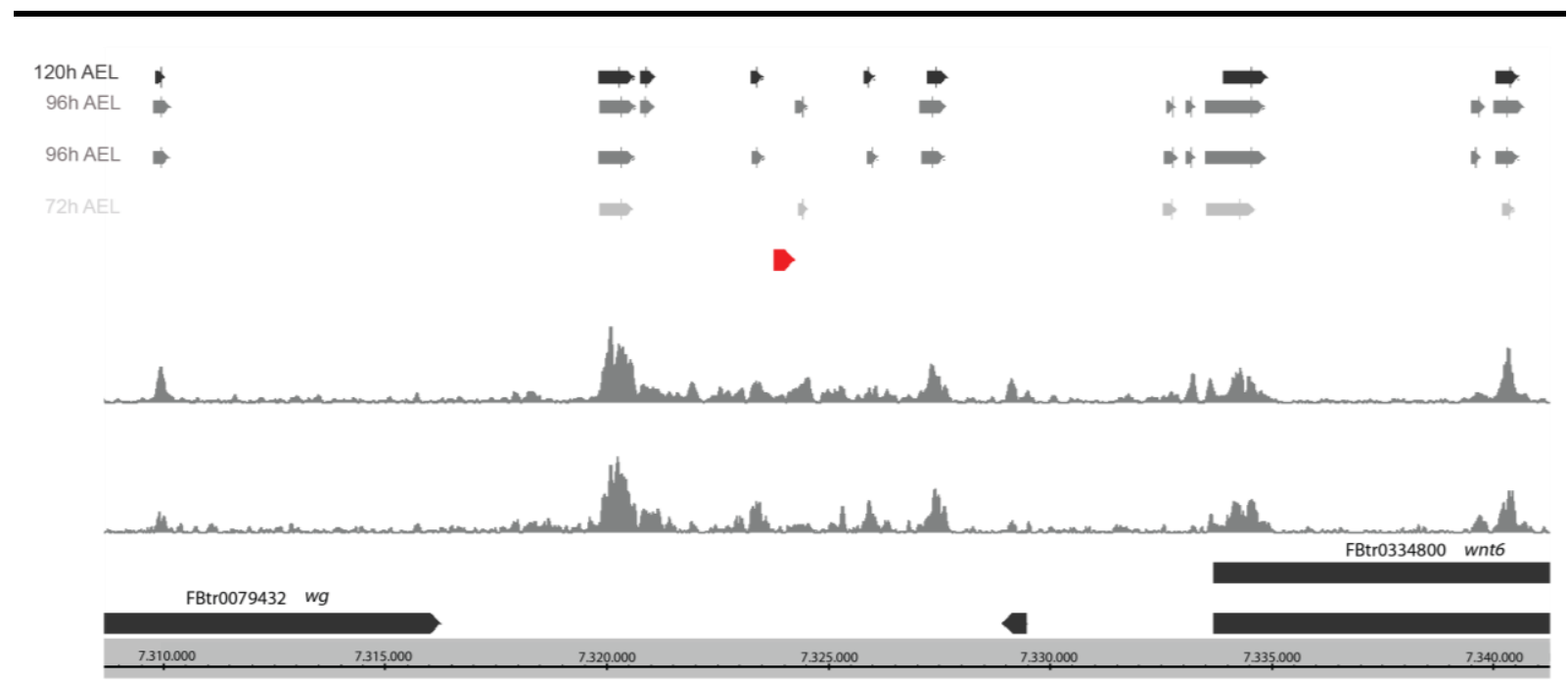

Supplementary Figure 13. Gene locus of $\boldsymbol{w g}$ and wnt6. The grey tracks show the depth graph of the ATAC-seq dataset at $96 \mathrm{~h} \mathrm{AEL}$. The grey bars are significantly called peaks at three timepoints (72h AEL - light grey, 96h AEL - grey, $120 \mathrm{~h} \mathrm{AEL} \mathrm{-} \mathrm{dark} \mathrm{grey).} \mathrm{The} \mathrm{red} \mathrm{bar} \mathrm{depicts} \mathrm{the} w g$-enhancer region containing two conserved GATA motifs, which are though not activated by Pnr to drive expression in the peripodial epithelium. 


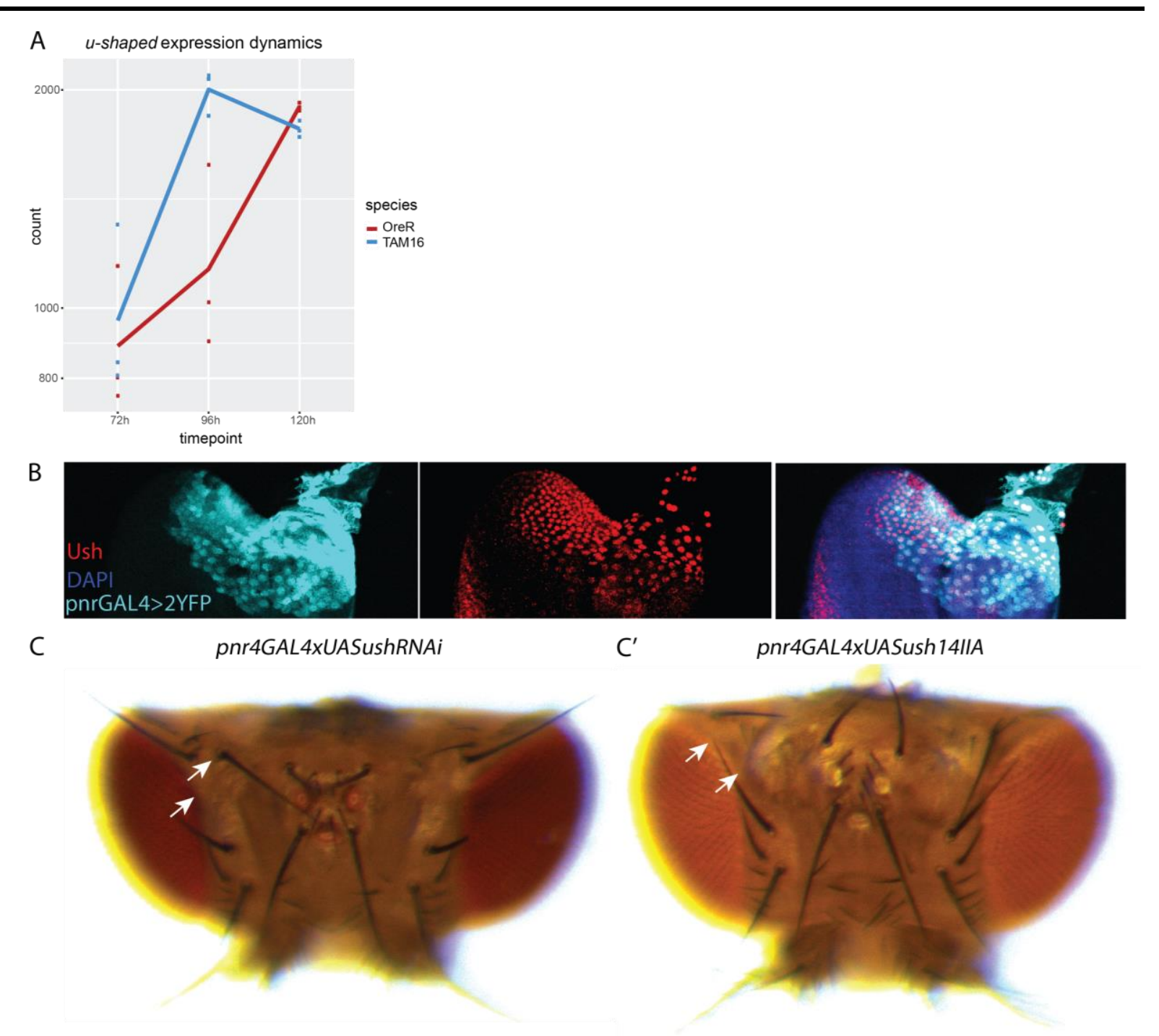

Supplementary Figure 14. A. Expression dynamics of the ush transcript in D. melanogaster (red) and $D$. mauritiana (blue). B. Overlap of pnr expression pattern visualized by pnrGAL4>2YFP line and Ush protein location, detected with an $\alpha$-Ush antibody. The two signals overlap in the dorsal part of the developing eye-antennal disc. C. Knock-down of ush using the VTO42374 driver line and UAS-ushRNAi. The head cuticle shows irregularities with loss of the posterior vertical bristles $\mathbf{C}^{\prime}$. Overexpression of ush using the VT042374 driver line and UASush14IIA line. As the knock-down of ush, upregulation affects the structure of the head cuticle and leads to an overgrowth of the occipital structures. Additionally, the bristle patterns are affected. The posterior vertical bristles as well as the bristles surrounding the eye area are lost. 


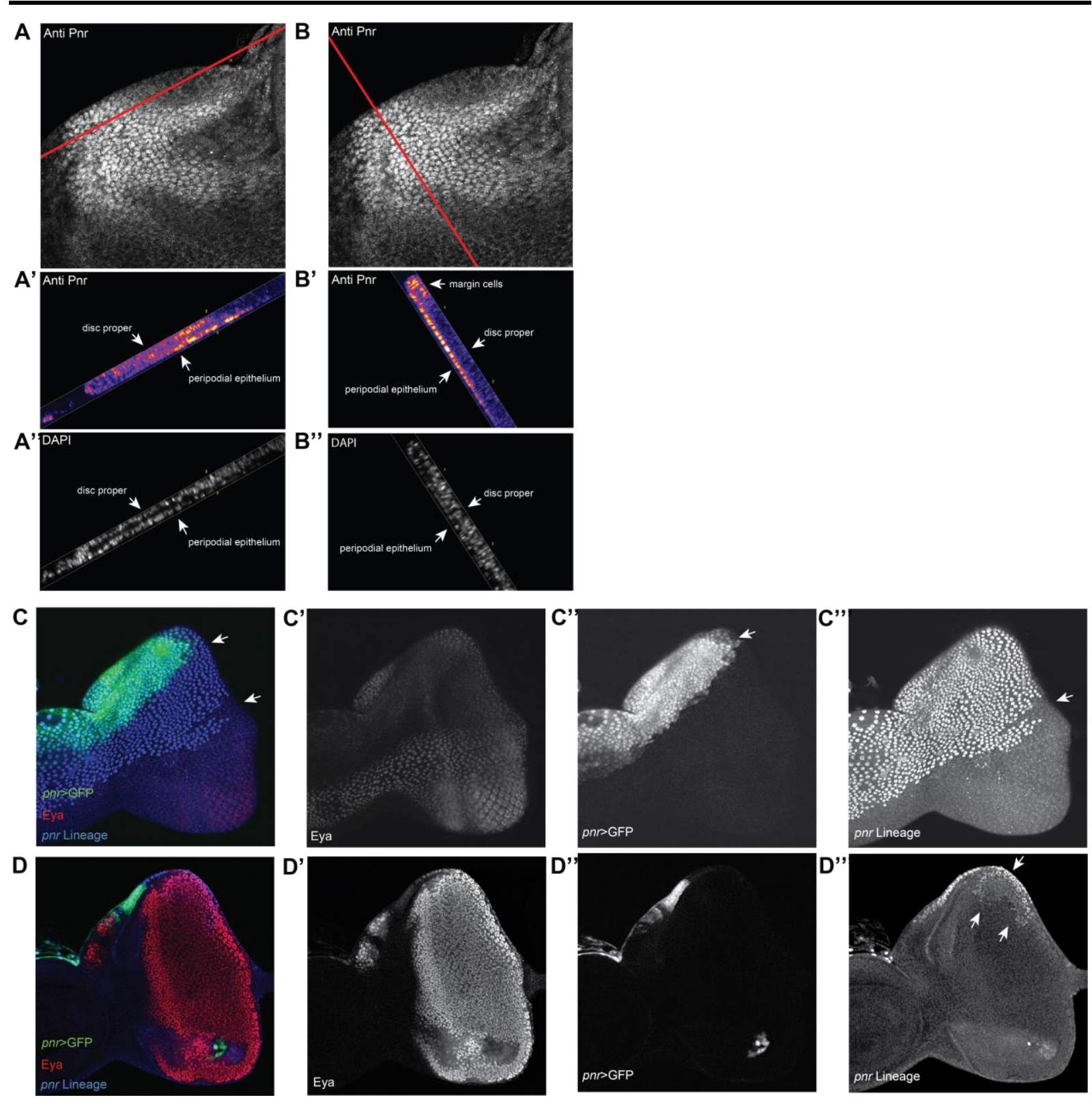

Supplementary Figure 15. A. Pnr protein location (detected with an $\alpha$-Pnr antibody) in the developing eyeantennal disc at $120 \mathrm{~h}$ AEL in D. melanogaster. $\mathbf{A}^{\prime}$. Vertical section of the same disc as depicted in $\mathbf{A}$ along the red line showing Pnr antibody staining. Pnr is expressed in the peripodial epithelium and in marginal cells reaching into the disc proper. The intensity of the Pnr signal is lower in the future ocelli region of disc. A". Vertical section of the same disc as in A along the red line showing the cell nuclei using DAPI staining. B. Pnr protein location (detected with an $\alpha$-Pnr antibody) in the same disc as in B. B'. Vertical section of the same disc as depicted in B along the red line showing Pnr antibody staining. Pnr is expressed in the peripodial epithelium and in the marginal cells of the disc proper. B". Vertical section of the same disc as in B along the red line showing the cell nuclei using DAPI staining. C-D. Lineage of pnr-expressing cells in the developing eye-antennal disc. C-C'”' pnr>GFP expression can be detected in the margin cells $\left(\mathbf{A}^{\prime \prime}\right)$ of the disc proper. Eyeless expression is shown in red ( $\mathbf{A}$ and $\left.\mathbf{A}^{\prime}\right), p n r$-GFP in green. The pnr-lineage shows (in blue) that a view cells that were initially pnr-expressing, are forming the dorsal-most cells of the developing retina ( $\left.\mathbf{A}^{\prime \prime \prime}\right)$. D- $\mathbf{D}^{\prime \prime \prime}$. pnr>GFP and the cells of the pnr-lineage in the peripodial membrane of the same disc as in A. D. Overlap of pnr>GFP cells (green) and the pnr-lineage in blue. Eyeless cannot be detected in the disc proper ( $\left.\mathbf{D}^{\prime}\right)$. The pnr-driver line drives expression of GFP in the dorsal most region of the developing eye-antennal disc ( $\left.\mathbf{D}^{\prime \prime}\right)$. Cells that initially expressed pnr cover the complete dorsal lineage of the eye-antennal disc including the retina (D'"). 

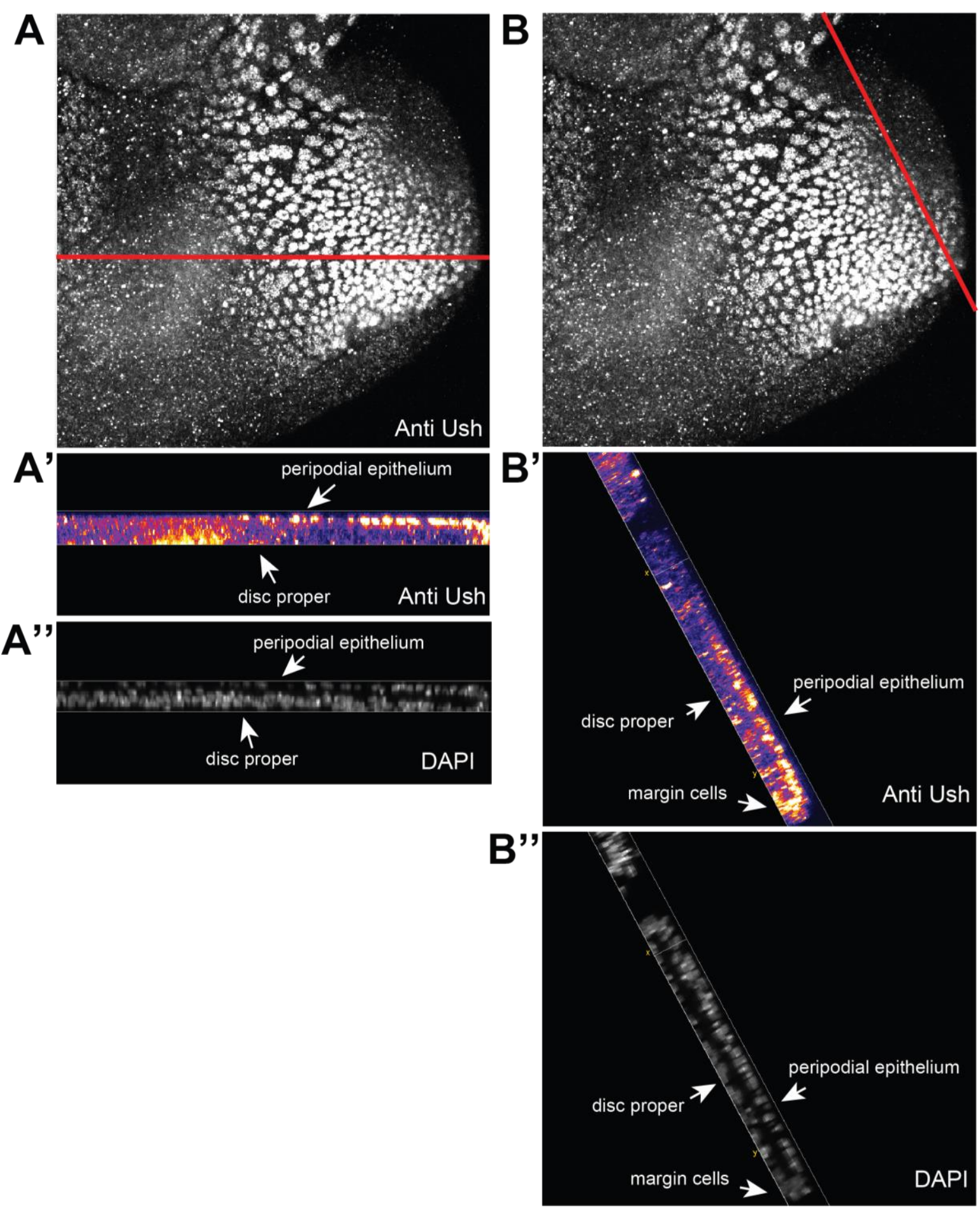

Supplementary Figure 16. A. Ush protein location (detected with an $\alpha$-Ush antibody) in the developing eyeantennal disc at $120 \mathrm{~h}$ AEL in D. melanogaster. $\mathbf{A}^{\prime}$. Vertical section of the same disc as depicted in $\mathbf{A}$ along the red line showing Ush antibody staining. Ush is expressed in the peripodial epithelium. A". Vertical section of the same disc as in $\mathbf{B}$ along the red line showing the cell nuclei using DAPI staining. B. Ush protein location (detected with an $\alpha$-Ush antibody) in the same disc as in A. B'. Vertical section of the same disc as depicted in $\mathbf{B}$ along the red line showing Ush antibody staining. Ush is expressed in the peripodial epithelium and in marginal cells reaching into the disc proper. B". Vertical section of the same disc as in B along the red line showing the cell nuclei using DAPI staining. 


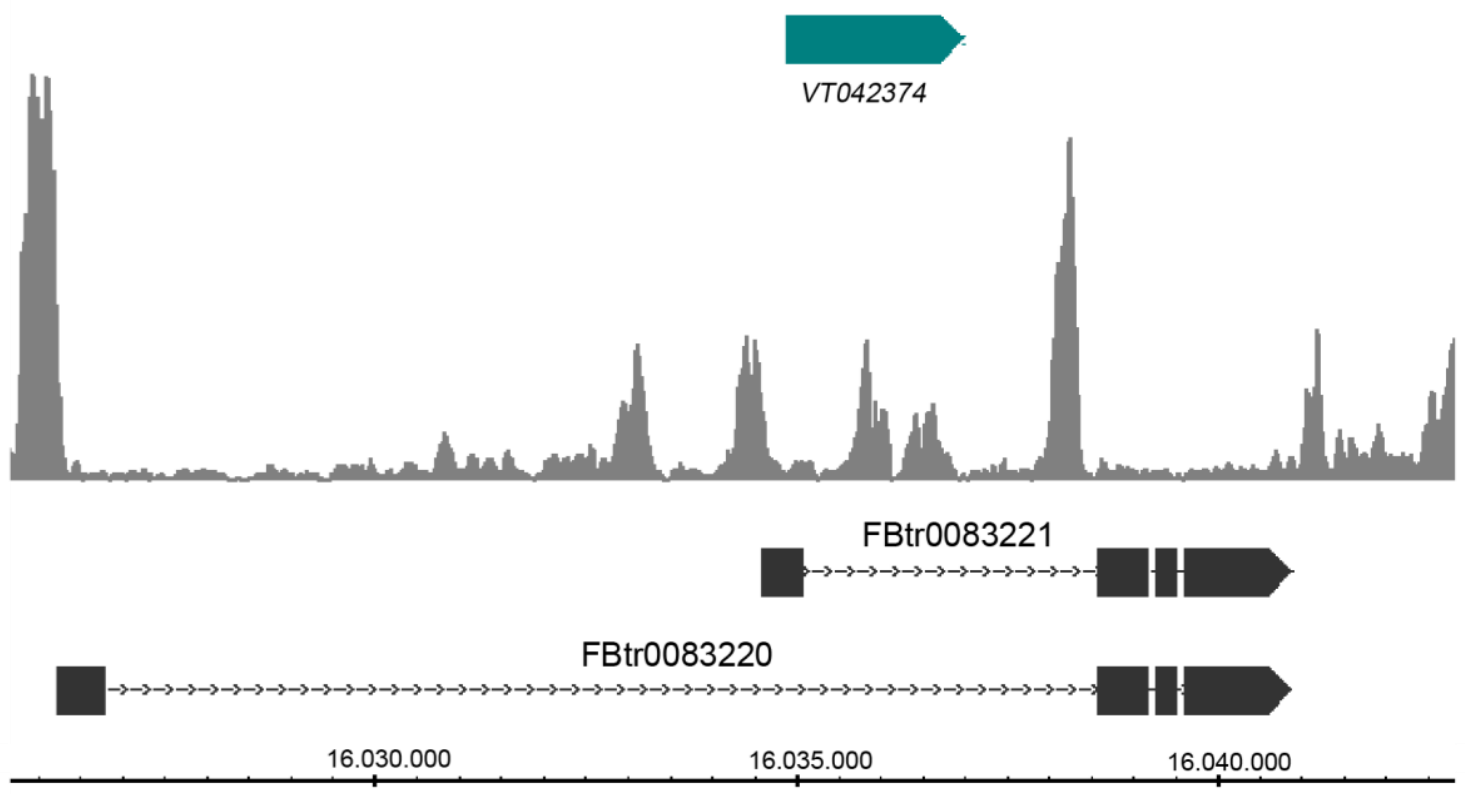

Supplementary Figure 17. pnr locus showing two isoforms, namely pnrA (FBtr0083221) and pnrB (FBtr0083220). The grey track shows the ATAC-seq data at $96 \mathrm{~h}$ AEL represented as a depth graph. The cyan bar represents the $2 \mathrm{~kb}$ DNA fragment that controls expression of GAL4 of the VT042374 driver line. It overlaps with two openchromatin peaks, which are potential regulatory regions for the expression of $p n r$ in the eye-antennal disc. 


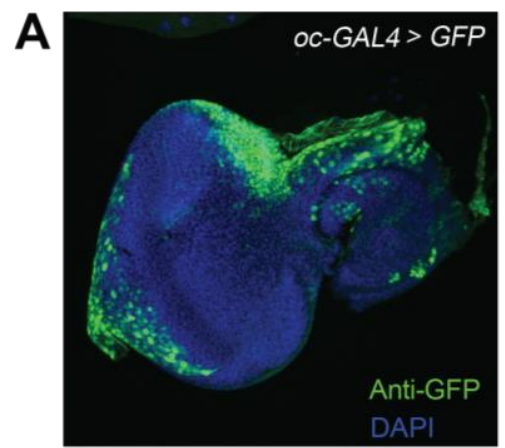

B

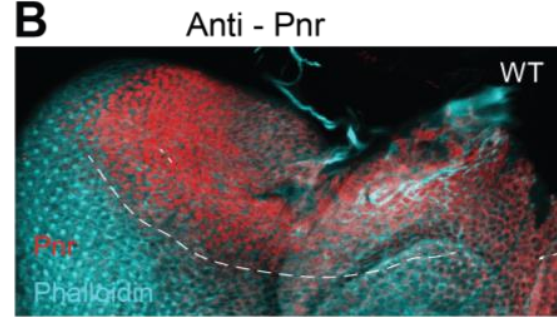

C

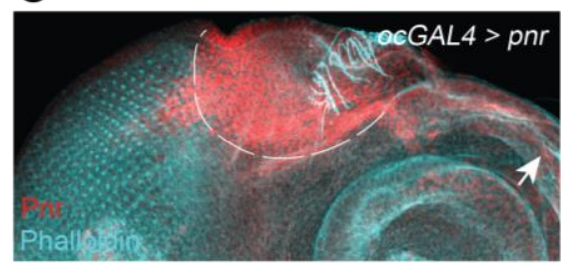

D

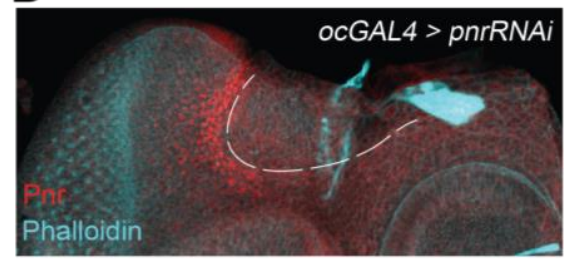

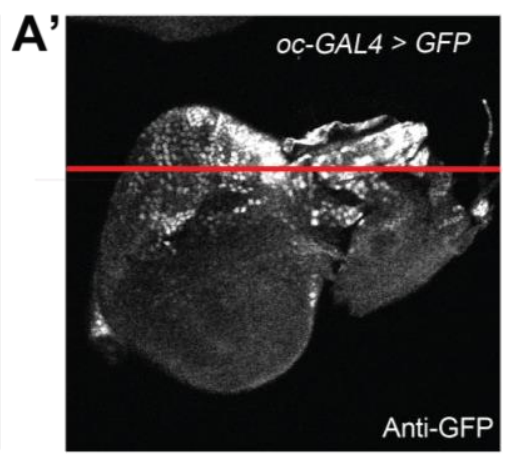

B' Anti - Ush

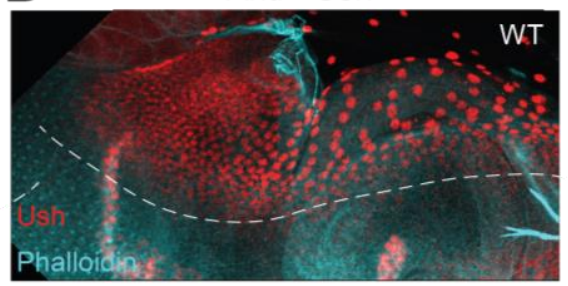

$C^{\prime}$

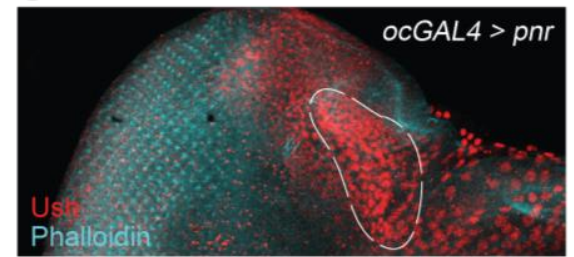

D'

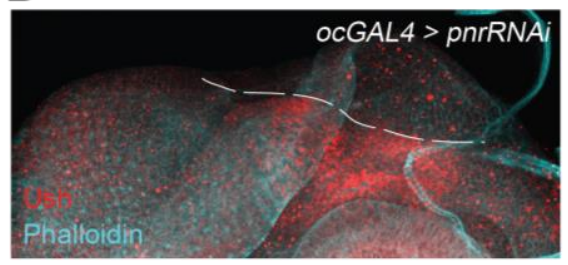

Supplementary Figure 18. A. oc-GAL4>UAS-GFP (Stingerll lline). A'. Vertical section along the red line in the same disc as in A showing GFP expression in a few cells of the disc proper and in the peripodial epithelium. B. Pnr location in D. melanogaster WT eye-antennal disc at $120 \mathrm{~h} \mathrm{AEL,} \mathrm{detected} \mathrm{with} \mathrm{an} \alpha$-Pnr antibody B'. Ush location in D. melanogaster WT eye-antennal disc at $120 \mathrm{~h} \mathrm{AEL.} \mathrm{C.} \mathrm{Overexpression} \mathrm{of} \mathrm{pnr} \mathrm{using} \mathrm{the} \mathrm{oc-GAL4} \mathrm{driver} \mathrm{line}$ leads to a stronger antibody signal in the future oc-region of the developing disc. C'. A slight upregulation of Ush signal can be detected in the future oc-region of the developing disc, upon upregulation of pnr using the oc-GAL4 driver line. White dotted lines mark the border between stronger antibody signals were pnr is overexpressed and weaker endogenous expression. D. Upon knock-down of pnr using the oc-GAL4 driver line, Pnr antibody staining is lost in the future oc-region. D'. Upon knock-down of pnr using the oc-GAL4 driver line, Ush antibody staining is lost in the future ocelli-region. Note that the dorsal part of the eye-antennal disc is folded in this picture. White dotted lines mark the border where antibody signal still can be detected and where it is lost, due to pnr RNAi. 


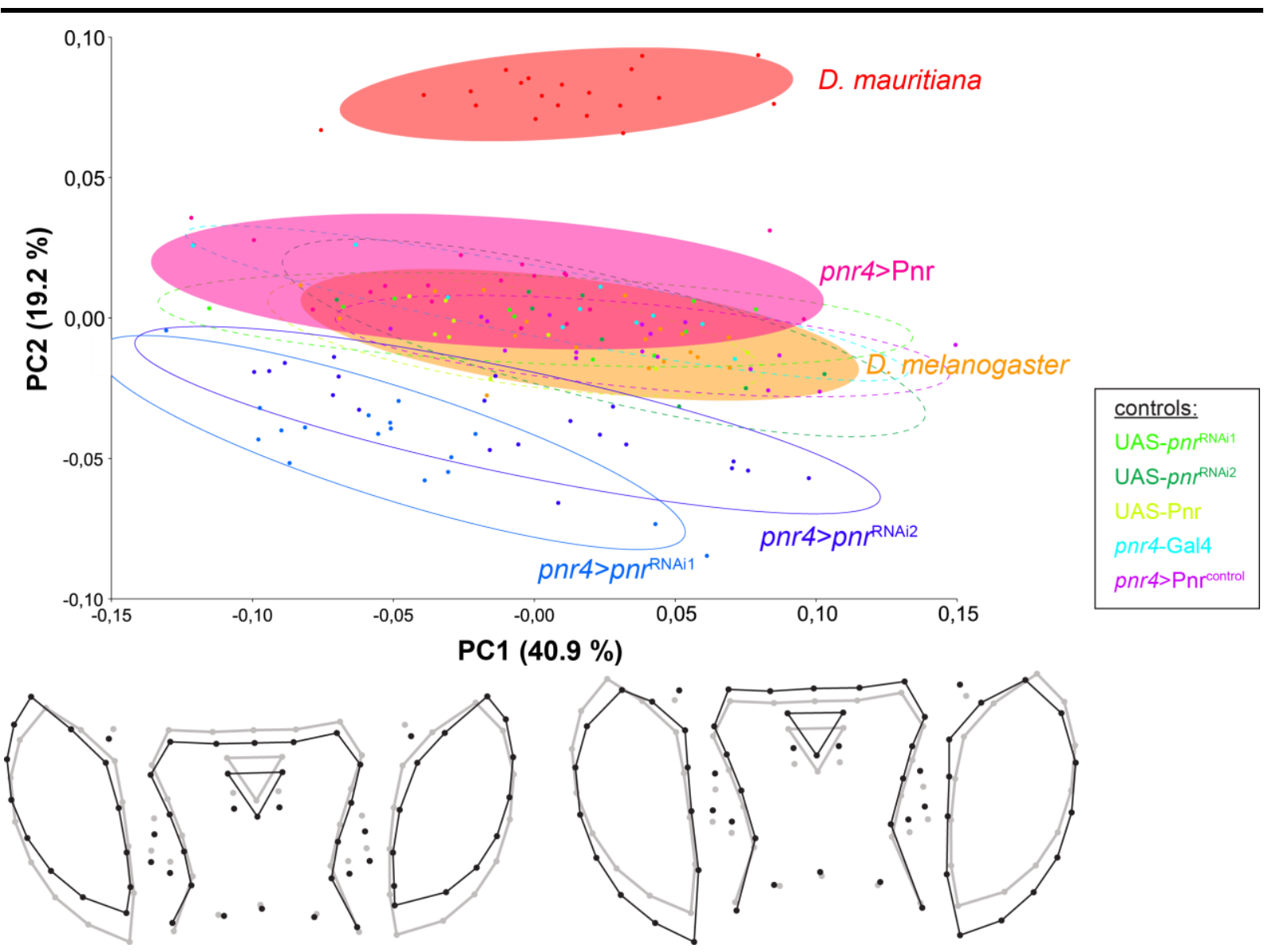

Supplementary Figure 19. Principle component analysis of dorsal head shapes. Shown are PC1 against PC2. Red and orange clouds represent the 'WT-like' head shapes ( $D$. melanogaster in orange and $D$. mauritiana in red). Overexpression of pnr is represented in pink. The blue empty circles represent knock-down of pnr, with a weak effector line in dark blue and a strong effector RNAi line in light blue. The dotted lined circles represent head shapes of the parental UAS- and GAL4 fly lines, that were used to set up the crosses for overexpression and knock-down of pnr. 


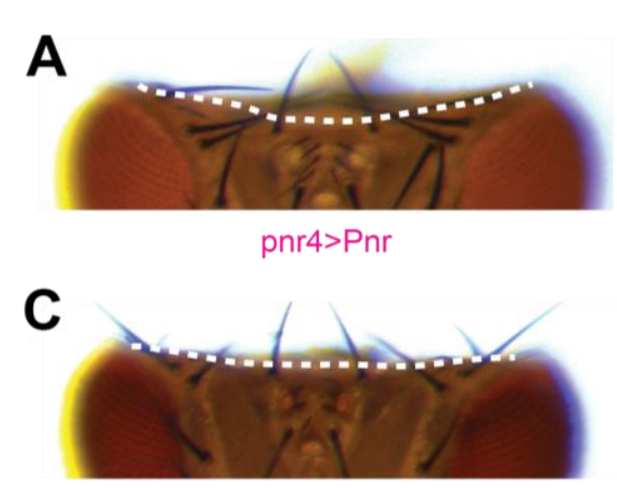

D. melanogaster

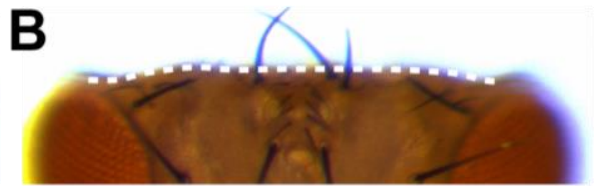

pnr4>pnrRNAi2

D

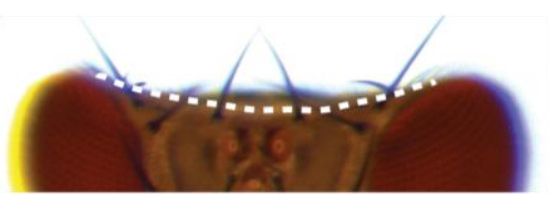

D. mauritiana

$\mathbf{E}$
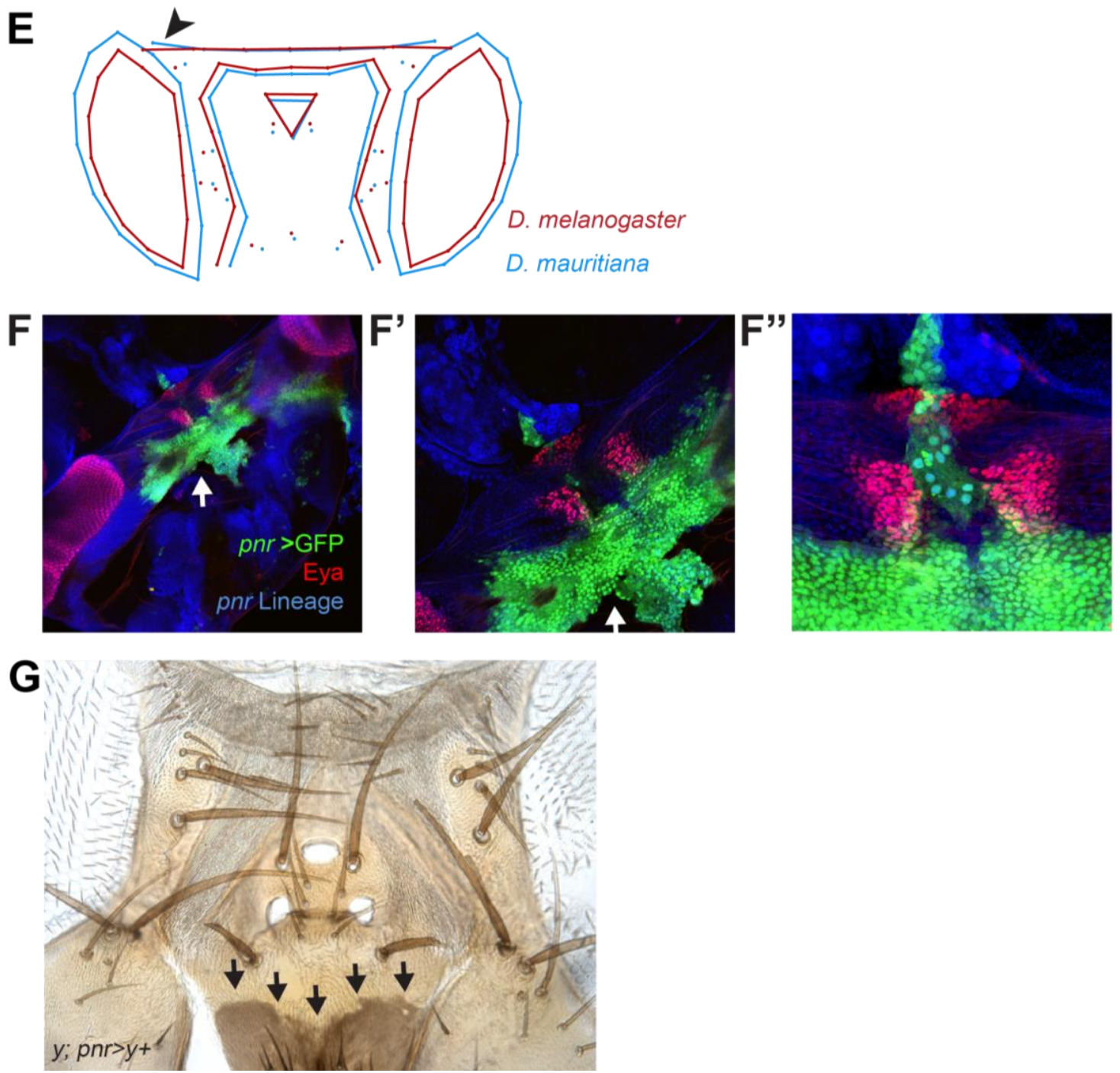

Supplementary Figure 20. A-D. Dorsal-most view of adult heads of D. melanogaster and D. mauritiana WT flies, VT042374>pnr and VT042374>pnr RNAi2 flies. The white dotted line represents the occipital region, showing the variation in this structure in the different lines: A: VT042374>pnr; overexpression of pnr. B. VTO42374>pnr RNAi2 knock-down of pnr. C. D. melanogaster D. D. mauritiana. E. Mean head shapes of D. melanogaster and $D$. mauritiana using 64 landmarks (instead of 57) including the occipital region. Discriminant function analysis clearly reveals the convex form of this region in D. mauritiana (see black arrowhead). F-F"'. pnr-expression in developing pupal head structures. Cells marked with $p n r>G F P$ are accumulating in the future occipital region (green), right behind the developing ocelli (red), and the head region where the two discs are fusing. D. The $y$-rescued area representing the pnr-domain, moves towards the occipital region (black arrows) in the adult Drosophila head. 


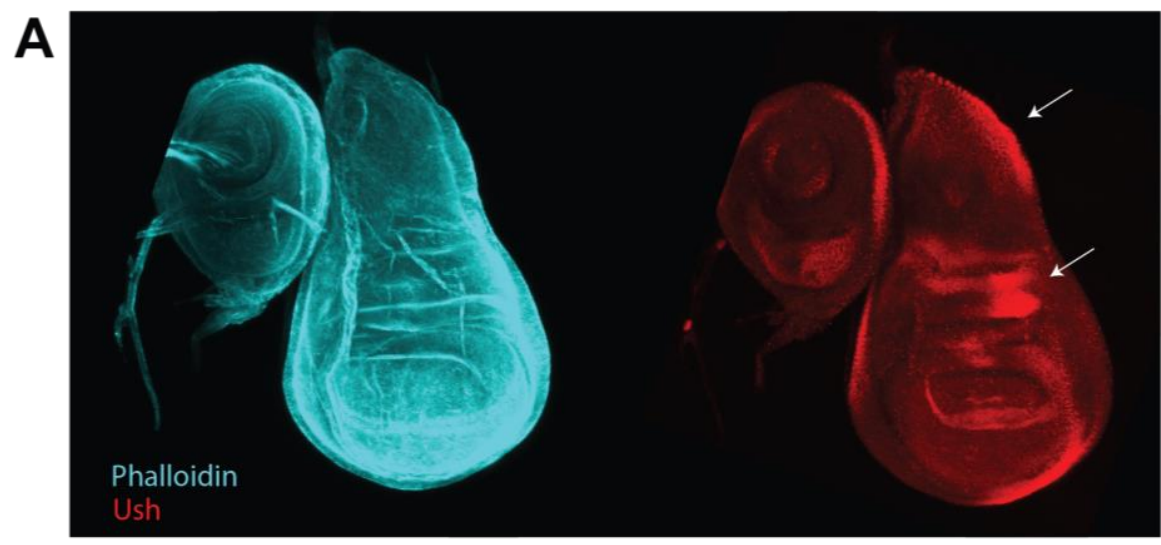

B
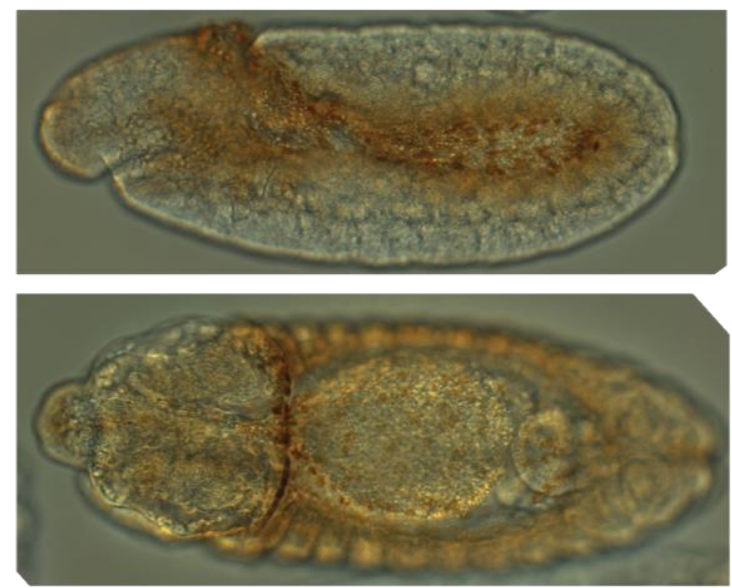

stage 9

$\sim$ stage 13

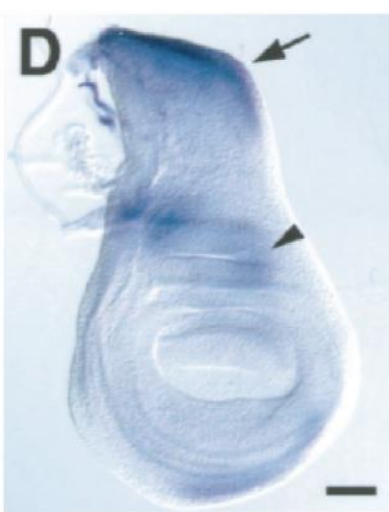

ush, wild-type

Tomoyasu et at. (2000)

Supplementary Material Figure 1. A. Ush protein location in the developing Drosophila wing disc, detected with the newly generated $\alpha$-Ush antibody. The regions where Ush can be detected is reminiscent of the region where ush mRNA was detected using in-situ hybridization in (Tomoyasu et al., 2000) (see white and black arrows). B. Ush protein location in the developing Drosophila embryo at $\sim$ stage 9 and $\sim$ stage 13, detected with an $\alpha$-Ush antibody. 


\subsection{Supplementary Tables}

Supplementary Table 19. List of putative Pnr target genes.

\begin{tabular}{|c|c|c|c|c|c|c|c|}
\hline FBgn & GeneSymbol & FBgn & GeneSymbol & FBgn & GeneSymbol & FBgn & GeneSymbol \\
\hline FBgn0027786 & Mtch & FBgn0015795 & Rab7 & FBgn0030610 & CG9065 & FBgn0004167 & kst \\
\hline FBgn0016984 & sktl & FBgn0264785 & $\mathrm{Hph}$ & FBgn0012051 & CalpA & FBgn0041188 & At $\times 2$ \\
\hline FBgn0053111 & CG33111 & FBgn0052423 & shep & FBgn0032901 & sky & FBgn0261270 & SelD \\
\hline FBgn0086856 & CG11555 & FBgn0011586 & $e(r)$ & FBgn0029840 & raptor & FBgn0263005 & CG43313 \\
\hline FBgn0038834 & RpS30 & FBgn0022029 & $\mathrm{l}(2) \mathrm{k} 01209$ & FBgn0038890 & CG7956 & FBgn0032988 & Tif-IA \\
\hline FBgn0266570 & N066 & FBgn0040212 & Dhap-at & FBgn0023519 & mRpL16 & FBgn0039633 & CG11873 \\
\hline FBgn0038504 & Sur-8 & FBgn0011817 & nmo & FBgn0004876 & cdi & FBgn0044020 & Roc2 \\
\hline FBgn0037358 & elm & FBgn0014020 & Rho1 & FBgn0083968 & CG34132 & FBgn0037363 & Atg17 \\
\hline FBgn0046704 & Liprin-alpha & FBgn0000611 & exd & FBgn0031310 & Vps29 & FBgn0030341 & p24-1 \\
\hline FBgn0036381 & CG8745 & FBgn0033649 & pyr & FBgn0261574 & kug & FBgn0015799 & $\mathrm{Rbf}$ \\
\hline FBgn0015279 & Pi3K92E & FBgn0263144 & bin3 & FBgn0030396 & CG2556 & FBgn0020261 & $\mathrm{pcm}$ \\
\hline FBgn0003660 & Syb & FBgn0038191 & CG9925 & FBgn0031174 & CG1486 & FBgn0027597 & CG17712 \\
\hline FBgn0029662 & CG12206 & FBgn0028484 & Ack & FBgn0003557 & $\mathrm{Su}(\mathrm{dx})$ & FBgn0032633 & Lrch \\
\hline FBgn0260748 & CG5004 & FBgn0000405 & $\mathrm{CycB}$ & FBgn0015789 & Rab10 & FBgn0038787 & CG4360 \\
\hline FBgn0026418 & $\mathrm{Hsc70Cb}$ & FBgn0005671 & Vha55 & FBgn0039929 & CG11076 & FBgn0051698 & CG31698 \\
\hline FBgn0029976 & snz & FBgn0261538 & CG42662 & FBgn0061198 & HSPC300 & FBgn0035989 & CG3967 \\
\hline FBgn0027342 & $\mathrm{fz} 4$ & FBgn0037696 & GstZ1 & FBgn0000017 & Abl & FBgn0003205 & Ras85D \\
\hline FBgn0283477 & SF2 & FBgn0052479 & Usp10 & FBgn0033340 & CG13751 & FBgn0040056 & CG17698 \\
\hline FBgn0030502 & tth & FBgn0032197 & CG5694 & FBgn0003371 & sgg & FBgn0036970 & Spn77Bc \\
\hline FBgn0025574 & $\mathrm{Pli}$ & FBgn0034091 & $m r j$ & FBgn0005659 & Ets98B & FBgn0036257 & RhoGAP68F \\
\hline FBgn0026533 & Dek & FBgn0039158 & TBC1d7 & FBgn0037906 & PGRP-LB & FBgn0031850 & Tsp \\
\hline FBgn0015791 & Rab14 & FBgn0034436 & CG11961 & FBgn0030030 & CG1636 & FBgn0031609 & CG15443 \\
\hline FBgn0035414 & CG14965 & FBgn0026206 & mei-P26 & FBgn0030503 & Tango2 & FBgn0034503 & MED8 \\
\hline FBgn0029944 & Dok & FBgn0000711 & flw & FBgn0052529 & Hers & FBgn0038551 & Odj \\
\hline FBgn0037551 & Arlo & FBgn0040087 & p115 & FBgn0263216 & CG43386 & FBgn0002715 & mei-S332 \\
\hline FBgn0086757 & cbs & FBgn0004837 & $\mathrm{Su}(\mathrm{H})$ & FBgn0037846 & CG6574 & FBgn0259176 & bun \\
\hline FBgn0263603 & Zn72D & FBgn0261524 & lic & FBgn0022787 & Hel89B & FBgn0000183 & $\mathrm{BicD}$ \\
\hline FBgn0050122 & CG30122 & FBgn0004888 & Scsalpha1 & FBgn0026196 & nop5 & FBgn0023143 & Uba1 \\
\hline FBgn0035088 & CG3776 & FBgn0030686 & $\mathrm{mRpL3}$ & FBgn0002645 & Map205 & FBgn0028509 & CenG1A \\
\hline FBgn0261609 & elF2alpha & FBgn0029689 & CG6428 & FBgn0035121 & Tudor-SN & FBgn0250843 & Prosalpha6 \\
\hline FBgn0053156 & CG33156 & FBgn0030873 & CG15814 & FBgn0264712 & CG1172 & FBgn0262117 & IntS3 \\
\hline 30616 & $7 a$ & 028 & CG16717 & 2354 & I(3)87Df & 25936 & Eph \\
\hline FBgn0027866 & CG9776 & FBgn0039233 & CG7006 & FBgn0015331 & abs & FBgn0001138 & grn \\
\hline FBgn0035540 & Syx17 & FBgn0267849 & Syx7 & FBgn0030581 & CG14408 & FBgn0000286 & $\mathrm{Cf} 2$ \\
\hline FBgn0011592 & fra & FBgn0004370 & Ptp10D & FBgn0023212 & EloB & FBgn0032817 & CG10631 \\
\hline FBgn0025864 & Crag & FBgn0263352 & Unr & FBgn0000179 & bi & FBgn0027532 & CG7139 \\
\hline FBgn0037978 & KLHL18 & FBgn0020279 & lig & FBgn0037082 & CG5664 & FBgn0031988 & CG8668 \\
\hline FBgn0011604 & Iswi & FBgn0027553 & NELF-B & FBgn0038156 & side-IV & FBgn0053653 & Cadps \\
\hline FBgn0014879 & Set & FBgn0034194 & CG15611 & FBgn0004177 & $\mathrm{mts}$ & FBgn0038454 & CG10324 \\
\hline FBgn0031253 & CG11885 & FBgn0033463 & CG1513 & FBgn0021874 & Nle & FBgn0035157 & CG13894 \\
\hline FBgn0016977 & spen & FBgn0038143 & CG9813 & FBgn0044826 & Pak3 & FBgn0028984 & Spn88Ea \\
\hline FBgn0037918 & CG6791 & FBgn0032725 & Nedd8 & FBgn0029504 & CHES-1-like & FBgn0038662 & Mpc1 \\
\hline
\end{tabular}




\begin{tabular}{|c|c|c|c|c|c|c|c|}
\hline FBgn0001087 & $\mathrm{g}$ & |FBgn0039140| & Miro & |FBgn0024734 & PRL-1 & |FBgn0031769 & CG9135 \\
\hline FBgn0041087 & wun2 & FBgn0032482 & Pect & FBgn0000015 & $A b d-B$ & FBgn0040344 & CG3711 \\
\hline FBgn0039641 & CG14511 & FBgn0024314 & Plap & FBgn0029801 & CG15771 & FBgn0017581 & Lk6 \\
\hline FBgn0031263 & Tspo & FBgn0039266 & CG11791 & FBgn0004395 & unk & FBgn0002774 & mle \\
\hline FBgn0003317 & sax & FBgn0052177 & Ndfip & FBgn0036926 & CG7646 & FBgn0030435 & CG4645 \\
\hline FBgn0061476 & Zwilch & FBgn0002973 & numb & FBgn0052708 & CG32708 & FBgn0001218 & $\mathrm{Hsc} 70-3$ \\
\hline FBgn0003447 & sn & FBgn0031474 & CG2991 & FBgn0038947 & Sar1 & FBgn0041706 & CG3253 \\
\hline FBgn0259202 & CG42306 & FBgn0032614 & CG13284 & FBgn0259113 & $\begin{array}{l}\text { DNApol- } \\
\text { alpha180 }\end{array}$ & FBgn0004587 & B52 \\
\hline FBgn0039068 & CG13827 & FBgn0002643 & mam & FBgn0027872 & rdgBbeta & FBgn0001995 & $\mathrm{mRpL} 4$ \\
\hline FBgn0002775 & $\mathrm{msl}-3$ & FBgn0039654 & Brd8 & FBgn0035159 & CG13896 & FBgn0016131 & Cdk4 \\
\hline FBgn0020653 & Trxr-1 & FBgn0033229 & CG12822 & FBgn0261550 & CG42668 & FBgn0003423 & $\operatorname{slg} A$ \\
\hline FBgn0037710 & CG9393 & FBgn0038928 & Fadd & FBgn0026375 & RhoGAPp190 & FBgn0030963 & CG7101 \\
\hline FBgn0002431 & hyd & FBgn0031023 & CG14200 & FBgn0030554 & CG1434 & FBgn0264089 & sli \\
\hline FBgn0015037 & Сур4p1 & FBgn0031143 & CG1532 & FBgn0250838 & roh & FBgn0039773 & CG2224 \\
\hline FBgn0261986 & RASSF8 & FBgn0005558 & ey & FBgn0051151 & wge & FBgn0030243 & CG2186 \\
\hline FBgn0015477 & Rme-8 & FBgn0085370 & Pde11 & FBgn0013272 & Gp150 & FBgn0003231 & $\operatorname{ref}(2) P$ \\
\hline FBgn0038981 & CG5346 & FBgn0038053 & CG18549 & FBgn0028688 & Rpn7 & FBgn0031053 & CG14223 \\
\hline FBgn0038870 & Oga & FBgn0039764 & CG15535 & FBgn0033762 & ZnT49B & FBgn0031768 & CG12393 \\
\hline FBgn0010303 & hep & FBgn0040283 & SMC1 & FBgn0015774 & NetB & FBgn0040237 & bor \\
\hline FBgn0039508 & CG3368 & FBgn0040660 & CG13551 & FBgn0027055 & CSN3 & FBgn0027779 & VhaSFD \\
\hline FBgn0024889 & Kap-alpha1 & FBgn0031992 & Acbp1 & FBgn0016693 & Past1 & FBgn0267252 & Ggamma30A \\
\hline FBgn0050338 & CG30338 & FBgn0262733 & Src64B & FBgn0037188 & CG7369 & FBgn0039737 & CG7920 \\
\hline FBgn0036890 & CG9368 & FBgn0051126 & CG31126 & FBgn0037021 & CG11399 & FBgn0022764 & $\sin 3 A$ \\
\hline FBgn0021895 & ytr & FBgn0051915 & CG31915 & FBgn0000536 & eas & FBgn0035094 & CG9380 \\
\hline FBgn0031118 & RhoGAP19D & FBgn0035449 & CG14971 & FBgn0041111 & lilli & FBgn0262517 & Ltn1 \\
\hline FBgn0015622 & Cnx99A & FBgn0003002 & opa & FBgn0261244 & inaE & FBgn0037841 & CG4565 \\
\hline FBgn0039904 & Hcf & FBgn0037468 & CG1943 & FBgn0086359 & Invadolysin & FBgn0039226 & Ude \\
\hline FBgn0015229 & glec & FBgn0029887 & CG3198 & FBgn0033052 & SCAP & FBgn0038578 & MED17 \\
\hline FBgn0003525 & stg & FBgn0000319 & Chc & FBgn0037900 & CG5276 & FBgn0032223 & GATAd \\
\hline FBgn0038745 & CG4538 & FBgn0036913 & Usp32 & FBgn0039938 & Sox102F & FBgn0263258 & chas \\
\hline FBgn0026257 & cav & FBgn0033961 & ND-B15 & FBgn0001105 & Gbeta13F & FBgn0260962 & pic \\
\hline FBgn0013954 & Fkbp12 & FBgn0003301 & rut & FBgn0003415 & skd & FBgn0038256 & CG7530 \\
\hline FBgn0030420 & CG12717 & FBgn0033089 & CG17266 & FBgn0033921 & tej & FBgn0032919 & CG9253 \\
\hline FBgn0086784 & stmA & FBgn0051075 & CG31075 & FBgn0283473 & S6KL & FBgn0038826 & Syp \\
\hline FBgn0013305 & Nmda1 & FBgn0263993 & CG43736 & FBgn0030082 & HP1b & FBgn0004657 & mys \\
\hline FBgn0026373 & Rpll33 & FBgn0037669 & Ibf2 & FBgn0034674 & CG9304 & FBgn0028476 & Usp1 \\
\hline FBgn0260632 & $\mathrm{dl}$ & FBgn0262656 & Myc & FBgn0034853 & Ice1 & FBgn0030572 & $\mathrm{mRpS} 25$ \\
\hline FBgn0034573 & CG3295 & FBgn0034878 & pita & FBgn0030786 & $\mathrm{mRpL} 22$ & FBgn0051436 & CG31436 \\
\hline FBgn0262614 & pyd & FBgn0037012 & Rcd2 & FBgn0023529 & CG2918 & FBgn0038953 & CG18596 \\
\hline FBgn0039160 & CG5510 & FBgn0032943 & Tsp39D & FBgn0030478 & CG1640 & FBgn0266696 & Svil \\
\hline FBgn0085377 & CG34348 & FBgn0041342 & Pcyt1 & FBgn0030744 & CG9992 & FBgn0265630 & sno \\
\hline FBgn0052133 & Ptip & FBgn0028375 & heix & FBgn0283499 & $\ln R$ & FBgn0020655 & ArfGAP1 \\
\hline FBgn0027280 & I(1)G0193 & FBgn0259749 & $\mathrm{mmy}$ & FBgn0038686 & CG5555 & FBgn0265434 & zip \\
\hline FBgn0001124 & Got1 & FBgn0087013 & Karybeta3 & FBgn0261799 & $d s x-c 73 A$ & FBgn0027621 & Pfrx \\
\hline FBgn0283724 & Girdin & FBgn0031779 & CG9175 & FBgn0004401 & Pep & FBgn0035824 & CG8281 \\
\hline FBgn0028662 & VhaPPA1-1 & FBgn0024973 & CG2701 & FBgn0026179 & siz & FBgn0033127 & Tsp42Ef \\
\hline
\end{tabular}




\begin{tabular}{|c|c|c|c|c|c|c|c|}
\hline FBgn0034997 & CG3376 & FBgn0029820 & CG16721 & FBgn0036697 & rogdi & FBgn0010621 & CCT5 \\
\hline FBgn0033770 & wuc & FBgn0037614 & TMEM216 & FBgn0004903 & Rb97D & FBgn0261439 & SdhA \\
\hline FBgn0264307 & orb2 & FBgn0030403 & CG1824 & FBgn0003274 & RpLP2 & FBgn0260970 & Ubr3 \\
\hline FBgn0031449 & CG31689 & FBgn0000810 & $\mathrm{fs}(1) \mathrm{K} 10$ & FBgn0003396 & shn & FBgn0025839 & ND-B14.5A \\
\hline FBgn0038659 & EndoA & FBgn0030505 & NFAT & FBgn0034657 & LBR & FBgn0035357 & MEP-1 \\
\hline FBgn0039155 & Kal1 & FBgn0035907 & GstO1 & FBgn0030973 & CG7332 & FBgn0039966 & Rab21 \\
\hline FBgn0028897 & CG4935 & FBgn0039215 & CG6695 & FBgn0038877 & CG3308 & FBgn0031505 & ND-B14.5B \\
\hline FBgn0031574 & TTLL4B & FBgn0261647 & Axud1 & FBgn0005648 & Pabp2 & FBgn0284250 & Oaz \\
\hline FBgn0028541 & TM9SF4 & FBgn0028695 & Rpn1 & FBgn0003134 & Pp1alpha-96A & FBgn0038471 & CG5220 \\
\hline FBgn0013983 & imd & FBgn0037944 & CG6923 & FBgn0025394 & inc & FBgn0003165 & pum \\
\hline FBgn0052141 & saturn & FBgn0037874 & Tctp & FBgn0033951 & CG10139 & FBgn0011606 & KIp3A \\
\hline FBgn0031549 & Spindly & FBgn0010265 & RpS13 & FBgn0030956 & CG18259 & FBgn0028968 & gammaCOP \\
\hline FBgn0004913 & Gnf1 & FBgn0020309 & crol & FBgn0015623 & Cpr & FBgn0267791 & HnRNP-K \\
\hline FBgn0031183 & CG14621 & FBgn0016685 & Nlp & FBgn0034914 & CG5554 & FBgn0027492 & $w d b$ \\
\hline FBgn0002590 & RpS5a & FBgn0028408 & Drep2 & FBgn0031682 & CG5828 & FBgn0261823 & Asx \\
\hline FBgn0266084 & Fhos & FBgn0024754 & Flo1 & FBgn0259876 & Cap-G & FBgn0031681 & Pgant5 \\
\hline FBgn0031161 & CG15445 & FBgn0265192 & Snp & FBgn0030055 & CG12772 & FBgn0029903 & pod1 \\
\hline FBgn0032821 & CdGAPr & FBgn0002873 & mud & FBgn0043903 & dome & FBgn0022153 & $\mathrm{I}(2) \mathrm{k} 05819$ \\
\hline FBgn0036373 & Tgi & FBgn0051683 & CG31683 & FBgn0030930 & Pgant7 & FBgn0029708 & CG3556 \\
\hline FBgn0029709 & CHOp24 & FBgn0061200 & Nup153 & FBgn0086361 & alph & FBgn0025741 & PlexA \\
\hline FBgn0010348 & Arf79F & FBgn0039213 & atl & FBgn0010488 & NAT1 & FBgn0000479 & dnc \\
\hline FBgn0051992 & gw & FBgn0035909 & ergic53 & FBgn0002638 & Rcc1 & FBgn0017567 & ND-23 \\
\hline FBgn0260780 & wisp & FBgn0025185 & az2 & FBgn0265784 & CrebB & FBgn0027343 & $\mathrm{fz3}$ \\
\hline FBgn0001169 & $\mathrm{H}$ & FBgn0031250 & Ent1 & FBgn0265052 & St3 & FBgn0011272 & RpL13 \\
\hline FBgn0032656 & CG5674 & FBgn0010408 & RpS9 & FBgn0033673 & CG8298 & FBgn0000404 & CycA \\
\hline FBgn0037912 & sea & FBgn0031144 & CG1529 & FBgn0003159 & CG2841 & FBgn0001941 & ifc \\
\hline FBgn0015024 & Cklalpha & FBgn0039705 & Atg16 & FBgn0039590 & CG10011 & FBgn0025724 & beta'COP \\
\hline FBgn0013749 & Arf102F & FBgn0039851 & mey & FBgn0028360 & Cdc7 & FBgn0036974 & eRF1 \\
\hline FBgn0028717 & Lnk & FBgn0036762 & CG7430 & FBgn0034528 & CG11180 & FBgn0035689 & CG7376 \\
\hline FBgn0265140 & Meltrin & FBgn0033154 & CG1850 & FBgn0033199 & CG17985 & FBgn0265298 & SC35 \\
\hline FBgn0263987 & spoon & FBgn0010198 & RpS15Aa & FBgn0010113 & hdc & FBgn0034240 & MESR4 \\
\hline FBgn0022213 & Cse1 & FBgn0000473 & Сур6а2 & FBgn0037238 & CG1090 & FBgn0024555 & $f|f|$ \\
\hline FBgn0030693 & CG8974 & FBgn0015320 & Ubc2 & FBgn0039635 & Pdhb & FBgn0037073 & Tsr1 \\
\hline FBgn0039026 & CG7029 & FBgn0022960 & vimar & FBgn0051989 & Cap-D3 & FBgn0037234 & CG9795 \\
\hline FBgn0039338 & XNP & FBgn0034570 & CG10543 & FBgn0025382 & Rab27 & FBgn0028331 & I(1)G0289 \\
\hline FBgn0023130 & a6 & FBgn0004907 & 14-3-3zeta & FBgn0053469 & CG33469 & FBgn0030592 & CG9514 \\
\hline FBgn0037354 & CG12171 & FBgn0052280 & CG32280 & FBgn0263392 & Tet & FBgn0027339 & jim \\
\hline FBgn0035558 & CG11357 & FBgn0264975 & $\mathrm{Nrg}$ & FBgn0039136 & CG5902 & FBgn0025335 & Cpes \\
\hline FBgn0025681 & CG3558 & FBgn0015270 & Orc2 & FBgn0028425 & Jhl-21 & FBgn0032029 & CG17292 \\
\hline FBgn0001075 & $\mathrm{ft}$ & FBgn0041585 & olf186-F & FBgn0011656 & Mef2 & FBgn0283657 & Tlk \\
\hline FBgn0263231 & bel & FBgn0034742 & CG4294 & FBgn0029679 & CG2901 & FBgn0086694 & Bre1 \\
\hline FBgn0034230 & CG4853 & FBgn0001269 & inv & FBgn0005655 & PCNA & FBgn0031037 & CG14207 \\
\hline FBgn0024184 & unc-4 & FBgn0038197 & foxo & FBgn0003139 & $\mathrm{PpV}$ & FBgn0036828 & CG6841 \\
\hline FBgn0024733 & RpL10 & FBgn0031145 & $\mathrm{Ntf}-2$ & FBgn0039665 & CG2310 & FBgn0028693 & Rpn12 \\
\hline FBgn0002044 & swm & FBgn0015218 & elF4E1 & FBgn0039908 & Asator & FBgn0032006 & Pvr \\
\hline FBgn0038320 & Sra-1 & FBgn0035046 & ND-19 & FBgn0032363 & Dlg5 & FBgn0041186 & Slbp \\
\hline FBgn0261885 & osa & FBgn0267912 & CanA-14F & FBgn0264294 & Cyt-b5 & FBgn0032475 & Sfmbt \\
\hline
\end{tabular}




\begin{tabular}{|c|c|c|c|c|c|c|c|}
\hline FBgn0031717 & Oscillin & FBgn0250786 & Chd1 & FBgn0003391 & shg & FBgn0032339 & Wdr59 \\
\hline FBgn0034313 & CG5726 & FBgn0025830 & IntS8 & FBgn0000719 & fog & FBgn0036566 & $\mathrm{ClC}-\mathrm{C}$ \\
\hline FBgn0020412 & JIL-1 & FBgn0283536 & Vha13 & FBgn0003042 & $\mathrm{Pc}$ & FBgn0024909 & Taf7 \\
\hline FBgn0021760 & chb & FBgn0003701 & thr & FBgn0003189 & $r$ & FBgn0050372 & Asap \\
\hline FBgn0085693 & CG41562 & FBgn0029006 & Smurf & FBgn0052212 & CG32212 & FBgn0039920 & CG11360 \\
\hline FBgn0001139 & gro & FBgn0010583 & dock & FBgn0031883 & Caper & FBgn0024251 & $b b x$ \\
\hline FBgn0036449 & $\mathrm{bmm}$ & FBgn0035452 & CG10359 & FBgn0029878 & Pat1 & FBgn0038755 & Hs6st \\
\hline FBgn0030349 & CG10353 & FBgn0260858 & Ykt6 & FBgn0030049 & Trf4-1 & FBgn0029095 & aru \\
\hline FBgn0038350 & AOX4 & FBgn0262114 & RanBPM & FBgn0262734 & elF4H1 & FBgn0032075 & Tsp29Fb \\
\hline FBgn0029990 & CG2233 & FBgn0037282 & CG14657 & FBgn0028394 & CG17834 & FBgn0033925 & CG8617 \\
\hline FBgn0001301 & kel & FBgn0040208 & Kat60 & FBgn0261268 & Cul3 & FBgn0032848 & nesd \\
\hline FBgn0004655 & wapl & FBgn0036476 & sstn & FBgn0051108 & TTLL5 & FBgn0003716 & tkv \\
\hline FBgn0030366 & Usp7 & FBgn0029825 & CG12728 & FBgn0033715 & CG8490 & FBgn0036240 & CG6928 \\
\hline FBgn0011754 & PhKgamma & FBgn0003731 & Egfr & FBgn0042134 & Capr & FBgn0036964 & FRG1 \\
\hline FBgn0037092 & M6 & FBgn0053181 & CG33181 & FBgn0031314 & IntS14 & FBgn0010620 & CG10939 \\
\hline FBgn0037549 & CG7878 & FBgn0260462 & CG12163 & FBgn0034418 & CG15118 & FBgn0085430 & CG34401 \\
\hline FBgn0283741 & prage & FBgn0000581 & $\mathrm{E}(\mathrm{Pc})$ & FBgn0034488 & $\mathrm{Hacl}$ & FBgn0052756 & CG32756 \\
\hline FBgn0037561 & CG9630 & FBgn0029685 & CG2938 & FBgn0261444 & CG3638 & FBgn0000261 & Cat \\
\hline FBgn0038853 & RhoGAP93B & FBgn0015790 & Rab11 & FBgn0260400 & elav & FBgn0031881 & MME1 \\
\hline FBgn0004959 & phm & FBgn0083984 & CG34148 & FBgn0041174 & Vhl & FBgn0001189 & hfw \\
\hline FBgn0029629 & elF3g1 & FBgn0000394 & $\mathrm{cv}$ & FBgn0037814 & CG6325 & FBgn0035533 & Cip4 \\
\hline FBgn0020440 & Fak & FBgn0038976 & Pfdn5 & FBgn0037391 & CG2017 & FBgn0035120 & wac \\
\hline FBgn0030996 & CG14194 & FBgn0039852 & nyo & FBgn0034854 & Golgin245 & FBgn0024957 & Irp-1B \\
\hline FBgn0023458 & Rbcn-3A & FBgn0036846 & MESR6 & FBgn0039065 & Rad60 & FBgn0033519 & CG11825 \\
\hline FBgn0034763 & RYBP & FBgn0265082 & Cdep & FBgn0031036 & CG14220 & FBgn0019637 & Atu \\
\hline FBgn0028506 & CG4455 & FBgn0004864 & hop & FBgn0261019 & moi & FBgn0029958 & Pdp \\
\hline FBgn0031492 & CG3542 & FBgn0267326 & $\mathrm{Ntl}$ & FBgn0027932 & Akap200 & FBgn0034225 & veil \\
\hline FBgn0035148 & CG3402 & FBgn0052831 & CG33695 & FBgn0028494 & CG6424 & FBgn0004397 & Vinc \\
\hline FBgn0028474 & CG4119 & FBgn0261705 & CG42741 & FBgn0035001 & Slik & FBgn0019968 & Khc-73 \\
\hline FBgn0030912 & CG6023 & FBgn0083167 & Neb-cGP & FBgn0053293 & CG33293 & FBgn0037138 & P5CDh1 \\
\hline FBgn0003117 & pnr & FBgn0015524 & otp & FBgn0260789 & $\mathrm{mxc}$ & FBgn0266111 & ana3 \\
\hline FBgn0033774 & CG12374 & FBgn0010328 & woc & FBgn0014388 & sty & FBgn0267390 & dop \\
\hline FBgn0020249 & stck & FBgn0262127 & kibra & FBgn0263705 & Myo10A & FBgn0052758 & CG32758 \\
\hline FBgn0259168 & $\mathrm{mnb}$ & FBgn0025615 & Torsin & FBgn0032444 & CCT4 & FBgn0035890 & CG13667 \\
\hline FBgn0265003 & koi & FBgn0025628 & CG4199 & FBgn0021856 & I(2)k14505 & FBgn0037218 & aux \\
\hline FBgn0262743 & Fs(2)Ket & FBgn0026259 & elF5B & FBgn0052767 & CG32767 & FBgn0034708 & Vps35 \\
\hline FBgn0001978 & stc & FBgn0031126 & Cyp6v1 & FBgn0030869 & Socs16D & FBgn0085220 & Ufm1 \\
\hline FBgn0052473 & CG32473 & FBgn0031488 & CG17265 & FBgn0261722 & fwe & FBgn0025637 & SkpA \\
\hline FBgn0025455 & Суст & FBgn0032341 & Reps & FBgn0086906 & sls & FBgn0037525 & CG17816 \\
\hline FBgn0067622 & LSm-4 & FBgn0031044 & MKP-4 & FBgn0035713 & velo & FBgn0262740 & Evi5 \\
\hline FBgn0004103 & Pp1-87B & FBgn0000283 & Cp190 & FBgn0032899 & CG9338 & FBgn0051184 & LSm3 \\
\hline FBgn0038737 & CG11447 & FBgn0034061 & Ufc1 & FBgn0017418 & ari-1 & FBgn0026376 & $\mathrm{Rgl}$ \\
\hline FBgn0003310 & $\mathrm{S}$ & FBgn0039914 & mav & FBgn0025693 & ZnT41F & FBgn0021825 & DCTN2-p5c \\
\hline FBgn0086368 & tw & FBgn0035644 & $\begin{array}{l}\text { DNApol- } \\
\text { epsilon58 }\end{array}$ & FBgn0040309 & Jafrac1 & FBgn0015772 & Nak \\
\hline FBgn0039265 & CG11790 & FBgn0039733 & CG11504 & FBgn0037892 & $\mathrm{mRpL} 40$ & FBgn0015379 & dod \\
\hline FBgn0041604 & dlp & FBgn0261570 & CG42684 & FBgn0015754 & Lis-1 & FBgn0263108 & BtbVII \\
\hline
\end{tabular}




\begin{tabular}{|c|c|c|c|c|c|c|c|}
\hline FBgn0025885 & Inos & FBgn0033033 & scaf & FBgn0259481 & Mob2 & FBgn0019925 & Surf4 \\
\hline FBgn0033259 & CG11210 & FBgn0260442 & rhea & FBgn0033766 & Nup188 & FBgn0263257 & Cngl \\
\hline FBgn0038769 & CG10889 & FBgn0029929 & CG4593 & FBgn0035432 & ZnT63C & FBgn0001248 & Idh \\
\hline FBgn0035213 & CG2199 & FBgn0025633 & CG13366 & FBgn0000259 & Ckllbeta & FBgn0039664 & CG2006 \\
\hline FBgn0035617 & I(3)psg2 & FBgn0030327 & FucT6 & FBgn0043900 & pygo & FBgn0087035 & AGO2 \\
\hline FBgn0036008 & CG3408 & FBgn0030121 & Cfp1 & FBgn0010531 & Ccs & FBgn0033005 & CG3107 \\
\hline FBgn0035771 & Sec63 & FBgn0027951 & MTA1-like & FBgn0010808 & Chchd3 & FBgn0033480 & $\mathrm{mRpL} 42$ \\
\hline FBgn0013987 & MAPk-Ak2 & FBgn0020312 & Tmtc3 & FBgn0002542 & Ids & FBgn0031540 & Pif1 \\
\hline FBgn0032833 & $\operatorname{cox} 4$ & FBgn0024556 & mEFTu1 & FBgn0265193 & Atf-2 & FBgn0036340 & $\mathrm{SRm} 160$ \\
\hline FBgn0053265 & Muc68E & FBgn0015621 & Clp & FBgn0011224 & heph & FBgn0035388 & CG2162 \\
\hline FBgn0003891 & tud & FBgn0265101 & Sgt1 & FBgn0052816 & CG32816 & FBgn0016926 & Pino \\
\hline FBgn0030218 & CG1628 & FBgn0022699 & D19B & FBgn0260392 & CG42518 & FBgn0052772 & CG32772 \\
\hline FBgn0035574 & RhoGEF64C & FBgn0066084 & RpL41 & FBgn0015269 & Nf1 & FBgn0035630 & CG10576 \\
\hline FBgn0028687 & Rpt1 & FBgn0052425 & CG32425 & FBgn0263395 & hppy & FBgn0064766 & CG7600 \\
\hline FBgn0033844 & $\mathrm{bbc}$ & FBgn0260450 & CalpC & FBgn0001491 & $\mathrm{I}(1) 10 \mathrm{Bb}$ & FBgn0039680 & Cap-D2 \\
\hline FBgn0016917 & Stat92E & FBgn0262126 & $\operatorname{Sec} 24 C D$ & FBgn0003392 & shi & FBgn0035356 & CG16986 \\
\hline FBgn0261573 & CoRest & FBgn0036309 & Hip1 & FBgn0029763 & Usp16-45 & FBgn0027066 & Eb1 \\
\hline FBgn0002283 & I(3)73Ah & FBgn0263237 & Cdk7 & FBgn0034049 & bdg & FBgn0034527 & CG9945 \\
\hline FBgn0034925 & CG5339 & FBgn0085451 & htk & FBgn0027865 & Tsp96F & FBgn0000289 & $c g$ \\
\hline FBgn0032751 & CG17343 & FBgn0086372 & lap & FBgn0011336 & Stt3B & FBgn0034500 & CG11200 \\
\hline FBgn0041789 & Pax & FBgn0045038 & Proc & FBgn0001316 & klar & FBgn0266717 & Bruce \\
\hline FBgn0031150 & bves & FBgn0052296 & Mrtf & FBgn0034542 & Fem-1 & FBgn0031874 & CG13775 \\
\hline FBgn0024998 & CG2685 & FBgn0040251 & Ugt302K1 & FBgn0261934 & dikar & FBgn0062413 & Ctr1A \\
\hline FBgn0037998 & $\operatorname{Cog} 1$ & FBgn0043884 & mask & FBgn0050389 & CG30389 & FBgn0030141 & Gga \\
\hline FBgn0026379 & Pten & FBgn0261477 & slim & FBgn0038269 & Rrp6 & FBgn0030809 & Ubr1 \\
\hline FBgn0035640 & $\operatorname{mad} 2$ & FBgn0038145 & Droj2 & FBgn0262527 & nsl1 & FBgn0003575 & su(sable) \\
\hline FBgn0031635 & tank & FBgn0039741 & CG7943 & FBgn0283500 & Sac1 & FBgn0035480 & CG14984 \\
\hline FBgn0000617 & $e(y) 1$ & FBgn0034504 & CG8929 & FBgn0003079 & Raf & FBgn0032050 & CG13096 \\
\hline FBgn0263968 & nonc & FBgn0051064 & CG31064 & FBgn0261456 & hpo & FBgn0266525 & CG45092 \\
\hline FBgn0040493 & grsm & FBgn0005585 & Calr & FBgn0260743 & GC1 & FBgn0024846 & p38b \\
\hline FBgn0036575 & CG5157 & FBgn0015371 & chn & FBgn0005427 & ewg & FBgn0031575 & Cep97 \\
\hline FBgn0261599 & RpS29 & FBgn0026679 & IntS4 & FBgn0011760 & $\operatorname{ctp}$ & FBgn0035024 & CG11414 \\
\hline FBgn0035942 & ValRS-m & FBgn0000635 & Fas2 & FBgn0036052 & CG10809 & FBgn0266917 & Sf3a1 \\
\hline FBgn0016983 & smid & FBgn0037215 & beta-Man & FBgn0052672 & Atg8a & FBgn0026149 & BCL7-like \\
\hline FBgn0053062 & CG33062 & FBgn0027505 & Rab3-GAP & FBgn0052264 & CG32264 & FBgn0243486 & rdo \\
\hline FBgn0035719 & tow & FBgn0024432 & Dlc90F & FBgn0035087 & CG2765 & FBgn0015737 & $\mathrm{Hmu}$ \\
\hline FBgn0037720 & CG8312 & FBgn0003204 & ras & FBgn0052451 & SPoCk & FBgn0086676 & spin \\
\hline FBgn0036376 & Liprin-beta & FBgn0039857 & RpL6 & FBgn0028686 & Rpt3 & FBgn0039293 & Alg9 \\
\hline FBgn0051324 & CG31324 & FBgn0039209 & REPTOR & FBgn0003360 & ses $B$ & FBgn0011573 & Cdc37 \\
\hline FBgn0022382 & Pka-R2 & FBgn0037270 & elF3f1 & FBgn0026316 & Ubc10 & FBgn0051457 & CG31457 \\
\hline FBgn0004107 & Cdk2 & FBgn0040334 & Tsp3A & FBgn0261108 & Atg13 & FBgn0028916 & CG33090 \\
\hline FBgn0010497 & dmGlut & FBgn0003261 & Rm62 & FBgn0031090 & Rab35 & FBgn0032378 & $\mathrm{CycY}$ \\
\hline FBgn0030674 & CG8184 & FBgn0001197 & His2Av & FBgn0030360 & CG1806 & FBgn0028689 & Rpn6 \\
\hline FBgn0004435 & Galphaq & FBgn0024811 & Crk & FBgn0083969 & CG34133 & FBgn0037702 & CG8176 \\
\hline FBgn0010288 & Uch & FBgn0267698 & Pak & FBgn0040239 & bc10 & FBgn0034067 & CG8399 \\
\hline FBgn0000625 & eyg & FBgn0051217 & $\bmod S P$ & FBgn0012037 & Ance & FBgn0026428 & HDAC6 \\
\hline FBgn0039620 & wat & FBgn0036932 & CG14184 & FBgn0033929 & Tfb1 & FBgn0039835 & $\mathrm{mRpL} 32$ \\
\hline
\end{tabular}




\begin{tabular}{|c|c|c|c|c|c|c|c|}
\hline FBgn0045866 & bai & FBgn0030808 & RhoGAP15B & FBgn0036483 & CG12316 & FBgn0086855 & CG17078 \\
\hline FBgn0037135 & CG7414 & FBgn0002936 & ninaA & FBgn0259483 & Mob4 & FBgn0004652 & fru \\
\hline FBgn0036341 & Syx13 & FBgn0028582 & lqf & FBgn0264075 & tgo & FBgn0020626 & Osbp \\
\hline FBgn0030519 & CG11151 & FBgn0004870 & bab1 & FBgn0033261 & udd & FBgn0058191 & CG40191 \\
\hline FBgn0031736 & CG11030 & FBgn0024236 & foi & FBgn0038055 & trus & FBgn0011771 & Hem \\
\hline FBgn0037911 & CG10898 & FBgn0031420 & Atxn7 & FBgn0019662 & qm & FBgn0039932 & fuss \\
\hline FBgn0033757 & muskelin & FBgn0034728 & $\operatorname{rad} 50$ & FBgn0028504 & CG12182 & FBgn0000044 & Act57B \\
\hline FBgn0035497 & CG14995 & FBgn0261954 & east & FBgn0025638 & Roc1a & FBgn0032886 & CG9328 \\
\hline FBgn0037440 & CRAT & FBgn0022349 & CG1910 & FBgn0261985 & Ptpmeg & FBgn0051279 & CG31279 \\
\hline FBgn0031320 & CG5126 & FBgn0026252 & msk & FBgn0029935 & CG4615 & FBgn0036058 & CG6707 \\
\hline FBgn0030753 & rngo & FBgn0039623 & CG1951 & FBgn0033668 & $\exp$ & FBgn0031374 & Wdr62 \\
\hline FBgn0024807 & DIP1 & FBgn0052262 & CG32262 & FBgn0040230 & dbo & FBgn0283468 & slmb \\
\hline FBgn0263974 & qin & FBgn0000721 & for & FBgn0260653 & serp & FBgn0034735 & CG4610 \\
\hline FBgn0032330 & Samuel & FBgn0086704 & stops & FBgn0267347 & squ & FBgn0004875 & enc \\
\hline FBgn0030114 & CG17754 & FBgn0037220 & CG14641 & FBgn0250830 & CG12547 & FBgn0038872 & Nelf-A \\
\hline FBgn0267975 & vib & FBgn0028956 & mthl3 & FBgn0029849 & Efr & FBgn0037831 & Cap-H2 \\
\hline FBgn0037743 & CG8412 & FBgn0002921 & Atpalpha & FBgn0004050 & z & FBgn0035147 & Gale \\
\hline FBgn0263106 & DnaJ-1 & FBgn0037680 & pasi2 & FBgn0011236 & ken & FBgn0030065 & CG12075 \\
\hline FBgn0001994 & crp & FBgn0031244 & CG11601 & FBgn0260659 & CG42542 & FBgn0035443 & CG12010 \\
\hline FBgn0012058 & $\mathrm{Cdc} 27$ & FBgn0051122 & CG31122 & FBgn0040238 & Best1 & FBgn0034722 & Rtf1 \\
\hline FBgn0037679 & Aduk & FBgn0035101 & p130CAS & FBgn0039487 & gb & FBgn0015396 & jumu \\
\hline FBgn0025743 & $\mathrm{mbt}$ & FBgn0030648 & CG6340 & FBgn0000163 & baz & FBgn0030137 & CG15317 \\
\hline FBgn0034180 & Ehbp1 & FBgn0026160 & tna & FBgn0032774 & CG17549 & FBgn0030321 & CG1703 \\
\hline FBgn0027561 & CG18659 & FBgn0034475 & Obp56h & FBgn0261592 & RpS6 & FBgn0039668 & Trc8 \\
\hline FBgn0026879 & CG13364 & FBgn0265998 & Doa & FBgn0031873 & Gas41 & FBgn0035393 & CG16753 \\
\hline FBgn0259704 & Nsun5 & FBgn0016754 & sba & FBgn0036254 & CG5645 & FBgn0030089 & AP-1gamma \\
\hline FBgn0032715 & CG17597 & FBgn0038956 & $\mathrm{CAH} 8$ & FBgn0266918 & CG32486 & FBgn0030354 & Upf1 \\
\hline FBgn0025639 & Hmt4-20 & FBgn0025463 & Bap60 & FBgn0051739 & AspRS-m & FBgn0035016 & CG4612 \\
\hline FBgn0032957 & CG2225 & FBgn0027497 & Madm & FBgn0034530 & Rcd6 & FBgn0036039 & Naa60 \\
\hline FBgn0050421 & Usp15-31 & FBgn0016694 & Pdp1 & FBgn0032815 & CG10462 & FBgn0033549 & mms4 \\
\hline FBgn0026250 & elF1A & FBgn0032949 & Lamp1 & FBgn0026239 & gukh & FBgn0026086 & Adar \\
\hline FBgn0028684 & Rpt5 & FBgn0038039 & CG5196 & FBgn0004654 & Pgd & FBgn0034918 & Pym \\
\hline FBgn0035021 & CG4622 & FBgn0015278 & Pi3K68D & FBgn0283509 & Phm & FBgn0037856 & Leash \\
\hline FBgn0046687 & Tre1 & FBgn0033100 & CG3420 & FBgn0260484 & HIP & FBgn0035270 & CG13933 \\
\hline FBgn0040964 & CG18661 & FBgn0005631 & robo1 & FBgn0053113 & Rtnl1 & FBgn0086613 & Ino80 \\
\hline FBgn0085481 & CG34452 & FBgn0036684 & CG3764 & FBgn0029893 & CG14442 & FBgn0028325 & Pdha \\
\hline FBgn0027546 & CG4766 & FBgn0262872 & milt & FBgn0051957 & CG31957 & FBgn0034300 & CG5098 \\
\hline FBgn0053977 & CG33977 & FBgn0036402 & CG6650 & FBgn0086680 & $\mathrm{vvl}$ & FBgn0003227 & rec \\
\hline FBgn0030217 & CG2124 & FBgn0029157 & ssh & FBgn0051158 & Efa6 & FBgn0266580 & Gp210 \\
\hline FBgn0024194 & rasp & FBgn0263006 & SERCA & FBgn0039858 & CycG & FBgn0001624 & dlg1 \\
\hline FBgn0044510 & mRpS5 & FBgn0284220 & Top2 & FBgn0003638 & $\operatorname{su}(w[a])$ & FBgn0264078 & Flo2 \\
\hline FBgn0265623 & $\operatorname{Su}(z) 2$ & FBgn0032586 & Tpr2 & FBgn0036577 & CG13073 & FBgn0035049 & Mmp1 \\
\hline FBgn0015773 & NetA & FBgn0036249 & CG11560 & FBgn0284256 & bsf & FBgn0053531 & Ddr \\
\hline FBgn0004854 & B-H2 & FBgn0037890 & CG17734 & FBgn0032600 & BuGZ & FBgn0003206 & Ras64B \\
\hline FBgn0014133 & bif & FBgn0037439 & CG10286 & FBgn0039827 & CG1544 & FBgn0038146 & CG9799 \\
\hline FBgn0030269 & CDK2AP1 & FBgn0026313 & $\mathrm{X} 11 \mathrm{~L}$ & FBgn0036825 & RpL26 & FBgn0263855 & BubR1 \\
\hline FBgn0262738 & norpA & FBgn0039528 & dsd & FBgn0039600 & CG1646 & FBgn0038100 & Paip2 \\
\hline
\end{tabular}


Chapter II - Variation in a pleiotropic regulatory module drives evolution of head shape and eye size in Drosophila

\begin{tabular}{|c|c|c|c|c|c|c|c|}
\hline FBgn0030240 & CG2202 & FBgn0027556 & CG4928 & FBgn0022985 & qkr58E-2 & FBgn0037336 & CG2519 \\
FBgn0028380 & fal & FBgn0028579 & phtf & FBgn0011584 & Trp1 & FBgn0052204 & CG32204 \\
\hline
\end{tabular}




\section{Chapter III - Regulatory Divergence in the Drosophila melanogaster subgroup}

The manuscript 'Regulatory Divergence in the Drosophila melanogaster subgroup' is the result of a side project that is initially based on allele specific expression analysis (ASE) started by Dr. Torres-Oliva, $\mathrm{M}$.

My contributions for this manuscript includes the following parts:

- Conceptualization of the project (together with Dr. Nico Ponsien)

- Bioinformatics analyses (ATAC-seq)

- Data interpretation (together with Dr. Nico Posnien)

- Writing of manuscript draft

- Visualization

\section{Contribution of other authors includes:}

- Dr. Torres-Oliva, M. and Dr. Almudi, I. generated the transcriptomic and ATAC-seq dataset. The Illumina sequencing was performed at the Transcriptome Analysis Lab (TAL) in Göttingen.

- Data prepared by Dr. Torres-Oliva, M. include the differential expression (ASE) analysis of the parental and hybrid RNA-seq datasets and the allele specific expression analysis (ASE), presented in Figure 1B. She also kindly provided the tables with all genes and the corresponding divergence types, which underlie GO analysis, presented in Figure 1C.

Status of the manuscript:

In preparation for submission 
Title

Regulatory divergence in closely related Drosophila species depends on the architecture of developmental gene regulatory networks.

\section{Authors}

Torres-Oliva, M. ${ }^{2+}$; Buchberger, E. ${ }^{1+} ;$ Almudi, I. ${ }^{3} ;$ Lu, $\mathrm{T}^{-H} .{ }^{1}$, Posnien, N. ${ }^{1 *}$ (in prep.):

1 Universität Göttingen, Department of Developmental Biology, Justus-von-Liebig-Weg 11, 37077 Göttingen, Germany

2 Institute of Clinical Molecular Biology, Christian-Albrechts-University of Kiel, University Hospital Schleswig-Holstein, Kiel, Germany

${ }^{3}$ CABD (CSIC/UPO/JA), DMC2 Unit, Pablo de Olavide University campus, Ctra. Utrera km1, 41013 Seville, Spain

+ equal contribution

* author for correspondence: nposnie@gwdg.de 


\subsection{Introduction}

In recent years it became clear that differences in gene expression levels contribute to a large extend to the diverse morphology of body plans that we can observe in the animal kingdom (e.g. (Carroll, 2005; Khaitovich et al., 2006; King and Wilson, 1975; Tautz, 2000)). The process of transcription i.e. gene expression must be tightly controlled to ensure the proper development and function of tissues and organs of an organism. Today, we have a very detailed understanding of transcriptional regulation that is at play on different levels. For instance, the interplay of transcription factors with co-factors and the accessibility of cis-regulatory regions, where these factors can bind to, represent central regulatory mechanisms (reviewed in (Buchberger et al., 2019)). But also, higher order chromatin structure (reviewed e.g. (Furlong and Levine, 2018) and post-translational processes, such as the action of regulatory RNA molecules (Bartel, 2018; Kittelmann and McGregor, 2019) work together to ensure time and tissue specific gene expression. Since evolutionary changes on each of these levels could cause natural variation in gene expression levels, the complexity of regulatory mechanisms contributes to the complications to pinpoint the exact cause of gene expression divergence between individuals and species (reviewed in (Buchberger et al., 2019)). The dissection of the molecular basis of gene expression differences and its impact on morphological evolution is further hampered by the polygenic nature of many phenotypes (Boyle et al., 2017; Mackay et al., 2009).

The number of differentially expressed genes is highly correlated with the phylogenetic distance of populations or species (Khaitovich et al., 2006; Rifkin et al., 2003). Variation in gene expression results from mutations in the genome, which can either affect the regulatory region of the differentially expressed gene itself (i.e. cis-regulatory changes) or an upstream regulator of the gene (for instance a transcription factor) (i.e. trans-regulatory changes)(Wittkopp, 2005). Even though it is under debate, how much of the overall gene expression divergence is caused by cis- or trans-regulatory changes, it is clear that both contribute to evolutionary changes within populations, strains or species (Genissel et al., 2007; Hoekstra and Coyne, 2007; Stern and Orgogozo, 2008; Tautz, 2000). The general idea is though that cis-regulatory changes are the main cause for divergent expression, since a change in these parts of the locus would only affect the respective gene, whereas an upstream trans-regulatory change would have major pleiotropic effects on its many target genes (Carroll, 2005; Prud'homme et al., 2007; Wray, 
2007, 2003). Interestingly, recent studies in various model organisms revealed that gene expression changes in cis and trans are context dependent, i.e. the respective tissue and environmental factors can affect the relative contribution of each divergence type (Duveau et al., 2017; Reuveni et al., 2018).

Since mutations affecting gene expression are heritable, allele specific expression analyses (ASE) have been used to understand the molecular mechanisms of divergent gene expression. This approach takes advantage of the possibility to obtain viable offspring from crosses among closely related strains or species (Cowles et al., 2002; Wittkopp et al., 2004). Comparing the gene expression levels in closely related parental strains to the expression of the respective allele in their offspring, allows to classify the modes of differential gene expression in the parental into cis- and trans-regulatory changes. ASE allows to deduce the mode of expression changes for each expressed gene in parental species but cannot provide information about the detailed genetic causes that underlie the observed expression divergence. The combination of open chromatin datasets revealing potential regulatory regions and RNA-seq datasets, helps nowadays to better draw the links between changes in these cisregulatory regions and the resulting differences in gene expression (e.g. (Hughes et al., 2017; Rendeiro et al., 2016; Starks et al., 2019)). Nevertheless, a genome-wide understanding of how cis- and trans-regulatory changes can be recapitulated on the level of accessible chromatin regions is missing up to now.

To address this open question, we use three species, D. melanogaster, D. mauritiana and $D$. simulans to understand the evolution of gene expression divergence during head and eye development in the Drosophila melanogaster subgroup. The three species vary remarkably in their eye sizes and head shapes. Differences in eye size between $D$. melanogaster and $D$. mauritiana are mainly due to variation in ommatidia number, and differences in eye size between $D$. mauritiana and $D$. simulans result mainly from variation in ommatidia size (Posnien et al., 2012). Previous gene-expression studies using these three species have shown, that a plethora of genes is differentially expressed between D. melanogaster and D. mauritiana (Buchberger et al. in prep.) and D. mauritiana and D. simulans (Almudi et al. in prep.). However, the underlying mechanisms of this gene expression divergence are completely unknown

We therefore performed ASE using the F1 hybrid generation of D. melanogaster $x D$. mauritiana and D. mauritiana $\times$ D. simulans to analyse the contribution of cis- and trans- 
regulatory changes. We further used a comparative ATAC-seq dataset to address the question how changes in the regulatory landscapes influence species-specific gene expression. We assessed and compared open chromatin regions between D. melanogaster, D. mauritiana and D. simulans in terms of differential cis-regulatory landscapes and sequence divergence and revealed that genes that were found to be differentially expressed due to cis-regulatory changes indeed exhibited a more divergent chromatin architecture. Additionally, orthologous regulatory sequences of cis-divergent genes showed a more pronounced sequence variation than regulatory regions of conserved genes or genes that are differentially expressed due to trans-changes. We suggest that both mechanisms contribute to cis-regulatory changes namely differential accessibility of regulatory regions, but also sequence divergence in potential promoters or enhancers.

\subsection{Results}

\subsubsection{Regulatory Divergence in the D. melanogaster subgroup}

To better understand the patterns of regulatory divergence in closely related Drosophila species, we obtained F1 hybrids of interspecific crosses between D. simulans and D. mauritiana and between $D$. melanogaster and D. mauritiana. The three species vary extensively in their adult head shapes ((Posnien et al., 2012), Figure 21A) and therefore provide an excellent model to understand how conserved GRNs evolve over time, being on the one hand tightly controlled to ensure proper organ development and function, and on the other hand flexible enough to allow evolution of size and shape of these structures. Since the adult head structures in Drosophila develop from two 2D larval epithelia, the eye-antennal discs (Haynie and Bryant, 1986) and variation in adult morphologies arise from differences during development of the respective structures, we applied RNA-seq to mid L3 developing eye-antennal discs (96h after egg laying, (AEL)) of the three parental species and the two hybrid crosses (Figure 21A). We first determined the number of genes, differentially expressed between the parental species. Subsequently, we analysed differential expression of the parental alleles in the two hybrid datasets to assess the type of regulatory divergence for each gene. When a gene was differentially expressed in the parental species as well as their alleles in the hybrids, its expression diverged due to cis-regulatory changes in its own locus. Cis-regulatory changes can have two underlying causes. First, mutations in either promoter or enhancer regions can change the regulation of a gene via, for instance, affecting the binding of transcription factors 
(e.g. (Prud'homme et al., 2006; Rogers et al., 2013)). Second, variation in the accessibility of such regulatory regions could alter gene regulation and hence gene expression (e.g. (Zhang and Borevitz, 2009)). If we found a gene to be differentially expressed between the parentals, but the two alleles in the hybrids were not, the gene expression in the parental species diverged due to upstream trans-regulatory changes. Trans-regulatory changes can arise due to nucleotide changes in the coding sequences (CDS) of an upstream regulator, but also due to cis-regulatory changes of an upstream transcription factor which changes the amount of available upstream regulators (Wittkopp, 2005). Genes that show neither significant differential expression between the parentals nor in the hybrid setting are considered conserved in expression. If genes with conserved expression levels in the parental species, showed differential allelic expression in the hybrids, we called the regulation type 'compensatory'.

Comparing D. melanogaster and D. mauritiana, we found that most genes were conserved (71\%, Figure 21B). Of the genes that showed divergent expression, most were differentially expressed due to variation in trans, i.e. due to variation in an upstream regulator (20\%, Figure 21B). Only 5\% of the differentially expressed genes showed regulatory divergence in cis (5\% Figure 21B). A nearly equal number of genes showed signatures of compensatory divergence (4\%, Figure 21B). Although the general trends are the same for both pairwise comparisons, we found more genes to be differentially regulated by cis-regulatory effects between D. melanogaster and D. mauritiana (Figure 21B), compared to the more closely related $D$. simulans and D. mauritiana (2\% vs. $5 \%$, Figure $21 B$ ). In the latter comparison we found even more genes to be differentially expressed due to trans-regulatory divergence (23\% vs. $20 \%$, Figure 21B).

We further assessed in which biological processes these genes were enriched and performed a GO enrichment analysis. Genes which are conserved between D. melanogaster and D. mauritiana were involved in basic processes like cell morphogenesis, cell proliferation, growth and developmental processes (Figure 21C). Genes that show regulatory divergence in trans, were enriched in morphological and developmental processes, and more specifically in transcription, neuronal processes or photoreceptor development (Figure 21C). We found genes that were differentially expressed due to cis-regulatory divergence to be enriched in metabolic and biosynthetic GO terms (Figure 21C). Genes with signs of compensatory regulation were mainly enriched in GO terms like cell fate, cell cycle or larval cuticle development (Figure 21C). 
We obtained a very similar pattern for the other species pair (D. simulans vs. D. mauritiana) (Supplementary Figure 21). Overall, we found that most genes that were differentially expressed in the developing eye-antennal disc in closely related species were different due to trans-regulatory changes. We showed that genes diverging in cis or trans take part in different processes, with the latter ones being enriched in developmental GO terms.

A

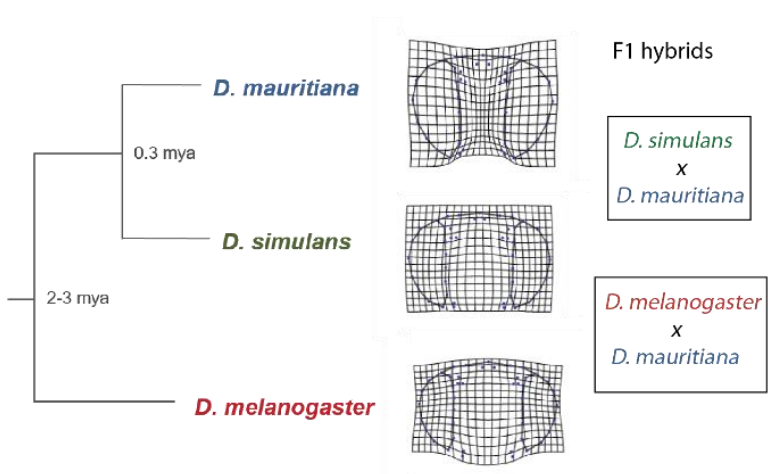

C

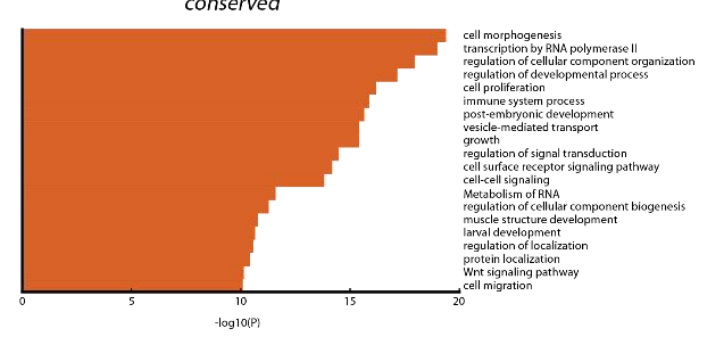

cis

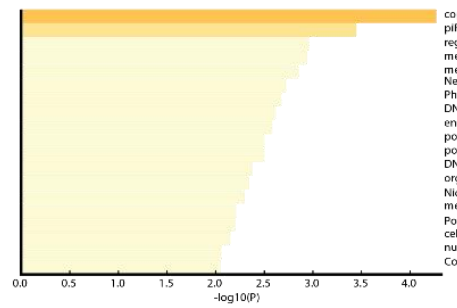

B

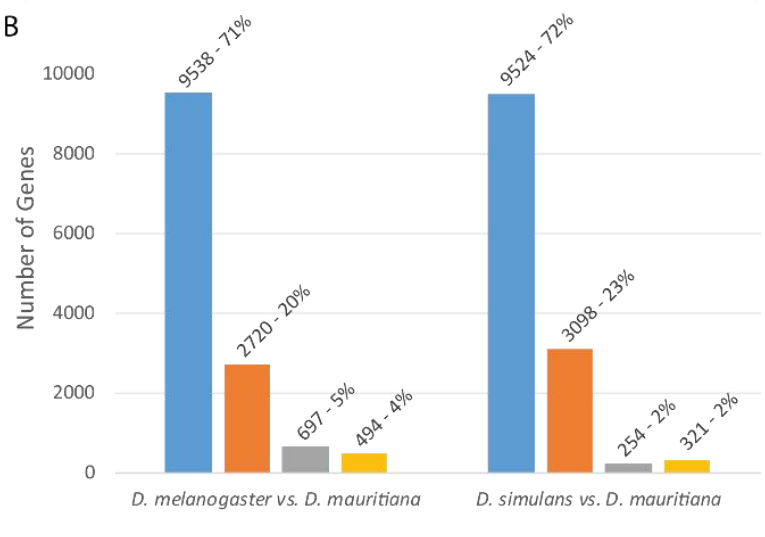

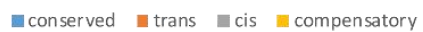

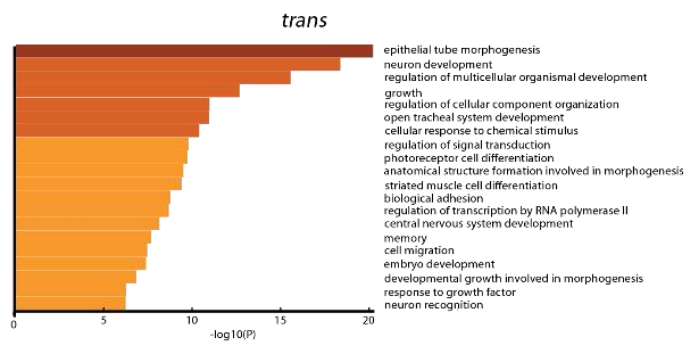

compensaory

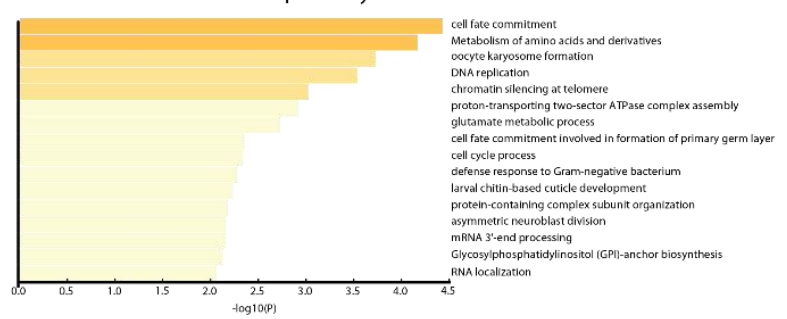

Figure 21. Regulatory divergence in three closely related Drosophila species. A. Phylogenetic relationship between the three Drosophila species used in this study and differences in their head shapes (Posnien et al., 2012). The boxes summarize the two crosses giving rise to viable F1 hybrids. RNA-seq was performed of developing eye-antennal discs of the three parental species and the two F1 hybrid offspring. B. Allele specific expression analysis between the two species pairs (D. melanogaster vs. D. mauritiana and D. simulans vs. $D$. mauritiana). Most genes are conserved between the species. Differentially expressed genes are predominantly differentially expressed due to changes in trans. We found more 'trans-genes' between $D$. simulans vs. $D$. mauritiana. A higher number of cis-regulatory changes was observed between D. melanogaster vs. D. mauritiana. C. A random subset of 3000 conserved genes (see Material and Methods) between D. melanogaster and D. mauritiana was highly enriched in processes like morphogenesis, cell proliferation, growth, larval development etc. Genes that were differentially expressed due to trans-regulatory changes were as well highly enriched in developmental and morphogenetic processes (especially neuronal development), whereas we found more biosynthetic and metabolic GO terms for cis-effect genes. Genes with compensatory expression in hybrids were mainly enriched in cell-cycle but also metabolic processes. 


\subsubsection{A comparative ATAC-seq dataset of three closely related Drosophila species}

To test if the different types of regulatory divergence during head development can also be recapitulated on the level of the regulatory landscape, we generated a comparative ATACseq dataset for eye-antennal discs (96h AEL) for the three species. We could significantly call 21,705 peaks in D. melanogaster, 21,499 peaks in D. mauritiana and 20,374 peaks in D. simulans (Figure 22A). These numbers of open chromatin regions in the developing eyeantennal disc is in concordance with previous studies (Davie et al., 2015).

For further quality assessment of the three ATAC-seq datasets, we calculated the insert size distribution. This is based on the assumption that the Tn5 transposase used for ATAC-seq can only insert adapters where the DNA is not covered by nucleosomes. Therefore, proper ATAC-seq library preparation should result in a clear peak at $\sim 100 \mathrm{bp}$ where the DNA is depleted of nucleosomes, hence most easily accessible to the transposase, and smaller peaks resulting from sequences that are wrapped around different-sized nucleosomes. All of our datasets showed the typical periodicity of $\sim 200$ bp (Figure 22B, Supplementary Figure 22, (Buenrostro et al., 2013; Davie et al., 2015)). We annotated open chromatin regions of $D$. melanogaster to the gene loci according to the closest transcription start site (TSS) and to the respective gene features. We found that open chromatin sites predominantly mapped to promoter regions ( 37\%), intronic ( 30\%) and intergenic regions ( 18\%) (Figure 22B). This demonstrates that we generated a reliable open chromatin dataset for developing eye-antennal discs at mid L3 larval stages in terms of peak number and annotation. 
A

\begin{tabular}{|c|c|c|c|}
\hline Species & original \# of Peaks & \# of Peaks afterCoordinate Conversion & \# of unmapped Peaks after Coordinate Conversion \\
\hline D.melanogaster & 21,705 & & \\
D. mauritiana & 21,499 & $20,678(96 \%)$ & $821(4 \%)$ \\
D. simulans & 20,374 & $19,732(97 \%)$ & $642(3 \%)$ \\
\hline
\end{tabular}

B

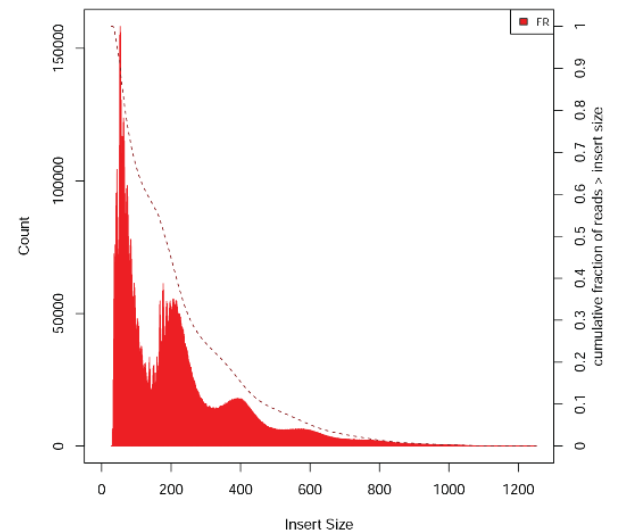

C

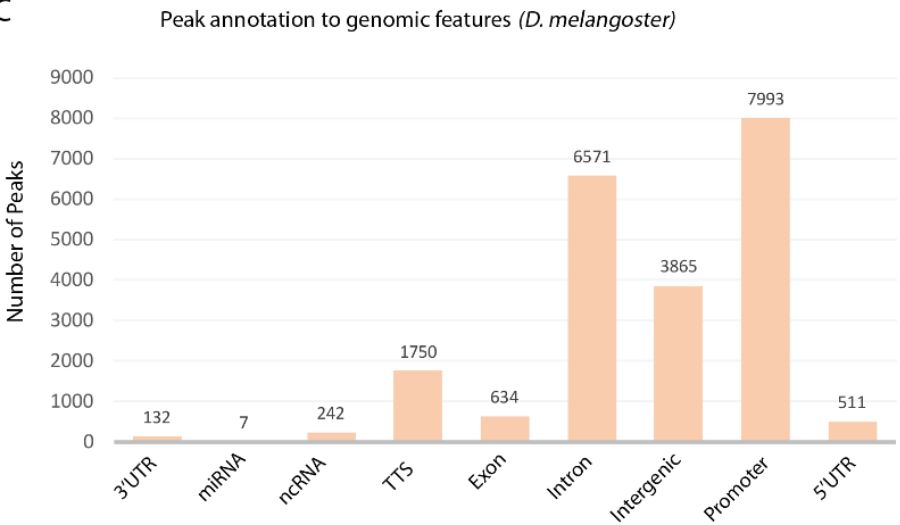

Figure 22. A comparative ATAC-seq dataset for the Drosophila melangaster subgroup. A. We were able to call a similar number of open chromatin peaks in all three species, namely between 20,300 and 21,700, which is comparable with previous ATAC-seq studies (Davie et al., 2015). We converted the peak coordinates of $D$. mauritiana and $D$. simulans into the $D$. melanogaster coordinate system. Using our customized pipeline, we were able to convert $96-97 \%$ of all peaks in $D$. mauritiana and $D$. simulans, respectively. B. The insert sizes of the $D$. melanogaster ATAC-seq dataset shows the typical periodicity of the expected 200 bp (Buenrostro et al., 2013). C. Annotation of the $D$. melanogaster peaks to gene features. Typically for open chromatin datasets, most peaks mapped to either promoter, intronic or intergenic regions.

To compare the open regulatory landscape of $D$. melanogaster with the ones of its sister species, we developed a pipeline to convert D. mauritiana and D. simulans peak coordinates into the $D$. melanogaster genome coordinate system. For this we adapted the workflow used by the UCSC coordinate conversion tool (http://genome.ucsc.edu/, see Material and Methods and Appendix). In short, split chromosomes of $D$. melanogaster were aligned to the $D$. mauritiana or the $D$. simulans genome and respective chain files were generated for each of the two species. We then used the liftOver tool (Hinrichs et al., 2006) to convert the coordinates. By this we were able to convert $96 \%$ and $97 \%$ of D. mauritiana and D. simulans peak regions, respectively into D. melanogaster coordinates (Figure 22A). Peaks that could not be reliably converted were mostly found at the centromeres of chromosomes (Supplementary Figure $23 \mathrm{~A}$ ), which is consistent with the suggested quick evolution of these genomic regions (Henikoff et al., 2001). To overcome this bias, we removed the centromeric regions in the genome of D. melanogaster for further analyses (Supplementary Figure 23B, Material and Methods). Annotation of the converted open chromatin regions to gene features showed that they mainly mapped to intronic, intergenic and promoter regions which is comparable to the annotation of D. melanogaster ATAC-seq peaks (Figure 22C, Supplementary Figure 23C and D). 
After applying this pipeline for peak coordinate conversion, we continued with a total number of 20,678 peaks in D. mauritiana and 19,732 peaks in D. simulans, compared to 21,705 peaks in D. melanogaster.

\subsubsection{Genes with species specific regulatory regions are more often regulated in cis}

To understand how the accessibility of regulatory regions influences the evolution of gene expression in the $D$. melanogaster subgroup, we sought to compare the open-chromatin landscape between each of the species pairs (D. melanogaster vs. D. mauritiana and D. simulans vs. D. mauritiana). For each species we first summarized the peaks that mapped either to a TSS/promoter region or an intronic region and excluded peaks that mapped to intergenic regions (also see Technical and other considerations). To find orthologous peak regions between D. melanogaster and D. mauritiana or D. simulans and D. mauritiana, we overlapped peak regions using the bedtools suite (Quinlan, 2014; Quinlan and Hall, 2010), which resulted in three peak sets per pairwise comparison: 1) Peaks shared between two species; 2) Speciesspecific peaks for one species; and 3) Species-specific peaks for the other species. We found 11,439 peaks, mapping to 6,159 genes that were shared between $D$. melanogaster and $D$. mauritiana, and 3,103 and 2,829 peaks being specific for D. melanogaster and D. mauritiana, respectively. Of these, a higher number of species-specific peaks are annotated to introns than promoters (Table 5). We found a very similar pattern between D. mauritiana and D. simulans, but a slightly smaller number of species-specific peaks between these two closer related species (Table 5).

Table 5. For each species in each species comparison we combined intronic and promoter/TSS peaks (excluding intergenic peaks) and overlapped the peak sets to find orthologous peaks, and species-specific peak sets. The table lists intronic peaks in each species, plus the overlapping intronic peaks in the fist column, and the same information for TSS/propmter peaks in the second column. The sum of species specific intronic, TSS/promoter and shared peaks is shown in the thrid column (please note, that this number does not correspond to the total peak number per species after peak conversion). In all comparisons we found more species-specific peaks mapping to intronic regions, than TSS/promoter regions. The \% is calculated by deviding the species specific intronic or TSS/promoter peaks by the sum of intronic or TSS/promoter peaks (species-specific plus shared).

\begin{tabular}{|c|c|c|c|}
\hline & intronic peaks & TSS/promoter peaks & $\begin{array}{c}\text { sum of TSS/promoter and intronic peaks / } \\
\text { species (species specific + shared) }\end{array}$ \\
\hline & \multicolumn{3}{|c|}{ D. melangoaster vs. D. mauritiana } \\
\hline D. melanogaster & $1696(12.2 \%)$ & $1407(10.1 \%)$ & 13898 \\
\hline D. mauritiana & $1718(12.6 \%)$ & $1111(8.2 \%)$ & 13624 \\
\hline shared & 4488 & 6307 & 12755 \\
\hline D. simulans & $1021(8 \%)$ & $854(6.7 \%)$ & 13640 \\
\hline D. mauritiana & $1537(11.3 \%)$ & $1223(9 \%)$ & \multicolumn{3}{|c|}{. simulans vs. D. mauritiana } \\
\hline shared & 4707 & 6173 & \\
\hline
\end{tabular}


A Set 1 - diverged regulatory landscape

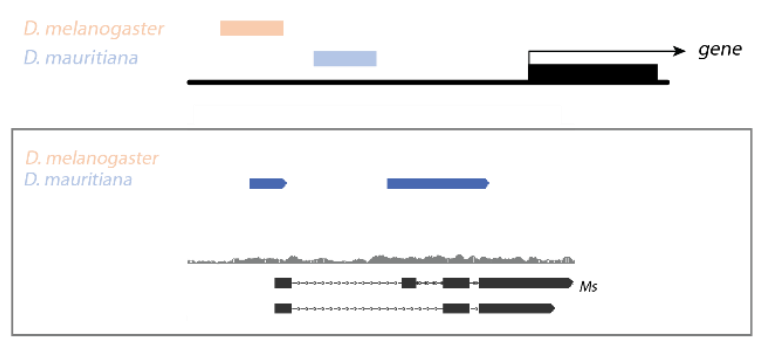

$A^{\prime} \quad$ Set 2 - conserved regulatory landscape

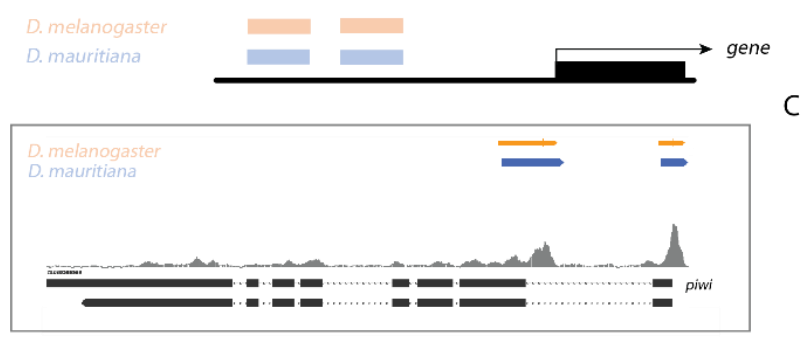

$A^{\prime \prime}$ Set 3 - conserved peaks and species specific peaks

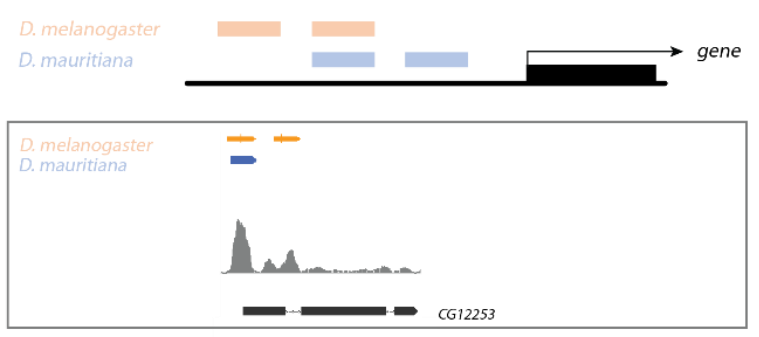

C

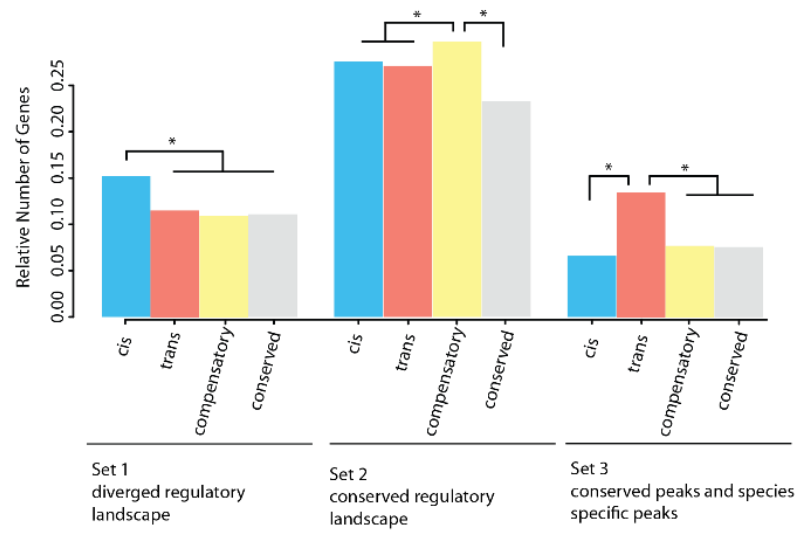

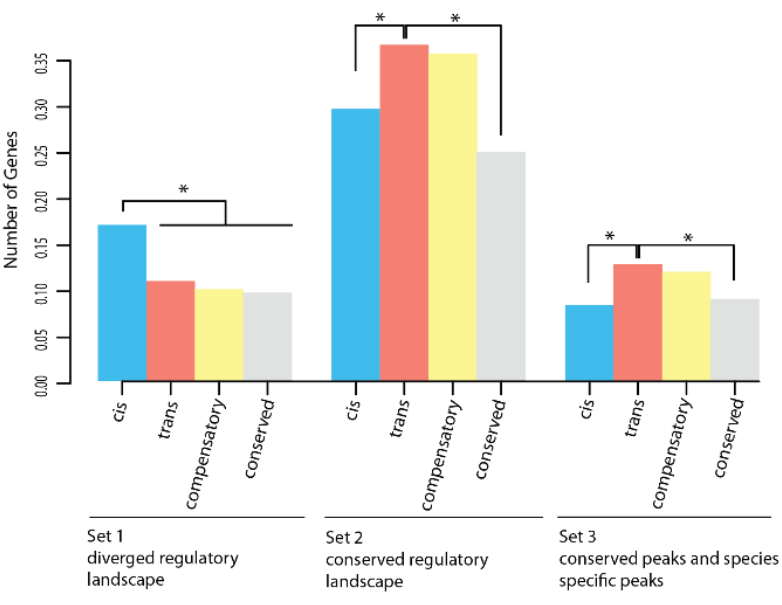

Figure 23. Comparison of the regulatory landscape. A-A". We summarized all gene loci according to their regulatory landscape into A) genes with highly diverged regulatroy landscape, with no overlapping peaks between the species. $\mathbf{A}^{\prime}$ ) genes with a very conserved regulatory landscape and no species-specific peaks and $\left.A^{\prime \prime}\right)$ genes with overlapping peaks but additional species specific ones. The loci in the rectangles show one randomly picked locus from each Set with the respective ATAC-seq peaks in the two species. orange: $D$. melanogaster, blue: D. mauritiana, grey: read densitiy of the D. melanogaster ATAC-seq dataset. B-C. Genes with a highly divergent regulatory landscape are significantly more often differentially expressed due to cis-regulatory changes. We find a high number of compensatory changes for genes with a very conserved regulatory landscape and significantly more genes that are differentially regulated in trans for genes with overlapping but also speciesspecific genes. Note that we provide all p-values between the pairwise comparisons in Supplementary Table 20A and B. B. D. melanogaster vs. D. mauritiana C. D. simulans vs. D. mauritiana.

Second, we assigned every gene locus to one of three genes sets (Figure 23A-A"): The first gene set included genes that showed a completely divergent regulatory landscape (i.e. no overlapping peaks between two species) (Figure 23A). The second set included genes, that had the same regulatory landscape in two species (i.e. only overlapping peaks between two species, Figure $23 \mathrm{~A}^{\prime}$ ) and third we pooled genes that had a similar open chromatin landscape in both species, but also putative species-specific regulatory regions (Figure 23A"). We then overlapped these sets with the differentially expressed genes, for which the type of regulatory divergence was known (Figure 21B). Interestingly, we found that genes that are differentially 
regulated due to cis-regulatory changes between the D. melanogaster and D. mauritiana, overlap predominantly with genes in Set1, i.e. genes that have only species-specific peaks (Figure $23 \mathrm{~B}$ and $\mathrm{C}$, e.g. cis vs. trans Fishers exact test, $\mathrm{p}=0.0095$ ), whereas genes with a conserved regulatory region, are mainly differentially expressed between species, due to compensatory mechanisms and surprisingly not necessarily conserved in expression levels (Figure 23B). Genes, which show conserved regulatory regions but also additional speciesspecific peaks (Set3), were predominantly differentially expressed due to trans mechanisms (Figure 23B).

To test, whether we find similar patterns in more closely related species, we also performed this analysis for the set of differentially expressed genes between $D$. mauritiana and D. simulans. As observed for D. melanogaster and D. mauritiana genes in Set 1 , summarizing genes with a divergent chromatin landscape, were mostly differentially regulated due to variation in cis. However, for genes with a conserved regulatory landscape we found that they were differentially expressed due to trans-regulatory changes. Genes in Set 3 showed the same pattern with genes being mostly differentially regulated due to upstream trans mechanisms (Figure 23C).

Overall, we show that genes with a highly divergent DNA accessibility landscape were significantly more often differentially expressed due to cis-regulatory changes, compared to genes that show a more conserved regulatory architecture.

\subsubsection{Regulatory regions of genes, diverging in cis, show a higher sequence divergence}

Since cis-regulatory divergence may not only arise due to differences in accessibility of the respective regulatory regions, but also due to sequence changes affecting for instance transcription factor binding, we focused in more detail on the sequence divergence of orthologous open chromatin regions between the species. We extracted the sequences of all orthologous intronic and TSS peaks and compared their sequences between the species pairs. Peak regions that were annotated to genes showing cis-regulatory divergence, showed a significantly lower percentage of sequence identity between $D$. melanogaster and $D$. mauritiana. Peaks assigned to genes showing compensatory divergence had similarly diverged regulatory sequences (Figure 24A). We found the same trend between D. mauritiana and D. simulans, although the differences in sequence divergence between different regulatory 
groups were not significant, reflecting the closer phylogenetic relationship between these two species (Supplementary Figure 24A).

A

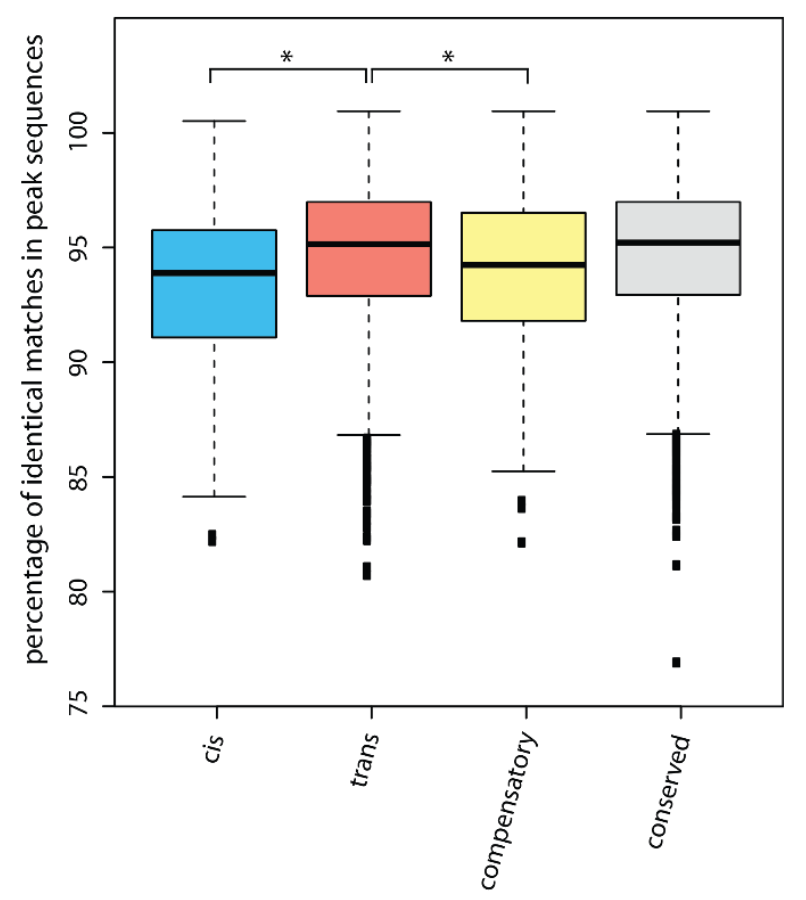

B

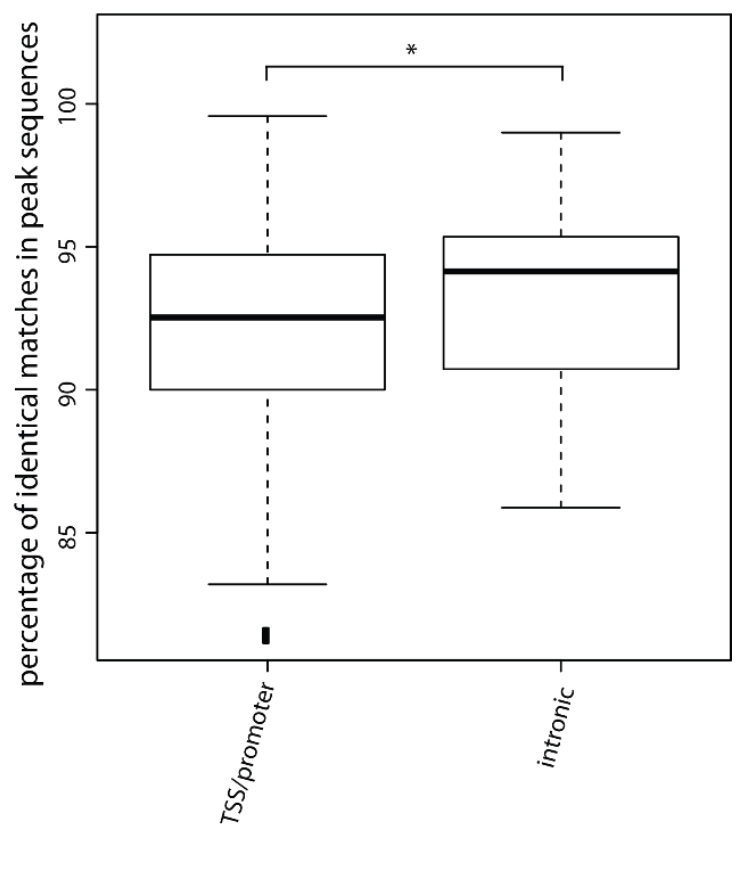

Figure 24. Cis-regulatory changes arise due to differences in DNA accesssibility but also due to sequence divergence. A. Genes that are differentially expressed due to cis-regulatroy changes, but also compensatory genes show more diverged peak sequences than conserved genes or genes with trans effects. Note that we provide all p-values between the pairwise comparisons in Supplementary Table 21. B. We separated TSS/promoter peaks from intronic peaks and showed that intronic peak sequences are on average more conserved than peaks mapping to promoter regions. Note that we provide all $p$-values between the pairwise comparisons in Supplementary Table 22.

We further wanted to test, whether TSS/ promoter regions and intronic regions evolve quicker in terms of nucleotide content. For this, we aligned the sequences of accessible orthologous promoter regions and intronic regulatory regions of $D$. melanogaster and $D$. mauritiana. Interestingly, we observed for all four classes of genes (cis, trans, compensatory and conserved) that intronic sequences seem to be more conserved in terms of nucleotide sequences, whereas peaks in the promoter regions show a lower sequence similarity (Figure 24B, Supplementary Figure 25, Supplementary Table 22).

We conclude, that orthologous regulatory sequences of genes, differentially expressed due to cis-regulatory changes show higher sequence divergence and that this pattern is more pronounced in peaks annotated to TSS/promoter regions. 


\subsubsection{Regulatory divergence in transcription factors}

Since the overall patterns seemed to be similar between the two species comparisons, we next asked, whether the same gene sets were composed of the same genes in the two comparisons. For this we focused on a set of 149 transcription factors which we overlapped with the gene sets shown in Figure 23A-A". Furthermore, we assessed if these transcription factors, if differentially expressed, showed divergence in cis, trans or compensatory expression in the hybrids. We found strikingly few transcription factors in Set 1 (i.e. genes with only species-specific peaks, Supplementary Table 23). The potential regulatory regions of most transcription factors were conserved (Set 2, 72 and 79 TFs from the D. melanogaster vs. D. mauritiana and D. simulans vs. D. mauritiana comparison, respectively; Supplementary Table 23) or only slightly diverged with additional species-specific peaks (Set 3, 51 TFs from the D. melanogaster vs. D. mauritiana and $46 \mathrm{TFs}$ from D. simulans vs. D. mauritiana comparison, respectively; Supplementary Table 23). Consistently, we found transcription factors mostly to be conserved in expression, and if differentially expressed, this was due to trans-regulatory changes, except in one case, where we found bicoid ( $b c d$ ) to be differentially regulated between D. melanogaster and D. mauritiana due to a cis-regulatory change.

Overall, we found that the expression and regulatory landscape of transcription factors is highly conserved between the two species pairs.

\subsection{Discussion}

\subsubsection{Regulatory divergence is context dependent}

Our differential expression analysis between three closely related Drosophila species revealed that most genes were conserved among species. This recapitulates previous data which showed that the overall gene expression dynamics in developing eye-antennal discs between the three species D. melanogaster, D. mauritiana and D. simulans are to a large extend conserved (Torres-Oliva, 2016). Still, we found a substantial number of genes to be differentially expressed during eye and head development (Buchberger et al. in prep; Chapter II, Almudi et al. in prep). Since changes in gene expression during development often correlate with variation in adult morphology and physiology (Carroll, 2005; Khaitovich et al., 2006; King and Wilson, 1975; Tautz, 2000), this observation most probably recapitulates the remarkable variation in size and shape of the head cuticle and the adjacent compound eyes (Posnien et al., 2012). However, in most cases it is not known which regulatory change underlies differential 
expression of a gene. ASE analysis provides a powerful tool to test this on a genome-wide level. Therefore, we generated F1 hybrids between D. melanogaster $\times D$. mauritiana and D. simulans $x$ D. mauritiana and performed ASE analysis do understand if the cause for differential expression in a developing epithelium can be mainly found in the gene's own regulatory region (cis) or is rather caused by changes in upstream factors (trans).

Interestingly, the majority of the differentially expressed genes was due to variation in trans. We applied the same analysis pipeline to two species comparisons differing in their divergence time using D. melanogaster vs. D. mauritiana, which diverged about 2-3 Mya, and D. simulans vs. D. mauritiana diverging about 0.3 Mya (Figure 21A). Even though patterns in terms of regulatory divergence were similar, the number of cis-regulatory changes increased with phylogenetic distance, whereas the number of trans-regulatory changes decreased (Figure 21B). This is consistent with the finding that usually more cis-regulatory changes accumulate throughout time (Metzger et al., 2017; Stern and Orgogozo, 2008; Wittkopp et al., 2008).

The excess of trans-regulatory changes contradicts most previous studies which reported a higher contribution of cis-regulatory changes, compared to trans (e.g. (Graze et al., 2009; Wittkopp et al., 2008, 2004). This has mainly been explained by the fact that a cisregulatory change would only affect the respective locus, whereas changes in an upstream regulator would have more widespread and pleiotropic effects on all of its target genes (Wittkopp et al., 2008). Nevertheless, other studies also found a slightly higher amount of transregulatory changes (McManus et al., 2010; Suvorov et al., 2013). More trans-acting changes were mostly found in intraspecific comparisons, explained by a larger mutational target size of trans-factors that correlates positively with mutational variance (i.e. increase in trait-variance introduced by mutations in each generation (Landry et al., 2007)) (Landry et al., 2007; Wittkopp et al., 2008). McManus and colleagues also found an increase in trans-regulatory changes between $D$. melanogaster and D. sechellia and explained that pattern with the small population size of the latter species. Consequently, mutations would rather get fixed due to random genetic drift than due to natural selection (McManus et al., 2010). We cannot exclude a similar scenario for D. mauritiana, which is endemic on the island of Mauritius (David et al., 1989). To test this, it would be necessary to produce an F1 hybrid generation between the two cosmopolitan species D. melanogaster and D. simulans and compare the ASE analysis for this comparison with the already existing ones, including D. mauritiana. 
We examined the role of the differentially expressed genes in the four divergence types to test, whether there may be functional constraints on the type of regulatory divergence. Indeed, we found that genes differentially expressed due to variation in trans were enriched in developmental processes. In contrast, genes showing cis-regulatory divergence were in general enriched in metabolic and biosynthetic processes. A similar pattern has been observed in Drosophila embryos, where genes with cis-effects were more enriched in housekeeping functions, and genes with trans-effects mainly functioned in developmental and gene regulatory processes (Cannavò et al., 2017), suggesting that the function of a gene product indeed has an impact on its evolvability.

Another source of constraints may be imposed by the excess of regulatory interactions of a gene within a gene regulatory network (GRN). We studied a developing tissue, whereas comparable ASE studies were mostly performed in whole-body adult flies (McManus et al., 2010; Suvorov et al., 2013). We therefore checked specifically for regulatory divergence of transcription factors to find out if upstream, developmental regulators are more constraint and as previously suggested, more likely to be affected by trans-regulatory changes (Luscombe et al., 2004; Wittkopp, 2005). Likewise, genes showing cis-regulatory changes display a lower average connectivity in mouse tissues (Mack et al., 2019) but also in plants (Mähler et al., 2017). In general, we found a low number of TFs to be differentially expressed between the species. If they showed divergent expression, this was due to upstream trans-regulatory changes, which suggests, that the loci of these important regulators are indeed kept highly conserved between the species. We did not specifically analyse the connectivity of these TFs but one can assume that most of developmentally important TFs are positioned at the top of the GRNs and are most probably highly interconnected (MacNeil and Walhout, 2011; Stern and Orgogozo, 2008). Therefore, an excess of trans-regulatory divergence may be a common feature of developing tissues.

Nevertheless, several important regulators in the eye-antennal disc were differentially expressed due to differences in an upstream regulator. Between D. melanogaster and $D$. mauritiana these include for instance sine oculis (so), pannier (pnr), ocelliless (oc), whereas we found that the alleles of eyeless (ey) were only differentially regulated in the hybrid (Supplementary Table 23). We could functionally show, that differential expression of pnr indeed underlies differences in head shape and eye size between these two species 
(Buchberger et al. in prep.; Chapter II). Finding the potential upstream trans-acting transcription factors, that lead to differential expression of these important developmental regulators might eventually reveal the true genetic causes of variation in adult morphologies that we observe between the here studied Drosophila species. Also, between D. simulans and D. mauritiana transcription factors involved directly in eye development were differentially expressed. Among these are interesting candidates like the proneural gene atonal (ato), scalloped ( $s d$ ), or ttk (tramtrack), for which a role in ommatidia development was reported (e.g. (Garg et al., 2007; Jarman et al., 1994; Li et al., 1997; Siddall et al., 2009)). In the light of the finding, that eye size between these two species varies due to changes in ommatidia size (Posnien et al., 2012), these could be additional candidates to test if they indeed impact the size of the individual facets.

5.3.2. cis-regulatory divergence is due to changes in chromatin accessibility and sequence divergence

We further tested, which mechanisms contribute to cis-regulatory divergence in our data. Two reasons can theoretically underlie cis-regulatory changes leading to subsequent gene expression divergence, namely either mutations directly in the regulatory regions or divergent accessibility of these regions.

Orthologous regulatory sequences might have experienced changes in their nucleotide sequence, which could, amongst other things, affect TF-binding (Wittkopp, 2013). Even if the regulatory regions of a gene are characterized, studying the influence of sequence changes on gene expression is not straightforward. In some reported cases only one nucleotide change is enough to alter the temporal expression of an important master regulator (Ramaekers et al., 2018), whereas other enhancer sequences keep their conserved function despite extensive reshuffling of TF binding sites (Khoueiry et al., 2017; Ludwig et al., 2000). However, some mechanistic insights have been gained in the last years, that may help interpreting the obtained data. It was for instance shown in Drosophila, that quantitative changes in enhancer strengths between species correlate linearly with sequence divergence (Arnold et al., 2014) and that sequence changes in regulatory regions may lead to differential functionality due to loss in transcription factor or co-factor binding (e.g. (Paris et al., 2013; Schmidt et al., 2010; Zheng et al., 2010)). However, how deleterious the loss of a certain TF binding motif is, seems to depend on the combinatorial binding of a TF collective (Khoueiry et al., 2017). In our genome wide comparison, orthologous sequence divergence is higher in open chromatin regions close to 
genes with cis-regulatory divergence between species or compensatory changes in hybrids. A higher rate of polymorphisms in promoter regions of cis-effect genes (compared to trans-effect genes) was for instance shown in plants (Zhang and Borevitz, 2009), but also in Drosophila (McManus et al., 2010). These studies and our results suggest higher purifying selection in regulatory regions of highly connected developmental genes, which we found to be more often differentially expressed due to upstream trans-effects or are conserved between the species.

Studies in Arabidopsis thaliana suggest that not only open chromatin regions with nucleotide changes, but also differentially accessible DNase hyperactive sites (DHS) are often found close to genes that show differential expression between ecotypes (Alexandre et al., 2018). Therefore, differential accessibility of regulatory regions very likely adds to expression variation in cis. Here, we found that indeed genes with highly divergent DNA accessibilities are significantly more often differentially expressed due to cis-regulatory changes. Chromatin remodelling and differential enhancer opening is prevalent during development (e.g. Bozek et al., 2019; Hughes et al., 2017; Kvon et al., 2014; McKay and Lieb, 2013; Uyehara et al., 2017), and in the last years an in-depth understanding of how 3D chromatin organization, epigenetic and histone modifications and chromatin accessibility interact has emerged (e.g. (Corrales et al., 2017; Cubeñas-Potts et al., 2017; Rennie et al., 2018b; Sexton et al., 2012)). How this though affects divergent DNA accessibility among species is still largely unclear.

We further checked for sequence divergence and accessibility of promoters and intronic regulatory regions separately. Regulatory sequences annotated to TSS and promoter regions showed a higher sequence divergence, whereas intronic regulatory sequences seemed to be more constraint. Intronic peaks were more often differentially accessible in both of our comparisons, suggesting that in general the accessibility of TSS/promoter peaks is more conserved, whereas accessibility of regulatory regions in introns seems to be more species specific. We could therefore observe the trend in which changes in DNA accessibility affect more often intronic regions, though their sequences seem to stay more conserved. Apart from the circumstance that intronic sequences are maybe more conserved due to their location in gene loci, higher sequence conservation was indeed observed in long introns, which are thought to harbour more functional elements (Haddrill et al., 2005). It will be important to compare these results with sequence divergence of more distant intergenic regulatory regions. 


\subsubsection{Compensation and conservation of gene expression}

It was suggested, that gene expression falls largely under stabilizing selection (e.g. (Landry et al., 2005; Lemos et al., 2005)), i.e. that a certain level of gene expression has to be kept stable. The rational is, that even though mutations in regulatory sequences accumulate over time, trans-regulatory factors co-evolve to buffer these changes (Landry et al., 2005). We found in our analysis a high number of compensatory effects, characterized by allelic misexpression in the F1 hybrid generation. Interestingly, regulatory regions of genes show a similar sequence divergence than genes that are affected by cis-regulatory changes, suggesting that indeed upstream trans-regulatory factors co-evolved to maintain the expression levels in the parental species. We found compensatory regulation in all three gene sets, predominantly though in genes that show no divergence in peak accessibility, therefore, the main mode of cisregulatory changes in these genes might be attributed to nucleotide changes. Nevertheless, compensatory changes are also found in genes with diverged accessibility of regulatory regions. One characteristic of enhancer function is that they usually work in a highly modular manner (reviewed for example in (Arnone and Davidson, 1997; Wray, 2003)). It was for instance estimated for Drosophila that each expressed gene is controlled by an average of four distinct enhancers (Kvon et al., 2014). This modularity allows also to control gene expression in a spatially and temporally controlled manner (reviewed for instance in (Prud'homme et al., 2007)). This has been elegantly shown in more simple traits like pigmentation patterns, in which the deletion of a 'spot enhancer' or an 'abdomen enhancer' leads to loss of wing pigmentation on a Drosophila wing or loss of dark abdomen coloration (Jeong et al., 2006; Prud'homme et al., 2006). Our dataset provides the opportunity to further analyse in more detail, how much of the compensatory coevolution is driven by differential combinatorial usage of such enhancer modules. Since DNA accessibility is highly dependent on the developmental stage and tissue, one can assume that this kind of compensatory regulation is in general highly context dependent and calls for a more thorough comparison with other developing tissues, like the wing disc for instance.

We found a high number of genes that show conserved expression in the parental species as well as in their F1 hybrids. These conserved genes were highly enriched in general developmental functions, like growth, proliferation or morphogenesis, which is consistent with our finding that most developmental TFs are conserved in expression between the species. Regulatory sequences of conserved genes were equally constraint in terms of sequence 
divergence than genes that showed trans-regulatory divergence. Nevertheless, in cases that show high sequence divergence, conservation of TF binding could be attributed for example to the topology but also the function of the GRN. It has been suggested that upstream genes in highly connected GRNs show a more conserved TF occupancy (Khoueiry et al., 2017) and have therefore a higher chance to balance sequence changes in their regulatory regions. For conserved genes that show a high divergence in peak accessibility between the species, the modularity of enhancer elements, as discussed for compensatory changes, might ensure the correct level of gene expression. In contrast to genes that show compensatory changes though, these mechanisms would not lead to misexpression in the hybrids, therefore they might be less dependent on the co-evolution of upstream trans-regulators.

Overall, the high number of compensatory and conserved genes that do show changes in DNA accessibility or enhancer and promoter sequence reflects the high potential of compensatory mechanisms, that ensure the correct level of expression despite substantial cisregulatory changes (Ludwig et al., 2000). In this study, we mainly concentrate on changes in regulatory regions and upstream transcription factors. Given the highly complex regulation of gene expression (reviewed in (Buchberger et al., 2019)) it remains to be studied how gene expression control on other levels, for instance miRNAs contribute to such compensatory mechanisms.

\subsubsection{Technical and other considerations}

The combination of RNA-seq and open chromatin datasets like ATAC-seq allows to deduce certain patterns in gene expression divergence and its correlation with regulatory regions. Apart from the high context dependency and compensatory mechanisms of gene expression, additional technical limitations must be considered. We assume here, that the annotation of a peak to the closest TSS does represent the true regulatory influence on the respective gene, which in Drosophila is often, but not always true. A systematic annotation of active enhancers in Drosophila revealed that about $88 \%$ are located in direct proximity of the target gene (Kvon et al., 2014). About 20\% of all enhancers, were found to be located in between $4 \mathrm{~kb}$ distance from the respective TSS; about the same fraction of enhancers showed though a distance $>100 \mathrm{~kb}$ (Kvon et al., 2014). The fact that we focus on only TSS/promoter and intronic peaks, might on the one hand reduce the chance to interpret intergenic peaks with ambiguous gene association, but on the other hand leads to wrong assumptions of peak 
number in a gene's regulatory landscape. Therefore, it will be important to repeat the analysis including these intergenic peaks. The annotation of peaks to a certain gene is particularly error prone for peaks in regions of the genome that contain many overlapping gene models. This is mostly due to the fact that an enhancer element might control only one gene in a multi-gene locus, or several ones. These drawbacks have started to be overcome in studies using annotation-unbiased approaches that base the characterization of regulatory regions rather on parameters related to transcriptional properties (Rennie et al., 2018a). Also, our focus on TSS/promoter and intronic peaks reduces the number of genes to be included in the analysis. While our study focuses on 6100-6200 genes, an earlier estimation using the same RNA-seq dataset as a basis resulted in about 9000 genes being transcribed in the eye (Torres-Oliva, 2016). This clearly shows, that to understand the complete picture, also intergenic peaks have to be included in this analysis.

Furthermore, we define here 'differential accessibility' as a peak being significantly called or not, i.e. we did not consider the height of ATAC-peaks. The height of peaks is defined by the number of reads that map to a specific peak region. This can be influenced for example by the number of cells in a heterogenous tissue that show the specific chromatin opening or the 'accessibility' of a specific regulatory region. To address questions like variation of DNA accessibility in an epithelium like the eye-antennal disc, which gives rise to a plethora of different head structures (Haynie and Bryant, 1986), one might learn a lot by applying single sell ATAC-seq (Buenrostro et al., 2015; Cusanovich et al., 2015).

\subsection{Conclusion}

In summary, we show that regulatory divergence can partly be recapitulated on the basis of DNA accessibility. This holds true, especially for cis-regulatory changes, where we found, that these are based on both, namely changes in DNA accessibility, as well as sequence divergence in orthologous regulatory regions. Comparing two different species pairs we confirm, that the amount of cis-regulatory divergence correlates with the phylogenetic distance in the Drosophila melanogaster subgroup. ASE expression analysis cannot reveal the causative genetic variants leading to differential expression, but with the combination of open chromatin datasets one can start to dissect the underlying genetic regulatory architecture. Our result that in general more trans-regulatory changes seem to underlie gene expression divergence between closely related species, calls for more tissue specific ASE studies in other animal 
groups. It will be also interesting to reveal how the here described patterns deviate in other tissues, like the developing thoracic or leg imaginal discs to learn more about the context dependency of regulatory divergence.

\subsection{Material and Methods \\ 5.5.1. RNA-seq}

The generation of RNA-seq datasets of developing eye-antennal discs (96h AEL) was performed as described for D. melanogaster in (Torres-Oliva et al., 2018). The same procedure was applied for datasets of D. mauritiana and D. simulans. In short, developing eye-antennal discs were dissected at $96 \mathrm{~h} \mathrm{AEL} \mathrm{for} \mathrm{D.} \mathrm{melanogaster} \mathrm{and} \mathrm{D.} \mathrm{mauritiana.} \mathrm{Please} \mathrm{note,} \mathrm{that} \mathrm{in} D$. simulans, the morphogenetic furrow progressed a bit slower than in the other two species, therefore disc were dissected at $98 \mathrm{~h} \mathrm{AEL}$, to ensure the same developmental time point (in the manuscript we still refer to ' $96 \mathrm{~h} \mathrm{AEL'} \mathrm{for} \mathrm{the} \mathrm{sake} \mathrm{of} \mathrm{clarity).} \mathrm{To} \mathrm{set} \mathrm{up} \mathrm{the} \mathrm{hybrid} \mathrm{crosses} 400$ D. melanogaster or D. simulans virgin females were crossed to 300 D. mauritiana males and the respective discs were dissected at 96h AEL. mRNA was extracted using the standard Trizol protocol and library preparation was prepared as described in (Torres-Oliva et al., 2018).

Differential expression analysis between parental strains and subsequent allele specific expression analysis (ASE) was performed by Dr. Torres-Oliva M. and is described in (TorresOliva, 2016).

We used the online tool Metascape (Zhou et al., 2019) to perform GO enrichment analysis for each group of genes (cis, trans, compensatory, conserved) in both pairwise settings (D. melanogaster vs. D. mauritiana and D. simulans vs. D. mauritiana.). In cases where more than 3000 genes were tested for enrichment, we randomly chose 3000 genes from the pool, since Metascape does not allow more genes as input.

\subsubsection{ATAC-seq}

\subsubsection{ATAC-seq library preparation}

For the generation of ATAC-seq datasets we followed (Buenrostro et al., 2013). Of all three species (D. melanogaster, D. mauritiana and D. simulans), developing eye-antennal discs were dissected in ice-cold PBS at 96h AEL. Please note, that in D. simulans, the morphogenetic furrow progressed a bit slower than in the other two species, therefore disc were dissected at $98 \mathrm{~h} \mathrm{AEL}$, to ensure the same developmental time point (in the manuscript we still refer to ' $96 \mathrm{~h} \mathrm{AEL'} \mathrm{for}$ 
the sake of clarity). PBS was removed and exchanged for $50 \mu$ lysis buffer $(10 \mathrm{mM}$ Tris- $\mathrm{HCl}$ $(\mathrm{pH}=7.4) ; 10 \mathrm{mM} \mathrm{NaCl} ; 3 \mathrm{mM} \mathrm{MgCl}$; $0.1 \%$ IGEPAL). The mixture was pipetted several times up and down to lyse the cells and then split into micro centrifuge tubes. Centrifugation for $10 \mathrm{~min}$ at $500 \mathrm{~g}$ and $4{ }^{\circ} \mathrm{C}$. The cell number was assessed in one of the samples and 50,000 to 80,000 nuclei were used in subsequent steps. The supernatant was removed and the pellet(s) dissolved in $47.5 \mu \mathrm{l}$ 1X tagmentation buffer (20 mM Tris- $\mathrm{CH} 3 \mathrm{COOH}$ ( $\mathrm{pH}=7.6) ; 10 \mathrm{mM} \mathrm{MgCl2}$; $20 \%$ (vol/vol) dimethylformamide) with $2.5 \mu \mathrm{l}$ Tn5 Transposase and then incubated for $30 \mathrm{~min}$ at $37^{\circ} \mathrm{C}$. For purification we used the QIAGEN MinElute Kit and eluted in $10 \mu$ l Elution Buffer (10 mM Tris, $\mathrm{pH}=8$ ). For the PCR amplification was done as follows:

- $10 \mu$ tagmented chromatin

- $10 \mu \mathrm{l} \mathrm{H} 2 \mathrm{O}$

- $2.5 \mu$ l Nextera PCR primer 1 *

- $2.5 \mu \mathrm{l}$ Nextera PCR primer $2^{* *}$

- 25 l NEBNext High-Fidelity 2X PCR Master Mix (Cat \#M0541)

We used the following program:

(8) $72{ }^{\circ} \mathrm{C} 5 \mathrm{~min}$

(9) $98^{\circ} \mathrm{C} 30 \mathrm{sec}$

(10) $\quad 98^{\circ} \mathrm{C} \quad 10 \mathrm{sec}$

(11) $\quad 63^{\circ} \mathrm{C} \quad 30 \mathrm{sec}$

(12) $72{ }^{\circ} \mathrm{C} \quad 1 \mathrm{~min}$

(13) repeat 3-5 13 times

(14) hold at $4{ }^{\circ} \mathrm{C}$

followed by another $2 \times$ purification step with the QIAGEN MinElute Kit: elution in $2 \times 10 \mu \mathrm{l}$ Elution Buffer (10 mM Tris, $\mathrm{pH}=8$ ).

\footnotetext{
* AATGATACGGCGACCACCGAGATCTACACTCGTCGGCAGCGTCAGATGTG

**Ad2.2 CGTACTAG Ad2.3_AGGCAGAA Ad2.4_TCCTGAGC Ad2.5_GGACTCCT Ad2.6_TAGGCATG Ad2.7_CTCTCTAC

CAAGCAGAAGACGGCATACGAGATCTAGTACGGTCTCGTGGGCTCGGAGATGT CAAGCAGAAGACGGCATACGAGATTTCTGCCTGTCTCGTGGGCTCGGAGATGT CAAGCAGAAGACGGCATACGAGATGCTCAGGAGTCTCGTGGGCTCGGAGATGT CAAGCAGAAGACGGCATACGAGATAGGAGTCCGTCTCGTGGGCTCGGAGATGT CAAGCAGAAGACGGCATACGAGATCATGCCTAGTCTCGTGGGCTCGGAGATGT CAAGCAGAAGACGGCATACGAGATGTAGAGAGGTCTCGTGGGCTCGGAGATGT
} 


\subsubsection{Bioinformatics}

We performed quality checks of the sequenced reads using FASTQC (https://www.bioinformatics.babraham.ac.uk/projects/fastqc/). The reads were trimmed, using Trimmomatic (version 0.36) (Bolger et al., 2014) appyling a sliding window trimming with the parameters slidingwindow 4:15 and minlen 30. Trimmed reads were mapped to the D. melanogaster genome (version 6.13) after discarding the mitochondrial genome, using Bowtie2 (version 2.3.4.3) (Langmead et al., 2009), with the commands: - - no-unal and X2000. Samtools version 1.9 (Li et al., 2009) were subsequently used to convert the sam to bam files, and to sort and index bam files. We removed duplicates using PICARD (version 2.1.1, https://github.com/broadinstitute/picard) with default parameters and converted the resulted bam files to bed files. Reads were then centered as described in (Buenrostro et al., 2013). We used MACS2 (version 2.1.2) (Zhang et al., 2008, p. 2) with the following commands -g dm -nomodel --shift -100 --extsize 200 - q 0.01 -bdg to call significant peaks. We used the Integrated Genome Browser (IGB, (Freese et al., 2016)) to visualize the read depth and peaks. Peaks were annotated to the closest gene using the annotatePeaks .pl program from the HOMER software package (v4.8.3) using dm6 as genomic input.

\subsubsection{Conversion of Coordinates}

To compare the open chromatin landscape of all three sister species, we converted peak coordinates of $D$. mauritiana and D. simulans into $D$. melanogaster coordinates. This required to create custom liftOver files, also called chain files which are usually used to convert annotations from one genome version to the other (here from one species to another).

First, the D. melanogaster genome was indexed and each chromosome arm was saved separately as a .fasta file, using the samtools faidx command (Li et al., 2009). The same was done for the D. mauritiana strain-specific genome (TAM16). Each chromosome arm sequence was then split into chunks using the following command to ensure an efficient BLAT alignment and a .lft file was directly created using the size parameters: fasplit lift=Dmel_x.lft -oneFile size Dmel/dem-x.fasta 3000 dmel-x_chunks, where $\mathrm{x}$ stands for each chromosome arm. The resulting sequence chunks were then aligned to the $D$. mauritiana genome sequences using the BLAT alignment tool (Kent, 2002) with default parameters and .psl files as output. The coordinates of the alignment were then changed to the D. melanogaster coordinate system using the liftUp tool (Hinrichs et al., 2006) and the . Ift files created in the chromosome split step (see above). The converted alignments were 
then chained together using the axtChain tool with the following parameters: -psl linearGap=medium -faQ -faT. The resulting chain file (of each chromosome arm) were then combined and sorted using the chainMergeSort program. Using the chromosome sizes, co-called nets were created form chains using chainNet and subsequently netChainSubset was used to create over.chain files which are the files used for the coordinate conversion of peaks. This pipeline was adapted to both species, D. mauritiana and D. simulans. To convert peak coordinates, the liftOver tool was used with the parameter: - minMatch $=0.1$. The script can be found in the Appendix. Peaks which could not be converted were visualized using the Integrated Genome Browser (IGB) (Freese et al., 2016) and since nearly all mapped to the centromere regions of the respective chromosomes, we removed this regions from the $D$. melanogaster genome as well for further analysis.

\subsubsection{Comparison of peak architectures}

To get the consensus peak set of two sister species we used the bedtools intersect tool from the Bedtools toolset (version 2.24) (Quinlan, 2014; Quinlan and Hall, 2010) with the following parameters: -wa -wb -wo. Species specific peaks were extracted using the same tool with the $-\mathrm{v}$ parameter. All further analyses were carried out using R version 3.3.3 or 3.5.2 (R Development Core Team, 2008). Gene sets were combined according to the following criteria: Set1 - genes did not have a single overlapping peak between two species, Set2 - genes that had only overlapping peaks, and Set3 - genes that had overlapping and additional nonoverlapping peaks. These genes were then overlapped with the information gained from our RNA-seq and ASE analysis, namely if the gene was differentially expressed between the two species, and if yes, which regulatory type was responsible for this differential expression. Fisher's exact tests was used to test for significances among the groups in the contingency tables.

\subsubsection{Sequence alignments}

To get the sequences of homologous peaks, the Bedtools (version 2.24) getfasta programm was used with default parameters. Peak sequences of $D$. mauritiana were then used to build a BLAST database (Camacho et al., 2009) using the -parse_seqids -dbtype nucl parameters. blastn $-d b$ was then used to blast the peak sequences in the two comparisons, with parameters -outfmt 6 -max_target_seqs 1 -evalue 0.01 . Wilcoxon Rank Sum test was applied to compare the percentage of identical matches between the groups (cis-, 
trans-, conserved or compensatory genes). To test if intronic sequences and TSS/promoter sequences show differences in sequences conservation, we split all peak sequences in the groups according to the respective annotation and repeated the analysis separately for both groups.

\subsubsection{Overlap with DrolD database}

To test for regulatory divergence specifically in transcription factors, we downloaded all entries from the Transcription Factor TF - Gene Interaction data file in the Drold database (version 2015-2) (Yu et al., 2008), including 157462 interactions and 12323 genes. We then overlapped the transcription factors with our RNA-seq dataset to filter those that are expressed in eye-antennal disc. Of these we retrieved the then the information about potential differential expression of the transcription factors between species, by comparing them with our differential expression analysis and further checked if differential expression was due to cis- or trans-regulatory changes. We additionally checked if the transcription factors fall into Gene Set 1,2 , or 3 , allowing us to categorize their regulatory landscape. 


\subsection{Supplementary Figures}
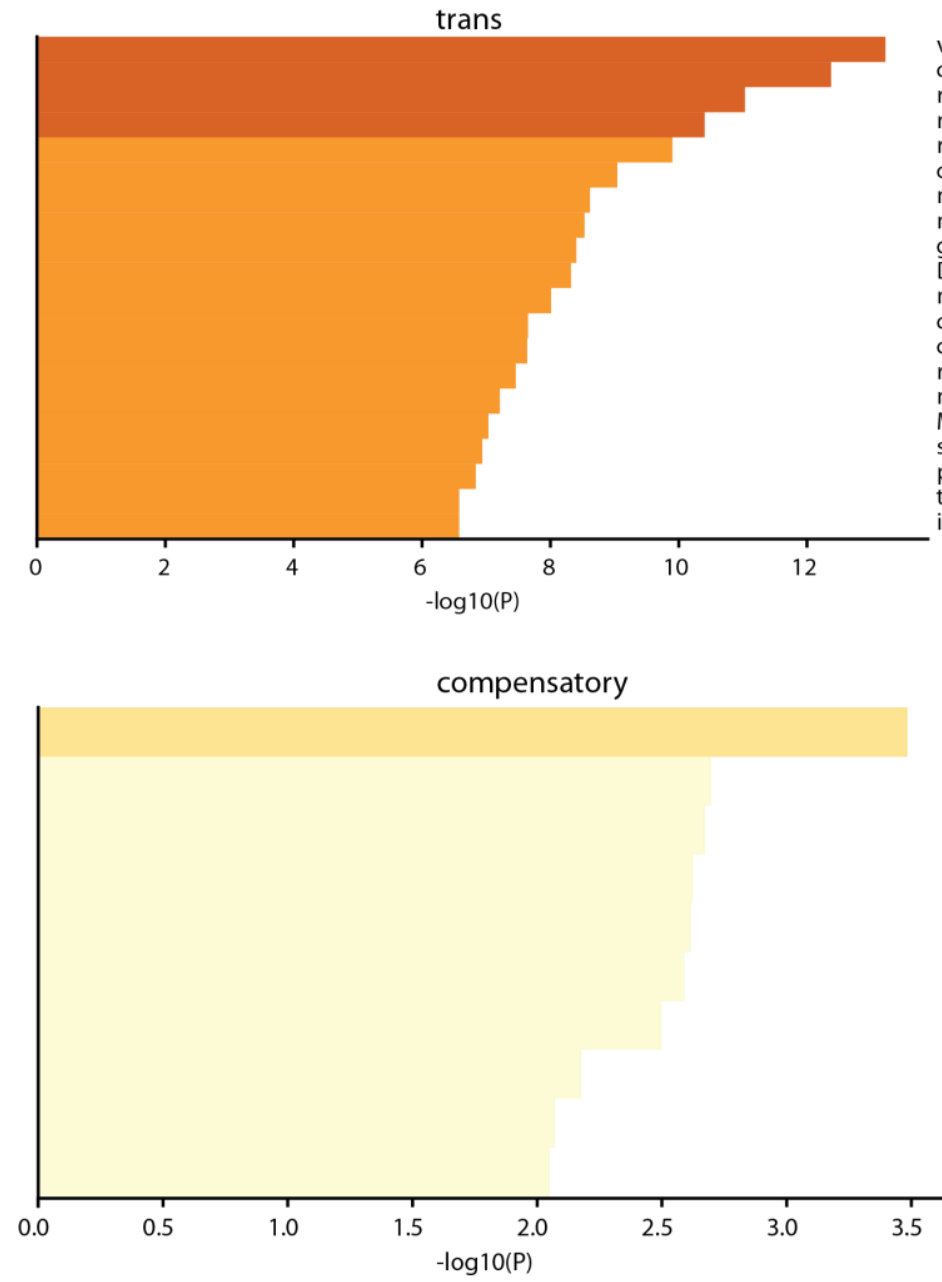

\section{-}

vesicle-mediated transport cell cycle process

regulation of cellular component organization neuron differentiation

regulation of developmental process

organelle localization

ncRNA metabolic process

mitochondrial translation

growth

DNA metabolic process

microtubule cytoskeleton organization

cell surface receptor signaling pathway

cell fate commitment

receptor-mediated endocytosis

morphogenesis of an epithelium

Metabolism of RNA

sensory organ development

positive regulation of transport

tRNA metabolic process immune system process

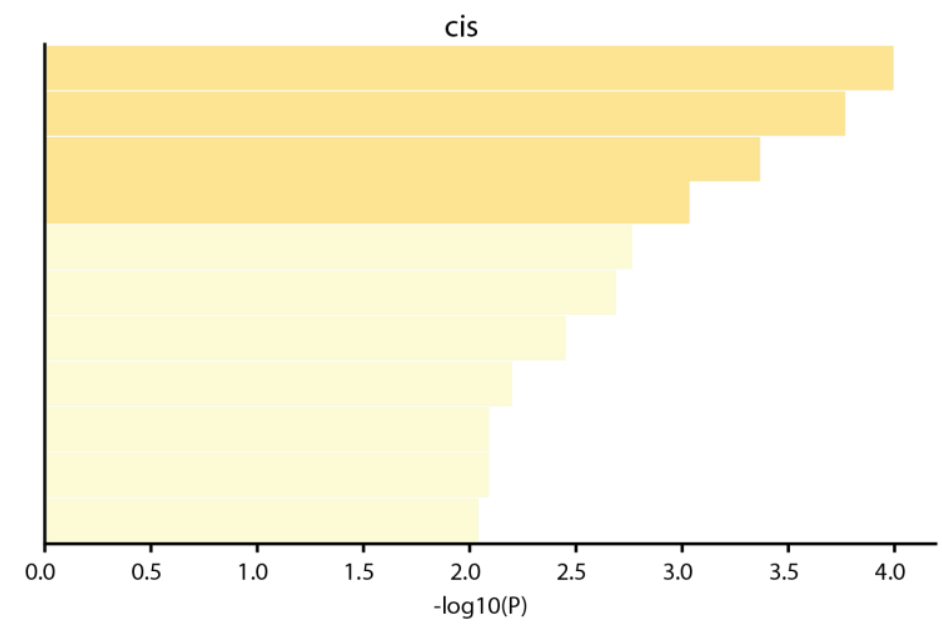

sulfur compound metabolic process

organic acid catabolic process

Basal transcription factors

proteasomal ubiquitin-independent protein catabolic process nucleoside monophosphate metabolic process

Nonsense-Mediated Decay (NMD)

regulation of gene silencing by RNA

Integration of energy metabolism

negative regulation of translation

isoprenoid biosynthetic process

nonribosomal peptide biosynthetic process

Metabolism of amino acids and derivatives

negative regulation of transposition

Phagosome

stem cell population maintenance

mitochondrial electron transport, cytochrome c to oxygen

regulation of compound eye retinal cell programmed cell death

Other glycan degradation

inorganic cation import across plasma membrane

regulation of pole plasm oskar mRNA localization

Synthesis of active ubiquitin: roles of E1 and E2 enzymes

Supplementary Figure 21. GO enrichment analysis following ASE analysis between D. simulans vs. D. mauritiana. Genes that were differentially expressed due to trans-regulatory changes were enriched in morphogenetic, cell cycle, growth and developmental GO terms. Genes, showing compensatory regulation in the hybrids were enriched in more metabolic processes. Gene with cis-regulatory divergence showed enrichment in similar processes, namely biosynthetic and metabolic processes, but also in more eye-specific processes, like 'retinal cell programmed cell death'. 
A

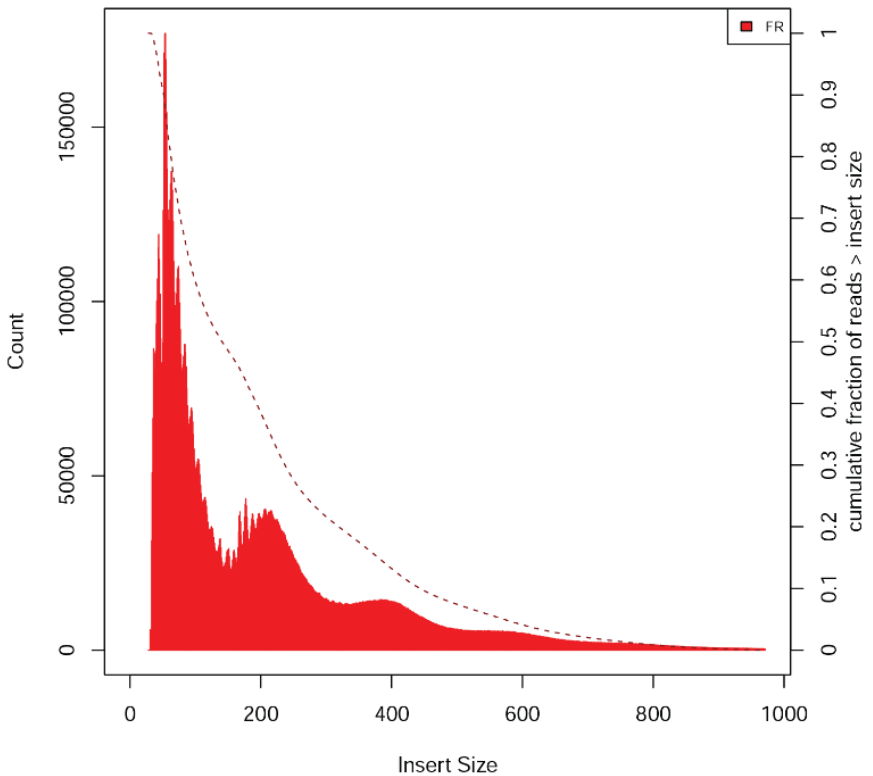

B

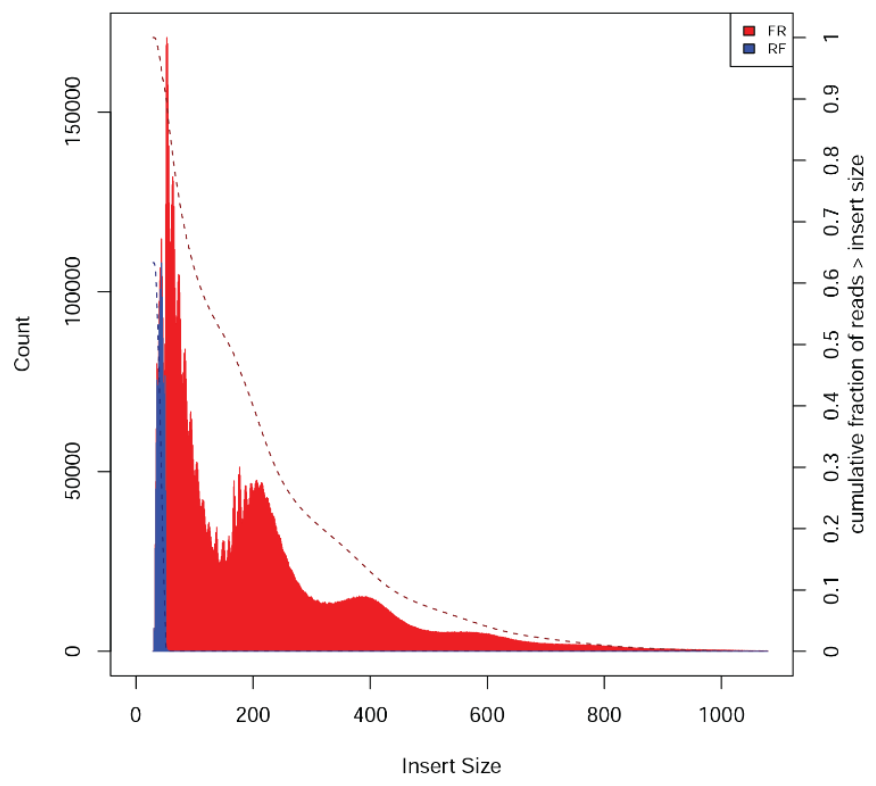

Supplementary Figure 22. Insert size distribution of ATAC-seq datasets of $D$. mauritiana and D. simulans. A. The insert size distribution of the $D$. mauritiana ATAC-seq dataset and $\mathbf{B}$. of $D$. simulans show the same typical periodicity of $\sim 200 \mathrm{bp}$ as the D. melanogaster dataset (Figure 22B.). 


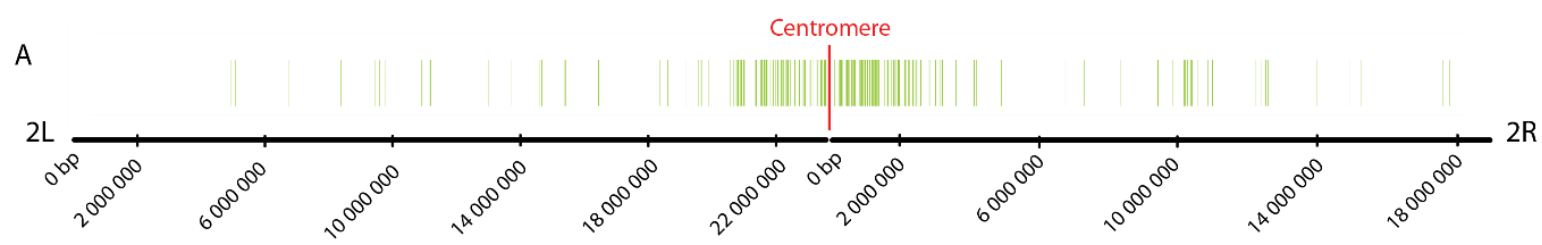

B

\begin{tabular}{|c|c|c|c|}
\hline & chromosome & peaks excluded & region excluded \\
\hline \multirow{6}{*}{ D.melanogaster } & Chr-2L & 0 & 0 \\
\hline & Chr-2R & 305 & $<4.100 .000$ \\
\hline & Chr-3L & 203 & $>24.000 .000$ \\
\hline & Chr-3R & 183 & $<4.160 .000$ \\
\hline & Chr-4 & 0 & 0 \\
\hline & Chr-X & 734 & $>18.270 .000$ \\
\hline \multirow{6}{*}{ D. mauritiana } & Chr-2L & 0 & \\
\hline & $\mathrm{Chr}-2 \mathrm{R}$ & 4 & \\
\hline & Chr-3L & 5 & \\
\hline & Chr-3R & 13 & \\
\hline & Chr-4 & 0 & \\
\hline & Chr-X & 0 & \\
\hline \multirow{6}{*}{ D. simulans } & Chr-2L & 0 & \\
\hline & Chr-2R & 99 & \\
\hline & Chr-3L & 133 & \\
\hline & Chr-3R & 111 & \\
\hline & Chr-4 & 0 & \\
\hline & Chr-X & 505 & \\
\hline
\end{tabular}
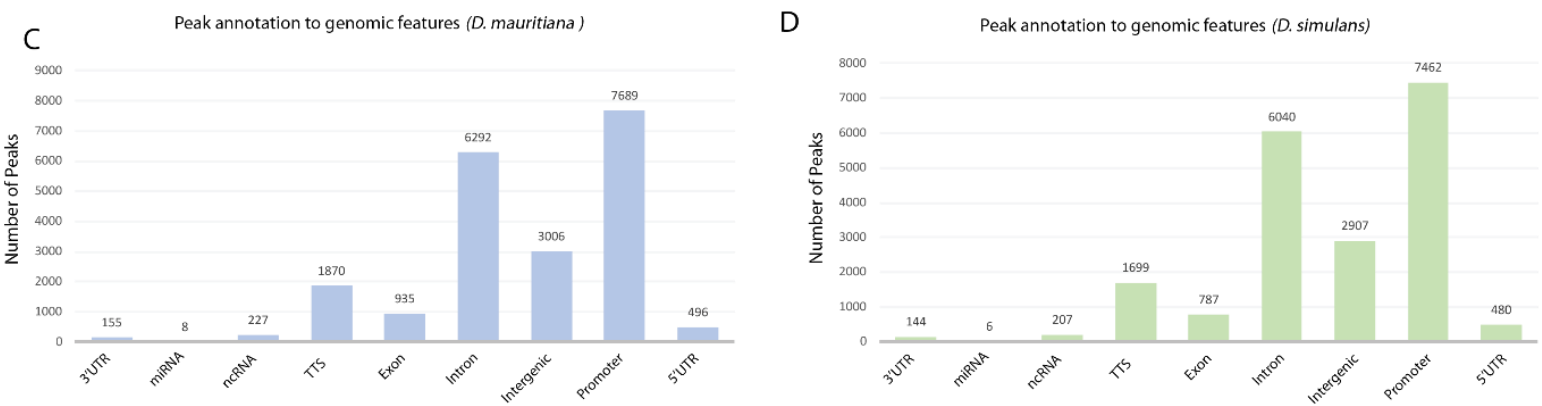

Supplementary Figure 23. Conversion of genomic coordinates. A. Peak coordinates that could not be converted from the D.simulans to the D. melanogaster genomic coordinate system mapped predominantly to the centromeric regions of the chromosomes. Shown here is the $2^{\text {nd }}$ chromosome of $D$. simulans. B. Peaks that were excluded by filtering centromeric regions and peaks that did not map in each species. The last column in $D$. melanogaster lists the regions that were excluded for each chromosome arm (in bp). C. Converted peaks were annotated to gene features. The pattern is comparable to D. melanogaster, where also most peaks were annotated to promoter regions, followed by intronic regions and intergenic regions. 


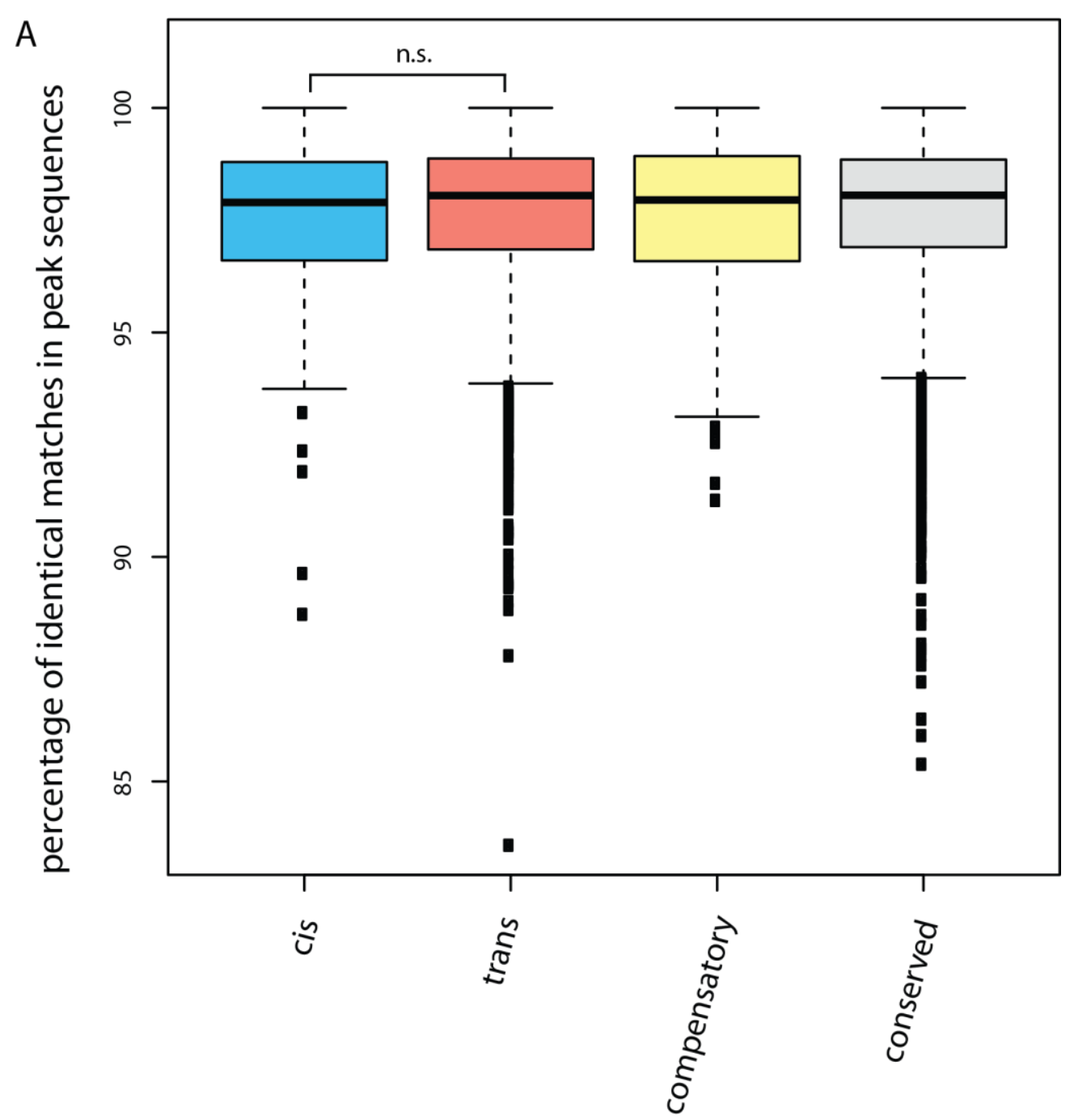

Supplementary Figure 24. A. The sequence divergence between peak sequences close to genes showing cisregulatroy divergence or 'compensatory - genes' in the hybrid do not show significantly more sequence changes than conserved genes or genes with trans-regulatory divergence when $D$. simulans is compared to $D$. mauritiana. 

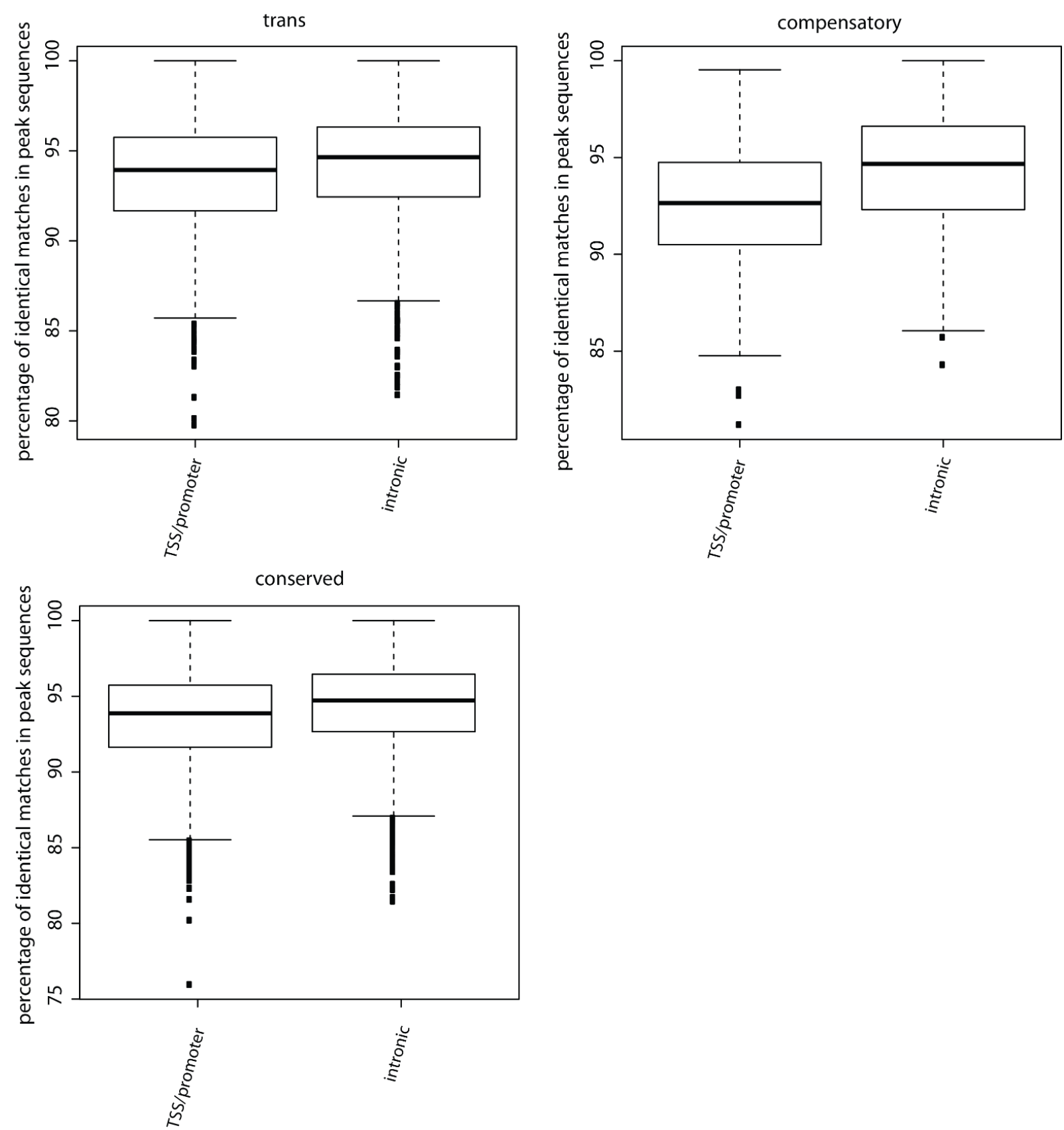

Supplementary Figure 25. As shown for genes with cis-regulatory divergence between $D$. melanogaster and $D$. mauritiana, TSS \promoter peaks of genes from all divergence groups show a significantly higher sequence divergence than intronic peaks. Note that we provide all $p$-values between the pairwise comparisons in Supplementary Table 22. 


\subsection{Supplementary Tables}

Supplementary Table 20. A. Fisher's Exact test ( $\mathrm{p}$-values) for pairwise comparisons of gene sets between $D$. melanogaster vs. D. mauritiana from Figure 25B. B. Fisher's Exact test ( $p$-values) for pairwise comparisons of gene sets between $D$. mauritiana vs. D. simulans from Figure $25 C$

A

\begin{tabular}{|c|l|l|r|}
\hline \multicolumn{3}{|c|}{ Fisher's Exact test - Gene Sets (ad. Figure 4A) } \\
\hline Set & \multicolumn{2}{|c|}{ Comparison } & p-value \\
\hline 1 & cis & trans & $9.54 \mathrm{E}-03$ \\
\hline 1 & cis & compensatory & $3.83 \mathrm{E}-02$ \\
\hline 1 & cis & conserved & $1.57 \mathrm{E}-03$ \\
\hline 1 & trans & compensatory & $7.59 \mathrm{E}-01$ \\
\hline 1 & trans & conserved & $5.35 \mathrm{E}-01$ \\
\hline 1 & compensatory & conserved & $1.00 \mathrm{E}+00$ \\
\hline & & & \\
\hline 2 & cis & trans & $8.12 \mathrm{E}-01$ \\
\hline 2 & cis & compensatory & $4.34 \mathrm{E}-01$ \\
\hline 2 & cis & conserved & $1.10 \mathrm{E}-02$ \\
\hline 2 & trans & compensatory & $2.28 \mathrm{E}-01$ \\
\hline 2 & trans & conserved & $4.76 \mathrm{E}-05$ \\
\hline 2 & compensatory & conserved & $1.31 \mathrm{E}-03$ \\
\hline & & & \\
\hline 3 & cis & trans & $1.60 \mathrm{E}-07$ \\
\hline 3 & cis & compensatory & $4.92 \mathrm{E}-01$ \\
\hline 3 & cis & conserved & $4.12 \mathrm{E}-01$ \\
\hline 3 & trans & compensatory & $2.17 \mathrm{E}-04$ \\
\hline 3 & trans & conserved & $2.20 \mathrm{E}-16$ \\
\hline 3 & compensatory & conserved & $8.62 \mathrm{E}-01$ \\
\hline & & & \\
\hline
\end{tabular}

Fisher's Exact test - Gene Sets (ad. Supplementary Figure 4A)

\begin{tabular}{|l|l|l|r|}
\hline Set & \multicolumn{2}{|c|}{ Comparison } & p-value \\
\hline 1 & cis & trans & $5.16 \mathrm{E}-03$ \\
\hline 1 & cis & compensatory & $1.75 \mathrm{E}-02$ \\
\hline 1 & cis & conserved & $3.52 \mathrm{E}-04$ \\
\hline 1 & trans & compensatory & $7.05 \mathrm{E}-01$ \\
\hline 1 & trans & conserved & $4.45 \mathrm{E}-02$ \\
\hline 1 & compensatory & conserved & $7.73 \mathrm{E}-01$ \\
\hline & & & \\
\hline 2 & cis & trans & $2.94 \mathrm{E}-02$ \\
\hline 2 & cis & compensatory & $1.53 \mathrm{E}-01$ \\
\hline 2 & cis & conserved & $9.16 \mathrm{E}-03$ \\
\hline 2 & trans & compensatory & $7.61 \mathrm{E}-01$ \\
\hline 2 & trans & conserved & $2.20 \mathrm{E}-16$ \\
\hline 2 & compensatory & conserved & $3.49 \mathrm{E}-05$ \\
\hline & & & \\
\hline 3 & cis & trans & $3.75 \mathrm{E}-02$ \\
\hline 3 & cis & compensatory & $1.69 \mathrm{E}-01$ \\
\hline 3 & cis & conserved & $8.23 \mathrm{E}-01$ \\
\hline 3 & trans & compensatory & $7.24 \mathrm{E}-01$ \\
\hline 3 & trans & conserved & $2.63 \mathrm{E}-09$ \\
\hline 3 & compensatory & conserved & $7.45 \mathrm{E}-02$ \\
\hline & & & \\
\hline
\end{tabular}


Supplementary Table 21. Wilcoxon Rank Sum Test ( $p$-values) of sequence alignments between regulatory regions between D. melanogaster and D. mauritiana (Boxplots are shown in Figure 24A).

\begin{tabular}{|c|c|c|}
\hline \multicolumn{3}{|c|}{ Wilcoxon Rank Sum Test - Sequence Alignment (ad. Figure 4B) } \\
\hline \multicolumn{2}{|c|}{ Comparison } & p-value \\
\hline cis & trans & 1.06E-12 \\
\hline cis & compensatory & 2.40E-02 \\
\hline cis & conserved & 1.07E-13 \\
\hline trans & compensatory & 3.61E-04 \\
\hline trans & conserved & $8.64 \mathrm{E}-01$ \\
\hline compensatory & conserved & 2.11E-04 \\
\hline
\end{tabular}

Supplementary Table 22. Wilcoxon Rank Sum Test ( $p$-values) of sequence alignments between intronic and TSS/promoter regulatory regions for each regulatory type. The boxplot for the cis-regulatroy changes is depicted in Figure 24B, whereas the three boxplots for trans-regulatry, compensatoy and conserved gene sets are shown in Supplementary Figure 25.

Wilcoxon Rank Sum Test -TSS/promoter vs. intronic (ad. Figure 4C and Supplementary Figure 5)

\begin{tabular}{|c|c|}
\hline regulatory divergence type & p-value \\
\hline cis & $9.19 \mathrm{E}-03$ \\
\hline trans & $7.81 \mathrm{E}-08$ \\
\hline compensatory & $1.48 \mathrm{E}-04$ \\
\hline conserved & $2.20 \mathrm{E}-16$ \\
\hline
\end{tabular}


Supplementary Table 23. Transcription Factors downloaded from the DrolD database were first overlapped with the Gene Sets 1-3 (highly diverged regulatory regions, conserved regulatory regions and sligthly diverged regulatory regions) and second with the information about their divergence type. Most transcription factors show a highly conserved regulatory region and their expression levels are conserved as well between the species. If differentially expressed the genes are differentially expressed due to upstream trans-regulatory changes.

\begin{tabular}{|c|c|c|c|c|c|}
\hline \multicolumn{6}{|c|}{ Transcription factors (DrolD) in the gene sets } \\
\hline \multicolumn{2}{|c|}{ Set 1} & \multicolumn{2}{|c|}{ Set 2} & \multicolumn{2}{|c|}{ Set 3} \\
\hline Dmel_Dmau & Dsim_Dmau & Dmel_Dmau & Dsim_Dmau & Dmel_Dmau & Dsim_Dmau \\
\hline ci & Jra & abd-A & abd-A & Abd-B & Antp \\
\hline CTCF & $\mathrm{sxc}$ & Adf1 & $A b d-B$ & Antp & ap \\
\hline \multicolumn{2}{|c|}{ E(spl)m5-HLHItgo } & Aef1 & Adf1 & ap & apt \\
\hline gsb & twi & ara & Aef1 & apt & bab1 \\
\hline hb & & ato & ara & bab1 & bin \\
\hline Jra & & bcd & ato & BEAF-32 & br \\
\hline sna & & brm & bcd & bin & byn \\
\hline tgo & & cf2 & BEAF-32 & br & chinmo \\
\hline twi & & Chro & brk & brk & cnc \\
\hline & & Cp190 & brm & byn & ct \\
\hline & & CtBP & cad & cad & CtBP \\
\hline & & D & CBP & CBP & Dfd \\
\hline compensato & & DCTN1-p150 & Cf2 & chinmo & DII \\
\hline cis & & Dfd & Chro & $\mathrm{cnc}$ & Dsp1 \\
\hline & & disco & Cp190 & CrebA & $d s x$ \\
\hline & & dl & CrebA & ct & E2f1 \\
\hline & & Doc1 & D & Deaf1 & $E \subset R$ \\
\hline & & $d p n$ & DCTN1-p150 & DII & Eip74EF \\
\hline & & Dref & Deaf1 & $d s x$ & en \\
\hline & & Dsp1 & disco & E2f1 & ftz-f1 \\
\hline & & $d w g$ & dl & EcR & grh \\
\hline & & dysf & Doc1 & Eip74EF & gro \\
\hline & & E(spl)m8-HLH & $d p n$ & en & gsb-n \\
\hline & & $E(z)$ & dwg & ey & $\mathrm{Hr} 39$ \\
\hline & & $E 2 \nmid 2$ & dysf & ftz-f1 & $\mathrm{H} \times 46$ \\
\hline & & ems & E(spl)m5-HLH & grh & inv \\
\hline & & eve & E(spl)m8-HLH & gsb-n & lab \\
\hline & & exd & $E(z)$ & Hr39 & Mad \\
\hline & & fkh & E2f2 & Hr46 & Mef2 \\
\hline & & $\mathrm{gcm}$ & ems & hth & oc \\
\hline & & gl & eve & inv & ора \\
\hline & & gro & exd & jumu & ovo \\
\hline & & h & ey & Iab & Pdp1 \\
\hline & & hkb & fkh & Iz & ph-p \\
\hline & & Hsf & $\mathrm{gcm}$ & Mad & pnt \\
\hline & & ind & gl & Mef2 & $s b b$ \\
\hline & & insv & gsb & oc & shn \\
\hline & & $\mathrm{kn}$ & $\mathrm{h}$ & opa & srp \\
\hline & & kni & hb & ovo & Stat92E \\
\hline & & $\mathrm{Kr}$ & hkb & pan & sv \\
\hline & & Med & Hsf & Pax & toy \\
\hline & & mip120 & hth & Pdp1 & trx \\
\hline & & MTF-1 & ind & ph-p & ttk \\
\hline & & Myb & insv & pnt & vnd \\
\hline & & NELF-B & jumu & sbb & z \\
\hline & & nub & $\mathrm{kn}$ & $\operatorname{shn}$ & zfh1 \\
\hline & & Pc & kni & Stat92E & \\
\hline & & pho & $\mathrm{Kr}$ & sv & \\
\hline & & phol & Iz & toy & \\
\hline & & pnr & Med & ttk & \\
\hline & & prd & mip120 & $z$ & \\
\hline & & sd & MTF-1 & & \\
\hline & & sens & Myb & & \\
\hline & & Sfmbt & NELF-B & & \\
\hline & & slbo & nub & & \\
\hline & & slp1 & pan & & \\
\hline & & Snr1 & Pax & & \\
\hline & & so & Pc & & \\
\hline & & srp & pho & & \\
\hline & & $\mathrm{Su}(\mathrm{H})$ & phol & & \\
\hline & & $\mathrm{su}(\mathrm{Hw})$ & pnr & & \\
\hline & & TfIIB & prd & & \\
\hline & & tin & $\mathrm{sd}$ & & \\
\hline & & tll & sens & & \\
\hline & & Top2 & Sfmbt & & \\
\hline & & Trl & slbo & & \\
\hline & & trx & slp1 & & \\
\hline & & tup & Snr1 & & \\
\hline & & Ubx & so & & \\
\hline & & vnd & $\mathrm{Su}(\mathrm{H})$ & & \\
\hline & & vvl & $\mathrm{su}(\mathrm{Hw})$ & & \\
\hline & & zfh1 & TfIIB & & \\
\hline & & & tin & & \\
\hline & & & tll & & \\
\hline & & & Top2 & & \\
\hline & & & $\mathrm{Trl}$ & & \\
\hline & & & tup & & \\
\hline & & & Ubx & & \\
\hline & & & vvl & & \\
\hline
\end{tabular}




\subsection{Appendix}

\#\#\#The script was written to generate chain files to convert open-chromatin peak coordinates from D. mauritiana to D. melanogaster

\#\#\#The same pipeline was applied to D. simulans

$\#$ ! /bin/bash

\#script was adapted from http://blog.windhager.io/2016/10/21/creatingliftover-chain-files/

\# requires UCSC genome browser 'kent' bioinformatic utilities

module load EMBOSS/6.5.7 UCSC/20160601

mkdir psl

mkdir chain

mkdir net

\#get all the chromosomes in extra files

mkdir Dmel

mkdir Dmau

samtools faidx dmel-all-chromosome-r6.13_woMito.fasta 2L > Dmel/dmel2L. fasta

samtools faidx dmel-all-chromosome-r6.13_woMito.fasta 2R > Dmel/dmel-

2R.fasta

samtools faidx dmel-all-chromosome-r6.13_woMito.fasta 3L > Dmel/dmel-

3L. fasta

samtools faidx dmel-all-chromosome-r6.13_woMito.fasta 3R > Dmel/dmel-

3R. fasta

samtools faidx dmel-all-chromosome-r6.13 woMito.fasta $4>$ Dmel/dmel-4.fasta

samtools faidx dmel-all-chromosome-r6.13-woMito.fasta $X>$ Dmel/dmel-X.fasta

samtools faidx TAM16_strainspecificGenome_woMito.fasta Dmau_2L > Dmau/dmau2L.fasta

samtools faidx TAM16_strainspecificGenome_woMito.fasta Dmau_2R > Dmau/dmau2R. fasta

samtools faidx TAM16_strainspecificGenome_woMito.fasta Dmau_3L > Dmau/dmau3L. fasta

samtools faidx TAM16_strainspecificGenome_woMito.fasta Dmau_3R > Dmau/dmau3R. fasta

samtools faidx TAM16_strainspecificGenome_woMito.fasta Dmau_4 > Dmau/dmau4. fasta

samtools faidx TAM16_strainspecificGenome_woMito.fasta Dmau_X > Dmau/dmauX.fasta

\# split new sequences for efficient BLAT alignment

faSplit -lift=Dmel_2L.lft -oneFile size Dmel/dmel-2L.fasta 3000 Dmel/dmel2L_chunks

fasplit -lift=Dmel_2R.Ift -oneFile size Dmel/dmel-2R.fasta 3000 Dmel/dmel$2 \mathrm{R}$ chunks

fasplit -lift=Dmel 3L.lft -oneFile size Dmel/dmel-3L.fasta 3000 Dmel/dmel3L chunks

fas̄plit -lift=Dmel_3R.lft -oneFile size Dmel/dmel-3R.fasta 3000 Dmel/dmel3R_chunks

fasplit -lift=Dmel_4.lft -oneFile size Dmel/dmel-4.fasta 3000 Dmel/dmel4 chunks

fāsplit -lift=Dmel_X.lft -oneFile size Dmel/dmel-X.fasta 3000 Dmel/dmelX_chunks 
\# align resulting sequence chunks to old sequence, which is in my case the Dmau genome

/home/uni05/ebuchbe/Programme/./blat

Dmau/TAM16_strainspecificGenome_woMito.fasta Dmel/dmel-2L_chunks.fa psl/chr2L $\bar{b} l$ at param.psl \&

/home/uni $\overline{0} 5$ /ebüchbe/Programme/./blat

Dmau/TAM16_strainspecificGenome_woMito.fasta Dmel/dmel-2R_chunks.fa

psl/chr2R_b̄lat_param.psl\&

/home/uni $\overline{0}$ /ebüchbe/Programme/./blat

Dmau/TAM16_strainspecificGenome_woMito.fasta Dmel/dmel-3L_chunks.fa

psl/chr3L_b̄lat_param.psi \&

/home/unīo5/ebüchbe/Programme/./blat

Dmau/TAM16_strainspecificGenome_woMito.fasta Dmel/dmel-3R_chunks.fa

psl/chr3R_blat_param.psl\&

/home/uni $\overline{0} 5 /$ ebüchbe/Programme/./blat

Dmau/TAM16 strainspecificGenome_woMito.fasta Dmel/dmel-4_chunks.fa

psl/chr4 blat param.psl \&

/home/unī05/ebuchbe/Programme/./blat

Dmau/TAM16_strainspecificGenome_woMito.fasta Dmel/dmel-X_chunks.fa psl/chrX_blat_param.psl\&

\# change alignment coordinates to parent coordinate system according to LFT file

\#Liftup can convert coordinates in most annotation files. It can add to positions and change the chromosome part of those files. It's main input is the lift-file that specifies how to convert the coordinates.

liftUp -psle psl/chr2L.psl Dmel_2L.lft warn psl/chr2L_blat param.psl

liftUp -psle psl/chr2R.psl Dmel_2R.lft warn psl/chr2R_blat_param.psl

liftUp -psle psl/chr3L.psl Dmel_3L.lft warn psl/chr3L_blat_param.psl

liftUp -pslQ psl/chr3R.psl Dmel_3R.lft warn psl/chr3R_blat_param.psl

liftup -psle psl/chr4.psl Dmel_4.lft warn psl/chr4_blāt_param.psl

liftup -psle psl/chrX.psl Dmel_X.lft warn psl/chrX_blat_param.psl

\# chain together alignments from PSL files

axtChain -psl -linearGap=medium -faQ -faT psl/chr2L.psl

Dmau/TAM16 strainspecificGenome woMito.fasta Dmel/dmel-2L.fasta

chain/chr2产_axtchain.chain

axtChain -psi -linearGap=medium -faQ -faT psl/chr2R.psl

Dmau/TAM16_strainspecificGenome_woMito.fasta Dmel/dmel-2R.fasta

chain/chr $2 \overline{\mathrm{R}}$ axtChain.chain

axtChain -psi -linearGap=medium -faQ -faT psl/chr3L.psl

Dmau/TAM16_strainspecificGenome_woMito.fasta Dmel/dmel-3L.fasta

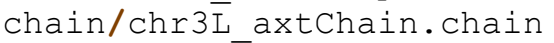

axtChain -psi -linearGap=medium -faQ -faT psl/chr3R.psl

Dmau/TAM16_strainspecificGenome_woMito.fasta Dmel/dmel-3R.fasta

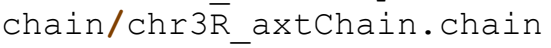

axtChain -psil -linearGap=medium -faQ -faT psl/chr4.psl

Dmau/TAM16_strainspecificGenome_woMito.fasta Dmel/dmel-4.fasta

chain/chr4_axtchain.chain

axtChain - $\mathrm{ps}$-linearGap=medium -faQ -faT psl/chrX.psl

Dmau/TAM16_strainspecificGenome_woMito.fasta Dmel/dmel-X.fasta

chain/chrX_axtchain.chain

\# combine and sort chain files

\#chainsort chain/chr1 axtchain.chain chain/chr2 axtchain.chain

chain/chr3 axtchain.chain | chainsplit chain stēin

chainMerge Sort chain/chr2L_axtChain.chain chain/chr2R_axtchain.chain

chain/chr3L axtChain.chain chain/chr3R axtchain.chain

chain/chr4_axtchain.chain chain/chrX_axtchain.chainl chainsplit chain stdin 
\# determine chromosome sizes

faToTwoBit Dmau/dmau-2L. fasta Dmau/dmau-2L.2bit

faToTwoBit Dmau/dmau-2R. fasta Dmau/dmau-2R.2bit

faToTwoBit Dmau/dmau-3L. fasta Dmau/dmau-3L.2bit

faToTwoBit Dmau/dmau-3R. fasta Dmau/dmau-3R.2bit

faToTwoBit Dmau/dmau-4. fasta Dmau/dmau-4.2bit

faToTwoBit Dmau/dmau-X. fasta Dmau/dmau-X.2bit

faToTwoBit Dmel/dmel-2L. fasta Dmel/dmel-2L.2bit

faToTwoBit Dmel/dmel-2R. fasta Dmel/dmel-2R.2bit

faToTwoBit Dmel/dmel-3L. fasta Dmel/dmel-3L.2bit

faToTwoBit Dmel/dmel-3R. fasta Dmel/dmel-3R.2bit

faToTwoBit Dmel/dmel-4.fasta Dmel/dmel-4.2bit

faToTwoBit Dmel/dmel-X.fasta Dmel/dmel-X.2bit

\{ twoBitInfo Dmau/dmau-2L.2bit stdout; twoBitInfo Dmau/dmau-2R.2bit stdout; twoBitInfo Dmau/dmau-3L.2bit stdout; twoBitInfo Dmau/dmau-3R.2bit stdout; twoBitInfo Dmau/dmau-4.2bit stdout; twoBitInfo Dmau/dmau-X.2bit stdout; \} > Dmau/chrom.sizes

\{ twoBitInfo Dmel/dmel-2L.2bit stdout; twoBitInfo Dmel/dmel-2R.2bit stdout; twoBitInfo Dmel/dmel-3L.2bit stdout; twoBitInfo Dmel/dmel-3R.2bit stdout; twoBitInfo Dmel/dmel-4.2bit stdout; twoBitInfo Dmel/dmel-X.2bit stdout; \} > Dmel/chrom.sizes

\# make alignment nets out of chains

mkdir net

chainNet chain/Dmau 2L. chain Dmau/chrom.sizes Dmel/chrom.sizes net/chr_2L.net/dev/null

chainNet chain/Dmau_2R.chain Dmau/chrom.sizes Dmel/chrom.sizes net/chr_2R. net/dev/null

chainNet chain/Dmau 3L.chain Dmau/chrom.sizes Dmel/chrom.sizes net/chr_3L.net/dev/null

chainNet chain/Dmau 3R. chain Dmau/chrom.sizes Dmel/chrom.sizes

net/chr 3R. net/dev/null

chainNet chain/Dmau_4.chain Dmau/chrom.sizes Dmel/chrom.sizes net/chr_4.net /dev/null

chainNet chain/Dmau_X.chain Dmau/chrom.sizes Dmel/chrom.sizes net/chr_X.net /dev/null

\# create over.chain

netchainsubset net/chr_2L.net chain/Dmau_2L.chain

chain/Dmau_2L_subset.chain

netchainsubsset net/chr 2R. net chain/Dmau 2R. chain

chain/Dmau_2R_subset.chain

netchainsub̄set net/chr_3L.net chain/Dmau_3L.chain

chain/Dmau 3L subset. chain

netChainsubset net/chr_3R.net chain/Dmau_3R.chain

chain/Dmau_3R_subset.chain

netchainsūbset̄ net/chr 4. net chain/Dmau 4. chain chain/Dmau 4 subset. chain netChainsubset net/chr_X.net chain/Dmau_X.chain chain/Dmau_X_subset.chain cat chain/Dmau_2L_subset.chain chain/Dmau_2R_subset.chain

chain/Dmau 3L subset.chain chain/Dmau 3R subset.chain

chain/Dmau_4_subset.chain chain/Dmau_ X_subset.chain > over_DmauToDmel.chain

rm -rf psl chain net

\# do the coordinate conversion with liftover

\# Usage:

\# liftover oldFile map.chain newFile unMapped

\#\#liftover the already called peaks for Dmau for comparison 
module load EMBOSS/6.5.7 UCSC/20160601

liftover -minMatch=0.1 TAM 96hA peaks.bed over DmauToDmel.chain

TAM_96hA_peaks_mapped.bed TAM_9 $\overline{6} \mathrm{hA} \_p e a k s \_u n m a p p e d . b e d \&$

\#\#\#grep the unmapped peaks in Dmau for visualization

grep "Dmau" TAM_96hA_peaks_unmapped.bed >

TAM_96hA_peaks_unmapped_IGBinput.bed 


\section{General Discussion and Outlook}

Evolutionary changes in phenotypes, including adult morphologies, life history or physiological traits are a prerequisite for a constant adaptation to an ever-changing environment and the result of heritable mutations in the genome. For variation in adult morphological structures it is widely accepted that such mutations often affect the developmental programs underlying the formation of the respective structures. Building a complex organism requires that an initially single cell differentiates into various cell types that make up a variety of tissues and eventually form functional organs. The instructions for these developmental processes are encoded in the genome and translated through stage- and tissuespecific gene expression, that allows a cell or a group of cells to acquire a specific fate. Consequently, a major goal of biological studies is to understand how a given genotype translates - on a molecular level - into relevant phenotypes ('genotype to phenotype map'). For morphological traits, the application of comparative developmental approaches has been proven to be a powerful way to achieve this goal.

\subsection{Integration of different datasets in comparative biological studies}

Historically, the relationships between animal lineages were often reconstructed by the comparison of adult morphological features (e.g. (Snodgrass, 1938)), resulting in numerous descriptions of morphological phenotypes and traits in a variety of organisms. Advances in molecular techniques and the establishment of genetic tools allowed a shift from comparative and descriptive studies, towards a more experimental discipline that made it possible to verify phylogenetic relationships on a molecular level. However, only the advent of high throughput sequencing technologies revolutionized the way to reconstruct such phylogenies (e.g. (Dunn et al., 2008; Oakley et al., 2013) for a very recent study see: (Laumer et al., 2019)). Approaches, that combine morphological data and genomic approaches have been used to resolve for example the relationships of Squamata (comprising lizards, snakes and amphisbaenia), including fossil taxa (Reeder et al., 2015) or to address the evolution of larger groups, like all deuterostomes (Swalla and Smith, 2008). Whole genome sequencing and/or transcriptomics also have the power to reveal major ancient evolutionary events like whole genome duplications, allowing for instance subsequent comparison of gene content and syntenies (eg. (Dehal and Boore, 2005; Schwager et al., 2017; Singh et al., 2015)). The finding that spiders and scorpions share an ancient genome duplication supports their close relationship compared to 
arachnids that do not show signatures of this duplication (Schwager et al., 2017). Apart from gaining more insights into phylogenetic relationships, such data are also highly valuable in answering questions about phenotypic evolution, including neofunctionalization of genes and the emergence of evolutionary novelties (e.g. (Moriyama and Koshiba-Takeuchi, 2018; Turetzek et al., 2017, 2016)).

Apart from the comparison of adult morphologies, classical comparative developmental approaches like the analysis of, for instance, Hox genes in several lineages, have brought major insights into the evolution of body plans (e.g. (Akam, 1995; Akam et al., 1994; Garcia-Fernàndez and Holland, 1994)). Tarazona and colleagues recently used the cuttlefish Sepia officinalis to study if the developmental processes underlying appendage development are conserved among Bilaterians. They could indeed find that, despite legs of vertebrates, arthropods and cephalopods not being homologous structures, the 'developmental mechanisms' of appendage formation seem to be highly conserved (Prpic, 2019; Tarazona et al., 2019).

Comparative embryology resulted in the suggestion that vertebrate embryogenesis goes through highly conserved stages, so-called phylotypic stages. Haeckel proposed, based on his observations that species look exceptionally similar during certain stages of embryonic development, his 'biogenetic law', suggesting that the phylogeny is recapitulated during development of an organism (Haeckl, 1879, 1867; Losos, 2014). Even though it is clear nowadays that the biogenetic law does not reflect reality, gene expression data has indeed shown that the transcriptome expressed at defined stages of zebrafish or Drosophila development shows signatures of an hourglass (Domazet-Lošo and Tautz, 2010). While molecular tools were for a long time only available for a few model systems, affordable sequencing technologies facilitated in recent years the establishment of genomic resources not only for classical, but also emerging model systems (Ellegren, 2014). Sequencing the genome and analyzing open chromatin datasets of Branchiostoma lanceolatum, the Mediterranean amphioxus, recently revealed that gene expression and the cis-regulatory architecture are highly conserved in all chordates during certain stages of development but showed that this phylotypic stage (i.e. the time point showing minimal transcriptomics divergence) between Branchiostoma and other vertebrates occurs at a slightly earlier time point compared to vertebrates (Marlétaz et al., 2018). Overall, it is relatively easy these days to provide a detailed description of the genotype for many different organisms for which detailed anatomical data 
has been revealed over the years. But even though many genomes are sequenced, and the morphology and development of many organisms are described, it remains often elusive to recapitulate how the genomic information is used to define the adult phenotype. Further, mechanistic insights are in most cases missing. In summary, one can assume that the integration of morphological, developmental and molecular datasets allows comprehensive insights in phylogenetic relationships (Lee and Palci, 2015) and the genotype-phenotype map.

Here I argue that the combination of various detailed datasets provides the means to establish genotype-phenotype associations. First, a thorough understanding of the phenotype of interest is necessary. Additionally, for morphological traits it is highly informative to gain insights into developmental differences. Second, a comprehensive overview of the gene content and the genome size/organization is helpful. This can be achieved by generating transcriptome and genome datasets. Third, a correlation between the genotype and the phenotype must be established. If closely related species, that do not yet result in sterile offspring, are studied this can be done by quantitative genetics approaches such as QTL mapping or GWAS. Also, gene expression has been extensively used as an intermediate phenotype, backed up by the fact, that many mapped variances were described that influence gene expression (e.g. (Chan et al., 2010; Coyle et al., 2007; Cresko et al., 2004; Dixon et al., 2007; Gilad et al., 2008; Jia and Xu, 2007; Rockman and Kruglyak, 2006; Shapiro et al., 2004)). Hence, establishing gene expression differences between species (independent of the phylogenetic distance) allows identifying candidate genes responsible for morphological diversification. For morphological traits, such approaches are most powerful if they are combined with developmental data and if they are studied throughout development.

In each chapter of this work we used a combination of different datasets to connect phenotypes on several levels. In Chapter I, we applied microscopy techniques like scanning electron microscopy (SEM) and transmission electron microscopy (TEM) to analyze the function of pleuropodia in Schistocerca gregaria and connected the obtained insights with stage specific gene expression datasets. This allowed us to study long standing questions about the function of these organs, revealing potential new functions and as discussed below, holds the possibility to ask more general questions about developmental processes. In Chapter II and III, we used the model species Drosophila melanogaster and its sister species Drosophila mauritiana and Drosophila simulans to understand how complex traits like organ size and shape can evolve. As 
in Chapter I, we used a comparative transcriptomic dataset as an intermediate phenotype to link this genetic readout to observable morphological changes. We applied geometric morphometrics to quantitatively compare adult head shapes and developed a semi-automated method to count individual ommatidia of single compound eyes. By adding a comparative ATAC-seq dataset, representing stage and tissue specific open chromatin landscapes, we were able to gain more genome-wide insights into the evolution of gene expression divergence and subsequently eye size and head shapes in these three closely related species.

The different types of transcriptomics and functional genomics datasets that I generated, will allow in the future to gain insights on a more global GRN level, going beyond a gene-centric approach. In the following two sections I will argue that a GRN-centric view will further result in new insights into development and phenotypic evolution.

\subsection{Comparative gene expression studies and gene regulatory networks in} development

Research in Evo-Devo has established that the development of diverse organisms as well as organs and tissues is based on a limited set of developmental genes, so-called 'toolkit genes' (Carroll et al., 2001). Intriguingly, many of these factors are highly conserved in different lineages (e.g. (Halder et al., 1995; King and Wilson, 1975)). Therefore, a central question is how this limited set of genes can control the development of different cell types and tissues? It is widely accepted nowadays, that differential expression of these genes and rewiring of regulatory interactions underlies the generation of differences between cells types and subsequently organs and that the proper development of organs and structures relies heavily on the correct temporal and spatial expression of genes. One of the best described examples exemplifying this is the development of the Drosophila nervous system. Initially identical precursor cells start to express distinct transcription factors in a spatially and temporally defined manner, leading to the formation of different neural identities (e.g. (Homem and Knoblich, 2012; Karcavich, 2005; Technau et al., 2006)). A great model to study how gene expression distinguishes organs are serially homologous structures, such as the insect appendages. We studied pleuropodia in the locust S. gregaria, small glandular structures that are apparent at the first abdominal segment of many insect embryos and are thought to be serially homologous to embryonic leg buds (Bennett et al., 1999; Lewis et al., 2000; Machida, 1981). These transient organs eventually mature and gain specific functions during 
embryogenesis, but in contrast to other appendages they degenerate already before hatching of the embryo (Bullière, 1970; Louvet, 1975, 1973; Stay, 1977). The comparison of developing leg buds and pleuropodia is therefore a valuable model to address the question of how differences in development, morphology and function of initially similar structures can arise. In our study we showed that especially in the early stages of embryonic development, legs and pleuropodia are not only morphologically extremely similar, but that this similarity is also recapitulated on a transcriptomic level. Genes which are known to be involved in leg development, for instance distal-less (dII) are also active in pleuropodia (Lewis et al., 2000; Yamamoto et al., 2004). In later stages we found that gene expression patterns become more and more divergent and gene set enrichment showed that the functional annotation of expressed transcripts gets more and more tissue specific. Our combinatorial approach revealed that pleuropodia of S. gregaria are indeed directly involved in the breakdown of the serosal cuticle and subsequently in the hatching of the insect embryo, supporting the result drawn by Slifer already 1937 (H. Slifer, 1937). Surprisingly, our GO-term enrichment analysis of differentially expressed genes points towards a role of pleuropodia in insect embryonic immunity, a function that is usually conferred by the extraembryonic serosa (Jacobs et al., 2014). It remains to be shown functionally if the pleuropodia take over immune protection of the embryo after degeneration of the serosa upon dorsal closure (Konopová et al., 2019; Panfilio, 2008). Overall, we demonstrate that the combination of thorough phenotyping of developing structures with the analysis of differential expression levels as an intermediate phenotype allows to gain major insights into function and developmental processes of embryonic structures.

While gene expression catalogs of various organs and expression dynamics of individual genes are being established for more and more developmental processes, it is not yet completely resolved how developmental genes are regulated in a tissue and stage specific manner. In recent years it became clear that the regulation of genes is not a simple hierarchical process but rather defined by an intricate interplay of gene products. These interactions are usually represented as so-called gene regulatory networks (GRNs) which describe genes or their products (transcription factors and other proteins) as nodes and the interaction among these (i.e. genetic interactions) as edges (Davidson and Levine, 2008; Thompson et al., 2015). GRNs provide therefore a logic and comprehensible cascade of the underlying developmental program. RNA profiling has been proposed as one of the main experimental procedures in 
reconstructing GRNs, since it provides the possibility to collect all nodes that theoretically have to be considered in the respective GRN (shown for instance in (Sonawane et al., 2017), reviewed in (Thompson et al., 2015)). This allows to mathematically describe global properties of biological networks: One hallmark seems to be, that GRNs are so-called scale-free networks, meaning that the majority of the nodes is poorly connected and that we can only find a few highly connected nodes, also called hubs (Barabasi and Albert, 1999). Other measures, like node betweenness can further provide information about the role of single nodes in the global network architecture (e.g.(Koschützki and Schreiber, 2008)). One question that arises is how GRNs confer tissue specificity, i.e. when and where initially similar GRNs change and get rewired. By comparing tissue specific GRNs from adult human organs, Sonawane and colleagues found a rather low number of tissue-specific transcription factors (Sonawane et al., 2017). They show that functional specificity is primarily ensured by tissue specific interactions and that the expression of transcription factors is less well correlated with the regulation of functions in specific organs. Instead, tissue specific target gene expression is rather accomplished by context dependent paths throughout the network (Sonawane et al., 2017). Also, during eye-and head development in Drosophila, it was shown, that the same genes are able to exert different functions, mainly via rewiring of existing nodes (Palliyil et al., 2018). Palliyil and colleagues suggested that the retinal determination gene network is first important for overall growth of the complete eye-antennal disc, whereas later on, it specifically promotes retinal development. The fact that GRNs are constantly rewired during development provides an explanation, how morphological diversification can be achieved despite the developmental toolkit genes being not only expressed in one organ but are crucial for the proper development of several structures.

To dissect in more detail how and when such a rewiring takes place, our comparative dataset of Schistocerca pleuropodia and legs provides an excellent starting point. The already existing transcriptomic dataset allows to deduce which nodes are present and will need to be considered for the reconstruction of the respective tissue and stage specific GRN. The generation of a GRN depends though not only the information which genes have to be considered as nodes, but one also has to establish where to draw the edges. To add the edges globally it will be necessary to combine the RNA-seq dataset with, for instance, open chromatin datasets. ATAC-seq allows to search for transcription factor binding motifs in accessible and therefore potential cis-regulatory regions in the whole genome. This can be used to predict 
direct genetic interactions between transcription factors and their target genes. While such a dataset remains to be established for pleuropodia, preliminary results of our ATAC-seq dataset of the eye-antennal disc suggests that the open chromatin landscape is dependent on the temporal context, since we found a number of stage specific peaks at each of the studied time points (72h, 96h and $120 \mathrm{AEL}$, data not shown). This is consistent with other studies conducted in Drosophila, that showed that the opening of regulatory regions is highly dynamic between stages during embryogenesis and also during larval stages (McKay and Lieb, 2013). Interestingly, the same study revealed also, that the accessibility of regulatory sequences in different developing appendages of Drosophila is exceptionally similar. The small number of tissue-specific open chromatin regions were annotated as regulatory regions of tissue specific master regulators (McKay and Lieb, 2013). In contrast, other studies found a highly unique and cell specific open chromatin landscape, for example in rods of murine retinas (Hughes et al., 2017). Hence, it will be interesting to investigate how changes in gene expression dynamics correlate with the open chromatin landscape in developing pleuropodia and legs. By comparing the output of this analysis between legs and pleuropodia at different stages, one might eventually be able to pinpoint the tissue and stage specific rewiring of the GRN that underlies the morphological differentiation of initially similar structures into two distinct organs. Overall, the combination of transcriptomic datasets with open chromatin datasets allows to tackle the question, how the rewiring of GRNs might be realized - on the level of chromatin accessibility and gene expression.

How the rewiring of existing GRNs is affecting direct gene interactions and impacts gene regulation on a mechanistic level, requires focusing on distinct nodes and edges. Apart from revealing global properties of biological networks, it has been shown, that GRNs are composed of smaller interaction entities or so-called circuits, which ensure certain gene expression outputs, like robustness or stochasticity (reviewed in (MacNeil and Walhout, 2011)). These are interactions between only a few nodes describe for instance feed-forward loops, autoregulatory loops or feed-back loops (reviewed in (MacNeil and Walhout, 2011)). In Chapter II of this work, we showed for the first time on a transcriptomics and protein level, that the cofactor of Pnr, called Ush is expressed in the eye-antennal disc. Subsequent validation of protein location and perturbation of pnr and ush expression levels allowed us in the following to analyze the small regulatory module of these two factors in more detail. Our results hint towards an auto-regulatory loop of Pnr, which is most probably kept in balance via the repressing function 
of the heterodimer Pnr/Ush. Additionally, an activating role of Pnr on the expression of ush is very likely, and together with the repressing function of Pnr/Ush on pnr expression this interaction might represent a feed-back loop. These interactions are highly similar to the ones described for the developing wing imaginal disc (Fromental-Ramain et al., 2010). In their earlier work, Fromental-Ramain and colleagues additionally showed, that two isoforms of Pnr (Pnr-A and Pnr-B) are differentially expressed (Fromental-Ramain et al., 2008). We could confirm on the basis of quantitative real-time PCR and RNA-seq that, similar to the wing disc, pnr-A is not or only weakly expressed in the developing eye-antennal disc (data not shown). Thus, it remains to be analyzed, if also in these imaginal discs, the isoforms take over a distinct function, which would eventually refine the understanding of this regulatory module. Overall, the combination of RNA-seq with classical genetic tools can be used to define these small circuits which provide further information about direct transcriptional interactions on a more mechanistic level.

In summary, implementing a GRN centric view in developmental studies has great potential to broaden our current understanding of the molecular control of developmental processes. Especially the analysis of stage- and tissue-specific regulatory modules allows to understand mechanistically how a limited number of developmental gene products governs the formation of different tissues and organs.

\subsection{Evolution of gene regulatory networks}

Up to now we established, that the development of distinct organ fates relies on differential wiring of GRNs and consequently on tissue and stage specific gene expression. Since the GRN architecture and the transcriptomic landscape is highly variable across different serially homologous organs, it is as well conceivable that such variation also underlies the evolution of adult organs. Assuming that changes in developmental GRNs cause variation in adult structures requires therefore to study how such GRNs can change and evolve, primarily via the loss of existing edges or the connection of previously unconnected nodes. In all cases, the readout of such changes is reflected in changes of the transcriptional landscape (Thompson et al., 2015).

In our work we assumed that the GRNs that underlie the development of head structures in $D$. melanogaster are highly conserved in its closely related sister species, $D$. simulans and D. mauritiana. Nevertheless, D. melanogaster and D. mauritiana differ extensively in their eye size and head shapes, and even though the genetic architecture of such complex 
traits has been started to be revealed (Arif et al., 2013a; Gaspar et al., 2019; Norry and Gomez, 2017), we lack - in most cases (for an exception see: (Ramaekers et al., 2018)) - functional data that validate single candidate loci in-vivo. We used comparative gene expression data to find nodes in the conserved eye and head developmental GRN of D. melanogaster and D. mauritiana that are flexible enough to lead to the observed differences in head shape. Using differential expression dynamics as a readout, we found that higher expression of pnr underlies the enlargement of the eye area, a higher number of ommatidia and a narrower face cuticle in D. mauritiana. However, using this approach it remains so-far unclear, where the causative mutation lies that leads to variation in pnr expression and subsequently to observed changes in adult morphologies. Studying if a gene is differentially expressed due to cis- or transregulatory divergence is a first step to reveal the causative variants (Wittkopp, 2013). Using our genome wide allele specific expression (ASE) dataset, we found that pnr itself shows very likely divergent expression due to changes in trans in the mid third instar disc (Chapter III). Therefore, Pnr most probably does not represent the evolving locus between D. melanogaster and $D$. mauritiana. We do not yet know which upstream factors activate pnr expression in the early eye-antennal disc. During early embryonic development pnr expression is under the control of Dpp signaling (Ashe et al., 2000; Winick et al., 1993), an interaction that had also been shown for the developing wing imaginal disc (Tomoyasu et al., 2000). Preliminary results that I gained combining our ATAC-seq datasets and transcription factor motif search tools, suggest that Jim, a zinc finger transcription factor or pMad, the transcription factor translating Dpp activity, might be good candidates which are currently tested in our lab.

A genome wide investigation of how the expression of highly connected transcription factors evolve (Chapter III) revealed that if they were not conserved between two species, they were almost exclusively divergent due to trans-regulatory changes. This underrepresentation of cis-regulatory changes in highly connected transcription factors suggests, that these toolkit genes are not only constraint on a coding sequence level (Halder et al., 1995; King and Wilson, 1975), but also on the level of cis-regulatory regions. We could support this hypothesis using our comparative ATAC-seq dataset, by showing that accessible regulatory regions of genes, divergent due to upstream trans-changes, are much more conserved on a sequence level than genes that are differentially expressed due to cis-regulatory changes. Consistent with this finding is that highly connected genes, have in general a lower log2 fold change between closely related species than genes displaying a low degree (Dr. Torres-Oliva, M.; personal 
communication). We conclude, that changes upstream in a developmental GRN are rather due to trans-regulatory changes, but that highly pleiotropic factors like Pnr still represent 'flexible nodes' in a conserved GRN, driving phenotypic variation.

It is still an open question if, during evolution of GRNs, we find changes in highly connected nodes, presumably in genes with highly pleiotropic functions, or rather changes in genes located at the endpoints of GRNs. Highly connected nodes can represent so-called 'transcription factor (TF)-hubs' and are defined as transcriptional regulators that impact an exceptionally large number of downstream target genes (MacNeil and Walhout, 2011). Our bioinformatics analysis of target genes suggests that Pnr potentially regulates more than 1000 genes, of which more than 700 were significantly differentially expressed between $D$. melanogaster and D. mauritiana during eye-antennal disc development. This high number of putative target genes is consistent with previous studies suggesting that Pnr takes over different functions during eye-and head development. Pnr defines the dorsal lineage of the eye-antennal disc and by this is involved in setting up the dorsal/ventral border (Maurel-Zaffran and Treisman, 2000; Singh et al., 2005; Singh and Choi, 2003). We confirmed this with our lineage tracing line, showing that the complete dorsal part of the eye-antennal disc, including the retinal part, stems from initially pnr-expressing cells. Pnr was also suggested to promote head cuticle fate via repression of members of the retinal determination network (Oros et al., 2010). Apart from these many roles during eye and head development, the function of Pnr during Drosophila wing development is well characterized (Sato and Saigo, 2000; Tomoyasu et al., 2000). The GATA factor is also crucial for the dorsal/ventral patterning of the embryo (Heitzler et al., 1996; Herranz and Morata, 2001; Winick et al., 1993). Pnr also plays a role in setting up proper sensory bristle patterns (Haenlin et al., 1997; Heitzler et al., 1996) and activates together with Tinman (Tin) D-mef2, promoting cardioblast fate in Drosophila (Gajewski et al., 1999; Lovato et al., 2015). Given these diverse functions it seems at a first glance counter-intuitive that such a highly pleiotropic factor underlies the evolution of adult morphologies.

A recent example which exemplifies that the wiring and evolution of GRNs is highly context dependent, was elegantly shown with the characterization of variation in another Drosophila GRN, namely the one underlying trichome development. These small actin-filled protrusions form in different stages at different positions of the developing fly, for instance on 
the larval cuticle, and during pupal stages on developing legs (reviewed in (Arif et al., 2015)). The causative changes that led to repeated loss of these small structures on legs and larval cuticle are though surprisingly different. Whereas changes in the regulatory regions of shavenbaby (svb) - a key player during trichome formation - lead to loss of these structures in the larva (McGregor et al., 2007; Sucena et al., 2003; Sucena and Stern, 2000), it seems that changes in the cis-regulatory regions of the microRNA miR-92a cause the appearance of the socalled naked valley on adult Drosophila legs (Arif et al., 2013b). Kittelmann and colleagues could show, that even though a similar set of genes governs trichome formation in both structures, some nodes and edges of the underlying GRN differ and that their variation in the wiring can lead to differences in which nodes and (sub-)networks eventually evolve (Kittelmann et al., 2018). Therefore, context-dependent wiring of important developmental nodes might be prevalent.

Our results, that variation in pnr expression affects the eye area but also the head cuticle suggests that Pnr is very likely involved in several sub-networks that participate in distinct developmental cascades. Context dependent integration into sub-networks and function might be ensured by spatiotemporal availability of co-factors like Ush, as for instance shown in the wing disc (Fromental-Ramain et al., 2010, 2008). Understanding the role of Pnr and architecture of potential sub-networks will require a more thorough dissection of the effects of Pnr up-or downregulation at distinct time points. The extension of the here used GAL4/UAS system with GAL80 (Jiang et al., 2009; Suster et al., 2004), providing additional temporal control of gene expression could be used in the future to address this question. In summary, with Pnr we found a highly pleiotropic TF-hub acting as a 'flexible node' underlying natural variation in eye size and head shape between D. melanogaster and D. mauritiana.

Phenotypes that evolved repeatedly, like the re-occurring loss of trichomes, provide a powerful tool to learn more about the evolvability, architecture and robustness of developmental GRNs, by studying if the same nodes or paths are evolving, given the assumption, that the structure of the GRN and therefore the position of a specific node influences where these switches can arise (Stern and Orgogozo, 2008). Categorizing phenotypes and their genetic basis revealed that often evolution at the same loci underlies the evolution of similar traits (Martin and Orgogozo, 2013). Simple traits like trichome patterns have proven to be an excellent model to study which nodes are likely to evolve, since the 
underlying GRN is extremely well understood. The shavenbaby (svb) gene has been proposed to act as a so called 'hot-spot' gene, since repeated changes in the expression of $s v b$ have been correlated with changes in trichome patterns on cuticles of Drosophila larva (McGregor et al., 2007; Sucena et al., 2003; Sucena and Stern, 2000). Stern and Orgogozo proposed that due to its specific position in the GRN, svb can act as a switch, turning the development of trichomes simply on or off (Stern and Orgogozo, 2008). Recently the genetic changes underlying repeated loss of pelvic appendages in stickleback fish have been revealed. Freshwater populations of these fish independently lost these structures and mapping data point towards repeated, independent deletions in the enhancer region of the Pitx1 gene, encoding a homeodomain transcription factor (Chan et al., 2010; Coyle et al., 2007; Cresko et al., 2004; Shapiro et al., 2004). Xie et al. elegantly showed, that the DNA sequence of the Pitx1 enhancer is exceptionally fragile and therefore prone to break more frequently (Xie et al., 2019), providing a clear mechanistic cause for repeated evolution at the same locus. These examples are cases, where relatively simple traits were studied, and the resulting phenotype is described with a discrete readout, namely loss or gain of a trait. It remains to be shown, which molecular changes underlie the repeated evolution of complex traits - like the here studied trade-off of head structures in different Drosophila species. This trade-off between eye size and head width was characterized in several species of the Drosophila melanogaster subgroup (Gaspar et al., 2019; Hilbrant et al., 2014; Norry et al., 2000; Posnien et al., 2012). A recent study describes a general inverse resource allocation between the visual system and the olfactory system in more than 60 species within the Drosophila genus (Keesey et al., 2019). The authors argued that this tradeoff evolved several times independently in the genus (Keesey et al., 2019) and therefore provides an excellent opportunity to study the repeated evolution of a complex trait. Characterization of the trade-off between D. melanogaster, D. simulans and D. mauritiana revealed, that evolution of eye size differences can have two different causes: First, the eye area can be changed by a different number of more or less equally sized single ommatidia. Second, the number of these single facets can be kept stable, but instead the size of the ommatidia can change. A single-nucleotide-polymorphism (SNP) in the regulatory region of ey has recently been linked to heterochronic changes in the expression of this master regulator and the authors could functionally validate, that this variant underlies natural variation in ommatidia number of various D. melanogaster populations (Ramaekers et al., 2018). Analyses of eye size differences between $D$. simulans and $D$. mauritiana have shown, that the number of 
ommatidia does not differ between these two species, but that the latter one develops larger ommatidia, leading to an increased eye ares (Posnien et al., 2012). Preliminary data suggests that changes in the expression of ocelliless (oc), causes the observed differences in facet size in the two species (Almudi et al. in prep.). Together with our results, this might indicate that in general expression changes in highly pleiotropic factors underlie repeated evolution of this trade-off between eye size and head width, but that the causative molecular mechanisms might be surprisingly different. 


\section{References}

Abzhanov, A., 2004. Bmp4 and Morphological Variation of Beaks in Darwin's Finches. Science 305, 14621465. https://doi.org/10.1126/science.1098095

Abzhanov, A., Kuo, W.P., Hartmann, C., Grant, B.R., Grant, P.R., Tabin, C.J., 2006. The calmodulin pathway and evolution of elongated beak morphology in Darwin's finches. Nature 442, 563567. https://doi.org/10.1038/nature04843

Adachi, Y., Hauck, B., Clements, J., Kawauchi, H., Kurusu, M., Totani, Y., Kang, Y.Y., Eggert, T., Walldorf, U., Furukubo-Tokunaga, K., Callaerts, P., 2003. Conserved cis-regulatory modules mediate complex neural expression patterns of the eyeless gene in the Drosophila brain. Mechanisms of Development 120, 1113-1126. https://doi.org/10.1016/j.mod.2003.08.007

Agawa, Y., Sarhan, M., Kageyama, Y., Akagi, K., Takai, M., Hashiyama, K., Wada, T., Handa, H., Iwamatsu, A., Hirose, S., Ueda, H., 2007. Drosophila Blimp-1 Is a Transient Transcriptional Repressor That Controls Timing of the Ecdysone-Induced Developmental Pathway. Molecular and Cellular Biology 27, 8739-8747. https://doi.org/10.1128/MCB.01304-07

Akagi, K., Ueda, H., 2011. Regulatory mechanisms of ecdysone-inducible Blimp-1 encoding a transcriptional repressor that is important for the prepupal development in Drosophila: Unique mode of Blimp-1 regulation. Development, Growth \& Differentiation 53, 697-703. https://doi.org/10.1111/j.1440-169X.2011.01276.x

Akam, M., 1995. Hox genes and the evolution of diverse body plans. Philos. Trans. R. Soc. Lond., B, Biol. Sci. 349, 313-319. https://doi.org/10.1098/rstb.1995.0119

Akam, M., Averof, M., Castelli-Gair, J., Dawes, R., Falciani, F., Ferrier, D., 1994. The evolving role of Hox genes in arthropods. Dev. Suppl. 209-215.

Akhtar, A., Becker, P.B., 2000. Activation of Transcription through Histone H4 Acetylation by MOF, an Acetyltransferase Essential for Dosage Compensation in Drosophila. Molecular Cell 5, 367-375. https://doi.org/10.1016/S1097-2765(00)80431-1

Alexandre, C.M., Urton, J.R., Jean-Baptiste, K., Huddleston, J., Dorrity, M.W., Cuperus, J.T., Sullivan, A.M., Bemm, F., Jolic, D., Arsovski, A.A., Thompson, A., Nemhauser, J.L., Fields, S., Weigel, D., Bubb, K.L., Queitsch, C., 2018. Complex Relationships between Chromatin Accessibility, Sequence Divergence, and Gene Expression in Arabidopsis thaliana. Molecular Biology and Evolution 35, 837-854. https://doi.org/10.1093/molbev/msx326

Alvarez, M., Schrey, A.W., Richards, C.L., 2015. Ten years of transcriptomics in wild populations: what have we learned about their ecology and evolution? Mol Ecol 24, 710-725. https://doi.org/10.1111/mec.13055

Alwine, J.C., Kemp, D.J., Stark, G.R., 1977. Method for detection of specific RNAs in agarose gels by transfer to diazobenzyloxymethyl-paper and hybridization with DNA probes. Proceedings of the National Academy of Sciences 74, 5350-5354. https://doi.org/10.1073/pnas.74.12.5350

Amore, G., Casares, F., 2010. Size matters: the contribution of cell proliferation to the progression of the specification Drosophila eye gene regulatory network. Dev. Biol. 344, 569-577. https://doi.org/10.1016/j.ydbio.2010.06.015

Ando, H., 1962. The comparative embryology of Odonata with special reference to a relic dragonfly Epiophlebia superstes Selys. Sugadaira Biological Laboratory of Tokyo Kyoiku University.

Andō, H., 1962. comparative embryology of Odonata with special reference to a relic dragonfly Epiophlebia superstes Selys.

Ando, H., Haga, K., 1974. Studies on the Pleuropodia of En1bioptera, 9.

Angelini, D.R., Liu, P.Z., Hughes, C.L., Kaufman, T.C., 2005. Hox gene function and interaction in the milkweed bug Oncopeltus fasciatus (Hemiptera). Developmental Biology 287, 440-455. https://doi.org/10.1016/j.ydbio.2005.08.010

Arif, S., Hilbrant, M., Hopfen, C., Almudi, I., Nunes, M.D.S., Posnien, N., Kuncheria, L., Tanaka, K., Mitteroecker, P., Schlötterer, C., McGregor, A.P., 2013a. Genetic and developmental analysis of differences in eye and face morphology between Drosophila simulans and Drosophila mauritiana: Evolution of eye and face morphology in Drosophila. Evolution \& Development 15, 257-267. https://doi.org/10.1111/ede.12027 
Arif, S., Kittelmann, S., McGregor, A.P., 2015. From shavenbaby to the naked valley: trichome formation as a model for evolutionary developmental biology. Evolution \& Development 17, 120-126. https://doi.org/10.1111/ede.12113

Arif, S., Murat, S., Almudi, I., Nunes, M.D.S., Bortolamiol-Becet, D., McGregor, N.S., Currie, J.M.S., Hughes, H., Ronshaugen, M., Sucena, É., Lai, E.C., Schlötterer, C., McGregor, A.P., 2013b. Evolution of mir-92a Underlies Natural Morphological Variation in Drosophila melanogaster. Current Biology 23, 523-528. https://doi.org/10.1016/j.cub.2013.02.018

Arnold, C.D., Gerlach, D., Spies, D., Matts, J.A., Sytnikova, Y.A., Pagani, M., Lau, N.C., Stark, A., 2014. Quantitative genome-wide enhancer activity maps for five Drosophila species show functional enhancer conservation and turnover during cis-regulatory evolution. Nature Genetics 46, 685692. https://doi.org/10.1038/ng.3009

Arnone, M.I., Davidson, E.H., 1997. The hardwiring of development: organization and function of genomic regulatory systems. Development 124, 1851-1864.

Ashburner, M., Ball, C.A., Blake, J.A., Botstein, D., Butler, H., Cherry, J.M., Davis, A.P., Dolinski, K., Dwight, S.S., Eppig, J.T., Harris, M.A., Hill, D.P., Issel-Tarver, L., Kasarskis, A., Lewis, S., Matese, J.C., Richardson, J.E., Ringwald, M., Rubin, G.M., Sherlock, G., 2000. Gene Ontology: tool for the unification of biology. Nat Genet 25, 25-29. https://doi.org/10.1038/75556

Ashe, H.L., Mannervik, M., Levine, M., 2000. Dpp signaling thresholds in the dorsal ectoderm of the Drosophila embryo. Development 127, 3305-3312.

Auerbach, C., 1936. The development of the legs, wings, and halteres in wild type and some mutant strains of Drosophila melanogaster. Proc. r. Soc. Edinb. 787-815.

Bailey, T.L., Boden, M., Buske, F.A., Frith, M., Grant, C.E., Clementi, L., Ren, J., Li, W.W., Noble, W.S., 2009. MEME SUITE: tools for motif discovery and searching. Nucleic Acids Research 37, W202W208. https://doi.org/10.1093/nar/gkp335

Baker, L.R., Weasner, B.M., Nagel, A., Neuman, S.D., Bashirullah, A., Kumar, J.P., 2018. Eyeless/Pax6 initiates eye formation non-autonomously from the peripodial epithelium. Development 145, dev163329. https://doi.org/10.1242/dev.163329

Barabasi, A.-L., Albert, R., 1999. Emergence of Scaling in Random Networks 286, 5.

Bartel, D.P., 2018. Metazoan MicroRNAs. Cell 173, 20-51. https://doi.org/10.1016/j.cell.2018.03.006

Bedford, G.O., 1978. The development of the egg of Didymuria violescens (Phasmatodea: Phasmatidae: Podacanthinae) - embryology and determination of the stage at shich first diapause occurs. Aust. J. Zool. 18, 155-169.

Bennett, R.L., Brown, S.J., Denell, R.E., 1999. Molecular and genetic analysis of the Tribolium Ultrabithorax ortholog, Ultrathorax. Dev. Genes Evol. 209, 608-619.

Bergman, P., Seyedoleslami Esfahani, S., Engström, Y., 2017. Chapter Two - Drosophila as a Model for Human Diseases - Focus on Innate Immunity in Barrier Epithelia, in: Pick, L. (Ed.), Current Topics in Developmental Biology, Fly Models of Human Diseases. Academic Press, pp. 29-81. https://doi.org/10.1016/bs.ctdb.2016.07.002

Bernays, E.A., 1971. The vermiform larva of Schistocerca gregaria (Forskål): Form and activity (Insecta, Orthoptera). Z. Morph. Tiere 70, 183-200. https://doi.org/10.1007/BF00277761

Berridge, M.J., Oschman, J.L., 1972. Transporting Epithelia. Elsevier. https://doi.org/10.1016/B978-012-454135-1.X5001-X

Bessa, J., 2002. Combinatorial control of Drosophila eye development by Eyeless, Homothorax, and Teashirt. Genes \& Development 16, 2415-2427. https://doi.org/10.1101/gad.1009002

Bird, A.P., Wolffe, A.P., 1999. Methylation-Induced Repression- Belts, Braces, and Chromatin. Cell 99, 451-454. https://doi.org/10.1016/S0092-8674(00)81532-9

Boell, L., Gregorova, S., Forejt, J., Tautz, D., 2011. A comparative assessment of mandible shape in a consomic strain panel of the house mouse (Mus musculus) - implications for epistasis and evolvability of quantitative traits. BMC Evolutionary Biology 11, 309. https://doi.org/10.1186/1471-2148-11-309 
Boell, L., Tautz, D., 2011. Micro-evolutionary divergence patterns of mandible shapes in wild house mouse (Mus musculus) populations. BMC Evolutionary Biology 11, 306. https://doi.org/10.1186/1471-2148-11-306

Bolger, A.M., Lohse, M., Usadel, B., 2014. Trimmomatic: a flexible trimmer for Illumina sequence data. Bioinformatics 30, 2114-2120. https://doi.org/10.1093/bioinformatics/btu170

Bonini, N.M., Choi, K.W., 1995. Early decisions in Drosophila eye morphogenesis. Curr. Opin. Genet. Dev. 5, 507-515.

Boyle, E.A., Li, Y.I., Pritchard, J.K., 2017. An Expanded View of Complex Traits: From Polygenic to Omnigenic. Cell 169, 1177-1186. https://doi.org/10.1016/j.cell.2017.05.038

Bozek, M., Cortini, R., Storti, A.E., Unnerstall, U., Gaul, U., Gompel, N., 2019. ATAC-seq reveals regional differences in enhancer accessibility during the establishment of spatial coordinates in the Drosophila blastoderm. Genome Res. 29, 771-783. https://doi.org/10.1101/gr.242362.118

Brennan, C.A., Ashburner, M., Moses, K., 1998. Ecdysone pathway is required for furrow progression in the developing Drosophila eye. Development 125, 2653-2664.

Brown, J.B., Boley, N., Eisman, R., May, G.E., Stoiber, M.H., Duff, M.O., Booth, B.W., Wen, J., Park, S., Suzuki, A.M., Wan, K.H., Yu, C., Zhang, D., Carlson, J.W., Cherbas, L., Eads, B.D., Miller, D., Mockaitis, K., Roberts, J., Davis, C.A., Frise, E., Hammonds, A.S., Olson, S., Shenker, S., Sturgill, D., Samsonova, A.A., Weiszmann, R., Robinson, G., Hernandez, J., Andrews, J., Bickel, P.J., Carninci, P., Cherbas, P., Gingeras, T.R., Hoskins, R.A., Kaufman, T.C., Lai, E.C., Oliver, B., Perrimon, N., Graveley, B.R., Celniker, S.E., 2014. Diversity and dynamics of the Drosophila transcriptome. Nature 512, 393-399. https://doi.org/10.1038/nature12962

Buchberger, Reis, Lu, Posnien, 2019. Cloudy with a Chance of Insights: Context Dependent Gene Regulation and Implications for Evolutionary Studies. Genes 10, 492. https://doi.org/10.3390/genes10070492

Buchon, N., Silverman, N., Cherry, S., 2014. Immunity in Drosophila melanogaster - from microbial recognition to whole-organism physiology. Nature Reviews Immunology 14, 796-810. https://doi.org/10.1038/nri3763

Buenrostro, J.D., Giresi, P.G., Zaba, L.C., Chang, H.Y., Greenleaf, W.J., 2013. Transposition of native chromatin for fast and sensitive epigenomic profiling of open chromatin, DNA-binding proteins and nucleosome position. Nature Methods 10, 1213-1218. https://doi.org/10.1038/nmeth.2688

Buenrostro, J.D., Wu, B., Litzenburger, U.M., Ruff, D., Gonzales, M.L., Snyder, M.P., Chang, H.Y., Greenleaf, W.J., 2015. Single-cell chromatin accessibility reveals principles of regulatory variation. Nature 523, 486-490. https://doi.org/10.1038/nature14590

Buffry, A.D., Mendes, C.C., McGregor, A.P., 2016. The Functionality and Evolution of Eukaryotic Transcriptional Enhancers, in: Advances in Genetics. Elsevier, pp. 143-206. https://doi.org/10.1016/bs.adgen.2016.08.004

Bullière, F., 1970. Development of pleuropoda during the embryonic development of Blabera craniifer (insect, Dictyoptera). Arch Anat Microsc Morphol Exp 59, 201-220.

Burgio, G., Baylac, M., Heyer, E., Montagutelli, X., 2009. Genetic analysis of skull shape variation and morphological integration in the mouse using interspecific recombinant congenic strains between C57BL/6 and mice of the mus spretus species. Evolution 63, 2668-2686. https://doi.org/10.1111/j.1558-5646.2009.00737.x

Bustin, S., 2000. Absolute quantification of mRNA using real-time reverse transcription polymerase chain reaction assays. Journal of Molecular Endocrinology 169-193. https://doi.org/10.1677/jme.0.0250169

Callaerts, P., Halder, G., Gehring, W.J., 1997. PAX-6 IN DEVELOPMENT AND EVOLUTION. Annu. Rev. Neurosci. 20, 483-532. https://doi.org/10.1146/annurev.neuro.20.1.483

Calleja, M., Moreno, E., Pelaz, S., Morata, G., 1996. Visualization of Gene Expression in Living Adult Drosophila. Science 274, 252-255. 
Camacho, C., Coulouris, G., Avagyan, V., Ma, N., Papadopoulos, J., Bealer, K., Madden, T.L., 2009. BLAST+: architecture and applications. BMC Bioinformatics 10, 421. https://doi.org/10.1186/1471-2105-10-421

Cannavò, E., Koelling, N., Harnett, D., Garfield, D., Casale, F.P., Ciglar, L., Gustafson, H.E., Viales, R.R., Marco-Ferreres, R., Degner, J.F., Zhao, B., Stegle, O., Birney, E., Furlong, E.E.M., 2017. Genetic variants regulating expression levels and isoform diversity during embryogenesis. Nature 541, 402-406. https://doi.org/10.1038/nature20802

Carroll, S.B., 2005. Evolution at Two Levels: On Genes and Form. PLoS Biol 3, e245. https://doi.org/10.1371/journal.pbio.0030245

Carroll, S.B., Grenier, J.K., Weatherbee, S.D., 2001. From DNA to Diversity: Molecular Genetics and the Evolution of Animal Design, 2nd Edition. Wiley-Blackwell.

Casares, F., Almudi, I., 2016. Fast and Furious 800. The Retinal Determination Gene Network in Drosophila, in: Castelli-Gair Hombría, J., Bovolenta, P. (Eds.), Organogenetic Gene Networks: Genetic Control of Organ Formation. Springer International Publishing, Cham, pp. 95-124. https://doi.org/10.1007/978-3-319-42767-6_4

Casasa, S., Moczek, A.P., 2018. Insulin signalling's role in mediating tissue-specific nutritional plasticity and robustness in the horn-polyphenic beetle Onthophagus taurus. Proceedings of the Royal Society B: Biological Sciences 9.

Chan, Y.F., Marks, M.E., Jones, F.C., Villarreal, G., Shapiro, M.D., Brady, S.D., Southwick, A.M., Absher, D.M., Grimwood, J., Schmutz, J., Myers, R.M., Petrov, D., Jónsson, B., Schluter, D., Bell, M.A., Kingsley, D.M., 2010. Adaptive evolution of pelvic reduction in sticklebacks by recurrent deletion of a Pitx1 enhancer. Science 327, 302-305. https://doi.org/10.1126/science.1182213

Chanut, F., Heberlein, U., n.d. Role of the morphogenetic furrow in establishing polarity in the Drosophila eye 10.

Chávez, V.M., Marqués, G., Delbecque, J.P., Kobayashi, K., Hollingsworth, M., Burr, J., Natzle, J.E., O'Connor, M.B., 2000. The Drosophila disembodied gene controls late embryonic morphogenesis and codes for a cytochrome P450 enzyme that regulates embryonic ecdysone levels. Development 127, 4115-4126.

Cheng, H., Wang, Y., Sun, M., 2018. Comparison of Gene Expression Profiles in Nonmodel Eukaryotic Organisms with RNA-Seq, in: Wang, Y., Sun, M. (Eds.), Transcriptome Data Analysis. Springer New York, New York, NY, pp. 3-16. https://doi.org/10.1007/978-1-4939-7710-9_1

Chintapalli, V.R., Wang, J., Herzyk, P., Davies, S.A., Dow, J.A.T., 2013. Data-mining the FlyAtlas online resource to identify core functional motifs across transporting epithelia. BMC Genomics 14, 518. https://doi.org/10.1186/1471-2164-14-518

Chyb, S., Gompel, N., 2013. Wild-type morphology, in: Atlas of Drosophila Morphology. Elsevier, pp. 123. https://doi.org/10.1016/B978-0-12-384688-4.00001-8

Ciapponi, L., 2001. Drosophila Fos mediates ERK and JNK signals via distinct phosphorylation sites. Genes \& Development 15, 1540-1553. https://doi.org/10.1101/gad.886301

Coolon, J.D., Stevenson, K.R., McManus, C.J., Yang, B., Graveley, B.R., Wittkopp, P.J., 2015. Molecular Mechanisms and Evolutionary Processes Contributing to Accelerated Divergence of Gene Expression on the Drosophila $X$ Chromosome. Mol Biol Evol 32, 2605-2615. https://doi.org/10.1093/molbev/msv135

Corrales, M., Rosado, A., Cortini, R., van Arensbergen, J., van Steensel, B., Filion, G.J., 2017. Clustering of Drosophila housekeeping promoters facilitates their expression. Genome Res. 27, 11531161. https://doi.org/10.1101/gr.211433.116

Cowles, C.R., Hirschhorn, J.N., Altshuler, D., Lander, E.S., 2002. Detection of regulatory variation in mouse genes. Nature Genetics 32, 432-437. https://doi.org/10.1038/ng992

Cox, M.P., Peterson, D.A., Biggs, P.J., 2010. SolexaQA: At-a-glance quality assessment of Illumina secondgeneration sequencing data. BMC Bioinformatics 11, 485. https://doi.org/10.1186/1471-2105$11-485$ 
Coyle, S.M., Huntingford, F.A., Peichel, C.L., 2007. Parallel Evolution of Pitx1 Underlies Pelvic Reduction in Scottish Threespine Stickleback (Gasterosteus aculeatus). Journal of Heredity 98, 581-586. https://doi.org/10.1093/jhered/esm066

Cresko, W.A., Amores, A., Wilson, C., Murphy, J., Currey, M., Phillips, P., Bell, M.A., Kimmel, C.B., Postlethwait, J.H., 2004. Parallel genetic basis for repeated evolution of armor loss in Alaskan threespine stickleback populations. Proceedings of the National Academy of Sciences 101, 6050-6055. https://doi.org/10.1073/pnas.0308479101

Cubadda, Y., Heitzler, P., Ray, R.P., Bourouis, M., Ramain, P., Gelbart, W., Simpson, P., Haenlin, M., 1997. $\mathrm{u}$-shaped encodes a zinc finger protein that regulates the proneural genes achaete and scute during the formation of bristles in Drosophila. Genes \& Development 11, 3083-3095. https://doi.org/10.1101/gad.11.22.3083

Cubeñas-Potts, C., Rowley, M.J., Lyu, X., Li, G., Lei, E.P., Corces, V.G., 2017. Different enhancer classes in Drosophila bind distinct architectural proteins and mediate unique chromatin interactions and 3D architecture. Nucleic Acids Research 45, 1714-1730. https://doi.org/10.1093/nar/gkw1114

Currea, J.P., Smith, J.L., Theobald, J.C., 2018. Small fruit flies sacrifice temporal acuity to maintain contrast sensitivity. Vision Research 149, 1-8. https://doi.org/10.1016/j.visres.2018.05.007

Cusanovich, D.A., Daza, R., Adey, A., Pliner, H.A., Christiansen, L., Gunderson, K.L., Steemers, F.J., Trapnell, C., Shendure, J., 2015. Multiplex single-cell profiling of chromatin accessibility by combinatorial cellular indexing. Science 348, 910-914. https://doi.org/10.1126/science.aab1601

David, J., McEvey, S., Solignac, M., Tsacas, L., 1989. Drosophila communities on Mauritius and the ecological niche of D. mauritiana (Diptera, Drosophilidae).

Davidson, E.H., 2001. Genomic Regulatory Systems. Academic Press, San Diego.

Davidson, E.H., Levine, M.S., 2008. Properties of developmental gene regulatory networks. Proceedings of the National Academy of Sciences 105, 20063-20066. https://doi.org/10.1073/pnas.0806007105

Davie, K., Jacobs, J., Atkins, M., Potier, D., Christiaens, V., Halder, G., Aerts, S., 2015. Discovery of Transcription Factors and Regulatory Regions Driving In Vivo Tumor Development by ATAC-seq and FAIRE-seq Open Chromatin Profiling. PLOS Genetics 11, e1004994. https://doi.org/10.1371/journal.pgen.1004994

Davis, G.K., Patel, N.H., 2002. Short, Long, and Beyond: Molecular and Embryological Approaches to Insect Segmentation. Annu. Rev. Entomol. 47, 669-699. https://doi.org/10.1146/annurev.ento.47.091201.145251

Dehal, P., Boore, J.L., 2005. Two Rounds of Whole Genome Duplication in the Ancestral Vertebrate. PLoS Biology 3, e314. https://doi.org/10.1371/journal.pbio.0030314

Deng, W., Nickle, D.C., Learn, G.H., Maust, B., Mullins, J.I., 2007. ViroBLAST: a stand-alone BLAST web server for flexible queries of multiple databases and user's datasets. Bioinformatics 23, 23342336. https://doi.org/10.1093/bioinformatics/btm331

Dermitzakis, E.T., 2008. From gene expression to disease risk. Nature Genetics 40, 492-493. https://doi.org/10.1038/ng0508-492

Dixon, A.L., Liang, L., Moffatt, M.F., Chen, W., Heath, S., Wong, K.C.C., Taylor, J., Burnett, E., Gut, I., Farrall, M., Lathrop, G.M., Abecasis, G.R., Cookson, W.O.C., 2007. A genome-wide association study of global gene expression. Nat Genet 39, 1202-1207. https://doi.org/10.1038/ng2109

Domazet-Lošo, T., Tautz, D., 2010. A phylogenetically based transcriptome age index mirrors ontogenetic divergence patterns. Nature 468, 815-818. https://doi.org/10.1038/nature09632

Domínguez, M., 1999. Hedgehog signalling in the Drosophila eye 126, 9.

Domínguez, M., Casares, F., 2005. Organ specification-growth control connection: new in-sights from the Drosophila eye-antennal disc. Dev. Dyn. 232, 673-684. https://doi.org/10.1002/dvdy.20311

Duboule, D., Dollé, P., 1989. The structural and functional organization of the murine HOX gene family resembles that of Drosophila homeotic genes. EMBO J. 8, 1497-1505.

Dunn, C.W., Hejnol, A., Matus, D.Q., Pang, K., Browne, W.E., Smith, S.A., Seaver, E., Rouse, G.W., Obst, M., Edgecombe, G.D., Sørensen, M.V., Haddock, S.H.D., Schmidt-Rhaesa, A., Okusu, A., 
Kristensen, R.M., Wheeler, W.C., Martindale, M.Q., Giribet, G., 2008. Broad phylogenomic sampling improves resolution of the animal tree of life. Nature 452, 745-749. https://doi.org/10.1038/nature06614

Duveau, F., Yuan, D.C., Metzger, B.P.H., Hodgins-Davis, A., Wittkopp, P.J., 2017. Effects of mutation and selection on plasticity of a promoter activity in Saccharomyces cerevisiae. Proceedings of the National Academy of Sciences 114, E11218-E11227. https://doi.org/10.1073/pnas.1713960115

Ellegren, H., 2014. Genome sequencing and population genomics in non-model organisms. Trends in Ecology \& Evolution 29, 51-63. https://doi.org/10.1016/j.tree.2013.09.008

Emilsson, V., Thorleifsson, G., Zhang, B., Leonardson, A.S., Zink, F., Zhu, J., Carlson, S., Helgason, A., Walters, G.B., Gunnarsdottir, S., Mouy, M., Steinthorsdottir, V., Eiriksdottir, G.H., Bjornsdottir, G., Reynisdottir, I., Gudbjartsson, D., Helgadottir, A., Jonasdottir, Aslaug, Jonasdottir, Adalbjorg, Styrkarsdottir, U., Gretarsdottir, S., Magnusson, K.P., Stefansson, H., Fossdal, R., Kristjansson, K., Gislason, H.G., Stefansson, T., Leifsson, B.G., Thorsteinsdottir, U., Lamb, J.R., Gulcher, J.R., Reitman, M.L., Kong, A., Schadt, E.E., Stefansson, K., 2008. Genetics of gene expression and its effect on disease. Nature 452, 423-428. https://doi.org/10.1038/nature06758

Fossett, N., Tevosian, S.G., Gajewski, K., Zhang, Q., Orkin, S.H., Schulz, R.A., 2001. The Friend of GATA proteins U-shaped, FOG-1, and FOG-2 function as negative regulators of blood, heart, and eye development in Drosophila. Proceedings of the National Academy of Sciences 98, 7342-7347. https://doi.org/10.1073/pnas.131215798

Fraulob, M., Beutel, R.G., Machida, R., Pohl, H., 2015. The embryonic development of Stylops ovinae (Strepsiptera, Stylopidae) with emphasis on external morphology. Arthropod Struct Dev 44, 42 68. https://doi.org/10.1016/j.asd.2014.10.001

Freese, N.H., Norris, D.C., Loraine, A.E., 2016. Integrated genome browser: visual analytics platform for genomics. Bioinformatics 32, 2089-2095. https://doi.org/10.1093/bioinformatics/btw069

Fristrom, D., Wilcox, M., Fristrom, J., 1993. The distribution of PS integrins, laminin A and F-actin during key stages in Drosophila wing development. Development 117, 509-523.

Fromental-Ramain, C., Taquet, N., Ramain, P., 2010. Transcriptional interactions between the pannier isoforms and the cofactor U-shaped during neural development in Drosophila. Mechanisms of Development 127, 442-457. https://doi.org/10.1016/j.mod.2010.08.002

Fromental-Ramain, C., Vanolst, L., Delaporte, C., Ramain, P., 2008. pannier encodes two structurally related isoforms that are differentially expressed during Drosophila development and display distinct functions during thorax patterning. Mechanisms of Development 125, 43-57. https://doi.org/10.1016/j.mod.2007.10.008

Fukamizo, T., Kramer, K.J., 1985. Mechanism of chitin hydrolysis by the binary chitinase system in insect moulting fluid. Insect Biochemistry 15, 141-145. https://doi.org/10.1016/00201790(85)90001-0

Furlong, E.E.M., Levine, M., 2018. Developmental enhancers and chromosome topology. Science 361, 1341-1345. https://doi.org/10.1126/science.aau0320

Gajewski, K., Fossett, N., Molkentin, J.D., Schulz, R.A., 1999. The zinc finger proteins Pannier and GATA4 function as cardiogenic factors in Drosophila. Development 126, 5679-5688.

Garcia-Fernàndez, J., Holland, P.W.H., 1994. Archetypal organization of the amphioxus Hox gene cluster. Nature 370, 563-566. https://doi.org/10.1038/370563a0

García-García, M.J., Ramain, P., Simpson, P., Modolell, J., 1999. Different contributions of pannier and wingless to the patterning of the dorsal mesothorax of Drosophila. Development 126, $3523-$ 3532.

Garg, A., Srivastava, A., Davis, M.M., O’Keefe, S.L., Chow, L., Bell, J.B., 2007. Antagonizing Scalloped With a Novel Vestigial Construct Reveals an Important Role for Scalloped in Drosophila melanogaster Leg, Eye and Optic Lobe Development. Genetics 175, 659-669. https://doi.org/10.1534/genetics.106.063966

Gaspar, P., Arif, S., Sumner-Rooney, L., Kittelmann, M., Stern, D.L., Nunes, M.D.S., McGregor, A.P., 2019. Characterisation of the genetic architecture underlying eye size variation within Drosophila 
melanogaster and Drosophila simulans (preprint). Evolutionary Biology. https://doi.org/10.1101/555698

Gautier, M., Yamaguchi, J., Foucaud, J., Loiseau, A., Ausset, A., Facon, B., Gschloessl, B., Lagnel, J., Loire, E., Parrinello, H., Severac, D., Lopez-Roques, C., Donnadieu, C., Manno, M., Berges, H., Gharbi, K., Lawson-Handley, L., Zang, L.-S., Vogel, H., Estoup, A., Prud'homme, B., 2018. The Genomic Basis of Color Pattern Polymorphism in the Harlequin Ladybird. Current Biology 28, 32963302.e7. https://doi.org/10.1016/j.cub.2018.08.023

Genissel, A., Mclntyre, L.M., Wayne, M.L., Nuzhdin, S.V., 2007. Cis and Trans Regulatory Effects Contribute to Natural Variation in Transcriptome of Drosophila melanogaster. Molecular Biology and Evolution 25, 101-110. https://doi.org/10.1093/molbev/msm247

Gibson, M.C., Schubiger, G., 2001. Drosophila peripodial cells, more than meets the eye? Bioessays 23, 691-697. https://doi.org/10.1002/bies.1098

Gibson, M.C., Schubiger, G., 2000. Peripodial cells regulate proliferation and patterning of Drosophila imaginal discs. Cell 103, 343-350. https://doi.org/10.1016/s0092-8674(00)00125-2

Gilad, Y., Rifkin, S.A., Pritchard, J.K., 2008. Revealing the architecture of gene regulation: the promise of eQTL studies. Trends in Genetics 24, 408-415. https://doi.org/10.1016/j.tig.2008.06.001

Gilbert, D., 2013. Gene-omes built from mRNA seq not genome DNA. 7th annual arthropod genomics symposium.

Giresi, P.G., Kim, J., McDaniell, R.M., Iyer, V.R., Lieb, J.D., 2007. FAIRE (Formaldehyde-Assisted Isolation of Regulatory Elements) isolates active regulatory elements from human chromatin. Genome Res. 17, 877-885. https://doi.org/10.1101/gr.5533506

Glaser-Schmitt, A., Parsch, J., 2018. Functional characterization of adaptive variation within a cisregulatory element influencing Drosophila melanogaster growth. PLOS Biology 16, e2004538. https://doi.org/10.1371/journal.pbio.2004538

Goltsev, Y., Rezende, G.L., Vranizan, K., Lanzaro, G., Valle, D., Levine, M., 2009. Developmental and evolutionary basis for drought tolerance of the Anopheles gambiae embryo. Developmental Biology 330, 462-470. https://doi.org/10.1016/j.ydbio.2009.02.038

Götz, S., García-Gómez, J.M., Terol, J., Williams, T.D., Nagaraj, S.H., Nueda, M.J., Robles, M., Talón, M., Dopazo, J., Conesa, A., 2008. High-throughput functional annotation and data mining with the Blast2GO suite. Nucleic Acids Res 36, 3420-3435. https://doi.org/10.1093/nar/gkn176

Gouy, M., Guindon, S., Gascuel, O., 2010. SeaView version 4: A multiplatform graphical user interface for sequence alignment and phylogenetic tree building. Mol. Biol. Evol. 27, 221-224. https://doi.org/10.1093/molbev/msp259

Graber, V., 1889. Über den Bau und die phylogenetische Bedeurung der embryonalen Bauchanähnge der Insekten. Biol. Zent. B1 9, 355-363.

Grabherr, M.G., Haas, B.J., Yassour, M., Levin, J.Z., Thompson, D.A., Amit, I., Adiconis, X., Fan, L., Raychowdhury, R., Zeng, Q., Chen, Z., Mauceli, E., Hacohen, N., Gnirke, A., Rhind, N., di Palma, F., Birren, B.W., Nusbaum, C., Lindblad-Toh, K., Friedman, N., Regev, A., 2011. Full-length transcriptome assembly from RNA-Seq data without a reference genome. Nat Biotechnol 29, 644-652. https://doi.org/10.1038/nbt.1883

Graham, A., Papalopulu, N., Krumlauf, R., 1989. The murine and Drosophila homeobox gene complexes have common features of organization and expression. Cell 57, 367-378. https://doi.org/10.1016/0092-8674(89)90912-4

Graveley, B.R., Brooks, A.N., Carlson, J.W., Duff, M.O., Landolin, J.M., Yang, L., Artieri, C.G., van Baren, M.J., Boley, N., Booth, B.W., Brown, J.B., Cherbas, L., Davis, C.A., Dobin, A., Li, R., Lin, W., Malone, J.H., Mattiuzzo, N.R., Miller, D., Sturgill, D., Tuch, B.B., Zaleski, C., Zhang, D., Blanchette, M., Dudoit, S., Eads, B., Green, R.E., Hammonds, A., Jiang, L., Kapranov, P., Langton, L., Perrimon, N., Sandler, J.E., Wan, K.H., Willingham, A., Zhang, Y., Zou, Y., Andrews, J., Bickel, P.J., Brenner, S.E., Brent, M.R., Cherbas, P., Gingeras, T.R., Hoskins, R.A., Kaufman, T.C., Oliver, B., Celniker, S.E., 2011. The developmental transcriptome of Drosophila melanogaster. Nature 471, 473479. https://doi.org/10.1038/nature09715 
Graze, R.M., McIntyre, L.M., Main, B.J., Wayne, M.L., Nuzhdin, S.V., 2009. Regulatory divergence in Drosophila melanogaster and D. simulans, a genomewide analysis of allele-specific expression. Genetics 183, 547-561, 1SI-21SI. https://doi.org/10.1534/genetics.109.105957

Grellet, P., 1971. Volume variations and DNA content of the nuclei ofScapsipedus marginatus Afz. and Br. (Orthoptera, Gryllidae) during embryogenesis. Wilhelm Roux Arch Entwickl Mech Org 167, 243-265. https://doi.org/10.1007/BF00584252

Grenier, J.K., Carroll, S.B., 2000. Functional evolution of the Ultrabithorax protein. Proceedings of the National Academy of Sciences 97, 704-709. https://doi.org/10.1073/pnas.97.2.704

Grossniklaus, U., Cadigan, K.M., Gehring, W.J., 1994. Three maternal coordinate systems cooperate in the patterning of the Drosophila head. Development 120, 3155-3171.

H. Slifer, E., 1937. The Origin and fate of the Membranes sur- rounding the Grasshopper Egg; together with some Experiments on the Source of the Hatching Enzyme.

Haas, B.J., Papanicolaou, A., Yassour, M., Grabherr, M., Blood, P.D., Bowden, J., Couger, M.B., Eccles, D., Li, B., Lieber, M., MacManes, M.D., Ott, M., Orvis, J., Pochet, N., Strozzi, F., Weeks, N., Westerman, R., William, T., Dewey, C.N., Henschel, R., LeDuc, R.D., Friedman, N., Regev, A., 2013. De novo transcript sequence reconstruction from RNA-seq using the Trinity platform for reference generation and analysis. Nat Protoc 8, 1494-1512. https://doi.org/10.1038/nprot.2013.084

Haddrill, P.R., Charlesworth, B., Halligan, D.L., Andolfatto, P., 2005. Patterns of intron sequence evolution in Drosophila are dependent upon length and GC content. Genome Biol. 6, R67. https://doi.org/10.1186/gb-2005-6-8-r67

Haeckl, E., 1879. Anthropogenie, Third Edition. ed. W. Engelmann, Leipzig.

Haeckl, E., 1867. Generelle Morphologie der Organismen. Georg Reimer, Berlin.

Haenlin, M., Cubadda, Y., Blondeau, F., Heitzler, P., Lutz, Y., Simpson, P., Ramain, P., 1997. Transcriptional activity of Pannier is regulated negatively by heterodimerization of the GATA DNA-binding domain with a cofactor encoded by the u-shaped gene of Drosophila. Genes \& Development 11, 3096-3108. https://doi.org/10.1101/gad.11.22.3096

Hagan, H.R., 1931. The embryogeny of the polyctenid, Hesperoctenes fumariius Westwood, with the reference to viviparity in insects. J. Morphol. Physiol. 51, 3-115.

Hagen, J.F.D., Mendes, C.C., Tanaka, K.M., Gaspar, P., Kittelmann, M., McGregor, A.P., Nunes, M.D.S., 2018. Tartan underlies the evolution of male genital morphology. bioRxiv. https://doi.org/10.1101/462259

Halder, G., Callaerts, P., Gehring, W., 1995. Induction of ectopic eyes by targeted expression of the eyeless gene in Drosophila. Science 267, 1788-1792. https://doi.org/10.1126/science.7892602

Hall, B.K., 2003. Evo-Devo: evolutionary developmental mechanisms. Int. J. Dev. Biol. 47, 491-495.

Haynie, J.L., Bryant, P.J., 1986. Development of the eye-antenna imaginal disc and morphogenesis of the adult head inDrosophila melanogaster. J. Exp. Zool. 237, 293-308. https://doi.org/10.1002/jez.1402370302

He, X., Zhang, J., 2006. Why Do Hubs Tend to Be Essential in Protein Networks? PLoS Genetics 2, 9.

Heberlein, U., Moses, K., 1995. Mechanisms of Drosophila retinal morphogenesis: the virtues of being progressive. Cell 81, 987-990. https://doi.org/10.1016/s0092-8674(05)80003-0

Heinz, S., Benner, C., Spann, N., Bertolino, E., Lin, Y.C., Laslo, P., Cheng, J.X., Murre, C., Singh, H., Glass, C.K., 2010. Simple Combinations of Lineage-Determining Transcription Factors Prime cisRegulatory Elements Required for Macrophage and B Cell Identities. Molecular Cell 38, 576589. https://doi.org/10.1016/j.molcel.2010.05.004

Heitzler, P., Haenlin, M., Ramain, P., Calleja, M., Simpson, P., 1996. A genetic analysis of pannier, a gene necessary for viability of dorsal tissues and bristle positioning in Drosophila. Genetics 143, 1271-1286.

Heming, B.S., 1993. Origin and fate of pleuropodia in embryos of Neoheegeria verbasci (Osborn) (Thysanoptera: Phlaeothripidae). Journal of Pure and Applied Zoology 4, 205-223.

Henikoff, S., Ahmad, K., Malik, H.S., 2001. The Centromere Paradox: Stable Inheritance with Rapidly Evolving DNA. Science 293, 1098-1102. https://doi.org/10.1126/science.1062939 
Herranz, H., Morata, G., 2001. The functions of pannier during Drosophila embryogenesis. Development $128,4837-4846$.

Herrmann, C., Van de Sande, B., Potier, D., Aerts, S., 2012. i-cisTarget: an integrative genomics method for the prediction of regulatory features and cis-regulatory modules. Nucleic Acids Research 40, e114-e114. https://doi.org/10.1093/nar/gks543

Hilbrant, M., Almudi, I., Leite, D.J., Kuncheria, L., Posnien, N., Nunes, M.D., McGregor, A.P., 2014. Sexual dimorphism and natural variation within and among species in the Drosophilaretinal mosaic. BMC Evol Biol 14, 240. https://doi.org/10.1186/s12862-014-0240-x

Hill, R.E., Favor, J., Hogan, B.L.M., Ton, C.C.T., Saunders, G.F., Hanson, I.M., Prosser, J., Jordan, T., Hastie, N.D., Heyningen, V. van, 1991. Mouse Small eye results from mutations in a paired-like homeobox-containing gene. Nature 354, 522. https://doi.org/10.1038/354522a0

Hinrichs, A.S., Karolchik, D., Baertsch, R., Barber, G.P., Bejerano, G., Clawson, H., Diekhans, M., Furey, T.S., Harte, R.A., Hsu, F., Hillman-Jackson, J., Kuhn, R.M., Pedersen, J.S., Pohl, A., Raney, B.J., Rosenbloom, K.R., Siepel, A., Smith, K.E., Sugnet, C.W., Sultan-Qurraie, A., Thomas, D.J., Trumbower, H., Weber, R.J., Weirauch, M., Zweig, A.S., Haussler, D., Kent, W.J., 2006. The UCSC Genome Browser Database: update 2006. Nucleic Acids Res 34, D590-D598. https://doi.org/10.1093/nar/gkj144

Hoekstra, H.E., Coyne, J.A., 2007. THE LOCUS OF EVOLUTION: EVO DEVO AND THE GENETICS OF ADAPTATION: THE LOCUS OF EVOLUTION. Evolution 61, 995-1016. https://doi.org/10.1111/j.1558-5646.2007.00105.x

Hoekstra, H.E., Hirschmann, R.J., Bundey, R.A., Insel, P.A., Crossland, J.P., 2006. A Single Amino Acid Mutation Contributes to Adaptive Beach Mouse Color Pattern. Science 313, 101-104. https://doi.org/10.1126/science.1126121

Hogenkamp, D.G., Arakane, Y., Kramer, K.J., Muthukrishnan, S., Beeman, R.W., 2008. Characterization and expression of the beta- $\mathrm{N}$-acetylhexosaminidase gene family of Tribolium castaneum. Insect Biochem. Mol. Biol. 38, 478-489. https://doi.org/10.1016/j.ibmb.2007.08.002

Homem, C.C.F., Knoblich, J.A., 2012. Drosophila neuroblasts: a model for stem cell biology. Development 139, 4297-4310. https://doi.org/10.1242/dev.080515

Huang, X., Warren, J.T., Buchanan, J., Gilbert, L.I., Scott, M.P., 2007. Drosophila Niemann-Pick type C-2 genes control sterol homeostasis and steroid biosynthesis: a model of human neurodegenerative disease. Development 134, 3733-3742. https://doi.org/10.1242/dev.004572

Hughes, A.E.O., Enright, J.M., Myers, C.A., Shen, S.Q., Corbo, J.C., 2017. Cell Type-Specific Epigenomic Analysis Reveals a Uniquely Closed Chromatin Architecture in Mouse Rod Photoreceptors. Sci Rep 7, 43184. https://doi.org/10.1038/srep43184

Hughes, C.L., Kaufman, T.C., 2002. Hox genes and the evolution of the arthropod body plan. Evol. Dev. 4, 459-499.

Hussey, P.B., 1926. Studies on the Pleuropodia of Belostoma Flumineum Say and Ranatra Fusca Palisot de Beauvois, wie a discussion of these organs in other insects. Entomol. Am. 1-82.

Imrichová, H., Hulselmans, G., Kalender Atak, Z., Potier, D., Aerts, S., 2015. i-cisTarget 2015 update: generalized cis-regulatory enrichment analysis in human, mouse and fly. Nucleic Acids Res 43, W57-W64. https://doi.org/10.1093/nar/gkv395

Jacobs, C.G.C., Braak, N., Lamers, G.E.M., van der Zee, M., 2015. Elucidation of the serosal cuticle machinery in the beetle Tribolium by RNA sequencing and functional analysis of Knickkopf1, Retroactive and Laccase2. Insect Biochem. Mol. Biol. 60, 7-12. https://doi.org/10.1016/j.ibmb.2015.02.014

Jacobs, C.G.C., Rezende, G.L., Lamers, G.E.M., van der Zee, M., 2013. The extraembryonic serosa protects the insect egg against desiccation. Proceedings of the Royal Society B: Biological Sciences 280, 20131082-20131082. https://doi.org/10.1098/rspb.2013.1082

Jacobs, C.G.C., Spaink, H.P., van der Zee, M., 2014. The extraembryonic serosa is a frontier epithelium providing the insect egg with a full-range innate immune response. elife 3, e04111. https://doi.org/10.7554/eLife.04111 
Jarman, A.P., Grell, E.H., Ackerman, L., Jan, L.Y., Jan, Y.N., 1994. atonal is the proneural gene for Drosophila photoreceptors. Nature 369, 398-400. https://doi.org/10.1038/369398a0

Jenny, A., 2010. Planar Cell Polarity Signaling in the Drosophila Eye, in: Current Topics in Developmental Biology. Elsevier, pp. 189-227. https://doi.org/10.1016/B978-0-12-385044-7.00007-2

Jeong, S., Rokas, A., Carroll, S.B., 2006. Regulation of Body Pigmentation by the Abdominal-B Hox Protein and Its Gain and Loss in Drosophila Evolution. Cell 125, 1387-1399. https://doi.org/10.1016/j.cell.2006.04.043

Jia, Z., Xu, S., 2007. Mapping Quantitative Trait Loci for Expression Abundance. Genetics 176, 611-623. https://doi.org/10.1534/genetics.106.065599

Jiang, F., Frey, B.R., Evans, M.L., Friel, J.C., Hopper, J.E., 2009. Gene Activation by Dissociation of an Inhibitor from a Transcriptional Activation Domain. Molecular and Cellular Biology 29, 56045610. https://doi.org/10.1128/MCB.00632-09

Johnson, D.S., Mortazavi, A., Myers, R.M., Wold, B., 2007. Genome-Wide Mapping of in Vivo ProteinDNA Interactions. Science 316, 1497-1502. https://doi.org/10.1126/science.1141319

Johnston, D.S., Nüsslein-Volhard, C., 1992. The origin of pattern and polarity in the Drosophila embryo. Cell 68, 201-219. https://doi.org/10.1016/0092-8674(92)90466-P

Jones, B.M., 1956. ENDOCRINE ACTIVITY DURING INSECT EMBRYO- GENESIS. CONTROL OF EVENTS IN DEVELOPMENT FOLLOWING THE EMBRYONIC MOULT (LOCUSTA MIGRATORIA AND LOCUST AN A PARDALINA, ORTHOPTERA). J Exp Biol 33, 685-696.

Junion, G., Spivakov, M., Girardot, C., Braun, M., Gustafson, E.H., Birney, E., Furlong, E.E.M., 2012. A Transcription Factor Collective Defines Cardiac Cell Fate and Reflects Lineage History. Cell 148, 473-486. https://doi.org/10.1016/j.cell.2012.01.030

Käll, L., Krogh, A., Sonnhammer, E.L.L., 2007. Advantages of combined transmembrane topology and signal peptide prediction--the Phobius web server. Nucleic Acids Res. 35, W429-432. https://doi.org/10.1093/nar/gkm256

Kamiya, A., Ando, H., 1985. External morphogenesis of the embryo of Ascalaphus ramburi (Neuroptera, Ascalaphidae)., in: Recent Advances in Insect Embryology in Japan. ISEBU Co.Led., pp. 203-213.

Karcavich, R.E., 2005. Generating neuronal diversity in the Drosophila central nervous system: a view from the ganglion mother cells. Dev. Dyn. 232, 609-616. https://doi.org/10.1002/dvdy.20273

Keesey, I.W., Grabe, V., Gruber, L., Koerte, S., Obiero, G.F., Bolton, G., Khallaf, M.A., Kunert, G., LavistaLlanos, S., Valenzano, D.R., Rybak, J., Barrett, B.A., Knaden, M., Hansson, B.S., 2019. Inverse resource allocation between vision and olfaction across the genus Drosophila. Nat Commun 10, 1162. https://doi.org/10.1038/s41467-019-09087-z

Kent, W.J., 2002. BLAT--the BLAST-like alignment tool. Genome Res. 12, 656-664. https://doi.org/10.1101/gr.229202

Kenyon, K.L., Ranade, S.S., Curtiss, J., Mlodzik, M., Pignoni, F., 2003. Coordinating Proliferation and Tissue Specification to Promote Regional Identity in the Drosophila Head. Developmental Cell 5, 403-414. https://doi.org/10.1016/S1534-5807(03)00243-0

Khaitovich, P., Enard, W., Lachmann, M., Pääbo, S., 2006. Evolution of primate gene expression. Nature Reviews Genetics 7, 693-702. https://doi.org/10.1038/nrg1940

Khan, A., Fornes, O., Stigliani, A., Gheorghe, M., Castro-Mondragon, J.A., van der Lee, R., Bessy, A., Chèneby, J., Kulkarni, S.R., Tan, G., Baranasic, D., Arenillas, D.J., Sandelin, A., Vandepoele, K., Lenhard, B., Ballester, B., Wasserman, W.W., Parcy, F., Mathelier, A., 2018. JASPAR 2018: update of the open-access database of transcription factor binding profiles and its web framework. Nucleic Acids Research 46, D260-D266. https://doi.org/10.1093/nar/gkx1126

Khoueiry, P., Girardot, C., Ciglar, L., Peng, P.-C., Gustafson, E.H., Sinha, S., Furlong, E.E., 2017. Uncoupling evolutionary changes in DNA sequence, transcription factor occupancy and enhancer activity. eLife 6. https://doi.org/10.7554/eLife.28440

Kim, A.-R., Choi, E.-B., Kim, M.-Y., Choi, K.-W., 2017. Angiotensin-converting enzyme Ance is cooperatively regulated by Mad and Pannier in Drosophila imaginal discs. Sci Rep 7, 13174. https://doi.org/10.1038/s41598-017-13487-w 
King, M., Wilson, A., 1975. Evolution at two levels in humans and chimpanzees. Science 188, 107-116. https://doi.org/10.1126/science.1090005

Kittelmann, McGregor, 2019. Modulation and Evolution of Animal Development through microRNA Regulation of Gene Expression. Genes 10, 321. https://doi.org/10.3390/genes10040321

Kittelmann, S., Buffry, A.D., Franke, F.A., Almudi, I., Yoth, M., Sabaris, G., Couso, J.P., Nunes, M.D.S., 2018. Gene regulatory network architecture in different developmental contexts influences the genetic basis of morphological evolution. PLoS Genet 14(5), 21. https://doi.org/10.1371/journal.pgen.1007375

Kjer, K.M., Simon, C., Yavorskaya, M., Beutel, R.G., 2016. Progress, pitfalls and parallel universes: a history of insect phylogenetics. J R Soc Interface 13. https://doi.org/10.1098/rsif.2016.0363

Klingenberg, C.P., 2011. MorphoJ: an integrated software package for geometric morphometrics: COMPUTER PROGRAM NOTE. Molecular Ecology Resources 11, 353-357. https://doi.org/10.1111/j.1755-0998.2010.02924.x

Kobayashi, Y., Ando, H., 1990. Early embryonic development and external features of developing embryos of the caddisfly, Nemotaulius admorsus (trichoptera: Limnephilidae). J. Morphol. 203, 69-85. https://doi.org/10.1002/jmor.1052030108

Kobayashi, Y., Suzuki, H., Ohba, N., 2003. Development of pleuropodia in the embryo of the glowworm Rhagophthalmus ohbai (Rhagophthalmidae, Coleoptera, Insecta), with comments on their probable function.

Konopová, B., Buchberger, E., Crisp, A., 2019. Transcriptomics supports that pleuropodia of insect embryos function in degradation of the serosal cuticle to enable hatching (preprint). Developmental Biology. https://doi.org/10.1101/584029

Konopová, B., Zrzavý, J., 2005. Ultrastructure, development, and homology of insect embryonic cuticles. J. Morphol. 264, 339-362. https://doi.org/10.1002/jmor.10338

Kopp, F., Mendell, J.T., 2018. Functional Classification and Experimental Dissection of Long Noncoding RNAs. Cell 172, 393-407. https://doi.org/10.1016/j.cell.2018.01.011

Koschützki, D., Schreiber, F., 2008. Centrality Analysis Methods for Biological Networks and Their Application to Gene Regulatory Networks. Gene Regul Syst Bio 2, GRSB.S702. https://doi.org/10.4137/GRSB.S702

Kouzarides, T., 2007. Chromatin Modifications and Their Function. Cell 128, 693-705. https://doi.org/10.1016/j.cell.2007.02.005

Kratochwil, C.F., Liang, Y., Gerwin, J., Woltering, J.M., Urban, S., Henning, F., Machado-Schiaffino, G., Hulsey, C.D., Meyer, A., 2018. Agouti-related peptide 2 facilitates convergent evolution of stripe patterns across cichlid fish radiations. Science 362, 457-460. https://doi.org/10.1126/science.aao6809

Krishnan, J., Rohner, N., 2016. Cavefish and the basis for eye loss. Phil.Trans. R. Soc. B 10. http://dx.doi.org/10.1098/rstb.2015.0487

Kumar, J.P., 2018. The fly eye: Through the looking glass: Future of The Fly Eye. Developmental Dynamics 247, 111-123. https://doi.org/10.1002/dvdy.24585

Kumar, J.P., 2009. The molecular circuitry governing retinal determination. Biochimica et Biophysica Acta (BBA) - Gene Regulatory Mechanisms 1789, 306-314. https://doi.org/10.1016/j.bbagrm.2008.10.001

Kumar, J.P., Jamal, T., Doetsch, A., Turner, F.R., Duffy, J.B., 2004. CREB Binding Protein Functions During Successive Stages of Eye Development in Drosophila. Genetics 168, 877-893. https://doi.org/10.1534/genetics.104.029850

Kumar, J.P., Moses, K., 2001. The EGF receptor and notch signaling pathways control the initiation of the morphogenetic furrow during Drosophila eye development. Development 128, 2689-2697.

Kvon, E.Z., Kazmar, T., Stampfel, G., Yáñez-Cuna, J.O., Pagani, M., Schernhuber, K., Dickson, B.J., Stark, A., 2014. Genome-scale functional characterization of Drosophila developmental enhancers in vivo. Nature 512, 91-95. https://doi.org/10.1038/nature13395

Lambiase, S., Grigolo, A., Morbini, P., 2003. Ontogenesis of pleuropodia in different species of Blattaria (Insecta): A comparative study. https://doi.org/10.1080/11250000309356518 
Landry, C.R., Lemos, B., Rifkin, S.A., Dickinson, W.J., Hartl, D.L., 2007. Genetic Properties Influencing the Evolvability of Gene Expression. Science 317, 118-121. https://doi.org/10.1126/science.1140247

Landry, C.R., Wittkopp, P.J., Taubes, C.H., Ranz, J.M., Clark, A.G., Hartl, D.L., 2005. Compensatory cistrans Evolution and the Dysregulation of Gene Expression in Interspecific Hybrids of Drosophila. Genetics 171, 1813-1822. https://doi.org/10.1534/genetics.105.047449

Langmead, B., Trapnell, C., Pop, M., Salzberg, S.L., 2009. Ultrafast and memory-efficient alignment of short DNA sequences to the human genome. Genome Biol. 10, R25. https://doi.org/10.1186/gb-2009-10-3-r25

Larink, O., 1983. Embryonic and postembryonic development of Machilidae and Lepismatidae (Insecta: Archaeognatha et Zygentoma).

Laumer, C.E., Fernández, R., Lemer, S., Combosch, D., Kocot, K.M., Riesgo, A., Andrade, S.C.S., Sterrer, W., Sørensen, M.V., Giribet, G., 2019. Revisiting metazoan phylogeny with genomic sampling of all phyla. Proc. R. Soc. B 286, 20190831. https://doi.org/10.1098/rspb.2019.0831

Lawrence, M., Daujat, S., Schneider, R., 2016. Lateral Thinking: How Histone Modifications Regulate Gene Expression. Trends in Genetics 32, 42-56. https://doi.org/10.1016/j.tig.2015.10.007

Lee, M.S.Y., Palci, A., 2015. Morphological Phylogenetics in the Genomic Age. Current Biology 25, R922R929. https://doi.org/10.1016/j.cub.2015.07.009

Lemaitre, B., Hoffmann, J., 2007. The host defense of Drosophila melanogaster. Annu. Rev. Immunol. 25, 697-743. https://doi.org/10.1146/annurev.immunol.25.022106.141615

Lemos, B., Meiklejohn, C.D., Cáceres, M., Hartl, D.L., 2005. Rates of divergence in gene expression profiles of primates, mice, and flies: stabilizing selection and variability among functional categories. Evolution 59, 126-137.

Lenaerts, C., Van Wielendaele, P., Peeters, P., Vanden Broeck, J., Marchal, E., 2016. Ecdysteroid signalling components in metamorphosis and development of the desert locust, Schistocerca gregaria. Insect Biochem. Mol. Biol. 75, 10-23. https://doi.org/10.1016/j.ibmb.2016.05.003

Lewis, D.L., DeCamillis, M., Bennett, R.L., 2000. Distinct roles of the homeotic genes Ubx and abd-A in beetle embryonic abdominal appendage development. PNAS 97, 4504-4509. https://doi.org/10.1073/pnas.97.9.4504

Li, D., Zhang, Jianqin, Wang, Y., Liu, X., Ma, E., Sun, Y., Li, S., Zhu, K.Y., Zhang, Jianzhen, 2015. Two chitinase 5 genes from Locusta migratoria: molecular characteristics and functional differentiation. Insect Biochem. Mol. Biol. 58, 46-54. https://doi.org/10.1016/j.ibmb.2015.01.004

Li, H., Handsaker, B., Wysoker, A., Fennell, T., Ruan, J., Homer, N., Marth, G., Abecasis, G., Durbin, R., 1000 Genome Project Data Processing Subgroup, 2009. The Sequence Alignment/Map format and SAMtools. Bioinformatics 25, 2078-2079. https://doi.org/10.1093/bioinformatics/btp352

Li, S., Li, Y., Carthew, R.W., Lai, Z.-C., 1997. Photoreceptor Cell Differentiation Requires Regulated Proteolysis of the Transcriptional Repressor Tramtrack. Cell 90, 469-478. https://doi.org/10.1016/S0092-8674(00)80507-3

Liang, P., Pardee, A.B., 2003. Analysing differential gene expression in cancer. Nature Reviews Cancer 3, 869. https://doi.org/10.1038/nrc1214

Lim, J., Choi, K.-W., 2004. Drosophila eye disc margin is a center for organizing long-range planar polarity. genesis 39, 26-37. https://doi.org/10.1002/gene.20022

Liu, H.-W., Wang, L.-L., Tang, X., Dong, Z.-M., Guo, P.-C., Zhao, D.-C., Xia, Q.-Y., Zhao, P., 2018. Proteomic analysis of Bombyx mori molting fluid: Insights into the molting process. J Proteomics 173, 115125. https://doi.org/10.1016/j.jprot.2017.11.027

Livak, K.J., Schmittgen, T.D., 2001. Analysis of relative gene expression data using real-time quantitative PCR and the 2(-Delta Delta C(T)) Method. Methods 25, 402-408. https://doi.org/10.1006/meth.2001.1262

Locke, M., Krishnan, N., 1973. The formation of the ecdysial droplets and the ecdysial membrane in an insect. Tissue Cell 5, 441-450. 
Lopes, C.S., Casares, F., 2010. hth maintains the pool of eye progenitors and its downregulation by Dpp and Hh couples retinal fate acquisition with cell cycle exit. Developmental Biology 339, 78-88. https://doi.org/10.1016/j.ydbio.2009.12.020

Losos, J.B. (Ed.), 2014. The Princeton guide to evolution. Princeton University Press, Princeton; Oxford.

Louvet, J.-P., 1983. Ultrastructure du pleuropode chez l'embryon du hanneton Rhizotrogus majalis razoum (Coleoptera: Melolonthidae). International Journal of Insect Morphology and Embryology 12, 97-117. https://doi.org/10.1016/0020-7322(83)90003-X

Louvet, J.P., 1975. Premieres observations sur l'ultrastructure du pleuropode chez le criquet migrateur. Comptes rendus hebdomadaires des seances. Serie D. Sciences naturelles.

Louvet, J.-P., 1973. L'ultrastructure du pleuropode et son ontogenèse, chez l'embryon du phasme arausius morosus Br. I. - Étude du pleuropode de l'embryon agé. Ann 948 Sci Nat Zool 12, 525-594.

Lovato, T.L., Sensibaugh, C.A., Swingle, K.L., Martinez, M.M., Cripps, R.M., 2015. The Drosophila Transcription Factors Tinman and Pannier Activate and Collaborate with Myocyte Enhancer Factor-2 to Promote Heart Cell Fate. PLOS ONE 10, e0132965. https://doi.org/10.1371/journal.pone.0132965

Love, M.I., Huber, W., Anders, S., 2014. Moderated estimation of fold change and dispersion for RNAseq data with DESeq2. Genome Biology 15. https://doi.org/10.1186/s13059-014-0550-8

Lübbe, A., Schaffner, W., 1985. Perspectives from Molecular Biology 5.

Ludwig, M.Z., Bergman, C., Patel, N.H., Kreitman, M., 2000. Evidence for stabilizing selection in a eukaryotic enhancer element. Nature 403, 564-567. https://doi.org/10.1038/35000615

Luscombe, N.M., Madan Babu, M., Yu, H., Snyder, M., Teichmann, S.A., Gerstein, M., 2004. Genomic analysis of regulatory network dynamics reveals large topological changes. Nature 431, 308 312. https://doi.org/10.1038/nature02782

Machida, R., 1981. External features of embryonic development of a jumping bristletail, Pedetontus unimaculatus Machida (Insecta, Thysanura, Machilidae). J. Morphol. 168, 339-355. https://doi.org/10.1002/jmor.1051680310

Machida, R., Tojo, K., Tsutsumi, T., Uchifune, T., Klass, K.-D., Picker, M.D., Pretorius, L., 2004. Embryonic development of heel-walkers: Reference to some prerevolutionary stages (Insecta: Mantophasmatodea).

Mack, K., Phifer-Rixey, M., Harr, B., Nachman, M., 2019. Gene Expression Networks Across Multiple Tissues Are Associated with Rates of Molecular Evolution in Wild House Mice. Genes 10, 225. https://doi.org/10.3390/genes10030225

Mackay, T.F.C., 2001. Quantitative trait loci in Drosophila. Nat Rev Genet 2, 11-20. https://doi.org/10.1038/35047544

Mackay, T.F.C., Stone, E.A., Ayroles, J.F., 2009. The genetics of quantitative traits: challenges and prospects. Nat Rev Genet 10, 565-577. https://doi.org/10.1038/nrg2612

MacNeil, L.T., Walhout, A.J.M., 2011. Gene regulatory networks and the role of robustness and stochasticity in the control of gene expression. Genome Research 21, 645-657. https://doi.org/10.1101/gr.097378.109

Maga, A.M., Navarro, N., Cunningham, M.L., Cox, T.C., 2015. Quantitative trait loci affecting the 3D skull shape and size in mouse and prioritization of candidate genes in-silico. Front. Physiol. 6. https://doi.org/10.3389/fphys.2015.00092

Magri, M.S., Domínguez-Cejudo, M.A., Casares, F., 2018. Wnt controls the medial-lateral subdivision of the Drosophila head. Biology Letters 14, 20180258. https://doi.org/10.1098/rsbl.2018.0258

Mähler, N., Wang, J., Terebieniec, B.K., Ingvarsson, P.K., Street, N.R., Hvidsten, T.R., 2017. Gene coexpression network connectivity is an important determinant of selective constraint. PLoS Genet 13, e1006402. https://doi.org/10.1371/journal.pgen.1006402

Marchal, E., Badisco, L., Verlinden, H., Vandersmissen, T., Van Soest, S., Van Wielendaele, P., Vanden Broeck, J., 2011. Role of the Halloween genes, Spook and Phantom in ecdysteroidogenesis in the desert locust, Schistocerca gregaria. J. Insect Physiol. 57, 1240-1248. https://doi.org/10.1016/j.jinsphys.2011.05.009 
Marchal, E., Verlinden, H., Badisco, L., Van Wielendaele, P., Vanden Broeck, J., 2012. RNAi-mediated knockdown of Shade negatively affects ecdysone-20-hydroxylation in the desert locust, Schistocerca gregaria. J. Insect Physiol. 58, 890-896. https://doi.org/10.1016/j.jinsphys.2012.03.013

Marlétaz, F., Firbas, P.N., Maeso, I., Tena, J.J., Bogdanovic, O., Perry, M., Wyatt, C.D.R., de la CalleMustienes, E., Bertrand, S., Burguera, D., Acemel, R.D., van Heeringen, S.J., Naranjo, S., HerreraUbeda, C., Skvortsova, K., Jimenez-Gancedo, S., Aldea, D., Marquez, Y., Buono, L., Kozmikova, I., Permanyer, J., Louis, A., Albuixech-Crespo, B., Le Petillon, Y., Leon, A., Subirana, L., Balwierz, P.J., Duckett, P.E., Farahani, E., Aury, J.-M., Mangenot, S., Wincker, P., Albalat, R., Benito-Gutiérrez, Ė., Cañestro, C., Castro, F., D’Aniello, S., Ferrier, D.E.K., Huang, S., Laudet, V., Marais, G.A.B., Pontarotti, P., Schubert, M., Seitz, H., Somorjai, I., Takahashi, T., Mirabeau, O., Xu, A., Yu, J.-K., Carninci, P., Martinez-Morales, J.R., Crollius, H.R., Kozmik, Z., Weirauch, M.T., Garcia-Fernàndez, J., Lister, R., Lenhard, B., Holland, P.W.H., Escriva, H., Gómez-Skarmeta, J.L., Irimia, M., 2018. Amphioxus functional genomics and the origins of vertebrate gene regulation. Nature $564,64-$ 70. https://doi.org/10.1038/s41586-018-0734-6

Martin, A., Orgogozo, V., 2013. THE LOCI OF REPEATED EVOLUTION: A CATALOG OF GENETIC HOTSPOTS OF PHENOTYPIC VARIATION: PERSPECTIVE. Evolution n/a-n/a. https://doi.org/10.1111/evo.12081

Mashimo, Y., Beutel, R.G., Dallai, R., Lee, C.-Y., Machida, R., 2014. Embryonic development of Zoraptera with special reference to external morphology, and its phylogenetic implications (Insecta). J. Morphol. 275, 295-312. https://doi.org/10.1002/jmor.20215

Maurel-Zaffran, C., Treisman, J.E., 2000. pannier acts upstream of wingless to direct dorsal eye disc development in Drosophila. Development 127, 1007-1016.

McGinnis, W., Krumlauf, R., 1992. Homeobox genes and axial patterning. Cell 68, 283-302. https://doi.org/10.1016/0092-8674(92)90471-N

McGregor, A.P., Orgogozo, V., Delon, I., Zanet, J., Srinivasan, D.G., Payre, F., Stern, D.L., 2007. Morphological evolution through multiple cis-regulatory mutations at a single gene. Nature 448, 587-590. https://doi.org/10.1038/nature05988

McKay, D.J., Lieb, J.D., 2013. A Common Set of DNA Regulatory Elements Shapes Drosophila Appendages. Developmental Cell 27, 306-318. https://doi.org/10.1016/j.devcel.2013.10.009

McManus, C.J., Coolon, J.D., Duff, M.O., Eipper-Mains, J., Graveley, B.R., Wittkopp, P.J., 2010. Regulatory divergence in Drosophila revealed by mRNA-seq. Genome Research 20, 816-825. https://doi.org/10.1101/gr.102491.109

Merzendorfer, H., 2013. Chitin synthesis inhibitors: old molecules and new developments. Insect Science 20, 121-138. https://doi.org/10.1111/j.1744-7917.2012.01535.x

Metzger, B.P.H., Wittkopp, P.J., Coolon, Joseph.D., 2017. Evolutionary Dynamics of Regulatory Changes Underlying Gene Expression Divergence among Saccharomyces Species. Genome Biology and Evolution 9, 843-854. https://doi.org/10.1093/gbe/evx035

Miller, A., 1940. Embryonic Membranes, Yolk Cells, and Morphogenesis of the Stonefly Pteronarcys Proteus Newman, (Plecoptera: Pteronarcidae). Ann Entomol Soc Am 33, 437-477. https://doi.org/10.1093/aesa/33.2.437

Milner, M.J., Bleasby, A.J., Kelly, S.L., 1984. The role of the peripodial membrane of leg and wing imaginal discs ofDrosophila melanogaster during evagination and differentiation in vitro. Wilhelm Roux' Archiv 193, 180-186. https://doi.org/10.1007/BF00848893

Mishra, A.K., Bargmann, B.O.R., Tsachaki, M., Fritsch, C., Sprecher, S.G., 2016. Functional genomics identifies regulators of the phototransduction machinery in the Drosophila larval eye and adult ocelli. Developmental Biology 410, 164-177. https://doi.org/10.1016/j.ydbio.2015.12.026

Miyakawa, K., 1979. Embryology of the dobsonfly, Protohermes grandis Thunberg (Megaloptera: Corydalidae). I. Changes in external form of the embryo during development. Konchu. Kontyu.

Mlodzik, M., 2002. Planar cell polarization: do the same mechanisms regulate Drosophila tissue polarity and vertebrate gastrulation? Trends in Genetics 18, 564-571. https://doi.org/10.1016/S01689525(02)02770-1 
Mohamed, A.A., Zhang, L., Dorrah, M.A., Elmogy, M., Yousef, H.A., Bassal, T.T.M., Duvic, B., 2016. Molecular characterization of a c-type lysozyme from the desert locust, Schistocerca gregaria (Orthoptera: Acrididae). Dev. Comp. Immunol. 61, 60-69. https://doi.org/10.1016/j.dci.2016.03.018

Moriyama, Y., Koshiba-Takeuchi, K., 2018. Significance of whole-genome duplications on the emergence of evolutionary novelties. Briefings in Functional Genomics 17, 329-338. https://doi.org/10.1093/bfgp/ely007

Muratoglu, S., Garratt, B., Hyman, K., Gajewski, K., Schulz, R.A., Fossett, N., 2006. Regulation of Drosophila friend of GATA gene, u-shaped, during hematopoiesis: a direct role for serpent and lozenge. Dev. Biol. 296, 561-579. https://doi.org/10.1016/j.ydbio.2006.04.455

Neto, M., Naval-Sánchez, M., Potier, D., Pereira, P.S., Geerts, D., Aerts, S., Casares, F., 2017. Nuclear receptors connect progenitor transcription factors to cell cycle control. Sci Rep 7, 4845. https://doi.org/10.1038/s41598-017-04936-7

Nijhout, F.J., 1998. Insect Hormones.

Niven, J.E., Laughlin, S.B., 2008. Energy limitation as a selective pressure on the evolution of sensory systems. J. Exp. Biol. 211, 1792-1804. https://doi.org/10.1242/jeb.017574

Niwa, R., Matsuda, T., Yoshiyama, T., Namiki, T., Mita, K., Fujimoto, Y., Kataoka, H., 2004. CYP306A1, a cytochrome P450 enzyme, is essential for ecdysteroid biosynthesis in the prothoracic glands of Bombyx and Drosophila. J. Biol. Chem. 279, 35942-35949. https://doi.org/10.1074/jbc.M404514200

Niwa, R., Niwa, Y.S., 2014. Enzymes for ecdysteroid biosynthesis: their biological functions in insects and beyond. Biosci. Biotechnol. Biochem. 78, 1283-1292. https://doi.org/10.1080/09168451.2014.942250

Noh, M.Y., Muthukrishnan, S., Kramer, K.J., Arakane, Y., 2018. A chitinase with two catalytic domains is required for organization of the cuticular extracellular matrix of a beetle. PLoS Genet. 14, e1007307. https://doi.org/10.1371/journal.pgen.1007307

Norling, U., 1982. Structure and ontogeny of the lateral abdominal gills and the caudal gills in Euphaeidae (Odonata: Zygoptera) larvae. Zoologische Jahrbucher. Abteilung fur Anatomie und Ontogenie der Tiere.

Norry, F.M., Gomez, F.H., 2017. Quantitative Trait Loci and Antagonistic Associations for Two Developmentally Related Traits in the Drosophila Head. Journal of Insect Science 17, 19. https://doi.org/10.1093/jisesa/iew115

Norry, F.M., Vilardi, J.C., Hasson, E., 2000. Negative genetic correlation between traits of the Drosophila head, and interspecific divergence in head shape. Heredity (Edinb) 85 ( Pt 2), 177-183. https://doi.org/10.1046/j.1365-2540.2000.00735.x

Novak, V.J.A., Zambre, S.K., 1974. To the problem of structure and function of pleuropodia in Schistocerca gregaria Forskal embryos. Zoologische Jahrbücher.

Oakley, T.H., Wolfe, J.M., Lindgren, A.R., Zaharoff, A.K., 2013. Phylotranscriptomics to Bring the Understudied into the Fold: Monophyletic Ostracoda, Fossil Placement, and Pancrustacean Phylogeny. Molecular Biology and Evolution 30, 215-233. https://doi.org/10.1093/molbev/mss216

Oros, S.M., Tare, M., Kango-Singh, M., Singh, A., 2010. Dorsal eye selector pannier (pnr) suppresses the eye fate to define dorsal margin of the Drosophila eye. Developmental Biology 346, 258-271. https://doi.org/10.1016/j.ydbio.2010.07.030

Ou, Q., Zeng, J., Yamanaka, N., Brakken-Thal, C., O'Connor, M.B., King-Jones, K., 2016. The Insect Prothoracic Gland as a Model for Steroid Hormone Biosynthesis and Regulation. Cell Rep 16, 247-262. https://doi.org/10.1016/j.celrep.2016.05.053

Pagès, H., Aboyoun, P., Gentleman, R., 2017. Biostrings: Efficient manipulation of biological strings. R package version 2.46.0.

Pallares, L.F., Harr, B., Turner, L.M., Tautz, D., 2014. Use of a natural hybrid zone for genomewide association mapping of craniofacial traits in the house mouse. Mol Ecol 23, 5756-5770. https://doi.org/10.1111/mec.12968 
Palliyil, S., Zhu, J., Baker, L.R., Neuman, S.D., Bashirullah, A., Kumar, J.P., 2018. Allocation of distinct organ fates from a precursor field requires a shift in expression and function of gene regulatory networks. PLoS Genet. 14, e1007185. https://doi.org/10.1371/journal.pgen.1007185

Pan, D., Rubin, G.M., 1995. cAMP-dependent protein kinase and hedgehog act antagonistically in regulating decapentaplegic transcription in drosophila imaginal discs. Cell 80, 543-552. https://doi.org/10.1016/0092-8674(95)90508-1

Panfilio, K.A., 2008. Extraembryonic development in insects and the acrobatics of blastokinesis. Dev. Biol. 313, 471-491. https://doi.org/10.1016/j.ydbio.2007.11.004

Pardue, M.L., Gall, J.G., 1969. MOLECULAR HYBRIDIZATION OF RADIOACTIVE DNA TO THE DNA OF CYTOLOGICAL PREPARATIONS. Proceedings of the National Academy of Sciences 64, 600-604. https://doi.org/10.1073/pnas.64.2.600

Paris, M., Kaplan, T., Li, X.Y., Villalta, J.E., Lott, S.E., Eisen, M.B., 2013. Extensive Divergence of Transcription Factor Binding in Drosophila Embryos with Highly Conserved Gene Expression. PLOS Genetics 9, e1003748. https://doi.org/10.1371/journal.pgen.1003748

Pereira, P.S., Pinho, S., Johnson, K., Couso, J.P., Casares, F., 2006. A 3'cis-regulatory region controlswingless expression in theDrosophila eye and leg primordia. Dev. Dyn. 235, 225-234. https://doi.org/10.1002/dvdy.20606

Pertea, G., Huang, X., Liang, F., Antonescu, V., Sultana, R., Karamycheva, S., Lee, Y., White, J., Cheung, F., Parvizi, B., Tsai, J., Quackenbush, J., 2003. TIGR Gene Indices clustering tools (TGICL): a software system for fast clustering of large EST datasets. Bioinformatics 19, 651-652. https://doi.org/10.1093/bioinformatics/btg034

Pesch, Y.-Y., Riedel, D., Patil, K.R., Loch, G., Behr, M., 2016. Chitinases and Imaginal disc growth factors organize the extracellular matrix formation at barrier tissues in insects. Sci Rep 6, 18340. https://doi.org/10.1038/srep18340

Petryk, A., Warren, J.T., Marqués, G., Jarcho, M.P., Gilbert, L.I., Kahler, J., Parvy, J.-P., Li, Y., DauphinVillemant, C., O'Connor, M.B., 2003. Shade is the Drosophila P450 enzyme that mediates the hydroxylation of ecdysone to the steroid insect molting hormone 20-hydroxyecdysone. Proc. Natl. Acad. Sci. U.S.A. 100, 13773-13778. https://doi.org/10.1073/pnas.2336088100

Ponting, C.P., Oliver, P.L., Reik, W., 2009. Evolution and Functions of Long Noncoding RNAs. Cell 136, 629-641. https://doi.org/10.1016/j.cell.2009.02.006

Pool, J.E., Aquadro, C.F., 2007. The genetic basis of adaptive pigmentation variation in Drosophila melanogaster: ADAPTIVE PIGMENTATION IN D. MELANOGASTER. Molecular Ecology 16, 2844 2851. https://doi.org/10.1111/j.1365-294X.2007.03324.x

Posnien, N., Hopfen, C., Hilbrant, M., Ramos-Womack, M., Murat, S., Schönauer, A., Herbert, S.L., Nunes, M.D.S., Arif, S., Breuker, C.J., Schlötterer, C., Mitteroecker, P., McGregor, A.P., 2012. Evolution of Eye Morphology and Rhodopsin Expression in the Drosophila melanogaster Species Subgroup. PLoS ONE 7, e37346. https://doi.org/10.1371/journal.pone.0037346

Potier, D., Davie, K., Hulselmans, G., Naval Sanchez, M., Haagen, L., Huynh-Thu, V.A., Koldere, D., Celik, A., Geurts, P., Christiaens, V., Aerts, S., 2014. Mapping Gene Regulatory Networks in Drosophila Eye Development by Large-Scale Transcriptome Perturbations and Motif Inference. Cell Reports 9, 2290-2303. https://doi.org/10.1016/j.celrep.2014.11.038

Prpic, N.-M., 2019. A lesson in homology. elife 8, e48335. https://doi.org/10.7554/eLife.48335

Prpic, N.-M., Wigand, B., Damen, W., Klingler, M., 2001. Expression of dachshund in wild-type and Distalless mutant Tribolium corroborates serial homologies in insect appendages. Development Genes and Evolution 211, 467-477. https://doi.org/10.1007/s004270100178

Prud'homme, B., Gompel, N., Carroll, S.B., 2007. Emerging principles of regulatory evolution. Proceedings of the National Academy of Sciences 104, 8605-8612. https://doi.org/10.1073/pnas.0700488104

Prud'homme, B., Gompel, N., Rokas, A., Kassner, V.A., Williams, T.M., Yeh, S.-D., True, J.R., Carroll, S.B., 2006. Repeated morphological evolution through cis-regulatory changes in a pleiotropic gene. Nature 440, 1050-1053. https://doi.org/10.1038/nature04597 
Qu, M., Ma, L., Chen, P., Yang, Q., 2014. Proteomic analysis of insect molting fluid with a focus on enzymes involved in chitin degradation. J. Proteome Res. 13, 2931-2940. https://doi.org/10.1021/pr5000957

Quinlan, A.R., 2014. BEDTools: The Swiss-Army Tool for Genome Feature Analysis. Curr Protoc Bioinformatics 47, 11.12.1-34. https://doi.org/10.1002/0471250953.bi1112s47

Quinlan, A.R., Hall, I.M., 2010. BEDTools: a flexible suite of utilities for comparing genomic features. Bioinformatics 26, 841-842. https://doi.org/10.1093/bioinformatics/btq033

Quiring, R., Walldorf, U., Kloter, U., Gehring, W., 1994. Homology of the eyeless gene of Drosophila to the Small eye gene in mice and Aniridia in humans. Science 265, 785-789. https://doi.org/10.1126/science.7914031

R Development Core Team, 2008. R: A language and environment for statistical computing. R Foundation for Statistical Computing, Vienna, Austria.

Raff, R.A., 2000. Evo-devo: the evolution of a new discipline. Nat Rev Genet 1, 74-79. https://doi.org/10.1038/35049594

Ramaekers, A., Weinberger, S., Claeys, A., Kapun, M., Yan, J., Wolf, R., Flatt, T., Buchner, E., Hassan, B.A., 2018. Altering the temporal regulation of one transcription factor drives sensory trade-offs (preprint). Developmental Biology. https://doi.org/10.1101/348375

Ramain, P., Heitzler, P., Haenlin, M., Simpson, P., 1993. pannier, a negative regulator of achaete and scute in Drosophila, encodes a zinc finger protein with homology to the vertebrate transcription factor GATA-1. Development 119, 1277-1291.

Rathke, H., 1844. Zur Entwicklungsgeschichte der Maulwurfsgrille (Gryllotalpa vulgaris). Arch Anat Physiol wiss Med 27-37.

Rau, A., Gallopin, M., Celeux, G., Jaffrézic, F., 2013. Data-based filtering for replicated high-throughput transcriptome sequencing experiments. Bioinformatics 29, 2146-2152. https://doi.org/10.1093/bioinformatics/btt350

Rau, A., Maugis-Rabusseau, C., 2017. Transformation and model choice for RNA-seq co-expression analysis. Briefings in Bioinformatics bbw128. https://doi.org/10.1093/bib/bbw128

Rebeiz, M., Pool, J.E., Kassner, V.A., Aquadro, C.F., Carroll, S.B., 2009. Stepwise Modification of a Modular Enhancer Underlies Adaptation in a Drosophila Population. Science 326, 1663-1667. https://doi.org/10.1126/science.1178357

Reeder, T.W., Townsend, T.M., Mulcahy, D.G., Noonan, B.P., Wood, P.L., Sites, J.W., Wiens, J.J., 2015. Integrated Analyses Resolve Conflicts over Squamate Reptile Phylogeny and Reveal Unexpected Placements for Fossil Taxa. PLoS ONE 10, e0118199. https://doi.org/10.1371/journal.pone.0118199

Rendeiro, A.F., Schmidl, C., Strefford, J.C., Walewska, R., Davis, Z., Farlik, M., Oscier, D., Bock, C., 2016. Chromatin accessibility maps of chronic lymphocytic leukaemia identify subtype-specific epigenome signatures and transcription regulatory networks. Nature Communications 7 , 11938. https://doi.org/10.1038/ncomms11938

Rennie, S., Dalby, M., Lloret-Llinares, M., Bakoulis, S., Dalager Vaagens $\varnothing$, C., Heick Jensen, T., Andersson, R., 2018a. Transcription start site analysis reveals widespread divergent transcription in D. melanogaster and core promoter-encoded enhancer activities. Nucleic Acids Research 46, 5455-5469. https://doi.org/10.1093/nar/gky244

Rennie, S., Dalby, M., van Duin, L., Andersson, R., 2018b. Transcriptional decomposition reveals active chromatin architectures and cell specific regulatory interactions. Nat Commun 9, 487. https://doi.org/10.1038/s41467-017-02798-1

Reuveni, E., Getselter, D., Oron, O., Elliott, E., 2018. Differential contribution of cis and trans gene transcription regulatory mechanisms in amygdala and prefrontal cortex and modulation by social stress. Scientific Reports 8. https://doi.org/10.1038/s41598-018-24544-3

Reynolds, S.E., Samuels, R.I., 1996. Physiology and Biochemistry of Insect Moulting Fluid, in: Evans, P.D. (Ed.), Advances in Insect Physiology. Academic Press, pp. 157-232. https://doi.org/10.1016/S0065-2806(08)60031-4 
Rifkin, S.A., Kim, J., White, K.P., 2003. Evolution of gene expression in the Drosophila melanogaster subgroup. Nature Genetics 33, 138-144. https://doi.org/10.1038/ng1086

Robertson, G., Hirst, M., Bainbridge, M., Bilenky, M., Zhao, Y., Zeng, T., Euskirchen, G., Bernier, B., Varhol, R., Delaney, A., Thiessen, N., Griffith, O.L., He, A., Marra, M., Snyder, M., Jones, S., 2007. Genome-wide profiles of STAT1 DNA association using chromatin immunoprecipitation and massively parallel sequencing. Nature Methods 4, 651-657. https://doi.org/10.1038/nmeth1068

Rockman, M.V., Kruglyak, L., 2006. Genetics of global gene expression. Nat Rev Genet 7, 862-872. https://doi.org/10.1038/nrg1964

Rogers, W.A., Salomone, J.R., Tacy, D.J., Camino, E.M., Davis, K.A., Rebeiz, M., Williams, T.M., 2013. Recurrent Modification of a Conserved Cis-Regulatory Element Underlies Fruit Fly Pigmentation Diversity. PLOS Genetics 9, e1003740. https://doi.org/10.1371/journal.pgen.1003740

Rong, S., Li, D.-Q., Zhang, X.-Y., Li, S., Zhu, K.Y., Guo, Y.-P., Ma, E.-B., Zhang, J.-Z., 2013. RNA interference to reveal roles of $\beta-\mathrm{N}$-acetylglucosaminidase gene during molting process in Locusta migratoria. Insect Sci. 20, 109-119. https://doi.org/10.1111/j.1744-7917.2012.01573.x

Roonwal Mithan Lal, Imms Augustus Daniel, 1936. X - Studies on the embryology of the African migratory locust, Locusta migratoria migratorioides R. and F. - I - The early development, with a new theory of multi-phased gastrulation among insects. Philosophical Transactions of the Royal Society of London. Series B, Biological Sciences 226, 391-421. https://doi.org/10.1098/rstb.1936.0011

Rost, M.M., Poprawa, I., Klag, J., 2004. Ultrastructure of the Pleuropodium in 8-d-old Embryos of Thermobia domestica (Packard) (Insecta, Zygentoma). Ann Entomol Soc Am 97, 541-547. https://doi.org/10.1603/0013-8746(2004)097[0541:UOTPID]2.0.CO;2

S. MILLAM STANLEY, M., W. GRUNDMANN, A., 1970. The Embryonic Development of Tribolium confusum. https://doi.org/10.1093/aesa/63.5.1248

Sarropoulos, I., Marin, R., Cardoso-Moreira, M., Kaessmann, H., 2019. Developmental dynamics of IncRNAs across mammalian organs and species. Nature. https://doi.org/10.1038/s41586-0191341-x

Sato, M., Saigo, K., 2000. Involvement of pannier and u-shaped in regulation of Decapentaplegicdependent wingless expression in developing Drosophila notum. Mechanisms of Development 93, 127-138. https://doi.org/10.1016/S0925-4773(00)00282-3

Schindelin, J., Arganda-Carreras, I., Frise, E., Kaynig, V., Longair, M., Pietzsch, T., Preibisch, S., Rueden, C., Saalfeld, S., Schmid, B., Tinevez, J.-Y., White, D.J., Hartenstein, V., Eliceiri, K., Tomancak, P., Cardona, A., 2012. Fiji: an open-source platform for biological-image analysis. Nature Methods 9, 676-682. https://doi.org/10.1038/nmeth.2019

Schluter, D., 2000. The Ecology of Adaptive Radiation, Oxford Series in Ecology and Evolution. Oxford University Press, Oxford, New York.

Schmidt, D., Wilson, M.D., Ballester, B., Schwalie, P.C., Brown, G.D., Marshall, A., Kutter, C., Watt, S., Martinez-Jimenez, C.P., Mackay, S., Talianidis, I., Flicek, P., Odom, D.T., 2010. Five-Vertebrate ChIP-seq Reveals the Evolutionary Dynamics of Transcription Factor Binding 328, 6.

Schmidt-Ott, U., 2000. The amnioserosa is an apomorphic character of cyclorrhaphan flies. Dev. Genes Evol. 210, 373-376. https://doi.org/10.1007/s004270050325

Schulz, M.H., Zerbino, D.R., Vingron, M., Birney, E., 2012. Oases: robust de novo RNA-seq assembly across the dynamic range of expression levels. Bioinformatics 28, 1086-1092. https://doi.org/10.1093/bioinformatics/bts094

Schwager, E.E., Sharma, P.P., Clarke, T., Leite, D.J., Wierschin, T., Pechmann, M., Akiyama-Oda, Y., Esposito, L., Bechsgaard, J., Bilde, T., Buffry, A.D., Chao, H., Dinh, H., Doddapaneni, H., Dugan, S., Eibner, C., Extavour, C.G., Funch, P., Garb, J., Gonzalez, L.B., Gonzalez, V.L., Griffiths-Jones, S., Han, Y., Hayashi, C., Hilbrant, M., Hughes, D.S.T., Janssen, R., Lee, S.L., Maeso, I., Murali, S.C., Muzny, D.M., Nunes da Fonseca, R., Paese, C.L.B., Qu, J., Ronshaugen, M., Schomburg, C., Schönauer, A., Stollewerk, A., Torres-Oliva, M., Turetzek, N., Vanthournout, B., Werren, J.H., Wolff, C., Worley, K.C., Bucher, G., Gibbs, R.A., Coddington, J., Oda, H., Stanke, M., Ayoub, N.A., 
Prpic, N.-M., Flot, J.-F., Posnien, N., Richards, S., McGregor, A.P., 2017. The house spider genome reveals an ancient whole-genome duplication during arachnid evolution. BMC Biology 15. https://doi.org/10.1186/s12915-017-0399-x

Scott, M.P., Weiner, A.J., 1984. Structural relationships among genes that control development: sequence homology between the Antennapedia, Ultrabithorax, and fushi tarazu loci of Drosophila. Proceedings of the National Academy of Sciences 81, 4115-4119. https://doi.org/10.1073/pnas.81.13.4115

Seeds, A.M., Tsui, M.M., Sunu, C., Spana, E.P., York, J.D., 2015. Inositol phosphate kinase 2 is required for imaginal disc development in Drosophila. Proc Natl Acad Sci USA 201514684. https://doi.org/10.1073/pnas.1514684112

Sexton, T., Yaffe, E., Kenigsberg, E., Bantignies, F., Leblanc, B., Hoichman, M., Parrinello, H., Tanay, A., Cavalli, G., 2012. Three-Dimensional Folding and Functional Organization Principles of the Drosophila Genome. Cell 148, 458-472. https://doi.org/10.1016/j.cell.2012.01.010

Shannon, P., Markiel, A., Ozier, O., Baliga, N.S., Wang, J.T., Ramage, D., Amin, N., Schwikowski, B., Ideker, T., 2003. Cytoscape: a software environment for integrated models of biomolecular interaction networks. Genome Res. 13, 2498-2504. https://doi.org/10.1101/gr.1239303

Shapiro, M.D., Marks, M.E., Peichel, C.L., Blackman, B.K., Nereng, K.S., Jónsson, B., Schluter, D., Kingsley, D.M., 2004. Genetic and developmental basis of evolutionary pelvic reduction in threespine sticklebacks. Nature 428, 717-723. https://doi.org/10.1038/nature02415

Shi, Y., Noll, M., 2009. Determination of cell fates in the R7 equivalence group of the Drosophila eye by the concerted regulation of D-Pax2 and TTK88. Developmental Biology 331, 68-77. https://doi.org/10.1016/j.ydbio.2009.04.026

Shutts, J.H., 1952. Some characteristics of the hatching enzyme in the eggs of Melanoplus differenitalis (Thomas). Proc S Dak Acad Sci. 31, 158-163.

Siddall, N.A., Hime, G.R., Pollock, J.A., Batterham, P., 2009. Ttk69-dependent repression of lozenge prevents the ectopic development of R7 cells in the Drosophila larval eye disc. BMC Dev Biol 9, 64. https://doi.org/10.1186/1471-213X-9-64

Simão, F.A., Waterhouse, R.M., loannidis, P., Kriventseva, E.V., Zdobnov, E.M., 2015. BUSCO: assessing genome assembly and annotation completeness with single-copy orthologs. Bioinformatics 31, 3210-3212. https://doi.org/10.1093/bioinformatics/btv351

Singh, A., Chan, J., Chern, J.J., Choi, K.-W., 2005. Genetic interaction of Lobe with its modifiers in dorsoventral patterning and growth of the Drosophila eye. Genetics 171, 169-183. https://doi.org/10.1534/genetics.105.044180

Singh, A., Choi, K.-W., 2003. Initial state of the Drosophila eye before dorsoventral specification is equivalent to ventral. Development 130, 6351-6360. https://doi.org/10.1242/dev.00864

Singh, P.P., Arora, J., Isambert, H., 2015. Identification of Ohnolog Genes Originating from Whole Genome Duplication in Early Vertebrates, Based on Synteny Comparison across Multiple Genomes. PLOS Computational Biology 11, e1004394. https://doi.org/10.1371/journal.pcbi.1004394

Siomava, N., Wimmer, E.A., Posnien, N., 2016. Size relationships of different body parts in the three dipteran species Drosophila melanogaster, Ceratitis capitata and Musca domestica. Development Genes and Evolution 226, 245-256. https://doi.org/10.1007/s00427-016-0543-6

Slifer, E.H., 1938. A cytological study of the pleuropodia of Melanoplus differentialis (Orthoptera, Acrididae) which furnishes new evidence that they produce the hatching enzyme. J. Morphol. 63, 181-205. https://doi.org/10.1002/jmor.1050630109

Snodgrass, R.E., 1938. Evolution of the annelida onychophora and arthropoda.

Sonawane, A.R., Platig, J., Fagny, M., Chen, C.-Y., Paulson, J.N., Lopes-Ramos, C.M., DeMeo, D.L., Quackenbush, J., Glass, K., Kuijjer, M.L., 2017. Understanding Tissue-Specific Gene Regulation. Cell Rep 21, 1077-1088. https://doi.org/10.1016/j.celrep.2017.10.001

Sorrentino, R.P., Tokusumi, T., Schulz, R.A., 2007. The Friend of GATA protein U-shaped functions as a hematopoietic tumor suppressor in Drosophila. Developmental Biology 311, 311-323. https://doi.org/10.1016/j.ydbio.2007.08.011 
Stanojevic, D., Small, S., Levine, M., 1991. Regulation of a segmentation stripe by overlapping activators and repressors in the Drosophila embryo. Science 254, 1385-1387. https://doi.org/10.1126/science.1683715

Starks, R.R., Biswas, A., Jain, A., Tuteja, G., 2019. Combined analysis of dissimilar promoter accessibility and gene expression profiles identifies tissue-specific genes and actively repressed networks. Epigenetics \& Chromatin 12, 16. https://doi.org/10.1186/s13072-019-0260-2

Stay, B., 1977. Fine structure of two types of pleuropodia in Diploptera punctata (Dictyoptera: Blaberidae) with observations on their permeability. International Journal of Insect Morphology and Embryology 6, 67-95. https://doi.org/10.1016/0020-7322(77)90013-7

Stern, D.L., 2014. Identification of loci that cause phenotypic variation in diverse species with the reciprocal hemizygosity test. Trends Genet. 30, 547-554. https://doi.org/10.1016/j.tig.2014.09.006

Stern, D.L., Orgogozo, V., 2008. THE LOCI OF EVOLUTION: HOW PREDICTABLE IS GENETIC EVOLUTION? Evolution 62, 2155-2177. https://doi.org/10.1111/j.1558-5646.2008.00450.x

Struhl, G., Basler, K., 1993. Organizing activity of wingless protein in Drosophila. Cell 72, 527-540. https://doi.org/10.1016/0092-8674(93)90072-x

Strutt, D.I., Wiersdorff, V., Mlodzik, M., 1995. Regulation of furrow progression in the Drosophila eye by cAMP-dependent protein kinase A. Nature 373, 705-709. https://doi.org/10.1038/373705a0

Sucena, E., Delon, I., Jones, I., Payre, F., Stern, D.L., 2003. Regulatory evolution of shavenbaby/ovo underlies multiple cases of morphological parallelism. Nature 424, 935-938. https://doi.org/10.1038/nature01768

Sucena, É., Stern, D.L., 2000. Divergence of larval morphology between Drosophila sechellia and its sibling species caused by cis-regulatory evolution of ovo/shaven-baby. PNAS 97, 4530-4534. https://doi.org/10.1073/pnas.97.9.4530

Sugahara, R., Tanaka, S., Shiotsuki, T., 2017. RNAi-mediated knockdown of SPOOK reduces ecdysteroid titers and causes precocious metamorphosis in the desert locust Schistocerca gregaria. Dev. Biol. 429, 71-80. https://doi.org/10.1016/j.ydbio.2017.07.007

Sui, Y.-P., Liu, X.-B., Chai, L.-Q., Wang, J.-X., Zhao, X.-F., 2009. Characterization and influences of classical insect hormones on the expression profiles of a molting carboxypeptidase A from the cotton bollworm (Helicoverpa armigera). Insect Mol. Biol. 18, 353-363. https://doi.org/10.1111/j.1365-2583.2009.00879.x

Suster, M.L., Seugnet, L., Bate, M., Sokolowski, M.B., 2004. Refining GAL4-driven transgene expression in Drosophila with a GAL80 enhancer-trap. genesis 39, 240-245. https://doi.org/10.1002/gene.20051

Suvorov, A., Nolte, V., Pandey, R.V., Franssen, S.U., Futschik, A., Schlötterer, C., 2013. Intra-Specific Regulatory Variation in Drosophila pseudoobscura. PLoS ONE 8, e83547. https://doi.org/10.1371/journal.pone.0083547

Swalla, B.J., Smith, A.B., 2008. Deciphering deuterostome phylogeny: molecular, morphological and palaeontological perspectives. Philosophical Transactions of the Royal Society B: Biological Sciences 363, 1557-1568. https://doi.org/10.1098/rstb.2007.2246

Tan, S., Amos, W., Laughlin, S.B., 2005. Captivity selects for smaller eyes. Current Biology 15, R540R542. https://doi.org/10.1016/j.cub.2005.07.019

Tanaka, M., Kobayashi, Y.K., Ando, H., 1985. Embryonic Development of the Nervous System and Other Ectodermal Derivatives in the Primitive Moth, Endoclita sinensis (Lepidoptera, Hepialidae) 15.

Tanizawa, T., Ando, H., Tojo, K., 2007. Notes on the Pleuropodia in the Giant Water Bug Appasus japonicus (Heteroptera, Belostomatida). Proc. Arthropod. Embryol. Soc. Jpn. 42, 9-11.

Tarazona, O.A., Lopez, D.H., Slota, L.A., Cohn, M.J., 2019. Evolution of limb development in cephalopod mollusks. eLife 8, e43828. https://doi.org/10.7554/eLife.43828

Tarazona, S., García-Alcalde, F., Dopazo, J., Ferrer, A., Conesa, A., 2011. Differential expression in RNAseq: a matter of depth. Genome Res. 21, 2213-2223. https://doi.org/10.1101/gr.124321.111

Tautz, D., 2000. Evolution of transcriptional regulation. Curr. Opin. Genet. Dev. 10, 575-579. 
Tear, G., Akam, M., Martinez-Arias, A., 1990. Isolation of an abdominal-A gene from the locust Schistocerca gregaria and its expression during early embryogenesis. Development 110, 915925.

Technau, G.M., Berger, C., Urbach, R., 2006. Generation of cell diversity and segmental pattern in the embryonic central nervous system of Drosophila. Dev. Dyn. 235, 861-869. https://doi.org/10.1002/dvdy.20566

The Gene Ontology Consortium, 2019. The Gene Ontology Resource: 20 years and still GOing strong. Nucleic Acids Research 47, D330-D338. https://doi.org/10.1093/nar/gky1055

Thompson, D., Regev, A., Roy, S., 2015. Comparative Analysis of Gene Regulatory Networks: From Network Reconstruction to Evolution. Annual Review of Cell and Developmental Biology 31, 399-428. https://doi.org/10.1146/annurev-cellbio-100913-012908

Todd, E.V., Black, M.A., Gemmell, N.J., 2016. The power and promise of RNA-seq in ecology and evolution. Mol Ecol 25, 1224-1241. https://doi.org/10.1111/mec.13526

Tomoyasu, Y., Ueno, N., Nakamura, M., 2000. The Decapentaplegic morphogen gradient regulates the notal wingless expression through induction of pannier and $\mathrm{u}$-shaped in Drosophila. Mechanisms of Development 96, 37-49. https://doi.org/10.1016/S0925-4773(00)00374-9

Torres-Oliva, M., 2016. Identification of the molecular changes underlying head morphology variation in closely related Drosophila species. Georg-August-Universität Göttingen, Göttingen.

Torres-Oliva, M., Almudi, I., McGregor, A.P., Posnien, N., 2016. A robust (re-)annotation approach to generate unbiased mapping references for RNA-seq-based analyses of differential expression across closely related species. BMC Genomics 17. https://doi.org/10.1186/s12864-016-2646-x

Torres-Oliva, M., Schneider, J., Wiegleb, G., Kaufholz, F., Posnien, N., 2018. Dynamic genome wide expression profiling of Drosophila head development reveals a novel role of Hunchback in retinal glia cell development and blood-brain barrier integrity. PLOS Genetics 14, e1007180. https://doi.org/10.1371/journal.pgen.1007180

Treisman, J.E., 2013. Retinal differentiation in Drosophila. WIREs Dev Biol 2, 545-557. https://doi.org/10.1002/wdev.100

Treisman, J.E., Heberlein, U., 1998. Eye development in Drosophila: formation of the eye field and control of differentiation. Curr. Top. Dev. Biol. 39, 119-158.

Treisman, J.E., Rubin, G.M., 1995. wingless inhibits morphogenetic furrow movement in the Drosophila eye disc. Development 121, 3519-3527.

Tsui, M.M., York, J.D., 2010. Roles of inositol phosphates and inositol pyrophosphates in development, cell signaling and nuclear processes. Advances in Enzyme Regulation 50, 324-337. https://doi.org/10.1016/j.advenzreg.2009.12.002

Tsutsumi, T., 2008. Embryonic development of a snakefly, Inocellia japonica Okamoto. Proc. Arthropod Embryol Soc Jpn.

Turetzek, N., Khadjeh, S., Schomburg, C., Prpic, N.-M., 2017. Rapid diversification of homothorax expression patterns after gene duplication in spiders. BMC Evolutionary Biology 17. https://doi.org/10.1186/s12862-017-1013-0

Turetzek, N., Pechmann, M., Schomburg, C., Schneider, J., Prpic, N.-M., 2016. Neofunctionalization of a Duplicate dachshund Gene Underlies the Evolution of a Novel Leg Segment in Arachnids. Molecular Biology and Evolution 33, 109-121. https://doi.org/10.1093/molbev/msv200

Turner, B.M., Birley, A.J., Lavender, J., 1992. Histone H4 isoforms acetylated at specific lysine residues define individual chromosomes and chromatin domains in Drosophila polytene nuclei. Cell 69, 375-384. https://doi.org/10.1016/0092-8674(92)90417-b

Uchifune, T., Machida, R., 2005. Embryonic development of Galloisiana yuasai Asahina, with special reference to external morphology (insecta: Grylloblattodea). J. Morphol. 266, 182-207. https://doi.org/10.1002/jmor.10373

UniProt: a worldwide hub of protein knowledge, 2019. . Nucleic Acids Res 47, D506-D515. https://doi.org/10.1093/nar/gky1049 
Uyehara, C.M., Nystrom, S.L., Niederhuber, M.J., Leatham-Jensen, M., Ma, Y., Buttitta, L.A., McKay, D.J., 2017. Hormone-dependent control of developmental timing through regulation of chromatin accessibility. Genes Dev. 31, 862-875. https://doi.org/10.1101/gad.298182.117

van Berkum, N.L., Lieberman-Aiden, E., Williams, L., Imakaev, M., Gnirke, A., Mirny, L.A., Dekker, J., Lander, E.S., 2010. Hi-C: A Method to Study the Three-dimensional Architecture of Genomes. Journal of Visualized Experiments. https://doi.org/10.3791/1869

Viscuso, R., Sottile, L., 2008. Fine structure of pleuropodia in three species of Insecta Orthoptera during embryonic development. Italian Journal of Zoology 75, 11-19. https://doi.org/10.1080/11250000701690475

Wang, C.-W., Sun, Y.H., 2012. Segregation of eye and antenna fates maintained by mutual antagonism in Drosophila. Development 139, 3413-3421. https://doi.org/10.1242/dev.078857

Wang, Y.-H., Huang, M.-L., 2009. Reduction of Lobe leads to TORC1 hypoactivation that induces ectopic Jak/STAT signaling to impair Drosophila eye development. Mechanisms of Development 126, 781-790. https://doi.org/10.1016/j.mod.2009.08.005

Wang, Z., Gerstein, M., Snyder, M., 2009. RNA-Seq: a revolutionary tool for transcriptomics. Nat Rev Genet 10, 57-63. https://doi.org/10.1038/nrg2484

Warren, J.T., Petryk, A., Marques, G., Jarcho, M., Parvy, J.-P., Dauphin-Villemant, C., O'Connor, M.B., Gilbert, L.I., 2002. Molecular and biochemical characterization of two P450 enzymes in the ecdysteroidogenic pathway of Drosophila melanogaster. Proc. Natl. Acad. Sci. U.S.A. 99, 1104311048. https://doi.org/10.1073/pnas.162375799

Warren, J.T., Petryk, A., Marqués, G., Parvy, J.-P., Shinoda, T., Itoyama, K., Kobayashi, J., Jarcho, M., Li, Y., O'Connor, M.B., Dauphin-Villemant, C., Gilbert, L.I., 2004. Phantom encodes the 25hydroxylase of Drosophila melanogaster and Bombyx mori: a P450 enzyme critical in ecdysone biosynthesis. Insect Biochem. Mol. Biol. 34, 991-1010. https://doi.org/10.1016/j.ibmb.2004.06.009

Waterhouse, R.M., Seppey, M., Simão, F.A., Manni, M., Ioannidis, P., Klioutchnikov, G., Kriventseva, E.V., Zdobnov, E.M., 2017. BUSCO applications from quality assessments to gene prediction and phylogenomics. Mol. Biol. Evol. https://doi.org/10.1093/molbev/msx319

Weber, U., Paricio, N., Mlodzik, M., 2000. Jun mediates Frizzled-induced R3/R4 cell fate distinction and planar polarity determination in the Drosophila eye. Development 127, 3619-3629.

Wei, Z., Yin, Y., Zhang, B., Wang, Z., Peng, G., Cao, Y., Xia, Y., 2007. Cloning of a novel protease required for the molting of Locusta migratoria manilensis. Development, Growth \& Differentiation 49, 611-621. https://doi.org/10.1111/j.1440-169X.2007.00957.x

Wheeler, W.M., 1890. On the appendages of the first abdominal segment of embryo insects. s.n.], [Madison.

Winick, J., Abel, T., Leonard, M.W., Michelson, A.M., Chardon-Loriaux, I., Holmgren, R.A., Maniatis, T., Engel, J.D., 1993. A GATA family transcription factor is expressed along the embryonic dorsoventral axis in Drosophila melanogaster. Development 119, 1055-1065.

Wittkopp, P.J., 2013. V.7. Evolution of Gene Expression, in: Losos, J.B., Baum, D.A., Futuyma, D.J., Hoekstra, H.E., Lenski, R.E., Moore, A.J., Peichel, C.L., Schluter, D., Whitlock, M.C. (Eds.), The Princeton Guide to Evolution. Princeton University Press, Princeton. https://doi.org/10.1515/9781400848065-058

Wittkopp, P.J., 2005. Genomic sources of regulatory variation in cis and in trans. Cellular and Molecular Life Sciences 62, 1779-1783. https://doi.org/10.1007/s00018-005-5064-9

Wittkopp, P.J., Haerum, B.K., Clark, A.G., 2008. Regulatory changes underlying expression differences within and between Drosophila species. Nat Genet 40, 346-350. https://doi.org/10.1038/ng.77

Wittkopp, P.J., Haerum, B.K., Clark, A.G., 2004. Evolutionary changes in cis and trans gene regulation. Nature 430, 85-88. https://doi.org/10.1038/nature02698

Wolpert, L., Tickle, C., 2011. Principles of development, 4th ed. / L. Wolpert, Cheryll Tickle. ed. Oxford : Oxford University Press.

Wray, G.A., 2007. The evolutionary significance of cis-regulatory mutations. Nature Reviews Genetics 8, 206-216. https://doi.org/10.1038/nrg2063 
Wray, G.A., 2003. The Evolution of Transcriptional Regulation in Eukaryotes. Molecular Biology and Evolution 20, 1377-1419. https://doi.org/10.1093/molbev/msg140

Xi, Y., Pan, P.-L., Ye, Y.-X., Yu, B., Xu, H.-J., Zhang, C.-X., 2015. Chitinase-like gene family in the brown planthopper, Nilaparvata lugens. Insect Mol. Biol. 24, 29-40. https://doi.org/10.1111/imb.12133

Xie, K.T., Wang, G., Thompson, A.C., Wucherpfennig, J.I., Reimchen, T.E., MacColl, A.D.C., Schluter, D., Bell, M.A., Vasquez, K.M., Kingsley, D.M., 2019. DNA fragility in the parallel evolution of pelvic reduction in stickleback fish. Science 363, 81-84. https://doi.org/10.1126/science.aan1425

Xiong, W.C., Montell, C., 1993. tramtrack is a transcriptional repressor required for cell fate determination in the Drosophila eye. Genes Dev. 7, 1085-1096. https://doi.org/10.1101/gad.7.6.1085

Yamamoto, D.S., Sumitani, M., Tojo, K., Lee, J.M., Hatakeyama, M., 2004. Cloning of a decapentaplegic orthologue from the sawfly, Athalia rosae (Hymenoptera), and its expression in the embryonic appendages. Dev. Genes Evol. 214, 128-133. https://doi.org/10.1007/s00427-004-0387-3

Yu, H., Luscombe, N.M., Qian, J., Gerstein, M., 2003. Genomic analysis of gene expression relationships in transcriptional regulatory networks. Trends in Genetics 19, 422-427. https://doi.org/10.1016/S0168-9525(03)00175-6

Yu, J., Pacifico, S., Liu, G., Finley, R.L., 2008. DrolD: the Drosophila Interactions Database, a comprehensive resource for annotated gene and protein interactions. BMC Genomics 9, 461. https://doi.org/10.1186/1471-2164-9-461

Zerbino, D.R., Birney, E., 2008. Velvet: algorithms for de novo short read assembly using de Bruijn graphs. Genome Res. 18, 821-829. https://doi.org/10.1101/gr.074492.107

Zhang, H., Shinmyo, Y., Mito, T., Miyawaki, K., Sarashina, I., Ohuchi, H., Noji, S., 2005. Expression patterns of the homeotic genes Scr, Antp, Ubx, and abd-A during embryogenesis of the cricket Gryllus bimaculatus. Gene Expr. Patterns 5, 491-502. https://doi.org/10.1016/j.modgep.2004.12.006

Zhang, J., Lu, A., Kong, L., Zhang, Q., Ling, E., 2014. Functional analysis of insect molting fluid proteins on the protection and regulation of ecdysis. J. Biol. Chem. 289, 35891-35906. https://doi.org/10.1074/jbc.M114.599597

Zhang, X., Borevitz, J.O., 2009. Global Analysis of Allele-Specific Expression in Arabidopsis thaliana. Genetics 182, 943-954. https://doi.org/10.1534/genetics.109.103499

Zhang, Y., Liu, T., Meyer, C.A., Eeckhoute, J., Johnson, D.S., Bernstein, B.E., Nussbaum, C., Myers, R.M., Brown, M., Li, W., Liu, X.S., 2008. Model-based Analysis of ChIP-Seq (MACS). Genome Biol 9, R137. https://doi.org/10.1186/gb-2008-9-9-r137

Zheng, L., Carthew, R.W., 2008. Lola regulates cell fate by antagonizing Notch induction in the Drosophila eye. Mechanisms of Development 125, 18-29. https://doi.org/10.1016/j.mod.2007.10.007

Zheng, W., Zhao, H., Mancera, E., Steinmetz, L.M., Snyder, M., 2010. Genetic analysis of variation in transcription factor binding in yeast. Nature 464, 1187-1191. https://doi.org/10.1038/nature08934

Zhou, Y., Zhou, B., Pache, L., Chang, M., Khodabakhshi, A.H., Tanaseichuk, O., Benner, C., Chanda, S.K., 2019. Metascape provides a biologist-oriented resource for the analysis of systems-level datasets. Nature Communications 10, 1523. https://doi.org/10.1038/s41467-019-09234-6

Zhu, J., Sanborn, J.Z., Diekhans, M., Lowe, C.B., Pringle, T.H., Haussler, D., 2007. Comparative Genomics Search for Losses of Long-Established Genes on the Human Lineage. PLoS Computational Biology 3, 12.

Zhu, K.Y., Merzendorfer, H., Zhang, W., Zhang, J., Muthukrishnan, S., 2016. Biosynthesis, Turnover, and Functions of Chitin in Insects. Annu. Rev. Entomol. 61, 177-196. https://doi.org/10.1146/annurev-ento-010715-023933

Zhu, Q., Arakane, Y., Beeman, R.W., Kramer, K.J., Muthukrishnan, S., 2008. Functional specialization among insect chitinase family genes revealed by RNA interference. Proc. Natl. Acad. Sci. U.S.A. 105, 6650-6655. https://doi.org/10.1073/pnas.0800739105 